\title{
Laser and Electron Beam Technology for Parity Violating Electron Scattering Measurements
}

\author{
Caryn A. Palatchi \\ Hamilton, Ohio \\ B.S. in Physics, California Institute of Technology, 2009 \\ M.S. in Physics, Ohio State University, 2013 \\ A Dissertation Presented to the Graduate Faculty \\ of the University of Virginia in Candidacy for the Degree of \\ Doctor of Philosophy \\ Department of Physics \\ University of Virginia \\ May, 2019 \\ Dr. Kent D. Paschke (Advisor) \\ Dr. Gordon D. Cates (Committee Member) \\ Dr. Cass A. Sackett (Committee Member) \\ Dr. G. Wilson Miller (GSAS Representative)
}


(C)Copyright by Caryn A. Palatchi

Hamilton, Ohio 2019

All Rights Reserved 


\section{Foreword}

I like to think of this thesis as actually 3 theses in one. I've had a wonderful experience here at UVa and I wanted to cover the breadth and depth of the research I've been fortunate enough to have participated in. I performed research in 3 areas during my tenure at UVa: preparation for the upcoming PREXII experiment, source development for the future MOLLER experiment, and analysis of a recently published ${ }^{12} \mathrm{C}$ transverse asymmetry measurement at Mainz. The Mainz measurement is presented in the manner of a traditional experimental nuclear physics thesis: physics context, experimental setup, data analysis, followed by published results and conclusion. The source work is for MOLLER is presented in a manner akin to an atomic physics thesis: the development of a novel precision laser-optics tool, followed by a demonstration of using that new tool successfully in a precision measurement. In this case the new tool is an RTP crystal Pockels cell with an innovative design. It was ultimately installed at JLab, a national accelerator facility, and was demonstrated to successfully generate polarized electron beam with the desired precision properties to meet the stringent experimental requirements for MOLLER. In the source development section of this thesis, the new RTP Pockels cell design and operating principles are described, its behaviors on the laser table fully predicted analytically and characterized empirically, and finally it is demonstrated to successfully produce electron beam at JLab with unprecedented properties: producing smaller position differences than have ever been documented at JLab previously and achieving precision beam position difference control at the 1nm-level. In nuclear physics thesis fashion, the physics case for MOLLER is presented, as well as the case for PREXII, the experimental design for both experiments is described (in greater detail for PREXII because the experiment is happening imminently), and the results of source work development on electron beam is shown for MOLLER while the results of PREXI and the impact PREXII results will have in the greater context of both nuclear and astrophysics are described for PREXII. The conclusion summarizes the progress made in all three experimental areas in the broader physics context. There are also number of subsidiary Appendices, primarily just to serve as detailed technical documentation for future students who will continue this work.

\section{Abstract}

Parity violating electron scattering (PVES) is a precision tool used in a broad program of experiments which include studying the structure of protons and nuclei and searching for new Beyond the Standard Model (BSM) physics. In parity violation electron scattering experiments, a longitudinally polarized electron beam is incident on an unpolarized target. The sign of the longitudinal polarization is changed (making a parity transformation), and the fractional rate difference between right and left helicity states, $d \sigma_{R}$ and $d \sigma_{L}$, is measured. An interference between the electromagnetic and weak amplitudes, $\mathcal{M}_{\gamma}$ and $\mathcal{M}_{Z}$ respectively, gives rise to a parity violating asymmetry $A_{P V}=\frac{\sigma_{R}-\sigma_{L}}{\sigma_{R}+\sigma_{L}}$, defined by the fractional rate difference between right and left helicity states. $A_{P V}$ can be measured to extraordinarily high precision and is proportional to the ratio of the weak and electromagnetic amplitudes. 
This thesis highlights three such PVES experiments as well as a new polarized beam source which will improve their precision. The PVES experiments are PREX-II, MOLLER, and a ${ }^{12} C$ transverse asymmetry measurement at Mainz. Chapters 1-3 of this thesis cover introductions to MOLLER and PREX-II, Chapter 4 contains the results of the ${ }^{12} C$ transverse asymmetry measurement at Mainz, and Chapters 5-6 contain the original work performed by this author. PREX-II, the Lead Radius Experiment, measures the weak skin of the ${ }^{208} \mathrm{~Pb}$ nucleus, provides a clean measurement of $R_{N}$, the RMS radius of neutrons in a heavy nucleus, and constrains the equation of state (EOS) of highly dense matter which is important for describing neutron star structure, heavy ion collisions, and atomic parity violation experiments. The ${ }^{12} C$ transverse asymmetry measurement at Mainz, and the future Mainz program of measuring the ${ }^{208} \mathrm{~Pb}$ transverse asymmetry as well, addresses an important systematic correction for PREX and other PVES experiments preformed with heavy-nuclei. MOLLER (Measurement Of Lepton Lepton Electroweak Reaction) is an extremely precise PVES experiment searching for new neutral currents in electron-electron scattering. It's called MOLLER because it will measure $A_{P V}$ in the Moller ee scattering process, and will infer the weak charge of the electron $Q_{W}^{e}$ to extremely high precision. MOLLER is sensitive to new Beyond the Standard Model physics at MeV and multi-TeV scales and will serve as an indirect complementary measurement to direct searches at high energy colliders. To achieve high precision measurements on $A_{P V}$ for MOLLER, we have developed an innovative Rubidium Titanyl Phosphate (RTP) Pockels cell in the polarized source to satisfy both statistical and systematic requirements as regards the electron beam produced. This new ultra-fast RTP cell design uses electric field gradients to provide unprecedented control over helicity correlated beam asymmetries and has been demonstrated to be capable of producing precisely controlled polarized electron beam at Jefferson Laboratory, controlling beam steering down to the nm-level. The precision reached with the RTP cell offers sufficient control over and minimization of helicity correlated beam asymmetries to perform PREX II. The RTP Pockels cell system will provide fast flipping and suitable control helicity correlated beam asymmetries and parity quality beam for the future MOLLER experiment, providing an unprecedented precision on the electron weak 
charge and electroweak mixing angle. 


\section{Acknowledgments}

I want to tell you the story of how I came up with the design for the new RTP Pockels cell, what happened afterwards, and what I learned from this experience. There were imperfections in the crystal, large gradients in the refractive index that came from the way the RTP crystals are grown, and when the electric field was applied, it steered the laser beam passing through the perfectly cut, imperfectly grown crystal like it was passing through a big, old, fat wedge. There was no getting around it, the crystals were what they were: not uniform. I was stuck with the beam steering... unless... the electric fields weren't uniform either! We could induce a gradient in the electric fields to counteract the intrinsic gradient in the crystal! And you can control electric fields with voltage, so you can control the laser beam steering with voltage to a very precise degree! We can control beam steering which is a thing we never control over before in our system! Thus the 8 high voltage design for the RTP Pockels cell for MOLLER was born.

I should note that when I first described my "crazy" idea to Kent to use 8 high voltages to control the Pockels cell instead of just the standard 2 high voltages, he called me "mad-scientist" and then proceeded to listen carefully, seriously considered the idea, and then almost immediately let me start ordering the parts to

build it. I want to take this opportunity to thank Kent for listening to his student and for not dismissing an unusual idea, which would have been so easy to do. In the process of coming up with this idea, I learned an important heuristic which is now permanently in my physics toolbox: when you see something that is causing "a problem", when you find a parameter that affects your results in a negative way, the glass isn't necessarily half-empty, because if you can find a way to adjust that "problem-causing" parameter, then you can harness it to control your system. When you discover a "problem" parameter, you actually just discovered a new knob you can turn gain another degree of freedom of control. Thus the source of your "problem" can in fact become your solution. There are no "problematic" results in physics, only the truth of the matter, and the truth of the matter always gives you more control over your system. So, the glass really is half-full. To summarize this heuristic of experimental physics: If the universe gives you lemons, make lemonade. Really, make lemonade. 
I want to thank my family, my friends, and my teachers for helping me get here. First and foremost, I want to thank my Mom for always listening, even when I'm rambling. I want to thank my twin brother whose humor never fails (wonder twin powers activate!). I want to thank my 7th grade teacher, the first person to tell me that I might be a "physical scientist in the making", for opening my eyes to the possibilities of what I might become. Finally, I want to thank my advisor, Kent, one of the best friends I've ever had. When reading other students' theses, I often think that the acknowledgements section acts as a kind of recommendation letter for their advisor. So, here's my recommendation letter for Kent to be read by future students who may consider working for him or who may already be working for him.

Kent is very easy to understand, because he's an open book. If you do something he thinks is good, he'll tell you. If you do something he thinks is not-so-good, he'll tell you. This straightforward feedback makes life very simple for a graduate student (simple, but not necessarily easy). Be advised that no matter how many plots you make, Kent will invariably ask for the one plot you didn't make. Kent's questions are like a game of fetch: he throws out the question, you go fetch the answer. Repeat ad infinitum. Beware you may hear many stories concerning dogs, Kent's three sons, Kent's three sons' hobbies, Kent's two dogs' hobbies, the Penguins, as well as a series of anecdotes potentially silly or meaningful or both which may be repeated multiple times, as well as references to Harry Potter, Hitchhiker's Guide, and Monty Python. Do not be concerned, this behavior is normal. Regarding the laboratory, in contrast to many, many other professors who though experienced have become hazards to their own laboratory, Kent is miraculously not hazardous to his own laboratory. When, after a prolonged period, Kent elects to appear in the lab and touch something, it does not immediately break. Kent is good in the lab, though he can be overly modest about it. In navigating the strange uncharted waters that is the research process, when you are surrounded by nothing but unknowns, Kent won't steer you wrong. You can trust him to ask the questions that need to be asked and that's more than enough to get you through.

You can also count on Kent to make something which should be stressful into 
something fun. This is largely due to his sense of humor and hyper-sensitive funny bone. If you are working hard and are really tired and under a lot of pressure, Kent will reliably become a goof-ball if necessary to motivate you to continue. In fact, Kent will reliably become a goof-ball even when not necessary. You, the student, should be adaptable to this and develop the ability to laugh (loliger!). If you don't have the ability to laugh, you will never survive as Kent's student.

This is my advice for having Kent as an advisor: be self-motivated, be considerate of your fellow students, and be considerate of Kent. Sometimes he is a little too willing to take too much upon himself. He tends to believe there is no such thing as a bad student, and may try to take your shortcomings upon himself as his responsibility to correct. If you are a student reading this, I urge you recognize your own shortcomings and take them upon yourself as your own responsibility to correct. Kent will point the way towards how you should be and what you should do, but it's ultimately up to you to actually do what you need to do. He, as your advisor, will lead you to water, but you, the horse, are the one who must drink. Stay calm, listen to Kent and try your best. Good luck, future students! 


\section{Contents}

1 Introduction 1

1.1 Parity Violation in the Weak Interaction . . . . . . . . . . . . 1

1.1.1 Birth of Glashow-Weinberg-Salam(GWS) theory . . . . . 3

1.1.2 Tests of the Standard Model: Three Regimes . . . . . . . . . 4

1.2 Motivation: Parity Violating Electron Scattering . . . . . . . . . . 10

1.2.1 Parity Experiments as a Precision Tool . . . . . . . . . . 11

2 MØLLER Experiment 15

2.1 Introduction . . . . . . . . . . . . . . . . . 15

2.1.1 Møller scattering .................. 16

2.1.2 BSM and Sensitivity to New Physics . . . . . . . . . . . 29

2.2 Parity Violation Experiments . . . . . . . . . . . . . . 41

2.2.1 Basics of PVeS Experiments . . . . . . . . . . . . . 41

2.2.2 Experimental Measurements of $\sin ^{2} \theta_{W} \ldots \ldots . . . . . .44$

2.3 MOLLER Experimental Design . . . . . . . . . . . . . . . . 49

2.3.1 Basic Conceptual Setup . . . . . . . . . . . . . 49

2.3.2 Figure of Merit and High Acceptance . . . . . . . . . . . . 50

2.3.3 Magnetic Spectrometer . . . . . . . . . . . . . . . . . . 52

2.3 .4 Detectors ....................... 55

2.3.5 Polarimetry .................... 56

2.3 .6 Target . . . . . . . . . . . . . . . . 58

2.3.7 Polarized Source . . . . . . . . . . . . . . . . . . . 59

2.3.8 Summary of Goals for MOLLER . . . . . . . . . . . . . 64 
$\begin{array}{llr}3 & \text { PREX-II } & 66\end{array}$

3.1 Parity Violation . . . . . . . . . . . . . . . . . 66

3.2 Principles . . . . . . . . . . . . . . . . . . . . . 67

3.2.1 Fundamental Considerations . . . . . . . . . . . . . 67

3.2.2 Neutron Skin, $A_{p v}$, Symmetry Energy, and EOS . . . . . . . 72

3.3 Seminal Texts and Experiments . . . . . . . . . . . . . 75

3.3.1 Neutron Density Theory and Corrections to $A_{p v} \ldots \ldots 75$

3.3.2 Other Neutron Radius Measurements . . . . . . . . . . . . . 80

3.3.3 Neutron Stars . . . . . . . . . . . . . . . . . . . . 83

3.3.4 R-process nucleosynthesis . . . . . . . . . . . . . 85

3.3.5 Gravity Waves and EOS . . . . . . . . . . . . . 86

3.3.6 PREX I . . . . . . . . . . . . . . . . . 89

3.4 Experimental Introduction . . . . . . . . . . . . . . . . . . 90

3.5 Primary Components of the Experiment . . . . . . . . . . . . 91

3.5.1 Helicity Reversal . . . . . . . . . . . . . . . . . . . 91

3.5.2 $\mathrm{FOM} \ldots \ldots \ldots \ldots \ldots \ldots . \ldots \ldots$

3.5.3 Targets . . . . . . . . . . . . . . . . 96

3.5.4 Collimator . . . . . . . . . . . . . . . . . 101

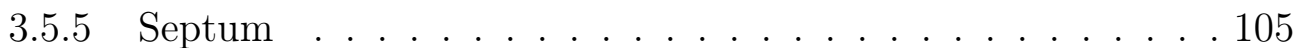

3.5.6 Spectrometers . . . . . . . . . . . . . . . . 108

3.5.7 Integrating Detectors . . . . . . . . . . . . . . . . 109

3.5.8 New Radiation Shielding . . . . . . . . . . . . . . . . . . 112

3.5.9 Compton Polarimeter . . . . . . . . . . . . . . . . 117

3.5.10 Møller Polarimeter . . . . . . . . . . . . . . . . 120

3.5.11 HCBA . . . . . . . . . . . . . . . . 125

3.6 PREX-II Summary of Goals . . . . . . . . . . . . . . . . . . 127

4 Mainz ${ }^{12} \mathrm{C}$ Transverse Asymmetry Analysis 129

4.1 Motivation . . . . . . . . . . . . . . . . . . . 129

4.2 Experimental Setup . . . . . . . . . . . . . . . . . . 131

4.3 Inversions . . . . . . . . . . . . . . . . . . . . . 132 
4.3 .1 Absolute Sign . . . . . . . . . . . . . . . . . . . . . 134

4.4 Detectors . . . . . . . . . . . . . . . . . . . 135

4.5 DAQ and Gate Length . . . . . . . . . . . . . . . . 137

4.6 Monitors . . . . . . . . . . . . . . . . . . . . . . . . . . 138

4.6.1 Current Monitor: PIMO . . . . . . . . . . . . . . . 138

4.6 .2 ENMO . . . . . . . . . . . . . . . . . . . . 139

$4.6 .3 \quad \mathrm{XYMO} \ldots \ldots \ldots \ldots \ldots \ldots$

4.6.4 Stabilization . . . . . . . . . . . . . . . 140

4.7 Calibrations and Optimizations . . . . . . . . . . . . 141

4.7 .1 Rotating $\lambda / 2$ plate . . . . . . . . . . . . . . 141

4.7 .2 Current calibration . . . . . . . . . . . . . . . . 142

4.7 .3 Energy calibration . . . . . . . . . . . . . . . . . 143

4.7.4 XYMO calibration ................. . . 146

4.7 .5 Polarization . . . . . . . . . . . . . . . . . . . . 148

4.8 Data Analysis . . . . . . . . . . . . . . . . . . . . 150

4.8.1 Beam Parameterization . . . . . . . . . . . . . . . . 150

4.8.2 False Asymmetries . . . . . . . . . . . . . . . . . . 154

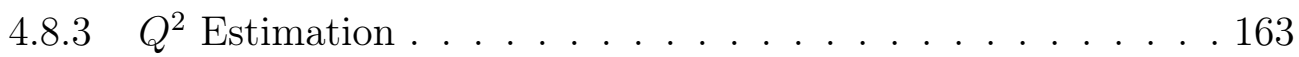

4.9 Non-linearities . . . . . . . . . . . . . . . . . . . . . . . 165

4.9 .1 Symptoms . . . . . . . . . . . . . . . 165

4.9.2 Recalibration Study . . . . . . . . . . . . . . . . . 168

4.9 .3 Unstabilized runs . . . . . . . . . . . . . . . . . . 170

4.9.4 A $A_{\text {false }}$ in Production Runs: from RMS method . . . . . . . . 180

4.9.5 $A_{\text {false }}$ in Production Runs: Summary of Error Estimates . . 184

4.9.6 Raw Data . . . . . . . . . . . . . . . . . . 185

4.10 Results $570 \mathrm{MeV}$ Data . . . . . . . . . . . . . . . . . 186

5 Beam Studies for PREXII and MOLLER 190

5.1 Parity Quality Beam . . . . . . . . . . . . . . 191

5.2 Beam Current Monitor . . . . . . . . . . . . . . . . . . . 192

5.2.1 BCM resolution . . . . . . . . . . . . . . . . . . 193 
5.2 .2 Current Dependence . . . . . . . . . . . . . . . . . 194

5.2.3 Small Angle Monitors . . . . . . . . . . . . . . . . . . . 195

5.2.4 Energy Dependence . . . . . . . . . . . . . . . 199

5.2.5 Frequency Analysis . . . . . . . . . . . . . . . . . 199

5.2.6 Frequency Dependence . . . . . . . . . . . . . . . . . 201

5.3 Digital Receivers . . . . . . . . . . . . . . . . . . 202

5.3 .1 Operating Principles . . . . . . . . . . . . . 202

5.3 .2 Cavity BPMs . . . . . . . . . . . . . . . . 204

5.3 .3 Digital Electronics . . . . . . . . . . . . . . . 205

5.3.4 Digital BCM receiver measurements . . . . . . . . . . . . 207

5.4 Beam Position Monitor . . . . . . . . . . . . . . . . . . . . . 212

5.4 .1 Introduction . . . . . . . . . . . . . . . . . . 212

5.4 .2 Calibration Derivation . . . . . . . . . . . 216

5.4 .3 Pedestal Error . . . . . . . . . . . . . . . . . . . . . . . . 219

5.4 .4 BPM widths . . . . . . . . . . . . . . . . 221

5.4.5 Helicity Patern . . . . . . . . . . . . . . . . . . 222

5.5 Measuring Spot Size Asymmetries with BPMs . . . . . . . . . . . 223

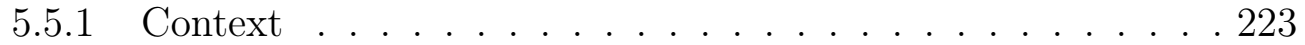

5.5.2 Measurement with BPM . . . . . . . . . . . . . . 224

5.5.3 Analytic Derivation of bpmelli . . . . . . . . . . . . 225

5.5.4 Numeric Derivation of bpmelli . . . . . . . . . . . . . . 226

5.5.5 Position Dependence of bpmelli . . . . . . . . . . . . . . 227

5.5.6 Pedestal Error in bpmelli . . . . . . . . . . . . . . . . . . . . 229

5.5.7 Position Difference Error in bpmelli . . . . . . . . . . . . 230

5.5.8 Spot-Size Scale Factor Error _ . . . . . . . . . . . . 231

5.5.9 Applying bpmelli in Data Analysis . . . . . . . . . . . . 231

5.6 Summary . . . . . . . . . . . . . . . . . . . . . . . . 244

6 RTP Pockels Cell: Parity Quality Beam for MOLLER 246

6.1 Introduction . . . . . . . . . . . . . . . . . 246

6.2 Parity Violation Experiments . . . . . . . . . . . . . . . . 249 
6.3 Helicity Correlated Beam Asymmetries . . . . . . . . . . . . . . . . 251

6.3 .1 Intensity Asymmetry . . . . . . . . . . . . . . . . 251

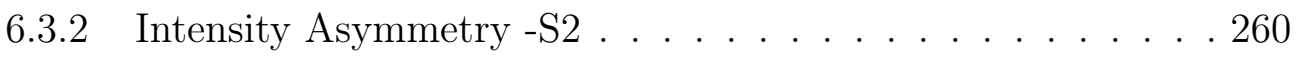

6.3.3 Position differences: Analyzing-like . . . . . . . . . . . . . . 264

6.3.4 Position differences: Steering . . . . . . . . . . . . . . . . . 272

6.3.5 RHWP scans . . . . . . . . . . . . . . . . . . 281

6.3.6 Analyzing/Angle-like Position Differences . . . . . . . . . . . 284

6.3.7 Spot size asymmetries . . . . . . . . . . . . . 287

6.3 .8 Interferometric-Etalon Effect . . . . . . . . . . . . . . . . 294

6.4 Design . . . . . . . . . . . . . . . . 296

6.4 .1 8-HV Design . . . . . . . . . . . . . . . . 296

6.4.2 Opto-Driver . . . . . . . . . . . . . . . . . . 302

6.4.3 Considerations for Fast Switching . . . . . . . . . . . . 305

6.4.4 PC-history effect/4-peak effect . . . . . . . . . . . . . 310

6.4.5 Lifetime - Mode of Failure . . . . . . . . . . . . . . . 313

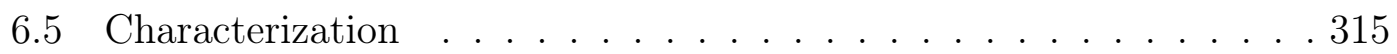

6.5.1 Alignment Procedure . . . . . . . . . . . . . 316

6.5.2 Translation scan . . . . . . . . . . . . . . . 317

6.5.3 Angle scans . . . . . . . . . . . . . . . . . . . . . . . . . . 319

6.5.4 Etalon scans . . . . . . . . . . . . . . . . . . . . . . . . 321

6.5.5 Steering control . . . . . . . . . . . . . . . 324

6.5.6 RHWP scans . . . . . . . . . . . . . . . . . . . 327

6.5.7 Spot size asymmetries - linear array measurements . . . . . 328

6.5.8 Temperature Sensitivity . . . . . . . . . . . . . . 332

6.6 Results: Electron beam measurements . . . . . . . . . . . . . . 335

6.6.1 Charge Asymmetries and Position Differences . . . . . . . . 335

6.6.2 Position Differences Transport: Adiabatic Damping \& Aper-

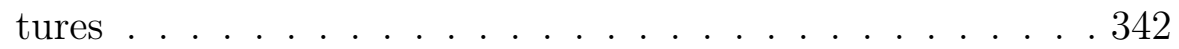

6.6.3 Spot Size asymmetries . . . . . . . . . . . . . . . . 347

6.6.4 Longitudinal Asymmetries . . . . . . . . . . . . . . . . 351 
6.7 Conclusion . . . . . . . . . . . . . . . . . . . . 361

7 Conclusion and Summary 362

A Derivation: Angle Dependence in RTP and KD*P 366

A.1 Angle Deflection Within Crystal: Inside vs Outside . . . . . . . . 366

A.2 Fast-axis direction angle dependence in $\mathrm{KD}^{*} \mathrm{P} \ldots$. . . . . . . . . . 368

A.3 Asymmetry from extraneous birefringent element . . . . . . . . . . 369

A.4 Refractive Index angle dependence in $\mathrm{KD}^{*} \mathrm{P}$. . . . . . . . . . . . 370

A.5 Asymmetry angle dependence in $\mathrm{KD}^{*} \mathrm{P}$. . . . . . . . . . . . . . . . 374

A.6 Fast-axis direction angle dependence in RTP . . . . . . . . . . . . . 375

A.7 Asymmetry angle dependence model in RTP . . . . . . . . . . . . . 376

A.8 Refractive Index angle dependence in RTP . . . . . . . . . . . . . 376

A.9 Asymmetry angle dependence in RTP . . . . . . . . . . . . . . . 378

B Tech Note: RTP Temperature Dependence and Aq Feedback 379

B.1 Thermal Compensation . . . . . . . . . . . . . . . . . . . . 379

B.2 Temperature Differences . . . . . . . . . . . . . . . . 380

B.3 Temperature Gradient . . . . . . . . . . . . . . . . . 383

B.3.1 Gradient Calculation . . . . . . . . . . . . . . . 384

B.3.2 Observed Temperature Gradient Fluctuations . . . . . . . . 387

B.4 Laser Heating . . . . . . . . . . . . . . . . . . . . . . . . . 388

B.5 Suppressing Effect of Temperature Drifts on e-beam . . . . . . . . . 393

B.5.1 Feedback . . . . . . . . . . . . . . . . 393

B.5.2 Depolarization . . . . . . . . . . . . . . 401

B.5.3 Pickoff Design . . . . . . . . . . . . . . . . . . 404

B.5.4 Temperature Feedback . . . . . . . . . . . . . . 405

C Tech Note: Interferometric/Etalon Effect 407

C.1 Temporal overlap . . . . . . . . . . . . . . . . . . 407

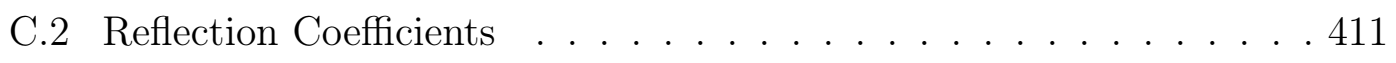

C.3 Non-Parallel Face Cuts . . . . . . . . . . . . . . . . . . . . . . . . . 411 
C.4 Laser Bandwidth . . . . . . . . . . . . . . . . . . . . 415

D Tech Note: KD*P Steering and General Pockels Cell Matters 417

D.1 KD*P . . . . . . . . . . . . . . . . . . . . . . . . . . . . .

D.1.1 Steering in $\mathrm{KD}^{*} \mathrm{P} \ldots \ldots \ldots$. . . . . . . . . . 417

D.1.2 The Etalon effect in $\mathrm{KD}^{*} \mathrm{P}$. . . . . . . . . . . . . . . 425

D.1.3 Intensity Asymmetry Angle Dependence in KD*P . . . . . 426

D.2 General Pockels Cell Considerations . . . . . . . . . . . . . . . . . 426

D.2.1 Formulation . . . . . . . . . . . . . . . 426

D.2.2 Angle-like Steering in KD*P . . . . . . . . . . . . . . . . 429

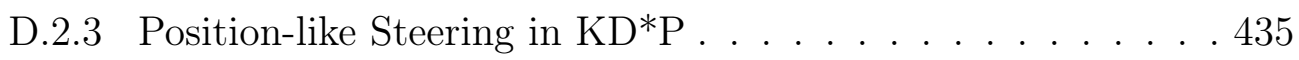




\section{List of Figures}

1-1 Cobalt weak beta decay _. . . . . . . . . . . . . . 2

1-2 Gargamelle Bubble chamber Experiment . . . . . . . . . . . . 6

$1-3 \quad$ E122 result . . . . . . . . . . . . . . . . 8

1-4 Parity violation experiment general description . . . . . . . . . . . . 10

1-5 Interference between weak and EM amplitudes, the weak interaction carrying signature of parity violation . . . . . . . . . . . . 10

1-6 Parity Experiment Blue Print . . . . . . . . . . . . . . . . 11

1-7 Precision of various parity experiments . . . . . . . . . . . . 12

1-8 JLab $12 \mathrm{GeV}$ upgrade . . . . . . . . . . . . . . . . 14

2-1 Møller scattering Tree level diagrams . . . . . . . . . . . . . . . 17

2-2 Significant 1-loop radiative corrections . . . . . . . . . . . 25

2-3 Box diagram contributions to Møller scattering involving two heavy

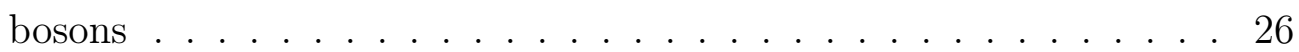

2-4 Running of $\sin ^{2} \theta_{W} \ldots \ldots \ldots \ldots$. . . . . . . . . . . . 27

2-5 Running of $\sin ^{2} \theta_{W}$ : Møller precision goal is shown compared with other past experiments . . . . . . . . . . . . . . . . . 28

$2-6 \sin ^{2} \theta_{W}$ vs $m_{H} \ldots \ldots \ldots \ldots$

2-7 The four best $\sin ^{2} \theta_{W}$ measurements and the projected error of the MOLLER proposal . . . . . . . . . . . . . . . . 32

2-8 Fermion contact interaction with unknown weak mediator X . . . . 34

2-9 Future constraints on chiral Z' couplings in two representative models for a $1.5 \mathrm{TeV}$ mass . . . . . . . . . . . . . . . . 37 
2-10 Exchange of a doubly-charges Higgs boson $\Delta^{++} \ldots$. . . . . . . . . 39

2-11 Running of $\sin ^{2} \theta_{W}$ with various dark Z mediator masses . . . . . . 40

2-12 $A_{P V}$ in Deep Inelastic Scattering from liquid Deuterium $Q^{2} \approx$ $1 G e V^{2} / c^{2} \ldots \ldots \ldots \ldots \ldots \ldots \ldots \ldots$

2-13 Experimental Layout of E122 (1978) . . . . . . . . . . . . . . . 41

2-14 Band structure of GaAs . . . . . . . . . . . . . . . . . . . 42

2-15 High luminosity polarized electron source . . . . . . . . . . . . . . . 42

2-16 Helicity States driven through the Pockels Cell voltage setting . . . 42

2-17 E122 Calorimeter . . . . . . . . . . . . . . . . . 44

2-18 Experimental layout of E158 . . . . . . . . . . . . . . . . 44

2-19 Qweak experiment results . . . . . . . . . . . . . . . 46

2-20 Experimental Design for Moller . . . . . . . . . . . . . . . 50

2-21 Moller $A_{P V}$ vs. $\theta_{C M} \ldots \ldots \ldots \ldots$. . . . . . . . . . . 51

2-22 Forward and Backscattered electrons are identical particles . . . . . 52

2-23 Toroid Spectrometer . . . . . . . . . . . . . . 53

2-24 Kinematic Focusing . . . . . . . . . . . . . . . . . . 53

2-25 Møller electron distributions in detector plane . . . . . . . . . . . 54

2-26 Proposed Møller Detector Segmentation . . . . . . . . . . . . 55

2-27 Quartz assembly of Cherenkov detectors . . . . . . . . . . . 56

2-28 Conceptual illustration of detector arrangements for the MOLLER

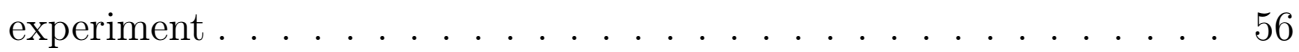

2-29 Compton uncertainties at $11 \mathrm{GeV}$. . . . . . . . . . . . . 57

2-30 Target Boiling noise vs. Helicity flip rate . . . . . . . . . . 58

2-31 Precision Goals for the MOLLER experiment . . . . . . . . . 65

3-1 Cross sections at $\mathrm{E}=502 \mathrm{MeV}$ as a function of effective momentum

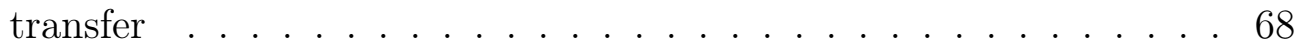

3-2 Electron scattering off ${ }^{208} \mathrm{~Pb}$ target nucleus with Z-boson and photon mediators . . . . . . . . . . . . . . . . . . . 69

3-3 Neutron and proton distributions in ${ }^{208} \mathrm{~Pb}$. . . . . . . . . . . . 71 
3-4 Parity-violating asymmetry for ${ }^{208} \mathrm{~Pb}$ at the kinematics of PREX against the neutron skin of ${ }^{208} \mathrm{~Pb} \ldots \ldots . \ldots . \ldots 73$

3-5 Models predicting the symmetry energy E/N vs. the neutron density 74

3-6 Neutron skin of ${ }^{208} \mathrm{~Pb}$ against slope of the symmetry energy L . . . 75

3-7 Neutron skin thickness for various nuclei of mass number A . . . . . 76

3-8 Flow chart of the physics data analysis of PREX . . . . . . . . . . . 80

3-9 Experimental constraints for symmetry energy parameters . . . . . 83

3-10 Equation of state pressure and energy density and mass vs. radius

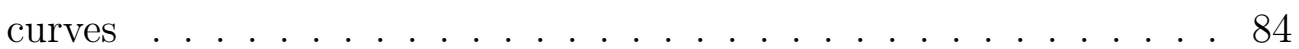

3-11 Atomic Abundances . . . . . . . . . . . . . . . . . . . 85

3-12 Abundance visualizer . . . . . . . . . . . . . . . . . 86

3-13 Quadrupole Moment . . . . . . . . . . . . . . . . . . 87

3-14 Neutron star inspiral . . . . . . . . . . . . . . . . 87

3-15 Tidal deformation of GW waveform . . . . . . . . . . 88

3-16 Tidal deformity - Mass NS curve . . . . . . . . . . . . . . 88

3-17 PREX-I result . . . . . . . . . . . . . . . . . . . . 89

3-18 Schematic of the injector setup at JLab . . . . . . . . . . . . . . 92

3-19 Double Wien . . . . . . . . . . . . . . . . . . . 93

3-20 Cross section, parity violating asymmetry, and sensitivity to Rn for ${ }^{208} \mathrm{~Pb}$ elastic scattering at $0.85 \mathrm{GeV}$. . . . . . . . . . . 95

3-21 Error in the neutron radius in $\mathrm{Pb}$ versus beam energy . . . . . . . . 96

3-22 PREX target: Diamond sandwich . . . . . . . . . . . . . . 96

3-23 PREX-I target degradation . . . . . . . . . . . . . . . . . 99

3-24 PREX target chamber . . . . . . . . . . . . . . 100

3-25 Targets required for PREX and CREX . . . . . . . . . . 100

3-26 PREX-II pivot region . . . . . . . . . . . . . . . . . . . 102

3-27 PREX-II collimator . . . . . . . . . . . . . . . 103

3-28 Collimator deinstallation concept [179] . . . . . . . . . . . . . . 103

3-29 Power deposited in beamline collimator . . . . . . . . . . . . . . . 103

3-30 Collimator Design . . . . . . . . . . . . . . . . . . 104 
3-31 Old vs. new collimator concepts . . . . . . . . . . . . . . . 104

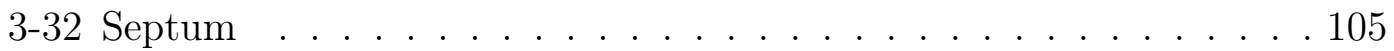

3-33 Scattering region . . . . . . . . . . . . . . 106

3-34 Tosca Model compared with measurements . . . . . . . . . . . 107

3-35 Septum Beam Pipe . . . . . . . . . . . . . . . . . . . . 108

3-36 Components of PREX experimental beamline . . . . . . . . . . 108

3-37 Sources of backgrounds during PREX, and the associated correction and systematic uncertainties in the parity-violating asymmetry . . . 109

3-38 PREX-II design for Integrating Quartz Detectors . . . . . . . . . 109

3-39 Flux integration technique . . . . . . . . . . . . . . . 110

3-40 Mainz beam tests on PREX-II detectors . . . . . . . . . . . . 111

3-41 Origin of photons hitting a plane detector downstream of the septum112

3-42 Neutron equivalent dose . . . . . . . . . . . . . . . . 113

3-43 Pivot Region Design Concept . . . . . . . . . . . . . . . . . . . 114

3-44 Sky-shine shielding blocks . . . . . . . . . . . . . . . . 115

3-45 Beam Dump and Shielding . . . . . . . . . . . . . . . 116

3-46 Drawing of the Compton Polarimeter . . . . . . . . . . . . . 118

3-47 Drawing of the Compton Photon Detector . . . . . . . . . . . . . 119

3-48 Hall A Møller polarimeter . . . . . . . . . . . . . . . . . . . . . 121

3-49 New Møller Target . . . . . . . . . . . . . . . . . . . . 122

3-50 Magnetization of pure iron as a function of applied magnetic field . 123

3-51 Hall A beam polarization 2015-2016 as measured by Møller polarimeter . . . . . . . . . . . . . . . . . . . 124

3-52 Injector position differences during PREX-I . . . . . . . . . . 126

3-53 Qweak: Horizontal position differences on successive injector BPMs 127

3-54 PREX error tables . . . . . . . . . . . . . . . . . . . 127

4-1 Extracted physics asymmetries $A_{n}$ vs. Q . . . . . . . . 130

4-2 Mainz A1 Collaboration Hall Spectrometer . . . . . . . . . . . . . 131

4-3 Obtaining vertical polarization . . . . . . . . . . . . . 133

4-4 Necessary vectors to define the absolute sign . . . . . . . . . . . . . 134 
4-5 Detectors SpekA (left) and SpekB (right) . . . . . . . . . . . 135

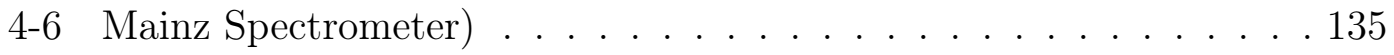

4-7 The excitation energy spectrum . . . . . . . . . . . . 136

4-8 Runs with beam stabilization off . . . . . . . . . . . . . . . 141

4-9 ENMO run 04-10-17-44-42 . . . . . . . . . . . . . . . . . . 145

4-10 ENMO run 04-10-17-44-42 . . . . . . . . . . . . . . . . 145

4-11 Raw detector B1 value vs. raw XYMO value . . . . . . . . . . . 146

4-12 Distances between the beam line components necessary to to perform XYMO calibrations . . . . . . . . . . . . . . . . 147

4-13 Projection of the vertical polarization on the normal of the scattering plane for one spectrometer setup . . . . . . . . . . . . . . 149

4-14 Mott measurements . . . . . . . . . . . . . . . . . . . . 150

4-15 Parameters used in describing measurement conditions . . . . . . 150

4-16 Non linearity of the current Calibration of spB0 from 0 to $20 \mu \mathrm{A}$. . 156

4-18 Carbon Form Factor and cross section . . . . . . . . . . . . . 160

4-19 Fit to Carbon form factor . . . . . . . . . . . . . . . . . . . 161

4-20 $Q^{2}$ distributions found from the counting-mode data for all of the setups in the experiment . . . . . . . . . . . . . . 164

4-21 2015-1 data showing PMT dying . . . . . . . . . . . . . . . . 166

4-22 Comparison between the charge asymmetry observed an unstabilized run with beam stabilization off/on . . . . . . . . . . . 167

4-23 Normalized detector asymmetries for each PMT . . . . . . . . . . . 168

4-24 Recalibration illustration . . . . . . . . . . . . . . . . . . 169

4-25 List of unstabilized runs . . . . . . . . . . . . . . . . . . 171

4-26 Example of correlations and cross-correlations in an unstabilized run 172

4-27 Comparison of observed systematic error contribution to calculated systematics found from noise correlations . . . . . . . . . . 178

$4-28$ RMS method . . . . . . . . . . . . . . . . . 181

4-29 Raw summed detector asymmetries for each spectrometer configuration. 
4-30 Extracted transverse asymmetries $A_{n}$ for the detectors . . . . . 188

5-1 Linear regression method for evaluating and correcting false asymmetries from HCBA . . . . . . . . . . . . . . . . . . . 191

5-2 The schematic diagram of the BCM system . . . . . . . . . . . . . 192

5-3 Correlations between beam asymmetry as measured by upstream and downstream $\mathrm{BCMs}$ for the analog $1 \mathrm{MHz}$ receivers and the digital receivers . . . . . . . . . . . . . . . . . . . . . 194

$5-4$ BCM resolution vs. current . . . . . . . . . . . . . . 195

5-5 Small Angle Monitors . . . . . . . . . . . . . . . . 196

5-9 Measurements of the beam asymmetry RMS width . . . . . . . . . 199

5-10 Sub-block analysis . . . . . . . . . . . . . . . . . 201

5-11 Functional block diagram of a basic superhet receiver . . . . . . . 202

5-12 Time domain description of harmonic sampling . . . . . . . . . . 204

5-13 Frequency domain description of harmonic sampling . . . . . . . . . 204

5-14 Cavity BPM . . . . . . . . . . . . . . . . . 205

5-15 Functional block diagram of a digital receiver system and BPM electronics . . . . . . . . . . . . . . . . . 206

$5-16$ Digital Latency . . . . . . . . . . . . . . . . . . . . . 208

5-17 Third-order intercept diagram . . . . . . . . . . . . . . . . 209

5-18 Digital Receiver Attenuation . . . . . . . . . . . . . . . . . 209

5-19 Digital BCM linearity test . . . . . . . . . . . . . . . . 210

5-20 Chain from BCM signal to receiver to ADC . . . . . . . . . 211

5-21 BPM cavity receiver configuration in 2016 . . . . . . . . . . . . 212

5-22 Schematic Drawing of M20 and M15 bpms . . . . . . . . . . . . . . 212

5-23 BPM readout electronics . . . . . . . . . . . . . . . . . . 213

5-24 Spot Size BPM current Derivatives . . . . . . . . . . . . . 217

$5-25 \mathrm{X}+$ wire response vs. beam position in $\mathrm{x}-\mathrm{y}$ plane $\ldots . . . .217$

5-26 Wire Channel Calculation X . . . . . . . . . . . . . . . 218

5-27 Stripline BPMs [106] . . . . . . . . . . . . . . . . . . . 219

5-28 Pedestal Error Calculation . . . . . . . . . . . . . . . . 220 
5-29 Injector BPM position difference widths at 30Hz . . . . . . . . . . . 221

5-30 HallA BPM position differences and position difference widths at

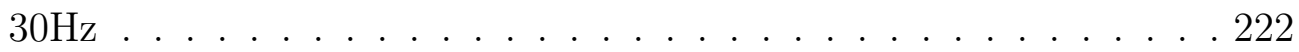

5-31 960Hz: large bpm widths observed in the injector due to a beat frequency with the $60 \mathrm{~Hz}$ line . . . . . . . . . . . . . . . 222

5-32 Wire Channel Calculation - Spot Size Asymmetry . . . . . . . . . . 227

5-33 Wire Channel Calculation for bpmelli . . . . . . . . . . . . . . 228

5-34 Wire Channel Calculation for Aelli . . . . . . . . . . . . . . . . 228

5-35 ELEGANT spot-size in gun region . . . . . . . . . . . . . 232

5-36 ELEGANT spot-sizes . . . . . . . . . . . . . . . . 233

5-37 Run3339 AlphaposU table . . . . . . . . . . . . . . . . . . . 234

5-38 Run3339 AlphaposV table . . . . . . . . . . . . . . . . . . . 234

5-39 Run3339 AlphaposU scan . . . . . . . . . . . . . . . . 235

5-40 Run3339 AlphaposVscan . . . . . . . . . . . . . . . . . 236

5-41 Couplings Angles 3339 . . . . . . . . . . . . . . . . . 237

5-42 RTP cell Run3331 with bpmelli . . . . . . . . . . . . . . . . 239

5-43 KDP Run3445 best-case with bpmelli . . . . . . . . . . . . . . . . 240

5-44 beam XY positions along beamline . . . . . . . . . . . . . . 241

5-45 Aelli scaled by scale factor error $\left(\sigma^{A} / \sigma^{e s t}\right)^{2}$ estimated from position difference sensitivity to AlphaposU/V scans . . . . . . . . . . . . . 241

6-1 Origin of analyzing power dependent beam asymmetries . . . . . . 251

6-2 RTP thermal compensation design . . . . . . . . . . . . 253

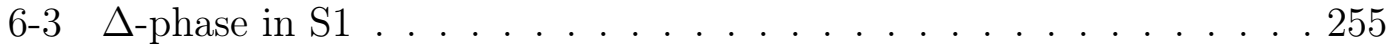

6-4 Laser table in the injector at JLab . . . . . . . . . . . . . . . . 256

6-5 Polarization ellipses for asymmetric S2 components on mostly circular polarization states. . . . . . . . . . . . . . . . 261

6-6 Relative Roll and $\mathrm{Aq}$ in $\mathrm{S} 2$. . . . . . . . . . . . . . . . . . . 264

6-7 4 crystal RTP cell . . . . . . . . . . . . . . . . . 270

6-8 Conceptual Diagram of E-field gradient control in Grounded SidePanel Design . . . . . . . . . . . . . . . . . . 271 
6-9 Birefringence Ray Separation _. . . . . . . . . . . . . . . . 273

6-10 4 Ray Separation: Steering . . . . . . . . . . . . . . . . . 273

6-11 RTP E-field Simulations . . . . . . . . . . . . . . . . . 280

6-12 Simulation - Steering Gradient . . . . . . . . . . . . . . . 281

6-13 RHWP scan example . . . . . . . . . . . . . . . . . . . . 283

6-14 RHWP scans RTP . . . . . . . . . . . . . . . . . . . . . 284

6-15 RHWP angle and analyzing/angle-like position differences . . . . . 286

6-16 RTP RHWP scans e-beam . . . . . . . . . . . . . . . . . 287

6-17 Reflection interference in a quarter-wave anti-reflection coating [252] 295

6-19 Electric field gradients . . . . . . . . . . . . . . . 298

6-20 Diagram of the 8HV driver configuration with optodriver . . . . . . 301

6-21 RTP cell design . . . . . . . . . . . . . . . . . . . . . 302

6-22 Assembled RTP mount . . . . . . . . . . . . . . . . . . . . 302

6-23 LED pulse . . . . . . . . . . . . . . . . . . . . . . . . . . . . . . . . . . . .

6-24 Annotated Pockels Cell Opto-driver Circuit Diagram [284] . . . . . 304

6-25 Transition Time . . . . . . . . . . . . . . . . . . 305

6-26 13us transition time with $<1 \%$ ringing . . . . . . . . . . 306

6-27 RTP Impulse response measurement . . . . . . . . . . . . . . . 308

6-28 Solid state switch with $50 \mathrm{kOhm}$ resistors on the HV lines to soften the transition down to $4 \mu \mathrm{s} \ldots \ldots$. . . . . . . . . . . . . . . . . . . . .

6-29 Solid state switch with no resistors . . . . . . . . . . . . . . 309

6-30 KD*P PC-history effect . . . . . . . . . . . . . . . . . . . . . 312

6-31 RTP PC-history effect . . . . . . . . . . . . . . . . . 313

6-32 RTP 4-peak effect . . . . . . . . . . . . . . . . . . 313

6-33 Tune Mode Generators Degradation . . . . . . . . . . . . . . . . . 314

6-34 Quad-photodiode layout . . . . . . . . . . . . . . 315

6-35 Translation scan . . . . . . . . . . . . . . . . . . . 318

6-36 RTP pitch-yaw scan . . . . . . . . . . . . . . 320

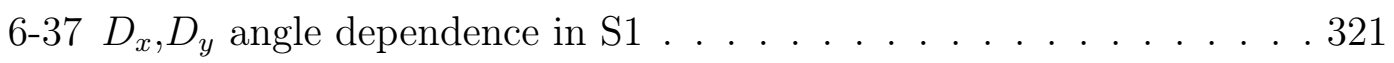

6-38 Etalon scans . . . . . . . . . . . . . . . . . . . . . . . 323 
6-39 Alpha-phase-U Voltage Scan . . . . . . . . . . . . . . . . . . 325

6-40 Alpha-Position Voltage Scans, Copper strips . . . . . . . . . . . . 326

6-41 RHWP scans with QPD . . . . . . . . . . . . . . 327

6-42 Linear-array photodiode (LAPD) detector [260] . . . . . . . . . . . 328

6-43 Spot-size asymmetry laser measurements . . . . . . . . . . . . . . . 329

6-44 e-beam spot-size asymmetry estimates . . . . . . . . . . . . 330

6-45 RHWP scans with linear array vertical . . . . . . . . . . . . 331

6-46 RHWP scans with linear array horizontal . . . . . . . . . . . . 332

6-47 Aq stability . . . . . . . . . . . . . . . . . . . . . 332

6-48 Temperature difference between crystals . . . . . . . . . . . . . . . 334

6-49 JLab table layout $[270] \ldots 335$

6-50 QE scan showing $\sim 0.69 \%[269] \ldots 337$

6-51 Using PC voltages, obtained 1-5nm convergence within 30min [257] 338

$6-52<30 \mathrm{~nm}$ Position Differences in 130keV region . . . . . . . . . . . . 339

6-53 Qweak horizontal position differences . . . . . . . . . . . . 339

6-54 Aq feedback . . . . . . . . . . . . . . . . . . . . . 340

6-55 Appearance of large position differences . . . . . . . . . . . . . . . 343

6-56 Aperture loss calculation . . . . . . . . . . . . . . . . . 344

6-57 Adiabatic Damping during Happex II Experiment - 100X . . . . . . 345

6-58 Adiabatic Damping - Good optical transport VS poor optical transport $[303] \ldots \ldots \ldots \ldots \ldots$

6-59 Emittance growth at $11 \mathrm{GeV}$ : factor of 5-10X [62] . . . . . . . . 348

6-60 RTP e-beam Aelli . . . . . . . . . . . . . . . . . . 350

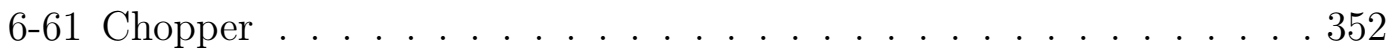

6-62 Qweak chopper scan . . . . . . . . . . . . . . . . 352

6-63 KD*P Chopper Scan 2018 . . . . . . . . . . . . . . . . . . . 354

6-64 Computations for KD*P Chopper Scan 2018 . . . . . . . . . . . . . 354

6-65 Chopper Scan: S2, bpm0I05, KD*P, Run4202, [257] . . . . . . . 356

6-66 Chopper Scan Computations: S2, bpm0I05, KD*P, Run4202, [257] . 357

6-67 KD*P Chopper scan with slit closed [62] . . . . . . . . . . . 358 
6-68 KD*P Chopper scan with slit open [62] . . . . . . . . . . . . . 358

A-1 Angular deviations and index ellipsoid . . . . . . . . . . . 366

A-2 Light refracting upon entering a birefringent material such as calcite 370

A-3 Defining Geometry . . . . . . . . . . . . . . . . 371

B-1 Thermal Compensation Design. RTP crystals in typical mount. Photograph by Raicol. . . . . . . . . . . . . . . . . . . . . . 380

B-2 Run4883, RTP cell, analyzed in S1 [321] . . . . . . . . . . . . . 381

B-3 dAq/dT calculation compared to measurement . . . . . . . . . . . 383

B-4 Thermal Gradient Calculation . . . . . . . . . . . . . . . . . . 385

B-5 Thermal induced Aq gradient for a 100\% analyzer along S1 . . . . . 385

B-6 Thermal induced Aq 1st and 2nd moments and the corresponding Dx and spot-size asymmetry for a $0.88 \mathrm{~mm}$ beam and $100 \%$ analyzer

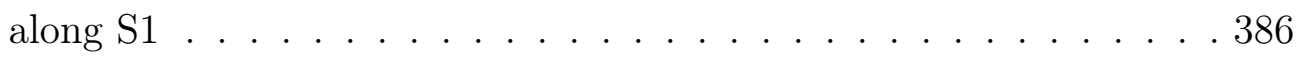

B-7 Dx,Dy measured with the qpd in $\mathrm{S} 1 \ldots$. . . . . . . . . . . 388

B-8 Laser induced thermal gradient for RTP . . . . . . . . . . . . . . 390

B-9 Laser induced difference in thermal gradient between the 2 RTP crystals with absorption coefficient . . . . . . . . . . . 391

B-10 Laser heating induced Aq for a 100\% analyzer along S1 . . . . . . . 391

B-11 Laser heating induced Aq 1st and 2nd moments and the corresponding Dx and spot-size asymmetry for a $0.88 \mathrm{~mm}$ beam and $100 \%$

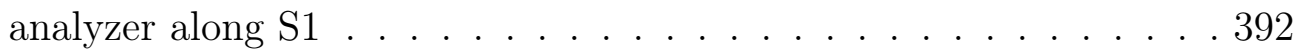

B-12 Active charge feedback . . . . . . . . . . . . . . . . 396

B-13 Modeling charge feedback on RTP cell . . . . . . . . . . . . . . . . 398

B-14 Modeling different settings for charge feedback on RTP cell . . . . . 400

B-15 Modeling charge feedback on RTP cell for $0.525 \%$ analyzer, $0.5 \mathrm{~s}$

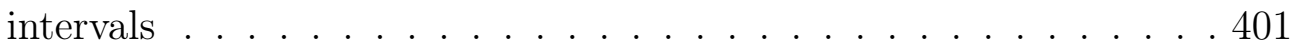

C-1 Etalon suppression factor: For a pure gaussian temporal distribution, for $L=10,12,14 \mathrm{~mm}$ crystals, for both RTP primary axes $n_{z}=1.867, n_{y}=1.783 \ldots \ldots \ldots \ldots$. . . . . . . . . . . . . . . 
C-2 Opposing face cuts: Face cuts $\theta_{f c 1}=\theta_{f c 2} \approx 0.5 \mathrm{mrad}$ within a desired tolerance of $\left|\theta_{f c 1}-\theta_{f c 2}\right|<0.05 \mathrm{mrad}$ and tolerance of length difference $\left|L_{1}-L_{2}\right|<2 \mu m$ (typical) . . . . . . . . . . . . . . . 444

D-1 Steering observed with different input polarization states, no analyzer, in translation scan for 'Princeton' Cell (commercial Fastpulse 10mm $1040 \mathrm{KD}^{*} \mathrm{P}$ [313]) [314]. Note that the sign of Dx,y on the qpd is arbitrary compared with the sign of the translation stage

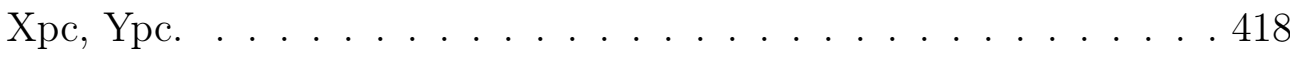

D-2 Intensity asymmetry in Translation scan [315], analyzing in S1, for ‘Princeton' Cell (commercial Fastpulse 10mm 1040 KD*P [313]) . . 419

D-3 Transmission in Translation scan [315], analyzing in S1, for 'Princeton' Cell (commercial Fastpulse 10mm 1040 KD*P [313]) . . . . . . 419

D-4 Etalon effect in KDP. These measurements were performed by setting input polarization along the fast axis or the slow axis $(+-45 \mathrm{deg})$ with a HWP, scanning the applied voltage, and examining the transmission without an analyzer . . . . . . . . . . . . . 4426

D-5 Charge asymmetry gradient for active $\mathrm{KD}^{*} \mathrm{P}$ with a vertical analyzer after cell("Gandalf",Run2815, elog 656) . . . . . . . . . . . . . 429

D-6 Ray Separation. Horizontally polarized beam splits in the crystal along the primary axes, angles not to scale. . . . . . . . . . . 430

D-7 Coordinates. Primary crystal axes are along the diagonal with respect to horizontal and vertical polarizations. . . . . . . . . . 430

D-8 4 Ray Separation. Horizontally polarized beam splits in the crystal along the primary axes, and voltage application split the beam further, angles not to scale.

D-9 RHWP scan. Isolating how angle-like steering in S1 and S2 contribute to position differences in RHWP scans.

D-10 4 Gaussians. Horizontally polarized beam splits in the crystal along the primary axes, and voltage application split the beam further, making 4 gaussian beam, 2 for each helicity state. 
D-11 Yaw and Steering. Even with no analyzer in, the PC angle can cause further steering in the beam (Run2861) . . . . . . . . . . 434

D-12 Wavefront. Propagation of wavefront of a gaussian beam. . . . . . 436

D-13 Radius-Angle Coupling. Wavefront determines Radius of Curvature which couples beam radius and direction. . . . . . . . . . . 437

D-14 Beam Profile. A 50cm lens is placed upstream of the Pockels cell. Due to laser astigmatism, the $\mathrm{x} / \mathrm{y}$ foci occur at different locations and the average divergence is not small. . . . . . . . . . . . 445

D-15 QE scans of photocathode showing a QE hole where the laser had

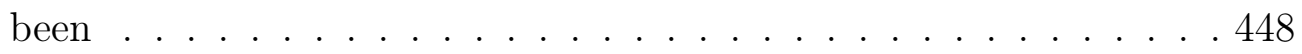

D-16 Cathode analyzing power at three locations (1) in a QE hole (2) on a QE gradient (3) in a high QE plateau uniform region. Polog $3470271 \ldots \ldots \ldots \ldots 4 \ldots \ldots \ldots$

D-17 Gradient in Analyzing Power on the photocathode combined with a linear polarization asymmetry leads to a position difference . . . . 449

D-18 Charge asymmetry gradient for active $\mathrm{KD}^{*} \mathrm{P}$ with a vertical analyzer after cell("Gandalf", Run2815, elog 656) . . . . . . . . . . . . 450

D-19 Charge asymmetry gradient for active $\mathrm{KD}^{*} \mathrm{P}$ with a vertical analyzer after cell("Gandalf",Run2857, elog 665) . . . . . . . . . . . . . 451

D-20 Rotation of the cube polarizer analyzer scan (equivalent to RHWP scan) for IHWP in and IHWP out. UVa KD*P Cell Gandalf. . . . 453

D-21 Predictions of RHWP scan for UVa KD*P Cell Gandalf. . . . . . . 454

D-22 RHWP scan for IHWP in and IHWP out. JLab KD*P cell. . . . . 454

D-23 UVa Spot Size Study Table Layout. . . . . . . . . . . . . . . 455

D-24 JLab Pockels Cell. . . . . . . . . . . . . . . . . . . . . 455

D-25 JLab Spot Size Study Table Layout. . . . . . . . . . . . . . . . 456

D-26 JLab Spot Size Study Results. . . . . . . . . . . . . . . . . . 456

D-27 M2 measurement of Hall A laser. Polog 3473100. . . . . . . . . . . 457 


\section{List of Tables}

1.1 E122 provided a definitive answer on gauge structure of electroweak interaction $[70] \ldots \ldots \ldots \ldots$

2.1 Helicity amplitude matrices for direct and crossed contributions to ee scattering via $\gamma$ and $\mathrm{Z}$ exchange . . . . . . . . . . . . 22

2.2 Electromagnetic and weak neutral charges . . . . . . . . . . . . 24

2.3 MOLLER Beam Goals . . . . . . . . . . . . . . . . . . . 60

2.4 Comparison of MOLLER PQB requirements to those achieved during HAPPEX-II . . . . . . . . . . . . . . . . . 61

2.5 Steps to achieving MOLLER PQB requirements compared with what has previously been achieved . . . . . . . . . . . . . 62

3.1 Electric and weak charges of spin-0 nucleus . . . . . . . . . . . . . 70

3.2 Septum running conditions . . . . . . . . . . . . . . . 107

3.3 Radiation summary tables . . . . . . . . . . . . . . . 117

4.1 PMT gain systematic errors from uncertainty in the recalibration method for each data configuration . . . . . . . . . . . . 170

4.2 Nonlinearity in unstabilized runs . . . . . . . . . . . . . . . . 179

4.3 Summary of $A_{\text {false }}(\mathrm{ppm})$ for different configurations . . . . . . . . . 180

4.4 Systematic error on $A_{n}(\mathrm{ppm})$ from non-linearity as estimated by change in RMS asymmetry distributions . . . . . . . . . . . . . 182

4.5 Summary of systematic errors (in ppm) due to non-linearity from various methods of estimation . . . . . . . . . . . . . . 184 
4.6 Systematic errors are added in quadrature to obtain total systematic error [203]. Values in parenthesis are uncertainties, values not in parenthesis are systematic corrections to measured asymmetries. 187

5.1 These are small beam charge asymmetry widths $(30 \mathrm{~Hz})$ [183] . . . 194

5.2 Verification of BCM resolution obtained by taking double-differences $\sigma_{B C M}^{D D}$ using SAM computations. . . . . . . . . . . . . . 198

5.3 The frequency dependence of the beam asymmetry $A q$ RMS width . 201

5.4 Double difference widths in $1 \mathrm{MHz}$ BCM system . . . . . . . . . . . 202

5.5 BPM types in injector and HallA [108] . . . . . . . . . . . . . 215

6.1 RTP refractive indices at $780 \mathrm{~nm}$. . . . . . . . . . . . . . . 259

6.2 HV\#, helicity, crystal, HV polarity, orientation, steering direction, equation . . . . . . . . . . . . . . . . . . . 298

6.3 Aperture losses of varying degrees coupling HCBAs for $\sigma=1 \mathrm{~mm}$ spot size e-beam [304] . . . . . . . . . . . . . . . . . . . . . . 345

D.1 Data from runs: 2841, 2838, 2850, 2848, 2847 and 2826, 2827, 2828, 2860, 2861, 2857, 2841, 2859 and 2857, 2857 elog714, elog714 (in

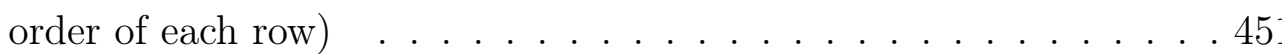





\section{Chapter 1}

\section{Introduction}

\subsection{Parity Violation in the Weak Interaction}

This history of parity violation in the weak interaction begins with Noether's theorem and the postulate of parity conservation. For every symmetry in nature, there exists a corresponding conserved quantity - so says Noether's Theorem of 1917 [51]. By Noether's theorem, symmetry of interactions under spatial inversion $(x \rightarrow-x)$ must lead to conservation of the parity quantum number of elementary particles [46]. The postulate, generally accepted prior to the mid-1950's, was that mirror symmetry in nature was self-evident and parity was therefore conserved. The sober view at the time was to assume parity was conserved in all physical processes including the weak interaction, as well as the strong and electromagnetic interactions, until proven otherwise [32].

In the early fifties, the so-called $\tau-\theta$ puzzle came along to challenge the postulate of parity conservation. The $\tau-\theta$ puzzle was a disturbing paradox in which two apparently identical mesons, which we now now as the $\mathrm{K}$, decayed into pionic states of opposite parity: $\theta^{+} \rightarrow \pi^{+}+\pi^{0}(P=1)$ and $\tau^{+} \rightarrow \pi^{+} \pi^{0}+$ $\pi^{0} \quad$ or $\pi^{+} \pi^{+}+\pi^{-}(P=-1)$. Lee and Yang addressed the $\tau-\theta$ puzzle by questioning the postulate of parity conservation: they suggested $\tau$ and $\theta$ were 
the same particle with parity just not conserved in one of the decay channels. A thorough study of previous experiments found no evidence of parity conservation in the weak interaction [32]. They proposed a test which lead directly to the experimental demonstration of parity violation later that year[50].

Parity was 'overthrown' in 1956 when Madame $\mathrm{Wu}$ [53] carried out the famous, decisive Co-60 experiment which proved that parity was not conserved in the weak interaction. ${ }^{60} \mathrm{Co}$ nuclei were polarized with the nuclear spins aligned parallel to the magnetic field of a solenoid[50], underwent beta decay to an excited state of ${ }^{60} \mathrm{Ni}$ via ${ }^{60} \mathrm{Co} \rightarrow{ }^{60} \mathrm{Ni}+e^{-}+\bar{\nu}_{e}$, and $\mathrm{Wu}$ recorded the direction of the emitted electron. Because the parity transformation reverses momentum $\vec{p}$ but not angular momentum $\vec{r} \times \vec{p}$ or spin, if parity were conserved in this process, the electron momentum would not depend on the nuclear spin. However, a 'forwardbackward decay asymmetry' was observed, and fewer electrons came out in the forward direction of nuclear spin than in the backward direction. This experiment established parity violation as a signature of the weak force [32].

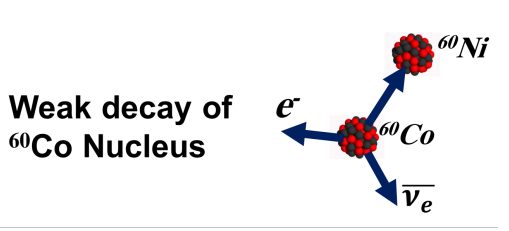

(a)

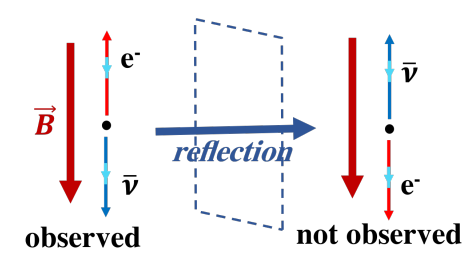

(b)

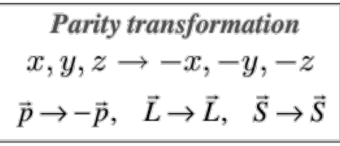

Parity transformation $x, y, z \rightarrow-x,-y,-z$ $\vec{p} \rightarrow-\vec{p}, \quad \vec{L} \rightarrow \vec{L}, \quad \vec{S} \rightarrow \vec{S}$

(c)

Figure 1-1: (a) Cobalt weak beta decay (b) Observation of 'forward-backward decay asymmetry' in Co-60 weak decay (c) Parity transformation reverses momentum $\vec{p}$ but not angular momentum $\vec{r} \times \vec{p}$ or spin [69] [70]

The discovery of parity violation was profound in the history of weak interactions because the effect is large. Once you look for it, parity violation is practically the signature of the weak force [32]. Understanding of weak interactions is impossible if parity violation is neglected [50]. 


\subsubsection{Birth of Glashow-Weinberg-Salam(GWS) theory}

The first theory of the weak interaction, presented by Fermi in 1933, contained no mediating particle, treating the weak process as simply a contact interaction occurring at a single point with no mediator exchange. It was widely recognized that this model was limited to low energy interactions and would fail at higher energies [32]. Any more general theory of the weak interaction would need to contain an 'intermediate vector boson', the $W \pm$ 's, and the challenge for theorists was to predict its properties. It was not until the emergence of the electroweak unification model that a really firm prediction of the weak intermediate vector mass was possible.

Armed with the new knowledge that parity was not conserved in the weak interaction, Glashow, Weinberg and Salam, in the 60's, formulated a theory unifying the weak and EM interaction - defining the electroweak interaction - which contained three weak intermediate vector bosons: $W^{ \pm}$and $Z_{0}$. Crucially, the Glashow-Weinberg-Salam (GWS) model was the first theory both containing parity violation in the weak interaction and containing weak neutral current reactions (the word 'neutral' referring to the charge of the exchange particle $Z_{0}$ in contrast to weak charged current reactions exchanging charged $W^{ \pm}$bosons). The theory made new predictions concerning the existence of a heavy neutral vector boson $Z_{0}$ and neutral current, the masses of the $W^{ \pm}$and $Z_{0}$ bosons, as well as the existence of a new spin-0 boson, the Higgs boson as we now know it [50].

Of the many remarkable successes of the unified theory, one of particular significance was the firm prediction relating the masses of $W^{ \pm}$and $Z_{0}$. The masses of the $\mathrm{W}$ and $\mathrm{Z}$ bosons were related through the so called "Weinberg angle"/"Weak-mixing angle" $\theta_{W}$ via $M_{Z}=M_{W} / \cos \theta_{W}$, where couplings in the model are determined by the single parameter $\theta_{W}$. The GWS model asserts that the $W \pm, Z_{0}$ and the photon $\gamma$ are produced by spontaneous symmetry breaking. The three weak isospin $T_{3}$ currents couple to a weak isotriplet of intermediate vector bosons $W^{+}, W^{0}$, 
and $W^{-}$, whereas the hypercharge $Y_{W}$ current couples to an isospin singlet intermediate vector boson $B^{0}$ via independent couplings $g$ and g'. The symmetry breaking means the two neutral states $W^{0}$ and $B^{0}$ mix, via the "Weak-mixing angle" $\theta_{W}$, to form the photon(massless linear combination) and $Z_{0}$ (orthogonal massive combination) [32].

$$
\begin{aligned}
& A=B^{0} \cos \theta_{W}+W^{0} \sin \theta_{W} \\
& Z=W^{0} \cos \theta_{W}-B^{0} \sin \theta_{W}
\end{aligned}
$$

In the early days it was hard to estimate the $\theta_{W}$ experimentally, hence the $Z_{0}$ mass was quite uncertain.

GWS electroweak unification theory became part of the Standard Model. Basic predictions of the GWS model were experimentally confirmed over the course of the 1970's. The Gargamelle bubble chamber experiment showed the existence of the neutral $Z_{0}$ in 1973 . The E122 experiment at SLAC, which was the pioneering parity violating electron scattering experiment, both verified parity non-conservation in the weak interaction and measured $\sin ^{2} \theta_{W}=0.2 \pm 0.03$ in 1978 [29]. Several other experiments in the late 70 's pointed to $\theta_{W} \approx 29^{\circ}$ and the weak force mediator masses were calculated to be $M_{W}=82 \pm 2 \mathrm{GeV} / c^{2}$ and $M_{Z}=92 \pm 2 \mathrm{GeV} / c^{2}$ [32]. Glashow, Weinberg and Salam were awarded the Nobel prize in 1979. Sometime later, finally in 1983 , CERN did officially observe the W (at $81 \mathrm{GeV}$ ) and the $\mathrm{Z}$ (at $95 \mathrm{GeV}$ ) as a direct confirmation of what was already established through indirect methods.

\subsubsection{Tests of the Standard Model: Three Regimes}

There was no direct evidence for weak neutral currents for some time. The trouble is that everything that the neutral $Z_{0}$ might couple to, the photon also couples to, and the weak force gets masked by EM force. Experimental strategies for 
detecting weak neutral currents can be understood by examining the amplitudes for the competing weak and EM interactions. The general form of the amplitude is

$$
\mathcal{M}\left(q^{2}\right)=\frac{g^{2} \hbar^{2}}{q^{2}-M_{X}^{2} c^{2}}
$$

where $M_{X}$ is the mass of the mediator and $\mathrm{g}$ is the coupling constant. In weak interactions, $M_{X}=M_{W, Z} \approx 80 \mathrm{GeV} / c^{2}$ and in the electromagnetic interaction, $M_{X}=M_{\gamma}=0$. So, even with comparable coupling $g_{\text {weak }} \approx g_{\text {em }}$, the amplitudes for the two interactions will only become of comparable size for $|q|^{2} \approx M_{X}^{2} c^{2}$ [50]. Electroweak unification only becomes clearly manifest at high energies. But experimentally reaching these high energies is usually very difficult and so indirect low energy methods were usually used first. One such method which can be employed at low energy is to use parity violation. There is a weak contamination in every EM process, and even though these effects are minute, they carry a telltale signature parity violation. The observation of these minute effects was further unambiguous evidence for electroweak unification [32]

Measurements of the neutral weak interaction in tests of the Standard Model followed a natural progression through what can be classified as 3 experimental regimes:

1. Neutrino $\left(\mathcal{M}_{\text {weak }} \neq 0, \mathcal{M}_{\gamma}=0\right)$ regime : First neutrino experiments were performed in a regime where there was low signal and no EM background.

2. Indirect $\left(\mathcal{M}_{\text {weak }}<<\mathcal{M}_{\gamma}\right)$ regime: Then indirect experiments were performed in a low energy regime, where the weak signal was small, EM background dominated, and parity properties were used to distinguish signal from background

3. High Energy $\left(\mathcal{M}_{\text {weak }} \sim \mathcal{M}_{\gamma}\right)$ regime : Finally ' direct' high energy experiments confirmed what had already been demonstrated indirectly. 


\section{Neutrinos: Low (Isolated) Signal Regime}

Neutral weak interactions were not detected for quite some time due to being obscured by electromagnetic processes. All observed weak interactions were limited to those mediated the charged boson $W^{ \pm}$only [50]. However, neutrinos, while difficult to detect, have no EM coupling, so the weak effects are not obscured [32] . So, neutrino scattering was used to first confirm the existence of neutral weak interactions in a regime where there was low signal and no EM background. It was predicted that hadronic neutral current reactions between a neutrino and a nucleon: $\nu_{\mu}+N \rightarrow \nu_{\mu}+X$, where $\mathrm{X}$ is any set of hadrons, although difficult to detect, could occur via the mechanism of neutral $Z_{0}$ exchange [50]. Leptonic neutral currents, events involving the interaction of a neutrino with an electron, were also predicted to occur. At last in 1973, the Gargamelle bubble chamber experiment [31] observed such reactions and showed the existence of the neutral $Z_{0}$.

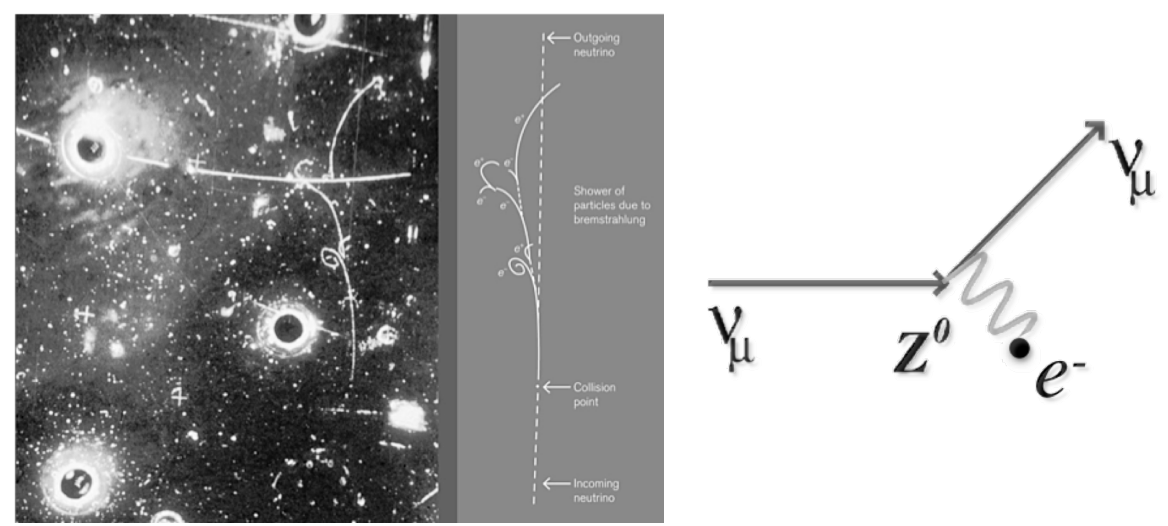

Figure 1-2: Gargamelle Bubble chamber Experiment: uncovers $\nu_{\mu}+e^{-} \rightarrow \nu_{\mu}+e^{-}$, shows neutral current exists. [70]

Fig. 1-2 shows the actual bubble chamber photograph where weak neutral currents were first observed. The measurement uncovered neutrino electron events where neutrino (or antineutrino) collides with an electron $\nu_{\mu}+e^{-} \rightarrow \nu_{\mu}+e^{-}$. The neutrino has no charge, so the interaction has no EM component and the mediator 
is just the $Z_{0}$. The scattering of neutrinos off electrons and nuclei was found to yield amplitudes comparable to those of other weak charged current processes [50].

The $\nu-e$ and $\nu-N$ experiments demonstrated the existence of the neutral current and $Z_{0}$, but not its nature, or its properties, or to what extent, or even whether, the weak neutral current was parity-violating. [46]

\section{Indirect: Low Energy Regime}

As stated previously, the Standard Model provided no way to calculate $\theta_{W}$, so the $Z_{0}$ mass was theoretically uncertain and required experimental inputs. After the existence of $Z_{0}$ weak mediator had been established, 'indirect' experiments were performed in the low energy regime. Even though the weak signal was small and EM background dominated, parity properties were used to distinguish signal from background. The clean separation can be made by making use of the parity violation in the weak interaction. Since the parity violating signal was predicted to be small, high precision was required. A common attribute of precision experiments is the interferometric nature of the measurement. In precision parity experiments, it is the interference term between the EM and weak interactions which gets detected and the parity violation property of the weak interaction which distinguishes it.

In 1978, the E122 experiment at SLAC [29], verified the Standard Model GWS electroweak theory, verified parity non-conservation in the weak interaction and determined $\theta_{W}$ by measuring the parity-violating asymmetry $A_{P V}$ between the Deuterium cross-section for incident right and left handed electron scattering. The asymmetry was predicted to be only a few parts per million. Nevertheless, a nonzero value was definitively established - verifying a weak neutral current at play and ruling out various left-right symmetric gauge theories (which predicted zero parity violation) [46]. $A_{P V}$ was measured to be $A_{P V}=100 \pm 10 \mathrm{ppm}$ (to $10 \%$ precision) and the weak mixing angle $\theta_{W}$ between the mass and flavor eigenstates of the photon and the $Z_{0}$ could be extracted from this asymmetry [46]. $A_{P V}$ 
was also modeled as a function of the fractional energy loss of the initial electron (Table 1.1). This is a function of the weak mixing angle and a value was found for $\sin ^{2} \theta_{W}=0.2 \pm 0.03$ (to $\sim 15 \%$ precision), consistent with GWS electroweak unification [46].

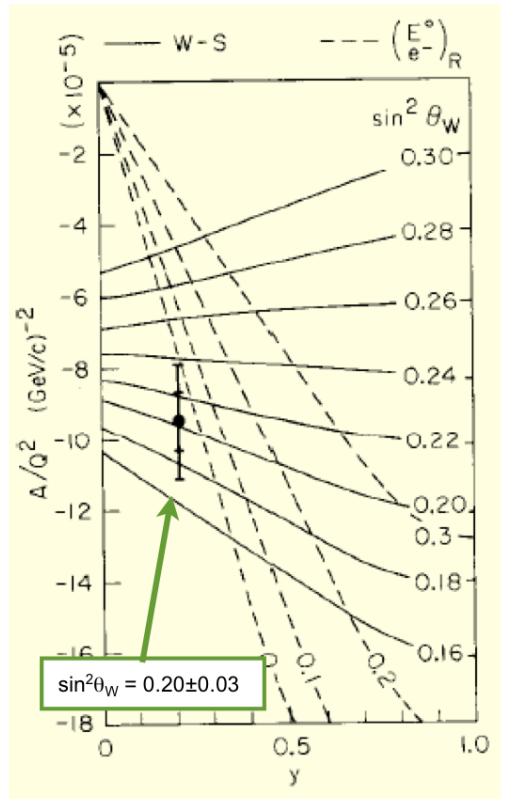

Figure 1-3: $\quad$ E122 result: $A_{P V}=100 \pm 10 \mathrm{ppm} \rightarrow \sin ^{2} \theta_{W}=0.2 \pm 0.03 . \quad A_{P V}$ modeled as a function of the fractional energy loss of the initial electron $y=$ $1-E^{\prime} / E$. Parity Non-Conservation in Inelastic Electron Scattering, C.Y. Prescott et. al., 1978 [70]

\begin{tabular}{|c|c|c|}
\hline & Left & Right \\
\hline$\gamma$ Charge & $q=0, \pm 1, \pm \frac{1}{3}, \pm \frac{2}{3}$ & $q=0, \pm 1, \pm \frac{1}{3}, \pm \frac{2}{3}$ \\
\hline W Charge & $T= \pm \frac{1}{2}$ & zero \\
\hline Z Charge & $T-q \sin ^{2} \theta_{W}$ & $-q \sin ^{2} \theta_{W}$ \\
\hline
\end{tabular}

Table 1.1: E122 provided a definitive answer on gauge structure of electroweak interaction [70]

The precision of the E122 experiment which allowed it to distinguish the GWS model from other gauge theories made it a foundational measurement upon which the Standard Model was built. The Nobel Prize in Physics was awarded in 1979, a 
year after E122, jointly to Glashow, Salam, and Weinberg "for their contributions to the theory of the unified weak and electromagnetic interaction between elementary particles, including, inter alia, the prediction of the weak neutral current". By measuring $\theta_{W}$, E122 and other experiments provided a definitive answer on gauge structure of electroweak interaction, demonstrated the VA (vector axial) - V (vector) structure of the weak current interaction, and showed that it is maximally parity violating [46]. To this day, precise calculation $\theta_{W}$ still currently stands as a major challenge for any theory going beyond the SM.[32]

\section{High Energy Regime}

At last in 1983, after the neutral weak force mediator had already been shown to exist, after $\mathrm{W}$ and $\mathrm{Z}$ masses had been determined by indirect measurements, CERN did officially observe the $\mathrm{W}$ (at $81 \mathrm{GeV}$ ) and the $\mathrm{Z}$ (at $95 \mathrm{GeV}$ ), directly confirmed what had already been demonstrated indirectly. These high energy measurements took such a long time to perform because a proton-antiproton collider designed specifically to produce these extremely heavy particles (nearly 100X the proton mass) had to be constructed first. The energy regime was specifically in the neighborhood of the $Z_{0}$ mass, and total energy hits $M_{Z}$ at the $Z_{0}$ pole where the denominator of the $Z_{0}$ propagator is small in Eq. 1.1 and the cross-section blows up.

CERN's measurement of the intermediate vector bosons $\mathrm{W}$ (at $81 \pm 5 \mathrm{GeV} / c^{2}$ ) and the $Z_{0}$ (at $95 \pm 3 \mathrm{GeV} / c^{2}$ ) [54] was a technical triumph, and the experiment added precision to the SM with mass and width measurements. The result was long awaited and served as a confirmation of a crucial aspect of the Standard Model[32].

Examining our scientific history informs our scientific future. At present, our community has begun this cycle yet again: there are a variety of new puzzles and a variety of new Beyond the Standard Model(BSM) theories. There are still these three experimental regimes at play: neutrino experiments, low energy precision 
experiments, and high energy direct searches. Further studies using parity violation have continued to test the structure of the Standard Model in the low energy precision regime. This regime is where the MOLLER (Measurement Of Lepton Lepton Electroweak Reaction) experiment resides, which of particular interest in this thesis.

\subsection{Motivation: Parity Violating Electron Scatter- ing}

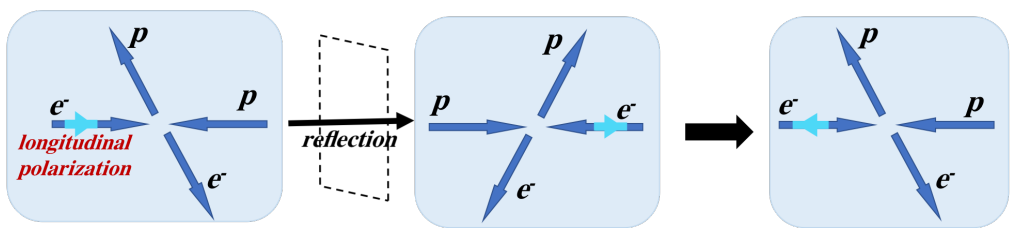

Figure 1-4: Parity violation experiment [70]

In electron scattering parity violation experiments, the longitudinally polarized electron beam is incident on an unpolarized target. Fig. 1-4 shows the COM interaction and Fig. 1-5 shows an interaction with a target. We change the sign of the longitudinal polarization (parity transformation), and measure the fractional rate difference between right and left helicity states, $d \sigma_{R}$ and $d \sigma_{L}$.

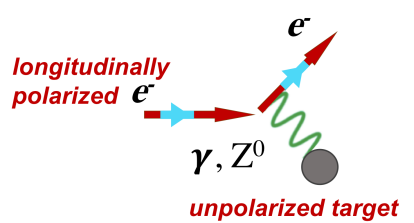

(a)

$$
\begin{aligned}
& \sigma \propto\left|M_{\gamma}+M_{\text {weak }}\right|^{2} \sim\left|M_{\gamma}\right|^{2} \\
& +M_{\gamma}\left(M_{\text {weak }}\right)^{*}+\left(M_{\gamma}\right)^{*} M_{\text {weak }}+\left|M_{\text {weak }}\right|^{2}
\end{aligned}
$$

Figure 1-5: Interference between weak and EM amplitudes, the weak interaction carrying signature of parity violation [70]

An interference between the electromagnetic and weak amplitudes, $\mathcal{M}_{\gamma}$ and 
$\mathcal{M}_{Z}$ respectively (Fig. 1-5), gives rise to a parity violating asymmetry

$$
A_{P V}=\frac{\sigma_{R}-\sigma_{L}}{\sigma_{R}+\sigma_{L}} \propto \frac{\left|\mathcal{M}_{Z}\right|}{\left|\mathcal{M}_{\gamma}\right|}
$$

defined by the fractional rate difference between right and left helicity states. $A_{P V}$ can be measured to extraordinarily high precision and is approximately proportional to the ratio of the weak and electromagnetic amplitudes.

\subsubsection{Parity Experiments as a Precision Tool}

The first parity violating electron scattering (PVeS) experiment, E122 in 1978, served as a blue print for parity electron scattering experiments - even for those experiments with much higher precision, probing BSM. A general description of a PVeS experiment is shown in Fig. 1-6. An $e^{-}$beam may interact with target by exchanging a mediator particle such as: the photon (EM force) or the $Z_{0}$ (weak force). The electron polarization is flipped, and the mirror image, parity transformed interaction is examined (Fig. 1-6). By looking at the differences between the two types of interactions one can extract the size of the $Z_{0}$ contribution. This can be compared to the SM and any deviations could be a hallmark of BSM.

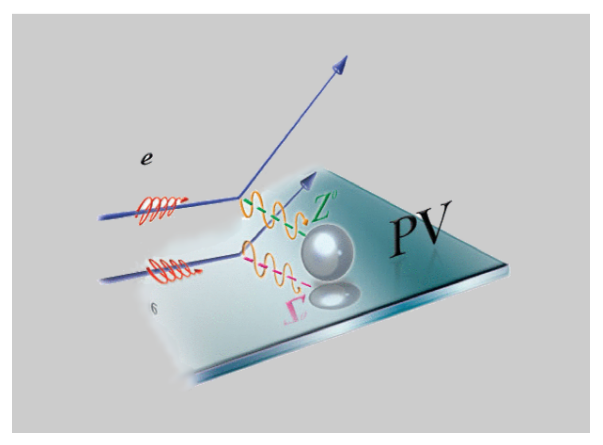

Figure 1-6: Parity Experiment Blue Print: Mirror Image Parity Transformation illustrated in electron scattering [73]

In the intervening years, PVeS has become a precision tool. There's a broad 
program of experiments, studying the structure of protons and nuclei, and searching for new BSM physics. Fig. 1-7 shows the precision of various experiments. The measured or predicted asymmetry $A_{P V}$ is plotted on the x-axis and the uncertainty on $A_{P V}$ is on the y-axis.

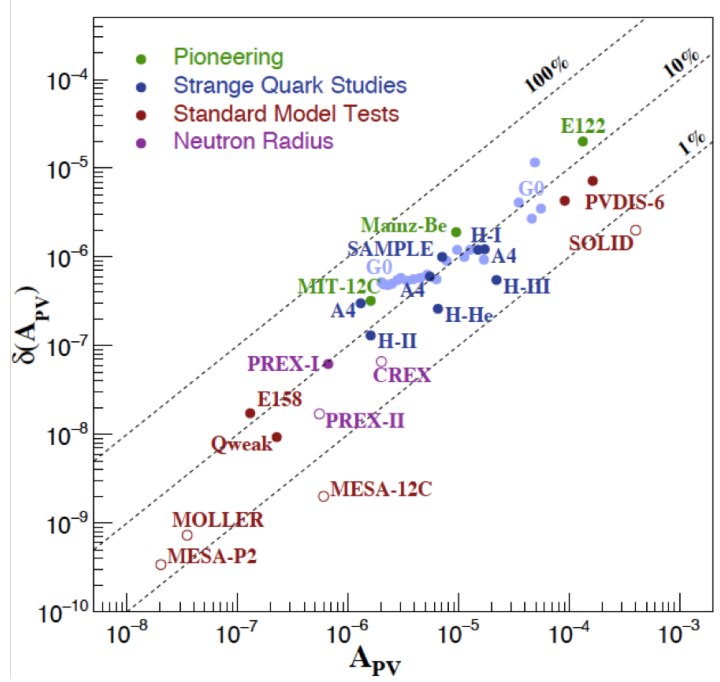

Figure 1-7: Precision of various parity experiments: measured or predicted asymmetry $A_{P V}$ on x-axis and the uncertainty on $A_{P V}$ on the y-axis [70]

E122 is shown at the top of Fig. 1-7 as one of the less precise experiments, being the first PVeS experiment after all. PREX-II, an experiment measuring the weak skin of the ${ }^{208} \mathrm{~Pb}$ nucleus, is also shown Fig. 1-7. PVES provides a clean measurement of $R_{N}$, the RMS radius of neutrons in a heavy nucleus, and constrains the equation of state (EOS) of highly dense matter which is important for describing neutron star structure, heavy ion collisions, and atomic parity violation experiments [164] . The Lead Radius Experiment (PREX) will measure the parity violating asymmetry $A_{P V}$ for $1 \mathrm{GeV}$ electrons scattering from ${ }^{208} \mathrm{~Pb}$ at $5^{\circ}$, and should be sensitive to the neutron radius of the nucleus to $1 \%( \pm 0.05 \mathrm{fm})$ precision [164]. An early iteration of this experiment, PREX-I, reached $\sim 9 \%$ precision on the neutron radius. PREX-II is expected to achieve higher precision than PREX-I which was statistics limited.

MOLLER(Measurement Of Lepton Lepton Electroweak Reaction), shown in 
Fig. 1-7, is a search for new neutral currents in electron-electron scattering experiments. It's essentially a more precise version of the SLAC E158 experiment [16] in Fig. 1-7, completed in 2005. As the name implies, this experiment will measure $A_{P V}$ in the Moller ee scattering process, and will infer the weak charge of the electron $Q_{W}^{e}$ to extremely high precision. MOLLER will extract $\sin ^{2} \theta_{W}$ at low momentum transfer $\left(Q^{2} \approx 0.0056 \mathrm{GeV}^{2}\right)$ by scattering a longitudinally polarized electron beam off electrons in a liquid hydrogen target and examining the resulting parity-violating asymmetry $A_{P V}$ to $\sim 0.8 \mathrm{ppb}$ precision. It will thereby measure the weak charge of the electron $Q_{W}^{e}$ to $2.4 \%$ precision.

Both PREX-II and MOLLER experiments will be performed at Jefferson Lab (JLab), a national electron beam accelerator facility in Newport News, Virginia. The upgraded $12 \mathrm{GeV}$ accelerator is depicted in Fig. 1-8. The electron beam is generated at the injector where it is accelerated from $130 \mathrm{keV}$ to $5 \mathrm{MeV}$ before entering the first pass and starting its first orbit. Magnets in the arcs steer the electron beam from the straight section of the tunnel to the next for up to five orbits (or passes), with each pass accelerating the electrons to successively higher energies up to $12 \mathrm{GeV}$. Each linear accelerator (LINAC) uses superconductors to drive acceleration and a refrigeration plant provides liquid helium for superconducting operation. The electron beam is delivered to the experimental halls where the experiments take place. 


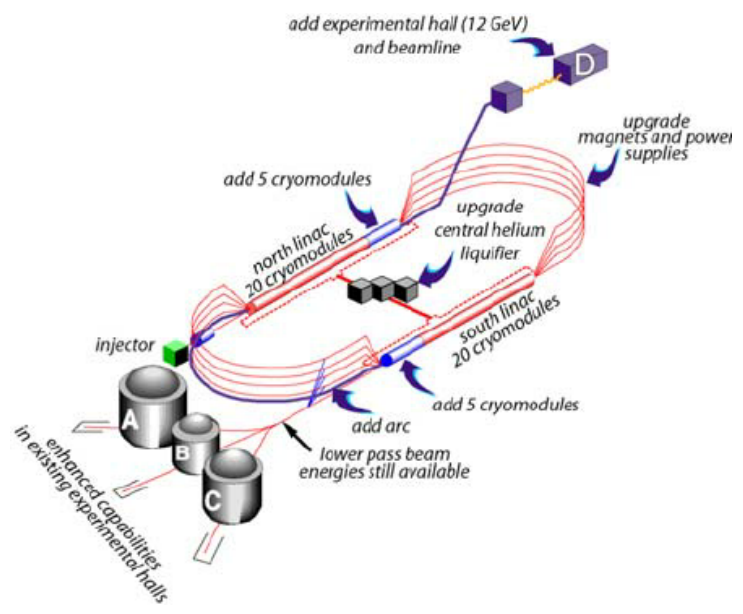

Figure 1-8: Jefferson Lab $12 \mathrm{GeV}$ upgrade [4]

\section{Summary}

This thesis highlights the PVES experiments PREX-II, MOLLER, and a ${ }^{12} C$ transverse asymmetry measurement at Mainz. Chapters 1-3 of this thesis cover introductions to MOLLER and PREX-II, Chapter 4 contains the results of the ${ }^{12} \mathrm{C}$ transverse asymmetry measurement at Mainz, and Chapters 5-6 contain the original work performed by this author developing important experimental equipment including an innovative Rubidium Titanyl Phosphate (RTP) Pockels cell in the polarized source 


\section{Chapter 2}

\section{MØLLER Experiment}

\subsection{Introduction}

MOLLER is an extremely precise parity violating electron scattering (PVeS) experiment searching for new neutral currents in electron-electron scattering. It's called MOLLER because it will measure $A_{P V}$ in the Møller ee scattering process, and will infer the weak charge of the electron $Q_{W}^{e}$ to extremely high precision. It is a low-energy test of the Standard Model, just as sensitive to new physics as complimentary high-energy colliders experiments [46]. MOLLER will measure $\sin ^{2} \theta_{W}$ at low momentum transfer by examining the parity-violating asymmetry $A_{P V}$ in the scattering of a longitudinally polarized electron beam off electrons in a liquid hydrogen target. This measurement will determine the weak charge of the electron $Q_{W}^{e}$ to very high precision, will be sensitive to new neutral current amplitudes, and has the potential to detect disagreement with the Standard Model [46] competitively with any proposed experiment measuring a flavor- and CP-conserving process over the next decade. MOLLER is sensitive to new physics at $\mathrm{MeV}$ and multi-TeV scales and will serve as an indirect complementary measurement to direct searches at high energy colliders [1]. 


\subsubsection{Møller scattering}

The idea to use Møller scattering as a clean probe of the weak mixing angle was pioneered at Princeton [3]. Unlike some high energy measurements in hadron colliders, there's no corrections due to hadron structure in ee scatting, so its less limited by theoretical interpretation. This makes it appealing to the fundamental symmetries subset of the physics community dedicated to precision measurements. The process is described by

$$
e_{1}^{-}\left(p_{1}, s_{1}\right)+e_{2}^{-}\left(p_{2}, s_{2}\right) \rightarrow e_{1^{\prime}}^{-}\left(p_{1}^{\prime}, s_{1}^{\prime}\right)+e_{2^{\prime}}^{-}\left(p_{2}^{\prime}, s_{2}^{\prime}\right)
$$

where $(\mathrm{p}, \mathrm{s})$ denote 4 momenta and helicity of electrons, $p_{1}, p_{2}\left(p_{1}^{\prime}, p_{2}^{\prime}\right)$ denote incoming (outgoing) momenta, and $s_{1}, s_{2}\left(s_{1}^{\prime}, s_{2}^{\prime}\right)$ denote incoming (outgoing) helicities. The helicity-independent differential cross-section for Møller scattering (derived in later sections) is approximately by

$$
\frac{d \sigma}{d \Omega}=\frac{\alpha^{2}}{2 m E} \frac{\left(3+\cos ^{2} \theta\right)^{2}}{\sin ^{4} \theta}=\frac{\alpha^{2}}{4 m E} \frac{1+y^{4}+(1-y)^{4}}{y^{2}(1-y)^{2}} y=1-E^{\prime} / E
$$

The tree level Feynman diagrams for Møller scattering can be seen in Fig. 2-1. Parity violating terms in Møller scattering arise from the interference between weak $\mathrm{Z}$ and electromagnetic $\gamma$ exchange amplitudes. This interference produces a parity violating asymmetry $A_{P V}$ which can be measured and the weak mixing angle inferred as follows:

$$
\begin{gathered}
A_{P V}=\frac{\sigma_{R}-\sigma_{L}}{\sigma_{R}+\sigma_{L}}=m E \frac{G_{F}}{\sqrt{2} \pi \alpha} \frac{16 \sin ^{2} \theta}{\left(3+\cos ^{2} \theta\right)^{2}} g_{e e}=m E \frac{G_{F}}{\sqrt{2} \pi \alpha} \frac{4 \sin ^{2} \theta}{\left(3+\cos ^{2} \theta\right)^{2}} Q_{W}^{e} \\
=m E \frac{G_{F}}{\sqrt{2} \pi \alpha} \frac{2 y(1-y)}{1+y^{4}+(1-y)^{4}} Q_{W}^{e}
\end{gathered}
$$

measured by comparing left and right helicity states of the electron beam. In Eq.2.1, $G_{F}$ is the Fermi coupling constant, $g_{e e}$ is the pseudoscaler weak neutral- 
current coupling for Møller scattering, and $Q_{W}^{e}$ is the electron weak charge. Within the context of the Standard Model, at tree level $g_{e e}$ is the product of a vector electron-photon vertex and an axial-vector electron photon vertex and takes the value

$$
g_{e e}=\rho \cdot g_{V_{e}} \cdot g_{A_{e}}=\frac{1}{4}-\sin ^{2} \theta_{W}=Q_{W}^{e} / 4
$$

where $\rho(=1$ within the SM $)$ is the relative strength of the weak neutral and charged current interactions and is defined as $\frac{M_{W}^{2}}{M_{Z}^{2} \cos ^{2} \theta_{W}}$ [46]. The measured asymmetry then relates directly to the electron weak charge and weak mixing angle $A_{P V} \rightarrow Q_{W}^{e} \rightarrow$ $\sin ^{2} \theta_{W}$ via the relationship

$$
Q_{W}^{e}=1-4 \sin ^{2} \theta_{W}
$$

Note that $g_{e e}$ is close to zero since $\sin ^{2} \theta_{W} \sim 0.023$. Thus a small (relative) change in $\sin ^{2} \theta_{W}$ introduces a much larger relative change in $g_{e e}$ and hence $A_{L R}^{e e}$.

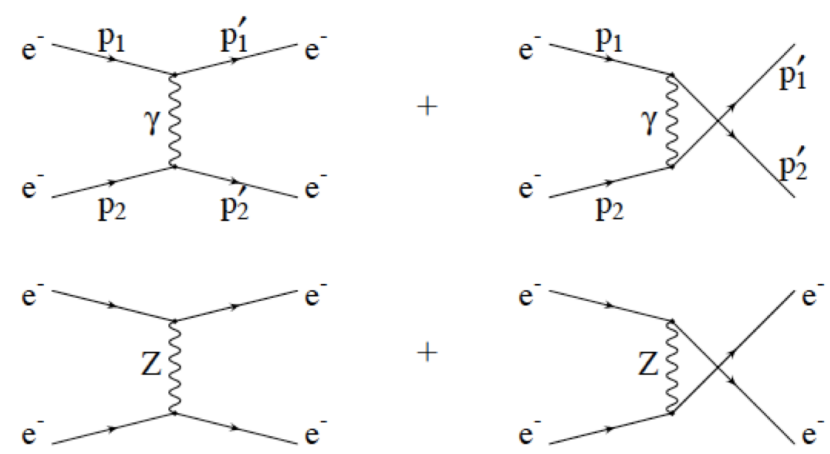

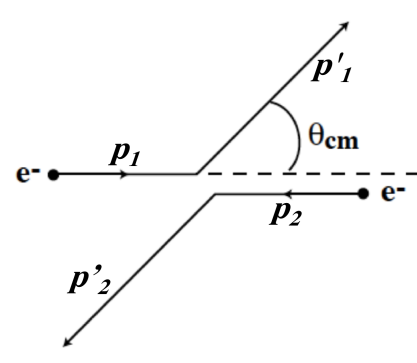

(b) COM frame

(a) Feynman diagrams

Figure 2-1: Møller scattering $e_{1}^{-}\left(p_{1}, s_{1}\right)+e_{2}^{-}\left(p_{2}, s_{2}\right) \rightarrow e_{1^{\prime}}^{-}\left(p_{1}^{\prime}, s_{1}^{\prime}\right)+e_{2^{\prime}}^{-}\left(p_{2}^{\prime}, s_{2}^{\prime}\right)$ : (a) Tree level diagrams for direct and crossed photon and $\mathrm{Z}$ boson exchange (b) Kinematic variables in center of momentum frame. Arrows denote incoming and outgoing electrons [1] 


\section{EM unpolarized cross section}

Here we show the derivation of the unpolarized cross section for EM Møller scattering from [46] [47]. The Møller cross section at low $Q^{2}$ is dominated by direct photon exchange between two electrons. The Feynman diagram for direct photon exchange between the two electrons is shown in Fig. 2-1 as well as the crossed diagrams and the $\mathrm{Z}$ exchange diagrams. The kinematic variables in centerof-momentum (COM) frame are also defined for the reaction:

$$
e_{1}^{-}\left(p_{1}, s_{1}\right)+e_{2}^{-}\left(p_{2}, s_{2}\right) \rightarrow e_{1^{\prime}}^{-}\left(p_{1}^{\prime}, s_{1}^{\prime}\right)+e_{2^{\prime}}^{-}\left(p_{2}^{\prime}, s_{2}^{\prime}\right)
$$

In the COM frame, the differential cross section is given by[47]:

$$
\left.\frac{d \sigma}{d \Omega}\right|_{C M}=\frac{1}{64 \pi^{2} s} \frac{p_{2}}{p_{1}}|\mathcal{M}|^{2}
$$

where the four-momentum transfer $q^{2}=\left(p_{1}-p_{1}^{\prime}\right)^{2}$, the invariant total energy of the system is $s=\left(p_{1}+p_{2}\right)^{2},|\mathcal{M}|^{2}$ is the invariant amplitude for the process and $p_{1}=\left|\overrightarrow{p_{1}}\right|$ and $p_{2}=\left|\overrightarrow{p_{2}}\right|$. The amplitude for the direct diagram is given by

$$
-i \mathcal{M}=\left[i e \bar{u}_{1^{\prime}} \gamma^{\mu} u_{1}\right] \frac{-i g_{\mu \nu}}{\left(p_{1}-p_{1}^{\prime}\right)^{2}}\left[i e \bar{u}_{2^{\prime}} \gamma^{\nu} u_{2}\right]-\left[i e \bar{u}_{2^{\prime}} \gamma^{\mu} u_{1}\right] \frac{-i g_{\mu \nu}}{\left(p_{1}-p_{2}^{\prime}\right)^{2}}\left[i e \bar{u}_{1^{\prime}} \gamma^{\nu} u_{2}\right]
$$

The cross diagram amplitude is found by interchange of $p_{1}^{\prime}$ and $p_{2}^{\prime}$. The amplitudes from the direct diagram and the crossed are combined with a relative minus sign following the antisymmetrization rule for exchange of identical fermions.

To calculate the unpolarized cross section, we average over the initial spin states and sum of the final spin states to obtain the spin-averaged amplitude squared.

$$
\begin{gathered}
\overline{|\mathcal{M}|^{2}}=\frac{1}{4} \sum_{s_{1}, s_{1^{\prime}}}|\mathcal{M}|^{2}=\frac{e^{4}}{4} \sum_{s_{1}, s_{1^{\prime}}}\{ \\
{\left[\left(\bar{u}_{1^{\prime}} \gamma^{\mu} u_{1}\right) \frac{g_{\mu \nu}}{\left(p_{1}-p_{1}^{\prime}\right)^{2}}\left(\bar{u}_{2^{\prime}} \gamma^{\nu} u_{2}\right)\right]\left[\left(\bar{u}_{1^{\prime}} \gamma^{\mu} u_{1}\right) \frac{g_{\mu \nu}}{\left(p_{1}-p_{1}^{\prime}\right)^{2}}\left(\bar{u}_{2^{\prime}} \gamma^{\nu} u_{2}\right)\right]^{*}}
\end{gathered}
$$




$$
\left.\begin{array}{l}
+\left[\left(\bar{u}_{2^{\prime}} \gamma^{\mu} u_{1}\right) \frac{g_{\mu \nu}}{\left(p_{1}-p_{2}^{\prime}\right)^{2}}\left(\bar{u}_{1^{\prime}} \gamma^{\nu} u_{2}\right)\right]\left[\left(\bar{u}_{2^{\prime}} \gamma^{\mu} u_{1}\right) \frac{g_{\mu \nu}}{\left(p_{1}-p_{2}^{\prime}\right)^{2}}\left(\bar{u}_{1^{\prime}} \gamma^{\nu} u_{2}\right)\right]^{*} \\
-\left[\left(\bar{u}_{1^{\prime}} \gamma^{\mu} u_{1}\right) \frac{g_{\mu \nu}}{\left(p_{1}-p_{1}^{\prime}\right)^{2}}\left(\bar{u}_{2^{\prime}} \gamma^{\nu} u_{2}\right)\right]\left[\left(\bar{u}_{2^{\prime}} \gamma^{\mu} u_{1}\right) \frac{g_{\mu \nu}}{\left(p_{1}-p_{2}^{\prime}\right)^{2}}\left(\bar{u}_{1^{\prime}} \gamma^{\nu} u_{2}\right)\right]^{*} \\
-\left[\left(\bar{u}_{2^{\prime}} \gamma^{\mu} u_{1}\right) \frac{g_{\mu \nu}}{\left(p_{1}-p_{2}^{\prime}\right)^{2}}\left(\bar{u}_{1^{\prime}} \gamma^{\nu} u_{2}\right)\right]\left[\left(\bar{u}_{1^{\prime}} \gamma^{\mu} u_{1}\right) \frac{g_{\mu \nu}}{\left(p_{1}-p_{1}^{\prime}\right)^{2}}\left(\bar{u}_{2^{\prime}} \gamma^{\nu} u_{2}\right)\right]^{*}
\end{array}\right\}
$$

where $s_{1}$ refers to the initial spin states and the superscripts $s_{i}$ in $u_{i}^{s_{i}}$ indicating the spin state of each electron are suppressed for clarity.

It is convenient to introduce the Mandelstam variables, which can be approximated in the ultrarelativistic limit as: $s=\left(p_{1}+p_{2}\right)^{2} \approx 2 p_{1} \cdot p_{2} \approx 2 p_{1}^{\prime} \cdot p_{2}^{\prime}$, $t=\left(p_{1}-p_{1}^{\prime}\right)^{2} \approx-2 p_{1} \cdot p_{1}^{\prime} \approx 2 p_{2} \cdot p_{2}^{\prime}$, and $u=\left(p_{1}-p_{2}^{\prime}\right)^{2} \approx-2 p_{1} \cdot p_{2}^{\prime} \approx 2 p_{2} \cdot p_{1}^{\prime}$.

Contracting indices,

$$
\begin{gathered}
\overline{|\mathcal{M}|^{2}}=\frac{e^{4}}{4} \sum_{s_{1}, s_{1^{\prime}}}\left\{\frac{1}{t^{2}}\left(\left[\bar{u}_{1^{\prime}} \gamma^{\mu} u_{1}\right]\left[\bar{u}_{1} \gamma^{\nu} u_{1^{\prime}}\right]\left[\bar{u}_{2^{\prime}} \gamma_{\mu} u_{2}\right]\left[\bar{u}_{2} \gamma_{\nu} u_{2^{\prime}}\right]\right)\right. \\
+\frac{1}{u^{2}}\left(\left[\bar{u}_{2^{\prime}} \gamma^{\mu} u_{1}\right]\left[\bar{u}_{1} \gamma^{\nu} u_{2^{\prime}}\right]\left[\bar{u}_{1^{\prime}} \gamma_{\mu} u_{2}\right]\left[\bar{u}_{2} \gamma_{\nu} u_{1^{\prime}}\right]\right)-\frac{1}{t u}\left(\left[\bar{u}_{1^{\prime}} \gamma^{\mu} u_{1}\right]\left[\bar{u}_{1} \gamma^{\nu} u_{2^{\prime}}\right]\left[\bar{u}_{2^{\prime}} \gamma_{\mu} u_{2}\right]\left[\bar{u}_{2} \gamma_{\nu} u_{2^{\prime}}\right]\right) \\
\left.+\frac{1}{t v}\left(\left[\bar{u}_{2^{\prime}} \gamma^{\mu} u_{1}\right]\left[\bar{u}_{1} \gamma^{\nu} u_{1^{\prime}}\right]\left[\bar{u}_{1^{\prime}} \gamma_{\mu} u_{2}\right]\left[\bar{u}_{2} \gamma_{\nu} u_{2^{\prime}}\right]\right)\right\}
\end{gathered}
$$

Applying the completeness relation $\sum_{s=1,2} u^{s}(p) \bar{u}^{s}(p)=\not p+m_{e}$ and neglecting the electron mass in the ultrarelativistic limit

$$
\begin{gathered}
\overline{|\mathcal{M}|^{2}}=\frac{e^{4}}{4}\left\{\frac{1}{t^{2}} \operatorname{Tr}\left[\not p_{1}^{\prime} \gamma^{\mu} \not p_{1} \gamma^{\nu}\right] \operatorname{Tr}\left[\not p_{2}^{\prime} \gamma_{\mu} \not p_{2} \gamma_{\nu}\right]\right. \\
+\frac{1}{u^{2}} \operatorname{Tr}\left[\not p_{2}^{\prime} \gamma^{\mu} \not p_{1} \gamma^{\nu}\right] \operatorname{Tr}\left[\not p_{1}^{\prime} \gamma_{\mu} \not p_{2} \gamma_{\nu}\right]-\frac{1}{t u} \operatorname{Tr}\left[\not p_{1}^{\prime} \gamma^{\mu} \not p_{1} \gamma_{\nu} \not p_{2}^{\prime} \gamma_{\mu} \not p_{2} \gamma^{\nu}\right] \\
\left.-\frac{1}{t u} \operatorname{Tr}\left[\not p_{2}^{\prime} \gamma^{\mu} \not \not_{1} \gamma^{\nu} \not p_{1}^{\prime} \gamma_{\mu} \not \not_{2} \gamma_{\nu}\right]\right\}
\end{gathered}
$$

Applying standard trace theorems yields 


$$
\begin{gathered}
\overline{|\mathcal{M}|^{2}}=\frac{e^{4}}{4}\left\{\frac{8}{t^{2}}\left[\left(p_{1}^{\prime} \cdot p_{2}^{\prime}\right)\left(p_{1} \cdot p_{2}\right)+\left(p_{1}^{\prime} \cdot p_{2}\right)\left(p_{1} \cdot p_{2}^{\prime}\right)\right]+\frac{8}{u^{2}}\left[\left(p_{1}^{\prime} \cdot p_{2}^{\prime}\right)\left(p_{1} \cdot p_{2}\right)+\left(p_{2}^{\prime} \cdot p_{2}\right)\left(p_{1} \cdot p_{1}^{\prime}\right)\right]\right. \\
\left.-\frac{8}{t u}\left[\left(p_{1} \cdot p_{2}\right)\left(p_{1}^{\prime} \cdot p_{2}^{\prime}\right)\right]-\frac{8}{t u}\left[\left(p_{1} \cdot p_{2}\right)\left(p_{1}^{\prime} \cdot p_{2}^{\prime}\right)\right]\right\}
\end{gathered}
$$

Using the Mandelstam variables, approximated in the ultrarelativistic limit, we obtain $\overline{|\mathcal{M}|^{2}}$ in Lorentz invariant form

$$
\overline{|\mathcal{M}|^{2}}=2 e^{4} \frac{s^{2}+u^{2}}{t^{2}}+\frac{s^{2}+t^{2}}{u^{2}}+\frac{2 s^{2}}{t u}
$$

Inserting $\overline{|\mathcal{M}|^{2}}$ into Equ. 2.2 for $\left.\frac{d \sigma}{d \Omega}\right|_{C M}$ as well as noting that, in the COM frame, $p_{1}=p_{2}$ and the Mandelstam variables are $s=4\left(p^{2}+m_{e}^{2}\right) \approx 4 p^{2}, t=$ $-2 p^{2}\left(1-\cos \theta_{C M}\right)$, and $u=-2 p^{2}\left(1+\cos \theta_{C M}\right)$, we obtain the differential cross section

$$
\left.\frac{d \sigma}{d \Omega}\right|_{C M}=\frac{e^{4}}{16 \pi^{2} s} \frac{\left(3+\cos ^{2} \theta_{C M}\right)^{2}}{\sin ^{4} \theta_{C M}}
$$

Expressed in terms lab frame variables, $s=2 m_{e} E_{2}$ and noting $\alpha=\frac{e^{2}}{4 \pi}$

$$
\left.\frac{d \sigma}{d \Omega}\right|_{C M}=\frac{\alpha^{2}}{2 m_{e} E_{2}} \frac{\left(3+\cos ^{2} \theta_{C M}\right)^{2}}{\sin ^{4} \theta_{C M}}
$$

\section{Parity violation in electron-electron scattering}

The differential cross section can be calculated using similar techniques as above or by the helicity amplitude method shown in this section. Both direct and crossed Feynman diagrams for identical particles for both the photon and the $\mathrm{Z}$ must be used in this calculation.

Here we show the derivation of $A_{P V}$ from Derman [48]. Considering the process

$$
e_{1}^{-}\left(p_{1}, s_{1}\right)+e_{2}^{-}\left(p_{2}, s_{2}\right) \rightarrow e_{1^{\prime}}^{-}\left(p_{1}^{\prime}, s_{1}^{\prime}\right)+e_{2^{\prime}}^{-}\left(p_{2}^{\prime}, s_{2}^{\prime}\right)
$$


where $(p, s)$ denote the 4-momenta and helicity states of the electrons. We use the approximation that $m_{e}$ is small compared with the CM energy $\sqrt{s}=$ $\sqrt{\left(p_{1}+p_{2}\right)^{2}}$. We define the variable $y=-\frac{\left(p_{1}-p_{1}^{\prime}\right)^{2}}{s}=\sin ^{2}\left(\theta_{C M} / 2\right)$, where $0 \leq$ $y \leq 1$, representing the fraction of incoming electron energy imparted to the other electron after collision, and where $\theta_{C M}$ is the CM scattering angle. This allows the definition of momentum transfer as $Q^{2}=-\left(p_{1}-p_{1}^{\prime}\right)^{2}=y s$.

In order to have a parity violating asymmetry, we must included both weak and electromagnetic interactions in our assumed Lagrangian $\mathcal{L}=|e| A_{\mu} \bar{e} \gamma^{\mu} e-$ $g_{0} Z_{\mu} \bar{e} \gamma^{\mu}\left(V+A \gamma_{5}\right) e$. Where the photon field is included by $A_{\mu}$, the neutral heavy boson field is included by $Z_{\mu},|e|$ denotes the electron charge, $e$ denotes the electron field, and $g_{0}$ the coupling strength between the electron and the Z-boson of mass $M_{z}$. The weak vector and axial vector components $V$ and $A$ must be included in this calculation since they give rise to the parity violating asymmetry defined by the helicity dependence in the differential cross-sections $A_{P V}=\frac{\sigma_{R}-\sigma_{L}}{\sigma_{R}+\sigma_{L}}$.

The individual amplitudes are denoted $\mathcal{M}_{i}^{j}$ where $i=Z, \gamma$ for the weak and electromagnetic amplitudes and $j=d, c$ for the direct and crossed Feynman diagrams. The total amplitude is given by

$$
\mathcal{M}=i\left(\mathcal{M}_{\gamma}^{d}+\mathcal{M}_{\gamma}^{c}+\mathcal{M}_{Z}^{d}+\mathcal{M}_{Z}^{c}\right)
$$

where we obtain crossed amplitudes by swapping $p_{2}^{\prime} \leftrightarrow p_{1}^{\prime}, y \leftrightarrow 1-y$ and performing Fermi sign change as follows (where the spin state of each electron is suppressed for clarity).

$$
\begin{gathered}
\mathcal{M}_{\gamma}^{d}=\frac{-e^{2}}{y s} \bar{u}_{1^{\prime}} \gamma_{\mu} u_{1} \cdot \bar{u}_{2^{\prime}} \gamma^{\mu} u_{2} \quad \mathcal{M}_{\gamma}^{c}=\frac{e^{2}}{(1-y) s} \bar{u}_{2^{\prime}} \gamma_{\mu} u_{1} \cdot \bar{u}_{1^{\prime}} \gamma^{\mu} u_{2} \\
\mathcal{M}_{Z}^{d}=\frac{-g_{0}^{2}}{M_{Z}^{2}} \bar{u}_{1^{\prime}}\left[V \gamma_{\mu}+A \gamma_{\mu} \gamma_{5}\right] u_{1} \cdot \bar{u}_{2^{\prime}}\left[V \gamma^{\mu}+A \gamma^{\mu} \gamma_{5}\right] u_{2} \\
\mathcal{M}_{Z}^{c}=\frac{g_{0}^{2}}{M_{Z}^{2}} \bar{u}_{2^{\prime}}\left[V \gamma_{\mu}+A \gamma_{\mu} \gamma_{5}\right] u_{1} \cdot \bar{u}_{1^{\prime}}\left[V \gamma^{\mu}+A \gamma^{\mu} \gamma_{5}\right] u_{2}
\end{gathered}
$$


We can approximately express the eigenspinors $u_{p}^{s= \pm 1}$ as

$$
u_{p}^{1}=\sqrt{\frac{E}{2 m}}\left(\begin{array}{c}
\cos \left(\theta_{C M} / 2\right) \\
\sin \left(\theta_{C M} / 2\right) \\
\cos \left(\theta_{C M} / 2\right) \\
\sin \left(\theta_{C M} / 2\right)
\end{array}\right) \quad u_{p}^{-1}=\sqrt{\frac{E}{2 m}}\left(\begin{array}{c}
-\sin \left(\theta_{C M} / 2\right) \\
\cos \left(\theta_{C M} / 2\right) \\
\sin \left(\theta_{C M} / 2\right) \\
-\cos \left(\theta_{C M} / 2\right)
\end{array}\right)
$$

where $\theta_{C M}$ is the center-of-momentum scattering angle and, neglecting the electron mass, $E=\sqrt{s} / 2$.

We organize the various contributions to $\mathcal{M}$ in the form of helicity amplitude matrices, where $\mathrm{R}(\mathrm{L})$ denote $+(-)$ electron helicity and matrix row(column) indicate the initial(final) electron helicities. Diagonal components correspond to interactions where helicity is conserved, the time reversal invariance is manifested in the matrix symmetry, and rotational invariance due to identical particles is expressed as $\mathcal{M}_{R L, R L}=\mathcal{M}_{L R, L R}$.

\begin{tabular}{|c|c|c|c|c|c|}
\hline & & $\mathrm{RR}$ & $\mathrm{RL}$ & $\mathrm{LR}$ & $\mathrm{LL}$ \\
\hline $\mathcal{M}_{\gamma}(d)=\frac{-e^{2}}{2 y m^{2}} \times$ & $\mathrm{RR}$ & 1 & 0 & 0 & 0 \\
& $\mathrm{RL}$ & 0 & $1-y$ & 0 & 0 \\
& $\mathrm{LR}$ & 0 & 0 & $1-y$ & 0 \\
& $\mathrm{LL}$ & 0 & 0 & 0 & 1 \\
\hline \multirow{3}{*}{$\mathcal{M}_{\gamma}(c)=\frac{-e^{2}}{2(1-y) m^{2}} \times$} & $\mathrm{RR}$ & 1 & 0 & 0 & 0 \\
& $\mathrm{RL}$ & 0 & 0 & $y$ & 0 \\
& $\mathrm{LR}$ & 0 & $y$ & 0 & 0 \\
& $\mathrm{LL}$ & 0 & 0 & 0 & 1 \\
$\mathcal{M}_{Z}(d)=\frac{-g_{0}^{2} s}{2 M_{Z}^{2} m^{2}} \times$ & $\mathrm{RR}$ & $(V+A)^{2}$ & 0 & 0 & 0 \\
& $\mathrm{RL}$ & 0 & $(1-y)\left(V^{2}-A^{2}\right)$ & 0 & $(1-y)\left(V^{2}-A^{2}\right)$ \\
& $\mathrm{LR}$ & 0 & 0 & 0 & 0 \\
& $\mathrm{LL}$ & 0 & 0 & 0 & 0 \\
$\mathcal{M}_{Z}(c)=\frac{-g_{0}^{2} s}{2 M_{Z}^{2} m^{2}} \times$ & $\mathrm{RR}$ & $(V+A)^{2}$ & 0 & $y\left(V^{2}-A^{2}\right)$ & 0 \\
& $\mathrm{RL}$ & 0 & 0 & 0 & $\left(V^{2}-A^{2}\right)$ \\
\hline
\end{tabular}

Table 2.1: Helicity amplitude matrices for direct and crossed contributions to ee scattering via $\gamma$ and $\mathrm{Z}$ exchange [48]. Row(column) denote initial (final) electron helicity states $\mathrm{R}(\mathrm{L}),+1(-1)$ 
Helicity is conserved in the $\gamma_{\mu}$ and $\gamma_{\mu} \gamma_{5}$ vertices and the vector and axial currents are given by

$$
\begin{aligned}
& \bar{u}_{p^{\prime}}^{s^{\prime}} \gamma_{\mu} u_{p}^{s}=\frac{\sqrt{s}}{2 m}\left(\cos \left(\frac{\theta-\theta^{\prime}}{2}\right),\right. \sin \left(\frac{\theta+\theta^{\prime}}{2}\right), \\
&\left.i(-1)^{(1+s) / 2} \sin \left(\frac{\theta-\theta^{\prime}}{2}\right), \cos \left(\frac{\theta+\theta^{\prime}}{2}\right)\right) \delta_{s, s^{\prime}} \\
& \bar{u}_{p^{\prime}}^{s^{\prime}} \gamma_{\mu} \gamma_{5} u_{p}^{s}=-(-1)^{(1+s) / 2} \bar{u}_{p^{\prime}}^{s^{\prime}} \gamma_{\mu} u_{p}^{s}
\end{aligned}
$$

Using the scattering cross-section for an electron of helicity $i$ incident on a target $\mathrm{j}, \sigma_{i j}=\sum_{k l}\left|\mathcal{M}_{i j, k l}\right|^{2}$, we can compute the scattering asymmetry for $\mathrm{R}(\mathrm{L})$ polarized electrons incident off of an unpolarized target where we note that by rotational invariance $\sigma_{R L}=\sigma_{L R}$ :

$$
\begin{aligned}
& A_{P V}=\frac{\left(\sigma_{R L}+\sigma_{R R}\right)-\left(\sigma_{L L}+\sigma_{L R}\right)}{\left(\sigma_{R L}+\sigma_{R R}\right)+\left(\sigma_{L L}+\sigma_{L R}\right)}=\frac{\sigma_{R R}-\sigma_{L L}}{\sigma_{R R}+\sigma_{L L}+2 \sigma_{L R}} \\
& =\frac{\left|\mathcal{M}_{R R, R R}\right|^{2}-\left|\mathcal{M}_{L L, L L}\right|^{2}}{\left|\mathcal{M}_{R R, R R}\right|^{2}+\left|\mathcal{M}_{L L, L L}\right|^{2}+2\left|\mathcal{M}_{L R, L R}\right|^{2}+2\left|\mathcal{M}_{L R, R L}\right|^{2}}
\end{aligned}
$$

From summing the elements of our table to leading order in $g_{0}^{2} / M_{z}^{2}$, assuming the coupling strength to the $\mathrm{Z}$ is small compared with $M_{z}$, valid for low $Q^{2}$ where CM energy $\sqrt{s}<<M_{z}$, we obtain

$$
A_{P V} \approx \frac{2 g_{0}^{2} V A Q^{2}}{\pi \alpha M_{z}^{2}} \frac{1-y}{1+y^{4}+(1-y)^{4}}
$$

In the GWS Standard Model, $g_{0}=\frac{2|e|}{\sin \left(2 \theta_{W}\right)}, V=\sin ^{2} \theta_{W}-1 / 4, A=1 / 4$ and the $\mathrm{Z}$ boson mass is given by $\frac{G_{F}}{\sqrt{2}}=\frac{|e|^{2}}{2 M_{z}^{2} \sin ^{2}\left(2 \theta_{W}\right)}$, giving $\frac{2 g_{0}^{2} V A}{\pi \alpha M_{z}^{2}}=\frac{G_{F}}{\sqrt{2} \pi \alpha}\left(4 \sin ^{2} \theta_{W}-1\right)$. 
So the Standard Model prediction for $A_{P V}$ is given by

$$
A_{P V}^{G W S} \approx \frac{G_{F} Q^{2}}{\sqrt{2} \pi \alpha}\left(4 \sin ^{2} \theta_{W}-1\right) \frac{1-y}{1+y^{4}+(1-y)^{4}}=m E \frac{G_{F}}{\sqrt{2} \pi \alpha} \frac{4 \sin ^{2} \theta}{\left(3+\cos ^{2} \theta\right)^{2}} Q_{W}^{e}
$$

\section{EM and weak charges}

$A_{P V}$ in electron-electron scattering probes the electron's neutral current. The charges for the electromagnetic and weak neutral currents are shown in Fig. 2.2. $q^{E M}$ is the electromagnetic charge of the particle, while $g^{A}, g^{V}, g^{R}$, and $g^{L}$, are the weak axial, vector, right-helicity and left helicity charges (with $g^{R}=g^{V}+g^{A}$ and $g^{L}=g^{V}-g^{A}$ ) [46]. Different experiments address different charge combinations and compliment each other. For example MOLLER addresses the weak charge of the electron, and Qweak addresses the proton weak charge.

\begin{tabular}{|c|c|c|c|c|c|}
\hline Particle & $q^{E M}$ & $g^{A}$ & $g^{V}$ & $g^{R}$ & $g^{L}$ \\
\hline$e^{-}$ & -1 & $\frac{1}{4}$ & $\begin{array}{c}-\frac{1}{4}+\sin ^{2} \theta_{W} \\
\sin ^{2} \theta_{W}\end{array}$ & $\begin{array}{c}-\frac{1}{2}+\sin ^{2} \theta_{W} \\
\end{array}$ \\
\hline$u$ & $\frac{2}{3}$ & $-\frac{1}{4}$ & $\begin{array}{c}\frac{1}{4}-\frac{2}{3} \sin ^{2} \theta_{W} \\
\sim 0.09\end{array}$ & $\begin{array}{c}-\frac{2}{3} \sin ^{2} \theta_{W} \\
\sim-0.16\end{array}$ & $\begin{array}{c}\frac{1}{2}-\frac{2}{3} \sin ^{2} \theta_{W} \\
\sim 0.34\end{array}$ \\
\hline$d, s$ & $-\frac{1}{3}$ & $\frac{1}{4}$ & $\begin{array}{c}-\frac{1}{4}+\frac{1}{3} \sin ^{2} \theta_{W} \\
\frac{1}{3} \sin ^{2} \theta_{W}\end{array}$ & $\begin{array}{c}-\frac{1}{2}+\frac{1}{3} \sin ^{2} \theta_{W} \\
\end{array}$ \\
\hline & & $\sim-0.17$ & $\sim 0.08$ & $\sim-0.42$ \\
\hline
\end{tabular}

Table 2.2: Electromagnetic and weak neutral charges [46]

\section{Radiative Corrections}

As previously stated in Eq. 2.1, the parity-violating asymmetry in Møller scattering at tree level (Fig. 2-1) is given by:

$$
A_{P V}=m E \frac{G_{F}}{\sqrt{2} \pi \alpha} \frac{2 y(1-y)}{1+y^{4}+(1-y)^{4}} Q_{W}^{e}
$$


where $Q_{W}^{e}=1-4 \sin ^{2} \theta_{W}$.

MOLLER experimental precision $\left(<0.8 \mathrm{ppb}\right.$ on $\left.A_{P V}\right)$ requires that the Standard Model predictions for $A_{P V}$ and $\sin ^{2} \theta_{W}$ must be carried out not only at tree level, as shown in previous sections, but with full treatment of one-loop and leading two-loop radiative corrections [33]. The 1st order contributions in the perturbative expansion Feynman treatment, are the tree-level diagrams in Fig. 2-1. Higher-order one-loop corrections consisting of $\gamma-Z$ mixing diagrams and the W-loop contribution to the anapole moment are shown in Fig 2-2 [46]. Leading box diagram contributions involving two heavy bosons, are shown in Fig. 2-3.

The weak mixing angle and the electron weak charge are initially defined by their relationship at tree-level $Q_{W}^{e}=1-4 \sin ^{2} \theta_{W}$. But when a measurement is performed, it probes a sum over all orders of the perturbative expansion. The relationship between $Q_{W}^{e}$ and $\sin ^{2} \theta_{W}$ is modified at the 1-loop level [5] [6] [7] and is dependent on the energy scale at which the experiment is carried out. In other words, $\sin ^{2} \theta_{W}$ "runs" with different $Q^{2}$.[1].

The error on the SM prediction due to uncertainty on radiative corrections is fairly small $<0.4 \mathrm{ppb}$. This is smaller than the expected MOLLER statistical error. Even so, to further reduce the error on the prediction to $<0.2 \mathrm{ppb}$ [11], there is an ongoing theoretical effort to investigate several classes of diagrams beyond one-loop [8] [9] [10] at MOLLER kinematics. [1]. Theoretical uncertainties for the Møller $A_{P V}$ are expected to be extremely well controlled by the time MOLLER runs.
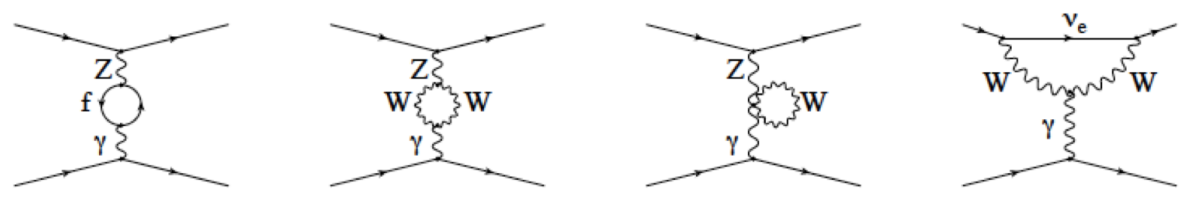

Figure 2-2: Significant 1-loop radiative corrections: $\gamma-Z$ mixing diagrams and W-loop contribution to the anapole moment (reproduced from Ref. [5]) [1] 


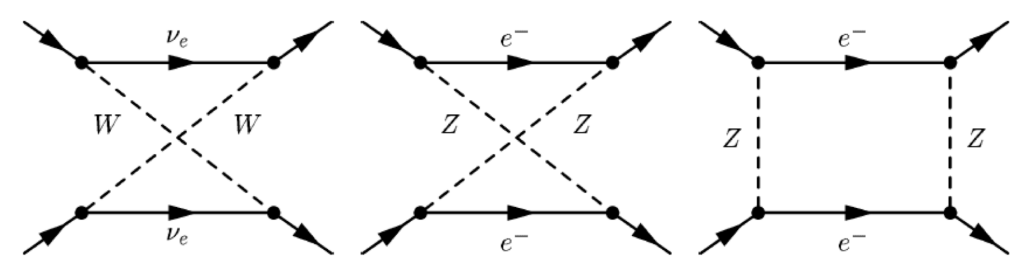

Figure 2-3: Box diagram contributions to Møller scattering involving two heavy bosons. Crossed diagrams also contribute [46]

\section{Running of $\sin ^{2} \theta_{W}$ and Renormalization}

When including high-order terms, it is important to avoid divergent behavior. In most cases, the high order terms aren't divergent because the coupling constant at tree level $\alpha / 4 \pi \sim 0.001$ is small and higher-order terms are suppressed. When there is divergent behavior, a renormalization procedure is employed which redefines the coupling constants, used at tree level, in terms of experimental values that inherently probe all orders. The weight given to certain higher-order diagrams in this scheme vary depending on the energy scale, defined by the $Q^{2}$ of the experiment used. This energy dependence can intuitively be understood as follows. Lower energies probe with poorer resolution than higher energies, they probe less deeply, and suffer from a "screening" of the electron's charge by a virtual particle cloud. Higher energies probe smaller distance scales with deeper penetration and so see a larger effective value of the electron's charge. As the energy scale is varied, contributions from higher-order diagrams evolve via a change in the effective value of the coupling constants [46].

This evolution of coupling constants in a renormalization scheme is shown in Fig.2-4b as the running of the weak mixing angle $\sin ^{2} \theta_{W}$ from its value at the Zpole $Q^{2}=M_{Z}^{2}$ to lower $Q^{2}$, referred to as "screening" in Fig. 2-4a. Czarnecki and Marciano [5] have calculated the 1-loop radiative corrections using the "Modified Minimal Subtraction" $(\overline{M S})$ scheme and defined the renormalized weak mixing 
angle at an arbitrary mass scale $\mu$ as [12]

$$
\sin ^{2} \theta_{W}(\mu)_{\overline{M S}}=e^{2}(\mu)_{\overline{M S}} / g^{2}(\mu)_{\overline{M S}}
$$

analogous to the definition in Sec. 1.1.1. Different $\sin ^{2} \theta_{W}$ experimental inputs at various $\mu=|Q|$ can be used in the $\overline{M S}$ renormalization scheme. If several experimental inputs are used with $Q^{2}$ determined from each experiment's average momentum conditions, the discontinuous $\sin ^{2} \theta_{W}$ curve is obtained as shown in Fig. 2-4a where discontinuities occur at $\mu=|Q|=$ particle masses.

A smooth curve can be obtained by only defining the renormalized weak mixing angle at one energy scale, $M_{Z}$, where Z-pole measurements have been performed and the weak mixing angle measured to be $\sin ^{2} \theta_{W}\left(m_{Z}\right) \overline{M S}=0.2314[13]$ [14] , and incorporating perturbative $\gamma-Z$ mixing through vacuum polarization. The running of $\sin ^{2} \theta_{W}$ by $3 \%$ of its Z-pole value from $Q^{2}=M_{Z}^{2}$ to $Q^{2}<<1$ arises primarily from fermion vacuum polarization effects as shown in Fig.2-4b, whereas the running beyond the Z-pole is dominated by boson vacuum polarization effects [12].

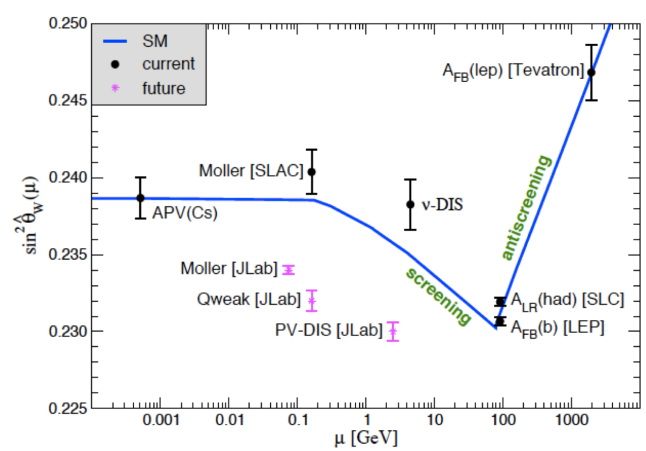

(a)

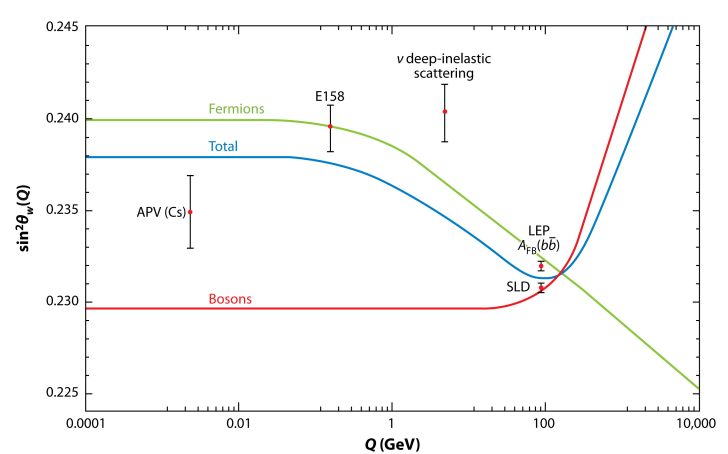

(b)

Figure 2-4: Running of $\sin ^{2} \theta_{W}$ (a) Ref. [33] (b) in 1-loop calculations by Marciano [12]

Derivation of the $Q^{2}$ dependence of the weak mixing angle $\theta_{W}$ is a challenge for any theory going beyond the SM. Measurement of $\sin ^{2} \theta_{W}$ at various $Q^{2}$ is 
an opportunity for parity experiments to extend the reach of new physics. The weak mixing angle played a central role in electroweak theory and testing it at the quantum loop level has been the central focus of precision electroweak physics over the past couple of decades[1].

Since $\sin ^{2} \theta_{W}$ runs as a function of $Q^{2}$ due to electroweak radiative corrections, one can use $\sin ^{2} \theta_{W}$ as a bookkeeping parameter to compare the consistency of the full $Q^{2}$ range of weak neutral current measurements, as shown in Fig. 2-5. New BSM mediators can not only affect the weak mixing angle at $Q^{2}$ near their mass scales, but at low $Q^{2}$ as well. Fig. 2-5b shows the running of $\sin ^{2} \theta_{W}$ with $Q^{2}$ as well as a series of past and future experiments which aim to measure the weak mixing angle at various $Q^{2}$ to very high precision.

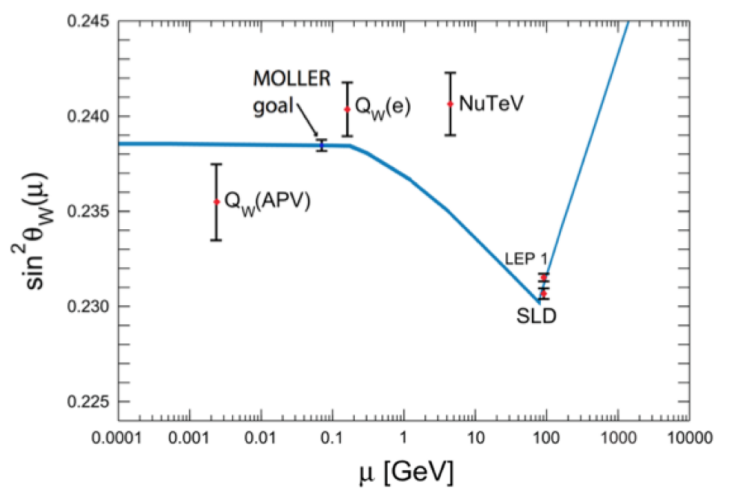

(a)

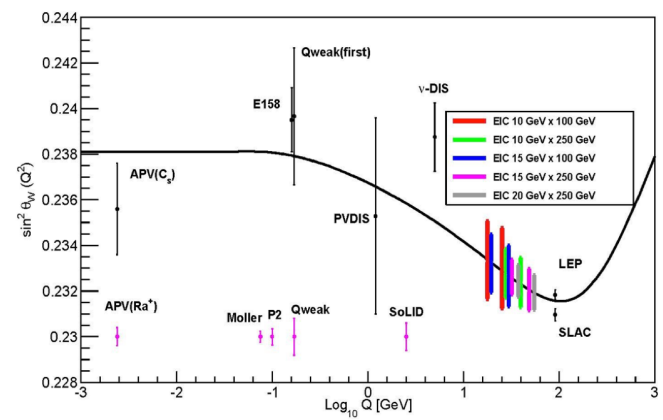

(b)

Figure 2-5: Running of $\sin ^{2} \theta_{W}$ (a) Møller precision goal is shown compared with other past experiments (base figure [12]) (b) Past experiments are shown in black. The precision goals for future experiments are shown in pink. EIC potential future measurements are shown in multicolor [70]

The goal of several parity experiments which measure $\sin ^{2} \theta_{W}$ is to extend the reach for new physics beyond the Standard Model by performing ultra-precise measurements sensitive to new parity-violating interactions. The best measurements of the weak mixing angle at lower energies are the SLAC E158 measurement [16], the measurement of the weak charge of ${ }^{133} \mathrm{Cs}$ [17] [18] via studies of table-top atomic parity violation, and the JLab Qweak measurement of the proton weak 
charge via electron proton scattering [15]. By comparison, the precision goal for MOLLER is quite high, matching the precision of the best collider (Z-pole) measurement shown in Fig. 2-5a.

\section{High energy vs. Low energy Measurements}

An important advantage of MOLLER is that it will be carried out at a low 4-momentum transfer scale $Q^{2}<<M_{Z}^{2}$, in contrast to the high energy SLC and CERN measurements, which were carried out at the Z-pole. Near the Z-pole, new physics can't interfere with the Z-amplitude, but at low $Q^{2}<<M_{Z}^{2}$, the interference term dominates such that $A_{P V} \sim \frac{1}{Q^{2}-M_{Z}^{2}}$. This difference of energy scales in the MOLLER experiment and the Z-pole measurements enhances the sensitivity of the MOLLER measurement dramatically to as yet undiscovered weak interactions at the $\mathrm{TeV}$ scale, as discussed in the next section.

\subsubsection{BSM and Sensitivity to New Physics}

Historically both direct and indirect searches for new physics have complimented one another in the development of electroweak theory [33]. The extreme precision of indirect searches at low energies, like MOLLER, makes for probes of new physics that would become dominantly manifest at super-massive high energy scales. The MOLLER precision on $A_{P V}$ and the electron weak charge $Q_{W}^{e}$ is capable of probing new neutral current amplitudes as weak as $10^{-3} G_{F}$ from Beyond the Standard Model (BSM) undiscovered dynamics. It will be the most sensitive probe of new flavor and CP conserving neutral current interactions in the leptonic sector until the advent of a linear collider or a neutrino factory [33].

The MOLLER experimental goal on the weak mixing angle is as precise as the best collider Z-pole measurements to date. This low $Q^{2}$ measurement compliments the Z-pole measurements because the two most precise independent determinations of the weak mixing angle $\sin ^{2} \theta_{W}$ differ by $3 \sigma$ from each other. Choosing 
one or the other experimental value leads to inconsistency with other electroweak measurements and constraints on the Higgs boson mass $m_{H}$, and implies very different new high-energy dynamics and MOLLER, being at the same level of precision, could address this issue [33]. It is worth mentioning that this is superseded a bit by expected LHC results which will be similarly precise at the Z-pole [22].

MOLLER is additionally complimentary to other precision low-energy experiments and the energy frontier efforts at the LHC because it has sensitivities to a specific linear combination of left- and right-handed four electron operators which collider measurements are relatively insensitive to [1]. While intuitively, there can be hidden physics at low E that only becomes manifest at high energies, there also may be hidden physics at high energies measurements which only become manifest in low-energy parity violation experiments. If the LHC sees agreement with the Standard Model at $14 \mathrm{TeV}$, MOLLER will help in the discovery of hidden weak scale BSM physics scenarios that could escape LHC detection: compressed supersymmetry [23], lepton number violating doubly charged scalar mediators [24], and light MeV-scale dark matter mediators such as the "dark" Z [20] [21]. If the LHC sees an anomaly, MOLLER will help provide constraints to choose between various BSM theories: Minimal Supersymmetric Standard Model observed through radiative loop effects (R-parity conserving) or tree-level interactions (R-parity violating) [25] [26] and TeV-scale $Z^{\prime}$ bosons which arise in many theories [27] [1]. These theories can be distinguished using low-energy precision measurements.

MOLLER has competitive sensitivity to a number of new plausible new-physics scenarios each of which are discussed below briefly. While standing alone, MOLLER measures a single number that cannot distinguish between various models, taken in conjunction with existing measurements, it will constrain the parameter space possible new physics scenarios[46]. It has a unique window to provide a glimpse of new physics beyond the SM and will compliment the information acquired in experiments at the high energy frontier.[46]. 


\section{Running of $\sin ^{2} \theta_{W}$ BSM}

As stated previously the weak mixing angle $\sin ^{2} \theta_{W}$ runs as a function of the energy scale at which measurements of it are carried out. The tree-level electroweak theory prediction $Q_{W}^{e}=1-4 \sin ^{2} \theta_{W}$ is modified as a function of $Q^{2}$ due to electroweak radiative corrections within the Standard Model. There are further corrections from Beyond the Standard Model dynamics which could become manifest in low $Q^{2}$ measurements of $\sin ^{2} \theta_{W}$ with enhanced sensitivity to new physics. "Oblique corrections" $[73,74]$ which come from very massive new particles could modify low-energy coupling constants, and hence $\sin ^{2} \theta_{W}$, through higher-order loop diagrams [46]. At mass scales $Q^{2} \leq M_{Z}^{2}$, these corrections can be described by a parameter $\mathrm{X}[77,78]$,

$$
\sin ^{2} \hat{\theta}_{W}\left(M_{Z}^{2}\right)-\sin ^{2} \hat{\theta}_{W}(0) \sim \alpha X
$$

where $\alpha$ is the fine structure constant and $\mathrm{X}$ can be interpreted as a measure of the running of $\sin ^{2} \theta_{W}$ due to BSM physics [46]. MOLLER would be sensitive to measuring the parameter $\mathrm{X}$ at an extremely high level of sensitivity, and if nonzero, would indicate that the mass scale for new physics is not much higher than $M_{Z}$ and that the new physics does not couple strongly to the $Z^{0}$ [46].

The weak mixing angle is a book-keeping parameter across various $Q^{2}$ which we can use to keep track of a variety of experiments at different energy scales (as in Fig. 2-5). But we can also compare the value of $\sin ^{2} \theta_{W}$ with other fundamental parameters such as the Higgs mass $m_{H}$ or top quark mass $m_{t}$ and define how individual experiments constrain the available parameter space, putting tighter limits on BSM theories. Fig. 2-6 shows a comparison, both measured and calculated, of $\sin ^{2} \theta_{W}$ vs. Higgs mass $m_{H}$. The yellow band is the world average of $\sin ^{2} \theta_{W}$ measurements. The black points are the two most precise measurements at $Q^{2}<<M_{Z}^{2}$. The projected MOLLER error is shown in red. In this plot, we observe how LHC experiments and MOLLER compliment one another in terms 
of confining the available parameter space. We also can clearly observe how the high precision of MOLLER might resolve the strain between two fairly precise experiments, E-158 and atomic PV shown here.

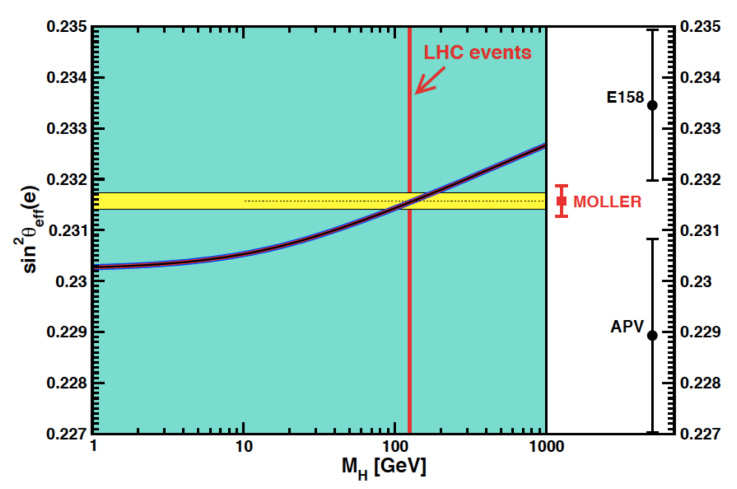

Figure 2-6: $\sin ^{2} \theta_{W}$ vs $m_{H}$. The yellow band is the world average. The black points are the two most precise measurements at $Q^{2}<<M_{Z}^{2}$. The projected MOLLER error is shown in red.[1]

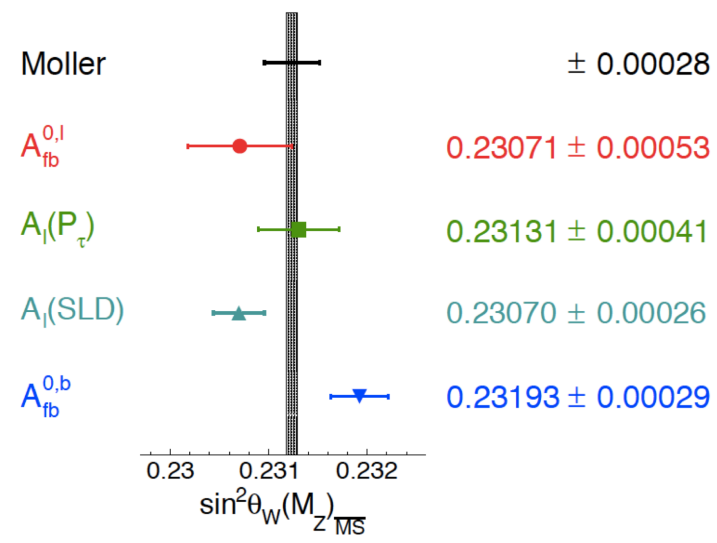

Figure 2-7: The four best $\sin ^{2} \theta_{W}$ measurements and the projected error of the MOLLER proposal. The black band represents the theoretical prediction for $m_{H}=126 \mathrm{GeV}$ (Measured value $m_{H}=124.98 \pm 0.28 \mathrm{GeV}$ [28]).[1]

Figure 2-7 shows further experiments, the four best measurements of $\sin ^{2} \theta_{W}$ from studies of $Z_{0}$ decays [37], the projected uncertainty for MOLLER, and the theoretical prediction for $m_{H}=126 \mathrm{GeV}$ (measured value $m_{H}=124.98 \pm 0.28 \mathrm{GeV}$ [28]). The top point is the MOLLER uncertainty, which would achieve a sensitivity 
on $\sin ^{2} \theta_{W}$ of $\pm 0.00028^{1}$. The bottom two points the Z-pole measurements of $\sin ^{2} \theta_{W}: A_{l}(S L D)$ the left-right asymmetry in Z production at SLC and $A_{f b}^{0 b}$ the forward-backward asymmetry in Z decays to b-quarks. We can see the strain between the Z-pole measurements, each of which implies different BSM dynamics [38]. Given the scatter in previous measurements, MOLLER will be a particularly useful measurement due to its comparable precision. MOLLER will be the first low $Q^{2}$ measurement to match the precision of the best high energy measurements at the Z-pole, extending the discovery reach for new physics to the multi- $\mathrm{TeV}$ scale [1].

\section{Precision of Moller}

MOLLER is expected to measure the left-right parity violating asymmetry $A_{P V} \sim 35.6 \mathrm{ppb}$ in Møller scattering to sub-ppb precision ( $\left.<0.8 \mathrm{ppb}\right)$. Including both statistical and systematic uncertainties, this is an effective $2.4 \%$ measurement on the electron weak charge $Q_{W}^{e}$, since $A_{P V}$ is directly proportional to the electron weak charge (Equ.2.3) :

$$
A_{P V}=m_{e} E \frac{G_{F}}{\sqrt{2} \pi \alpha} \frac{4 \sin ^{2} \theta_{C M}}{\left(3+\cos ^{2} \theta_{C M}\right)^{2}} Q_{W}^{e}
$$

We infer the precision on the weak mixing angle by examining the tree-level relationship between $Q_{W}^{e}$ and $\sin ^{2} \theta_{W}$

$$
Q_{W}^{e}=4 g_{e e}=4 \rho \cdot g_{V_{e}} \cdot g_{A_{e}}=1-4 \sin ^{2} \theta_{W}
$$

where $\rho=\frac{M_{W}^{2}}{M_{Z}^{2} \cos ^{2} \theta_{W}}$. As the weak mixing angle runs due to radiative corrections, $\sin ^{2} \theta_{W}$ increases by $3 \%$ from its Z-pole value, but the electron weak charge $Q_{W}^{e}$ decreases to $45 \%$ of its original $\mathrm{Z}$ pole value, due to its being very near zero. A small fractional change in $\sin ^{2} \theta_{W}$ introduces a much larger fractional change

\footnotetext{
${ }^{1}$ Note that estimates of this sensitivity are subject to change
} 
in $Q_{W}^{e}$ and hence $A^{P V}$. Conversely, MOLLER's sensitivity to $\sin ^{2} \theta_{W}$ is greatly enhanced compared to the fractional sensitivity on $Q_{W}^{e}$. As previously stated in Sec. 2.1.1, the closer $A_{P V}$ and $Q_{W}^{e}$ are to zero, the closer $\sin ^{2} \theta_{W}$ is to $1 / 4$, the greater the enhancement of the corresponding fractional sensitivity on $\sin ^{2} \theta_{W}$ : a $2.4 \%$ measurement of $A_{P V}$ yields a $\sim 0.1 \%$ measurement of $\sin ^{2} \theta_{W}$ [46].

\section{Mass Reach}

In quantifying the significance of a precision low $Q^{2}$ experiment, we speak in terms of "mass reach". To compare various experiments, there is a need for a model independent way to: quantify the effects of new high energy dynamics in low-energy processes, translate the high precision at low-energy into the high energy regime, and express the 'reach' of a precision experiment in a standard way. The typical standard way is to treat new low-energy neutral current interactions, with $Q^{2}<<M_{x}^{2}$ of unknown mediator X, as contact interactions, where the denominator in the $A_{P V, X} \sim \frac{1}{Q^{2}-M_{X}^{2}} \sim \frac{4 \pi}{\Lambda^{2}}$, is $\sim Q^{2}$ independent, and is quantified at a mass scale $\Lambda$, where here we have switched to using natural units.

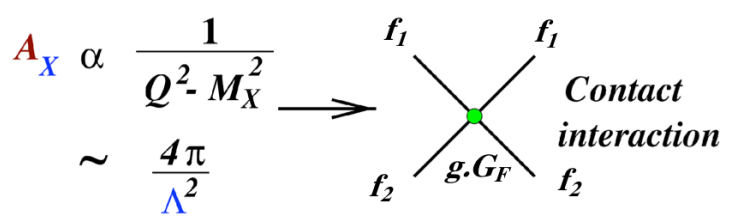

Figure 2-8: Fermion contact interaction with unknown weak mediator X [1] [70]

For each fermion and handedness combination, the interaction is characterized by a ratio of the strength of the coupling $g$ to the mass scale $\Lambda$ [36] [33]

$$
\mathcal{L}=\sum_{i, j=L, R} \frac{\left(g_{i j}^{12}\right)^{2}}{2 \Lambda_{i j}^{2}} \bar{e}_{1 i} \gamma_{\mu} e_{1 i} \bar{e}_{2 j} \gamma_{\mu} e_{2 j}
$$

where $e_{L / R}=\frac{1}{2}\left(1 \mp \gamma_{5}\right) \psi_{e}$

Taking the Møller experiment as an example, a precision of $2.4 \%$ gives a sensitivity of $7.5 \mathrm{TeV}$ for the ratio of mass to coupling strength. 


$$
\frac{\Lambda}{\sqrt{\mid g_{R R}^{2}-g_{L L}^{2}}}=\frac{1}{\sqrt{\sqrt{2} G_{F}\left|\Delta Q_{W}^{e}\right|}}=7.5 \mathrm{TeV}
$$

To define conventional "mass limits" for new contact interactions, to get at a mass scale for the new mediator, we must assume some nominal coupling constant. Based on models of lepton compositeness characterized by strong coupling dynamics we let $\sqrt{\mid g_{R R}^{2}-g_{L L}^{2}}=2 \pi$. We should emphasize this is a standard coupling constant used for contact interactions which gives the mass reach scale for comparison with other measurements [2]. This standard coupling strength gives mass reach scales of up to $47 \mathrm{TeV}$ for the new mediator from the MOLLER precision on $A_{P V}$.

Based on certain assumptions on isospin structure and strong coupling other comparative estimates [35] for mass reach of various past and future precision experiments are as follows: E158 17 TeV, PV- 8 TeV, Qweak $\sim 33 \mathrm{TeV}$, Møller $\sim 39 \mathrm{TeV}, \mathrm{P} 2 \sim 49 \mathrm{TeV}$, Solid $\sim 22 \mathrm{TeV}$. Compared with other experiments, the mass reach scale of Møller is quite high, matching the precision of the best collider (Z-pole) measurement shown in Fig. 2-5.

\section{Complimentary Measurements}

Certain BSM dynamics can have a high impact on low $Q^{2}$ observables while having a suppressed impact collider observables. The low-energy measurements are sensitive to interference effects which are suppressed at the Z-pole [1], so the low-energy and high energy measurements can be complimentary. We take as an example, the dynamics of a potential new Z' boson at contact interaction scales.

\section{Case: LHC observes an anomaly}

If the LHC observes an anomaly, then MOLLER will have the sensitivity to constrain various possible BSM scenarios to explain the anomaly [1]. MOLLER will be sensitive to new, super-massive $Z^{\prime}$ bosons, new particles predicted by the 
Minimal Supersymmetric Standard Model (MSSM), as well as electron compositeness scenarios.

\section{$Z^{\prime}$ bosons}

Many BSM theories predict the existence of new, supermassive Z' bosons with masses in the TeV range. Many GUTs and certain string theory models, allowing for one or more large extra dimensions, predict additional Z' bosons which are Kaluza-Klein excitations of the SM photon and $Z^{0}$ with masses at the $\mathrm{TeV}$ scale [46]. The LHC, while able to detect and measure the mass of such particles up to $5 \mathrm{TeV}$, is unable to subject Z'-bosons to detailed measurements to learn about their properties. If the LHC were to detect a super-massive Z', the low-energy precision electroweak MOLLER measurement would help to decipher what has actually been discovered [33]. MOLLER would help disentangle all of the chiral $Z^{0}$ couplings to SM particles [39] and providing important constraints [1].

Collider Z-pole measurements are only sensitive to the chiral couplings $g_{R L}^{2}$ and the combination $g_{R R}^{2}+g_{L L}^{2}$. At best, LHC measurements would be able to measure the ratio of the chiral leptonic couplings. Whereas MOLLER $A_{P V}$ is sensitive to the parity violating coupling constant combination $g_{R R}^{2}-g_{L L}^{2}$. For example, for the Z' boson appearing in $\mathrm{SO}(10)$ Grand Unified Theories,

$$
\sqrt{\left|g_{R R}^{2}-g_{L L}^{2}\right|}=\sqrt{\frac{4 \pi \alpha}{3 \cos ^{2} \theta_{W}}} \approx 0.2
$$

implying MOLLER is sensitive to Z' bosons with masses up to about 1.5 TeV [33]. In left-right symmetric models, the $Z_{L R}$ boson couples with strength

$$
\sqrt{\left|g_{R R}^{2}-g_{L L}^{2}\right|}=\sqrt{\frac{\pi \alpha}{\cos ^{2} \theta_{W}\left(1-2 \sin ^{2} \theta_{W}\right)}} \approx 0.24
$$

corresponding to a $1.8 \mathrm{TeV}$ MOLLER mass reach [33].

Fig. 2-9 depicts, in two specific models [40], how the proposed $A_{P V}$ mea- 
surement and the SLHC would impact constraints on chiral Z' couplings. The difference in the parameter space constrained by MOLLER vs the SLHC data shows how MOLER would provide an independent determination of the left- and right-handed leptonic Z' couplings, helping to distinguish between models [33]. ${ }^{2}$

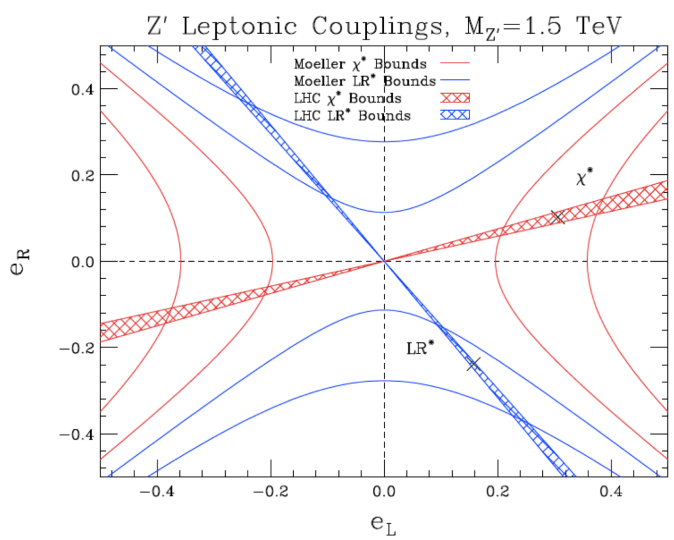

Figure 2-9: Future constraints on chiral Z' couplings in two representative models for a $1.5 \mathrm{TeV}$ mass. The hyperbolas are from a potential $A_{P V}$ measurement while the hatched regions are from an SLHC. The latter were obtained assuming a given model with the parameters as discussed including statistical errors and uncertainties from parton distribution functions. There is a reflection symmetry (a doubling of the bands) because of an overall unphysical sign ambiguity. For technical reasons, masses and couplings have been scaled in the figure by a factor of 1.25. (Figure courtesy of F. Petriello et al.)[33]

\section{Electron Compositeness}

Electron compositeness can be parameterized as a contact interaction, at an energy scale where the internal dynamics of the electron have become important, with a Lagrangian of the form [72] [46]

$$
\mathcal{L}=\frac{4 \pi}{2 \Lambda_{e e}^{2}}\left[\eta_{L L}\left(\bar{\psi}_{L} \gamma_{\mu} \psi_{L}\right)^{2}+\eta_{R R}\left(\bar{\psi}_{R} \gamma_{\mu} \psi_{R}\right)^{2}+2 \eta_{L R}\left(\bar{\psi}_{R} \gamma_{\mu} \psi_{R}\right)\left(\bar{\psi}_{L} \gamma_{\mu} \psi_{L}\right)\right]
$$

\footnotetext{
${ }^{2}$ We note that the $1.5 \mathrm{TeV}$ mass shown in Fig.2-9 has been excluded. While new "general" Z's are no longer in reach for MOLLER, it could be useful for "designer" Z's which would not be seen at the LHC but would be light enough/couple strongly enough for MOLLER
} 
where $\Lambda_{e e}$ is the energy scale and $\left|\eta_{i j}\right| \leq 1$. MOLLER has a large sensitivity to any parity violating couplings in the contact interaction

$$
g_{e e}^{\text {meas }}-g_{e e}^{S M}= \pm \frac{\pi}{G_{F} \sqrt{2}} \frac{\eta_{R R}-\eta_{L L}}{\Lambda_{e e}^{2}}
$$

For $\eta_{R R}$ or $\eta_{L L}$ equal to \pm 1 , MOLLER sensitivity to electron compositeness reaches $\mathrm{TeV}$ energy scales.

\section{Minimal SUSY}

MOLLER could be sensitive to new BSM particles predicted by Minimal Supersymmetric Standard Models (MSSM) via radiative loop effects or tree-level interactions. Radiative loop effects are R-parity conserving (RPC) and tree-level interactions are R-parity violating (RPV) [25] [26]. If nature is supersymmetric, RPC and RPV interactions would affect the electron weak charge $Q_{W}^{e}$ with opposite sign. So SM deviations, as measured by MOLLER $A_{P V}$ would be able to distinguish RPC and PRV versions of SUSY. Interestingly, while the presence RPV interactions would suggest that neutrinos are Majorana particles, the presence of RPC interactions would suggest that the lightest supersymmetric particle is stable, making it a non-baryonic dark matter candidate [1].

\section{Case: LHC agrees with the Standard Model}

There is a variety of BSM physics that could escape LHC detections. If the LHC continues to agree with the Standard Model up to 14TeV, MOLLER could help in the discovery of hidden weak scale scenarios such as compressed supersymmetry, light $\mathrm{MeV}$-scale dark matter mediators, and lepton-number violating processes, such as doubly-charged scaler exchange [1]. 


\section{Doubly charged scalars}

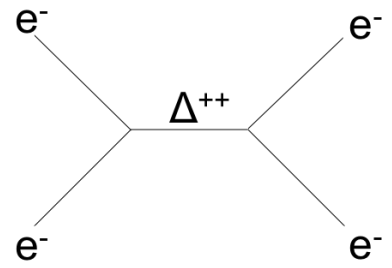

Figure 2-10: Exchange of a doubly-charges Higgs boson $\Delta^{++}$

MOLLER will be able to probe lepton-number violating amplitudes mediated by exchange of a doubly-charges Higgs boson $\Delta^{++}$with a high level of sensitivity. [46]. Doubly charged scalars can arise in certain extended Higgs sector models. For example, the left-right symmetric model contains $\Delta_{L}$ and $\Delta_{R}$ triplets with doubly-charged components $\delta_{L, R}^{++}$that can couple to two charged leptons via the Lagrangian:

$$
\mathcal{L}_{\text {matter }}^{\delta^{++}} \sim h_{L}^{i j} \delta_{L}^{++} \bar{\ell}_{i}^{C} P_{L} \ell_{j}+h_{R}^{i j} \delta_{R}^{++} \bar{\ell}_{i}^{C} P_{R} \ell_{j}+h . c .
$$

The Møller scattering process is unique because it is sensitive to an amplitude that violates lepton number by 2 units:

$$
\mathcal{M}^{P V} \sim \frac{\left|h_{L, R}^{e e}\right|^{2}}{2 M_{\delta_{L}}^{2}} \bar{e}_{L} \gamma_{\mu} e_{L} \bar{e}_{L} \gamma^{\mu} e_{L}
$$

which is equivalent to a contact interaction with $\Lambda=M_{\delta_{L}},\left|g_{L}^{2} L\right|=\left|h_{L}^{e e}\right|^{2} / 2$ and $g_{R R}=g_{L R}=0$. Hence MOLLER would have a mass reach of [1]

$$
\frac{M_{\delta_{L}}}{\left|h_{L}^{e e}\right|} \sim 5.3 \mathrm{TeV}
$$

in the left-right symmetric model, making MOLLER one of the most stringent probes of the left-handed charged scalar,above the LEP 2 constraint of $\sim 3 \mathrm{TeV}$, as well as being a complimentary measurement to other lepton-flavor violation and neutrinoless double-beta decay searches [24] [1]. 


\section{Compressed SUSY}

Compressed supersymmetry [23] is a hidden weak scale scenario in which one of the super-partner masses is relatively light. In that scenario, the LHC signatures would be very challenging to disentangle from QCD backgrounds whereas MOLLER would be sensitive to such a scenario [1].

\section{$\operatorname{Dark} Z_{d}^{0}$}

It has been postulated [20] [21] that a dark matter mediator, denoted $Z_{d}$, with $\mathrm{MeV}$ scale mass $m_{Z_{d}}$, might exist and could couple to SM particles. In the presence of a combination of kinetic and mass mixing with the photon and the $Z^{0}$, with couplings $\epsilon$ and $\epsilon_{Z}=\frac{m_{Z_{d}}}{m_{Z}} \delta$, if $\delta \neq 0$ 'dark' parity violation arises [20] which is negligible in high energy measurements of shifts in the weak mixing angle, but apparent at low $Q^{2}[21]$.

Figure 2-11 shows the range of possible deviations of $\sin ^{2} \theta_{W}(Q)$ for $Z_{d}$ mass of 100 and $150 \mathrm{MeV}$. A precise low $Q^{2}$ measurement on the weak mixing angle, like MOLLER, has obvious discovery potential in this light dark matter mediator scenario [1].
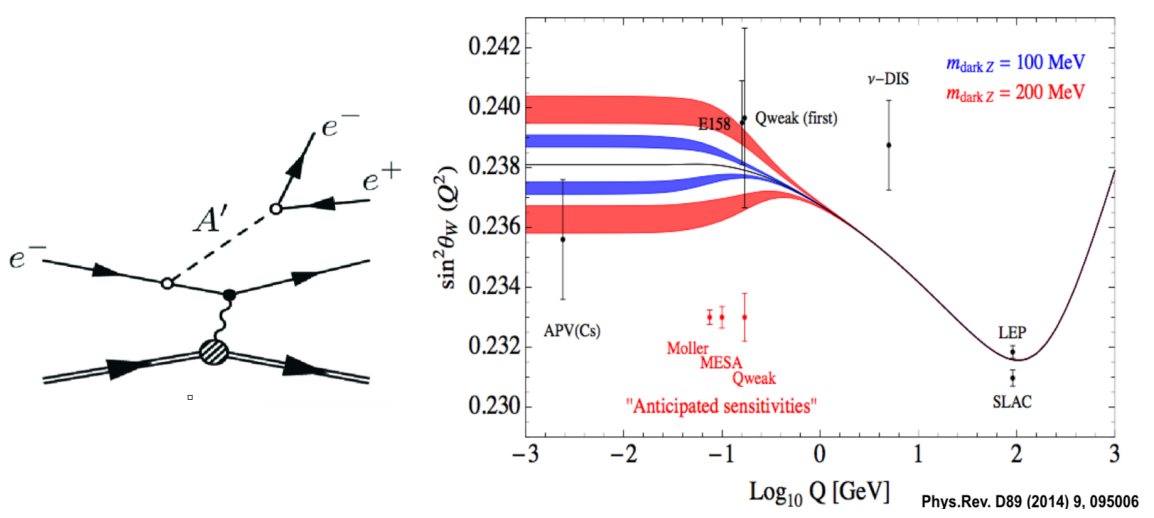

Figure 2-11: Running of $\sin ^{2} \theta_{W}$ with various dark $\mathrm{Z}$ mediator masses [41] [42] [43] [44] 


\subsection{Parity Violation Experiments}

\subsubsection{Basics of PVeS Experiments}

Fig. 2-13 illustrates the layout of the first PVeS experiment E122 that provided further unambiguous evidence for electroweak unification. It measured the parity violating asymmetry in deeply inelastic scattering of an $e^{-}$from a liquid deuterium target at $Q^{2} \approx 1 G e V^{2} / c^{2}$, detecting the scattered electron.

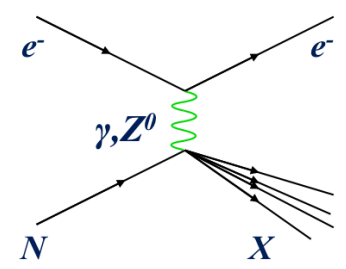

Figure 2-12: $A_{P V}$ in Deep Inelastic Scattering from liquid Deuterium $Q^{2} \approx$ $1 \mathrm{GeV}^{2} / \mathrm{c}^{2}$. Inclusive measurement which detected scattered electron only. [70]

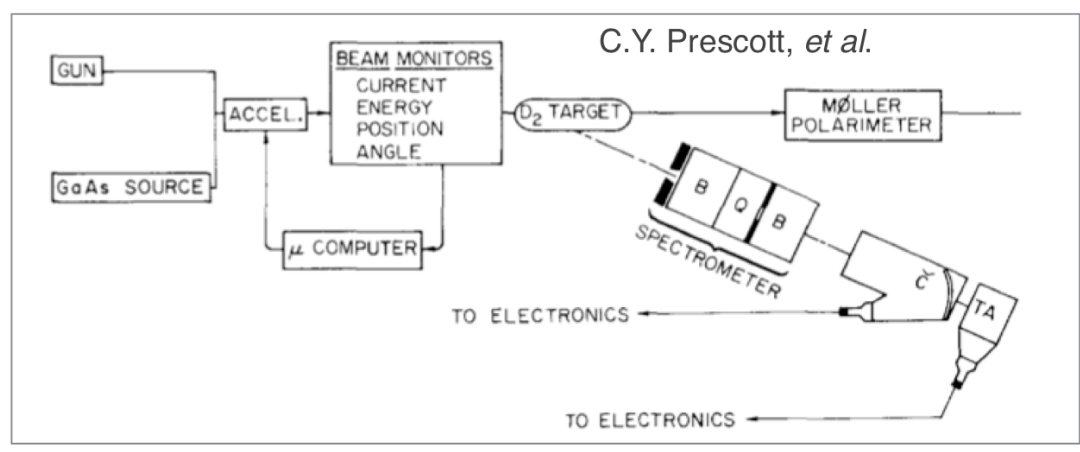

Figure 2-13: Experimental Layout of E122 (1978) [70]

To produce polarized electrons is a complicated multistage process. The Right and Left handed longitudinally polarized electrons for this experiment come from Right and Left Circularly polarized light. It starts with linearly polarized photons from a laser source that are then converted to states with circular polarization with a pockels cell electro-optic device. The light helicity (and the corresponding electron polarization) is flipped rapidly with the pockels cell. At present, $1 \mathrm{kHz}$ 
fast flip rate is possible. This polarized light is used to pump a GaAs photocathode to produce the polarized electrons, which was first developed for E122. The current state-of-the-art superlattice cathodes produce electron beams of roughly 90\% polarization, with high quantum efficiency and long lifetimes. The electrons are accelerated and then sent into the experimental hall.

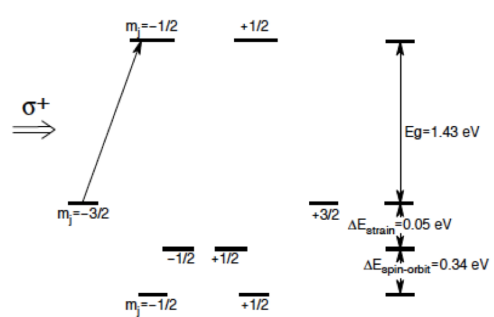

Figure 2-14: Band structure of GaAs, showing how circularly polarized laser light produces polarized electrons.[33]

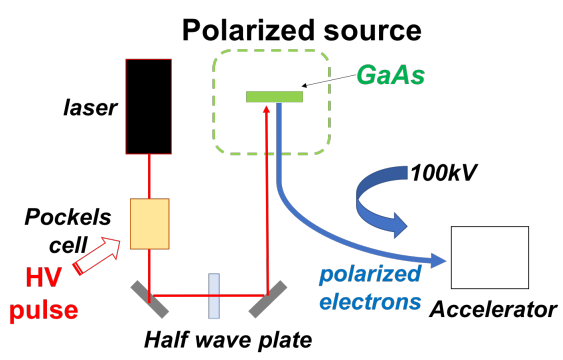

Figure 2-15: High luminosity polarized electron source - GaAs photocathode [70]

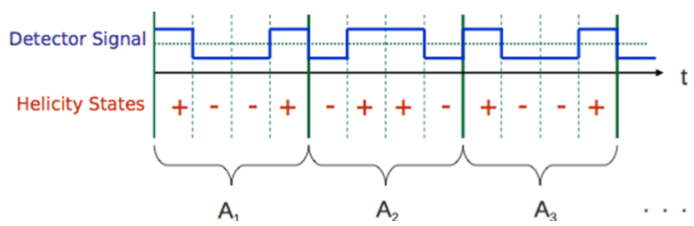

Figure 2-16: Helicity States driven through the Pockels Cell voltage setting [70]

Since a scattering asymmetry is being measured in this experiment, it is important to keep the electron beam symmetric between the two polarization states, to minimize the "helicity correlated beam asymmetries". Nowadays, through both precision configuration and feedback, charge asymmetry $A_{q}$ in the $e^{-}$beam can 
be kept down at ppb level and position differences $\Delta_{x}$ between helicity states can be kept down at the nanometer level on target. E122 employed beam monitors to measure the helicity dependent changes in current and position. Now they usually are RF antenna or RF resonant cavities and can measure with precision charge at $30 \mathrm{ppm}$ and position at micron level at 250Hz. Even in 1978, a computer was used for fast feedback to control beam asymmetries, a method which we still employ now.

A high-power cryogenic target was used: $30 \mathrm{~cm}$ of liquid deuterium for high luminosity. Currently the high power target can handle $2300 \mathrm{~W}$ of deposited beam power, and noise from density fluctuations from "boiling" of the cryogenic fluid are $<40 \mathrm{ppm}$ at $250 \mathrm{~Hz}$ helicity flip rate. In the future MOLLER experiment, we will use a $1.5 \mathrm{~m}$ LH2 target, exposed to $4 \mathrm{~kW}$, with stability better than $25 \mathrm{ppm}$ at $1 \mathrm{kHz}$ flip rate.

Knowing the degree of $e^{-}$beam polarization was an important experimental parameter. In E122 a Møller polarimeter was used. Now multiple polarimeters are used to $1 \%$ precision: Mott, Møller and Compton (a continuous measurement at $0.7 \%$ precision). For MOLLER, we hope to have $0.5 \%$ precision on the polarimetry measurements.

A magnetic spectrometer directs scattered $e^{-}$flux to a background-free region and defines the kinematic acceptance. The electron flux was detected by quartz bars and photomultiplier tubes. More generally the electron flux can be detected by Cherenkov light in an optically transparent media like lucite or quartz, often with a heavy material like lead or copper to create showering of the incident election as shown in Fig. 2-17 for E158. The signal gets integrated during the helicity window and is sent to ADCs. In E122, the detectors measured high $100 \mathrm{kHz}$ rates. Currently JLab Hall A parity experiments regularly have rates that can approach $7 \mathrm{GHz}$. For MOLLER, we hope to detect rates of $500 \mathrm{GHz}$. 


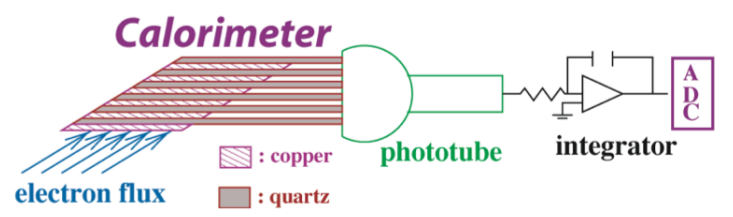

Figure 2-17: Concept of E158 Calorimeter - Integrating Detection of light from quartz-copper sandwich [70]

\subsubsection{Experimental Measurements of $\sin ^{2} \theta_{W}$}

\section{E158}

E158 (1997-2004) measured the electron weak charge to high precision through parity violating Møller scattering at low $Q^{2}$. It was the first measurement of the electron-electron weak interaction.

E158 bore similarity to E122 in the overall experimental layout (Fig. 2-18). A highly polarized, high current electron beam was incident on an unpolarized, high luminosity target of $\mathrm{LH}_{2}$ with $\sim 1 k W$ of beam power deposited. The target was an 18\% radiator. E158 employed a magnetic spectrometer system so the detectors could distinguish between signal and background, in this case distinguish Møller electrons from ep backgrounds. The spectrometer system consisted of a dipole chicane to separate the primary and scattered beam and a quadrupole spectrometer to separate the Møller signal from the ep background. Beam energies of $45 \mathrm{GeV}$ and $48 \mathrm{GeV}$ were used. The spectrometer accepted electrons from 47 mrad scattering angles corresponding to about $60-90^{\circ}$ scattering angles in the center of mass frame.

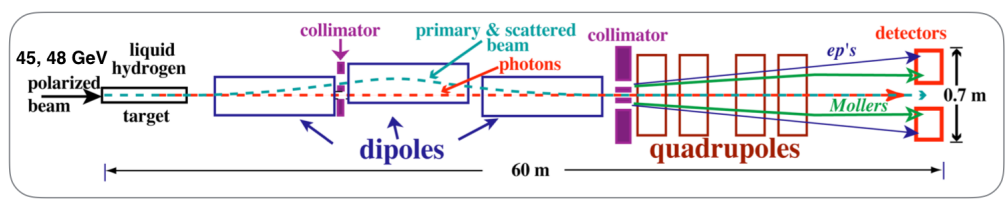

Figure 2-18: Experimental layout of E158 [70] 
The parity violating asymmetry was measured to be

$$
A_{P V}=(-131 \pm 14 \pm 10) p p b
$$

which implied a precision on the weak mixing angle of

$$
\frac{\delta\left(\sin ^{2} \theta_{W}\right)}{\sin ^{2} \theta_{W}} \sim 0.5 \%
$$

and mass-reach scales on the order of $\Lambda_{R R-L L}^{e e} \sim 17 \mathrm{TeV}$.

\section{$Q_{\text {weak }}$ Experiment}

$Q_{\text {weak }}$ was a measurement of the parity-violating asymmetry in elastic ep scattering of longitudinally polarized electrons from protons in HallC [56]. It compliments MOLLER because it measures the weak charge of the proton and is therefore sensitive to hadronic couplings of the $Z^{0}$ rather than leptonic couplings and therefore has a different response to new physics scenarios [46]. The experiment measured a parity-violating asymmetry $A_{e p}=-226.5 \pm 7.3($ stat $) \pm 5.8($ syst $)$ ppb [57].

The weak charge of the proton $Q_{w}^{p}$ was determined from a global fit, shown in Fig. 2-19a of parity-violating elastic scattering (PVES) results from nuclear targets, since uncertainties of hadronic structure could be constrained by higher $Q^{2}$ measurements [56]. The value extracted from the global fit is in agreement with the standard model prediction $Q_{w}^{p}(S M)=0.0710 \pm 0.0007$ [56]. The weak neutral current quark vector couplings $C_{1 d}$ and $C_{1 u}$ as constrained by $Q_{\text {weak }}$ and the APV measurement in ${ }^{133} \mathrm{Cs}$ [18] are shown in Fig.2-19b. The exclusion limits are $\lambda / g \approx$ $7.5 \mathrm{TeV}, \lambda \approx 27 \mathrm{TeV}[57]$ with the usual convention for contact interactions $g=$ $\sqrt{4 \pi}$ (see Sec.2.1.2). Finally, the $Q_{\text {weak }}$ result for $\sin ^{2} \theta_{W}$ is shown in Fig. 2-19c. Qweak result is plotted in red at the energy scale of the Qweak experiment, and also in green as interpreted using lattice QCD strange quark results for form factors 
of the proton. Error bars (1 s.d.) include statistical and systematic uncertainties. The modified-minimal subtraction (MS) scheme is shown as the solid curve [27] together with experimental determinations. The result is consistent with SM predictions.

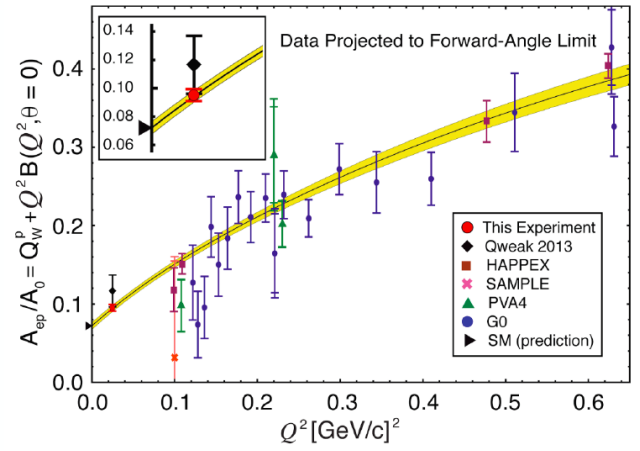

(a)

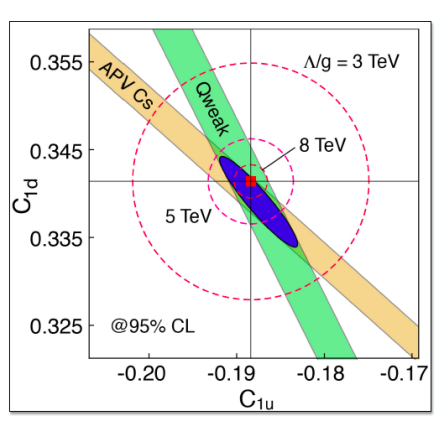

(b)

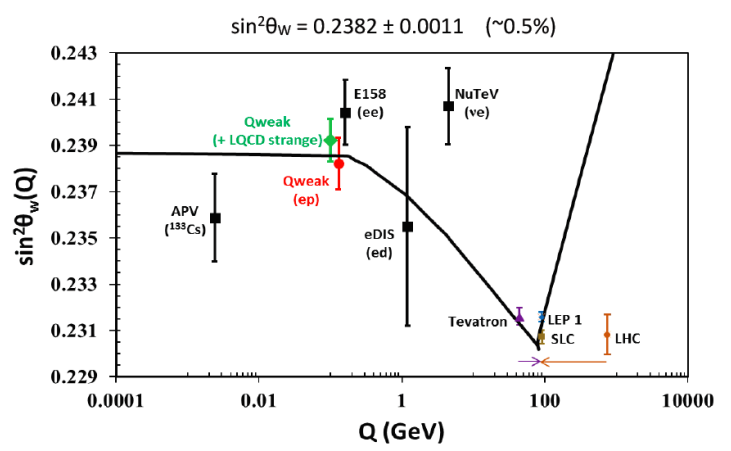

(c)

Figure 2-19: Qweak experiment results: (a) $Q_{w}^{p}$ as determined from a global fit (b) Weak Neutral Current Quark Vector Couplings constraints (c) Weak mixing angle result interpreted from the Qweak measurement. Qweak result is plotted in red at the energy scale of the Qweak experiment, $\mathrm{Q}=0.158 \mathrm{GeV}$. Error bars (1 s.d.) include statistical and systematic uncertainties. The modified-minimal subtraction (MS) scheme is shown as the solid curve together with experimental determinations. [19] [15] [57]

\section{Atomic Parity Violation}

Atomic parity violation (APV) experiments, also known as parity non-conservation (PNC), measure the weak mixing angle using, for example, Cesium atoms [58]. The first APV SM test was performed with on ${ }^{133}$ Cs in 1983 by Bouchiat \& 
Bouchiat [63]. Other atoms besides ${ }^{133} \mathrm{Cs}$ have also been used, such as Ytterbium, Radium, ${ }^{208} \mathrm{~Pb}$ [64] [65] , ${ }^{209} \mathrm{Bi}$, and molecules [63]. The best APV measurement of the weak mixing angle at lower energies are those extracted from the measurement of the weak charge of ${ }^{133} \mathrm{Cs}, Q_{W}^{C s}$, via studies of table-top atomic parity violation obtained by Wieman in 1999 [17]. In a nucleus, the SM prediction for the weak charge including EW corrections is given by [58 $]^{3}$

$$
Q_{W}^{S M+r a d . c o r r .} \approx-2\left[Z\left(g_{A V}^{e p}\right)+N\left(g_{A V}^{e n}\right)\right]\left(1-\frac{\alpha}{2 \pi}\right) \approx Z\left(1-4 \sin ^{2} \theta_{W}^{S M}\right)-N
$$

where $g_{A V}^{e p} \approx-\frac{1}{2}+2 \sin ^{2} \theta_{W}^{S M}$ and $g_{A V}^{e n} \approx \frac{1}{2}$. In an APV experiment, the transition energy $E_{A P V}$, an energy state that is shifted by the weak charge in an EM field, is measured via an interference term in excited state transitions ("APV lightshift") and this energy is related to the nuclear weak charge

$$
E_{A P V} \approx k Q_{W}
$$

The transition energy $E_{A P V}$ is measured, $\mathrm{k}$ is calculated from atomic wavefunctions, and thus $Q_{W}$ is inferred. Constraining theoretical calculations of $\mathrm{k}$ is a crucial steps towards higher precision in the interpretation of APV experiments. The weak mixing angle measurement from the atomic parity violation (APV) in ${ }^{133} \mathrm{Cs}$ can be compared with the Standard Model prediction at low $Q^{2}$. Deviation from the SM prediction implies new BSM physics:

$$
Q_{W}=Q_{W}+\text { rad.corr. }+ \text { BSMphysics }
$$

For example, an extra Z' boson in $\mathrm{SO}(10)$ GUTs produces a change to the nucleus $Q_{W}$ of [63]

$$
\delta Q_{W} \approx(2 N+Z) a_{e}^{\prime}(\xi) \nu_{d}^{\prime}(\xi) \frac{M_{Z}^{2}}{M_{Z^{\prime}}^{2}}
$$

$$
{ }^{3} Q_{W}^{S M+\text { rad.corr. }}=-2\left[Z\left(g_{A V}^{e p}+0.00005\right)+N\left(g_{A V}^{e n}+0.00006\right)\right]\left(1-\frac{\alpha}{2 \pi}\right)
$$


While APV measurements are sensitive to additional Z (Z') bosons predicted in grand unified theories (GUTs) [58], they are insensitive to SUSY loops (cancellation of neutron and proton effects) [63]. As shown in Fig. 2-19b, the $C_{1 u}, C_{1 d}$ sensitivity is complimentary to PVES experiments like Qweak or MOLLER, because due to the dominance of the neutron weak charge, APV probes orthogonally to PVES [63] using proton targets.

The largest uncertainty in APV measurement is the theoretical interpretation and the calculation of $\mathrm{k}$ from atomic wavefunctions. Calculations of the atomic wavefunctions evolve every few years, as additional atomic structure effects are incorporated into the calculations and other measurements, such neutron radius measurements, are taken into account [58]. So, historically the APV extracted result on the weak mixing angle has moved significantly over the years, frequently being updated [66] [18] [1], being mostly lower than the SM prediction [58]. The level of agreement with the SM prediction has changed with subsequent analyses [67] [18] [56].

\section{$\mathrm{NuTeV}$}

The NuTeV Collaboration extracted the electroweak parameter $\sin ^{2} \theta_{W}$ from the measurement of the ratio of weak neutral current to charged current $\nu$ and $\bar{\nu}$ cross sections in neutrino-nucleus deep inelastic scattering $(\nu D I S)$ [68] [56]. The original reported value was $\sin ^{2} \theta_{W}^{(\text {on-shell })}=0.2277 \pm 0.0013($ stat $) \pm .0009($ syst $), 3$ standard deviations above the SM prediction [68], dubbed "the NuTeV anomaly" [56]. While such a discrepancy would indicate new BSM physics, there are theoretical interpretability issues related to atomic and nuclear structure effects. The issues highlight the importance of a theoretically clean experimental probe [56] like MOLLER. 


\section{Z-pole measurements}

The two most precise measurements of $\sin ^{2} \theta_{W}$ at the Z-pole $\left(\sin ^{2} \theta_{W}^{e f f}\left(M_{Z}\right)\right)$ are the SLC collider measurement of the left-right asymmetry in Z production $A_{I}(S L D)=0.23070 \pm 0.00026$ and the LEP1 collider measurement of the forwardbackward asymmetry in $\mathrm{Z}$ decays to b-quarks $A_{f b}^{0, b}(L E P)=0.23193 \pm 0.00029$ [1]. The two measurement aren't consistent with one another, independently implying very different BSM dynamics [38] but averaging to the Standard Model value. The main systematic uncertainty is the interpretability of the measurement with Parton Distribution Functions (PDFs), although recently Bodek et al. [49] have found a method to reduce the PDF uncertainties in $p p$ collisions, tightening up the theoretical interpretations and enabling precision results from the LHC. This, future planned hadron experiments, like $\mathrm{LHC} 14 \mathrm{TeV}, 300 \mathrm{fb}-1$ with precision of $\delta \sin ^{2} \theta_{W} \sim 0.00036$, will not be theoretically limited with PDF uncertainties. One of the past appealing aspects of MOLLER would be to provide the reach of the Z-pole measurements, with a precision of $\delta \sin ^{2} \theta_{W} \sim 0.00028$, comparable to the Z-pole measurements uncertainties, but without the systematic uncertainty from PDFs. Now that the PDF uncertainty has been reduced for $p p$ collisions, the appeal of MOLLER lies largely in that it is a low $Q^{2}$ measurement, off of the Z-pole, with improved sensitivity to new BSM physics.

\subsection{MOLLER Experimental Design}

\subsubsection{Basic Conceptual Setup}

MOLLER will improve on the precision of E158 by about a factor of 5 . There are a broad range of technical challenges to achieve high precision for MOLLER. The experiment will be performed at $11 \mathrm{GeV}$ at JLab, with high luminosity and high stability $e^{-}$beam, so that this large improvement on E158 is possible. The design for MOLLER is shown in Fig. 2-20. In the $Q^{2}$ regime for this design, $A_{P V}$ 
is expected to be $35.6 \mathrm{ppb}$. The goal is to reach a statistical precision of $0.73 \mathrm{ppb}$ on $A_{P V}$, to measure $\sin ^{2} \theta_{W}$ with uncertainties of \pm 0.00026 (stat) \pm 0.00013 (syst), and obtain mass reach scales of up to $47 \mathrm{TeV}$. In short, the goal is to perform an experiment at low $Q^{2}$ with a precision on $\sin ^{2} \theta_{W}$ matching collider Z-pole measurements.

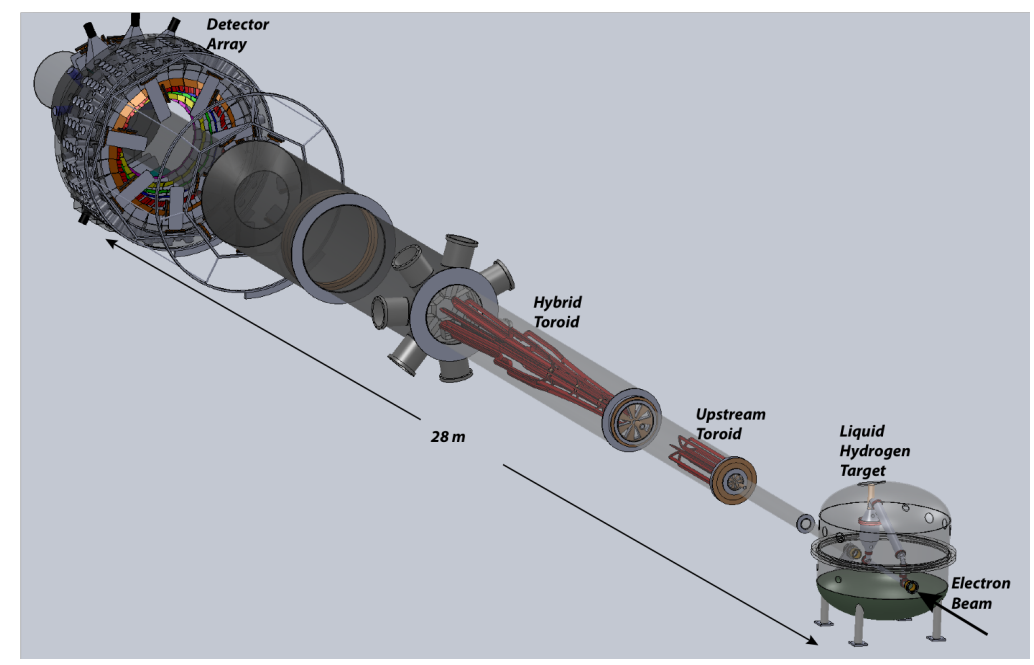

Figure 2-20: Experimental Design for Moller [70]

The basic conceptual design is shown in Fig. 2-20. There is a $\mathrm{LH}_{2}$ target, followed by an upstream magnet that pre-focuses the Møller scattered elections so that they make it through the downstream hybrid toroid which is designed to focus and maximize the kinematic acceptance.

\subsubsection{Figure of Merit and High Acceptance}

To obtain high statistical precision, high luminosity, high rates, high acceptance, and optimal figure of merit (FOM) are required. The figure-of-merit is defined as $F O M=R \times A_{P V}^{2}$, where $\mathrm{R}$ is the detected rate $R \sim \frac{d \sigma}{d \Omega}$, and where $A_{P V} \sim E_{l a b} Q_{W}^{e}$ rises with $E_{l a b}$ and the cross-section $\sigma \sim \frac{1}{E_{l a b}}$ decreases with $E_{l a b}$. The FOM is proportional the beam power and the experiment need high energy but also high luminosity. The $11 \mathrm{GeV}$ JLab beam can be more precise than the $45 \mathrm{GeV}$ SLAC measurements because of luminosity and systematic precision. 
The goal of the optimization of MOLLER kinematics is to minimize the runtime required to obtain a high accuracy of $0.72 \mathrm{ppb}$ on $A_{P V} \approx 35.6 \mathrm{ppb}$. The spectrometer is designed to separate Møller electrons from ep scattered background. There is a large phase space of relevant properties which go into the FOM such as Møller and elastic ep rate and asymmetry, and ee and ep focus and separation.

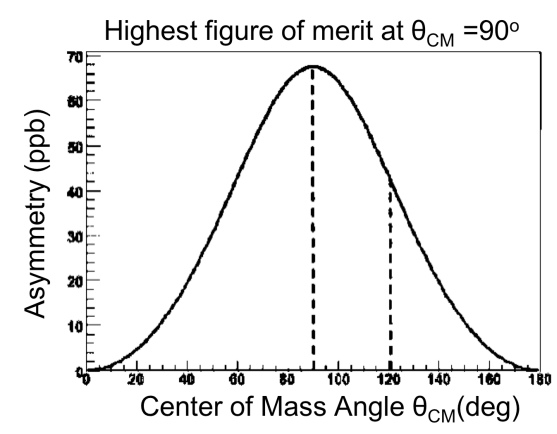

Figure 2-21: Moller $A_{P V}$ vs. Center of Mass Scattering Angle $\theta_{C M}$ [71]

In this ee scattering experiment, the FOM contribution is highest for $E_{l a b}=$ $11 \mathrm{GeV}$ and an $e^{-}$scattering angle of $90^{\circ}$ in COM frame with optimum acceptance from about $90^{\circ}-120^{\circ}$ back scattered electrons as depicted in Fig. 2-21. This is because, independent of gauge model [48], the maximal asymmetry occurs at $y=0.5$ due to $y \leftrightarrow 1-y$ symmetry for identical particles, which corresponds to a CM scattering angle $\theta_{C M}=90^{\circ}$. In the lab frame the two outgoing electrons (Fig.2-22) from $\theta_{C M}=90^{\circ}$ scattering emerge at a scattering angle $\theta_{l a b} \approx \sqrt{2 m_{e} / E}$ where $\mathrm{E}$ is the incoming electron energy and each outgoing electron carries energy $\mathrm{E} / 2$. This approximately corresponds to the optimum FOM region (yellow) in Fig.2-22 which shows that the optimal detector would cover energies from 2.5$5.5 \mathrm{GeV}$ at $10-17 \mathrm{mrad}$ to detect electrons backscattered in the center-of-mass frame which are easier to isolate due to higher laboratory angles and lower energy compared to their forward scattered partners. 

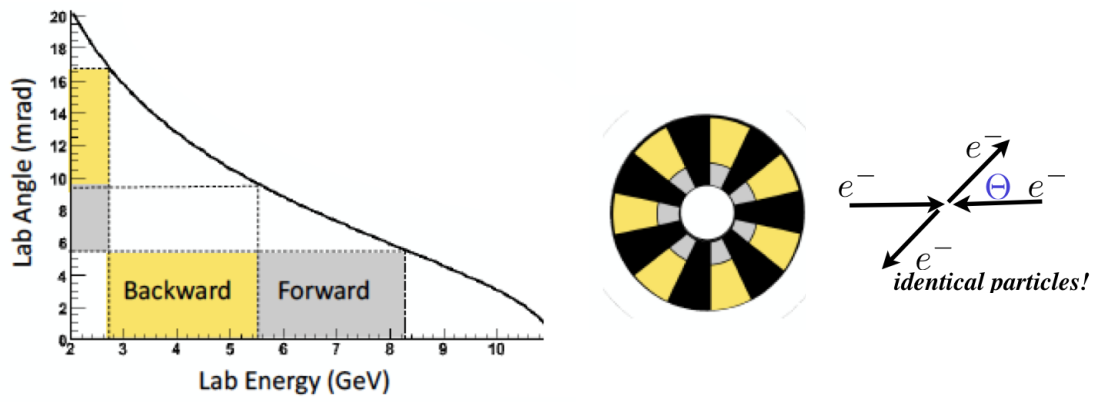

Figure 2-22: The FOM is highest at $\theta_{C M}=90^{\circ}$, with optimum acceptance $\left[90^{\circ}, 120^{\circ}\right]$ in highly boosted lab frame. Forward and Backscattered electrons are identical particles [71]

Ideally, all of the backscattered electrons would be accepted, but complete $360^{\circ}$ azimuthal coverage is not possible. The magnets and collimator in the spectrometer must be placed somewhere and supported so azimuthal acceptance must be lost. However, because these are identical particles, we can use a clever idea to get an effective 100\% acceptance shown in Fig. 2-22 : Any region where back scattered electrons (black) are lost, detect forward scattered electrons instead (grey) at the opposite azimuthal angle. In this way, one of the electrons (either yellow or gray) is always detected from each event. An odd number of octants are used with only $50 \%$ azimuthal coverage, but $100 \%$ acceptance is achieved for seeing at least one electron from each elastic event in the optimal FOM region.

A conceptual description of the components of the experiment follows.

\subsubsection{Magnetic Spectrometer}

The spectrometer must separate Møller scattered electrons from backgrounds while maintaining high acceptance. The dominant backgrounds are elastic and inelastic ep scattering. The toroid spectrometer is designed to use the energyangle correlation of ee elastic scattering by focusing kinematic acceptance from $E_{l a b}=2.5-8.5 \mathrm{GeV}$ and $\theta_{l a b}=0.3^{\circ}-1.1^{\circ}$ onto a small region for detection. It consists of two resistive ( $\mathrm{Cu}$ non-superconducting) toroids each with 7 magnets 


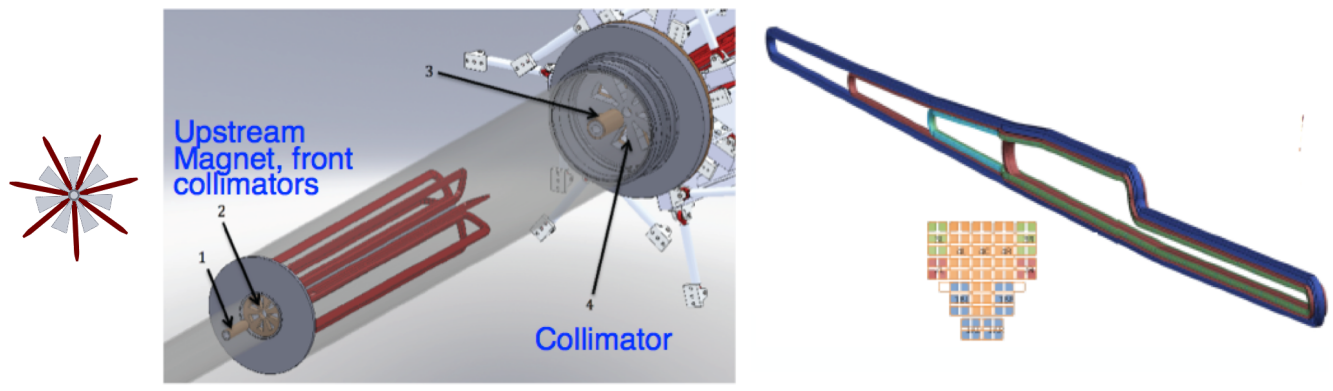

Figure 2-23: Toroid Spectrometer: Upstream Magnet and Downstream Toroidal Magnet [71]
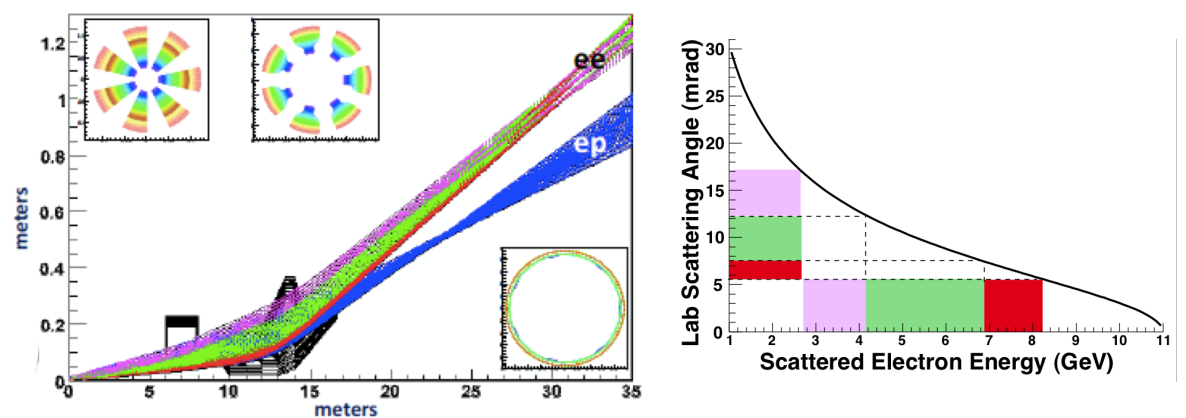

Figure 2-24: Illustration of the radial and azimuthal focusing of the MOLLER spectrometer. The figure on the right illustrates the colors for the forward and back scattered events as used on the left [71].

arrayed azimuthally. The upstream magnet starts bending small scatters out to larger angles. A second toroid continues this out bending, but uses 6 different segments with progressively more magnet turns to vary the effective $\int B \cdot d l$ over the range of acceptance, to bend the highest accepted energies without overbending the lowest. The hybrid's 6 segments are shown in Fig.2-23 where regions with different NI's corresponds to different magnet segments.

As shown in Fig. 2-24, in addition to radial focusing from azimuthal fields, radial fields defocus azimuthally the accepted distributions. The accepted events, which are neat wedges just at the collimator, occupy the entire azimuth at a radius of about $1 \pm 0.1 \mathrm{~m}$, approximately $35 \mathrm{~m}$ downstream of the target. Critical factors determining the desired optics are the ee focus at detector plane, the $e p$ focus at the detector plane, and the foci separation.

The Møller electron distribution in the detector plane is shown in black, az- 


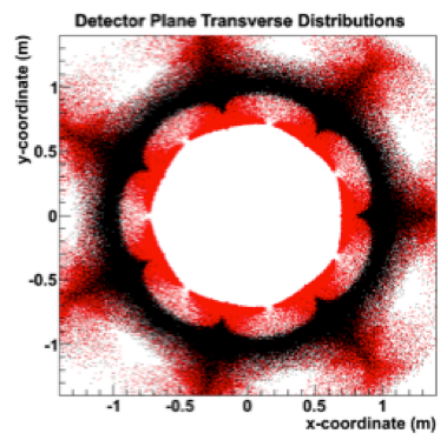

(a)

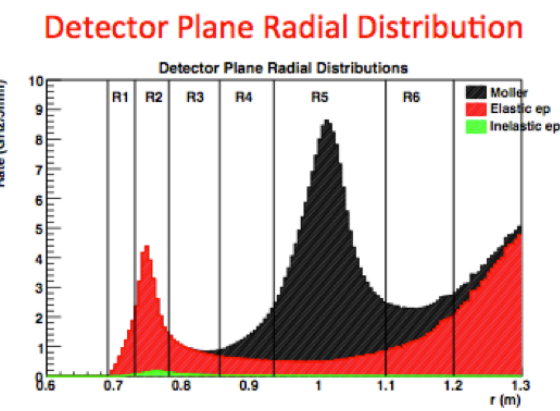

(b)

Figure 2-25: Møller electron distributions in detector plane: (a) Transverse distributions showing azimuthal defocusing - bins for different $\phi$, different $\theta_{C M}$ (b) Radial distributions - main Møller peak is in Region 5 [71]

imuthally in Fig. 2-25a. Elastic ep backgrounds are shown in red. The background for $e p$ processes $e+p(+\gamma) \rightarrow e+p(+\gamma)$ contributes $<0.3 \%$ fractional error to the final result. The spectrometer succeeds in achieving high signal to noise ratio at the main Møller peak in the radial Møller electron distribution shown in Fig. $2-25 b$ at a $1 m$.

A prototype of the toroidal magnet has been built and is being tested at MIT-Bates. The current density in the magnets (up to $20 \mathrm{~A} / \mathrm{mm}^{2}$ [59]) requires more careful engineering. While the optics design is complete, the engineering of the magnets is still in progress. There is a large phase space for the design: strength of the field integral (from current density, length), coil positions (z, radius),collimator location/orientation/size, choice of primary collimator, detector location/orientation. The system must keep the acceptance high, which allows the un-scattered beam to exit to the beam dump ${ }^{4}$, and must keep the current density as low as possible. In principle, small changes can be made to the current density or otherwise improve the mechanical integrity at the magnets, perhaps by exploring the phase space. It is worth noting that if higher current densities were practically possible, better focus and separation could be achieved [59].

\footnotetext{
${ }^{4}$ The constraints on the magnet design are: obeying keep in zones (half azimuth between tracks, full azimuth under tracks, no closer than 5X the multiple scattering radius)
} 


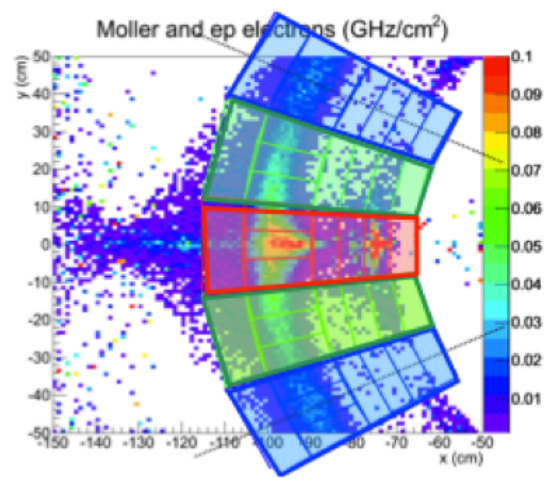

Figure 2-26: Proposed Detector Segmentation [71]

\subsubsection{Detectors}

The main Cherenkov detectors consist of quartz bars and photomultiplier-tubes (PMT's). Because of the azimuthal defocusing in radial fields, the detectors must cover the full azimuth and have radial and azimuthal segmentation to detect both signal and backgrounds. The radial segmentation is indicated in Fig. 2-25b and the azimuthal segmentation is indicated in Fig. 2-26.

There are planned to be 28 azimuthal channels per radial bin in most regions, with the except of the Møller peak (region 5) which will have 84 azimuthal channels per radial bin, giving a grand total of 224 channels. The rate per channel is expected to vary considerable from a few $\mathrm{MHz}$ to several $\mathrm{GHz}$, with a total rate of $159 \mathrm{GHz}$. Each detector consists of quartz with air light guides and PMT's as shown in Fig. 2-27. One interesting consideration: to avoid introducing a polarization sensitivity to the detectors, it is prudent to avoid excessive clamping force on the quartz causing birefringence in the detector and potentially differential light collection efficiency for polarized Cherenkov light.

In addition to the assembly of integrating quartz Cherenkov detectors, there are a variety of auxiliary detectors surrounding the setup as shown in Fig. 228. Pions and muons will be detected with a quartz sandwich behind shielding. Luminosity monitors downstream will detect beam and target density fluctuations. GEM-tracking chambers will be used upstream of the integrating detectors in a 

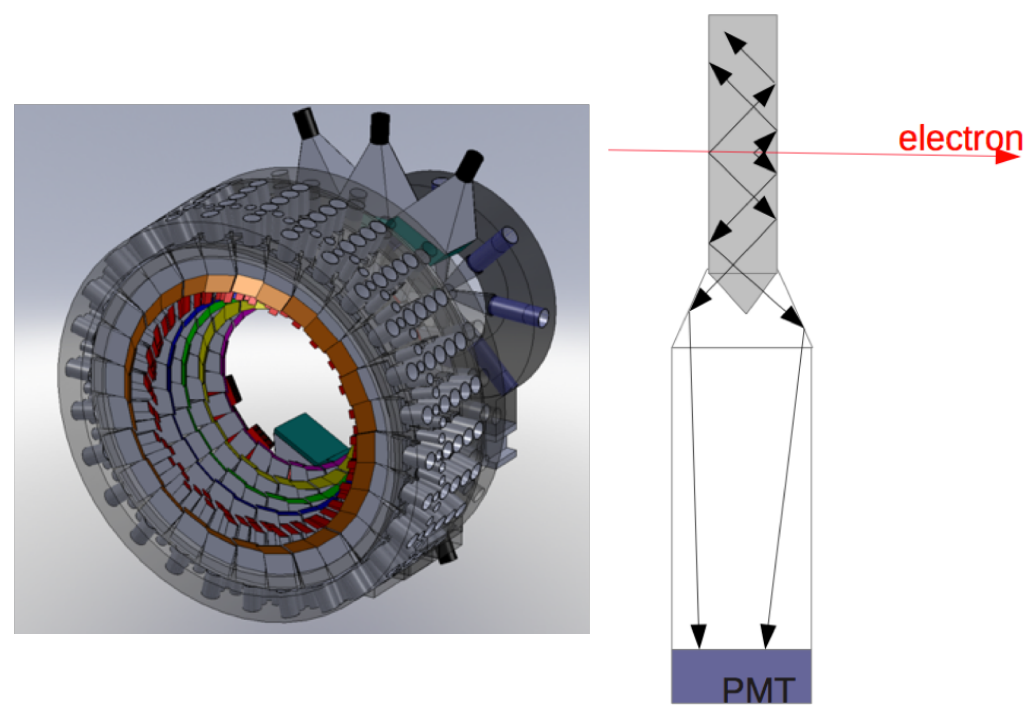

Figure 2-27: Quartz assembly of Cherenkov detectors with air light guides and PMTs. [71]
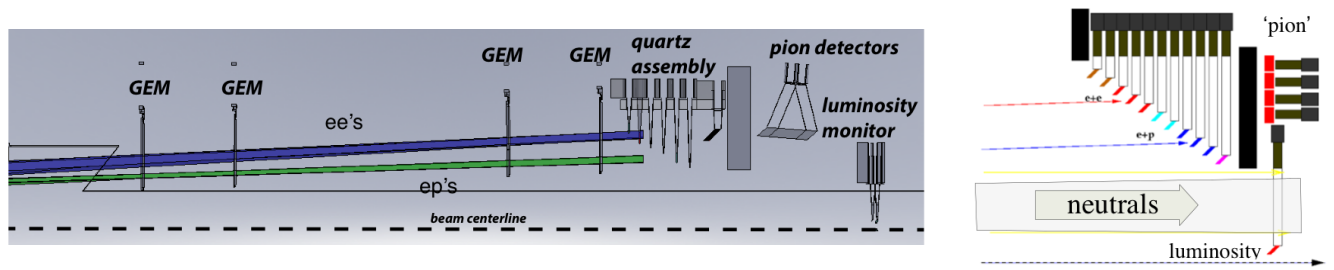

Figure 2-28: Conceptual illustration of detector arrangements for the MOLLER experiment including Cherenkov detectors, GEMs, pion detectors, and luminosity monitors [71]

special low beam-current calibration mode.

\subsubsection{Polarimetry}

The goal for MOLLER is to obtain robust $0.4 \%$ polarimetry using at least 2 different methodologies: Compton polarimetry and Møller polarimetry Transverse polarization uncertainties must also contribute $<0.2 \%$. More detailed descriptions of the Møller and Compton polarimeters can be found in Sec. 3.5.10 and Sec. 3.5.9, respectively. Here we will address the status of the equipment and error budgets required for MOLLER.

The Compton polarimeter measures the degree of longitudinal electron beam 
polarization via Compton scattering from polarized laser light. The electron beam crosses a laser beam in a high-gain optical cavity and some fraction of the electrons Compton scatter with the laser photons. The scattered electrons are separated from the primary $e^{-}$beam and get detected along with the scattered photons. There are two detectors in the Compton: a tracking electron detector and a photon calorimeter. For full capabilities at $11 \mathrm{GeV}$ during MOLLER, a radiation hard electron detector is needed, as well as a large, dense, linear detector without phosphorescence, and control of synchrotron light and BremBremsstrahlungstrahlung background at $11 \mathrm{GeV}$ [61]. While Qweak achieved $0.6 \%$ precision polarimetry with the electron detector at $1 \mathrm{GeV}$, and HAPPEX-3 achieved $1.0 \%$ precision polarimetry with the photon detector at $3 \mathrm{GeV}$, for MOLLER, operation at high energy (11 $\mathrm{GeV}$ ) with $0.4 \%$ precision is a very different challenge [61]. The error budget for various uncertainties in the Compton at $11 \mathrm{GeV}$ is shown in Fig. 2-29.

\begin{tabular}{|l|c|c|}
\hline Relative Error (\%) & electron & photon \\
\hline Position Asymmetries & \multicolumn{2}{|c|}{-} \\
\hline Ebeam and $\lambda_{\text {laser }}$ & \multicolumn{2}{|c|}{0.3} \\
\hline Radiative Corrections & \multicolumn{2}{|c|}{0.5} \\
\hline Laser Polarization & \multicolumn{2}{|c|}{0.2} \\
\hline Background/Deadtime/Pileup & 0.2 & 0.2 \\
\hline $\begin{array}{l}\text { Analyzing Power Calibration / } \\
\text { Detector Linearity }\end{array}$ & 0.25 & 0.35 \\
\hline Total & 0.38 & 0.45 \\
\hline
\end{tabular}

Figure 2-29: Compton uncertainty goals for MOLLER at $11 \mathrm{GeV}$ [61]

The Møller polarimeter measures longitudinal electron beam polarization based on Møller scattering from ferromagnetic foil targets. A double-polarization spin asymmetry in Møller scattering is measured and employed to infer the electron beam polarization.

To achieve $<0.5 \%$ polarimetry, the Møller polarimeter requirements include knowing the target polarization to $0.25 \%$ with small temperature corrections due to demagnetization from target heating of $<0.05 \%$. The upgraded Møller polarimeter should achieve high fields, where the electrons in the ferromagnetic target 
foil are polarized by a strong magnetic field. A 4Tesla magnetic field will saturate the magnetization of the iron foil target, for which the electron polarization is well determined [55]. The simulation of the Møller optics (the spectrometer tune and magnet currents) and acceptance must be accurate. The analyzing power averaged over the acceptance must be known to $0.2 \%$ and the Levchuk correction must contribute $<0.2 \%$ uncertainty.

\subsubsection{Target}

MOLLER requires a long, high-luminosity target with minimal noise from target boiling/density fluctuations $(<25 \mathrm{ppm})$. The target will be a $1.5 \mathrm{~m}$ long, LH2 target with a beam current up to $85 \mathrm{uA}$ and a deposited energy of up to $5 \mathrm{~kW}$. The difficulty with high luminosity/high power is that while you get high rates, you also get higher noise from target boiling. When it comes to statistics, it's possible to be self-defeating with high-luminosity. However, one can get around target boiling, by taking data quickly with fast helicity flipping, making fast comparisons in measuring the asymmetry. This is the helicity flipping equivalent to using a high speed camera. The choice of helicity flip rate can strongly impact the ultimate precision of a parity experiment. Fig. 2-30, from a measurement by Qweak,shows the luminosity noise level (in blue) decreasing at high frequencies. The density fluctuations in the target get cancelled when asymmetries are formed at high flip rates. For MOLLER, helicity reversal at $1-2 \mathrm{kHz}$ is required.

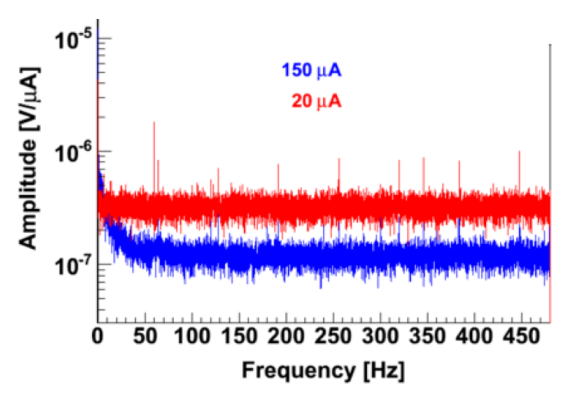

Figure 2-30: Target boiling noise vs. frequency [74] [71] 


\subsubsection{Polarized Source}

To achieve high precision measurements on $A_{P V}$ for MOLLER, the Pockels cell in the polarized source must satisfy both statistical and systematic requirements as regards the electron beam produced. The asymmetry measurement is based on the helicity switching, in which time windows are generated in the electron bunch train at a selected flip rate, with the sign of the beam's longitudinal polarization in each window assigned on a pseudo random basis. Frequency selection for helicity flipping affects the noise, and statistical errors significantly. Sec. 2.3.6 motivated the need for a high helicity flip rate during high data rate experiments, like the future MOLLER experiment, which requires a flip rate of $\sim 2 \mathrm{kHz}$. However, when data is taken at a high helicity flip rate for improved statistics, it is also desirable to have a short settle time for each transition to prevent downtime data losses. The Pockels cell which controls the electron beam must switch helicity states very quickly within 10us, with minimal dead-time $<2 \%$, to obtain sufficient statistical precision at high data rates. We have developed a new Pockels cell for the polarized source using RTP (Rubidium Titanyl Phosphate) crystals, described in Chapter 6.

Regarding systematics, the differential cross-section asymmetries are extremely small in parity experiments, and so the symmetry between incident right and left helicity beams is of increased importance. Since this type of measurement compares electrons of opposite helicity and looks for changes in scattering, any change in the polarized beam correlated with the helicity reversal can be a potential source for systematic error, or a false asymmetry. This includes energy changes, position changes, intensity changes, or spot-size changes. To first order, this can be written as:

$$
A_{\text {raw }}=A_{\text {det }}-A_{Q}+\alpha \Delta E+\sum \beta_{i} \Delta x_{i}
$$

where $A_{\text {raw }}$ is the beam current normalized detector asymmetry, $A_{Q}$ is the beam charge asymmetry, $\Delta E$ is the helicity correlated energy difference, $\Delta x_{i}$ are the 
helicity correlated position differences, and $\alpha, \beta_{i}$ are the coupling constants, both calculated and measured through cross correlations and linear regression in data analysis. Additionally, helicity correlated changes to the spot size of the beam can also give rise to systematic errors. For precise comparisons to be made, the two helicity state beams must be extremely symmetric: their intensity, position, and spot-size must be very nearly identical.

\begin{tabular}{|c|c|c|c|c|c|}
\hline $\begin{array}{c}\text { Beam } \\
\text { Property }\end{array}$ & $\begin{array}{c}\text { Assumed } \\
\text { Sensitivity }\end{array}$ & $\begin{array}{c}\text { Accuracy of } \\
\text { Correction }\end{array}$ & $\begin{array}{c}\text { Required 2kHz } \\
\text { random fluctuations }\end{array}$ & $\begin{array}{c}\text { Required cumulative } \\
\text { HCBA }\end{array}$ & $\begin{array}{c}\text { Systematic } \\
\text { Contribution }\end{array}$ \\
\hline Intensity & $1 \mathrm{ppb} / \mathrm{ppb}$ & $\sim 1 \%$ & $<1000 \mathrm{ppm}$ & $<10 \mathrm{ppb}$ & $\sim 0.1 \mathrm{ppb}$ \\
Energy & $-1.4 \mathrm{ppb} / \mathrm{ppb}$ & $\sim 10 \%$ & $<108 \mathrm{ppm}$ & $<0.7 \mathrm{ppb}$ & $\sim 0.05 \mathrm{ppb}$ \\
Position & $0.85 \mathrm{ppb} / \mathrm{nm}$ & $\sim 10 \%$ & $<47 \mu \mathrm{m}$ & $<1.2 \mathrm{~nm}$ & $\sim 0.05 \mathrm{ppb}$ \\
Angle & $8.5 \mathrm{ppb} / \mathrm{nrad}$ & $\sim 10 \%$ & $<4.7 \mu \mathrm{rad}$ & $<0.12 \mathrm{nrad}$ & $\sim 0.05 \mathrm{ppb}$ \\
Spot-size & $12 \mathrm{ppb} / \mathrm{ppt}$ & - & - & $<10^{-6}-10^{-5}$ & $\sim 0.012-0.12 \mathrm{ppb}$ \\
\hline
\end{tabular}

Table 2.3: MOLLER Beam Goals [71]

For MOLLER, there are more stringent requirements on the systematic error as shown in Table 2.3 with goals of a few parts-per-billion for the intensity asymmetry, about $1 \mathrm{~nm}$ for position differences, and spot-size asymmetries at the $<10^{-5}$ level. For each type of HCBA (helicity correlated beam asymmetry), the table shows the assumed sensitivity of $A_{P V}$ on the beam parameter, the accuracy of the corresponding beam correction which will be made to $A_{P V}$ in data analysis, the required statistical noise given as asymmetry and helicity-pair difference widths, and the implied correction for the expected helicity asymmetry. The last column is the expected contribution to the systematic uncertainty on $A_{P V}$ given the anticipated correction precision achieved in the results of Qweak (2011) and HAPPEX-II (in 2005). Minimizing systematic experimental uncertainty begins at the Pockels cell source and was the motivation for the design of a new RTP Pockels cell to ensure both fast transition times and small helicity-correlated asymmetries

Table 2.4 shows a comparison of the HCBA goals for MOLLER with those previously achieved during HAPPEX-II. The intensity asymmetry $I_{A}$ must be 
kept 3X smaller. Position differences, angle differences, and energy differences all need about factor of $2 \mathrm{X}$ improvement on HAPPEX-II. The goal for MOLLER is a $2 \mathrm{kHz}$ fast helicity flip rate with $10 \mu \mathrm{s}$ settle time, whereas previously, Qweak achieved $1 \mathrm{kHz}$ with a $60 \mu$ s settle time using a $\mathrm{KD}^{*} \mathrm{P}$ Pockels cell. This thesis is focused on the RTP polarized source goals: (1) to develop a source that can flip helicity states quickly, so we don't lose statistics through deadtime (waiting for the polarization to flip); and (2) also to get the beam stable - at the nm level on target - and to control HCBA's.

\begin{tabular}{|c|c|c|}
\hline & MOLLER & previously achieved \\
\hline Intensity Asymmetry & $<10 \mathrm{ppb}$ & $30 \mathrm{ppb}$ (Qweak) \\
Energy Asymmetry & $<0.7 \mathrm{ppb}$ & $0.2 \mathrm{ppb}$ (H-II) \\
Position Differences & $<1.2 \mathrm{~nm}$ & $2 \mathrm{~nm}$ (H-II) \\
Angle Differences & $<0.12 \mathrm{nrad}$ & $0.25 \mathrm{nrad}$ (H-II) \\
Spot-size Asymmetries & $<1 \times 10^{-5}$ & $<1 \times 10^{-4}$ (PREX-I,Qweak) \\
\hline
\end{tabular}

Table 2.4: Comparison of MOLLER PQB requirements to those achieved during HAPPEX-II, Qweak, and PREX-I [62]

\section{Meeting MOLLER goals for HCBA}

Table 2.5 shows the path towards achieving the small HCBA's required for MOLLER. As regards the intensity asymmetry requiring a $3 \mathrm{X}$ improvement over the past, this can be improved with better feedback, the longer run period, and more strict data cuts for good long-term cancellation [62]. Nominally 10ppb in the Hall is achieved from obtaining $<10 \mathrm{ppm}$ in the injector, which is then reduced by a factor of 10X through slow helicity reversals such as the insertable half-wave plate (IHWP) and the double Wien (see Sec. 3.5.1), and reduced again by another factor of $100 \mathrm{X}$ by using feedback on the Pockels cell voltages to minimize $A_{q}$, finally giving 10ppm/10/100 10ppb. We note the main hurdle for the RTP Pockels cell was maintaining $A_{q}$ stability as there were slow drifts ( $100 \mathrm{ppm}$ in $30 \mathrm{~min}$ for $e^{-}$beam, larger for laser) due to fluctuations in temperature difference between the two RTP crystals in the cell $\left(\sim 50 \mathrm{kppm} /{ }^{\circ} \mathrm{C}\right.$ for $\Delta T$ and $100 \%$ analyzing $)$. 
However, these slow drifts are easily corrected with $A_{q}$ PITA-voltage feedback on the cell. Summer 2018 injector studies showed that feedback could used on intensity fluctuations to cancel slow drifts.

\begin{tabular}{|c|c|c|c|c|}
\hline & Source & Adiabatic Damping & Slow Reversals & Feedback \\
\hline Intensity & $<10 \mathrm{ppm}(\mathrm{inj})$ & - & $\sim 10 \times$ & $100 \times$ \\
\hline $\begin{array}{c}\text { Position/angle } \\
\text { (Past) }\end{array}$ & $\sim 20 \mathrm{~nm}(\mathrm{inj})$ & $\sim 100 \times$ & $\sim 10 \times$ & $\sim 10 \times$, control jitter \\
\hline Spot-size & $<10^{-5}$ (laser) & (synch light & $\sim 10 \times($ IHWP, $\mathrm{g}-2, \mathrm{ISM})$ & - \\
(Past) & $\left(<10^{-4}\right)$ & dilution $)$ & $(\sim 10 \times$ from ISM $)$ & - \\
\hline
\end{tabular}

Table 2.5: Steps to achieving MOLLER PQB requirements compared with what has previously been achieved [62]

Regarding position/angle differences, the ultimate goal is to achieve $<1.2 \mathrm{~nm}$, $<0.12 \mathrm{nrad}$ in the Hall and this can be done by a combination of improvements to position differences in the injector, adiabatic damping, slow helicity reversals, and position difference feedback. The first goal is for position differences to be kept $<20-30 \mathrm{~nm}$ in the injector before acceleration. We have made significant strides towards this goal using the an RTP design which utilizes new degree of position difference control. We estimated the effect of the electric field gradients in a polarization dependent steering model, calculated the E-field gradients for different geometries with the dielectric crystals, and found a way to control the E-field gradient to steer the beam. We have demonstrated the ability to use the RTP cell for active, voltage controlled, position feedback. Summer 2018 injector studies showed that in a particular selected bpm, electron beam position differences could be controlled to within 1-5nm (Sec. 6.6). Overall in the injector, before the chopper, the RTP achieved electron beam position differences $<70 \mathrm{~nm}$ in 2018 and $<30 \mathrm{~nm}$ in 2019. Both charge and position asymmetries were well controlled, the RTP cell shows promise for MOLLER, and it is being considered for installation during PREXII.

In adiabatic damping, the area of the beam distribution in phase space is inversely proportional to the momentum. If the electron beam is aligned well, 
acceleration from momentum $p_{0}$ to $p$ can reduce position differences by a factor of $\sqrt{p / p_{0}}$. In the past adiabatic damping has been shown to reduce position differences by a factor of 10-30X, at lower $(3 \mathrm{GeV})$ energies. In principle, MOLLER $11 \mathrm{GeV}$ beam energy should provided up to $150 \mathrm{X}$. However realizing the full benefit requires improved injector performance and good optical transport through the accelerator that has yet to be demonstrated (which is why only 10-30X damping was observed instead 100X previously). We note that the improvements shown in Fig. 2.5 in each of these steps are in total, overkill by a factor of 500X: for position $20 \mathrm{~nm} / 100 / 10 / 10 \sim 0.002 \mathrm{~nm}>1.2 \mathrm{~nm}$. So, if for example, very small position differences are achieved in the injector, 100X adiabatic damping is unnecessary, and vice versa. Furthermore, if the factor of $2 \mathrm{X}$ improvement (as compared with HAPPEX-II) in beam position asymmetry were not achieved, it would be a small hit on total error bar, or even zero net loss if correction precision can exceed modest assumptions [62].

Spot size asymmetry contributions to the systematic error are nominally given by $12 \mathrm{ppm} \times \delta \sigma / \sigma$ [1]. Based on this assumption, the spot size asymmetry for MOLLER requires 10X improvement over the past $\left(<1 \times 10^{-5}\right.$ vs $10^{-4}$ quoted in Qweak and PREX), but incoherent emittance growth provides this factor. The suppression between spot size asymmetry in the injector and spot-size asymmetry contribution in the Hall, is due to the assumption of synchrotron light emittance growth greatly suppressing the spot size asymmetry by a factor of $A_{\sigma, i n j} / A_{\sigma, \text { synch }} \sim$ $25 X-100 X$. We note that coherent beam size changes (damping, raster, focusing) don't affect the dilution, only stochastic processes help dilute the asymmetry. With the RTP cell, $A \sigma$ (on laser, estimated for $e^{-}$beam) was as good as $10^{-4}$ (for the laser) and $10^{-5}-10^{-4}$ in the $e^{-}$beam off the cathode.

MOLLER also depends on qualities of the beam beyond standard PQB requirements shown in the above tables, most notably beam "halo". Beam halo was observed during Qweak in the small angle monitors as a beam asymmetry which correlated with the BCM asymmetry, but which was not cancelled by normalizing 
to the $\mathrm{BCM} A_{q}$. It was theorized this was due to a small current density outside the main beam profile RMS which had a large charge asymmetry, not reflected in the central portion of the beam current as detected by the BCMs. However, the asymmetry "halo" is not yet well understood and the collaboration needs to identify which parameters require development. A program of test plans on beam halo should be defined before MOLLER and various ideas for "halo" monitors are in development. Additionally, there are some suggestive beam test results as regards the longitudinal structure of the $e^{-}$beam pulse. During beam studies in summer 2018, longitudinal bunch length asymmetries were observed to develop from induced charge asymmetries via space-charge effects. The longitudinal asymmetries in the $e^{-}$beam may potentially couple into the transverse HCBA's that MOLLER depends on minimizing.

To summarize: source considerations for MOLLER include fast flipping, short transition times, noise assessments, injector position differences, injector spot size asymmetries, adiabatic damping, couplings in accelerator, coherent emittance growth, apertures, halo, longitudinal bunch length asymmetries, and longitudinal position differences. ${ }^{5}$

\subsubsection{Summary of Goals for MOLLER}

MOLLER presents a number of experimental challenges to achieve high precision including stringent requirements on the properties for the polarized electron beam, a high luminosity liquid hydrogen target with extremely small pulse-to-pulse density fluctuations, and control of contributions from background scattering pro-

\footnotetext{
${ }^{5}$ We should note that before MOLLER, there will be an injector upgrade. A $200 \mathrm{keV}$ gun has been installed, though it not used at that voltage yet. The new gun should improve cathode lifetime and should improve transmission without RF pre-bunching. Two new source BPMs 2I01 and 2I02 have been installed just after photocathode. Other upgrades in the near future include improvements to injector spin manipulation (double Wien) which will be upgraded to work at $200 \mathrm{keV}$ with improved optics elements/apertures, new electrodes and coils, and new HV supplies [62]. The plan is to remove the prebuncher between elements for more reliable operation and quicker setup. There will be a new $1 / 4$ cryomodule $(10 \mathrm{MeV}$, integrates RF capture) which will reduce $\mathrm{x} / \mathrm{y}$ coupling that has in the past been major impediment to optics match to design.
} 
cesses [46]. Fig. 2-31 shows a summary of the statistical and systematic error goals for MOLLER : $2.1 \%$ statistical and $1.1 \%$ systematic.

\begin{tabular}{|l|c|}
\hline Error Source & Fractional Error (\%) \\
\hline \hline Statistical & $\mathbf{2 . 1}$ \\
\hline Absolute Normalization of the Kinematic Factor & 0.5 \\
Beam (second order) & 0.4 \\
Beam polarization & 0.4 \\
$e+p(+\gamma) \rightarrow e+X(+\gamma)$ & 0.4 \\
Beam (position, angle, energy) & 0.4 \\
Beam (intensity) & 0.3 \\
$e+p(+\gamma) \rightarrow e+p(+\gamma)$ & 0.3 \\
$\gamma^{(*)}+p \rightarrow(\pi, \mu, K)+X$ & 0.3 \\
Transverse polarization & 0.2 \\
Neutral background (soft photons, neutrons) & 0.1 \\
\hline \hline Total systematic & $\mathbf{1 . 1}$ \\
\hline
\end{tabular}

Figure 2-31: Precision Goals for the MOLLER experiment [71]. All systematics are required at the sub-1\% level.

This work is primarily focused on the beam intensity asymmetry, position differences, and 2nd order effects like spot size asymmetry. These goals translate into helicity correlated beam asymmetry minimization down to the ppb-level in intensity, and $1.2 \mathrm{~nm}$ level in position difference between left and right helicity states. If these HCBA goals are achieved, MOLLER is expected to measure $A_{P V} \sim$ $35.6 \mathrm{ppb}$ with a goal to reach a precision of $0.73 \mathrm{ppb}$ on $A_{P V}$, as precise as the best collider Z-pole measurement and probe new BSM physics.

To summarize, source considerations for MOLLER include ambitious, but achievable goals. The polarized source achievements using the RTP cell will be discussed in Chapter 6 and in Chapter 5 beam studies. 



\section{Chapter 3}

\section{PREX-II}

\subsection{Parity Violation}

Our picture of the atomic nucleus comes from nuclear charge densities which have been well measured by electron scattering experiments [76]. However, neutron densities in the nucleus are not so well known because the neutron is uncharged, thus it is not probed directly in typical electron scattering measurements, as well as due to uncertainties in the strongly interacting probes [164]. Donnelly, Dubach, and Sick [78] suggested that parity violating electron scattering can measure neutron densities [77] in nuclei. As described in Sec. 1.2, in PVES experiments a longitudinally polarized electron beam is incident on an unpolarized target, the sign of the longitudinal polarization is changed making a parity transformation. The fractional rate difference between right and left helicity states is measured, forming an asymmetry $A_{P V}=\left(\sigma_{R}-\sigma_{L}\right) /\left(\sigma_{R}+\sigma_{L}\right)$ arising from interference term between the electromagnetic and weak amplitudes, $\mathcal{M}_{\gamma}$ and $\mathcal{M}_{Z}$, which, in the Born approximation, is proportional to the ratio of the weak and electromagnetic form factors $F_{\text {weak }} / F_{E M}$, closely related to the Fourier transform of the neutron density. The reason PVES can provide information regarding neutron densities in nuclei is that the $\mathrm{Z} 0$ boson, that carries the weak force, couples primarily to the 
neutron, rather than the proton, at low $Q^{2}$, while $\mathcal{M}_{\gamma}$ is dominated by the electric charge (proton) distribution, which is already well measured by electron scattering. Hence, the weak-charge density, and the closely related neutron density, can be deduced from parity-violating asymmetry measurement in PVES experiments [77]. PVES provides a clean measurement of $R_{N}$, the RMS radius of neutrons in a heavy nucleus, and constrains the equation of state (EOS) of highly dense matter which has impact on neutron star structure, heavy ion collisions, and atomic parity violation experiments [164] . The Lead Radius Experiment (PREX) will measure the parity violating asymmetry $A_{P V}$ for $1 \mathrm{GeV}$ electrons scattering from ${ }^{208} \mathrm{~Pb}$ at five degrees, and should be sensitive to the neutron radius of ${ }^{208} \mathrm{~Pb}$ to $1 \%$ $( \pm 0.05 \mathrm{fm})$ precision [164]

\subsection{Principles}

\subsubsection{Fundamental Considerations}

The differential cross section for electron scattering off of a nucleus is dominated by the electromagnetic interaction of the electron with the with proton distribution in the nucleus, characterized by the form factor $F_{p}$. In the born approximation, the differential cross section can be written as [77]

$$
\frac{d \sigma}{d \Omega}=\frac{d \sigma}{d \Omega}_{M o t t}\left|F_{p}\left(Q^{2}\right)\right|^{2}
$$

where the Mott cross section consists of the electron Rutherford cross section, appropriate for low energies, and additionally takes into account the election magnetic moment effect and nuclear recoil effect in the terms $(1+\cos \theta) / 2$ in the numerator and $(1-\cos \theta) K E / M c^{2}$ in the denominator, respectively.

$$
\frac{d \sigma}{d \Omega}_{M o t t}=\frac{d \sigma}{d \Omega} \frac{(1+\cos \theta) / 2}{R} \frac{\pi}{1+\frac{(1-\cos \theta) K E}{M c^{2}}} Z^{2} \alpha^{2}\left(\frac{\hbar c}{K E}\right)^{2} \frac{1}{(1-\cos \theta)^{2}}
$$


which can be written as

$$
\frac{d \sigma}{d \Omega}_{M o t t}=\frac{4 Z^{2} \alpha^{2} E^{2}}{Q^{2}}
$$

Our picture of nuclei comes from the electric charge distribution, which has been very well measured already. As Q increases, nuclear size becomes an important correction to the differential scattering cross section. Neglecting relativistic recoil, the form-factor $\mathrm{F}(\mathrm{q})$ is the Fourier Transform of charge density $\rho(r)$. The proton and neutron form-factors are defined in terms of the density distributions as $[77]$

$$
\begin{aligned}
& F_{p}\left(Q^{2}\right)=\frac{1}{4 \pi} \int d^{3} r j_{0}(q r) \rho_{p}(r) \\
& F_{n}\left(Q^{2}\right)=\frac{1}{4 \pi} \int d^{3} r j_{0}(q r) \rho_{n}(r)
\end{aligned}
$$

where $j_{0}$ is the $0 t h$ spherical Bessel function. The form factor is the Fourier transform of charge density: The edge of the electric charge distribution in nuclei gives rise to this diffraction as a function of $\mathrm{Q}$ (analogous to an airy pattern in optics) as shown in Fig. 3-1 [76].

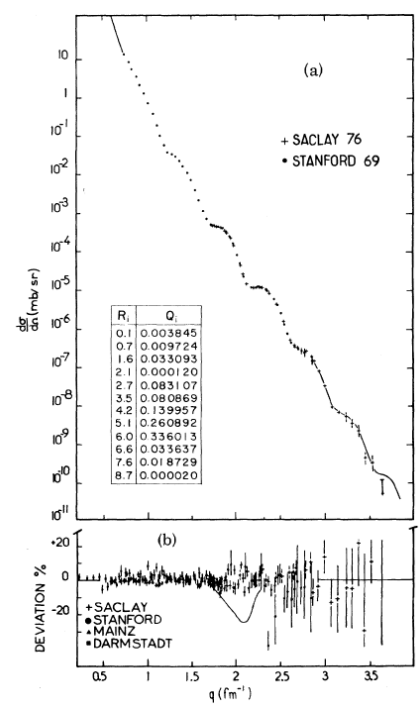

Figure 3-1: (a) Cross sections at $\mathrm{E}=502 \mathrm{MeV}$ as a function of effective momentum transfer. (b) Deviation between fit and data used. [76]

PREX and CREX are parity violating electron scattering experiments which 
aim to map the weak charge distribution in nuclei, which is much harder to measure than the electric charge distribution. Longitudinally polarized electrons scattering off an unpolarized target produce a scattering cross-section containing an interaction term between the electromagnetic and weak amplitudes.

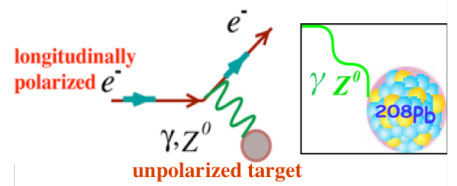

Figure 3-2: Electron scattering off ${ }^{208} \mathrm{~Pb}$ target nucleus with Z-boson and photon mediators [73]

$$
\sigma \sim\left|M_{\gamma}+M_{\text {weak }}\right|^{2} \sim\left|M_{\gamma}\right|^{2}+2 M_{\gamma}\left(M_{\text {weak }}\right)^{*}+\ldots
$$

The resulting parity-violating asymmetry is measured by comparing left and right helicity states, and can be shown to be proportional to the ratio of the weak and electric charge form factors.

$$
A_{P V}=\frac{\sigma_{R}-\sigma_{L}}{\sigma_{R}+\sigma_{L}}
$$

${ }^{208} \mathrm{~Pb}$ is the chosen target for the PREX experiment because it is notable as the heaviest known stable isotope of any element , with $\mathrm{Z}=82, \mathrm{~N}=126$, and nuclear spin $0^{1}$. This choice of a spinless target and low $Q^{2}$ makes for a more straightforward calculation of $A_{P V}$, as angular momentum can't be absorbed by the nucleus.

In the electron scattering process, the predominant couplings between the electron and the nucleus occur via the photon and z-boson mediators at tree-level with propagators of the form [77]

$$
\frac{1}{Q^{2}+M_{Z}^{2}} \approx \frac{1}{M_{Z}^{2}}
$$

\footnotetext{
${ }^{1}$ all nuclei with even $\mathrm{Z}$ and even $\mathrm{N}$ have nuclear spin $\mathrm{I}=0$
} 


$$
\frac{1}{Q^{2}+M_{\gamma}^{2}}=\frac{1}{Q^{2}}
$$

. The photon couples largely to the protons in the nucleus, and the Z-boson couples largely to the neutrons in the nucleus.

\begin{tabular}{l|r|r} 
& proton & neutron \\
\hline electric charge & 1 & 0 \\
weak charge & $\sim 0.08$ & 1
\end{tabular}

Table 3.1: Electric and weak charges of spin-0 nucleus [73]

The weak form factor almost entirely couples to the neutron distribution and the electric charge form factor entirely couples to the proton distribution, due to the small weak charge of the proton and non-existent electric charge of the neutron. In a spinless target, the photon, with its pure vector couplings, has no contribution from magnetic components, and the Z-boson's net axial vector coupling is absent [77]. The choice a a spinless, closed-shell, isospin 0 target nucleus simplifies matters, and $A_{p v}$ is unaffected by relativistic corrections to the cross section. In this scenario, the asymmetry is given by [79]

$$
A_{P V}=A_{P V}^{0}\left[g_{V}^{p}+g_{V}^{n} \frac{F_{n}\left(Q^{2}\right)}{F_{p}\left(Q^{2}\right)}\right]
$$

2. Neglecting radiative corrections, at tree level (in $\bar{M} S$ scheme),

$$
\begin{gathered}
g_{V}^{p}=1-4 \sin ^{2} \theta_{W} \\
g_{V}^{n}=-1
\end{gathered}
$$

Hence we can write the asymmetry as [77]

$$
\begin{aligned}
& A_{P V}=\frac{G_{F} Q^{2}}{4 \pi \alpha \sqrt{2}}\left(4 \sin ^{2} \theta_{W}-1+\frac{F_{n}\left(Q^{2}\right)}{F_{P}\left(Q^{2}\right)}\right) \approx \frac{G_{F} Q^{2}}{4 \pi \alpha \sqrt{2}} \frac{F_{n}\left(Q^{2}\right)}{F_{P}\left(Q^{2}\right)} \\
& { }^{2} \text { where } A_{P V}^{0}=-\frac{G_{F} Q^{2}}{4 \pi \alpha \sqrt{2}}
\end{aligned}
$$


where $G_{F}$ is the Fermi coupling, $\mathrm{Q}$ is the 4-momentum transfer in the process 3. Though not useful for realistic calculations, it is worth recalling the Born approximation to Apv, as it nicely illustrates that Apv relates to the neutron and proton nuclear form factors [117]. Measurement of the parity violating asymmetry $A_{P V}$ is a direct measure of the Fourier transform of the neutron density at choice of $Q^{2}$.

The Fourier transform of the proton density at a particular choice of $Q^{2}$ has been determined by previous electron scattering experiments [76] as shown in Fig. 3-1 .The weak form factor (and corresponding neutron radius) are not as well understood as the proton nuclear radius (and corresponding electric charge form factor). However this ratio of weak to electromagnetic form factors is directly related to the neutron skin thickness on heavy nuclei predicted by nuclear theory as shown in this plot of proton/electric charge density vs radius in red and neutron/weak charge density in black.

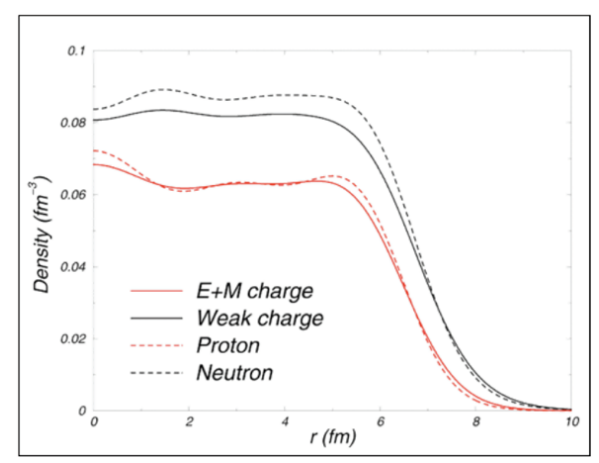

Figure 3-3: Neutron and proton distributions in ${ }^{208} \mathrm{~Pb}[73]$

This neutron skin thickness is highly sensitive to the pressure of pure neutron matter: the greater the pressure, the thicker the skin as neutrons are pushed out against surface tension. Apv provides a clean measure of the neutron skin thickness.

\footnotetext{
${ }^{3}$ We note that coulomb distortion effects must be accounted for in the calculation for ${ }^{208} \mathrm{~Pb}$, since the born approximation isn't valid for the heavy nucleus [79]. The coulomb distortion effects have been calculated([80],[81],[82],[83],[84],[79]) and that and other effects discussed [77].
} 


\subsubsection{Neutron Skin, $A_{p v}$, Symmetry Energy, and EOS}

The basic property of the size of a heavy nucleus, both it's neutron radius and proton radius, is an important feature of all heavy nuclei [77]. However, the size cannot be fully inferred from measured charge radii because of a neutron skin of uncertain thickness [77]. We have derived above that the measured asymmetry in polarized electron scattering directly relates to the ratio of neutron and proton form factor distributions at particular $Q^{2}$. How does this inform the neutron skin thickness? At a single $Q^{2}$, how can this measurement determine the skin thickness at a particular radius $\mathrm{r}$ where the edge of the nucleus in ${ }^{208} \mathrm{~Pb}$ is? $\left(r \sim r_{0} A^{1 / 3}\right.$ where $r_{0}=1.2 \mathrm{fm}$ ) How does one choose the $Q^{2}$ ? These questions have been theoretically explored extensively, and reveal a model independent solution. The appropriate choice of $Q^{2}$ has been determined and the optimal kinematics of PREX are an electron beam energy is $1.06 \mathrm{GeV}$ and a scattering angle of $5^{\circ}$ [121]. At appropriate choice of $Q^{2}$, the neutron skin thickness is related to $A_{p v}$ via a linear relationship. The neutron skin is furthermore related to the density slope of the symmetry energy also via a linear relationship, thereby constraining the equation of state in a model independent manner. The PREX measurement is unique in that it constrains L more than it constrains $\mathrm{S}$ at saturation density, and other experiments constrain a different portion of the parameter space, that PREX cuts across.

Qualitatively it makes sense that the neutron skin should relate to $A_{p v}$ : the greater the pressure of pure neutron matter, the thicker the skin as neutrons are pushed out against surface tension. Apv provides a clean measure of the neutron skin thickness. Furthermore theoretically, quantitatively it does relate by a simple, direct, linear relationship as shown in Fig. 3-4. Every existing model that predicts a weak charge density and an electric charge density demonstrate that neutron skin thickness and the parity-violating asymmetry are by no means independent parameters. Rather, they are firmly dependent parameters. For sensitive $Q^{2}$, 
there is robust correlation between ${ }^{208} \mathrm{~Pb} A_{P V}$ and the neutron skin over existing nuclear structure models. Asymmetry in PVES provides a clean probe of the neutron distribution, one is a direct measure of the other, and the precision on one directly translates to a precision on the other. Note that in principle, the weak radius is related to the derivative of the form factor evaluated at zero $Q^{2}$ : $\left.\partial F_{W}\left(Q^{2}\right)\right|_{Q^{2}=0} \sim R_{W}$ [77], though in practice the measurement is at a non-zero $Q^{2}$, so since $A_{p v}$ accesses the weak form factor, it makes sense it should relate to the neutron radius directly. For PREX, an Apv precision of $3 \%$ directly translates to precision on the neutron radius in ${ }^{208} \mathrm{~Pb}$ of $0.06 \mathrm{fm}$. For CREX, an $A_{P V}$ precision of $2.5 \%$ directly translates to precision on the neutron radius in $48 \mathrm{Ca}$ of $0.02 \mathrm{fm}$.

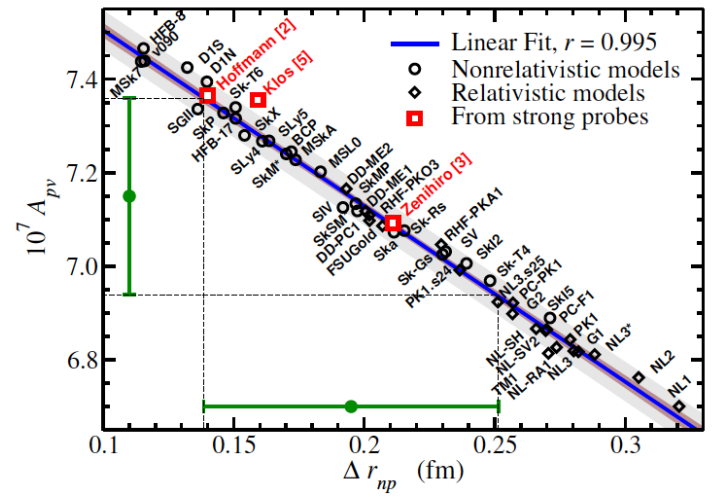

Figure 3-4: Parity-violating asymmetry for ${ }^{208} \mathrm{~Pb}$ at the kinematics of PREX against the neutron skin of ${ }^{208} \mathrm{~Pb}$. The linear fit is $10^{7} A_{p v}=7.88-3.75 \Delta r_{n p}$. The inner/outer colored regions depict the loci of the $95 \%$ confidence/prediction bands of the regression. Also shown are the points calculated with the neutron densities deduced from experiment. [117]

The manner in which $A_{p v}$, the experimental result, is related to the weak form factor and the neutron skin thickness through calculation is as follows. Since the charge density is known, a Born approximation equivalent weak form factor $F_{W}\left(Q^{2}\right)$ can be deduced from the measured asymmetry $A_{p v}$ at the $Q^{2}$ of the experiment. One way this can be done is to adjust a range of model weak densities until full distorted wave calculations reproduce $A_{p v}$, then take the Fourier transform of the weak charge density to calculate $F_{W}\left(Q^{2}\right)$. Since full distorted wave 
calculations need some information on $F_{W}\left(Q^{2}\right)$ for q different from the single measurement, this procedure is slightly model dependent, but the model dependence is expected to be very small [77].

Intuitively, the density distribution of protons and neutrons within a nucleus must be energetically favorable, it must relate to the symmetry energy or energy penalty for breaking $\mathrm{N}=\mathrm{Z}$ symmetry. As the radius reaches the edge of the nucleus, as the density falls off as in Fig. 3-3, the symmetry energy as a function of density must play an important role in the determining the thickness of the neutron skin. The slope of the symmetry energy at saturation density (corresponding to nuclear densities) is defined as $\mathrm{L}$

$$
\left.L \sim \frac{\partial S(\rho)}{\partial \rho}\right|_{\rho_{0}}
$$

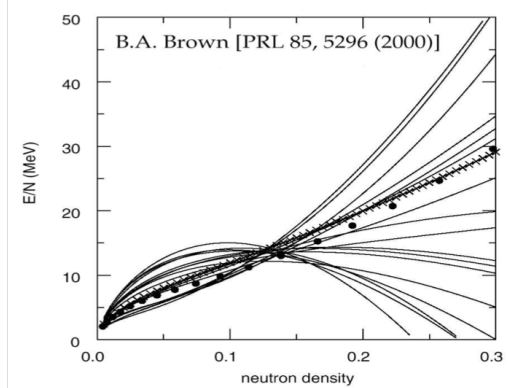

Figure 3-5: A variety of models predicting the symmetry energy E/ $\mathrm{N}$ vs. the neutron density with the constraint of the well characterized value of the symmetry energy at saturation density, but allowing for a range of L (slope os symmetry energy at saturation density) that leads to large divergence of theoretical predictions at high densities, such as in neutron stars [118]

The value of $\rho_{0}$ is found from the central density of heavy nuclei, such as ${ }^{208} \mathrm{~Pb}$, corrected for surface tension and Coulomb interactions, inferring the saturation density for infinite systems [77]. The saturation density is relevant parameter in nuclear structure models, the nature of the interactions between nucleons, models of heavy ion collisions, and applications of dense matter in astrophysics [77].

Quantitatively, mean-field predictions show a clear correlation between neutron skin of a heavy nucleus and L the density slope of the symmetry energy as shown 
in Fig. 3-6. Just as the $A_{p v}$ and the neutron skin are deterministically linked, so the neutron skin and the slope of the symmetry energy are deterministically linked. So far the probes for stable medium and heavy nuclei have been strongly interacting, having a somewhat more complicated interpretation. In contrast, the neutron radius calibrates the EOS of neutron rich matter directly, and constrains and guides models needed for heavy nuclei via L, the slope of the symmetry energy at saturation density as shown in Fig. 3-5.

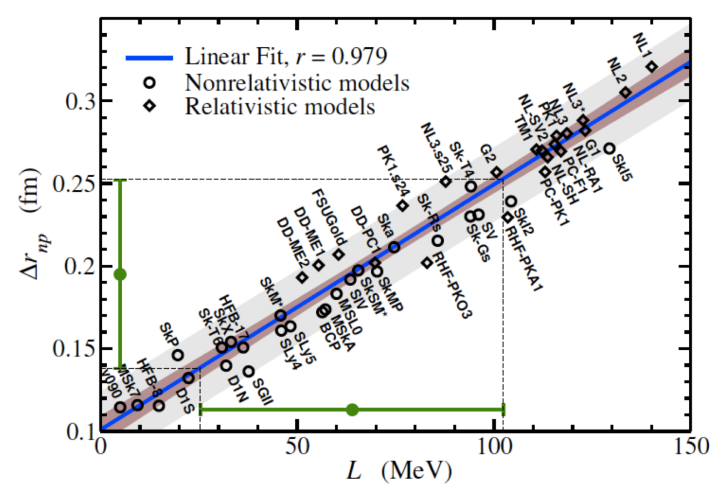

Figure 3-6: Neutron skin of ${ }^{208} \mathrm{~Pb}$ against slope of the symmetry energy L. The linear fit is $\Delta r_{n p}=0.101+0.00147 L$. A sample test constraint from a $3 \%$ accuracy in $A_{p v}$ (as in PREX-I) is drawn[117]

\subsection{Seminal Texts and Experiments}

\subsubsection{Neutron Density Theory and Corrections to $A_{p v}$}

Nuclear charge densities and charge radii can be calculated using various mean field theory models. Two typical interactions used in MF models are a nonrelativistic zero-range Skyrme force and a relativistic mean field calculation, the predictions of which are shown in Fig. 3-7 [126]. Across nuclei of various mass number A, the relativistic MF calculation predicts a smaller neutron density and larger neutron radius than the Skyrme interaction calculation. Such MF models are not currently well constrained by measurements of neutron radii. But if a neutron radius measurement, of ${ }^{208} \mathrm{~Pb}$ for example, were performed precisely, it 
would constrain the MF models and allow for a variety of predications in other highly dense matter scenarios, such as in unstable, exotic nuclei important for astrophysics and radioactive isotope beams [77].

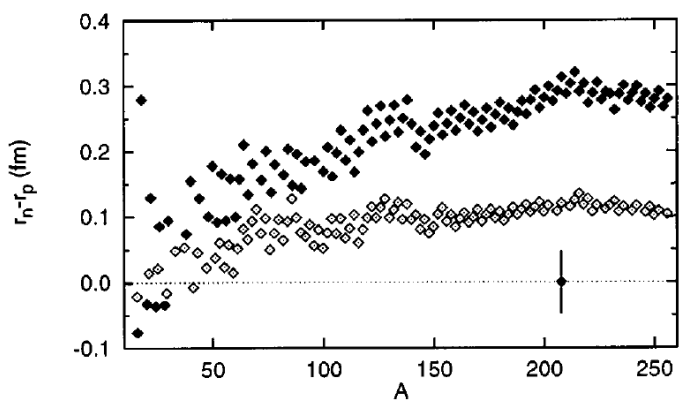

Figure 3-7: Neutron skin thickness for various nuclei of mass number A. Black diamonds are a calculation from the nonrelativistic zero range Skyrme skiii interaction and white diamonds are from the relativistic mean field NL1 interaction [126]

PVES is a very clean measure of the neutron skin thickness, as can be demonstrated by examining several corrections to $A_{p v}$ and demonstrating small uncertainties on these corrections, as is done in the following section. The corrections to the asymmetry are considered extensively by Horowitz, Pollock, Souder, and Michaels [77]. Their analysis is summarized here.

Coulomb distortions: The most significant correction to $A_{p v}$ comes from Coulomb distortions, which arise from repeated EM interactions of the electron with the ground-state nucleus. These corrections are on the order of $\sim Z \alpha / \pi \sim 20 \%$ for ${ }^{208} \mathrm{~Pb}$ [77]. While Coulomb distortion corrections are larger than the expected experimental error, they have been calculated [132] to an accuracy much smaller than the experimental error, solving the Dirac equation numerically for an electron in axial-vector weak and Coulomb potentials, as confirmed by many cross-checks [137] [138] [139].

Strangeness and neutron electric form factors: The strange quarks contribution to the neutron electric form factor, while relevant, are not significant for a neutron radius measurement. The experimental result is the weak form factor $F_{W}$ which 
can be used to determine the weak radius $R_{W}$, which, only after appropriate corrections for strange quark contributions, can be related to the neutron radius $R_{n}$. For a spin-0 nucleus, only the electric form factors for Z-coupling to the neutron and proton need be considered

$$
\begin{aligned}
G_{p}^{Z} & =\frac{1}{4}\left(G_{p}-G_{n}\right)-\sin ^{2} \theta_{W} G_{p}-\frac{1}{4} G_{s} \\
G_{n}^{Z} & =-\frac{1}{4}\left(G_{p}-G_{n}\right)-\sin ^{2} \theta_{W} G_{n}-\frac{1}{4} G_{s}
\end{aligned}
$$

The relationship between the weak distribution radius $R_{W}$ and neutron distribution radius $R_{n}$ can be derived as [77]

$$
\begin{aligned}
& R_{W} \approx R_{n}+\frac{Z\left(1-4 \sin ^{2} \theta_{W}\right)}{N+\left(4 \sin ^{2} \theta_{W}-1\right) Z}\left(R_{n}-R_{p}\right) \\
& +\frac{1}{2 R_{n}}\left(r_{p}^{2}+\frac{Z+\left(4 \sin ^{2} \theta_{W}-1\right) N}{N+\left(4 \sin ^{2} \theta_{W}-1\right) Z} r_{n}^{2}+\frac{N+Z}{N+\left(4 \sin ^{2} \theta_{W}-1\right) Z} r_{s}^{2}\right)
\end{aligned}
$$

where $r_{p}^{2}$ is the mean square charge radius of the proton, $r_{n}^{2}$ is the mean square of the neutron charge radius, and $r_{s}^{2}$ is the mean square strangeness radius. The strangeness contribution to $R_{n}$ in ${ }^{208} \mathrm{~Pb}$ is $<0.4 \%$ [140] [141] [142] [77]. It can be shown [77] the neutron radius can be related to $<1 \%$ accuracy to the weak radius via

$$
R_{n} \approx R_{W}-0.06 \mathrm{fm}
$$

demonstrating the neutron radius $R_{n}$ of a heavy nucleus can be accurately determined from the measured weak radius $R_{W}$.

Parity admixtures: For elastic scattering from a spin zero nucleus, parity admixtures are a non-issue. A parity admixture refers to the ground state of ${ }^{208} \mathrm{~Pb}$ not being an spin zero parity eigenstate, but rather some admixture of $0^{-}$. This doesn't affect the parity violating asymmetry as long as the initial and final states are spin zero, and furthermore as long as the virtual photon exchanged is spin 
zero, there's no PV interference. [77]

Meson exchange currents (MEC): Parity violating meson exchange currents aren't expected to change the measured $R_{n}$ significantly. This is because mesons are expected to carry weak charge over just a short distance $r_{M E C}<<R_{n}$, where $r_{M E C}^{2}$ the square of the mean distance the weak charge is moved by MEC. By the above equation relating $R_{W}$ and $R_{n}$, the change to the neutron radius should only be of order $r_{M E C}^{2} / R_{n}$ which should be quite small. Additionally, the effect of MEC just slightly changes the surface thickness, softening the edge on the neutron distribution, but not extending much further out in radius, so negligibly affecting $R_{n}[77]$

Dispersion corrections: Dispersion corrections are from multiple EM or weak interactions where the nucleus is excited to intermediate states. At low $Q^{2}$, the elastic cross-section should be of order $Z^{2}$, a coherently sum over Z protons, and the inelastic transitions in an incoherent sum should be of order Z, so dispersion corrections are expected to be $\sim \alpha / Z<<1$, insignificant in ${ }^{208} \mathrm{~Pb}$.

Shape dependence and surface thickness: The weak radius as extracted from the form factor may be sensitive to the surface thickness, or that shape of the neutron distribution, as the density falls off. The weak density can be modeled in ${ }^{208} \mathrm{~Pb}$ as

$$
\rho_{W}(r)=\rho_{0} /(\exp [(r-c) / z]+1)
$$

where $\mathrm{c}$ is the radius and $z \approx 0.55 \mathrm{fm}$ is related to the surface thickness. To achieve $1 \%$ precision on $R_{W}$, at $Q^{2} \sim 0.008 \mathrm{GeV}^{2}$, the uncertainty on the surface thickness parameter must be $\sim 0.14 \mathrm{fm}, 25 \%$ of its calculated value, which is entirely feasible. All mean field models have a spread in $z$ of much less than $25 \%$, cause they are constrained well in this regard by known binding energies in various nuclei and a too small surface thickness, a fast density change, results in a too high surface energy. Furthermore, the surface thickness of the charge density is known, and that further constrains the neutron surface thickness [77]. 
Inelastic contributions: The separation of the inelastic and elastic peaks in the high resolution spectrometer is planned to be very clean in PREX-II, as it was during PREX-I. However, in principle one could run in such a way as to attempt to increase rates at the expense of allowing for some inelastic contamination. We consider the inelastic asymmetry. The first excited state in ${ }^{208} \mathrm{~Pb}$ is at $2.6 \mathrm{MeV}$ and has spin and parity $3^{-}$. The first excited state can be modeled as a deformation of the ground state density. The elastic neutron density radius is $R_{n}^{0}$, and the the excited state has can be described by a density parameter $R_{n}^{0}(\theta)$. The asymmetry in this scenario can be derived [77] as

$$
A\left(3^{-}\right) \approx D \frac{G_{F} Q^{2}}{4 \pi \alpha \sqrt{2}}\left(4 \sin ^{2} \theta_{W}-1+\frac{N}{Z}\left(\frac{R_{n}}{R_{p}}\right)^{J=3}\right)
$$

where $D \approx 0.74 \pm 0.26$ is a correction factor for Coulomb distortions at $850 \mathrm{MeV}$ and $6^{\circ}$. For $R_{n} \approx R_{p}$, we obtain

$$
A\left(3^{-}\right) \approx D \frac{G_{F} Q^{2}}{4 \pi \alpha \sqrt{2}}\left(4 \sin ^{2} \theta_{W}-1+\frac{N}{Z}\right)
$$

But this is very similar to the equation that the elastic asymmetry also reduces to, so the asymmetry for excited states is similar to the elastic asymmetry.

$$
\left.A\left(3^{-}\right) \approx 1.25 \text { A(elastic }\right)
$$

The inelastic cross sections at low $Q^{2}$ are considerably smaller than the elastic cross sections, so the contamination is already expected to be small. Additionally, as we just demonstrated, the asymmetry for the first excited state in ${ }^{208} \mathrm{~Pb}$ is similar to the elastic asymmetry, therefore the inelastic correction is reduced even further.

Isospin violation: In the formalism used to treat a heavy nucleon, isospin symmetry in the nucleon is assumed, and isospin symmetry in a heavy nucleus with $N>Z$ is not assumed. Since good isospin in the nucleon is a good assumption, 
with only very small corrections, and since a formalism is used which treats protons and neutron separately, with independent densities, separately adding their contributions to the weak charge density, with no isospin violation corrections, the results aren't impacted much by isospin violation [77].

Radiative corrections: Radiative corrections could potentially come into play via axial current, vector weak current, or bremsstrahlung radiation. However, axial current doesn't contribute in a spin-0 target, vector weak current radiative corrections at low $Q^{2}$ are constrained by current conservation, and the change in beam polarization from bremsstrahlung is $\sim \Delta E / E$ (where $\Delta E$ is the energy resolution) which is only about $0.5 \%$. Hence, radiative corrections are negligible.

To conclude, we show a flow chart of the physics data analysis of PREX, summarizing the corrections made in Fig. 3-8

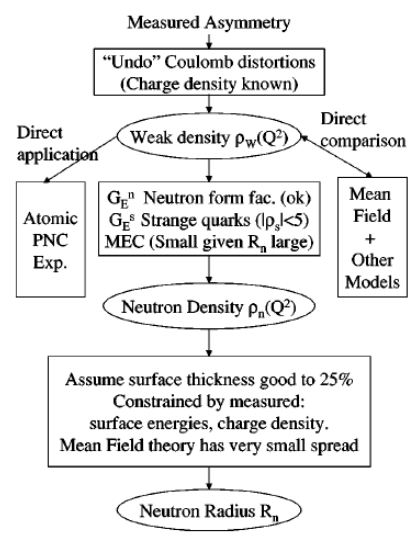

Figure 3-8: Flow chart of the physics data analysis of PREX [77]

\subsubsection{Other Neutron Radius Measurements}

Despite many measurements of neutron densities with strongly interacting probes, due to theoretical systematic errors, there is no established consensus in the community that any existing measurements of neutron densities or radii are accurate to within 1\%. Hadronic probes have uncertainty in the reaction mechanism and therefore interpretation issues when inferring the neutron radius $R_{n}$ [77]. Alternative measurements include Coulomb energy differences, pion or 
proton elastic scattering off heavy nuclei, Compton scattering, elastic magnetic electron scattering, and neutron star observations.

Coulomb energy difference measurements [130] were initially used to infer neutron radii, but isospin violating interactions preclude accurate interpretation. Pion elastic scattering off heavy nuclei ([127] and pion production at Mainz), comparing positive and negative pions, suffer from analysis uncertainties [129] [128] when inferring indirectly the neutron density [77]. Elastic magnetic electron scattering cannot directly determine the neutron radius because, while sensitive to the neutron magnetic moment, most of the neutrons in heavy nuclei don't contribute to the magnetization as they are are coupled to spin zero [77]. Proton elastic scattering experiments (such as in FRIB) could feasibly prove useful, but are limited by theoretical analysis of the impulse approximation where nucleon-nucleon (NN) interaction is folded with the nucleon density [131], as well as off-shell ambiguities and distortion effects [124]. Enormous ambiguities yield an energy dependent neutron skin [124]. For extracting neutron distribution from proton scattering, or Compton scattering, in a variety of nuclei, one needs to accurately calibrate to the neutron density in a stable nucleus using the electroweak technique, using PREX-II and CREX as calibrating anchors (or full folding calculations which mitigate the necessary corrections from multiple scattering and modifications to the NN interaction) [77] [124]. Hence, no hadronic measurement of neutron densities has been generally accepted by the field due to uncertain systematic errors, and modern mean field interactions are typically fit without using any neutron density information[77].

There has been progress in experiment, theory, and observation. Fig. 3-9 shows the constraints on the equation of state of highly dense matter as interpreted from various types of measurements. Many of these constraints have uncontrolled systematics. The nuclear symmetry energy $S_{v}$ is shown on the horizontal axis, and $L=\partial S /\left.\partial \rho\right|_{\rho_{0}}$ the slope of the symmetry energy with respect to density evaluated at saturation density is shown on the vertical axis. It should be noted that many 
assumptions go into the making of this plot, as interpreting certain measurements to define these constraints is not altogether straightforward, so quantitatively, this plot should be taken with skepticism. However, it is qualitatively and conceptually useful in demonstrating how electroweak neutron skin measurements, like PREX, cut across the phase space constrained by other measurements, and PREX is almost orthogonal to these. Lattimer and Lim [149], by studying a wide range of plausible density functionals, and Roca-Maza et al. [172], by studying a series of relativistic and nonrelativistic interactions, deduced a relationship and constraint between dipole polarizability $\alpha_{D}$, neutron skin thickness $r_{n p}$, bulk symmetry parameter $S_{v}$, and slope of the symmetry energy $L$ [148]. In Fig. 3-9, the black dashed region corresponds to astrophysical measurements of neutron star radii. The yellow region, corresponds to the electric dipole polarizability $\alpha_{D}=20.1 \pm 0.6 \mathrm{fm}^{3}$. of ${ }^{208} \mathrm{~Pb}$ by Tamii et al. [173]. The red region corresponds to measurement of the centroid energy of the giant dipole resonance (GDR) of ${ }^{208} \mathrm{~Pb}$ by Trippa et al. [175]. From the measured energy they inferred a symmetry energy $\mathrm{S}$ at a particular density from which can be inferred a correlation between $\mathrm{S}$ and $\mathrm{L}$ shown in the figure. The green region corresponds to Heavy-ion collision isospin diffusion measurements (HIC) [176]. The purple region corresponds to excitation energies to isobaric analog states (IAS) [174]. The orange region corresponds to measured nuclear masses is taken from Hartree-Fock calculations which imply a correlation between L and Sv. The dark blue regions labeled G and $\mathrm{H}$ refer to the neutron matter studies of Gandolfi et al. [150] and Hebeler et al. [151], respectively [148]. The light blue region corresponds to constraints for the neutron skin thickness of ${ }^{208} \mathrm{~Pb}$ taken from a study by Chen et al. [177], who converted experimental results for $\mathrm{Sn}$ isotopes into an equivalent value for ${ }^{208} \mathrm{~Pb}$ and performed a series of Skyrme Hartree-Fock calculations. The white region corresponds to overlap, or agreement, six of these experimental constraints giving a for the slope of the symmetry energy: $44 \mathrm{MeV}<\mathrm{L}<66 \mathrm{MeV}$. Constraining the nuclear symmetry energy and the slope of the symmetry energy is crucial for 
understanding the NN interaction near saturation and going beyond saturation density to impact astrophysical systems [148].

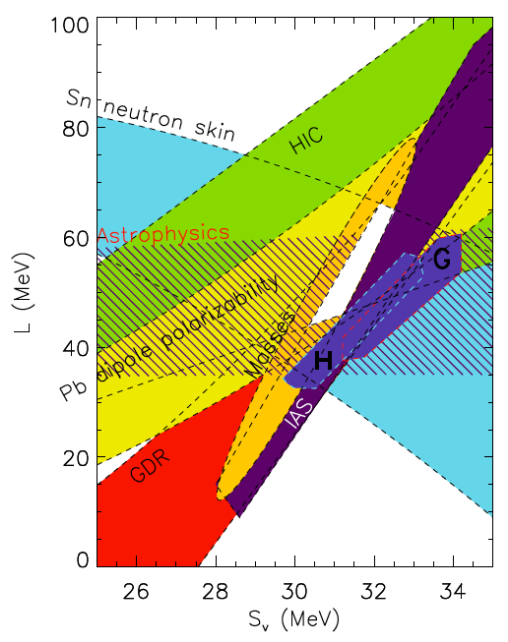

Figure 3-9: Experimental constraints for symmetry energy parameters, adapted and revised from [149] [148].

\subsubsection{Neutron Stars}

Neutron stars are an excellent laboratory for nuclear physics. Neutron star properties such as spin, mass, radius, crust, tidal deformability place constraints on nuclear physics. Conversely, neutron density measurements have implications for nuclear structure and neutron-rich matter in astrophysics [77]. There is a complementarity between neutron radius measurements in a finite nucleus and measurements of the neutron radius of a neutron star. Neutron stars have a strong analogy to nuclei: symmetry pressure pushes against gravity in neutron stars, whereas symmetry pressure pushes against surface tension in nuclei. Both measurement of the neutron radius in heavy nuclei and of measurement of neutron star mass-radius curves provide information on the equation of state of dense matter [77].

Neutron stars have a maximum mass limit before they collapse. Thus far, there have been just two neutron stars observed to have $\sim 2$ solar mass. Neutron stars 
(to better than 10\%) all lie on one universal mass-radius curve [186]. There is 1:1 correspondence between the Mass-Radius curves, shown in Fig. 3-10b, and the EOS as defined by pressure P and energy density $\epsilon$ curves, shown in Fig. 3-10a, where $\epsilon$ refers to energy density of neutron-rich matter in beta equilibrium [125]. All observed neutron star radii lie between 10.4 and $12.9 \mathrm{~km}$ on the neutron star mass - radius curves, as shown in Fig. 3-10c. which suggests suggests a neutron skin thickness of $R_{n}\left({ }^{208} \mathrm{~Pb}\right)<0.2 \mathrm{fm}$. Conversely, PREX informs neutron star size vs. mass.

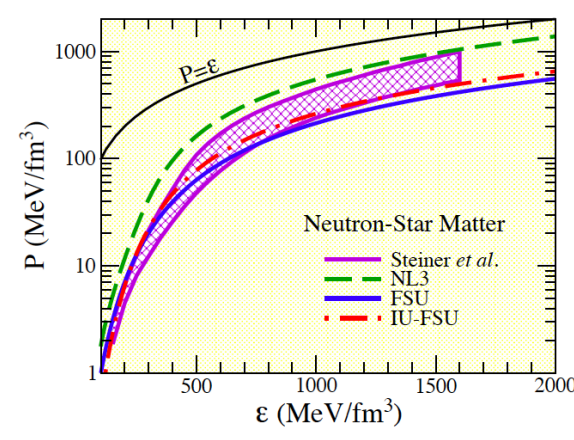

(a)

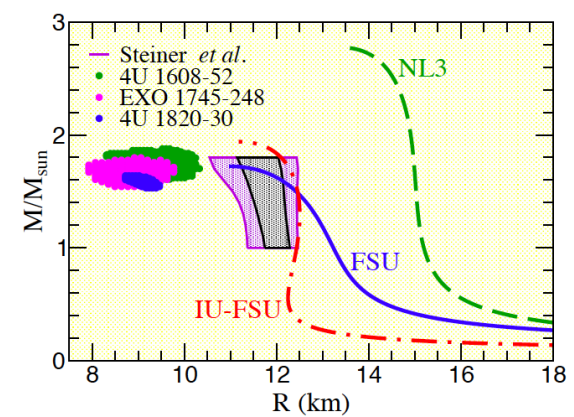

(b)

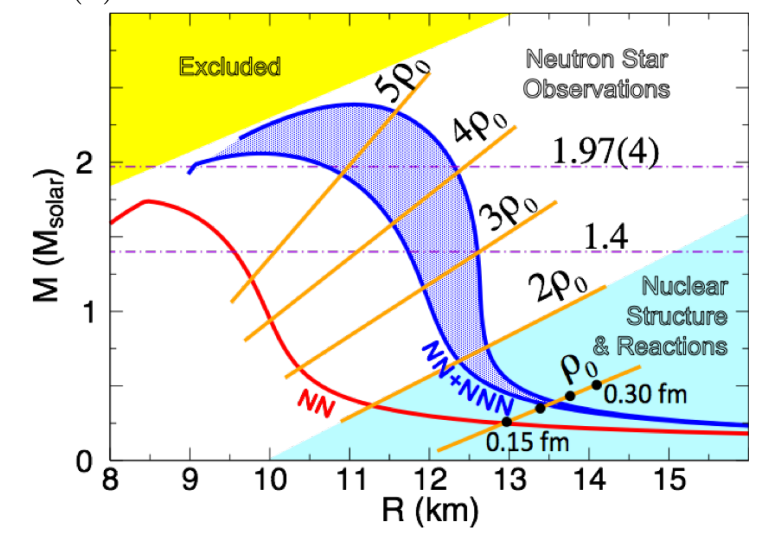

(c)

Figure 3-10: (a) Equation of state pressure vs energy density of neutron-rich matter in beta equilibrium. The shaded region displays observational constraints. The solid black line $(P=\epsilon)$ denotes the stiffest possible equation of state consistent with causality [125] . (b) Mass-vs-Radius relation predicted by the three relativistic mean-field models. (c) NS Mass-vs-Radius curve and connection to PREX measurements at nuclear density $\rho_{0}[147]$ 


\subsubsection{R-process nucleosynthesis}

R-process stands for rapid neutron-capture process, a predominant mechanism for the nucleosynthesis of heavy elements. It is rapid in that the neutron capture occurs faster than the nuclei have time to decay. when the increasingly neutron-rich nuclei cannot physically retain another neutron, the sequence of neutron capture ceases. There are two ways of synthesizing heavy elements: s-process and r-process. The r-process contrasts with the s-process, the slow captures of neutrons, which primarily occurs within ordinary stars. The r- and s-processes account for almost the entire abundance of chemical elements heavier than iron.

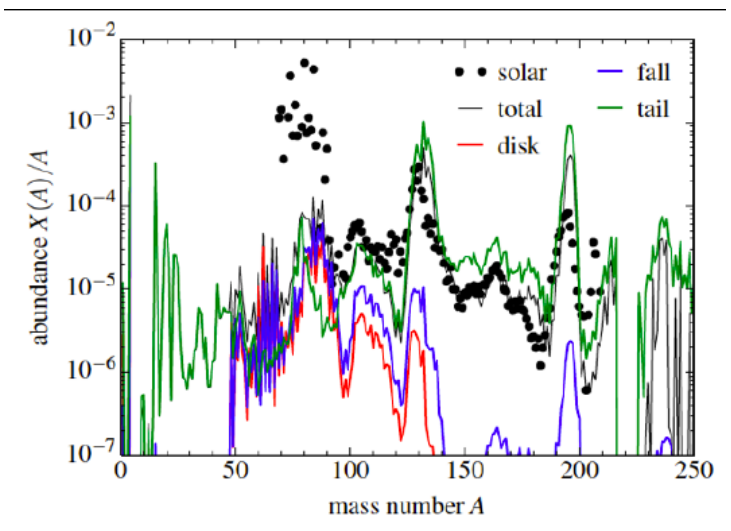

Figure 3-11: Abundance vs Atomic Mass: Solar system abundances shown in black, Dynamical ejecta produces heavy elements, Disk outflows produce lighter elements [194] [195]

Abundances of the chemical elements in the Solar System, as shown in Fig.3-11 is determined by the mechanism of synthesis. Nucleosynthesis can occur either by nuclear fusion (including both r- and s-process multiple neutron capture) or nuclear fission followed by beta decay. What is the astrophysical site of the r-process? Neutron star mergers are believed to be a main source of r-process elements. A visualizer predictions showing the development of chemical abundances vs mass number over time is shown in Fig.3-12. 


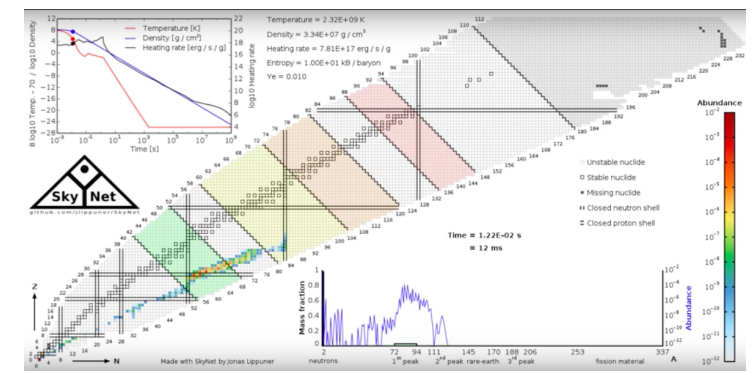

(a)

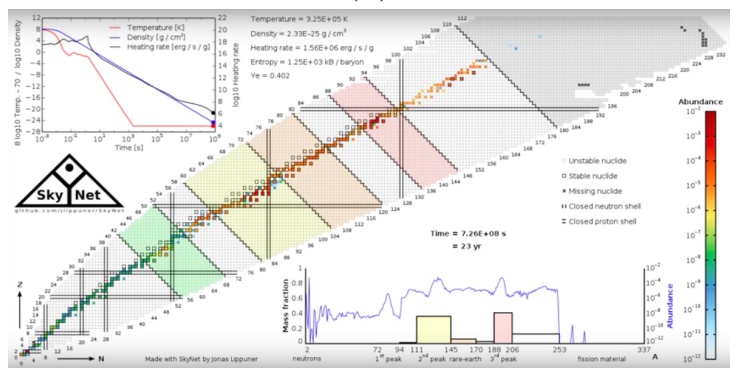

(b)

Figure 3-12: Abundance visualizer: Video of nucleosynthesis [188] (a) Beginning of nucleosynthesis of lighter elements (b) Convergence of nucleosynthesis on heavy elements

Neutron star properties affect potential nucleosynthesis. Smaller NS radii lead to higher electron fraction of the ejected material, more universal r-process production, and larger amounts of ejected r-process material. Nucleosynthesis is nearly independent of the electron fraction of the ejected material, however it is dependent on the amount of material ejected, thus it depends on the EOS [197] [198]. PREX-II/CREX could assist in furthering our understanding of stellar processes as well as helping us understand the formation of heavy elements in neutron stars and their abundances in the universe.

\subsubsection{Gravity Waves and EOS}

In general relativity, mass-energy creates spacetime curvature and masses moving in curved space generate gravity waves. The recent gravitational wave detections of black hole and neutron star inspirals and have generated a lot of excitement and have made the ability to interpret and understand the state of dense matter 
in neutron stars increasingly desirable.

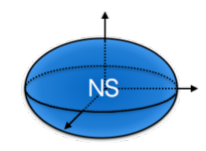

Figure 3-13: Quadrupole

The gravitation wave luminosity, to first order, goes by the quadrupole formula [189]

$$
L_{G W} \sim \sum_{i, j}\left(\partial_{t}^{3} Q_{i j}\right)^{2}
$$

For binary NS mergers, there is strong emission for very compact systems

$$
L_{G W} \sim M^{2} \Omega^{6} d^{4} \sim(M / d)^{5}
$$

where $\mathrm{d}$ is the orbital distance, $\mathrm{M}$ is the NS chirp mass, and $\Omega$ is the frequency.
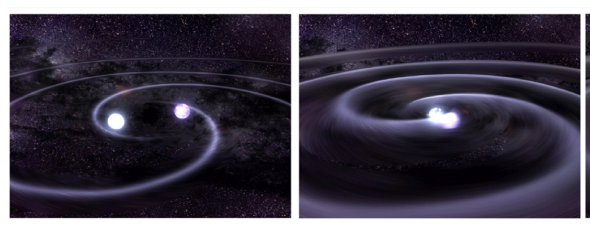

(a)
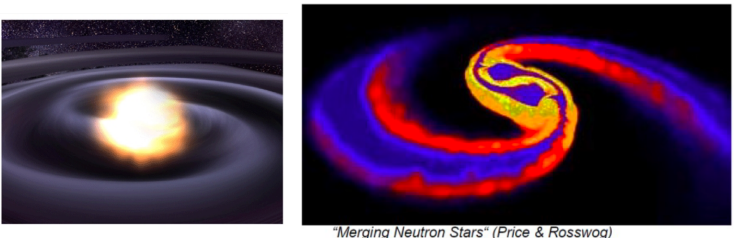

(b)

Figure 3-14: Neutron star inspiral [201]

In NS mergers, the tidal field $\epsilon_{i j}$ created by companion stars induces a quadrupole moment $Q_{i j}$. Tides in neutron stars cause large stars to merge faster [189]. An example of the tidal induced phase shift is shown in Fig. 3-15. Waveform behaves as if NS are point masses early on, then deformation occurs when inspiral rate reaches $100 \mathrm{~Hz}-1 \mathrm{kHz}$ range. The tidal dephasing (to leading order in $\Omega$ ), assuming a perfect waveform model [190] [191], is given by

$$
d \Phi \sim R^{5} \Omega^{5 / 3}
$$


Gravitational wave signal from a NS merger measures the tidal deformity $\lambda$. The amount of deformation depends on stiffness of EOS via the tidal deformability $\lambda$ and so the waveform is sensitive to the nuclear EOS for neutron stars. Additionally, the post merger signal is at high frequency, more difficult to detect, may provide info about the EOS and NS structure, including radius, etc. [186]. GW measurements are of tidal deformity-mass, as shown in Fig. 3-16 are nearly equivalent to performing radius-mass measurements.

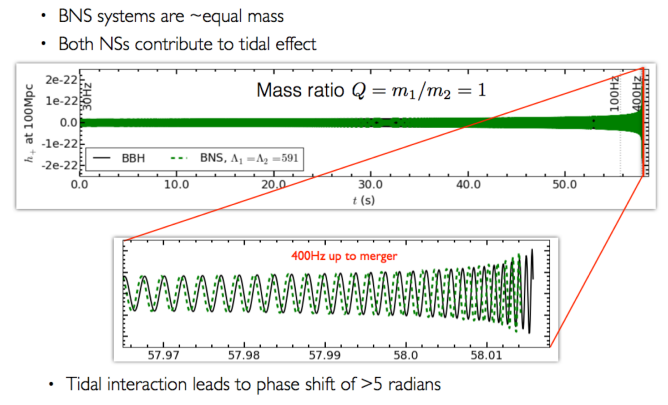

(a)

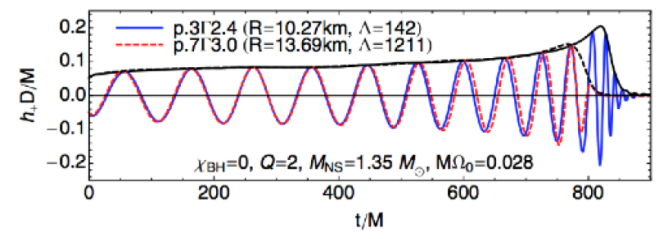

(b)

Figure 3-15: Tidal deformation of GW waveform [192]

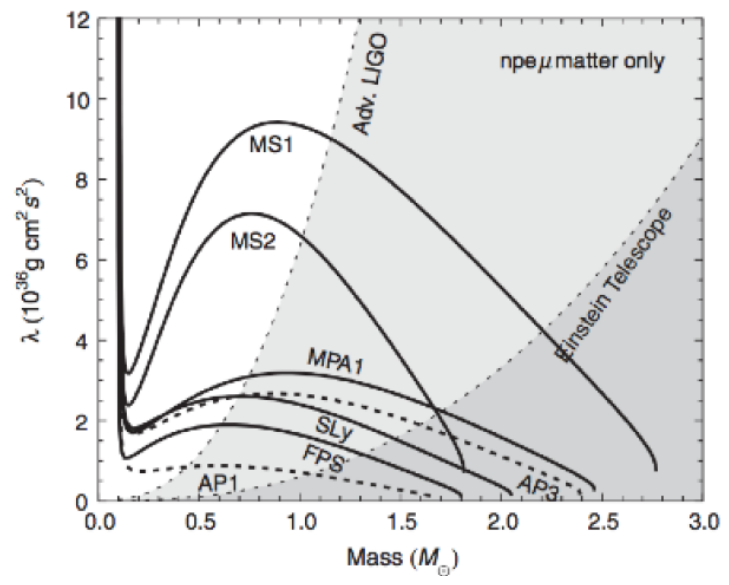

Figure 3-16: Tidal deformity - Mass NS curve [193]

The PREX and CREX measurements are directly related to the equation of state of highly dense matter and are important for modeling the collision of neutron stars, which can now be detected by LIGO. It is potentially exciting to make a comparison of PREX-II with LIGO observations. Measurement of the radius 
of the neutron distributions in nuclei has significance in stellar explosions and in calibrating the equation of state for highly dense matter, which is an important input parameter for modeling neutron stars and will undoubtedly enter into neutron star inspiral collisions.

\subsubsection{PREX I}

PREX-I, which ran in 2010, measured the parity-violating asymmetry in the elastic scattering of electrons off ${ }^{208} \mathrm{~Pb}$. It was performed at $1.0 \mathrm{GeV}$ and at a $5^{\circ}$ scattering angle. It measured an asymmetry of $0.6 \mathrm{ppm}$ and for the very first time made the electroweak observation the the weak charge density is more extended than the electric charge density, establishing there is indeed a weak skin around a heavy nucleus at the $95 \%$ confidence level.[164].

PREX-I demonstrated successful control of systematic errors, as well as the technologies needed for PREX-II. The systematic error goals $(2 \%)$ were achieved [164], however PREX-I was statistics limited and only $15 \%$ of the planned statistics were taken because of various experimental difficulties. [164]. The results of PREX-I are shown in Fig. 3-17

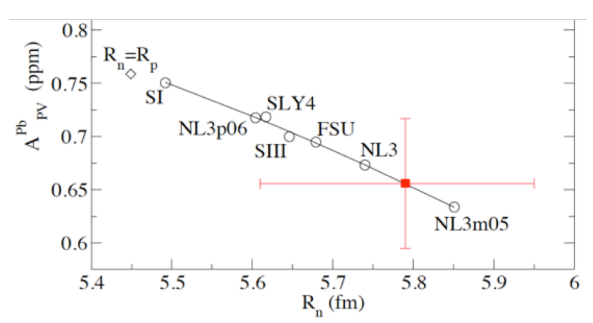

(a)

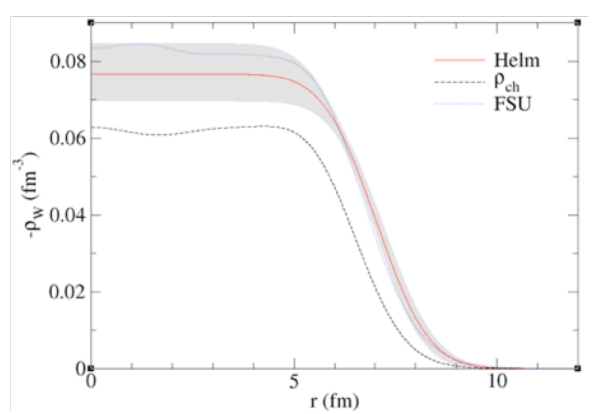

(b)

Figure 3-17: PREX-I result [119] [120]

PREX-I measured the parity violating asymmetry in ${ }^{208} \mathrm{~Pb}$ to be

$$
A_{P V}=0.657 \pm 0.060(\text { stat }) \pm 0.014(\text { sys }) \text { ppm }
$$


The corresponding neutron skin thickness was found to be $R_{n}-R_{p}=0.33_{-0.18}^{+0.16} \mathrm{fm}$. Interestingly the PREX-I central value is not consistent with measured neutron star properties and existing models (though it is within $\sigma$ ). Measurement of the PREX quantity inspires significant interest from the community (the 2012 papers collecting $250+$ references on Inspire as of 2018). PREX-II expects to achieve a factor of 3X improvement on the neutron skin thickness uncertainty.

\subsection{Experimental Introduction}

PREX-I was a major accomplishment as the first electroweak proof of neutron skin with many milestones successfully achieved [164]. However, it ended before reaching the proposed precision. PREX-II is a followup measurement to PREX-I which will achieve $3 \mathrm{X}$ improvement on the Rn-Rp uncertainty. PREX-II is designed with several improvements to achieve the originally proposed experimental precision in $R_{N}$ of $\pm 1 \%$ [164].

One issue for PREX-I that reduced the running efficiency, which will be rectified in PREX-II, is the vacuum system. The vacuum system ran into difficulties with radiation. Radiation caused the failure of a soft O-Ring in the pivot region that was part of the vacuum coupling of the scattering chamber to the exit beam pipe. It was a primary source of downtime during PREX-I, compromising the statistical precision reached. This pivot region is redesigned so that the seals are all-metal, not only for radiation hardness but also durability in thermal cycling [164].

To reach the proposed precision, PREX-II will run at $1 \mathrm{GeV}$, using the septum magnet to attain the desired $5^{\circ}$ scattering angle, for 35 days of running, including 5 days for commissioning and 5 days for polarimetry and auxiliary measurements [164] 


\subsection{Primary Components of the Experiment}

\subsubsection{Helicity Reversal}

There are several methods to reverse the helicity of the electron. There are both fast helicity reversals, controlled by the Pockels Cell, and slow helicity reversals (the insertable half wave plate and the double Wien). Fig. 3-18 shows a diagram of the polarized source. The Right and left handed longitudinally polarized electrons for this experiment will come from Right and Left Circularly polarized light. A Pockels cell is fed a randomized helicity control signal which applies either positive or negative high voltages, producing either $\mathrm{R}$ or L circularly polarized light, which is incident on a photocathode, producing consecutive windows of R-handed and Lhanded electrons. The electrons are accelerated and then sent into the experimental hall. In helicity switching, time windows are generated in the electron bunch train at a selected flip rate, with the sign of the beam's longitudinal polarization in each window assigned on a pseudo random basis. Frequency selection for helicity flipping affects the noise, measurement widths, and statistical errors significantly. The PREX-II flip rate will be $120 \mathrm{~Hz}$ (or $240 \mathrm{~Hz}$ ) in quartet (octet) randomized helicity pattern, RLLR or LRRL (RLLRLRRL or LRRLRLLR). These multiplets are formed at multiples of $60 \mathrm{~Hz}$ to cancel out $60 \mathrm{~Hz}$ noise. 


\section{Injector Laser Table Layout}

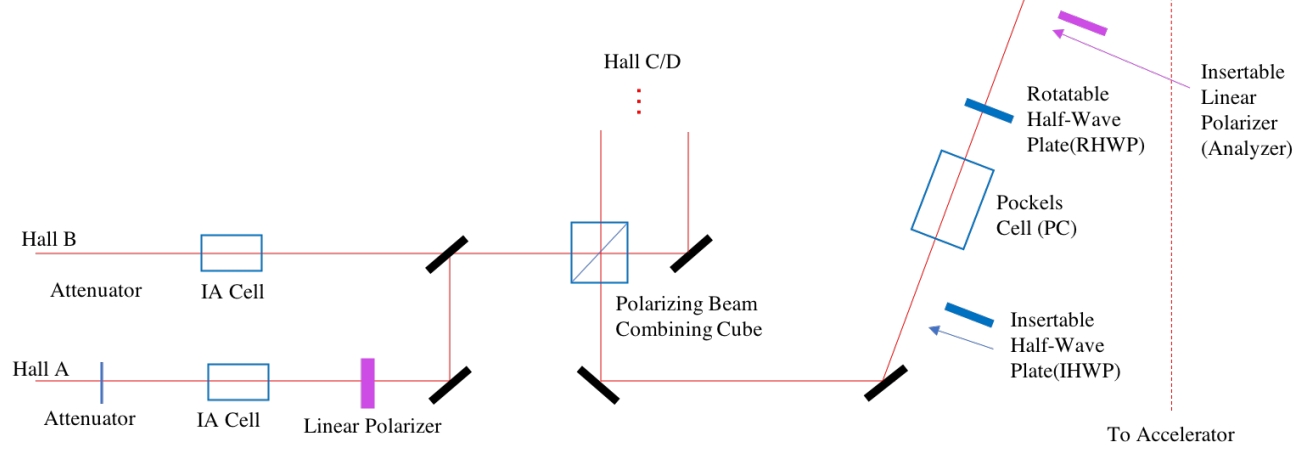

Figure 3-18: The schematic of the injector setup at JLab. The Pockels Cell (PC) was used to convert linearly polarized light into circularly polarized light. The insertable mirror was used to guide the beam onto a quad-photodiode (QPD) or a linear array photodiode (LAPD) detector during laser table studies. The insertable lens and the insertable linear polarizer were only used during laser table studies. The insertable mirror and the insertable linear polarizer were retracted during the electron beam source studies and the experiments. The insertable half-wave plate (IHWP) was inserted and retracted during laser table studies, electron beam source studies and the experiments.[200]

Regarding slow helicity reversals, there is the IHWP shown in Fig. 3-18 and the double Wien in the electron beamline. A slow reversal is where the electron beam helicity is reversed relative to both the electronic helicity control signals and the voltage applied to the PC [152] and are used to help cancel systematics. The IHWP take the incoming horizontally polarized light, which gets converted to $\mathrm{R}(\mathrm{L})$ circular polarization by the Pockels cell when fed a $+(-)$ helicity signal, and makes the incoming polarization vertical, which gets converted to $\mathrm{L}(\mathrm{R})$ circular polarization by the Pockels cell when fed a $+(-)$ helicity signal, thereby performing a 'slow' reversal on the helicity state. It should be noted that in principle, the IHWP could be placed downstream of the Pockels cell and this method of reversal 
would still work.

The second slow helicity reversal is the double Wien. As shown in Fig. 319 it consists of two solenoids and two Wien filters. The beam polarization is oriented vertically by the first solenoid and the vertical Wien filter. The beam polarization is rotated $\pm 90^{\circ}$ by the second solenoid. Finally, to optimize the degree of longitudinal polarization in the experimental hall, the horizontal Wien is used [152].

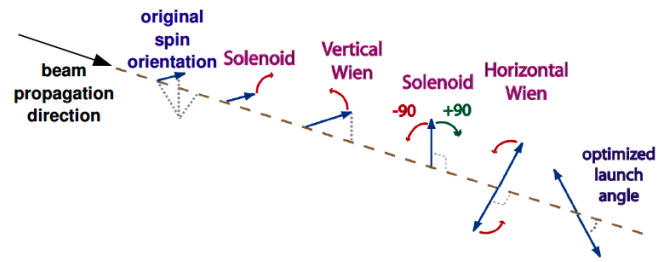

Figure 3-19: Double Wien [152]

There are several requirements on the helicity reversal that must be considering in parity experiments to maintain systematic errors associated with helicity reversal at the $10^{-} 8$ level. For the fast helicity reversals, during an experiment, many reversals are needed and to avoid any correlation with noise, they should follow a rapid and random sequence [77]. To minimize systematic errors, false asymmetries must be minimized. False asymmetries can also arise from electronic pickup from reporting of the helicity signal to the DAQ, so the helicity signal has delayed reporting and is randomized. False asymmetries can also arise from deadtime if the pockels cell transition is mistakenly left within the integration window. Helicity correlated beam asymmetries can give rise to false asymmetries. The helicity reversals should be uncorrelated to other parameters affecting the cross section (i.e. beam intensity asymmetry, position differences, and spot size asymmetries). Experiments must measure the sensitivity of the cross section to HCBA as discussed in Sec. 3.5.11 .

On the injector laser table, it is important to characterize the laser parameters 
at the Pockels cell and on the photocathode. Important parameters include the spot size and divergence at the cell, the effective throw distance to the cathode (including the effect of the steering lens used), and spot-size on the photocathode as estimated by the "spiricon-cathode" (a camera at the same effective throw distance as the cathode). One should measure the analyzing power and the QE of the photocathode as well. During an experiment, degradation of QE often correlates with degradation in the degree of polarization of the electron beam, so longevity of the cathode is important, and spot moves may be necessary during the course of an experiment. One can obtain smaller HCBA's (especially position differences), by reducing the spot size at the pockels cell and the photocathode (as discussed in the RTP chapter), but it is important to maintain a small beam divergence within the pockels cell as well as preserve the longevity of the photocathode by not making the beam too small.

\subsubsection{FOM}

The appropriate choice of kinematics is a balance between maximizing the parity violating signal and minimizing the signal to noise ratio. The goal of the optimization is to minimize the runtime required to obtain a $1 \%$ accuracy on $R_{n}$. This is not just maximizing the figure-of-merit (FOM), but accounting for sensitivity to the parameter $R_{n}$ as well. The quantity to maximize is

$$
F O M \times \epsilon^{2}=R \times A^{2} \times \epsilon^{2}
$$

where the figure-of-merit is defined as $F O M=R \times A^{2}$, the detected rate is $R \sim \frac{d \sigma}{d \Omega}$, sensitivity to neutron radius is $\epsilon=\frac{d A}{A}=(A 1-A) / A$ where $\mathrm{A}$ is computed from a MFT calculation [132] and A1 is the same calculation but with the neutron radius increased by $1 \%$. $A_{P V}$ increases with $Q^{2}$ and the cross-section decreases with $Q^{2}$ as shown in the calculation in Fig. 3-20 [77]. 

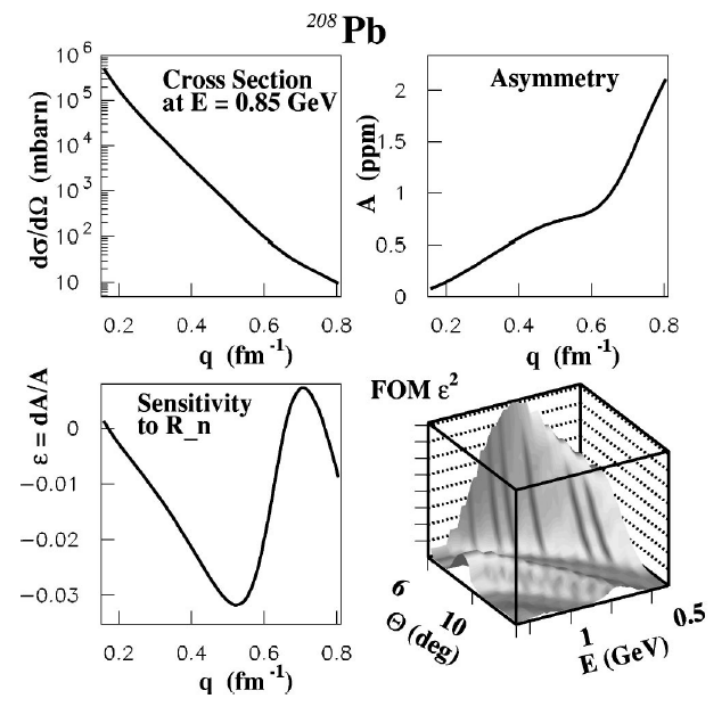

Figure 3-20: Cross section, parity violating asymmetry, and sensitivity to Rn for ${ }^{208} \mathrm{~Pb}$ elastic scattering at $0.85 \mathrm{GeV}$. The fourth plot shows the variation of $F O M \times \epsilon^{2}$ with energy and angle [77]

The fourth plot in Fig. 3-20 shows optimal $\epsilon^{2} F O M$ at $E=0.85 \mathrm{GeV}$ for $\theta=6^{\circ}$ and $q=0.45 \mathrm{fm}^{-} 1$. The runtime to achieve the desired uncertainty of $1 \%$ is given in this case by

$$
T \approx 7 /\left(P^{2} I \Omega\right)
$$

where $P$ is the polarization $(P \approx 0.8-0.9$ achievable $), I$ is the average beam current $(I \approx 70 \mu A)$ and $\Omega$ is the solid angle acceptance of the spectrometer. PREX-I ran at $\mathrm{E}=1 \mathrm{GeV}$ and $\theta=5^{\circ}$ with a measured 4-momentum transfer of $Q^{2}=0.00880 \pm 0.00011 \mathrm{GeV}^{2}[133]$ where $Q^{2}=2 E_{\text {beam }} E^{\prime}(1-\cos \theta)$. PREX-II will run at $\mathrm{E}=0.95 \mathrm{GeV}$ [134] and $\theta=5^{\circ}$ degrees. The optimum kinematics is insensitive to the value of $R_{n}$ and its shape. As illustrated in Fig. 3-21, the optimum energy for a measurement is about the same for the two models shown, providing an indication of the insensitivity to the model. 


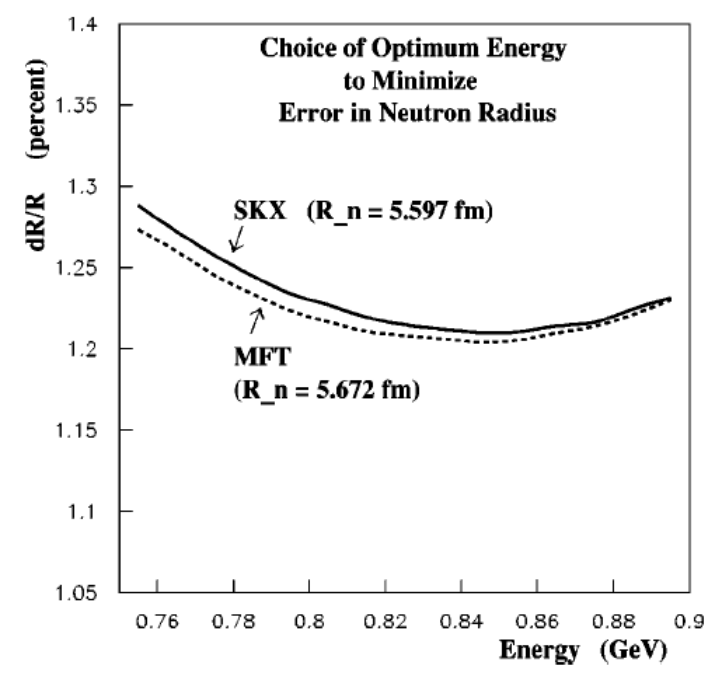

Figure 3-21: Error in the neutron radius in $\mathrm{Pb}$ versus beam energy[77], for two models of the neutron radius SKX [135] and MFT [136]

\subsubsection{Targets}

The design of the lead targets for PREX-II will be the same as for the socalled "thick diamond" targets of PREX-I. As shown in Fig. 3-22, each lead target consists of a $0.5 \mathrm{~mm}$ thick lead square sandwiched between two foils of diamond which are each $0.25 \mathrm{~mm}$ thick and 1 inch square. The thermal contact between the diamond and lead is improved with a thin layer of vacuum grease (Apiezon L, a pure hydrocarbon with high thermal conductivity) and spring-like washers ( Belleville) clamp the assembly together to ensure good thermal contact as changes in temperature occur when exposed to the electron beam [144].

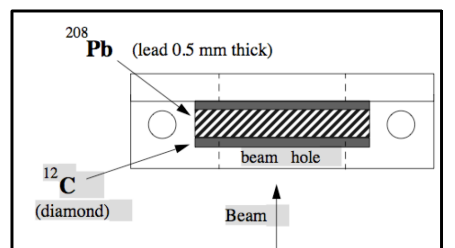

Figure 3-22: Diamond sandwich [143]: 0.5mm lead, 0.25mm diamond, 1 sqin

There are several considerations which go into determining target thickness. The obvious advantage of a thicker target is increased rates and reduced runtime. 
However, there are several issues which must be considered. There is an optimal thickness at which the rate is maximized, beyond which, given the energy resolution in the detector, the rate actually decreases due to radiative losses. This correspond to approximately $10 \%$ of the radiation length. The rate may also be increased by including more of the radiative tail, up to $4 \mathrm{MeV}$, into the detector, including some inelastic contributions. Lead has low melting point, and low thermal conductivity, whereas diamond foils have excellent thermal conductivity. the high thermal conductivity of the diamond will efficiently transfer the beam power and keep the target from melting [77]. Diamond may be used to improve the heat load capacity of the He cooled target, enabling higher current running, but the amount of diamond which can be used is determined by the systematics on $A_{p v}$ from ${ }^{12} \mathrm{C}$, which must be well understood. ${ }^{12} \mathrm{C}$ is an isoscaler, spin-0 nucleus, with well-measured $A_{p v}$, so the background is benign, as explained in discussion below [77].

Target impurities: The ${ }^{12} \mathrm{C}$ target impurities are benign. At low $Q^{2}$, because $\mathrm{Pb}$ is much larger than $\mathrm{C}$, the experiment is much less sensitive to the ${ }^{12} \mathrm{C}$ density. Additionally, excited states are a non-issue because ${ }^{12} \mathrm{C}$ 's first excited state is high, above $4 \mathrm{MeV}$. Furthermore, the parity violating asymmetry for ${ }^{12} \mathrm{C}$, a light nucleus with $\mathrm{N}=\mathrm{Z}$, is well known. For example, at $\sim 850 \mathrm{MeV}$ and $6^{\circ}$,

$$
A\left({ }^{12} C\right)=0.66 p p m
$$

with an uncertainty on the order of $0.4 \%$. The elastic cross section for ${ }^{12} \mathrm{C}$ is $2 \%$ of the $\mathrm{Pb}$ cross section at these kinematics and carbon could contribute about $4 \%$ to the rate in the composite target. For a $0.4 \%$ error in a $4 \%$ impurity, the uncertainty in the asymmetry contribution from ${ }^{12} \mathrm{C}$ is two orders of magnitude smaller than needed for the $3 \%$ asymmetry measurement [77].

PREX-I showed the necessity of dealing with target non-uniformities which developed after prolonged beam exposure, due to localized beam heating, on the 
"thin diamond" targets. FIg. 3-23a shows the degradation over time of the "thin diamond" target during PREX-I, where a raster scan was used to measure density loss. The substantial target non-uniformity resulted in luminosity fluctuations that increased the asymmetry width by as much as $40 \%$. The failure mode of the targets was degradation in rate, followed by a hole at the center of the target, followed by target melting. The solution to deal with the non-uniformity was to lock the raster pattern frequency to a multiple of the helicity frequency, forcing the raster to execute the same orbit between adjacent helicity cycles, thereby eliminating asymmetry noise arising from target non-uniformity. PREX-II will also use a synchronized $4 \mathrm{x} 4 \mathrm{~mm}$ raster to handle potential non-uniform lead thickness. Fig. 3-23c shows the effect of synched rastering on the correlation plots of the asymmetries measured between the two HRSs, which characterize the extent of target degradation. The correlation getting stronger as the target non-uniformity increases. The left correlation plot was acquired without the raster synch, and the plot on the right was acquired with the raster synched where the effects of the target non-uniformity are clearly suppressed [146]. It should be noted the "thick diamond" targets appeared to maintain their uniformity during PREX-I, as shown in Fig. 3-23b. Whereas, the "thin diamond" target survived $>1$ week production running, with a rate drop of $92 \%$, the "thick diamond" target never degraded and lasted 4 days at $70 \mu A$ [147]. We know how to handle target degradation with a synched raster, and we have plenty of extra targets (as described below), so we should be prepared to deal with target non-uniformities during PREX-II. 


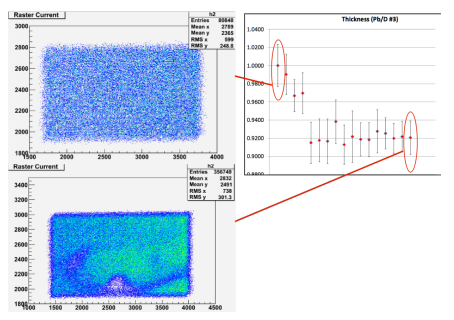

(a) thin diamond

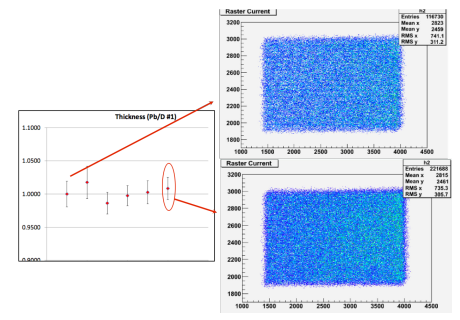

(b) thick diamond

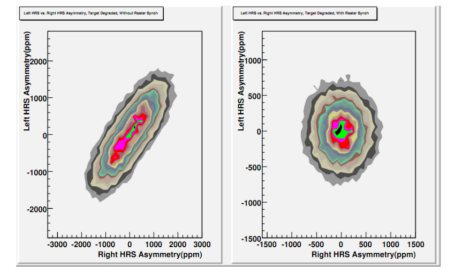

(c) Rastering

Figure 3-23: [146] (a) thin diamond target degradation during PREX-I (b)thick diamond target lack of degradation during PREX-I (c) Correlation plots of the asymmetries measured between the two HRSs. On the left is without the raster synch, on the right is with the raster synched [143] [146]

During PREX-I, the lead-diamond targets were established to have a minimum survival lifetime of $>25 \mathrm{C}$ exposure. 82 Coulombs of beam was collected on 3 targets, a "thin diamond", a medium diamond, and a "thick diamond", which suggests an an average lifetime of $27 \mathrm{C}$, a minimum lifetime of the "thick diamond" lead targets given that it never degraded. PREX-2 should collect $150 \mathrm{C}$ total charge and it is expected that 5-6 isotopically-enriched ${ }^{208} \mathrm{~Pb}$ targets may be needed, so the number of production targets available is planned to be 10, double what is needed, with a $66 \%$ safety margin based on PREX performance [147]. Fig. 3-24 shows the He cooled target ladder where the lead-diamond targets will be housed. Fig. 3-24c is a photograph of the PREX-I target ladder. There will be a cooled target ladder (coming in horizontally) and a warm target ladder (coming it at the diagonal). The spacing between these 1 " targets on the cooled ladder is about $1 / 4$ ". A silver-based paste will be applied between the diamond and the copper housing, outside of the central area where the beam intercepts the target. Thermal calculations have been performed which demonstrate target functionality with a 4 x 4 $\mathrm{mm}$ rastered beam at $100 \mu \mathrm{A}$ assuming good thermal contact [144]. 


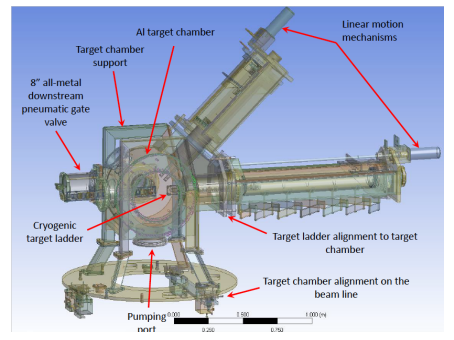

(a) Target chamber

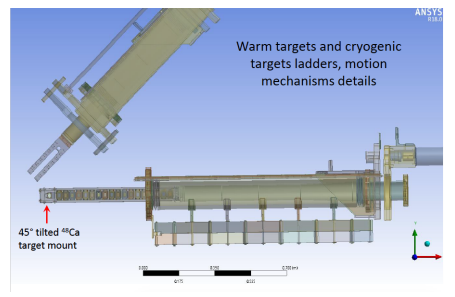

(b) Target ladder

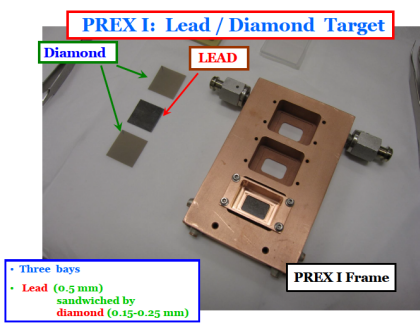

(c) PREXI ladder

Figure 3-24: (a)Target chamber and (b) ladder [145] (c)Photograph of the PREX-I target ladder [144] [143]

Table 3-25 shows a comprehensive list of the targets required for PREX and CREX physics and optics calibrations. Optics targets include a carbon hole target, thin carbon foil, and a water cell. There is also a thick carbon target. For studies of the target damage, targets with un-enriched lead have been added. A graphite backing, with lower thermal conductivity but with more robustness against radiation damage, will be used for one isotopic and one natural lead target. In addition to the CREX 48Ca target for CREX, obviously, there will be a 40Ca target.

\begin{tabular}{|c|c|}
\hline \multicolumn{2}{|c|}{ Optics Ladder } \\
\hline \hline Carbon Hole & $\sim 0.1 \mathrm{~g} / \mathrm{cm}^{2}$ \\
\hline Watercell & \\
\hline Tantalum foil or thin C foil & $0.1 \pm 0.05 \mathrm{~g} / \mathrm{cm}^{2}$ \\
\hline thin natural Pb & $0.05 \pm 0.01 \mathrm{~g} / \mathrm{cm}^{2}$ \\
\hline thin $^{40} \mathrm{Ca}$ & $0.05 \pm 0.01 \mathrm{~g} / \mathrm{cm}^{2}$ \\
\hline
\end{tabular}

\begin{tabular}{|c|c|}
\hline \multicolumn{2}{|c|}{ Production Ladder } \\
\hline \hline Carbon Hole & $\sim 0.1 \mathrm{~g} / \mathrm{cm}^{2}$ \\
\hline$(9 \mathrm{X}){ }^{208} \mathrm{~Pb} /$ Diamond & $0.5 \mathrm{~mm}$ \\
\hline${ }^{208} \mathrm{~Pb} /$ Graphite & $0.5 \mathrm{~mm}$ \\
\hline${ }^{48} \mathrm{Ca}$ (tilted) & $1 \pm 0.1 \mathrm{~g} / \mathrm{cm}^{2}$ \\
\hline${ }^{40} \mathrm{Ca}$ & $1 \pm 0.1 \mathrm{~g} / \mathrm{cm}^{2}$ \\
\hline thick $\mathrm{C}$ & $0.5 \pm 0.1 \mathrm{~g} / \mathrm{cm}^{2}$ \\
\hline $\mathrm{Pb} /$ Diamond & $0.5 \mathrm{~mm}$ \\
\hline $\mathrm{Pb} /$ Graphite & $0.5 \mathrm{~mm}$ \\
\hline
\end{tabular}

Figure 3-25: Targets required for PREX and CREX physics and optics calibrations [144] 


\subsubsection{Collimator}

The collimator is located between the scattering chamber and the septum magnet as shown in Fig. 3-26. The purpose of the PREX-II collimator is two-fold: (1) to optimize the HRS acceptance for the figure of merit and (2) to mitigation radiation. The part of the collimator that defines the spectrometer acceptance is shown in purple and blue in Fig. 3-26c. It consists of a hole in the center for the unscattered electron beam, and two carefully designed curved holes on the left and right which define the acceptance. This portion of the collimator is similar in design to PREX-II. In addition, there is a new collimator, shown in orange in Fig. 3-26c, designed to intercept scattered electrons so they do not intercept the beam pipe downstream, thereby isolating the radiation to one region in the beamline, and trapping it by shielding that region to mitigate EM and neutron radiation in the Hall. Sieve slit collimators for optics calibration are also shown in Fig. 3-26c shown in white. These are alike to those used during PREX-I. Remote actuation will swing the sieve slit between a "beam out" and "beam in" position [144]. Housing the collimator is the collimator box shown in yellow in Fig. 3-26c, and the box includes housing above the collimator for deinstallation. The collimator box connects the scattering chamber to the center beam pipe running through the septum and to the left and right spectrometer vacuum boxes penetrating the septum bore. Critically, only metal seals will be used in the pivot region, there should be no rubber seals in order to avoid radiation damage to the seals, causing vacuum leaks [144]. 


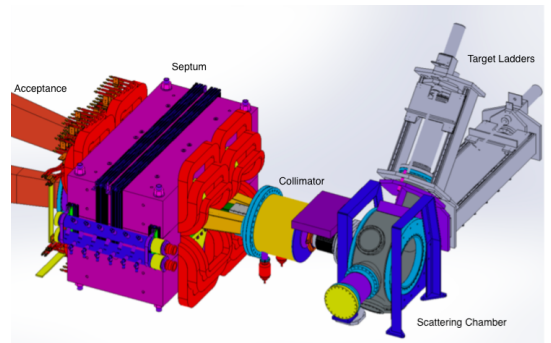

(a)

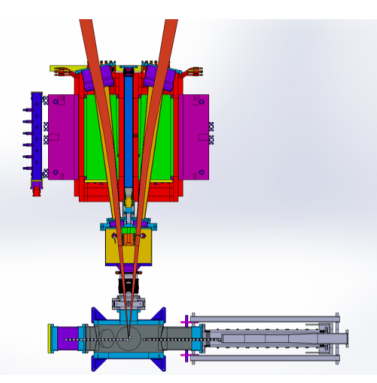

(b)

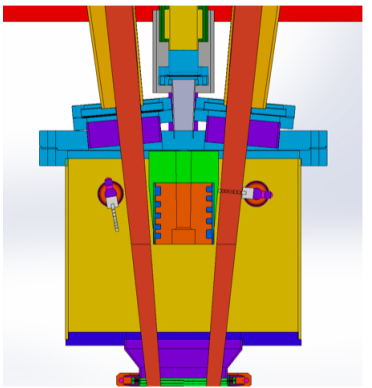

(c)

Figure 3-26: (a) PREX-II pivot region (b) Top View (c) Top View zoomed in [159]

The new collimator front face is $\sim 85 \mathrm{~cm}$ from the target and designed to intercept electrons with $>0.78^{\circ}$ scattering angles. The power deposited is expected to be $2.1 \mathrm{~kW}$ at $70 \mu A$ as shown in Table $3-29$. In contrast, the PREX-I collimator had a much larger opening angle of $1.27^{\circ}$ and $<1 / 3$ of the power deposition $500 \mathrm{~W}$. The inner cylinder is composed a $70 \% \mathrm{~W} / 30 \% \mathrm{Cu}$ alloy collimator, with cooling water channel spirals attached to water lines, with a $\mathrm{Cu}$ brazed sleeve. To put the peak beam power deposition inside the tungsten collimator, rather than close to the front face, there is a cylinder removed from the front of the inner collimator. This new collimator has been built and Fig. 3-27b shows the brazed, water tight collimator. However, the collimator was machined upside down, so the water cooling lines will have to come from underneath instead of from the top.

The inner cylinder is housed within a sintered tungsten jacket. The jacket traps EM power and self-shields the neutrons produced in the collimator [147]. The tungsten jacket is flush with the front of the inner collimator, not extending all the way to the front of the inner collimator on the sides [144]. After operation, to simplify the dis-assembly, the collimator gets lifted into the housing, where further shielding limits the radiation dose from the activated collimator for faster deinstallation [144] as shown in Fig 3-28 [179]. 


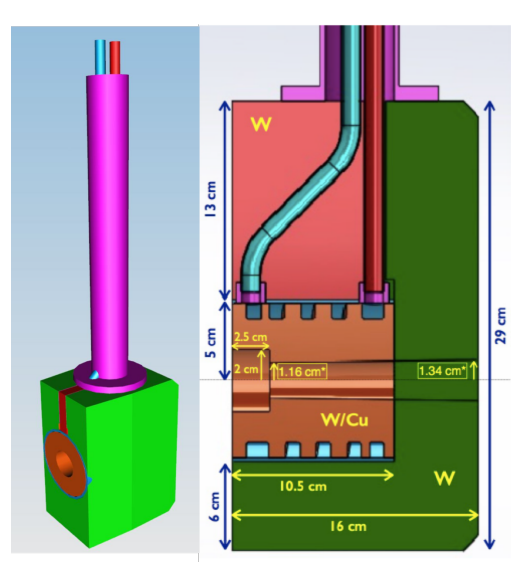

(a)

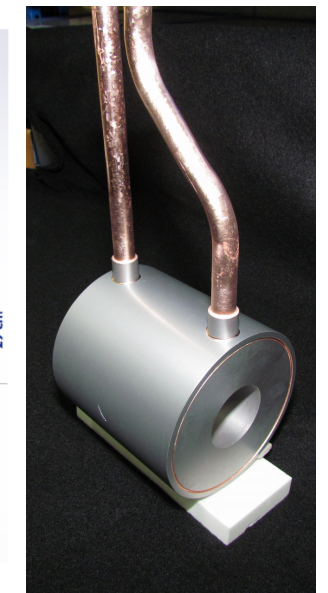

(b)

Figure 3-27: (a) $\mathrm{Cu} / \mathrm{W}$ collimator conceptual design [147] (b) PREX-II collimator [157]

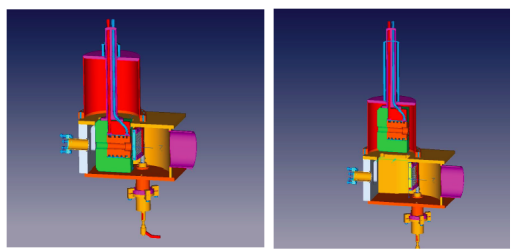

Figure 3-28: Collimator deinstallation concept [179]

\begin{tabular}{|c|c|c|}
\hline & PREX & CREX \\
\hline \hline Current & $70 \mu \mathrm{A}$ & $150 \mu \mathrm{A}$ \\
\hline Power $/ \mu \mathrm{A}$ & $28.8 \mathrm{~W} / \mu \mathrm{A}$ & $6.8 \mathrm{~W} / \mu \mathrm{A}$ \\
\hline Total power & $2020 \mathrm{~W}$ & $1020 \mathrm{~W}$ \\
\hline
\end{tabular}

Figure 3-29: Power deposited in beamline collimator, for PREX and CREX at 5deg scattering angle, $1.05 \mathrm{GeV}$ and $2.0 \mathrm{GeV}$ beam energies, respectively.[144]

Downstream of the $\mathrm{Cu} / \mathrm{W}$ collimator is the acceptance defining collimator. Fig. 3-30 shows the PREX-I collimator and the PREX-II preliminary collimator design. It is the connection between the end of the collimator box and the front end of both the central beam pipe running through the septum and to the vacuum boxes to the the right and left HRSs. In contrast to PREX-I, in PREX-II only metal seals, no rubber seals, will be used in the pivot region to avoid radiation damage induced vacuum leaks. 


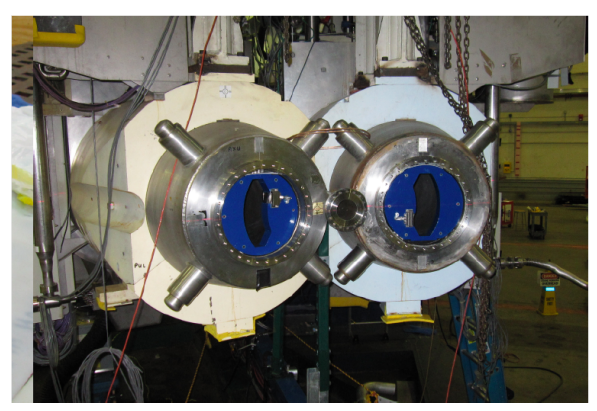

(a)

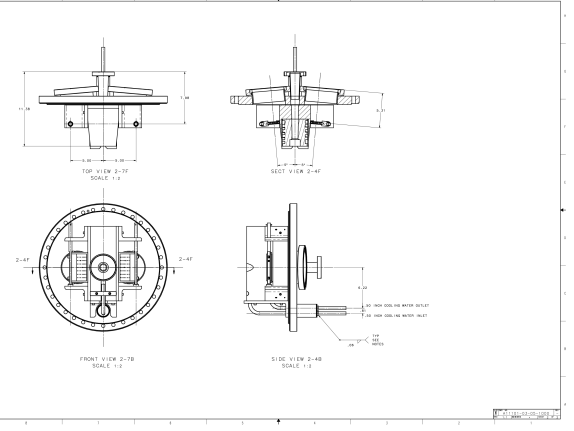

(b)

Figure 3-30: (a)PREX-I collimator [160] (b) PREX-II collimator design (preliminary) [158]

The difference in the design for the acceptance cuts in PREX-I vs PREX-II is shown in Fig. 3-31. The problems with the old concept include [153]: (1) the circle center must shift for a constant $\theta$ cut (2) the constant $\phi$ chamfer cuts out too much acceptance and (3) merely adjusting $r_{\min }$ is insufficient to resolve the troublesome inner edge. In contrast, the new concept has a fixed inner and outer radius with constant $\theta$ cut. It revert to the softer PREX-I chamfer on the corners with an additional chamfer on the inner edge, the slope and intercept of which depends on $y_{\text {inner }}$ to maximize the acceptance [153].

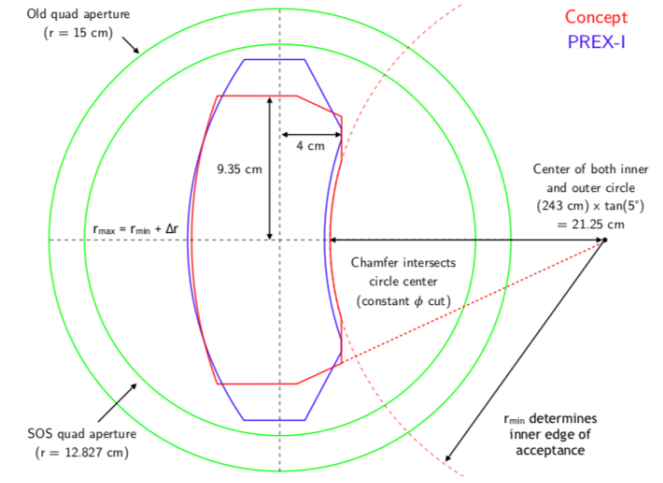

(a)

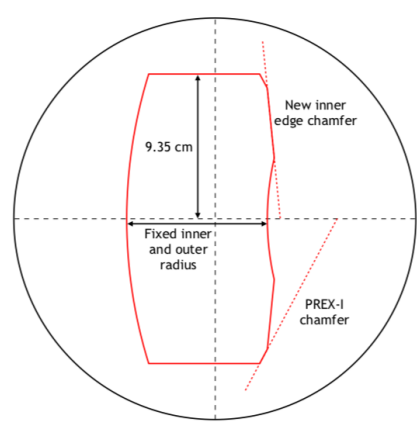

(b)

Figure 3-31: (a)Old collimator concept (b) New collimator concept [153] 


\subsubsection{Septum}

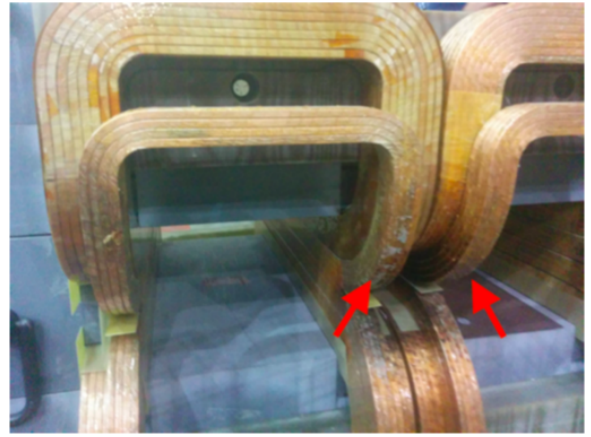

(a)

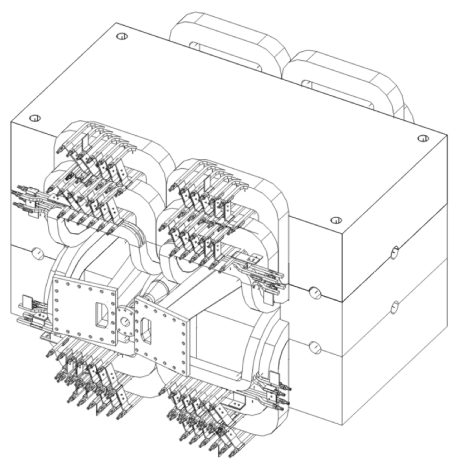

(b)

Figure 3-32: (a) Septum Coils [153] (b) Septum Diagram [147]

The septum is a non-superconducting magnet, the purpose of which is to overcome the physical constraints of the HRS's. The HRSs have a finite width and cannot be set to scattering angles smaller than $12.5^{\circ}$, so the septum magnets bend electrons elastically scattered at small angles, like $5^{\circ}$, into the HRSs. The $Q^{2}$ for PREX-I was approximately $0.009 \mathrm{GeV}^{2}$ and the small scattering angle of $5^{\circ}$ necessitates the use of a septum magnet to deflect the low angle of incidence into the the two HRS's which can only come so close together [147]. The septum is shown in Fig. 3-32. There are coils on the right and left of the central beam line which induce magnetic fields up and down, which bend the forward moving scattering electrons left or right into the $12.5^{\circ}$ spectrometers. Unscattered electrons pass through the central beam line and a set of separate coils generate magnetic fields to transport the electrons onto each of the HRSs [156]. The septum is located between the target scattering chamber, after the collimator, and the first quadrupole of the HRSs (Q1) [156] as shown in Fig. 3-33. The septum is required for $5^{\circ}$ scattering angle used in both PREX and CREX (with same target position for both). 


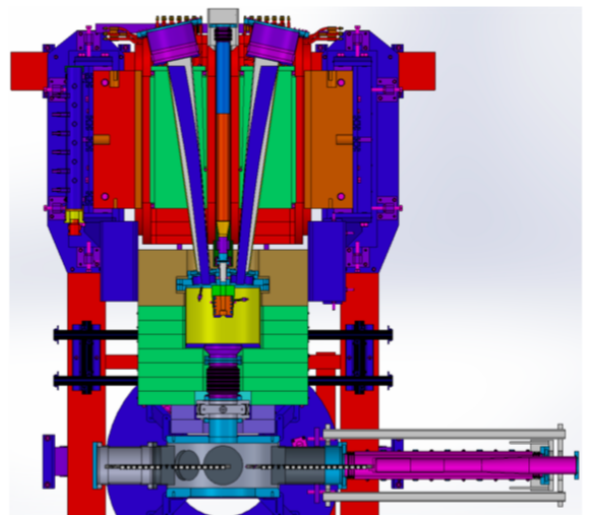

(a)

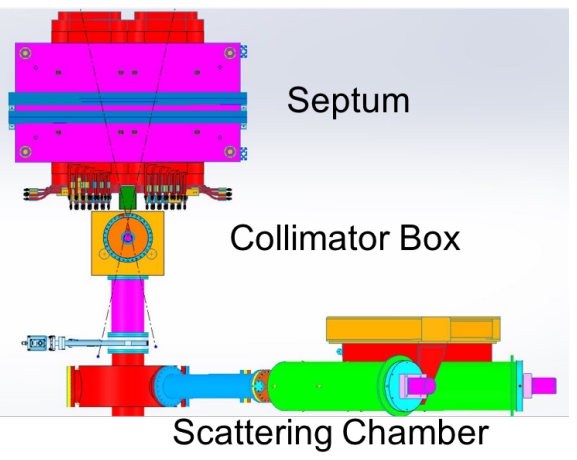

(b)

Figure 3-33: (a) Detailed Top View of scattering region [153] (b) Top View of scattering region [147]

The septum magnet worked during PREX-I and we know how to tune it to optimize the FOM. The configuration parameters are well known for this magnet. The alignment tolerance is $2 \mathrm{~mm}$ in the horizontal, to avoid interfering with acceptance on the front end and to align the beam pipe with the primary beam on the back end [144].

PREX-I ran with 2 coils, whereas PREX-II will run in the 3 coil configuration, reducing the power supply current necessary to reach the desired field strength, and reducing the flow rate of the cooling water needed by a factor of 2 . This change was important, not necessarily for the running of PREX-II, but rather for the running of CREX, at a higher energy and current. CREX has $2 \mathrm{X}$ the $\int B d l$ of PREX, and requires twice the current and cooling flow rate, these requirements are still less than they were for $\mathrm{g} 2 \mathrm{p}$. The $\int B d l$, currents, and flow rates are summarizing in Fig. 3.2 [154]. 


\begin{tabular}{l|c|c|c|c|c|} 
Experiment & PREX-I & g2p & PREX-II & CREX & CREX \\
\hline Conditions & $\begin{array}{c}(2 \text { coils }) \\
1 \mathrm{GeV}\end{array}$ & & $\begin{array}{c}(3 \mathrm{coils}) \\
\sim 1 \mathrm{GeV}\end{array}$ & $\begin{array}{c}(3 \mathrm{coils}) \\
2.2 \mathrm{GeV}\end{array}$ & $\begin{array}{c}(3 \mathrm{coils}, 1.9 \mathrm{GeV}) \\
1.9 \mathrm{GeV}\end{array}$ \\
\hline Bdl & 0.46 & 1.3 & 0.46 & 0.96 & 0.83 \\
Current in PS (A) & 756 & 1050 & 377 & 805 & 718 \\
Voltage (V) & 176 & 427 & 153 & 327 & 292 \\
Power (kW) & 133 & 448 & 58 & 264 & 210 \\
Flow Rate (gal/min) & 25 & 85 & 11 & 50 & 40
\end{tabular}

Table 3.2: Table of Septum running conditions [154]

PREX-II/CREX running will use existing power supplies and water-cooling systems for the gap-shimmed, 3-coil septum. PREX-II will run at a nominal septum current, with no change to performance as compared with PREX-I [147] [153]. For CREX, because it runs at a higher current, and energy $(150 \mathrm{uA}, 2.2 \mathrm{GeV}$, $1 \mathrm{x} 4 \mathrm{~mm}$ raster, $1.1 \mathrm{~g} / \mathrm{cm} 2,0.6 \mathrm{~cm}$ thick target), the 5 degree configuration for CREX brings the current density into a same regime as g2p. CREX will use the same water-cooling system, the same 3-coil configuration, and the same shims as g2p [147]. The shims will be used reduce the necessary coil current and thermal power to achieve the required field integral by reducing the vertical bore dimension [144]. The values in Table 3.2 are obtained from the Tosca model, checked against measurements, which characterizes the B-field behavior near saturation, as shown in Fig. 3-34 [154].

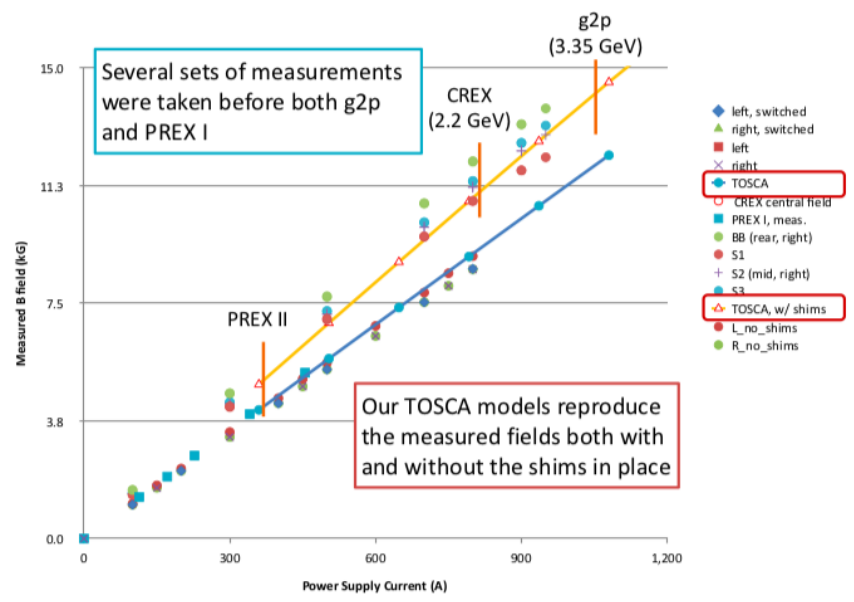

Figure 3-34: Tosca Model compared with measurements [154] 
Both the septum and the quads result in a stray quadrupole field along the beamline (not a dipole) [154], but this will be mitigated by magnetic shielding by the septum beam pipe design, a preliminary drawing of which is shown in Fig. $3-35[155]$.

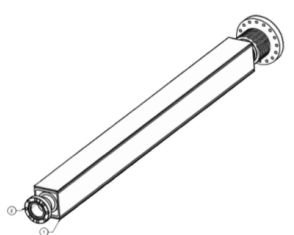

Figure 3-35: Septum Beam Pipe (preliminary diagram) [155]

\subsubsection{Spectrometers}

The nuts and bolts of PREX and CREX at Jlab are shown in FIg. 3-36. The target and septum magnet, used for the small scattering angle, are followed by the usual HRS magnets (Q1,Q2,HRS Dipole, and Q3) which focus and guide the beam into the spectrometer. The electrons which are detected by Vertical Drift Chambers (VDC) and quartz integrating detectors have been demonstrated to be largely elastic events due to the very clean separation of elastic events by the HRS optics.

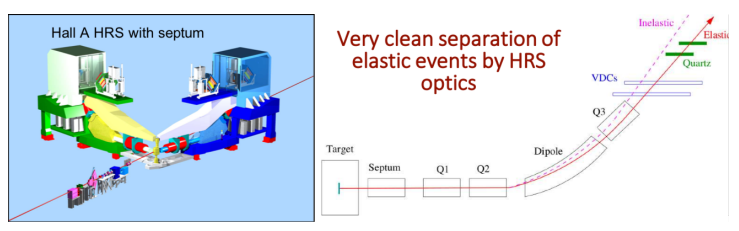

Figure 3-36: Components of PREX experimental beamline [147]

The HRS acceptance and optics are designed for the separation of elastic and inelastic peaks. During PREX-I, the background asymmetries $A_{i}^{b k}$, where i refers to different sorts of backgrounds, and corresponding systematic uncertainty $\sigma_{A_{i}^{b k}}$ on $A_{P V}$ due to inelastically scattered electrons was negligible. The acceptance of the first-excited states of both ${ }^{12} \mathrm{C}$ and ${ }^{208} \mathrm{~Pb}$ in the lead-diamond target was 
very small [161]. Fig. 3-37 shows the fractional contribution $f_{i}$ from various background sources, the associated correction to $A_{P V}$, and the systematic uncertainties on that correction. Inelastic scattering systematics are negligible as well as are elastically scattered electrons that rescattered inside the HRSs [161]. The dominant background correction comes from the elastics scattered off ${ }^{12} \mathrm{C}$ in the lead-diamond target sandwich, as described in Sec. 3.5.3 [161] .

\begin{tabular}{lccc}
\hline \hline Source & Frac.(\%) & Correction (ppb) & Syst. Uncer. (ppb) \\
\hline Carbon Backing & $6.3 \pm 0.6$ & -8.8 & 2.6 \\
Inelastics & $<0.1$ & 0.0 & 0.0 \\
Rescattering & $<0.1$ & 0.0 & 0.0 \\
\hline TOTAL & - & -8.8 & 2.6 \\
\hline \hline
\end{tabular}

Figure 3-37: The sources of backgrounds during PREX, and the associated correction and systematic uncertainties in the parity-violating asymmetry, APV. The dominant source of background was the elastically scattered electrons from the carbon backing (diamond cooling foils). [161]

For PREX-II, thick diamond targets will be used, to the asymmetry correction for ${ }^{12} \mathrm{C}$ scattered electrons may be larger than it was for PREX-I. However the systematic uncertainty is extremely small in either case, because the asymmetry for elastic scattering off of ${ }^{12} \mathrm{C}$ is extremely well known, as outlined in Sec. 3.5.3.

\subsubsection{Integrating Detectors}

The PREX and CREX measurements use thin quartz integrating electron detectors, consisting of photomultiplier tubes (PMT) to detect Cherenkov radiation from particles traversing the quartz [144]. They are shown in Fig.3-38.

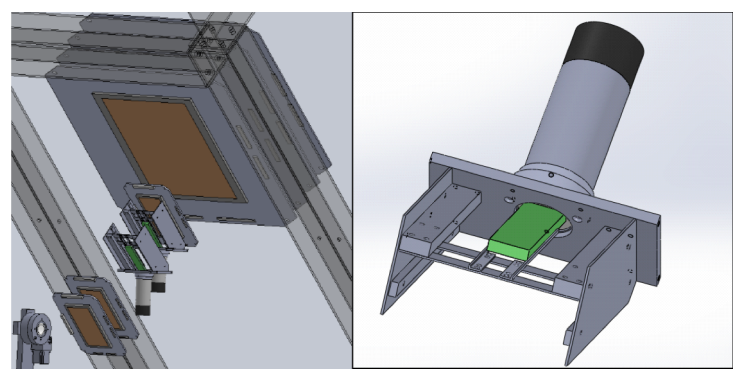

Figure 3-38: PREX-II design for Integrating Quartz Detectors consisting of quartz bars and a photomultiplier tube [147] 
A general description of the flux integration technique is shown if Fig. 3-39. The detectors perform an analog integration over a helicity window of everything that comes off the quartz and through the PMT. The thickness of the quartz is carefully chosen to balance shower fluctuations and photon statistics to optimize the detected asymmetry widths.

\section{Flux integration Technique}

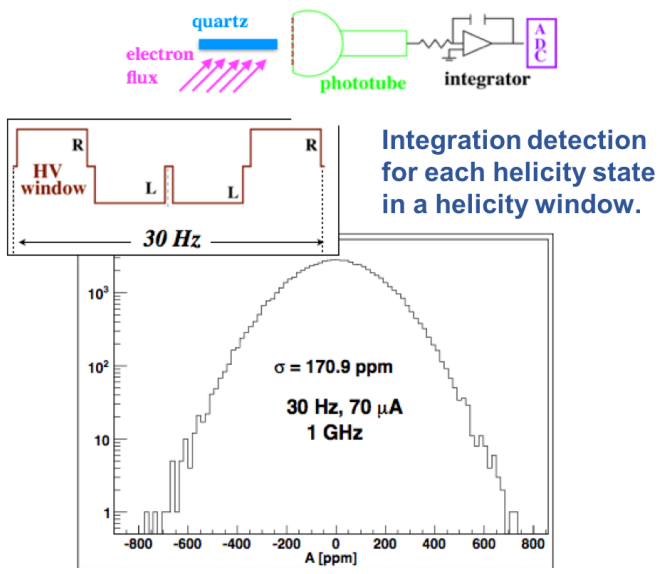

Figure 3-39: General description of the flux integration technique. Analog integration over a helicity window. PREX-I asymmetry width is shown. [147]

As shown in Fig. 3-39, PREX-I observed an asymmetry width of 170ppm (with $A 0.6 \mathrm{ppm})$ at a rate of $1 \mathrm{GHz}$, which are the conditions that the collaboration expects to achieve in PREX-II. The asymmetry width is determined by the rates and associated counting statistics $\sigma_{\text {stat }}$ as well as the detector signal to noise ratio $R M S / \mu=r m s /($ mean - pedestal $)$ where $\mu$ is proportional to the number of photoelectrons produced by the detector for every event and the event rate.

The statistical width is given by counting statistics $\sigma_{\text {stat }}=1 / \sqrt{N}=1 / \sqrt{2 T_{\text {window }} R}$, where $\mathrm{N}$ is the number of particles detected over a the duration of a helicity window $T_{\text {window }}$ at a rate $\mathrm{R}$. The expansion factor (or penalty of how much larger width gets) is given by $\sigma_{r}=\sqrt{1+(R M S / \mu)^{2}}$, and if there are $\mathrm{n}$ photoelectrons for every event, then $\sigma_{r}$ is approximately given by $\sqrt{n} / n$. The total asymmetry 
width is given by

$$
\sigma=\sigma_{\text {stat }} \sqrt{1+\sigma_{r}^{2}}=\frac{\sqrt{1+(R M S / \mu)^{2}}}{\sqrt{2 T_{\text {window }} R}}
$$

During PREX-I, the detectors had $R M S / \mu \sim 50 \%$, incurring a $\sim 12 \%$ penalty on the asymmetry width. The detectors worked, achieving suitable energy resolution and the new detectors for PREX-II are expected to do better. For PREXII, the finalized design was tested at Idaho, Stony Brook, and Mainz, and both simulation and data benchmarked the quartz properties. The Mainz beam tests demonstrated the PREX-II detectors have $R M S / \mu \sim 19 \%$, incurring a mere $\sim 2 \%$ penalty.

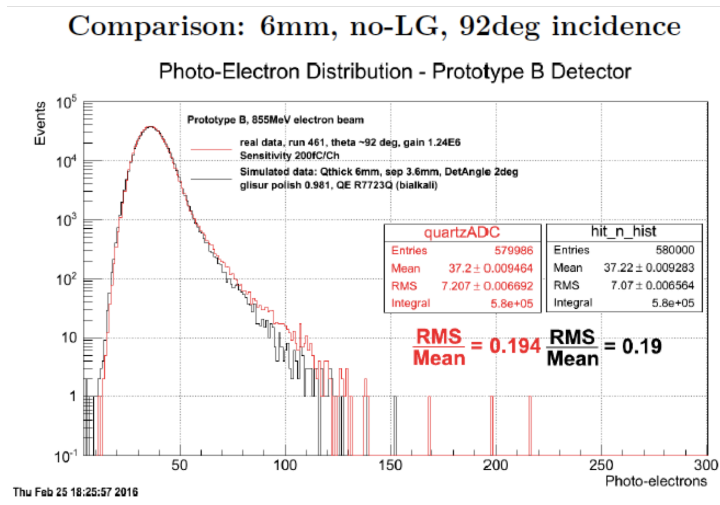

Figure 3-40: Mainz beam tests on PREX-II detectors [147]

In an experiment which uses this integration technique, where the detected particles must be integrated in order to get the desired accuracy in a reasonable time, the linearity of the linearity of the detectors, avoiding pedestal error, is of significant importance [77]. So, in PREX-II, each detector will also include an LED system for studying system linearity [144].

In addition to quartz detectors, both PREX and CREX experiments will use the VDC trackers. To avoid radiation damage the standard Cherenkov detectors, calorimeters, and S2 scintillator plane should be removed [144] during the course of the experiment. 


\subsubsection{New Radiation Shielding}

PREX-II Radiation Strategy: During PREX-I, control systems failures and loss of running efficiency was caused by the radiation inside the hall [164]. Elastics from the thick, high-Z target at low energy have a significant rate outside the dump. For PREX-II, the beamline must be designed to mitigate the large radiation dose and stop the PREX-I sources of radiation. The goal is to reduce the radiation deposited in the hall by $5-10 \mathrm{X}$ compared to PREX-I. The plan is to use a single collimator to stop everything that misses the dump and then shield around that collimator [179]. Compared with PREX-I, the collimator inner diameter will be decreased.

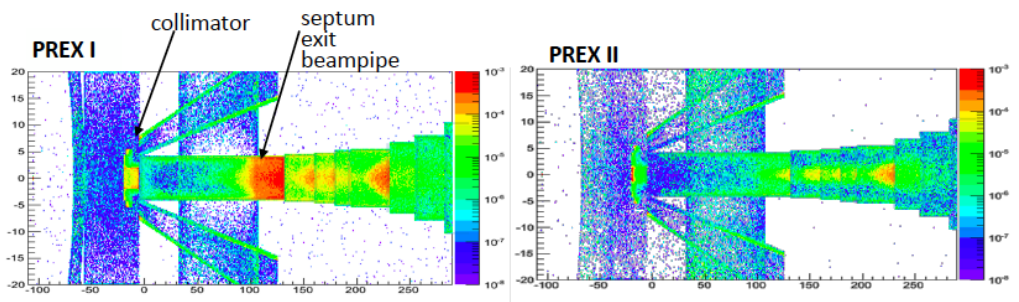

Figure 3-41: Origin of photons hitting a plane detector downstream of the septum [179]

Fig. 3-41 shows the origin of photons hitting a plane downstream of the septum for PREX-I and PREX-II simulations. G4 Monte Carlo simulations have shown collimation and shielding strategy reduces the expected radiation load in PREX-II to the level of previous successful experiments. For PREX-II the power distributed into the hall down by factor of 6 [179], the integrated dose will be down an order of magnitude from PREX-I and will not exceed optocoupler damage threshold in sensitive areas in the Hall where electronics are located (such as under the HRS platform).

Neutrons: While the boundary dose is sensitive to high energy neutrons (> $30 \mathrm{MeV}$ ), electronics are more sensitive to low energy neutrons. Fig. 3-42 shows relative silicon damage, in units of $1 \mathrm{MeV}$ neutron-equivalent (NIEL) metric, with respect to neutron energy. This is the damage function relative to a $1 \mathrm{MeV}$ neutron. 
Commercial off-the-shelf electronics are typically robust up to about $10^{13} 1 \mathrm{MeV}$ neq $/ \mathrm{cm}^{2}$ and optocouplers damage threshold is $\sim 10^{11} \mathrm{neq} / \mathrm{cm}^{2}$ [179]. The goal for PREX-II and CREX is to not exceed the optocoupler NIEL damage thresholds in regions of the Hall where electronics are stored.

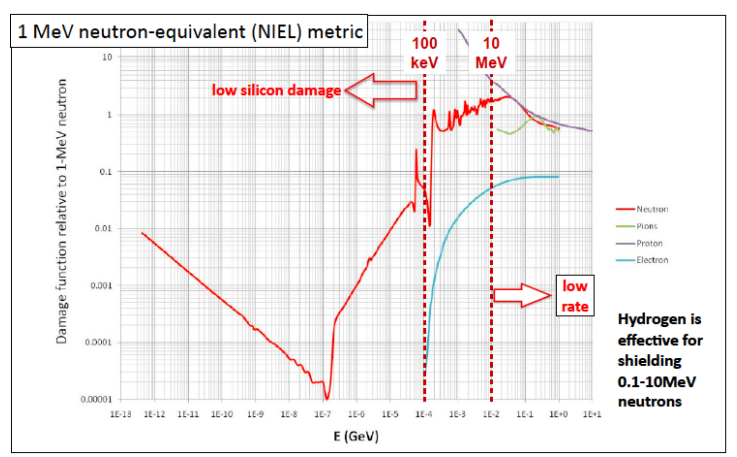

Figure 3-42: Neutron equivalent dose [179]

Since neutrons do a lot of elastic scattering, they behave more like a gas than a flux of particles, and reducing the neutron radiation is challenging. For $0.1-10 \mathrm{MeV}$ neutrons, Hydrogen is an effective shielding, so polyethylene is used. Shielding high and low energy neutrons usually takes at least $0.5-1 \mathrm{~m}$ of concrete or poly. There are space and weight problems with the large amount of material required and so its challenging to design effective shielding [164].

Pivot Region Shielding: The shielding in the pivot region is conceptually shown in Fig. 3-43b. The new tungsten collimator, described in Sec. 3.5.4, intercepts electrons with scattering angles greater than $0.78^{\circ}$, so that whatever gets past it, gets to the dump [179]. It stops everything that misses the dump. The power deposited on the $30 \% \mathrm{Cu}-70 \% \mathrm{~W}$ inner cylinder is $2.1 \mathrm{~kW}$ at 70 , which will be water-cooled. We shield around that collimator with an outer tungsten cover (jacket), which traps EM power. So the collimator as a whole self-shields produced neutrons. During deinstallation, there is shielded housing to enable the quick removal of the activated hot bore which gets covered at the run completion. 


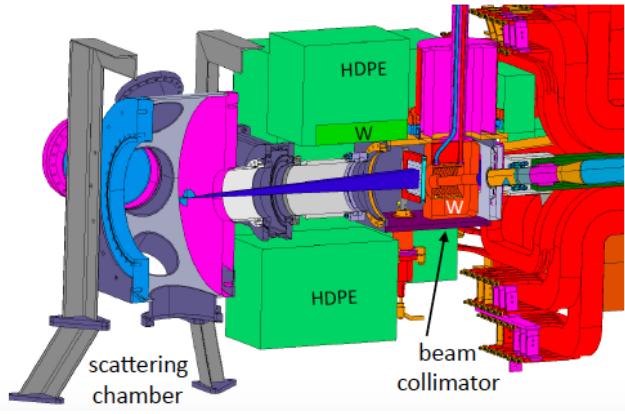

(a)

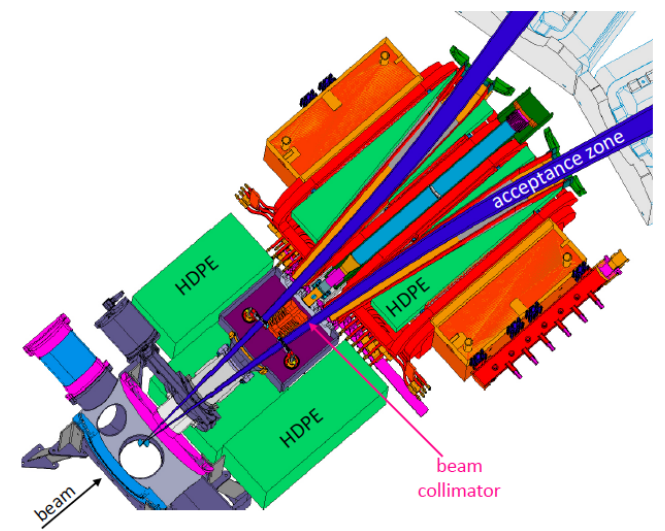

(b)

Figure 3-43: Preliminary designs that have since been modified. Here to show general concept (a) Side view of pivot region, with vertical midplane cut, showing location of the sky-shine shield relative to the scattering chamber and collimator [180] (b) Top view [179] (note: ignore the tungsten block, it is no longer present in the design))

A high-density polyethylene (HDPE) neutron shield is placed around the collimator region [144]. The general rule is that 20-30 cm thick HDPE is needed to moderate neutrons. The HDPE shield as shown consists of the following components: side curtains on either side of the collimator box, a roof above the beamline and collimator box and below the concrete target skyshine shield, a piece above back of collimator between housing and septum water connections, a block between the scattering chamber assembly and the collimator box, and wedges are also inserted into the septum bore, to attenuate forward going neutrons [144] [179].

Skyshine Shielding: Site-boundary dose mostly is caused by upward going, high-energy neutrons. For PREX, the largest source of skyshine neutrons is from the collimator region $(\sim 60 \%)$ and the target region contribute $\sim 20 \%$. For CREX, the largest source of skyshine neutrons is the target region $(\sim 70 \%)$ and collimator region only contributes $\sim 15 \%$ [144]. The skyshine shield is shown in Fig. 3-44. Two concrete blocks will be used, one of which is over the collimator region to further attenuate neutrons from the collimator region, and the other is over the target region to attenuate neutrons from the calcium target. The concrete is $40 \mathrm{~cm}$ 
thick covering roughly $1.2 \mathrm{~m} \times 3.3 \mathrm{~m}$, weighing several tons, with a wedge cutout to allow space for the target chamber mover. Together, the two blocks attenuate about $55 \%$ of high energy neutron power through the hall roof for CREX, and about $40 \%$ for PREX-II [144].

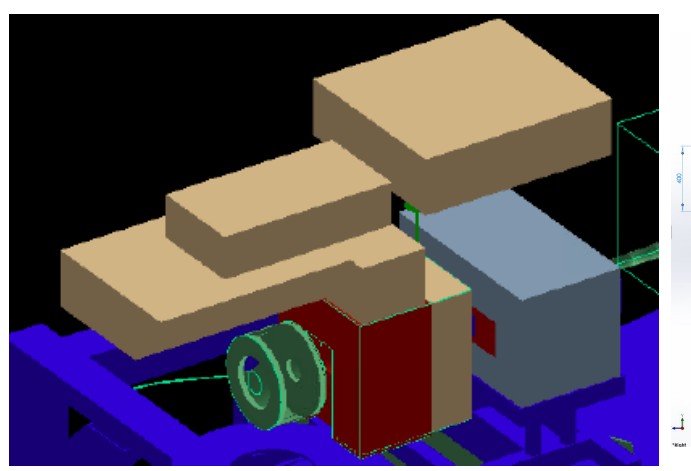

(a)

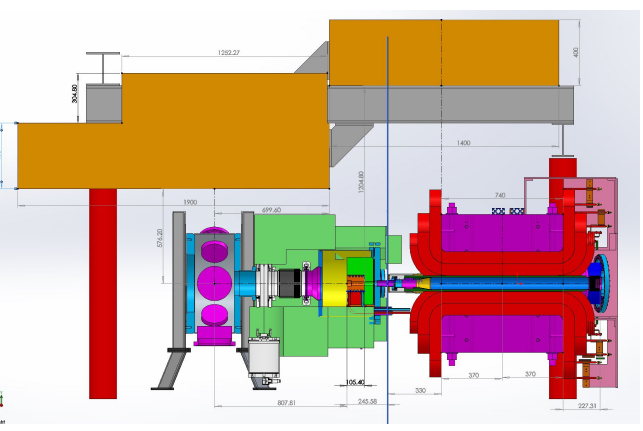

(b)

Figure 3-44: (a) Perspective view of pivot region with sky-shine shielding blocks [178] (b) Zoom in [179]

The goal for PREX and CREX is to stay under the JLab boundary dose limit: the DOE limit is $100 \mathrm{mrem} / \mathrm{yr}$ at boundary and the JLab limit is $10 \mathrm{mrem} / \mathrm{yr}$, to provide a 10X safety margin. To estimate the effect of configuration changes on the site-boundary dose, we use our G4 simulation and consider power of neutrons with E $>30 \mathrm{MeV}$. Results of simulation usually overestimate of measured site-boundary dose rates (the RSAD calculation estimated 13mrem for combined PREX-II and CREX with shielding, although PREX is happening in 2019 and CREX in 2020 so they count for separate years). Overestimates of site-boundary by factors of 2-3 are common. Scaling directly to measurements of site boundary dose during PREX-I, in which $82 \mathrm{C}$ on $\mathrm{Pb}$ target measured 1.34 mrem, the unshielded total for PREX-II and CREX would be a manageable $5.8 \mathrm{mrem}$, and this can be shielded to 2.7 mrem (13mrem), well within limits [179].

Dump Shielding: For the $12 \mathrm{GeV}$ upgrade, to allow for a diffuser, the dump beam line was reconfigured. The beam pipe diameter has been reduced and a $4 \mathrm{~cm}$ radius $\mathrm{Al}$ aperture (donut) was added $\sim 2 m$ inside the dump along with with ion 
chambers to protect against beam excursions [178]. Fig. 3-45 and Fig. 3-45b show the new dump with its reduced pipe size, a neck-down, and the donut. Fig. 3-45c shows the radiation coming from the dump and contributing to radiation in the hall, in particular the region where electronics are stored underneath the HRS. While most of the problem for CREX is the donut, for PREX the neck down, the pipe and donut all contribute to radiation underneath the HRS .

The solution is to modify the beam pipe and shield the dump. The beam pipe will be modified so (1) the aperture(donut) can be removed and (2) increase the hole to at least 12" diameter. For PREX-II/CREX we will most likely run the donut completely removed. Additionally the HRS platforms must be shielded from particles streaming from the beam interception at the neck down at the exit of the hall. Fig. 3-45d shows the concrete dump shield. A $45 \mathrm{~cm}$ thick concrete $\mathrm{U}$ shield is placed between the HRS arms where the middle section is stationary while the sides are to be attached to the HRS and poured in place [178].

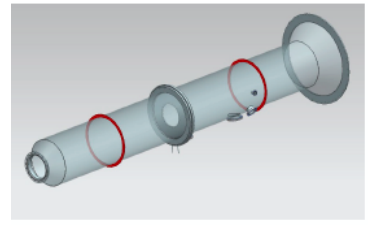

(a)

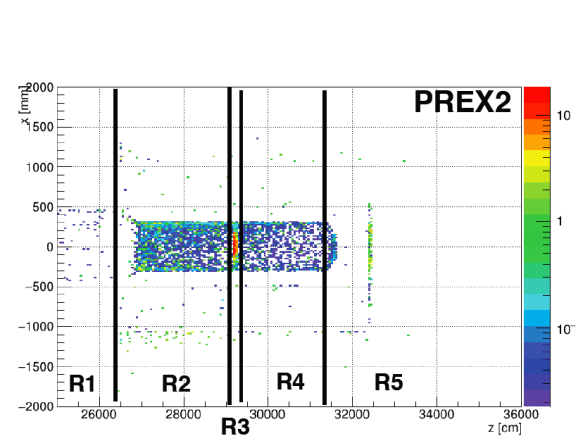

(c)

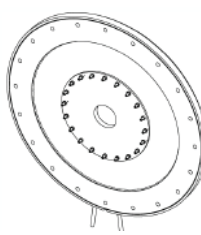

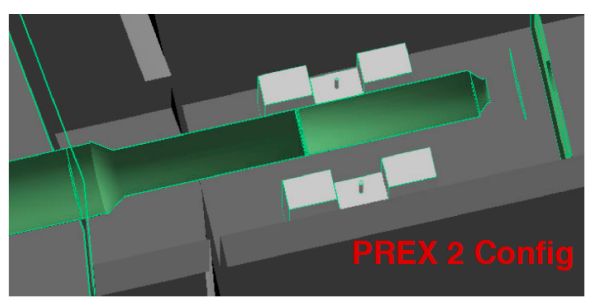

(b)

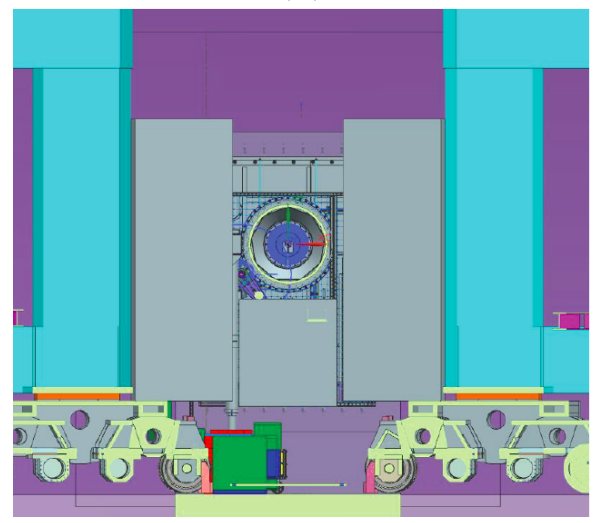

(d)

Figure 3-45: Beam Dump and Shielding [178] 
Summary: A summary of the NEIL dose estimates in various critical hall locations is shown in Table 3.3 for PREX-II and CREX. The goal for PREX-II and CREX is a level of boundary dose radiation that is only $2 \mathrm{X}$ times larger than PREX-I for both experiment ( 2.7mrem), an HRS NEIL dose of 25\% (9\% PREX2, $16 \%$ CREX) of the PREX-I dose, and a factor of 5X-10X reduction in radiation deposited in the hall.

\begin{tabular}{l|c|c|} 
& PREX-II NEIL $/ \mathrm{cm}^{2}$ & CREX NEIL $/ \mathrm{cm}^{2}$ \\
\hline Left HRS & $1.07 \mathrm{e}-5$ & $8.42 \mathrm{e}-7$ \\
Right HRS & $1.13 \mathrm{e}-5$ & $9.29 \mathrm{e}-7$ \\
Under Left HRS & $1.02 \mathrm{e}-6$ & $9.45 \mathrm{e}-8$ \\
Left Side HRS & $7.48 \mathrm{e}-7$ & $8.21 \mathrm{e}-8$ \\
US green wall & $3.44 \mathrm{e}-7$ & $5.46 \mathrm{e}-8$ \\
$10 \mathrm{~m} \mathrm{~W}$ & $1.43 \mathrm{e}-6$ & $8.88 \mathrm{e}-7$ \\
$10 \mathrm{~m} \mathrm{NW}$ & $1.46 \mathrm{e}-6$ & $2.05 \mathrm{e}-7$ \\
Hall Lid $\left(>10{\left.\mathrm{MeV} e^{-} \text {'s }\right)}^{6.61 \mathrm{e}-6}\right.$ & $1.48 \mathrm{e}-7$
\end{tabular}

Table 3.3: Radiation summary tables [178] at various critical locations in the Hall

\subsubsection{Compton Polarimeter}

Both the Møller and the Compton polarimeters demonstrated $1.3 \%$ and $1.2 \%$ precision at low energy and we expect to do even better for upcoming PREX-II and CREX, which require $\sim 1 \%$ polarimetry.

The Compton polarimeter acts on principles based on Compton scattering from polarized laser light. It measures the degree of longitudinal electron beam polarization, which is largely limited by the photocathode (but can be reduced when there are polarization components in the transverse direction), and provides a continuous, non-invasive beam polarization measurement concurrently with data acquisition [85].

A diagram of the Hall A Compton is shown in Fig. 3-46. When in use, the electron beam passes through a chicane into the interaction region, where a green $(532 \mathrm{~nm})$ laser has been amplified in a Fabry-Perot cavity so it reaches $\sim 2 \mathrm{~kW}$ power as the photon target. The electron beam crosses the photon beam at a 
small angle and some fraction of the electrons Compton scatter with the photons in the cavity [86] ${ }^{4}$. The scattered electrons are separated from the primary beam and get detected along with the scattered photons. Downstream of the electronphoton interaction region, the electron beam is bent by the last chicane, restoring the beamline [33].

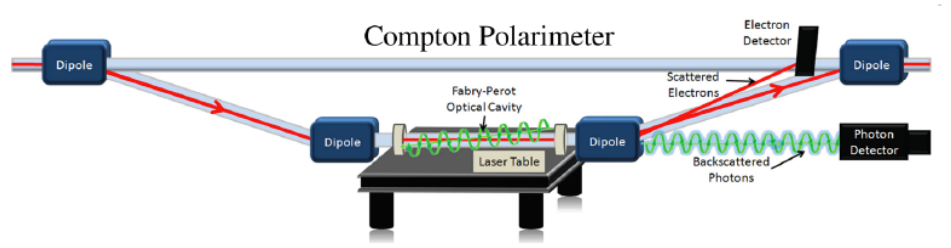

Figure 3-46: Drawing of the Compton Polarimeter. We see the four dipole chicane diverting the beam towards the laser table. The third dipole selects the Compton scattered electrons and allows the photons to pass through to the photon detector. The fourth dipole then diverts the remainder of the beam towards the target. [88]

There is an asymmetry in scattering rate in the Compton interaction between right and left helicity states scattering of right and left circularly polarized light. This asymmetry, with well known dependent on the scattered photon energy, is measured by the polarimeter. The electron beam polarization $P_{b}$ is then determined from [86]

$$
A_{\text {exp }}=P_{b} P_{\gamma} A_{t h}=\frac{S^{+}-S^{-}}{S^{+}+S^{-}}
$$

where $P_{b}$ is the polarization of the electron beam, $P_{\gamma}$ is the polarization of the photon, $A_{t h}$ is the theoretical asymmetry is Compton scattering rate for perfectly polarized electrons and photons, $A_{\text {exp }}$ is the measured Compton asymmetry [86], and where $S^{+(-)}$is the scattering rate flux for the right and left helicity states of electron beam. The circular polarization state of the laser, $P_{\gamma}$, is determined by a quarter-wave plate upstream of the cavity [86] ${ }^{5}$.

\footnotetext{
${ }^{4}$ at the nominal dipole settings and photon-electron beam crossing angle of $23.5 \mathrm{mrad}$, and the left-right Compton asymmetry was about $1.7 \%$ [89].

${ }^{5}$ The polarization of the light is flipped between left and right circularly polarized light using an insertable quarter wave plate. There are plans to use a Pockels cell to switch the laser polarization so that this process can be done quickly, on a similar time scale to that of the
} 
The theoretical asymmetry $A_{t h}$ as a function of scattered photon energy is well known. The asymmetry is below zero at low photon energies, there's a zero crossing at a nominal photon energy, and the asymmetry becomes positive for higher photon energies [87] and increases up to the "Compton edge", the maximum allowable photon energy given the kinematic constraints. Higher photon energy corresponds to greater scattering angle of the electron, so polarimetry can be performed by measuring the scattering rate asymmetry and either measuring the photon energy or measuring the electron scattering angle or both. So the Compton polarimeter has detectors for both electrons and photons.

The electrons are detected by a silicon microstrip detector which measures asymmetry as a function of position. The calculated analyzing power as a function of energy is well known. The position of the electron in the detector is converted to energy [33]. The low beam energy PREX experiment will be unable to detect the asymmetry zero-crossing in the electron detector and so will rely the integrating photon method for polarimetry [33].

Most photons in the cavity backscatter with very small angles, and the highly collimated photons are detected by a calorimeter. Photon detector is positioned downstream of interaction point. The response function is calibrated using the electron detector in conjunction to infer the energy of the coincidence photons [33]. A diagram of the photon detector concept is show in Fig 3-47.
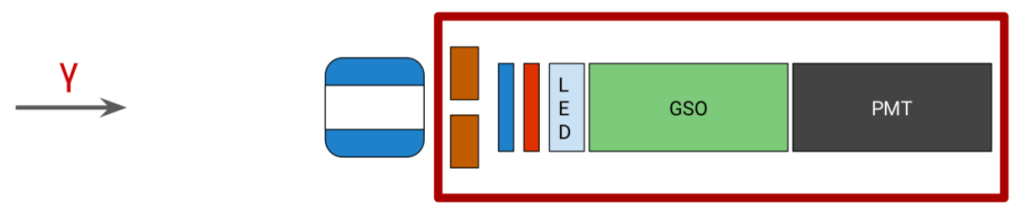

Figure 3-47: Drawing of the Compton Photon Detector [88]

The material used for the calorimeter is a single GSO crystal (manufactured by Hitachi Chemical, 0.5\% Ce-doped Gd2SiO5, $6 \mathrm{~cm}$ diameter x $15 \mathrm{~cm}$ length electron beam polarization flip rate, so that polarization measurements can be done separately on the DoLP of the right-handed electron beam and the DoLP of the left-handed electron beam. 
[87]), which is acceptable when used at lower energies and has high light yield detected by the PMT. Several LED's are positioned at the front of the GSO are used to monitor detector linearity. Two "finger" scintillators positioned in front of main detector and behind tungsten "fingers" are used to center scattered photons on detector (shown in Fig. 3-47 in red). A lead collimator serves as synchrotron shielding with apertures of $0.5 \mathrm{~cm}-2 \mathrm{~cm}$ permanently mounted in front of detector and additional pieces of thin lead plates (of a few mm's each) are placed in front of detector (shown in Fig. 3-47 in blue). An additional remote-controllable Tungsten collimator is used with aperture controllable from $1 \mathrm{~mm}$ to $5 \mathrm{~cm}$ (brown), can also be used to center scattered photons on the detector. All these components are mounted on detector stand and sits on a remote-controllable table with $\mathrm{x}, \mathrm{y}$ motion control.

During PREX-I, the average beam polarization measured by the Compton polarimeter was

$$
P_{b}=88.20 \pm 0.12(\text { stat }) \pm 1.04(\text { syst }) \%
$$

The PREX Compton polarimeter measurements were very clean for PREX-I, and measured the beam polarization with a total systematic error of $1.1 \%$ at $1 \mathrm{GeV}$ was achieved [89] and further improvements are expected for PREX-II and CREX.

\subsubsection{Møller Polarimeter}

The Møller polarimeter measures longitudinal electron beam polarization based on Møller scattering from ferromagnetic foil targets. A ferromagnetic polarized target is used, incident polarized electrons are Møller scattered off the target, and the double-polarization spin asymmetry measured by left-right counting rate asymmetry is employed to infer the electron beam polarization. In contrast to the Compton polarimeter, the Møller polarimeter is invasive and so cannot be run continuously. Several measurements are taken over the course of the running experiment and compared to the Compton measurements. The measured left- 
right counting rate $\mathrm{M} \varnothing l$ ler asymmetry measured by the Møller polarimeter $A_{\text {Moller }}$ is given by

$$
A_{\text {Moller }}=P_{b} P_{\text {tgt }} \cos \theta A_{z z}
$$

where $P_{b}$ is the polarization of the electron beam, $P_{t g t}$ is the polarization of the target foil, $A_{z z}$ is the analyzing power, $\theta$ is the angle subtended by the beam on target. The rate detected is given by [93]

$$
R^{ \pm}=\int \frac{d \sigma^{0}}{d \Omega} d \Omega+P_{b}^{ \pm} P_{t g t} \int \frac{d \sigma^{ \pm}}{d \Omega} d \Omega
$$

where it is assumed $P_{b}^{ \pm}= \pm P_{b}$. The measured asymmetry is then given by

$$
A_{\text {Moller }}=\frac{R^{+}-R^{-}}{R^{+}+R^{-}}=P_{b} P_{t g t} \frac{\left\langle\sigma^{+}\right\rangle-\left\langle\sigma^{-}\right\rangle}{2\left\langle\sigma^{0}\right\rangle}=\left\langle A_{z z}\right\rangle P_{b} P_{t g t}
$$

In the standard treatment, $A_{\text {Moller }}$ is measured by comparing rates, $P_{t g t}$ is believed to be accurately known, and $A_{z z}$ is calculated where many corrections pile into the calculation of this parameter [93].

A diagram of the Møller polarimeter is shown in Fig. 3-48. The Hall A Møller polarimeter scatters longitudinally in ferromagnetic iron target foils, and uses the left-right counting rate asymmetry to extract the beam polarization. The quads adjust the beam focus, and the dipole bends the beam vertically out of the scattering plane onto the detectors [92].

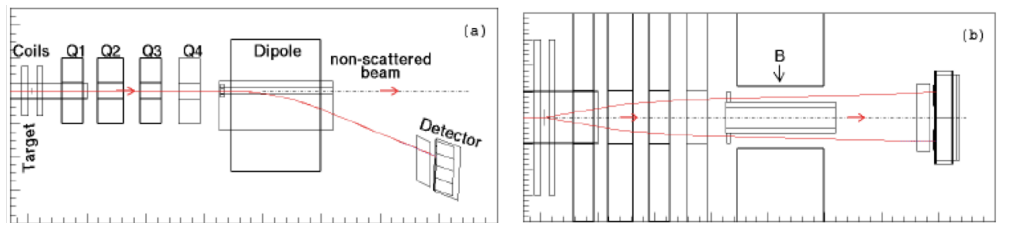

Figure 3-48: The Hall A Møller polarimeter scatters longitudinally polarized electrons polarized along the beam propagation direction in ferromagnetic target foils, and exploits the left-right counting rate asymmetry to extract the beam polarization. The quads adjust the beam focus, and the dipole bends the beam vertically out of the scattering plane onto the detectors [92]. Reproduced from talk given by Don Jones. 
As shown in Fig. 3-48 b , this magnetic spectrometer focuses the scattered electron-pair onto two separate lead-glass calorimeter detectors that detects the electrons in coincidence [92]. A vertical steel plate at the dipole midplane, with a hole at the center to allows the non-scattered electrons through, acts as a collimator for the scattered electrons and acts as a magnetic shield for the non-scattered electrons in the beam [92].

A new target system has been designed for PREX-II which includes a new magnet, new target frame, 4 iron foils of various thicknesses, a rigid target rotator assembly, as well as a frame design incorporating the potential for Kerr monitoring (an in situ measure of the iron magnetization $M_{F e}$ ) which is important for preliminary R\&D for the MOLLER experiment. Components of this new system are shown in Fig. 3-49b.

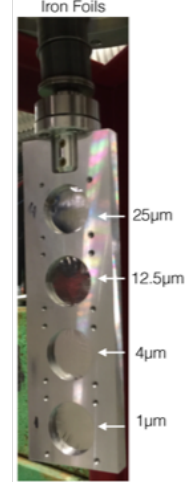

(a) Moller Foils

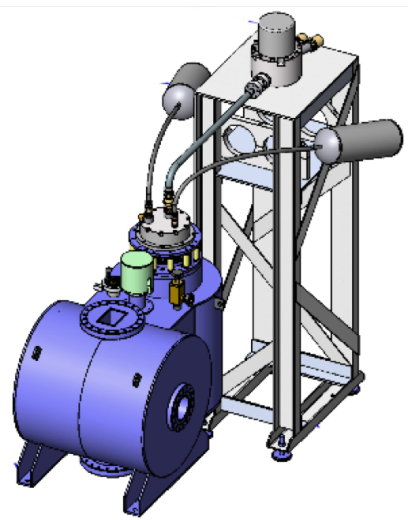

(b) Moller magnet

Figure 3-49: New Møller Target : Four targets, in frame designed for rotation and Kerr monitoring

During PREX-I target foil thicknesses were from 7-30 $\mu \mathrm{m}$ to 1-10 $\mu \mathrm{m}$ for beam polarization measurements at currents of up to $50 \mathrm{uA}$. The new target foils have thicknesses of $1 \mu \mathrm{m}, 4 \mu \mathrm{m}, 12.5 \mu \mathrm{m}$, and $25 \mu \mathrm{m}$. Other improvements to the system during PREX included introduction of a beam duty cycle in order to reduce target heating at high beam currents, new data acquisition system (DAQ) based on Flash ADCs (FADCs) to handle higher scattering rates and the introduction of 
segmented detectors to handle higher scattering rates [90] [91]. During PREX-I, the Møller polarimeter used a high-field magnet that provided a magnetic field of up to $4 \mathrm{~T}$, although only $3 \mathrm{~T}$ was used during PREX-I.[90]. The upgraded Møller polarimeter should also achieve high fields during PREX-II, where the electrons in the ferromagnetic target foil are nearly 100\% polarized, and the foil magnetization is near saturated. As shown in Fig. 3-50, when the iron is in saturation, uncertainty in the applied magnetic field has a less significant effect in the uncertainty on the degree of magnetization of the iron. So, the target polarization should be determined with much greater precision near saturation at 3-4T than for lower field targets. During PREX-I, the target foil polarization was inferred with an uncertainty of $0.25 \%$ [90] and this should be further reduced in PREX-II.

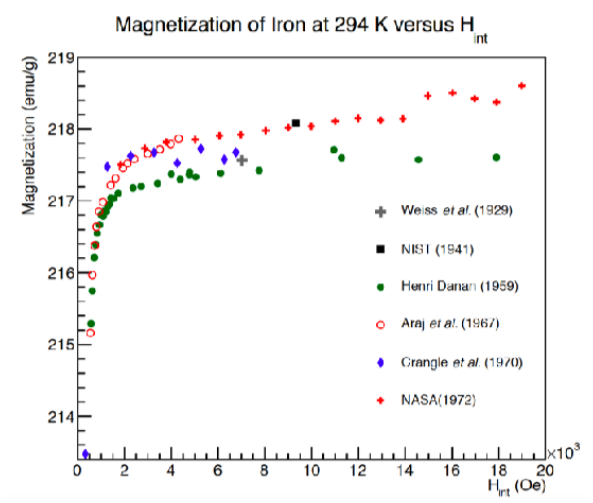

Figure 3-50: Magnetization of pure iron as a function of applied magnetic field. Reproduced from [93]

Several systematic uncertainties that contribute to this measurement include: the magnetization of iron at near saturation, spin vs. orbital component of the magnetization, target foil angle with respect to the holding field, the spectrometer tune and magnet currents, the analyzing power averaged over the acceptance, the Levchuk effect, demagnetization from target heating, background contributions to rate, electronic deadtime corrections, radiative corrections, deviation from perfect polarization reversal, and effects from transverse polarization. In PREX-I, the 
dominant error in the polarization measurement was a systematic of $1.7 \%$, with this uncertainty dominated by the target foil polarization uncertainty of $1.5 \%$ [92]. The new Møller polarimeter will eventually allow in situ Kerr measurements to nail down more precisely this foil polarization uncertainty, as well a the study of possible systematic effects, in data and simulation (such as the Levchuck effect etc.) $[93]$.

\section{5/16 operation}

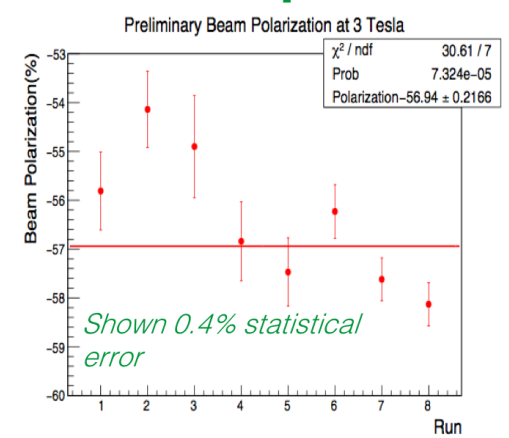

Figure 3-51: Hall A beam polarization 2015-2016 as measured by Møller polarimeter before upgrade [95]

New data collected in 2015-2016 run period shows $0.4 \%$ statistical uncertainty in measurements taken in reasonable amount of time (a few hours). During PREXI, the Møller polarimeter made nine measurements of the beam polarization the average of which was

$$
P_{b}=90.32 \pm 0.07(\text { stat }) \pm 1.12(\text { syst }) \%
$$

The precision of the polarization measurement for PREX-II is expected to be $<1 \%$ level (both systematic and statistical) given the many improvements to the new Møller target system. 


\subsubsection{HCBA}

As previously stated in Sec. 2.3.7, since this measurement compares electrons of opposite helicity and looks for changes in scattering rates, any change in the polarized beam correlated with the helicity reversal, including energy changes, position changes, or position changes, can be a potential source for systematic error, or a false asymmetry. These false asymmetries are described by Eq. 2.4, reiterated here for convenience:

$$
A_{\text {raw }}=A_{\text {det }}-A_{Q}+\alpha \Delta E+\sum \beta_{i} \Delta x_{i}
$$

where $A_{\text {raw }}$ is the beam current normalized detector asymmetry, $A_{Q}$ is the beam charge asymmetry, $\Delta E$ is the helicity correlated energy difference, $\Delta x_{i}$ are the helicity correlated position differences, and $\alpha, \beta_{i}$ are the coupling constants, both calculated and measured through cross correlations and linear regression in data analysis.

In PREX-I, beam asymmetries contributed $1.1 \%$ to the measured asymmetry $A_{P V}\left({ }^{208} \mathrm{~Pb}\right) \sim 0.6 \mathrm{ppm}$, or less than $7 \mathrm{ppb}$. Experience suggests that leading systematic errors can be improved beyond that in PREX-II. For CREX, beam asymmetries must contribute less then $0.3 \%$ to the measured asymmetry $A_{P V}\left({ }^{48} \mathrm{Ca}\right) \sim 2.3 \mathrm{ppm}$, or less than 70ppb. PREX-II expects helicity correlated changes in the beam of less than $7 \mathrm{ppb}$. To achieve such a small systematic error goal, the beam trajectory must remain unchanged with respect to to the sign of the electron beam polarization at the $\mathrm{nm}$ and nrad level. The beam position differences in the injector before acceleration must also be small, $\sim 300 \mathrm{~nm}$. The helicity correlated laser spot size asymmetries must be small too on the order $\sim 10^{-4}$. Achieving these goals all depends on the laser and on the Pockels cell. An intensity asymmetry in the electron beam can arise from a polarization asymmetry in the laser beam when incident on a polarizing element such as the photocathode. A position difference in the electron beam can arise from a polarization gradient 
in the pockels cell, a 1st moment effect producing a shift in central laser beam position. A spot size asymmetry can arise from a 2nd moment in polarization gradient, which can broaden or narrow the beam distribution.

The position differences in the injector during PREX-I are shown in Fig. 3-52. Position differences in the injector were $\sim 200-300 \mathrm{~nm}$. In the experimental Hall, position differences were observed to be $\sim 30-100 \mathrm{~nm}$, approximately $60 \mathrm{~nm}$ overall, implying adiabatic damping of 3X-10X, compared with the predicted damping factor given by $\sqrt{p / p_{0}}=\sqrt{1 G e V / 130 k e V}=87 X$ as defined from the $130 \mathrm{keV}$ region and $\sqrt{1 \mathrm{GeV} / 5 \mathrm{MeV}}=14 \mathrm{X}$ as defined from the $5 \mathrm{MeV}$ region. The cumulative average position differences in the Hall during PREX-I was $<5 \mathrm{~nm}$, much smaller due to cancellations as a consequence of the slow helicity reversal [163].

PREX-II expects to achieve even smaller position differences in the injector, possibly at the sub-100nm Qweak level (Fig. 3-53), which given similar adiabatic damping, will produces smaller position differences in the Hall and therefore smaller beam systematics after slow reversal cancellations.

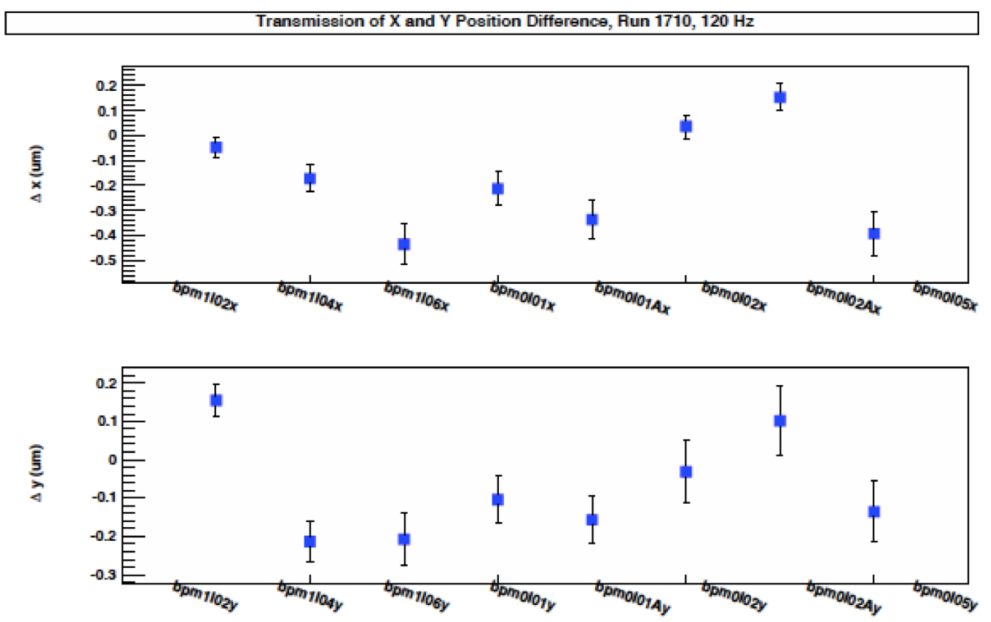

Figure 3-52: Injector position differences during PREX-I [162] 


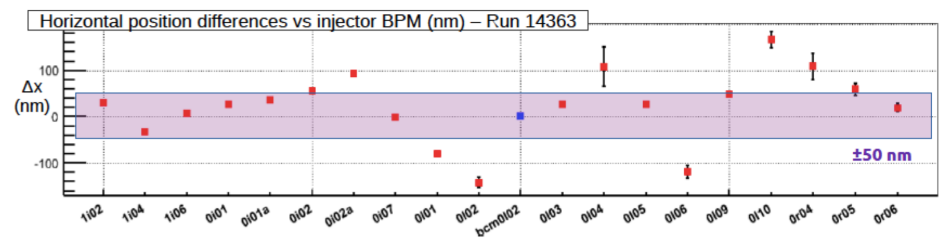

Figure 3-53: Qweak: Horizontal position differences on successive injector BPMs. The purple band corresponds to $\pm 50 \mathrm{~nm}$ of position differences [56] by the Qweak definition $\Delta x_{\text {qweak }}=\left(x^{0}-x^{1}\right) / 2$ which corresponds to $\pm 100 \mathrm{~nm}$ by the PREX and HAPPEX definition which we use in this thesis $\Delta x=x^{0}-x^{1}$

\subsection{PREX-II Summary of Goals}

The systematic and statistical error goals for PREX-II and CREX are shown in Fig. 3-54 as well as those achieved in PREX-I.

\begin{tabular}{|c|c|}
\hline \multicolumn{2}{|c|}{$\begin{array}{c}\text { PREX-I } \\
\mathrm{E}=1.1 \mathrm{GeV}, 5^{\circ} \\
\mathrm{A}=0.6 \mathrm{ppm}\end{array}$} \\
\hline Charge Normalization & $0.2 \%$ \\
\hline Beam Asymmetries & $1.1 \%$ \\
\hline Detector Non-linearity & $1.2 \%$ \\
\hline Transverse Asym & $0.2 \%$ \\
\hline Polarization & $1.3 \%$ \\
\hline Target Backing & $0.4 \%$ \\
\hline Inelastic Contribution & $<0.1 \%$ \\
\hline Effective $Q^{2}$ & $0.5 \%$ \\
\hline Total Systematic & $2.1 \%$ \\
\hline Total Statistical & $9 \%$ \\
\hline
\end{tabular}

\begin{tabular}{|c|c|}
\hline \multicolumn{2}{|c|}{$\begin{array}{c}\text { PREX-II } \\
\mathrm{E}=0.95 \mathrm{GeV}, 5^{\circ} \\
\mathrm{A}=0.6 \mathrm{ppm} \\
70 \mu \mathrm{A}, 25+10 \text { days }\end{array}$} \\
\hline Charge Normalization & $0.1 \%$ \\
\hline Beam Asymmetries* & $1.1 \%$ \\
\hline Detector Non-linearity* & $1.0 \%$ \\
\hline Transverse Asym & $0.2 \%$ \\
\hline Polarization* & $1.1 \%$ \\
\hline Target Backing & $0.4 \%$ \\
\hline Inelastic Contribution & $<0.1 \%$ \\
\hline Effective $Q^{2}$ & $0.4 \%$ \\
\hline Total Systematic & $2 \%$ \\
\hline Total Statistical & $3 \%$ \\
\hline
\end{tabular}

\begin{tabular}{|c|c|}
\hline \multicolumn{2}{|c|}{$\begin{array}{c}\text { CREX } \\
\mathrm{E}=2.2 \mathrm{GeV}, 5^{\circ} \\
\mathrm{A}=2.3 \mathrm{ppm} \\
150 \mu \mathrm{A}, 35+10 \text { days }\end{array}$} \\
\hline Charge Normalization & $0.1 \%$ \\
\hline Beam Asymmetries & $0.3 \%$ \\
\hline Detector Non-linearity & $0.3 \%$ \\
\hline Transverse Asym & $0.1 \%$ \\
\hline Polarization & $0.8 \%$ \\
\hline Target Contamination & $0.2 \%$ \\
\hline Inelastic Contribution & $0.2 \%$ \\
\hline Effective $Q^{2}$ & $0.8 \%$ \\
\hline Total Systematic & $1.2 \%$ \\
\hline Total Statistical & $4 \%$ \\
\hline
\end{tabular}

Figure 3-54: PREX error tables: PREX-I was a statistics limited result, with most systematics well under control. *Experience suggests that leading systematic errors such as beam asymmetries can be improved further [147]

PREX-I did not achieve its statistical precision goal of $3 \%$ due to equipment failure as a result of high radiation [171], but the systematics were well under control. Though experience suggests, beam systematics could be controlled even better than they were during PREX-I. And PREX-II expects to achieve a factor of 3 improvement on the neutron skin thickness uncertainty. [147]

Despite the statistical uncertainty, PREX-I made the first measurement of the parity-violating asymmetry, APV, in the elastic scattering of polarized electrons 
from ${ }^{208} \mathrm{~Pb}$ and provided the first electroweak evidence the the weak charge density is more extended than the electric charge density. It showed there is indeed a weak skin around a heavy nucleus. PREX-I measured an asymmetry of $0.6 \mathrm{ppm}$, and inferred a neutron skin thickness, a difference between the neutron and proton distribution radii in the ${ }^{208} \mathrm{~Pb}$ nucleus, of $R_{n}-R_{p}=0.33_{-0.18}^{+0.16} \mathrm{fm}$. Interestingly, current PREX central value not consistent with measured neutron star properties and existing models (though it is within sigma). Measurement of this quantity has inspired significant interest from the community. PREX informs neutron star size vs. mass curves and the gravitational waveform is sensitive to the nuclear EOS for neutron stars. PREX-II and CREX will have impact on nuclear physics and astrophysics, including LIGO. 



\section{Chapter 4}

\section{Mainz ${ }^{12} \mathrm{C}$ Transverse Asymmetry}

\section{Analysis}

This chapter presents the results of the Mainz measurement of the beam-normal single spin asymmetry for elastic scattering off ${ }^{12} C$ at various $Q^{2}$ as presented in [208]. The data analysis was performed by the A1 collaboration at Mainz and we present here a cross-check of that analysis.

\subsection{Motivation}

Measurements of the transverse asymmetry $A_{n}=\left(\sigma_{\uparrow}-\sigma_{\downarrow}\right) /\left(\sigma_{\uparrow}+\sigma_{\downarrow}\right)$ presents a useful theoretical challenge for the description of elastic ep scattering. The transverse asymmetry in ${ }^{208} \mathrm{~Pb}$ is important for PREX because the parity-violating asymmetry $A_{P V}=\left(\sigma_{+}-\sigma_{-}\right) /\left(\sigma_{+}+\sigma_{-}\right)$in elastic electron scattering has a systematic uncertainty which comes from a non-zero transverse component of the beam polarization. The transverse asymmetry is defined by $A_{n}=\left(\sigma_{\uparrow}-\sigma_{\downarrow}\right) /\left(\sigma_{\uparrow}+\sigma_{\downarrow}\right)$, where $\sigma_{\uparrow(\downarrow)}$ is the elastic scattering cross-section for electrons with spin $\vec{P}_{e}$ parallel (or antiparallel) to the normal vector defined by the scattering plane $\hat{n}=$ $\left(\vec{k} \times \overrightarrow{k^{\prime}}\right) /\left|\vec{k} \times \overrightarrow{k^{\prime}}\right|$, where $\vec{k}$ and $\overrightarrow{k^{\prime}}$ correspond to the the incident and scattered electron momenta [208]. 
$A_{n}$ can contribute to the extracted $A_{P V}$ if the electron beam polarization has a transverse component. In precision scattering experiments like PREX, the contributed false measured asymmetry $A_{n}^{m}$ relates to the transverse asymmetry via $A_{n}^{m}=A_{n} \vec{P}_{e} \cdot \hat{k}[209]$.

For light nuclei, like ${ }^{1} \mathrm{H},{ }^{4} \mathrm{He}$ and ${ }^{12} \mathrm{C}$, this false asymmetry can be accounted for by contributions from two-photon exchange processes in e- scattering. The interference of the one-photon and two-photon exchange amplitudes gives rise to the transverse asymmetry $A_{n}$. Calculations [211] [210] of $A_{n}$ in a two-photon exchange approximation, but including a full range of intermediate excited states, predict that $A_{n}$ scales roughly as the ratio $\mathrm{A} / \mathrm{Z}$, and is not strongly Z-dependent. These theoretical calculations for light nuclei at low $Q^{2}$ are found to be in good agreement with data (Fig. 4-1).

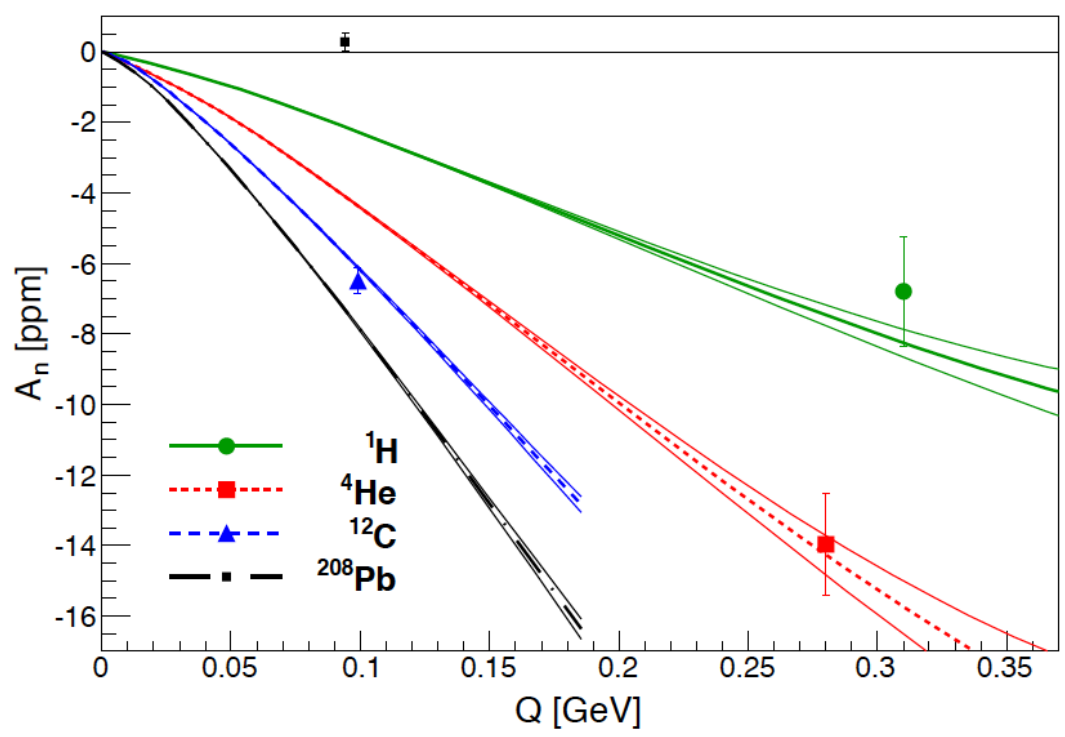

Figure 4-1: Extracted physics asymmetries $A_{n}$ vs. Q [209]. Each curve, specific to a particular nucleus as indicated, is a theoretical calculation from Ref. [210].

However, for heavy nuclei, like ${ }^{208} \mathrm{~Pb},{ }^{48} \mathrm{Ca}$ the accounting for the transverse asymmetry remains a challenge. The two-photon exchange calculations, which account for dispersion corrections but neglect Coulomb distortions, fail for the heavier ${ }^{208} \mathrm{~Pb}$ nucleus, as measured during the PREX-I experiment [209]. Ad- 
ditional calculations [212] for Coulomb distortion effects that work to all orders in photon exchanges by numerically solving the Dirac equation, albeit for only the elastic intermediate state, predict small contributions to $A_{n}$ which increase strongly with Z . Understanding the $Q^{2}$ dependence and the energy dependence is also important since the weight of dispersion corrections varies with the incident beam energy. Hence, the measurement of $A_{n}$ in scattering off heavy nuclei, for a range of $\mathrm{Z}$ at various beam energies, might lead to new insights into the structure of heavy nuclei and might also help with systematic errors on measurements of the $A_{P V}$ in precision experiments such as PREXII [209].

To inform theoretical calculations, an experiment has been performed at MAMI on ${ }^{12} \mathrm{C}$ at various $Q^{2}$ at constant $570 \mathrm{MeV}$ beam energy. Similar experiments for heavier nuclei, such as Calcium, Lead or Tin have also been considered, but have not been attempted with this apparatus.

\subsection{Experimental Setup}

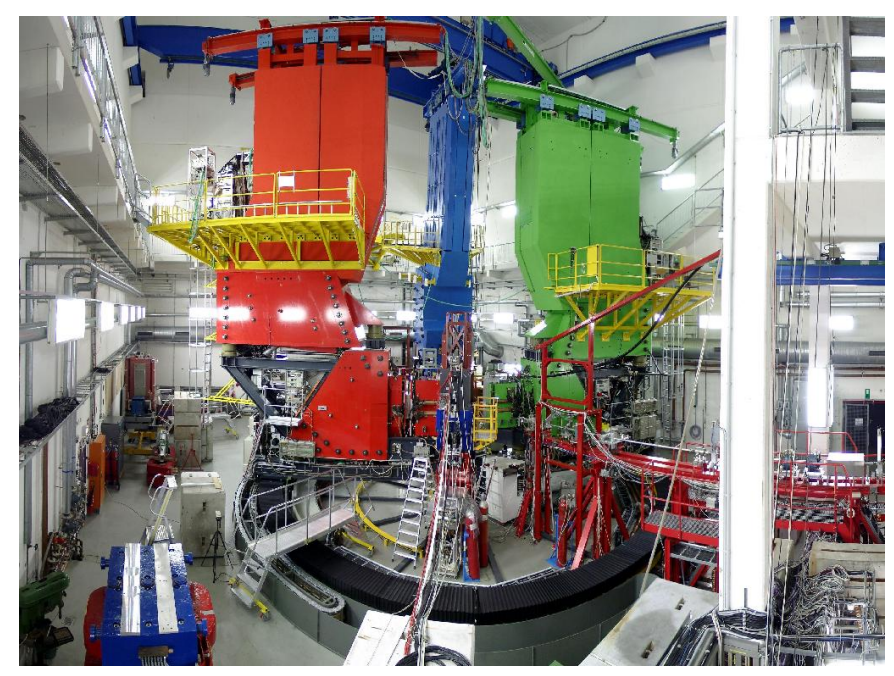

Figure 4-2: Mainz A1 Collaboration Hall Spectrometer [213], SpekA is beam left and $\mathrm{SpekB}$ is beam right 
The experiment for the measurement of the beam-normal single spin asymmetry $A_{n}$ in elastic $e^{-12} \mathrm{C}$ scattering was performed at the the Mainz Microtron MAMI with the spectrometer setup of the A1 Collaboration.

A $20 \mu \mathrm{A}$ vertically polarized continuous-wave (CW) e-beam was scattered off a $2.27 \mathrm{~g} / \mathrm{cm}^{2}$ carbon target and elastics were focused onto two fused-silica detectors, one on beam left(SpekA) and one on beam right(SpekB), in the focal planes of the high-resolution spectrometers (HRS) [222].

Several $Q^{2}$ measurements were performed at the same beam energy of $570 \mathrm{MeV}$ by changing the spectrometer angles: spectrometer $\mathrm{A}$ (left) was placed an angle of $23.5^{\circ}\left(Q^{2}=0.04 \mathrm{GeV}^{2} / \mathrm{c}^{2}\right)$ and also at $25.90^{\circ}\left(Q^{2}=0.05 \mathrm{GeV}^{2} / \mathrm{c}^{2}\right)$; spectrometer $\mathrm{B}$ (right) was placed at angles $20.61^{\circ}\left(Q^{2}=0.04 \mathrm{GeV}^{2} / \mathrm{c}^{2}\right), 17.65^{\circ}\left(Q^{2}=\right.$ $\left.0.03 \mathrm{GeV}^{2} / \mathrm{c}^{2}\right)$, and $15.11^{\circ}\left(Q^{2}=0.02 \mathrm{GeV}^{2} / \mathrm{c}^{2}\right)$, where the calculated momentum transfer takes into account the differing sizes of focal plane acceptance for the detectors in spekA and spekB.

\subsection{Inversions}

MAMI, a normal-conducting CW (continuous-wave) electron accelerator, consists of a three race-track microtrons and a harmonic double-sided microtron. A source of longitudinally polarized electrons is produced with circularly polarized light incident on a GaAs/GaAsP photocathode [216] [217]. MAMI did not normally provide vertical e-beam polarization in its conventional operation, which ordinarily produces longitudinal e-beam polarization, so modifications to the existing accelerator were made in order to perform these transverse asymmetry experiments with vertically polarized beam for the first time. [215]. To obtain vertical polarization, the electrons emitted off the cathode with longitudinal polarization first pass through a Wien filter in the $100 \mathrm{keV}$ region of the injector linac, which rotates the polarization vector by $90^{\circ}$ horizontally. Then a double solenoid, located shortly after the Wien, rotates the polarization vector into the vertical 
orientation (Fig.4-3).

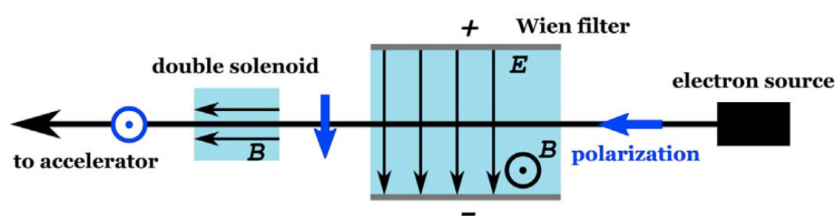

Figure 4-3: Obtaining vertical polarization: Electrons emitted off cathode with longitudinal polarization. Wien filter rotates the polarization vector by $90^{\circ}$ horizontally. Double solenoid field rotates the polarization vector into the vertical orientation. [215]

Both fast polarization flips and slow polarization reversal are employed in the process of measuring the helicity correlated asymmetry in the electron scattering off the Carbon target. A slow reversal is performed by inserting a $\lambda / 2$ plate (know as GVZ) in the laser path before the photocathode, reversing the polarization relative to the helicity signal. This allows for cancellation of possible false asymmetries and suppression of systematic effects, such as position differences. One fast polarization reversal is induced by alternating the polarity of the high voltage of a Pockels cell in the circularly polarized laser source which in turn alternates the orientation of the electron beam polarization vector up and down at $25 \mathrm{~Hz}$, creating windows of fixed polarization, $20 \mathrm{~ms}$ in duration to cancel $50 \mathrm{~Hz}$ line pickup. Another fast reversal is the choice of helicity pattern. A gate generator reversed helicity in pseudo-random quartet patterns $\uparrow \downarrow \downarrow \uparrow$ and $\downarrow \uparrow \uparrow \downarrow$.

A final "reversal" was performed in the detection of the scattered electrons for one of the $Q^{2}$ configurations. This "reversal" was a flipping of the $\hat{n}$ direction, the normal vector defined by the scattering plane. The $\operatorname{left}(\operatorname{SpekA})$ and right(SpekB) spectrometers were respectively set to angles corresponding to the same $Q^{2}=0.04 G e V^{2} / c^{2}$ at $570 \mathrm{MeV}$, but due to their scattering directions $\hat{k}^{\prime}$ being of opposite sign, the normal vector defined by the scattering plane $\hat{n}$ is reversed and the transverse asymmetries $A_{n}$ obtained with the left and right detector systems were of opposite sign. In this configuration, SpekA was placed at 
$23.5^{\circ}$ and SpekB was placed at $20.61^{\circ}$. Due to the smaller acceptance of SpekB, the two spectrometers covered same range of momenta and accepted the same average $Q^{2}$. This symmetric measurement allowed for detection of potential false asymmetries due to polarization correlated beam asymmetries. The $A_{n}$ results for $Q^{2}=0.04 \mathrm{GeV}^{2} / \mathrm{c}^{2}$ for SpekA and SpekB and were found to be consistent with one another.

\subsubsection{Absolute Sign}

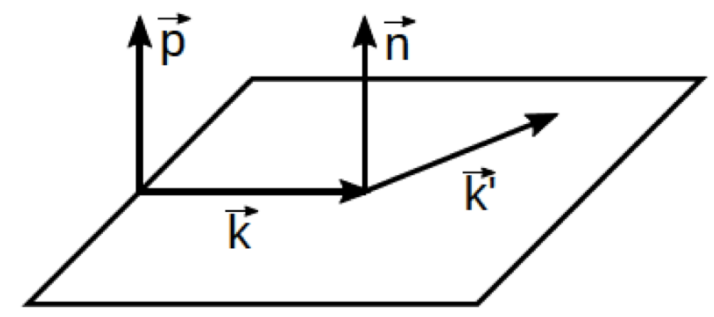

Figure 4-4: Necessary vectors to define the absolute sign [203]. Note $\vec{p}$ and $\vec{n}$ are depicted in the same direction for simplicity, but this is not the general case.

To define the beam-normal asymmetry, the vectors as shown in Fig.4-4 must be defined. $\vec{k}$ is the vector of the incident electron, $\overrightarrow{k^{\prime}}$ is the scattered electron, $\hat{n}=\left|\vec{k} \times \overrightarrow{k^{\prime}}\right|$ is the normal to the scattering plane and $\vec{p}$ the polarization direction of the incident electron. The transverse spin dependent cross section can be written

$$
\sigma=\sigma_{0}\left(1+A_{n} \vec{p} \times \hat{n}\right)
$$

with $A_{n}$ being the transverse analyzing power. SpekA and SpekB have acceptance in the horizontal plane with SpekA on beam left and SpekB on beam right. The detector asymmetries are defined as:

$$
A_{n}^{\text {SpekA }}=\frac{\sigma^{+}-\sigma^{-}}{\sigma^{+}+\sigma^{-}}=A_{n}
$$




$$
A_{n}^{S p e k B}=\frac{\sigma^{-}-\sigma^{+}}{\sigma^{-}+\sigma^{+}}=A_{n}
$$

where $\sigma^{+(-)}$refers to the vertical polarization of the beam being vertical up (down),

$$
\sigma^{+}=\vec{p} \uparrow \uparrow \vec{n}, \quad \sigma^{-}=\vec{p} \uparrow \downarrow \vec{n}
$$

\subsection{Detectors}
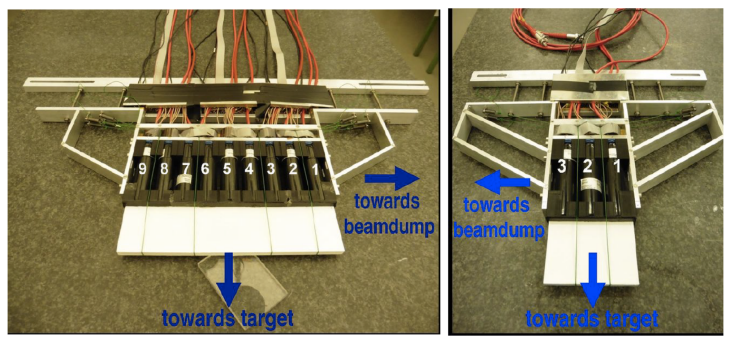

Figure 4-5: Detectors SpekA (left) and SpekB (right) [203]

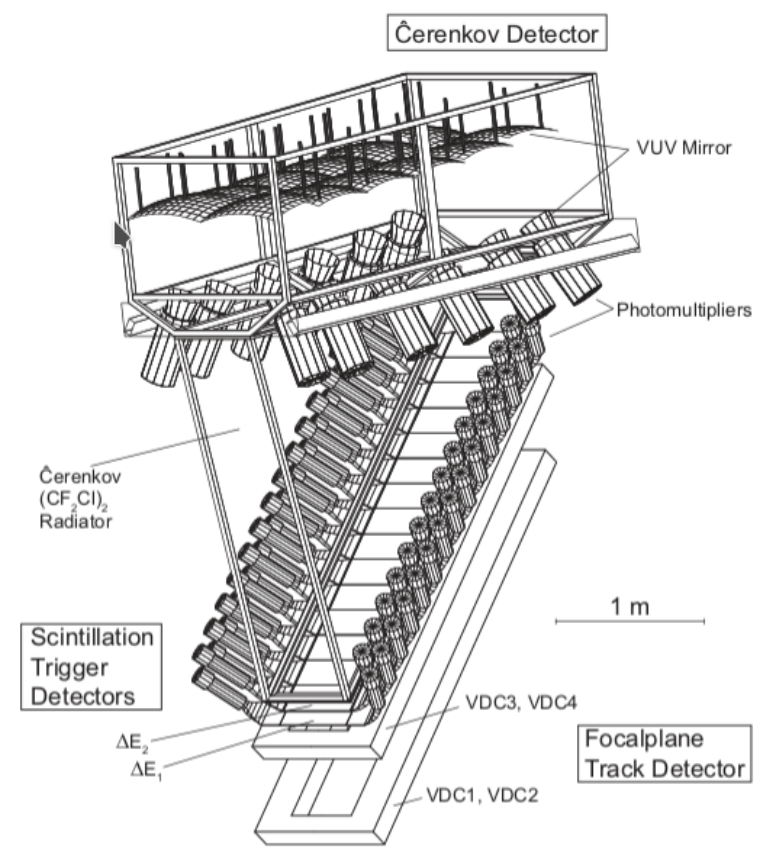

Figure 4-6: Spectrometer B [222] 
The detectors consisted of fused-silica bars which produced Cherenkov light detected by fused silica window photomultipliers attached to the bars. The size of the fused-silica bars was $30 \times 7 \times 1 \mathrm{~cm}^{3}$ for SpekA and 10x7 $1 \mathrm{~cm}^{3}$ for SpekB (Fig. 4-5), due to the differing focal plane geometries of the left and right spectrometers [203]. Five $25 \mathrm{~mm}$ fused silica-window photomultipliers were directly attached to the fused-silica bars in SpekA and three in SpekB. The detectors were oriented at $45^{\circ}$ with respect to the scattered electrons in the spectrometers.

The Cherenkov detector position was aligned to the elastic line by tuning the magnetic field. For calibration, the detectors were readout in coincidence with vertical drift-chambers (VDCs) (Fig. 4-5). The excitation energy spectrum (Fig.4-7) measured in this calibration mode shows separation between elastics and inelastics from the first $4.4 \mathrm{MeV}$ excited state.

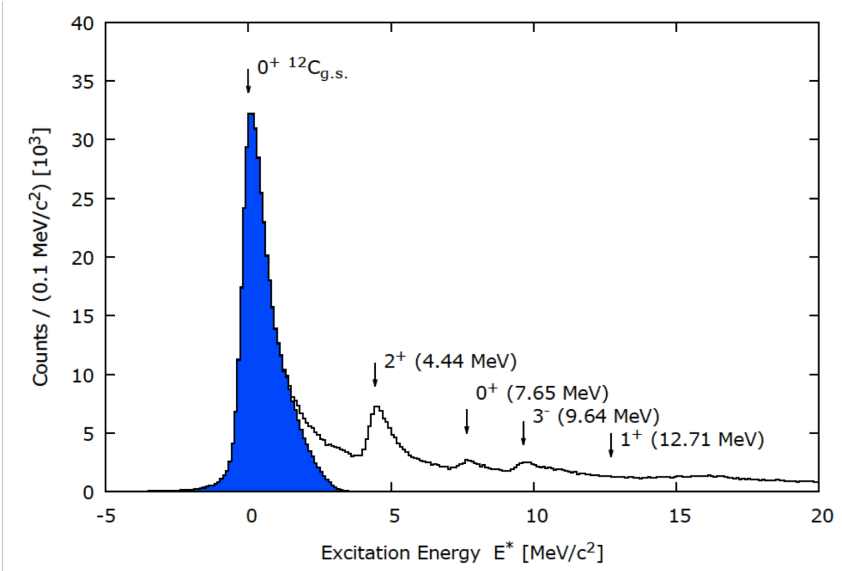

Figure 4-7: The excitation energy spectrum: the acceptance of the spectrometer (black line) and of the Cherenkov detector only (blue area)

After the detector position was aligned, the high voltages were adjusted in order to obtain useful signals during experimental readout. The triggered readout (counting-mode) differed from the experimental readout (integrating mode) in several ways (1) in counting-mode the PMT signals are only readout when triggered, while in integrating-mode the current from PMT was integrated during the entire $20 \mathrm{~ms}$ helicity window (2) the current was low $(50 \mathrm{nA})$ in counting mode (3) the 
amplification of the PMTs was reduced in integrating-mode to avoid non-linear response to rate or pulse height.

\subsection{DAQ and Gate Length}

A gate generator provided $20 \mathrm{~ms}$ measurement periods where the polarization was reversed in pseudo-random quartet patterns $\uparrow \downarrow \downarrow \uparrow$ and $\downarrow \uparrow \uparrow \downarrow$. The detector channels and beam monitoring channels were read out with parts of the former A4 experiment data acquisition system [218]. The signals were integrated during the $20 \mathrm{~ms}$ integration windows by gated integrators and digitized by 16-bit ADCs. The precise window length was synched to the power grid frequency of $50 \mathrm{~Hz}$ at Mainz by a phase locked loop (PLL). As the power grid frequency can vary by $\pm 0.150 H z( \pm 0.3 \%)[219]$, so can the integration window and signal acquired vary. Both the grid frequency and the gate length were continuously measured by the frequency being counted by a micro-controller and by a constant current signal input to an integrating DAQ channel, respectively [218]. Initially, in data acquisition, the gate length was calibrated by changing the scale value to set the mean of the gate length histogram to approximately $20 \mathrm{~ms}$. The gate length was measured for each helicity for normalization.

In addition to gate length, there is also a gate generator delay, a time period in-between each measurement window where the high voltage of the Pockels cell is changed and an transition between polarization states is allowed to occur while data is not being recorded. For this short transition period (80 $\mu s$ during the A4 experiment [218]), the polarization of the beam is changing and the the beam intensity increases or decreases drastically. In the data from 2015 and 2016, there was a fault in the control software, the order in which the VME-commands were issued, which did not set the delay of the gate generator correctly. The issue was resolved by the beginning of 2017. Asymmetries on the order of several tens of ppm were observed between identical helicity states which differed only in that 
one went through a transition and the other did not. This was a symptom the intensity variation in the transition was not entirely cut out by the gate delay [220] (it is also a symptom of the well known 4 peak effect Sec.6.4.4). However, even though every polarity flip suffered false beam asymmetry, it should be cancelled by the pseudo random helicity pattern (to the extent that the effect matched between helicity states).

The beam monitor signals corresponding to the current, energy, and beam position are also recorded by the A4 data acquisition system

\subsection{Monitors}

\subsubsection{Current Monitor: PIMO}

The current is measured by a beam phase and intensity monitor (PIMO). A PIMO consists of an RF resonator which the beam excites, producing RFmonitor signals which demodulated by a mixer with a reference RF-signal. The demodulated single input signal has two outputs, one related to beam current and the other related to phase as follows [221]:

$$
\begin{gathered}
U_{\text {intensity }}=U_{0, \text { int. }} \sin \left(\phi+\phi_{\text {ref,int. }}\right) \\
U_{\text {phase }}=U_{0, p h .} \sin \left(\phi+\phi_{\text {ref,ph. }}\right)
\end{gathered}
$$

where $U_{0, p h}$. and $U_{0, i n t}$. depend linearly on the beam current [203] and $\phi$ is the phase relative to the reference phase $\phi_{r e f}$. The phase difference $\phi_{r e f, i n t .}-\phi_{r e f, p h}$. is chosen to be $\pm 90^{\circ}$ to make the signals independent. The beam phase and intensity can then be extracted from $U_{\text {phase }}$ and $U_{\text {intensity }}$. Since the PIMO is sensitive to the phase as well as the intensity or a superposition of both, MAMI adjusts the PIMO-phase $\phi_{\text {ref,int }}$ is adjusted in an initial calibration step, so the PIMO measures primarily the intensity [203]. 
For the ${ }^{12} \mathrm{C}$ transverse asymmetry measurement, two PIMOs were used:: PIMO13 and PIMO21 between the Møller polarimeter and target [203]. PIMO21 was used as the main beam current monitor in the experiment since it was considered to give the correct value for the current on the target, whereas PIMO13 might not give as accurate a value on target due to losses in the accelerator.

The intensity monitors are used for the beam stabilization (see Sec.4.6.4). PIMO 21 is used to normalize the monitors and detectors and to correct the asymmetry of the beam current in data analysis.

\subsubsection{ENMO}

Deviation in the electron beam energy is measured with an energy monitor called ENMO. The energy change is determined through a time-of-flight measurement. It is measured with two high-frequency cavities and phase monitors placed before and after a dipole magnet. The time of travel (or phase deviation) between both cavities is related to the electron energy. A master oscillator provides the reference frequency which then is mixed with each PIMO signal, outputting a phase deviation signal which is linear with the beam current. If both PIMOs which make up the ENMO are used in their linear range, the ENMO-signal should scale with the square of the current.

In theory, each of the two PIMO signals should be linear with beam current and the ENMO signal should be quadratic with the beam current [223]. However, during the experimental running, the ENMO signal was not quadratic with beam current, it was observed to be linear with beam current. Probably, the highfrequency electronics of one of the PIMOs used in the ENMO was operated in saturation mode, such that it provided a reference high-frequency signal phase without current dependence. Then it was mixed with the other current dependent signal and so only linear behavior was observed [220]. 


\subsubsection{XYMO}

The beam position monitors, known as XYMOs, operate by mixing a master oscillator reference frequency with the beam-induced RF XYMO signal which is proportional both to the beam current and the beam position. In the A4 experimental halls, there are two beam position monitors used for this experiment: XYMO21 and XYMO26, placed at $8.28 \mathrm{~m}$ and $0.78 \mathrm{~m}$ before the target respectively. The XYMO signals are integrated in the A4 DAQ. These two XYMO measurements are normalized to PIMO21, then converted into position and angle measurements for each integration window.

\subsubsection{Stabilization}

Four stabilization systems at MAMI were dedicated to minimizing both beam fluctuations and, by extension, helicity-correlated beam-fluctuations: beam current stabilization, beam energy stabilization, slow position (DC), and fast position (AC) stabilization. Fig. 4-8 illustrates an example of the effect that beam current stabilization had on the charge asymmetry [208]

The beam parameters were measured by several monitors in the A1 beamline. PIMOs, intensity and phase monitors, were used for the beam current stabilization. Deviations were corrected at the source with the master oscillator of the laser diode [207]. The ENMO measurement was employed for beam energy stabilization. The energy correction resulted in polarization correlated differences of $<10 \mathrm{eV}$, keeping energy induced false asymmetries under control [207] [203]. XYMOs were used for beam position stabilization. All position jitter was suppressed via feedback. This feedback also reduces any polarization correlated beam position differences [207]. 


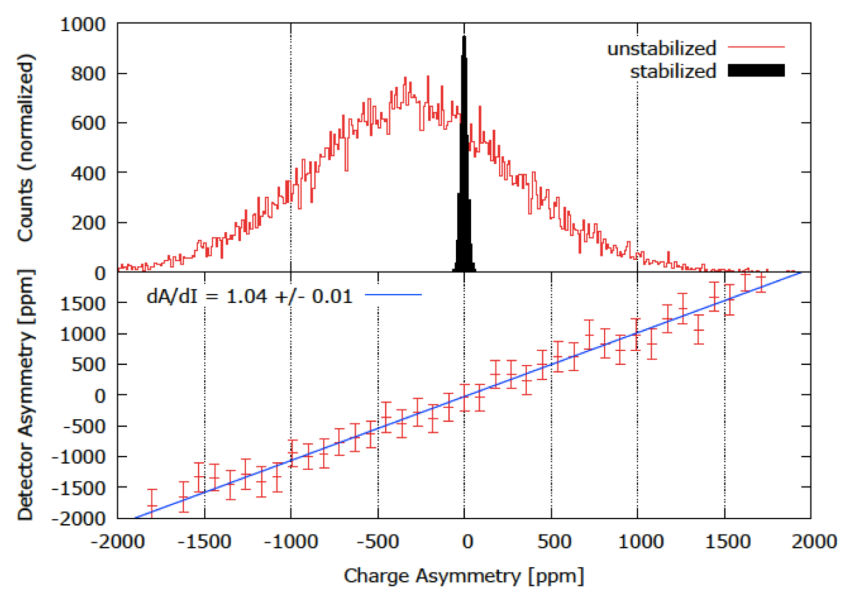

Figure 4-8: Top: Charge asymmetry observed with beam stabilization off(red) and with beam stabilization on (black). Bottom: A run without beam stabilization off showing raw asymmetry as measured by one PMT of the Cherenkov detector in spectrometer B vs. the charge asymmetry as measurement by the beam current monitor PIMO

\subsection{Calibrations and Optimizations}

\subsubsection{Rotating $\lambda / 2$ plate}

In the optical system of the MAMI source, in the region of the Pockels cell, there are two $\lambda / 2$ wave-plates: an insertable waveplate (GVZ/general sign changer 1) and a rotatable waveplate.

The second $\lambda / 2$-plate is mounted such that it can be rotated around the laser beam axis by a motor. This rotating half-wave-plate is used to rotate residual linear polarization components and control the helicity correlated charge asymmetry in the electron beam current [203]. The procedure for setting the angle of this rotating $\lambda / 2$-plate is referred to as the "Lambda Rotation". The Lambda Rotation procedure is as follows:

1. Insert or remove the GVZ (as required)

2. Set the Pimo-Phase

\footnotetext{
${ }^{1}$ Possible German translation is allGemeines Zeichen Verandern
} 
3. Reduce the PIMO beam current asymmetry by rotating the lambda half plate by several degrees. Let the operator set an angle, then measure for 3 minutes, check the current asymmetry and iterate different angles until the asymmetry is smaller in magnitude than $\sim 2 \mathrm{ppm}$ (this measurement is always performed with the current stabilization activated) [220].

After this rotating waveplate angle is set, a current calibration is performed.

\subsubsection{Current calibration}

Several devices along the beam line measure the beam current: PIMO intensity monitors and the Foerster probe. The intensity monitors do not measure absolute values and must be calibrated with the additional Foerster probe ${ }^{2}$, a monitor which is "claimed" to readback $1 \%$ absolute precision on beam current at $0.1 \mathrm{~Hz}$ bandwidth [207]. During the experiment, PIMO21 was calibrated with the Foerster probe in current calibration runs which varied the beam current at several fixed levels near $20 \mu \mathrm{A}$, the running current. The Foerster probe is readout using an analog-to-frequency-converter such that it emits 10 counts/s per $\mu A$. With the accelerator using 4 recirculations in the RTM3, for example, the Foerster measures 4 times the actual current [220]. To convert the Foerster data(given in units of counts/sec) to current, the following conversion is performed:

$$
\text { Current }[\mathrm{nA}]=\text { counts }[\text { Foerster }] * 100 / \text { MamiTurns }
$$

where the MamiTurns for the different energies are: $210 \mathrm{MeV}: 4$ turns, $315 \mathrm{MeV}: 18$ turns, 420MeV:32 turns, 570MeV:52 turns.

Current calibration runs are also used in the calibration of the PMTs, the ENMO, and the XYMOs. The assumption of linearity with beam current is used to obtain pedestals for the PMTs, ENMO and XYMOs. During experimental

\footnotetext{
${ }^{2}$ This probe is named after someone called Foerster
} 
running, current calibration runs over the full beam current range as well as in a narrow region around $20 \mu \mathrm{A}$ were performed regularly to monitor the functioning of the PMTs.

\subsubsection{Energy calibration}

The energy in each run is calculated using ENMO and current monitor data, information from current calibration runs, ENMO runs, and the assumption of a particular model relating ENMO response to energy and current. To understand the energy calibration, it is important to note a couple of observations regarding the ENMOs. The ENMOs do not measure absolute energy. It is a TOF type of measurement where the energy is determined using two high frequency cavities placed before and after a dipole magnet and the time necessary for the electrons to pass through the dipole is assessed [207]. At a given current and at a given beam energy, the ENMO phase is set, so that the ENMO reading is near 0. Deviations from this nominal TOF are what gets measured. So, the ENMO is only sensitive to energy fluctuations. Furthermore, while the magnitude of the ENMO is fixed, the sign of the ENMO in not fixed, since the MAMI operator rotates the phase of the ENMO in an arbitrary direction after the calibration [203].

Furthermore, the linearity of the raw ENMO signal with respect to current observed in the 2017 data set is not normal, in principle it should be quadratic with beam current. The linearity of the raw ENMO signal may potentially the result of one PIMO being saturated, though this explanation is questionable. However, the calibrations performed for the ENMO during this experiment stipulate the observed linearity with beam current and the data analysis was performed accounting for that functional form.

By modulating the energy by a known value, at a known current, the ENMO calibration can be performed for that particular current. The ENMO calibration is performed at a particular current, in this case $20 \mu \mathrm{A}$. The helicity signal is sent 
to MAMI causing a polarization correlated modulation of the signal by \pm 11.3 $\mathrm{keV}$. The difference of the raw ENMO readout in two helicity states at $20 \mu \mathrm{A}$ is shown in 4-9 for such a calibration.

The current calibration runs were used to find the PIMO pedestal and the energy calibration runs were used to find the scale factor in the equation

$$
\begin{gathered}
E r=I_{1}\left(E-E_{0}\right) / \text { scale }_{E}+\operatorname{ped}_{E} \\
E=\left(E r-\operatorname{ped}_{E}\right) * \operatorname{scale}_{E} / I_{1}+E_{0}
\end{gathered}
$$

where Er is the raw output from the ENMO and $I_{1}$ is the current obtained from PIMO21. The parameter ped ${ }_{E}$ can be understood as the value the ENMO would read when the current is 0 if the signal was linear with current. The parameters of this equation are found from the current calibration runs and ENMO runs, with the exception of $E_{0}$. ENMO is differential phase monitor, nominally set to 0 at some running energy $E_{0} . E_{0}$ is nominal energy which gives no signal in the ENMO. As such, $E_{0}$ is not accessible via ENMO calibration runs or current calibration runs. For simplicity, we set $E_{0}=0$ in analysis, though in principle it should be somewhere near $570 \mathrm{MeV}$.

The ENMO calibration runs are used to find scale $_{E}$ in the equation $E=$ $\left(E r-\operatorname{ped}_{E}\right) *$ scale $_{E} / I 1$ by inducing small helicity correlated energy changes of $11.3 \mathrm{keV}$ around a central energy at a particular current $d E=d E r * \operatorname{scale}_{E} / I 1$. To analyze the ENMO runs, we first apply the offset found in the current calibration run without normalizing to I1, treat scale $_{E}$ as 1 , examine $\mathrm{dE}$ (which is effectively $\mathrm{dEr}$ ), then set scale $E_{E}$ such that $\mathrm{dE}$ will equal $2 * 11.3 \mathrm{keV}$ for a given mean value of I1.

As an example, here we show the $20 \mathrm{uA}$ ENMO run at $210 \mathrm{MeV}$ where have not normalized $E$ to current in analysis $E=\left(E r-\operatorname{ped}_{E}\right)$. 

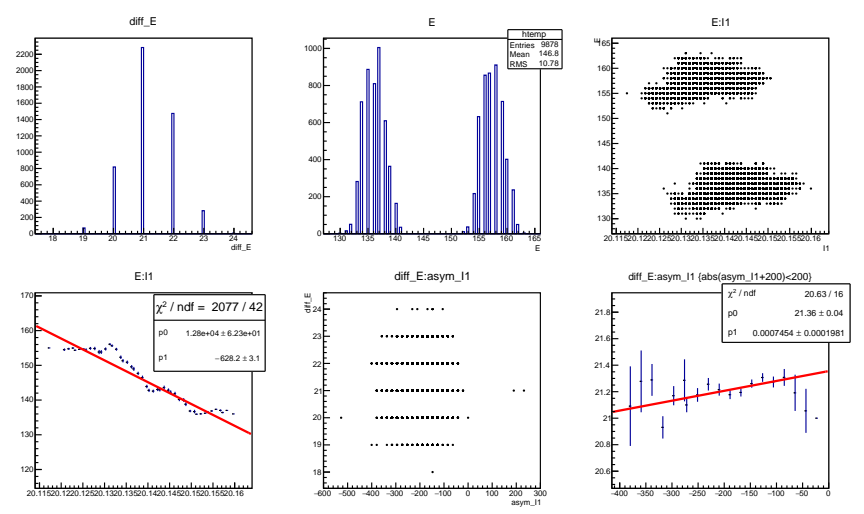

Figure 4-9: ENMO run 04-10-17-44-42 at 210MeV where E has been current normalized $E=\left(E r-\operatorname{ped}_{E}\right) / I 1$

Here we show the $20 \mathrm{uA}$ ENMO run at $210 \mathrm{MeV}$ where we've normalized E to current in analysis $E=\left(E r-\operatorname{ped}_{E}\right) / I 1$.
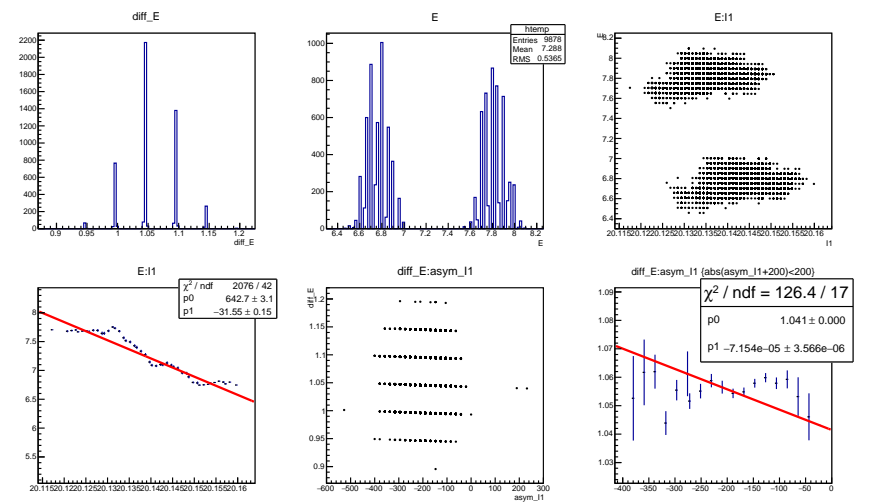

Figure 4-10: ENMO run 04-10-17-44-42 at 210MeV where E has been current normalized $E=\left(E r-\operatorname{ped}_{E}\right) / I 1$

A difference in energy is used to calibrate how $\Delta E r$, normalized to current, is proportional $\Delta E$, and the ENMO calibration runs are performed with this assumption. In general $E r=f(E, I), \frac{d E r}{d E}=\frac{\partial E r}{\partial I} \frac{d I}{d E}+\frac{\partial E r}{\partial E}$, for small changes $\Delta E r=\frac{d E r}{d E} \Delta E$, we define $\beta$ as $\Delta E r=\beta \Delta E$. And all you obtain from and ENMO calibration run is the scale factor. With the available information you can't turn an ENMO signal into an actual energy, only assess deviations from some nominal energy value. Furthermore, you can't correct for helicity correlated 
energy deviations because the sign of the energy signal is not known, since the MAMI operator rotates the phase of the ENMO in an arbitrary direction after the calibration [203].

\subsubsection{XYMO calibration}

The A1 Collaboration performed an XYMO calibration procedure. We mention it here because the result is used analysis to bound the systematic uncertainty arising from beam position differences.

In the XYMO, a master oscillator provides the reference frequency which then is mixed with the XYMO signal, which is proportional to the beam current. So the XYMO calibration is performed in conjunction with a current calibration. First proper pedestals are obtain for the raw XYMO signals with a current calibration run. Then for the XYMO calibration, a wire target is used (or a carbon strip target as of 2016). The carbon strip target consists of 3 strips of thickness $d=$ $0.5125 \pm 0.0015 \mathrm{~mm}$ spaced equidistantly [203]. The target is used in either vertical and horizontal orientation. The beam position is rastered slowly over the target while data is acquired. The signal in the PMT detectors is plotted with respect to the measured beam offset and a triple gaussian fit is performed for the calibration as shown in Fig. 4-11.

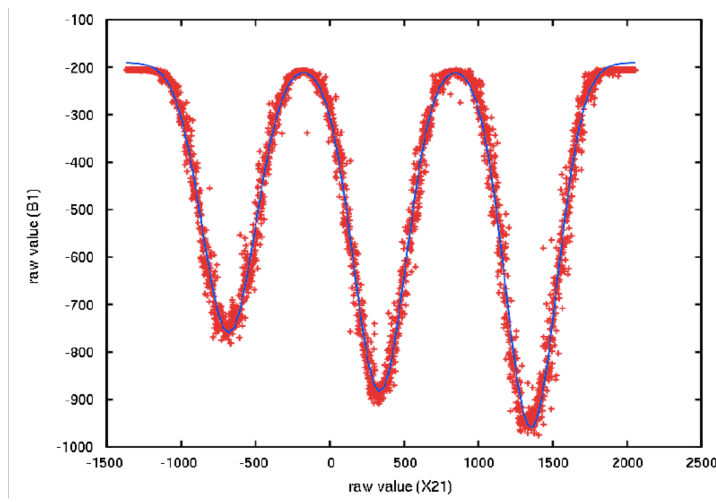

Figure 4-11: Raw detector B1 value vs. raw XYMO value. The three minima correspond to the beam positions in the XYMO where the carbon plates are hit [203] 
For the XYMO calibration, the geometry of the beamline components [220] (and the setting of some quadrupoles can also be important)

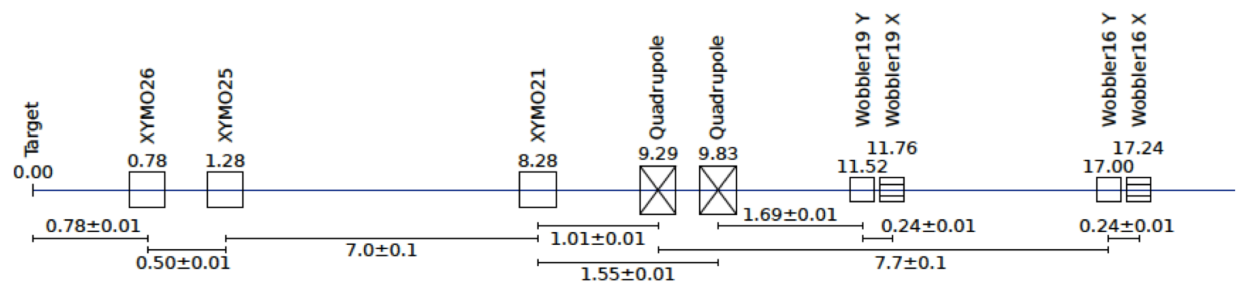

Figure 4-12: Distances between the beam line components necessary to to perform XYMO calibrations [203]

The target positions and angles are then calculated from the beam position in the XYMOs as follows [203]:

$$
\begin{gathered}
\theta=\arctan (X 26-X 21) \times X y m o D i \text { stance } \\
\phi=\arctan (Y 26-Y 21) \times X y m o D i \text { stance } \\
X=X 26+\tan (\theta) \times X y m o \text { TargetDistance } \\
Y=X 26+\tan (\phi) \times X \text { ymoTargetDistance }
\end{gathered}
$$

where the distances are obtained from Fig. 4-12.

\section{Coordinate System}

The coordinate system of the Hall as follows for XYMOs 21, 25 and 26[203]:

- The XYMOs are positive in $\mathrm{X}$, if the beam is shifted to the left when looking along the beam direction.

- The XYMOs are positive in $\mathrm{Y}$, if the beam is shifted downwards.

- The $\theta$ angle is defined on the horizontal plane between the nominal and 
the actual beam direction with positive values, when the horizontal beam inclination points towards SpekA (to the left)

- The $\phi$ angle is defined on the vertical plane on which the nominal beam line lies, with positive values, when the beam inclination points downwards.

\subsubsection{Polarization}

The electron beam polarization measurement [215] is very important for this experiment. Uncertainty in the determination of polarization is the one largest contributors to the systematic error. The degree of polarization and it's orientation were measured using a combination of Mott and Compton polarimeters downstream of the injector and a Møller polarimeter in the hall [207]. This measurement differs from the typical polarimetry measurements in that the beam is vertically polarized instead of longitudinally polarized, so several measurements must be performed. Firstly, the total beam polarization was measured with the beam polarization oriented longitudinally in the experimental hall using the Møller polarimeter [215]. Then the polarization vector was rotated in the vertical direction and the Møller polarimeter measured the residual component of longitudinal polarization. Møller measurements were performed fairly regularly throughout the run period.

The degree of the vertical polarization was deduced by subtracting the residual longitudinal and horizontal polarization components from the total polarization [207] and was on average $P e=82.7 \pm 0.3 \%$ (stat.) $\pm 1.1 \%$ (syst.) [208].

A few aspects distinguish this experiment from one with longitudinal polarization. For one, the the Møller split-coil magnet focuses the beam as well as rotating the transverse polarization by $\sim 20^{\circ}$. So, the magnet must be ramped down after each Møller measurement and the downstream quadrupoles are readjusted. Another differing aspect is that the effective polarization depends on $\Phi_{0}$, the angle of the scattered electron rotation around the beam axis. If the beam is scattered 
horizontally, the vertical polarization is perpendicular to the scattering plane, but when the plane is tilted by an angle $\Phi_{0}$, the projection of the polarization on the normal of the scattering plane is reduced. The effective polarization must be reduced by this factor, which is on the order of $<1 \%$. The distribution of the factor is shown in Fig. 4-13 [203]

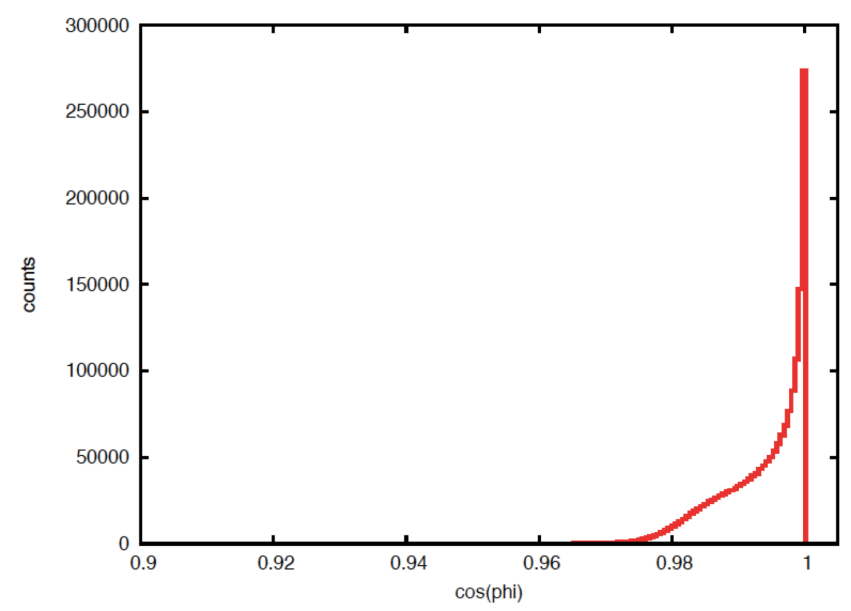

Figure 4-13: Projection of the vertical polarization on the normal of the scattering plane for one spectrometer setup [203]

Polarization measurements were also performed intermittently with the Mott and Compton, allowing for a comparison with the Møller measurements, to understand systematic errors in measurements. While the Møller polarimeter was not sensitive to vertical polarization components, the Mott polarimeter was not sensitive to longitudinal polarization components. So, it provided a complimentary polarimetry measurement. These measurements were performed intermittently during the running and the polarization were interpolated linearly between the few consecutive Mott measurements. Additionally the absolute value of the Mott measurements were corrected to the Møller measurements and the result is shown in Fig. 4-14 


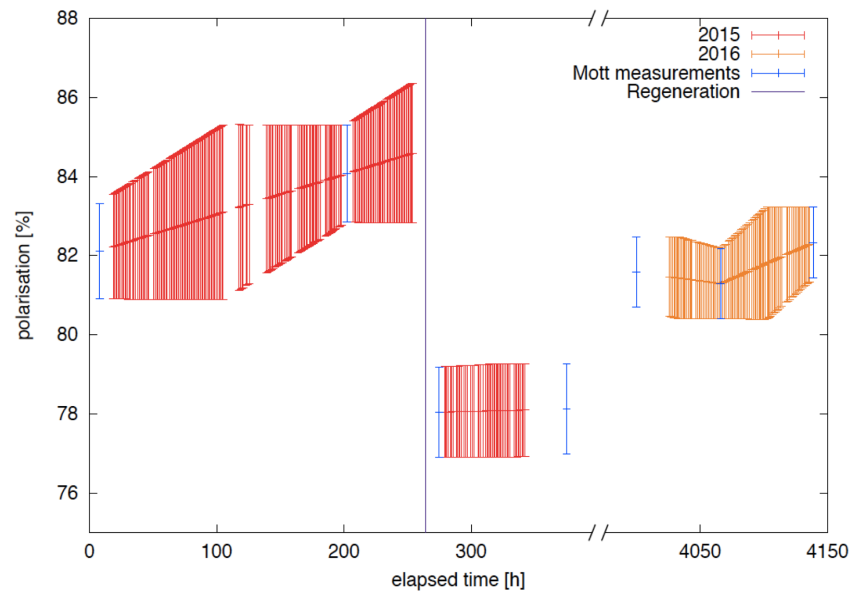

Figure 4-14: The polarization is interpolated linearly between consecutive Mott measurements [203]

\subsection{Data Analysis}

\subsubsection{Beam Parameterization}

The following table shows all the beam parameters acquired in addition to the integrated detector signals [203]:

\begin{tabular}{|l|l|c|c|}
\hline parameter & measurement point & abbreviation & unit \\
\hline \hline beam current & PIMO21 & $\mathrm{I} 21$ & $\mu \mathrm{A}$ \\
beam current & PIMO13 & $\mathrm{I} 13$ & $\mu \mathrm{A}$ \\
beam current & target* & $\mathrm{I}$ & $\mu \mathrm{A}$ \\
hor. beam position & XYMO21 & $\mathrm{X} 21$ & $\mu \mathrm{m}$ \\
vert. beam position & XYMO21 & $\mathrm{Y} 21$ & $\mu \mathrm{m}$ \\
hor. beam position & XYMO25** & $\mathrm{X} 26$ & $\mu \mathrm{m}$ \\
vert. beam position & XYMO25** & $\mathrm{Y} 26$ & $\mu \mathrm{m}$ \\
hor. beam position & target & $\mathrm{X}, \mathrm{x}$ & $\mu \mathrm{m}$ \\
vert. beam position & target & $\mathrm{X}^{\prime}, \mathrm{x}^{\prime}$ & $\mu \mathrm{rad}$ \\
hor. beam inclination & target & $\mathrm{Y}^{\prime}, \mathrm{y}^{\prime}$ & $\mu \mathrm{rad}$ \\
vert. beam inclination & target & $\Theta$ & $\mu \mathrm{rad}$ \\
hor. beam angle & target & $\Phi$ & $\mu \mathrm{rad}$ \\
vert. beam angle & target & $\mathrm{E} 0, \mathrm{E}$ & $\mathrm{eV}$ \\
deviation from nominal beam energy & RTM 3 & $\mathrm{GL}$ & $\mathrm{ms}$ \\
integration gate length & DAQ & & \\
\hline
\end{tabular}

Figure 4-15: Parameters used in describing measurement conditions. (* the beam current at the target is assumed to be the same as in PIMO21; ** initially, XYMO26 was connected to the DAQ electronics) [203] 
The order of calculations are as follows (used by Mainz collaborators [203]):

1. Scaling the gate length to its physical value by a simple multiplication.

$$
G L_{\text {phys }}=G L_{\text {raw }} \times \text { scale }
$$

2. The signal raw values are corrected for the gate-length:

$$
\text { val }_{\text {corr }}=v_{\text {al }} \text { raw } \times 20 \mathrm{~ms} / G L
$$

3. Scaling the beam current to the physical value.

$$
I_{\text {phys }}=I_{\text {corr }} \times \text { scale }- \text { offset }
$$

4. Some signals(monitors) are later normalized to beam current after pedestal subtraction

$$
v a l_{\text {phys }}=\left(v_{\text {al }} l_{\text {corr }} \times \text { scale }- \text { offset }\right) / I_{\text {phys }}
$$

we note the energy monitor is treated linearly also

$$
E=\left(E r-\operatorname{ped}_{E}\right) * \operatorname{scale}_{E} / I_{1}+E_{0}
$$

5. Some signals(detectors) may remain unnormalized to beam current. Scaling the detector signals to an approximate value of 1000 . The scale cancels out in the asymmetry calculation. For the detector signals only the offset is of importance, therefore the value is calculated as:

$$
v_{\text {phys }}=\left(\text { val }_{\text {corr }}-\text { offset }\right) / \text { scale }
$$

6. The target positions and angles are then calculated from the beam position in the XYMOs as shown in Sec.4.7.4 [203]: 
7. The raw asymmetries for each PMT are calculated, for the mean asymmetry of a spectrometer the sums of all the tubes $(\mathrm{A} 0, \mathrm{~A} 1, \mathrm{~A} 2, \mathrm{~A} 3, \mathrm{~A} 4)$ for $\mathrm{spA}$ and (B0,B1,B2) for spB are used as values. The asymmetry for the current, gate length, and position differences and energy differences are also calculated:

$$
\begin{gathered}
A_{\text {raw }}=\frac{v a l_{\text {phys }}^{+}-v a l_{\text {phys }}^{-}}{v a l_{\text {phys }}^{+}+v a l_{\text {phys }}^{-}} \\
A_{\text {raw }, \text { spA }}=\frac{\sum_{i} s p A_{i, p h y s}^{+}-\sum_{i} s p A_{i, p h y s}^{-}}{\sum_{i} s p A_{i, p h y s}^{+}+\sum_{i} s p A_{i, p h y s}^{-}} \\
A_{\text {raw }, s p B}=\frac{\sum_{i} s p B_{i, p h y s}^{+}-\sum_{i} s p B_{i, p h y s}^{-}}{\sum_{i} s p B_{i, p h y s}^{+}+\sum_{i} s p B_{i, p h y s}^{-}} \\
\Delta v a l=v a l_{\text {phys }}^{+}-v a l_{\text {phys }}^{-}
\end{gathered}
$$

where $A_{i, \text { phys }}$ refers to the physics asymmetry as measured in PMT $i$ and +/corresponding to the polarization state. The sign for the SpekB asymmetry is corrected later to be consistent with Equ. 4.1

8. After asymmetries are formed, the beam current asymmetry can be subtracted out to obtain an asymmetry that is normalized to current in first order:

$$
A_{\text {norm }}=A_{\text {raw }}-A_{I}
$$

9. These raw asymmetries are then corrected for beam polarization and polaritycorrelated fluctuations:

$$
A_{n}=\frac{A_{\text {raw }}-A_{I}-A_{G L}-\frac{\partial \sigma}{\partial X} \Delta X-\frac{\partial \sigma}{\partial Y} \Delta Y-\frac{\partial \sigma}{\partial \Theta} \Delta \Theta \ldots}{P_{\perp}}
$$

Here we describe an alternative method of parameterization (used by myself):

- No gate length correction is made, its asymmetry is simply calculated later and subtracted out 
- The beam current is pedestal subtracted and scaled to physical values

$$
I_{\text {phys }}=\left(I_{\text {raw }}-\text { ped }\right) \times \text { scale }
$$

- The monitors are normalized to beam current after pedestal subtraction

$$
v a l_{\text {phys }}=\left(v a l_{\text {raw }}-\text { ped }\right) * \text { scale } /\left(I_{\text {phys }}\right)
$$

we note, as previously stated, that the energy monitor is also treated linearly and is reiterated here for convenient reference $E_{\text {phys }}=\left(E r-\operatorname{ped}_{E}\right) *$ scale $_{E} / I_{1}+E_{0}$

- The detectors are just pedestal subtracted and not normalized to beam current

$$
v_{\text {det }}=v_{\text {al }}-\text { ped }
$$

- The raw asymmetries for each PMT and for the summed PMT signals for spA and spB are calculated. The raw asymmetries for each PMT in a given spectrometer are also averaged with equal weights ${ }^{3}$ to form total raw asymmetries. Further, the asymmetry for the current, gate length, and position differences and energy differences:

$$
\begin{aligned}
& A_{\text {raw }}=\frac{v a l_{\text {det }}^{+}-v a l_{\text {det }}^{-}}{v a l_{\text {det }}^{+}+v a l_{\text {det }}^{-}} \quad A_{I}=\frac{I_{\text {phys }}^{+}-I_{\text {phys }}^{-}}{I_{\text {phys }}^{+}+I_{\text {phys }}^{-}} A_{G L}=\frac{G L_{\text {raw }}^{+}-G L_{\text {raw }}^{-}}{G L_{\text {raw }}^{+}+G L_{\text {raw }}^{-}} \\
& A_{\text {raw }, \text { sp } A}=\frac{\sum_{i} s p A_{i, p h y s}^{+}-\sum_{i} s p A_{i, p h y s}^{-}}{\sum_{i} s p A_{i, p h y s}^{+}+\sum_{i} s p A_{i, p h y s}^{-}} \\
& A_{\text {raw }, \text { spB }}=\frac{\sum_{i} s p B_{i, p h y s}^{+}-\sum_{i} s p B_{i, p h y s}^{-}}{\sum_{i} s p B_{i, p h y s}^{+}+\sum_{i} s p B_{i, p h y s}^{-}}
\end{aligned}
$$

\footnotetext{
${ }^{3}$ this choice was made by the A1 Collaboration despite each PMT having very different widths, and here we conform to their convention.
} 


$$
\begin{gathered}
A_{\text {raw }, \text { tot } A}=\frac{1}{5} \sum_{i=0}^{4} A_{\text {raw,spekAi }} \quad A_{\text {raw }, \text { tot } B}=\frac{1}{3} \sum_{i=0}^{2} A_{\text {raw }, \text { spek } B i} \\
\Delta E=E_{\text {phys }}^{+}-E_{\text {phys }}^{-} \Delta X=X_{\text {phys }}^{+}-X_{\text {phys }}^{-} \cdots
\end{gathered}
$$

- These raw asymmetries for each PMT in a given spectrometer are averaged with equal weights and are then corrected for beam polarization and polarization-correlated fluctuations:

$$
A_{n}=\frac{A_{\text {raw }, t o t}-A_{I}-A_{G L}-\frac{\partial \sigma}{\partial X} \Delta X-\frac{\partial \sigma}{\partial Y} \Delta Y-\frac{\partial \sigma}{\partial \Theta} \Delta \Theta \ldots}{P_{\perp}}
$$

We note that in summing the PMT's with equal weights, the varying rates and gains are not accounted for.

\subsubsection{False Asymmetries}

There is an influence of residual polarity correlated asymmetries in the measured beam parameters on the measured asymmetry, giving rise to false asymmetries. To verify these false asymmetries are negligible, calculation of false asymmetries needed be be performed for the following parameters: Beam Current (I), beam position (X and Y), beam angles $(\Theta$ and $\Phi)$, integration gate (GL), beam polarization, and PMT nonlinearities. From the measured raw asymmetry $A_{\text {raw }}$, the physical asymmetry is calculated [203] by subtracting out polarity correlated beam asymmetries:

$$
\begin{gathered}
A_{\text {exp }}=A_{\text {raw }}-c 1 A_{I}-c 2 \Delta X-c 3 \Delta Y-c 4 \Delta X^{\prime}-c 5 \Delta Y-c 6 \Delta E \\
A_{n}=\frac{A_{\text {raw }}-A_{I}-A_{G L}-\frac{\partial \sigma}{\partial x} \Delta X-\frac{\partial \sigma}{\partial y} \Delta Y-\frac{\partial \sigma}{\partial X^{\prime}} \Delta X^{\prime}-\frac{\partial Y^{\prime}}{\partial Y^{\prime}} \Delta X^{\prime}-\frac{\partial \sigma}{\partial E} \Delta E}{P_{\perp}}
\end{gathered}
$$


where the correction factors $c_{i}$ and the raw asymmetry were calculated, taken from a simulation or measured.

\section{Current Correction}

An important false asymmetry to correct is the current asymmetry $A_{I}$. It is subtracted from the measured asymmetry in the last step of the calculation with the correction factor set to $c 1=1$ since luminosity and count rate change linearly with the beam current.

\section{PMT non-linearity correction}

While the detector response can be modeled as linear around the run current, there is a clear non-linearity between 0 and $20 \mu \mathrm{A}$ as shown in Fig.4-16. The scaled detector value is calculated as

$$
v a l_{\text {phys }}=\left(v a l_{\text {corr }}-\text { offset }\right) / \text { scale }
$$

where the scaling factor does not affect the asymmetry, however the offset does. Non-linearities can be corrected by appropriate choice of offset or "pedestal". An incorrect choice of pedestal,offset $=\operatorname{ped}_{\text {true }}-\Delta$, leads to a so called "pedestal error" which manifests itself in the calculated detector asymmetry:

$$
\begin{gathered}
A=\frac{v a l_{\text {phys }}^{+}-v a l_{\text {phys }}^{-}}{v a l_{\text {phys }}^{+}+v a l_{\text {phys }}^{-}}=\frac{\left(v a l_{\text {raw }}^{+}-\text {offset }\right)-\left(v a l_{\text {raw }}^{-}-\text {offset }\right)}{\left.v a l_{\text {raw }}^{+}-\text {offset }\right)-\left(v a l_{\text {raw }}^{-}-\text {offset }\right)} \\
=\frac{v a l_{\text {raw }}^{+}-v a l_{\text {raw }}^{-}}{v a l_{\text {raw }}^{+}+v a l_{\text {raw }}^{-}-2 \text { offset }}=\frac{v a l_{\text {raw }}^{+}-v a l_{\text {raw }}^{-}}{v a l_{\text {raw }}^{+}+v a l_{\text {raw }}^{-}-2\left(\text { ped }_{\text {true }}-\Delta\right)}=A_{\text {true }}(1-\alpha)
\end{gathered}
$$

A pedestal error would overall scale down (or scale up) the measured asymmetry seen by the detector. 


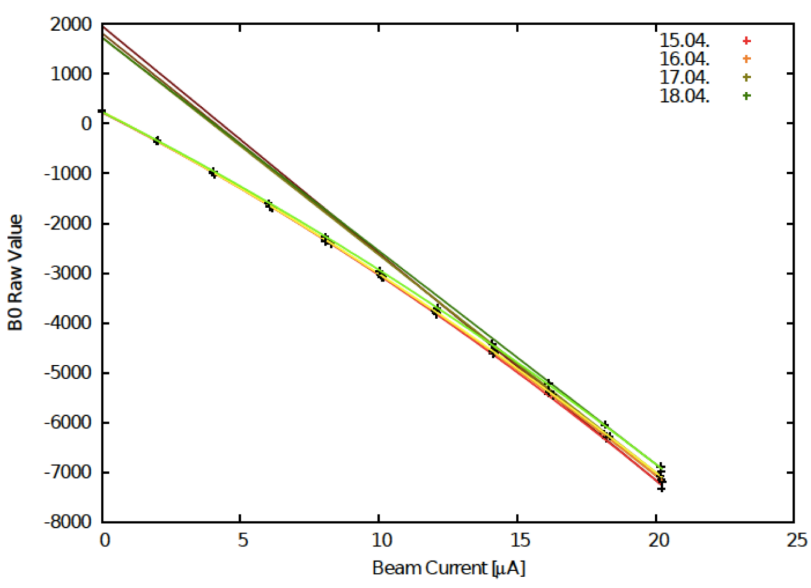

Figure 4-16: Non linearity of the current Calibration of spB0 from 0 to $20 \mu \mathrm{A}$ [203]

During the run period, the raw values of the PMTs decreased in magnitude over time (especially during the initial set-up for the detectors in SpekB ) as shown in Fig.4-16. The decrease was due to PMT aging (50\% gain loss due to aging after 250C has been drawn from its anode). A change in amplification can also change the pedestal offset. This results in a "pedestal error" as described in as shown in Fig.4-17.

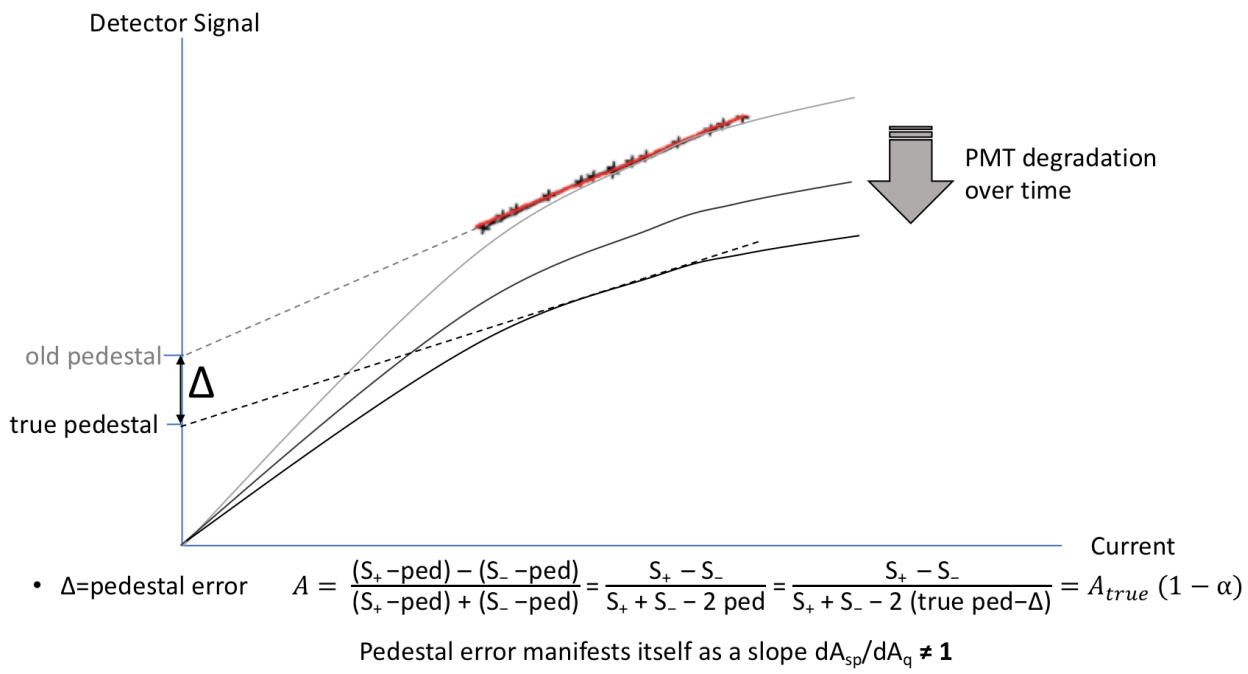

Figure 4-17: Defining Pedestal Error from PMT aging

If there is a pedestal error in the spekA and spekB signals and the PMTs had 
became more non-linear, it would affect all asymmetries coming into the detectors, whether from the current asymmetry, from physics asymmetry, etc. A pedestal error would overall scale down (or scale up) the measured asymmetry seen by the detector. The correction would be as follows:

$$
\begin{aligned}
A_{\text {raw }} & =\frac{d A_{\text {raw }}}{d A_{\text {raw }, \text { pedOK }}} A_{\text {raw,pedOK }} \\
= & \frac{d A_{\text {raw }}}{d A_{\text {true }}}\left(A_{\text {exp }}+A_{I}+c 2 \Delta X+c 3 \Delta Y+c 4 \Delta X^{\prime}+c 5 \Delta Y+c 6 \Delta E\right) \\
& A_{\text {exp }}=\frac{A_{\text {raw }}}{\frac{d A_{\text {raw }}}{d A_{\text {true }}}}-A_{I}-c 2 \Delta X-c 3 \Delta Y-c 4 \Delta X^{\prime}-c 5 \Delta Y-c 6 \Delta E
\end{aligned}
$$

We can obtain $\left(\frac{d A_{\text {raw }}}{d A_{\text {true }}}\right)^{-1}$ from correlations between the detector and a monitor. In a run with plenty of charge noise, and very little energy and position noise, we can correlate the detector asymmetry with the current asymmetry as measured fro each quartet. If the detector is close to being properly calibrated, then $\frac{d A_{\text {raw }}}{d A_{I}} \approx 1$. We estimate the factor for $\frac{d A_{\text {raw }}}{d A_{I}} \approx 1$, as

$$
\left(\frac{d A_{\text {raw }}}{d A_{\text {true }}}\right)^{-1} \approx\left(\frac{d A_{\text {raw }}}{d A_{I}}\right)^{-1} \approx 1-\left(\frac{d A_{\text {raw }}}{d A_{I}}-1\right)
$$

Once $\left(\frac{d A_{\text {raw }}}{d A_{\text {true }}}\right)^{-1}$ is obtained, most reliably from runs with sufficient charge noise, it can be applied to other runs to obtain the systematic error contribution as follows:

$$
A_{\text {exp }} \approx A_{\text {raw }}-\left(\frac{d A_{\text {raw }}}{d A_{I}}-1\right) A_{\text {raw }}-A_{I}-c 2 \Delta X-c 3 \Delta Y-c 4 \Delta X^{\prime}-c 5 \Delta Y-c 6 \Delta E
$$

We incorporate the partial polarization of the beam via:

$$
\begin{aligned}
& A_{n}=\frac{A_{\text {exp }}-A_{G L}}{P_{\perp}} \\
& =\frac{A_{\text {raw }}-\left(\frac{d A_{\text {raw }}}{d A_{I}}-1\right) A_{\text {raw }}-A_{I}-A_{G L}-c 2 \Delta X-c 3 \Delta Y-c 4 \Delta X^{\prime}-c 5 \Delta Y-c 6 \Delta E}{P_{\perp}}
\end{aligned}
$$




\section{Energy,Angle and Position Correction}

The Rutherford cross-section for a spin-0 nucleus is given by

$$
\left(\frac{d \sigma}{d \Omega}\right)_{\text {Rutherford }}=\frac{Z^{2} \alpha^{2}(\hbar c)^{2}}{4 E^{2} \sin ^{4}(\theta / 2)}
$$

The Mott scattering cross section is given by

$$
\left(\frac{d \sigma}{d \Omega}\right)_{M o t t}=\frac{Z^{2} \alpha^{2}(\hbar c)^{2}}{4 E^{2} \sin ^{4}(\theta / 2)}\left(1-v^{2} / c^{2} \sin ^{2}(\theta / 2)\right) \approx \frac{Z^{2} \alpha^{2}(\hbar c)^{2} \cos ^{2}(\theta / 2)}{4 E^{2} \sin ^{4}(\theta / 2)}
$$

The scattering cross section of a nucleus of mass $\mathrm{M}$ can be approximated by the Mott cross-section with recoil factor E'/E

$$
\left(\frac{d \sigma}{d \Omega}\right)_{\text {exp }} \approx \frac{Z^{2} \alpha^{2}(\hbar c)^{2} \cos ^{2}(\theta / 2)}{4 E^{2} \sin ^{4}(\theta / 2)} \frac{E^{\prime}}{E}=\frac{Z^{2} \alpha^{2}(\hbar c)^{2} \cos ^{2}(\theta / 2)}{4 E^{2} \sin ^{4}(\theta / 2)} \frac{1}{1+\frac{E}{M}(1-\cos \theta)}
$$

The experimental scattering cross section is more accurately described by

$$
\begin{gathered}
\left(\frac{d \sigma}{d \Omega}\right)_{\exp }=\left(\frac{d \sigma}{d \Omega}\right)_{M o t t}\left|F\left(q^{2}\right)\right|^{2} \\
q^{2}=-4 E^{2} \sin ^{2}(\theta / 2)
\end{gathered}
$$

The polarization dependent asymmetry detected at a particular angle is given by

$$
A=\frac{\frac{d \sigma_{+}}{d \Omega}-\frac{d \sigma_{-}}{d \Omega}}{\frac{d \sigma_{+}}{d \Omega}+\frac{d \sigma_{-}}{d \Omega}} \approx \frac{\frac{d \sigma_{+}}{d \Omega}-\frac{d \sigma_{-}}{d \Omega}}{2 \frac{d \sigma}{d \Omega}} \approx \frac{\Delta\left(\frac{d \sigma}{d \Omega}\right)}{2 \frac{d \sigma}{d \Omega}}
$$

The systematic error contribution to the measured asymmetry from energy is given by

$$
\Delta A=\frac{\Delta \frac{d \sigma}{d \Omega}}{2 \frac{d \sigma}{d \Omega}}=\frac{\frac{d}{d E} \frac{d \sigma}{d \Omega} \Delta E}{2 \frac{d \sigma}{d \Omega}}
$$




$$
\frac{d A}{d E}=\frac{\frac{d}{d E} \frac{d \sigma}{d \Omega}}{2 \frac{d \sigma}{d \Omega}}
$$

The systematic error contribution to the measured asymmetry from angle is likewise given by

$$
\frac{d A}{d \theta}=\frac{\frac{d}{d \theta} \frac{d \sigma}{d \Omega}}{2 \frac{d \sigma}{d \Omega}}
$$

The asymmetry dependence on energy and angle can be split up into a portion from the Mott cross section and a correction from the FF dependence on energy and angle

$$
\begin{aligned}
& \frac{d A}{d E} \approx \frac{1}{2} \frac{\frac{d}{d E} \frac{d \sigma}{d \Omega}}{\frac{d \sigma}{d \Omega}} \approx\left(\frac{d A}{d E}\right)_{M o t t}+\left(\frac{d A}{d E}\right)_{F F} \\
& \frac{d A}{d \theta} \approx \frac{1}{2} \frac{\frac{d}{d \theta} \frac{d \sigma}{d \Omega}}{\frac{d \sigma}{d \Omega}} \approx\left(\frac{d A}{d \Omega}\right)_{M o t t}+\left(\frac{d A}{d \Omega}\right)_{F F}
\end{aligned}
$$

The energy dependence is given by

$$
\begin{gathered}
\frac{d}{d E}\left(\frac{d \sigma}{d \Omega}\right)_{e x p}=\frac{d}{d E}\left(\frac{d \sigma}{d \Omega}\right)_{M o t t}\left|F\left(q^{2}\right)\right|^{2} \\
=\left|F\left(q^{2}\right)\right|^{2} \frac{d}{d E}\left(\frac{d \sigma}{d \Omega}\right)_{M o t t}+\left(\frac{d \sigma}{d \Omega}\right)_{M o t t} \frac{d}{d E}\left|F\left(q^{2}\right)\right|^{2} \\
=\frac{-2}{E}\left|F\left(q^{2}\right)\right|^{2}\left(\frac{d \sigma}{d \Omega}\right)_{M o t t}+\left(\frac{d \sigma}{d \Omega}\right)_{M o t t} \frac{d\left|F\left(q^{2}\right)\right|^{2}}{d q} \frac{d q}{d q^{2}} \frac{d q^{2}}{d E} \\
=-\left(\frac{2}{E}\left|F\left(q^{2}\right)\right|^{2}-\frac{d\left|F\left(q^{2}\right)\right|^{2}}{d q} 2 \sin (\theta / 2)\right)\left(\frac{d \sigma}{d \Omega}\right)_{M o t t} \\
=\frac{d}{d E}\left(\frac{d \sigma}{d \Omega}\right)_{0}+\frac{d}{d E}\left(\frac{d \sigma}{d \Omega}\right)_{F F}
\end{gathered}
$$

The angle dependence is given by

$$
\begin{gathered}
\frac{d}{d \theta}\left(\frac{d \sigma}{d \Omega}\right)_{\exp }=\frac{d}{d \theta}\left(\frac{d \sigma}{d \Omega}\right)_{M o t t}\left|F\left(q^{2}\right)\right|^{2} \\
=\left|F\left(q^{2}\right)\right|^{2} \frac{d}{d \theta}\left(\frac{d \sigma}{d \Omega}\right)_{M o t t}+\left(\frac{d \sigma}{d \Omega}\right)_{M o t t} \frac{d}{d \Theta}\left|F\left(q^{2}\right)\right|^{2}
\end{gathered}
$$




$$
\begin{gathered}
=-\left|F\left(q^{2}\right)\right|^{2} \frac{Z^{2} \alpha^{2}(\hbar c)^{2}(7 \cos (\theta / 2)+\cos (3 \theta / 2))}{16 E^{2} \sin ^{5}(\theta / 2)}+\left(\frac{d \sigma}{d \Omega}\right)_{M o t t} \frac{d\left|F\left(q^{2}\right)\right|^{2}}{d q} \frac{d q}{d \theta} \\
=-\left|F\left(q^{2}\right)\right|^{2} \frac{Z^{2} \alpha^{2}(\hbar c)^{2}(7 \cos (\theta / 2)+\cos (3 \theta / 2))}{16 E^{2} \sin ^{5}(\theta / 2)}+\left(\frac{d \sigma}{d \Omega}\right)_{M o t t} \frac{d\left|F\left(q^{2}\right)\right|^{2}}{d q} E \cos (\theta / 2) \\
=\frac{d}{d \theta}\left(\frac{d \sigma}{d \Omega}\right)_{0}+\frac{d}{d \theta}\left(\frac{d \sigma}{d \Omega}\right)_{F F}
\end{gathered}
$$

The form factor for Carbon can be inferred from the following xsec measurement [204]

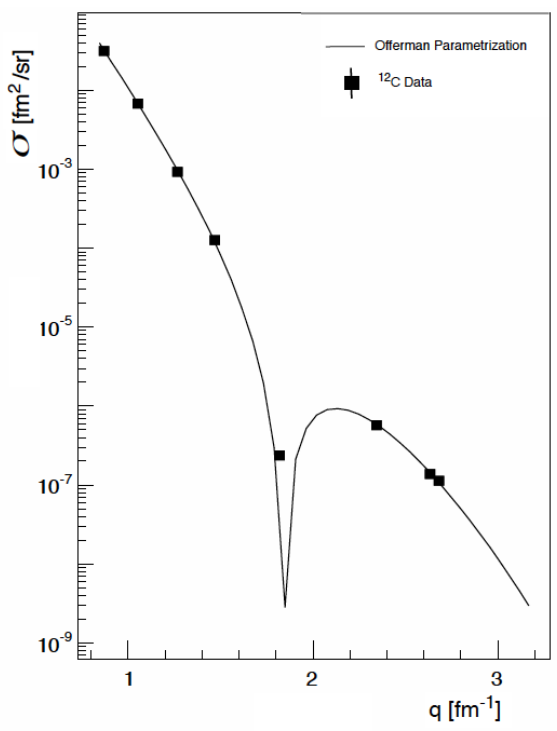

(a) Carbon cross section

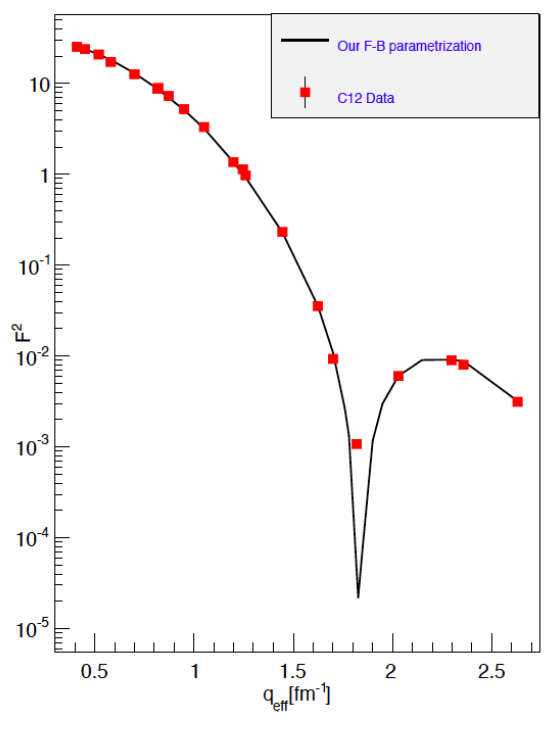

(b) Carbon Form Factor

Figure 4-18: $q_{\text {eff }}=q\left(1+4 / 3 \frac{Z \alpha}{E\left\langle r_{r m s}\right\rangle}\right),\left\langle r_{r m s}\right\rangle=2.45 \mathrm{fm}$ (a) The curve corresponds to the Offerman parametrization model [204],[205](b) Comparison between the experimental values of reduced cross section and the results obtained via the FourierBessel analysis [204].

We extracted the form factor data from these plots and parameterized it with an exponential fit in the region $\mathrm{q}=0.6-1.2 \mathrm{fm}-1$. We obtained the fit $|F(q)|^{2} \approx$ $90.43 e^{-2.489 f m q}-3.36$ also shown. 


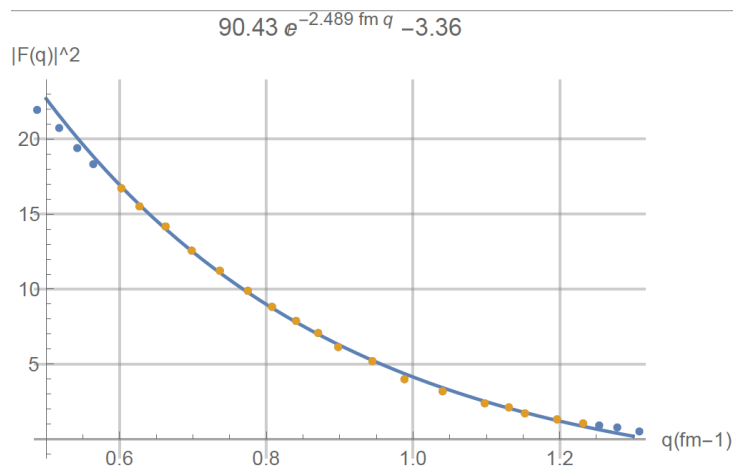

Figure 4-19: Fit to Carbon form factor $|F(q)|^{2} \approx 90.43 e^{-2.489 f m q}-3.36$

From this parameterization, we obtain the differential

$\frac{d F(q)^{2}}{d q} \approx-225.15 f m e^{-2.489 f m q} \times \frac{1}{197 M e V f m} \approx-1.1423 M e V^{-1} e^{-0.0126 M e V^{-1} q}$ in the range $q=0.6-1.2 \mathrm{fm}^{-1}$.

The $Q^{2}$ and $\theta$ setting for each configuration of the $570 \mathrm{MeV}$ data set shown in the following table along with the energy dependence of the asymmetry after the FF correction. For comparison $\left(\frac{d A}{d E}\right)_{M o t t}=-\frac{1}{2} 3.509 \mathrm{ppm} / \mathrm{keV}=-1.755 \mathrm{ppm} / \mathrm{keV}$, which differs from Mainz collaborator's value by a factor of 2 .

To obtain position dependences from angle dependencies, knowledge of the monitor, target, detector geometry is required. We took the ratio's of $\frac{d A}{d \theta}$ to $\frac{d A}{d x}$ obtained by Mainz collaborators and applied that ratio to our calculated angle sensitivity to obtain our calculated position sensitivity. 


\begin{tabular}{|c|c|c|c|c|c|}
\hline config & $\begin{array}{c}\mathrm{spB} \\
\text { data3 }\end{array}$ & $\begin{array}{c}\mathrm{spB} \\
\mathrm{data} 2\end{array}$ & $\begin{array}{c}\mathrm{spB} \\
\text { data1 }\end{array}$ & $\begin{array}{c}\mathrm{spA} \\
\text { data1 }\end{array}$ & $\begin{array}{c}\mathrm{spA} \\
\text { data2 \& 3 }\end{array}$ \\
\hline$Q^{2}\left(\mathrm{GeV}^{2} / c^{2}\right)$ & 0.023 & 0.03 & 0.041 & 0.039 & 0.049 \\
$q\left(\mathrm{fm}^{-1}\right)$ & 0.770 & 0.879 & 1.028 & 1.002 & 1.124 \\
$\theta$ & $15.11^{\circ}$ & $17.65^{\circ}$ & $20.61^{\circ}$ & $23.5^{\circ}$ & $25.9^{\circ}$ \\
$\left|F\left(q^{2}\right)\right|^{2}$ & 9.95 & 6.77 & 3.64 & 4.10 & 2.15 \\
$\frac{d F(q)^{2}}{d q} /\left|F\left(q^{2}\right)\right|^{2}(\mathrm{MeV}-1)$ & -0.0169 & -0.0189 & -0.0243 & -0.0230 & -0.0323 \\
$\left(\frac{d A}{d E}\right)_{\text {Mainz }}(\mathrm{ppm} / \mathrm{keV})$ & \pm 3.5 & \pm 3.5 & \pm 3.5 & \pm 3.5 & \pm 3.5 \\
$\left(\frac{d A}{d E}\right)_{\text {Mott }}(\mathrm{ppm} / \mathrm{keV})$ & $-3.51 / 2$ & $-3.51 / 2$ & $-3.51 / 2$ & $-3.51 / 2$ & $-3.51 / 2$ \\
$\frac{d A}{d E}(\mathrm{ppm} / \mathrm{keV})$ & -3.98 & -4.65 & -6.10 & -6.44 & -4.13 \\
$\left(\frac{d A}{d \theta}\right)_{\text {Mainz }}(\mathrm{ppm} / \mu \mathrm{rad})$ & -15.226 & -13.53 & -11.2 & 9.84 & 8.95 \\
$\left(\frac{d A}{d \theta}\right)_{\text {Mott }}(\mathrm{ppm} / \mu \mathrm{rad})$ & $-15.34 / 2$ & $-13.19 / 2$ & $-11.36 / 2$ & $10.03 / 2$ & $9.16 / 2$ \\
$\frac{d A}{d \theta}(\mathrm{ppm} / \mu \mathrm{rad})$ & -12.45 & -11.92 & -12.49 & 11.4 & 7.52 \\
$\left(\frac{d A}{d x}\right)_{\text {Mainz }}(\mathrm{ppm} / \mu \mathrm{m})$ & -3.86 & -3.841 & -3.787 & 19.472 & 19.942 \\
$\frac{d A}{d x} / \frac{d A}{d \theta}(\mu \mathrm{rad} / \mu \mathrm{m})$ & 0.253 & 0.284 & 0.338 & 1.979 & 2.228 \\
$\frac{d A}{d x}(\mathrm{ppm} / \mu \mathrm{m})$ & -3.150 & -3.016 & -4.222 & 22.561 & 16.755 \\
\hline
\end{tabular}

\section{Absolute Sign of Energy Correction}

Since the operator rotates the phase of the ENMO in an arbitrary direction after the calibration, the sign of the ENMO in not fixed. Therefore it is not possible to correct for energy deviations, only to estimate the potential systematic error. Due to the energy stabilization, the effect of the energy on the asymmetry was limited to a value of $\approx 0.01-0.03 \mathrm{ppm}$.

\section{Gate Length Correction}

A false asymmetry could be created by a variation in the gate length which is helicity correlated. The gate length is coupled to the line frequency so changes in the power grid frequency result in changes in all the detector and monitor signals.

There are two ways to correct the detector signals and monitor signals (with 
linear dependence on the beam current): (1) normalize all signals, including the beam current, with the gate-length and then normalize with the gate-length corrected beam current (2) Normalize all signals to the beam current raw value

Here we describe the 1st method. The gate length is scaled to its physical value by a simple multiplication. The gate length is initially tweaked it by hand to approximately $20 \mathrm{~ms}$ to set a scale factor and it is locked to the line frequency. It is not necessary to set this scale factor with highest precision, since the absolute value cancels out in the asymmetry calculation: $G L_{\text {phys }}=G L_{\text {raw }} \times$ scale. The signal raw values, including the beam current, are corrected for the gate-length: $v a l_{\text {corr }}=v_{a l} l_{\text {raw }} \times 20 \mathrm{~ms} / G L$. The gate-length corrected beam current is used to normalize the monitors and the gate length correction beam current asymmetry is subtracted out from the detector asymmetry.

Here we describe the 2nd method. No gate-length normalization is performed. The measured beam current (which depends on the gate length) is pedestal subtracted and scaled to physical values $I_{\text {phys }}=\left(I_{\text {raw }}-\right.$ ped $) \times$ scale . The monitor signals are normalized to beam current after pedestal subtraction $v l_{\text {norm }}=$ $\left(v_{\text {al }}\right.$ raw $\left.-p e d\right) /\left(I_{\text {phys }}\right)$. The detector signals are corrected for (gate-dependent) beam current asymmetry as well as the gate-length asymmetry by subtracting them out from the detector asymmetry: $A_{\text {corrected }}=A_{\text {unnorm }}-A_{q}-A_{G L}$

\subsection{3 $Q^{2}$ Estimation}

For calibration, the detectors were readout in coincidence with vertical driftchambers (VDCs) (Fig. 4-5) in counting-mode, where the detector PMT signals are only readout when triggered. The current was low $(50 \mathrm{nA})$ in counting mode and the PMT's were used with higher amplification than for data running integrating-mode. Each set-up was analyzed separately to obtain $Q^{2}$ from the spectrometer counting-mode data.

The following figures are the $Q^{2}$ distributions found from the counting-mode 
data for all of the setups in the experiment:

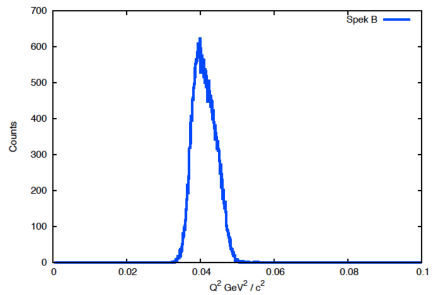

(a) $Q^{2} 2015-1$, SpekB

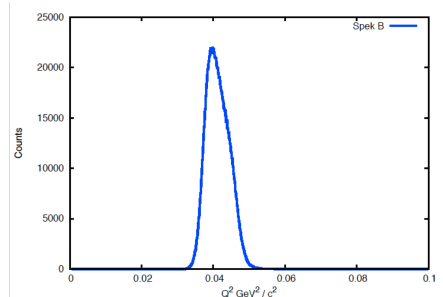

(d) $Q^{2} 2016$, SpekB

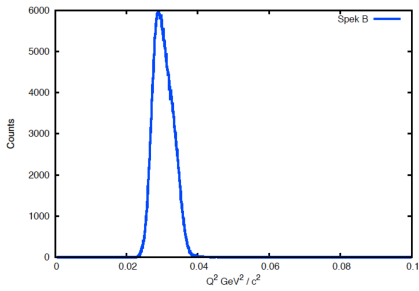

(b) $Q^{2} 2015-2$, SpekB

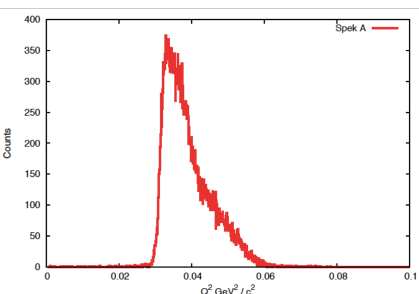

(e) $Q^{2} 2015-1$, SpekA

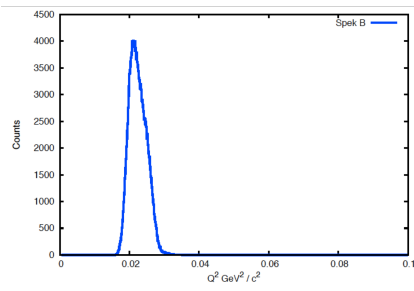

(c) $Q^{2} 2015-3, \mathrm{SpekB}$

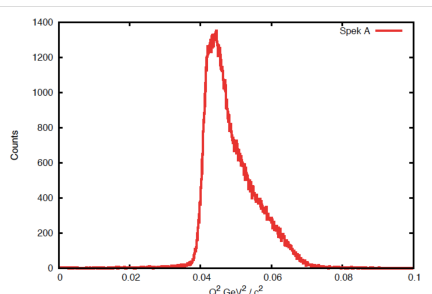

(f) $Q^{2} 2015-1$, SpekA

Figure 4-20: $Q^{2}$ distributions found from the counting-mode data for all of the setups in the experiment

The $Q^{2}$ range is given in terms of arithmetic mean of the histogram, together with the lower and upper point of the FWHM. It should be noted that the asymmetry analysis does not account for different rates for different individual PMTs, but averages all PMTs in each spectrometer together. The correct $Q^{2}$ averages are taken from plots such as Fig. 4-20, which treats the counts from each tube equally in forming the total acceptance spectrum.

\begin{tabular}{|c|c|c|c|c|c|}
\hline \multicolumn{6}{|c|}{ Spectrometer B } \\
\hline Setup & Angle & $Q^{2}$ Range $(\min )$ & $Q^{2}$ Range(avg) & $Q^{2}$ Range(max) & $Q^{2}$ uncertainty \\
\hline $2015-1$ & $20.61^{\circ}$ & $0.03709 \mathrm{GeV}^{2} / \mathrm{c}^{2}$ & $0.04127 \mathrm{GeV}^{2} / \mathrm{c}^{2}$ & $0.04503 \mathrm{GeV}^{2} / \mathrm{c}^{2}$ & $\pm 0.00002 \mathrm{GeV}^{2} / \mathrm{c}^{2}$ \\
\hline $2015-2$ & $17.65^{\circ}$ & $0.02674 \mathrm{GeV}^{2} / \mathrm{c}^{2}$ & $0.03044 \mathrm{GeV}^{2} / \mathrm{c}^{2}$ & $0.03380 \mathrm{GeV}^{2} / \mathrm{c}^{2}$ & $\pm 0.0005 \mathrm{GeV}^{2} / \mathrm{c}^{2}$ \\
\hline $2015-3$ & $15.11^{\circ}$ & $0.01919 \mathrm{GeV}^{2} / \mathrm{c}^{2}$ & $0.02250 \mathrm{GeV}^{2} / \mathrm{c}^{2}$ & $0.02545 \mathrm{GeV}^{2} / \mathrm{c}^{2}$ & $\pm 0.0003 \mathrm{GeV}^{2} / \mathrm{c}^{2}$ \\
\hline 2016 & $20.62^{\circ}$ & $0.03599 \mathrm{GeV}^{2} / \mathrm{c}^{2}$ & $0.04354 \mathrm{GeV}^{2} / \mathrm{c}^{2}$ & $0.04712 \mathrm{GeV}^{2} / \mathrm{c}^{2}$ & $\pm 0.002 \mathrm{GeV}^{2} / \mathrm{c}^{2}$ \\
\hline \multicolumn{6}{|l|}{ Spectrometer A } \\
\hline Setup & Angle & $Q^{2}$ Range $(\min )$ & $Q^{2}$ Range(avg) & $Q^{2}$ Range(max) & $Q^{2}$ uncertainty \\
\hline $2015-1$ & $23.51^{\circ}$ & $0.03141 \mathrm{GeV}^{2} / \mathrm{c}^{2}$ & $0.03909 \mathrm{GeV}^{2} / \mathrm{c}^{2}$ & $0.04106 \mathrm{GeV}^{2} / \mathrm{c}^{2}$ & $\pm 0.002 G e V^{2} / c^{2}$ \\
\hline $2015-2 \& 3$ & $25.9^{\circ}$ & $0.04066 \mathrm{GeV}^{2} / \mathrm{c}^{2}$ & $0.04873 \mathrm{GeV}^{2} / \mathrm{c}^{2}$ & $0.05090 \mathrm{GeV}^{2} / \mathrm{c}^{2}$ & $\pm 0.003 \mathrm{GeV}^{2} / \mathrm{c}^{2}$ \\
\hline
\end{tabular}




\subsection{Non-linearities}

\subsubsection{Symptoms}

There was significant evidence that the detectors were 'dying' during running. There are several symptoms which were indicative of a non-linearity in the detectors. The first symptom which was noticed during running (in configuration1) was the RMS widths of the detector asymmetry distributions, in spekB PMTs, were narrower than widths predicted by counting statistics with the observed rates (obtained in counting-mode data). The second symptom was a gradual decrease in the PMT signal, and therefore the PMT gain, over the run period shown in Fig. 4-21a for the spekB0 tube. A third symptom was that this decrease in gain coincided with a narrowing of RMS widths in asymmetry distributions, indicating a pedestal error that was gradually becoming worse. When there is a pedestal error, it shows up in the RMS widths. During config-1 running, there was an overall gradual decrease in gains by $\sim 9-16 \%$ and spB (sum of B0,B1, and B2) RMS gradually decreases by $15 \%$ going from $\sim 3300$ ppm to $\sim 2800$ ppm as shown in Fig. 4-21b. 


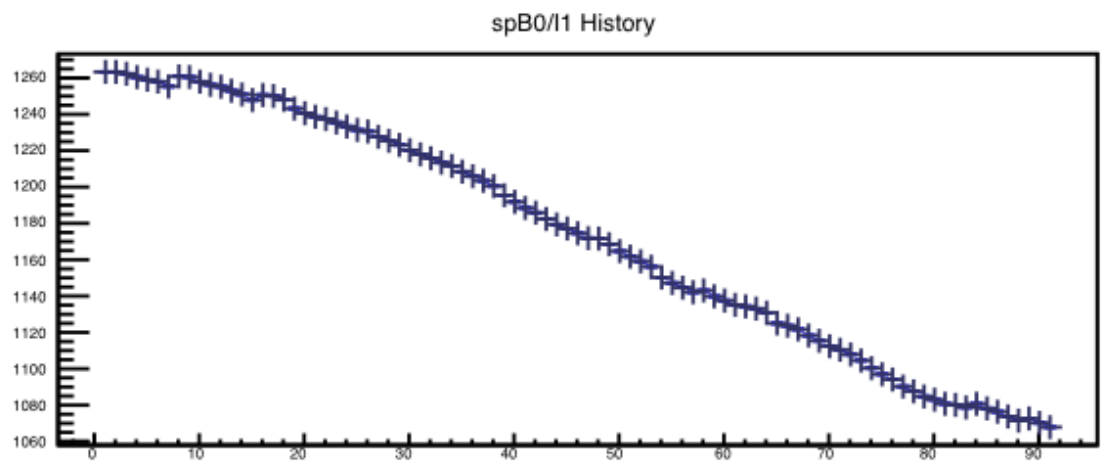

(a)

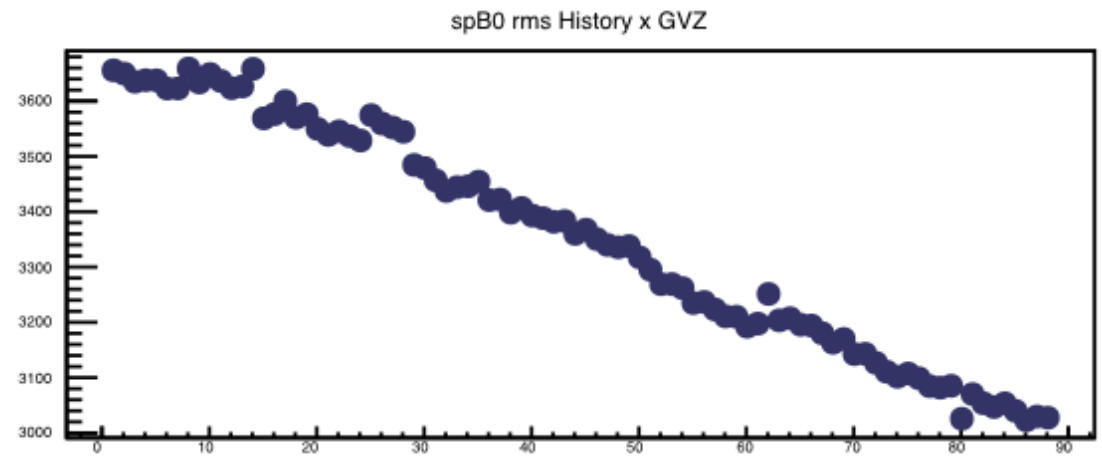

(b)

Figure 4-21: 2015-1 data showing PMT dying: (a) PMT spB0 current normalized signal vs Run\# (b) PMT spB0 Asymmetry RMS width vs Run\#

A fourth symptom was from beam-current unstabilized runs which showed a significant deviation in detector response to charge variation, indicative of a pedestal error. For example, Fig. 4-22 shows a 4\% deviation from 1 in the slope of the asymmetry in a spekB detector with respect to the measured charge asymmetry. 


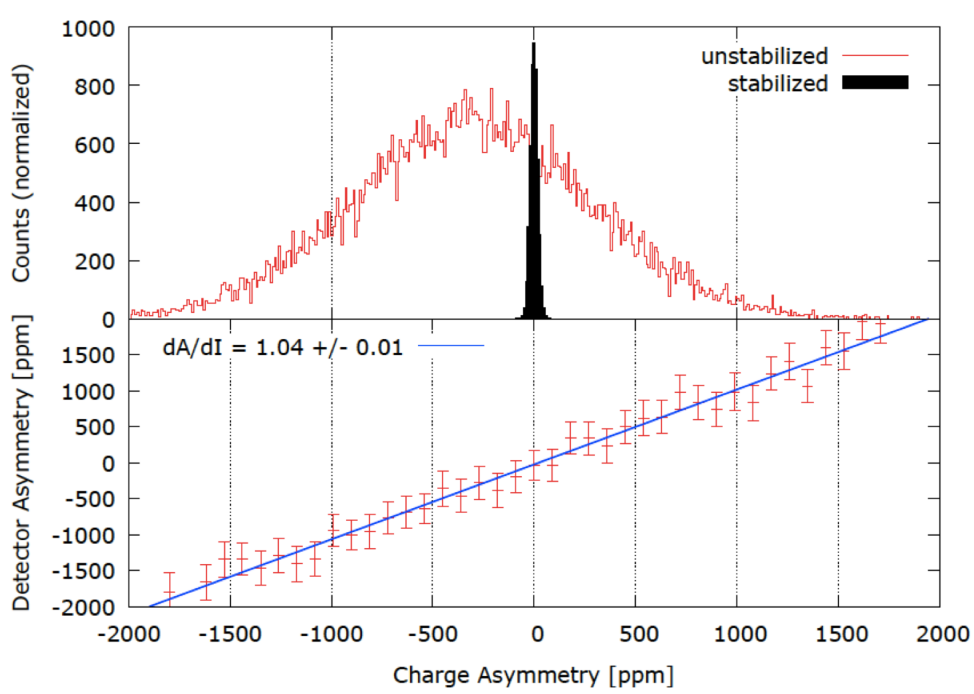

Figure 4-22: Top: Comparison between the charge asymmetry observed an unstabilized run with beam stabilization off (red) and with beam stabilization on (black). Bottom: Raw asymmetry determined for one PMT of the Cherenkov detector in spectrometer B as a function of the charge asymmetry for a run without beam stabilization off. [208]

A fifth symptom was that during unstabilized runs, the detector asymmetry was not equal to the sum of the expected physics asymmetry plus the measured charge asymmetry, which was quite large (hundreds of ppm), further indicative of a pedestal error. Stabilization was off for runs 9-11 for data configuration1 taken on 10/30/2015. As shown in FIg. 4-23 there is an observed shift in spB detector asymmetries when stabilization was turned off as compared to the other runs when stabilization was on. Spectrometer B detectors $0,1,2$ are shown in blue and spectrometer A detectors 0,1,2,3,4 are shown. The spB normalized detector asymmetries for the current unstabilized runs show a significant deviation in the inferred physics asymmetry of several tens of ppm, indicating a failure to normalize to charge and hence a non-linearity. 


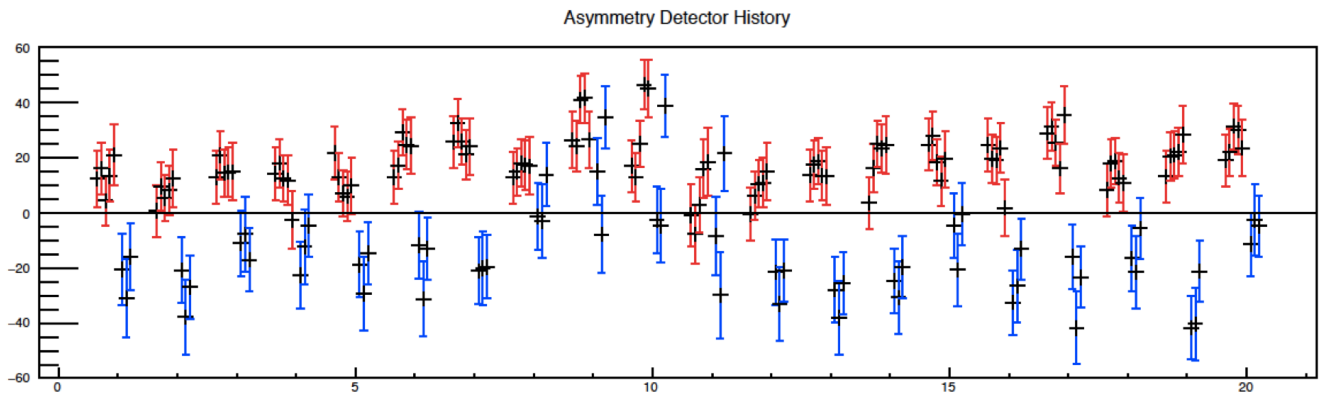

Figure 4-23: Normalized detector asymmetries for each PMT (normalized to charge asymmetry $A_{\text {phys }} \approx A_{\text {det }}-A_{I}$ ) for spA0,1,2,3,4 (red) and spB0,1,2(blue) for a sample of 20 runs of spectrometer configuration1. Runs 9,10,11 in the figure are current unstabilized runs with charge asymmetry in the hundreds of ppm. The spB normalized detector asymmetries for the current unstabilized runs show a significant deviation in the inferred physics asymmetry of several tens of ppm, changing sign from negative to positive.

Additionally, we note a couple of other anomalous behaviors: (1) The PMTs in spekB appeared to have an auto correlations coefficient which was not consistent with zero, indicating a possible 'smoothing out' of detected asymmetries. This phenomenon would look exactly like a pedestal error, except it would not be correctable by performing a current calibration. (2) The raw signal for the different PMTs suffered from fluctuations ${ }^{4}$ and this was observed by the A4-Collaboration previously ${ }^{5}$. It is possible this fluctuation was an indication of something amiss with these PMTs [220].

\subsubsection{Recalibration Study}

During 2016 running, a study was performed in which current calibration runs were performed very frequently to help characterize the manner in which the PMT's were dying and how to account for it in the pedestals. The goal was to find a model for the relationship between the PMT raw signal magnitude (the PMT gain) and the pedestals for each PMT. It was confirmed that the PMTs

\footnotetext{
${ }^{4}$ alternating between a higher and a lower value, with rise and fall times of $\sim 200 \mathrm{~ms}$

${ }^{5}$ but was dismissed for unknown reasons as being caused by a "beam halo scraping somewhere"
} 
were dying and a relationship between the PMT gain and PMT pedestal was established for a particular PMT voltage setting. Our Mainz collaborators used an ad hoc model for the relationship between the PMT pedestal and the PMT gain: data could be pedestal corrected by fixing a point on the linear calibration function, and rotating the function around it with the second point coming from the detector response at the nominal beam current [226] [227] as shown in Fig. 4-24.

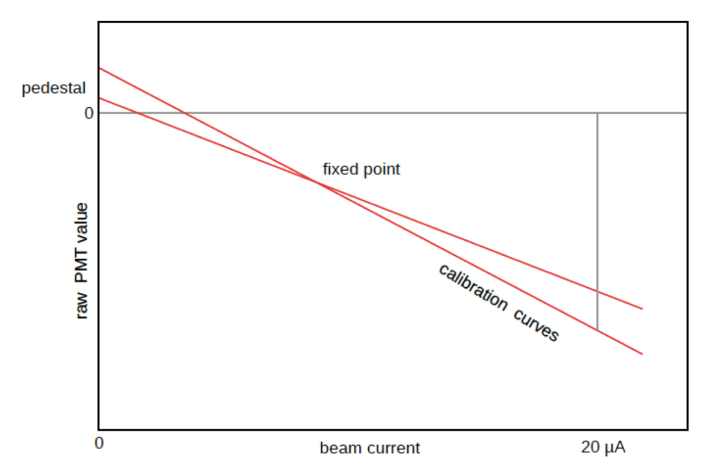

Figure 4-24: Recalibration illustration. When the raw value at $20 \mu \mathrm{A}$ decreases in magnitude, the calibration function rotates around a fixed point, and the pedestal is changed as well [227].

With this model, the data from 2015 was retroactively corrected with the data from 2016 by fixing a point on the linear calibration function as described above. However since the PMT voltages were changed throughout running for different configurations, applying this relationship retroactively to configurations with different PMT voltage settings was not sufficient to fully correct the earlier pedestal errors, as evidenced in Sec.4.9.3.

There were uncertainties in the recalibration method described in [226]. These uncertainties give rise to potential systematic PMT gain errors in each data configuration which are summarized in the following table: 


\begin{tabular}{|c|c|c|c|c|c|}
\hline configuration & $3-\mathrm{B}$ & $2-\mathrm{B}$ & $1-\mathrm{B}$ & $1-\mathrm{A}$ & $2,3-\mathrm{A}$ \\
\hline PMT gain error $(\mathrm{ppm})$ & 0.380 & 0.130 & 1.100 & 0.170 & 0.030 \\
\hline
\end{tabular}

Table 4.1: PMT gain systematic errors from uncertainty in the recalibration method for each data configuration

\subsubsection{Unstabilized runs}

While production runs had several forms of stabilization on, there were some runs with select stabilizations off. These runs could be used to test the calibrations, look for non-linearities or pedestal errors, and asses the coupling between various monitors and the detectors. Unstabilized runs reveal relationships by examining correlations between detectors and monitors. For example, runs with current stabilization off had significant enough fluctuations in Aq to determine the slope $\frac{d A_{s p B}}{d A q}$, relating detector asymmetry to charge asymmetry. This slope should nominally be equal to 1 , if the pedestals are correct. However, even after attempting to apply pedestal corrections as prescribed in Sec.4.9.2, some beam-current unstabilized runs showed significant deviation in detector response to charge variation. This implied a potential systematic error in the measurement - a false asymmetry.

There were a variety of unstabilized runs with different sets of stabilizations turned off. There are a variety of runs in which stabilizations were shut off. Some runs were taken without current stabilization, without position stabilized, without energy stabilization, or some combination of the three. Here is a compiled list of unstabilized runs taken in the $570 \mathrm{MeV} 2015$ data in kinematics configurations 1,2, and 3 . 


\begin{tabular}{|c|c|c|c|}
\hline \multicolumn{2}{|l|}{ run } & \multirow{2}{*}{\begin{tabular}{|l|} 
name \\
Longitudinal PV data
\end{tabular}} & \multirow{2}{*}{\begin{tabular}{|l|} 
calibration used \\
HighPrecisionCalib2810 \\
\end{tabular}} \\
\hline run_2015-10-28-01-03-45 & I|data|withoput DC pos. stabil. & & \\
\hline run_2015-10-30-14-05-41 & |withOUT current stab. (both)|data| & data1 run1 & HighPrecisionCalib3010 \\
\hline \multicolumn{2}{|c|}{ run_2015-10-30-23-37-56.txt noisy, stabilization off, GVZ IN?, transverse } & data1 run2 & HighPrecisionCalib3010 \\
\hline \multicolumn{2}{|c|}{ run_2015-10-31-00-39-34.txt noisy, stabilization off, GVZ IN?, transverse } & data1 run3 & HighPrecisionCalib3010 \\
\hline \multicolumn{2}{|c|}{ run_2015-10-31-01-52-46.txt noisy, stabilization off, GVZ IN?, transverse } & data1 run 4 & HighPrecisionCalib3010 \\
\hline run_2015-11-03-06-43-07 & |withOUT current stab. (both)|data| & data1 run5 & HighPrecisionCalib0311 \\
\hline run_2015-11-03-07-14-53 & |withouT position stab. (both)|data| & data1 run6 & HighPrecisionCalib0311 \\
\hline run_2015-11-03-08-07-11 & |withOUT energy stab.|data| & data1 run7 & HighPrecisionCalib0311 \\
\hline run_2015-11-06-07-17-14 & |withoUT current stab. (both)|data| & data2 run1 & HighPrecisionCalib0611 \\
\hline run_2015-11-06-09-05-13 & |withOUT current stab. (both)|data| & data2 run2 & HighPrecisionCalib0611 \\
\hline run_2015-11-10-15-44-07 & |withoUT current stab. (both)|data| & data3 run 1 & HighPrecisionCalib0911 \\
\hline run_2015-11-10-16-21-35 & |withoUT current stab. (both)|data| & data3 run2 & HighPrecisionCalib0911 \\
\hline run_2015-11-10-20-27-48 & | withOUT position stab. (both)|data|Run without position AC\&DC stabilization & data3 run3 & HighPrecisionCalib0911 \\
\hline run_2015-11-12-06-27-52 & |production data3 |data|NO AC pos.stab. in the second half & data3 run 4 & HighPrecisionCalib0911 \\
\hline run_2015-11-12-07-35-01 & |withOUT pos. stab. (both)|data| & data3 run5 & HighPrecisionCalib0911 \\
\hline run_2015-11-12-08-07-09 & | withouT current stab. (both)|data| plus NO AC pos. stab. & data3 run6 & HighPrecisionCalib0911 \\
\hline run_2015-11-12-08-40-29 & | withOUT energy stab. |data| plus NO AC pos. stab. & data3 run7 & HighPrecisionCalib0911 \\
\hline run_2015-11-12-08-53-58 & |withOUT energy stab.|data| & data3 run8 & HighPrecisionCalib0911 \\
\hline
\end{tabular}

Figure 4-25: List of unstabilized runs

For runs with only current stabilization off, the relationship between current and the detector signals could be accessed directly through correlation as in Fig. 4-26a. However, in runs with multiple stabilizations turned off, both direct and indirect relationships are at play. There are three ways to make $d A_{s p} / d A_{q} \neq 1$ : (1) poor Aq resolution (2) detector or current monitor pedestal error (3) indirect coupling through another parameter (i.e $A_{q} \rightarrow D_{x} \rightarrow A_{d e t}$ ) as shown in Fig. 426b. For runs with multiple stabilizations turned off, because of cross correlations, simultaneous multiple linear regression (Sec.4.9.3) was required for interpretation. 


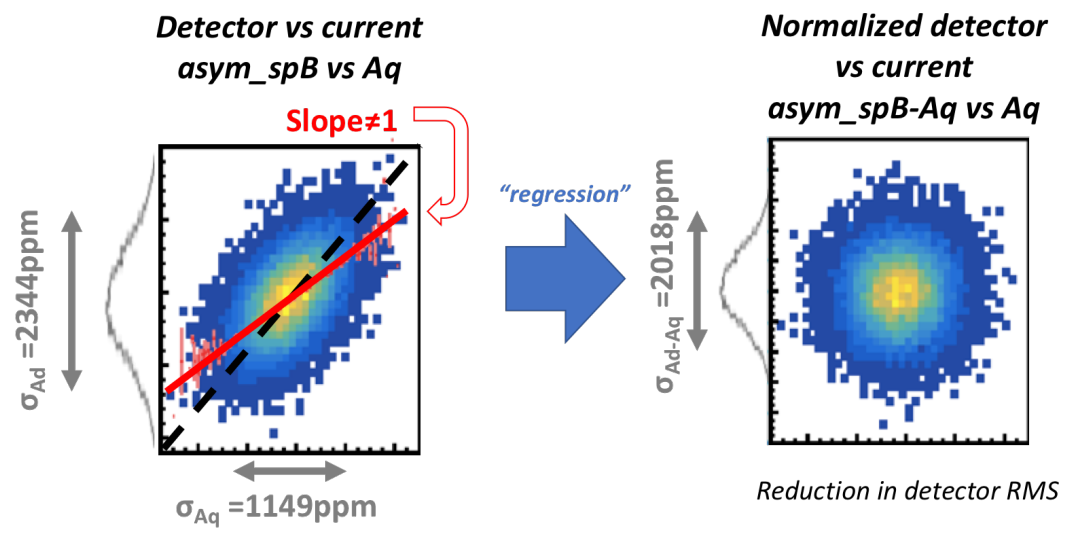

(a) $d A_{s p B} / d A_{q}$
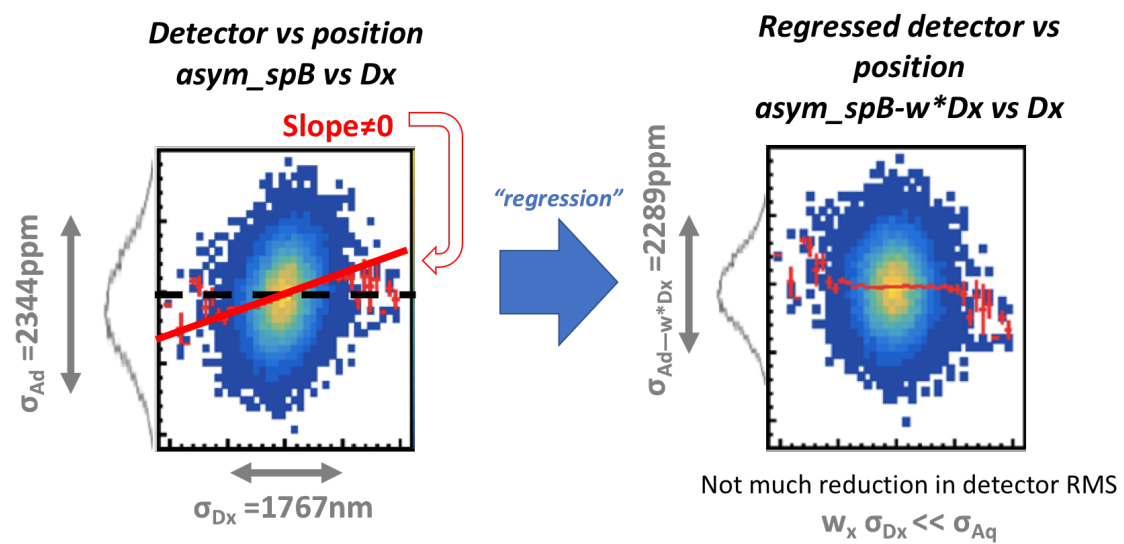

(b) $d A_{s p B} / d D_{x}$

Figure 4-26: Example of correlations in an unstabilized run

\section{Correlations and Linear Regression}

Linear regression is used to find the relationship between the detector signal $Y[i]$ and monitor signals $X_{0}[i], X_{1}[i], X_{2}[i] \ldots X_{N}[i]$, where i corresponds to discrete data points in time. We wish to focus on correlating the the noise in the signals, so let us define mean subtracted signals $y[i]=Y[i]-\langle Y\rangle$ and $x_{n}[i]=X_{n}[i]-\left\langle X_{n}\right\rangle$ which are have mean zero by definition. The noise in detector signal $y[i]$ can be filtered by the monitor signals $x_{0}[i], x_{1}[i], x_{2}[i] \ldots x_{N}[i]$ with regression coefficients $w_{0}, w_{1}, w_{2} \ldots w_{N}$. The residual $y_{\text {res }}[i]$, which is the portion of $y[i]$ uncorrelated with any of the monitors, will have a narrower width than $y[i]$. 
The detector signal is composed of some portion which is correlated with monitor signals and a residual portion uncorrelated with any monitor

$$
y[i]=\sum_{n=0}^{N} w_{n} x_{n}[i]+y_{\text {res }}[i]
$$

The regressed signal is given by

$$
y_{\text {res }}=y[i]-\sum_{n=0}^{N} w_{n} x_{n}[i]
$$

The above equations contain mean subtracted signals centered on zero. Rewriting the equations for the true non-zero mean detector and monitor signals, we obtain

$$
Y_{\text {res }}=y_{\text {res }}+\left\langle Y_{\text {res }}\right\rangle=y[i]+\langle Y\rangle-\sum_{n=0}^{N} w_{n}\left(x_{n}[i]-\left\langle X_{n}\right\rangle\right)
$$

Hence, the detector signal systematic errors can be subtracted out using the obtained regression coefficients.

$$
\left\langle Y_{\text {res }}\right\rangle=\langle Y\rangle-\sum_{n=0}^{N} w_{n}\left\langle X_{n}\right\rangle
$$

To obtain the regression coefficients, we use correlation coefficients defined as

$$
\begin{gathered}
R_{x_{n}, x_{m}}=E\left[x_{n}, x_{m}\right]=\frac{1}{N_{t o t}} \sum_{i=0}^{N_{t o t}} x_{n}[i] x_{m}[i] \\
p_{x_{n}, y}=E\left[x_{n} y\right]=\frac{1}{N_{\text {tot }}} \sum_{i=0}^{N_{\text {tot }}} x_{n}[i] y[i]
\end{gathered}
$$

The correlation matrix of independent monitor variables $\mathbf{R}$ and the cross correlation vector $\vec{p}$ of dependent detector signal y and monitor signals relate to the 
vector of regression coefficients $\vec{w}$ via the equation:

$$
\begin{gathered}
\mathbf{R} \vec{w}=\vec{p} \\
{\left[\begin{array}{cccc}
R_{x_{0}, x_{0}} & R_{x_{0}, x_{1}} & \ldots & R_{x_{0}, x_{N}} \\
R_{x_{1}, x_{0}} & R_{x_{1}, x_{1}} & \ldots & R_{x_{1}, x_{N}} \\
\vdots & \vdots & \ddots & \vdots \\
R_{x_{N}, x_{0}} & R_{x_{N}, x_{0}} & \ldots & R_{x_{N}, x_{N}}
\end{array}\right]\left[\begin{array}{c}
w_{0} \\
w_{1} \\
\vdots \\
w_{N}
\end{array}\right]=\left[\begin{array}{c}
p_{x_{0}, y} \\
p_{x_{1}, y} \\
\vdots \\
p_{x_{N}, y}
\end{array}\right]}
\end{gathered}
$$

The matrix elements along the diagonal are autocorrelation coefficients. When we are just assessing the relationship between the detector y and a single monitor $\mathrm{x}$ (i.e. $\mathrm{N}=0$ ) equation for the regression coefficient simplifies to the ratio of the crosscorrelation coefficient between the detector and monitor to the autocorrelation coefficient of the monitor.

$$
\begin{gathered}
R_{x x} w=p_{x y} \\
w=p_{x y} / R_{x x}=\frac{E[x y]}{E\left[x^{2}\right]}=\frac{\sum_{i=0}^{\infty} x[i] y[i]}{\sum_{n=0}^{\infty} x[i]^{2}}
\end{gathered}
$$

A simple way to understand this equation is that this regression coefficient $w$ is equivalent to the slope $\mathrm{m}$ obtained when fitting $\mathrm{y}$ vs $\mathrm{x}$ with a linear function $\mathrm{y}=\mathrm{mx}+\mathrm{b}$, if the uncorrelated noise in both signals were very small and the offset $\mathrm{b}$ were 0 . Recall $\mathrm{x}[\mathrm{n}]$ and $\mathrm{y}[\mathrm{n}]$ must be centered on 0 for this method to work, so that is why the generic signals $\mathrm{X}[\mathrm{n}]$ and $\mathrm{Y}[\mathrm{n}]$ must have their mean values subtracted out. The equation can be generalized to the non-zero mean detector and monitor signals as follows.

$$
w=\frac{E[(X-\langle X\rangle)(Y-\langle Y\rangle)]}{E\left[(X-\langle X\rangle)^{2}\right]}=\frac{E[X Y]-E[X] E[Y]}{E\left[X^{2}\right]-E[X]^{2}}=\frac{E[X Y]-E[X] E[Y]}{\sigma_{x}^{2}}
$$


The above equation is related to the correlation coefficient

$$
\rho_{X, Y}=\frac{\operatorname{cov}(X, Y)}{\sigma_{x} \sigma_{y}}=\frac{E[X Y]-E[X] E[Y]}{\sqrt{E\left[X^{2}\right]-E[X]^{2}} \sqrt{E\left[Y^{2}\right]-E[Y]^{2}}}=\frac{E[X Y]-E[X] E[Y]}{\sigma_{x} \sigma_{y}}
$$

with standard deviation on the correlation coefficient [202] given by

$$
\sigma_{\rho_{X, Y}}=\sqrt{\frac{1-\rho_{X, Y}^{2}}{n}}
$$

So we conclude the appropriate slope relating the two signals $\mathrm{Y}$ and $\mathrm{X}$ is given by the w-coefficient

$$
w=\frac{E[X Y]-E[X] E[Y]}{\sigma_{X}^{2}}=\frac{\sigma_{Y}}{\sigma_{X}} \frac{E[X Y]-E[X] E[Y]}{\sigma_{X} \sigma_{Y}}=\frac{\sigma_{Y}}{\sigma_{X}} \rho_{X, Y}
$$

with standard deviation on the w-coefficient given by

$$
\sigma_{w}=\sqrt{\frac{1-w^{2}}{n}}
$$

To understand tis equation, we note if the RMS of $\mathrm{y}$ is very large and the RMS of $\mathrm{x}$ is small, either the slope relating $\mathrm{x}$ to $\mathrm{y}$ is very large, or $\mathrm{y}$ is quite noisy and the correlation coefficient will be low, making for a reasonable slope. The w-coefficient effectively describes $\frac{d y}{d x}$.

We note the slope of $\mathrm{x}$ vs $\mathrm{y}$ should be $1 / w$. In that case our equation would become

$$
w_{\text {inverse }}=\frac{E[X Y]-E[X] E[Y]}{\sigma_{y}^{2}}=\frac{\sigma_{X}}{\sigma_{Y}} \frac{E[X Y]-E[X] E[Y]}{\sigma_{X} \sigma_{Y}}=\frac{\sigma_{X}}{\sigma_{Y}} \rho_{X, Y}
$$

If $\mathrm{X}$ and $\mathrm{Y}$ were $100 \%$ correlated, $\rho_{X, Y}=1$ and $w_{\text {inverse }}=\frac{\sigma_{x}}{\sigma_{y}}=1 / w$. Hence a simple way to obtain the correlation coefficient is to plot y vs $\mathrm{x}$ and plot $\mathrm{x}$ vs $\mathrm{y}$, fit a linear function to each, and multiply the slopes together. The result is the correlation coefficient. 


\section{Wiener Filters}

As a aside, we note a useful form of feedback commonly employed is a Wiener filter, which relates a detector signal at time interval $t_{n}$ to a monitor signal at $t_{n}$ and also at times in the past $t_{n-1}, t_{n-2} \ldots$ A Wiener filter is used to find the relationship between the correlated portion of signal $y[n]$ and monitor $\mathrm{x}[\mathrm{n}]$ where $\mathrm{n}$ corresponds to discretized data points in time and $\mathrm{y}[\mathrm{n}], \mathrm{x}[\mathrm{n}]$ are both centered on 0 . The signal $y[n]$ is filtered by the monitor signals $x[n], x[n-1], \ldots$ with filter coefficients $w_{0}, w_{1}, \ldots w_{N}$ and the residual $\mathrm{e}[\mathrm{n}]$ is that in $\mathrm{y}[\mathrm{n}]$ which uncorrelated with $\mathrm{x}[\mathrm{n}]$.

$$
\begin{aligned}
& y_{n}=\sum_{i=0}^{N} w_{i} x_{n+i} \\
& R_{x}^{j}=E\left[x_{n} x_{n+j}\right] \\
& p_{x y}^{j}=E\left[x_{n} y_{n+j}\right]
\end{aligned}
$$

Autocorrelation matrix $\mathbf{R}$ and cross correlation vector $\vec{p}$ are related to the vector of coupling filter coefficients $\vec{w}$.

$$
\begin{gathered}
\mathbf{R} \vec{w}=\vec{p} \\
{\left[\begin{array}{cccc}
R_{x}^{0} & R_{x}^{1} & \ldots & R_{x}^{N} \\
R_{x}^{1} & R_{x}^{2} & \ldots & R_{x}^{N-1} \\
\vdots & \vdots & \ddots & \vdots \\
R_{x}^{N} & R_{x}^{N-1} & \ldots & R_{x}^{0}
\end{array}\right]\left[\begin{array}{c}
w_{0} \\
w_{1} \\
\vdots \\
w_{N}
\end{array}\right]=\left[\begin{array}{c}
p_{0} \\
p_{1} \\
\vdots \\
p_{N}
\end{array}\right]}
\end{gathered}
$$

If a filter like this is employed as a feedback mechanism, it could give rise to autocorrelations in the monitors between the signal at time $t_{n}$ and the signal at time $t_{n-1}$ for example. 


\section{Testing Non-linearity Model}

We used 10 current unstabilized runs to test the whether the detector signal systematic errors can be accounted for through regression/correlation coefficients. We calculated the systematic uncertainty contribution in two ways to obtain: (1) a derived systematic and (2) an empirically observed systematic.

For the derived systematic, we used correlations to get a slope and predict false asymmetries the unstabilized data. In unstabilized runs, there is more noise in $A_{I}$ so the RMS is larger, allowing us to obtain slopes $d A_{s p} / d A_{I}$ with more accuracy allowing for a systematic uncertainty estimate. We have calculated a systematic uncertainty contribution from taking the correlation coefficient $\alpha=\left(\frac{d A_{\text {raw }}}{d A_{I}}-1\right)$ and the measured asymmetry $A_{\text {raw }}$, and multiplying them to obtain our derived error $A_{\text {sys. }}^{\text {calc }}=\alpha A_{\text {raw }}$. We note that the coefficient $w=d A_{\text {spek }} / d A_{I}$ obtained from noise in beam-current unstabilized runs was observed have as large as a $20 \%$ deviation from 1 . This slope deviation from 1 combined with the the physics asymmetry $A_{\text {perp }}$ is $\sim 20 \mathrm{ppm}$, implies the systematic correction for stabilized production runs could be as large as $\sim 20 \mathrm{ppm} \times 20 \%=5 \mathrm{ppm}$. We can check that we observe this systematic error in the noisy data.

For the empirically observed systematic, we look for significant deviation from the average $A_{p} h y s$ from stabilized production runs. In unstabilized runs, there is also a non-zero and sizable $\left\langle A_{I}\right\rangle$, so that in principle $\left\langle A_{\text {raw }}\right\rangle=A_{\text {phys }}+\left\langle A_{I}\right\rangle$ if $d A_{s p} / d A_{I}=1$, but this is not quite the case and we observe a systematic error $A_{\text {sys }}=A_{\text {raw }}-A_{\text {phys }}-A_{I}$. We examine the mean value seen by the detector $A_{\text {raw }}$ and the charge monitor $A_{I}$, attempt to normalize to charge using the usual method to obtain the inferred physics asymmetry $A_{\text {phys }}^{*}=A_{\text {raw }}-A_{I}$, and then look for a systematic error by comparing the inferred asymmetry to the actual physics asymmetry $A_{s y s}^{\text {obs }}=A_{\text {phys }}^{*}-A_{\text {phys }}=A_{\text {raw }}-A_{I}-A_{\text {phys }}$.

We tabulated the false asymmetries implied by the anomalous detector vs. charge slopes for the beam-current unstabilized runs, and compared to the ap- 
parent false asymmetries measured during those unstabilized runs. We compare these observed systematic errors $A_{\text {sys }}^{\text {obs }}=A_{\text {raw }}-A_{I}-A_{\text {phys }}$ to our calculated systematics found from noise correlations $A_{\text {sys. }}^{\text {calc }}=\alpha A_{\text {raw }}$. In the unstabilized runs, the raw asymmetry and charge asymmetries can be very large, $\sim 300 \mathrm{ppm}$ and the systematic corrections can be as large as $\sim 60 \mathrm{ppm}$. We observe consistency between the observed and predicted systematic error contributions to within $2 \sigma$. These estimates are well correlated as shown in Fig. 4-27b.

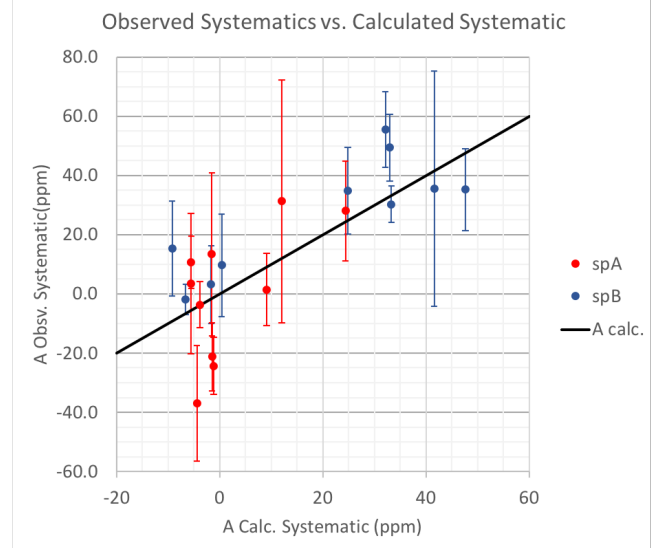

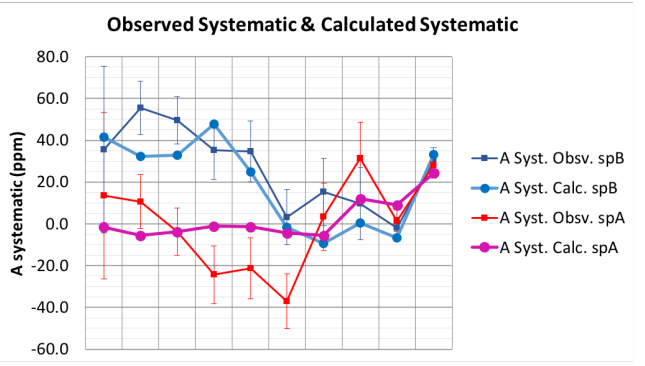

(b)

(a)

Figure 4-27: Comparison of observed systematic error contribution $A_{s y s}^{\text {obs }}=$ $A_{\text {exp }}-A_{I}-A_{\text {phys }}$ to calculated systematics found from noise correlations $A_{\text {sys. }}^{\text {calc }}=$ $\left(\frac{d A_{\text {raw }}}{d A_{I}}-1\right) A_{\text {raw }}=(w-1) A_{\text {raw }}$ shown for each current unstabilized run. Slope of $d A_{\text {raw }} / d A_{I}$ predicts the false asymmetry for unstabilized runs, the systematic error is $\left(d A_{\text {raw }} / d A_{I}-1\right) A_{\text {raw }} . A_{\text {raw }} \sim A_{\text {phys }}+A_{I}$, if $A_{I}$ is large, it dominates, if $A_{I}$ is small we still have $A_{\text {phys }}$.

\section{$A_{\text {false }}$ in Production Runs: from Nonlinearity in Unstabilized Runs}

The false asymmetry contribution to the transverse asymmetry $A_{n}$ from detector nonlinearities can be estimated by $A_{\text {false }}=\left(1-\frac{d A_{\text {raw }}}{d A_{I}}\right) A_{n}$ (note: systematic on $A_{\text {exp }} \sim A_{n} \times P$ is given by $\left(d A_{\text {raw }} / d A_{I}-1\right) A_{n} \times P$, so the systematic on $A_{n}$ is given by $\left.\left(d A_{\text {raw }} / d A_{I}-1\right) A_{n}\right)$. The slope scale factor $\left(1-\frac{d A_{\text {raw }}}{d A_{I}}\right)$ is obtained from only the unstabilized runs with energy and position stabilization on and current stabilization off. The current unstabilized runs to allow us to obtain decent 
precision on the slopes. The slope percent deviations from unity are in Tab. 4.2

\begin{tabular}{|c|c|c|c|c|c|c|c|}
\hline $\begin{array}{c}\text { data } \\
\text { set }\end{array}$ & run \# & time & $\begin{array}{c}\text { calib. } \\
\text { date }\end{array}$ & $\begin{array}{c}\frac{d A_{s p A}}{d A_{I}} \\
\text { original } \\
\text { peds }\end{array}$ & $\begin{array}{c}\frac{d A_{s p B}}{d A_{I}} \\
\text { original } \\
\text { peds }\end{array}$ & $\begin{array}{c}\frac{d A_{s p A}}{d A_{I}} \\
\text { recalib. } \\
\text { peds }\end{array}$ & $\begin{array}{c}\frac{d A_{s p B}}{d A_{I}} \\
\text { recalib. } \\
\text { peds }\end{array}$ \\
\hline \multirow[t]{5}{*}{ config 1} & run1 & 10-30-14:05 & \multirow[t]{5}{*}{$10-30$} & $-1.9 \%$ & $-6.4 \%$ & $0.45 \%$ & $-11.3 \%$ \\
\hline & run2 & $10-30-23: 37$ & & $-0.9 \%$ & $-3.8 \%$ & $1.6 \%$ & $-9.1 \%$ \\
\hline & run3 & 10-31-00:39 & & $-1.4 \%$ & $-3.6 \%$ & $1.1 \%$ & $-9.2 \%$ \\
\hline & run4 & $10-31-01: 52$ & & $-2.2 \%$ & $-7.3 \%$ & $0.3 \%$ & $-12.8 \%$ \\
\hline & run5 & $11-03-06: 43$ & & $-1.5 \%$ & $-13.1 \%$ & $1.2 \%$ & $-22.3 \%$ \\
\hline \multirow[t]{2}{*}{ config 2} & run1 & 11-06-07:17 & \multirow[t]{2}{*}{$11-06$} & $1.9 \%$ & $0.9 \%$ & $2.0 \%$ & $0.7 \%$ \\
\hline & run2 & 11-06-09:05 & & $2.7 \%$ & $4.1 \%$ & $2.9 \%$ & $4.0 \%$ \\
\hline \multirow[t]{3}{*}{ config 3} & run1 & $11-10-15: 44$ & \multirow[t]{2}{*}{ 11-09 } & $-4.4 \%$ & $1.5 \%$ & $-4.8 \%$ & $-0.1 \%$ \\
\hline & run2 & $11-10-16: 21$ & & $-2.8 \%$ & $3.7 \%$ & $-3.2 \%$ & $2.0 \%$ \\
\hline & run6* & 11-12-08:07 & & $-7.68 \%^{*}$ & $3.74 \% *$ & $-8.0 \% *$ & $0.2 \%^{*}$ \\
\hline
\end{tabular}

Table 4.2: Nonlinearity in unstabilized runs for spA and spB, for original pedestals taken from current calibration runs and for pedestals after the recalibration procedure was performed. The recalibration procedure mitigated some of the larger nonlinearities some, but not fully. (*)this run had position also unstabilized as well as current and slopes were obtained from $6 \mathrm{D}$ regression

We tabulate the implied false-asymmetries in the stabilized production runs. Below is a table (Tab. 4.3) showing that the results are significant, but also roughly consistent with the PMT gain error estimates from the recalibration procedure. 


\begin{tabular}{|c|c|c|c|c|c|c|c|}
\hline config & $3-\mathrm{B}$ & $2-\mathrm{B}$ & $1-\mathrm{B}$ & $1-\mathrm{A}$ & $2-\mathrm{A}$ & $3-\mathrm{A}$ & $2,3-\mathrm{A}$ \\
\hline$A_{n}$ & -15.98 & -20.67 & -21.94 & -23.87 & $-27.35^{*}$ & $-30.43^{*}$ & -28.3 \\
\hline Non- & $-3 \%$ & $2.5 \%$ & $-5.3_{ \pm 1.9 \%}$ & $0.9 \%$ & $2.3 \%$ & $-5 \%$ & $-0.24 \%$ \\
linearity & & {$[1-4 \%]$} & {$[$ up to $-13 \%]$} & & {$[2-3 \%]$} & {$[-3.6$ to-7.7\%] } & \\
\hline$A_{\text {false }}$ & 0.5 & -0.5 & $1.1_{\text {[up to } 1.7]}$ & -0.22 & -0.65 & 1.4 & 0.06 \\
\hline
\end{tabular}

Table 4.3: Summary of $A_{\text {false }}(p p m)$ for different configurations using unstabilized run slopes. $A_{n}$ are taken from final analysis shown in [208] $\left(^{*}\right) A_{n}$ taken from raw analysis

\subsection{4 $A_{\text {false }}$ in Production Runs: from RMS method}

One of the symptoms of non-linearity in the detectors was that the decrease in gain coincided with a narrowing of RMS widths in asymmetry distributions. When there is a pedestal error, it shows up in the RMS widths. Just as $A_{\exp }=$ $A_{\text {raw }} /\left(d A_{\text {raw }} / d A_{\text {true }}\right)$, also $A_{\text {rms,true }}=A_{\text {rms }, \text { raw }} /\left(d A_{\text {raw }} / d A_{\text {true }}\right)$. So we can estimate the non-linearity as $d A_{\text {raw }} / d A_{\text {true }}-1 \approx A_{\text {rms,raw }} / A_{\text {rms,true }}-1$ and we can estimate the false asymmetry contribution to the transverse asymmetry as:

$$
A_{\text {false }} \approx A_{\text {raw }}\left(A_{\text {rms,raw }} / A_{\text {rms }, \text { true }}-1\right)
$$

So, instead of using correlations from infrequent, intermittent unstabilized runs to estimate the nonlinearities through $d A_{d e t} / d A_{I}$, we can continuously use the RMS detector asymmetries in all the stabilized production runs. For $A_{\text {rms,true }}$, we assume that immediately after a current calibration run, our pedestal are correct and the RMS $A_{r m s, 0}$ is the 'true' RMS appropriate for the rates. This assumption puts a lower bound on the magnitude of false asymmetries calculated. The false asymmetry could be larger than this method estimates.

The following figures show the RMS ratios $A_{r m s} / A_{r m s, 0}$ and the false asymmetry $A_{\text {false }} \approx A_{\text {raw }}\left(A_{\text {rms }} / A_{\text {rms }, 0}-1\right)$ over time for every production run in each configuration. On the left, spekA RMS ratio is shown in pink and the spekB 
RMS ratio is shown in green. On the right, the spekA systematic false asymmetry estimate for each run is shown in red, and the spekB systematic false asymmetry is shown in blue.
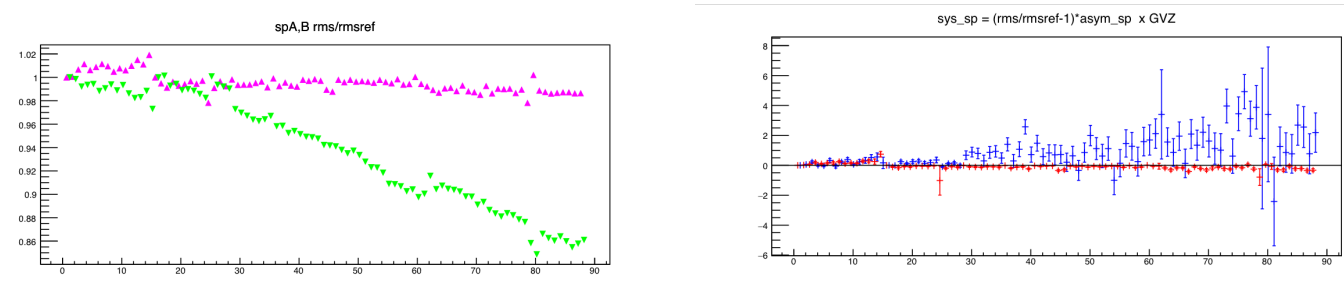

(a) configuration 1: $\operatorname{spA} 23.5^{\circ}\left(Q^{2}=0.039 G e V^{2} / c^{2}\right) \& \operatorname{spB} 20.61^{\circ}\left(Q^{2}=0.041 G e V^{2} / c^{2}\right)$
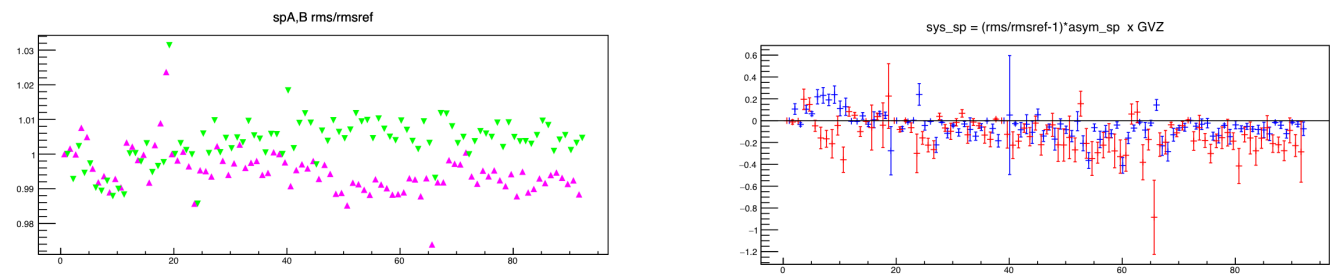

(b) configuration 2: $\operatorname{spA} 25.9^{\circ}\left(Q^{2}=0.049 \mathrm{GeV}^{2} / \mathrm{c}^{2}\right) \& \mathrm{spB} 17.65^{\circ}\left(Q^{2}=0.03 \mathrm{GeV}^{2} / \mathrm{c}^{2}\right)$
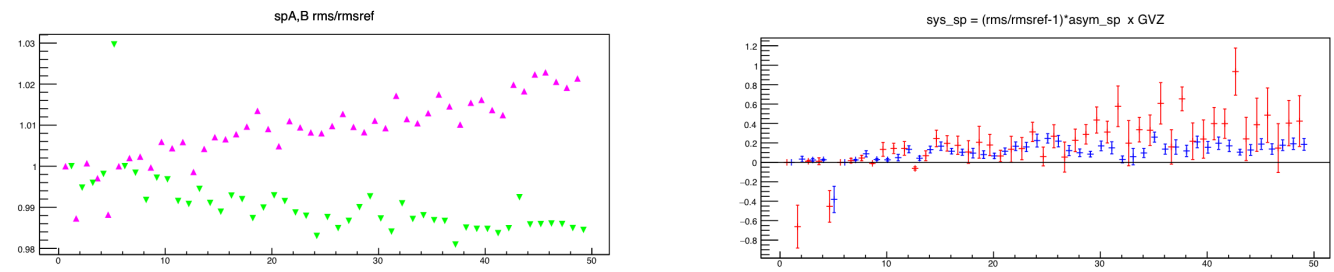

(c) configuration 3: $\operatorname{spA} 25.9^{\circ}\left(Q^{2}=0.049 \mathrm{GeV}^{2} / \mathrm{c}^{2}\right) \& \operatorname{spB} 15.11^{\circ}\left(Q^{2}=0.023 \mathrm{GeV}^{2} / \mathrm{c}^{2}\right)$

Figure 4-28: RMS method : spA systematic in red, spB systematic in blue, spA rms ratio in pink, spB rms ratio in green.

For each configuration the overall false asymmetry is estimated by combining all the runs together, weighted by their uncertainties, and the results are shown in Table 4.4. For the combined 2,3 -A configuration, we weight configuration 2-A and 3-A by the number of runs in each, there being approximately twice as many runs in configuration 2-A as there are in 3-A resulting in a near cancellation in the false asymmetry estimate. Because of the assumption that the RMS immediately after a current calibration run is the 'true' RMS, these estimates should be treated as a lower bound on the false asymmetry due to non-linearity. 


\begin{tabular}{|c|c|c|c|c|c|c|c|}
\hline config & $3-\mathrm{B}$ & $2-\mathrm{B}$ & $1-\mathrm{B}$ & $1-\mathrm{A}$ & $2-\mathrm{A}$ & $3-\mathrm{A}$ & $2,3-\mathrm{A}$ \\
\hline$A_{\text {false }}$ & 0.144 & -0.0532 & 1.25 & 0.102 & 0.154 & -0.274 & 0.005 \\
\hline
\end{tabular}

Table 4.4: Systematic error on $A_{n}$ (ppm) from non-linearity as estimated by change in RMS asymmetry distributions

\section{Autocorrelations}

An auto correlation coefficient $\mathrm{R}$ is defined as

$$
R(k)=\frac{1}{(n-k) \sigma_{y}^{2}} \sum_{t=1}^{n-k}(y[t]-\bar{y})(y[t+k]-\bar{y})
$$

where the first order auto correlation coefficient is given by $\mathrm{R}(1)$

$$
R(1)=\frac{1}{(n-1) \sigma_{y}^{2}} \sum_{t=1}^{n-1}(y[t]-\bar{y})(y[t+k]-\bar{y})
$$

A non-zero autocorrelation coefficient $R(1)$, which correlates a data point with the one before it, is symptomatic of a 'smoothing' of our detector signal between successive helicity states. Autocorrelation decreases the magnitude of the measured asymmetry. It could occur in the detector signals, if for example the integration window was set incorrectly, so each window would not correspond to a single helicity state. Or it could occur if the PMT response had a very long time components, possibly due to the PMT 'dying'. This is a potentially important quantity to consider. It manifests itself as a suppression of the measured asymmetry, in a manner nearly identical to a non-linearity and the methods we used to account for non-linearity should cover any effects associated with auto-correlations.

The effect of the auto-correlation coefficient $\mathrm{R}(1)$ on data can be described as follows:

$$
Y[t]=(1-w) X[t]+w[X[t-1]
$$

where $\mathrm{Y}$ is the smoothed detector signal and $\mathrm{X}$ is what the detector signal would be 
if autocorrelation were properly 0 .This results in the measured asymmetry being suppressed by a factor of:

$$
A_{Y}=A_{X}(1-R)
$$

for helicity sequences ...0)(01) or ...1)(10).

$$
A_{Y}=A_{X}(1-2 R)
$$

for helicity sequences ...1)(01) or ...0)(10).

The prevalence of these sequences is determined by the helicity pattern: pairs, quartets, octets, etc. So, there is a range of correction factors $1 \mathrm{R}-2 \mathrm{R}$. $A_{\text {meas }}$ is $80-90 \%$ of what it should be for autocorrelation coefficient for 0.1 for example. In this case, the helicity pattern is formed in quartets.

Hence, $A_{\text {measured }}=(1-R) A_{\text {true }}$ to $(1-2 R) A_{\text {true }}$. Autocorrelation coefficients were observed to be greater for spectrometer B. First order autocorrelation coefficients of 0.1 were observed in spectrometer B data in configuration 1 [224] [225], where coefficients for $\mathrm{k}=1$ (i.e 1 window behind) for 17 runs using $\mathrm{N}=10,000$ data points were determined. For these 17 runs, the spBs were $7 \% \pm(2 \% / \sqrt{17})$ correlated with previous window and spA's were $2 \% \pm(1 \% / \sqrt{17})$. This implies the spekB PMT detectors could suffer a $10-20 \%$ systematic error in the measured asymmetry. For $A_{s p B}=-20 \mathrm{ppm}, \mathrm{sp} B_{R}=0.08$, the systematic error for $\mathrm{spB}$ is 1.6-3.2ppm.

The autocorrelation manifests in a manner identical to the way a pedestal error manifests itself. So the diagnostics we used and corrections we made for pedestal error also would fix this potential autocorrelation issue. 


\subsection{5 $A_{\text {false }}$ in Production Runs: Summary of Error Esti- mates}

We accounted for systematic errors in the transverse asymmetry measurement due to detector non-linearities using several different methods: (1) through recalibration uncertainties, (2) using unstabilized run correlations and (3) with an RMS tracking method described in previous sections. Table 4.5 summaries all the results from these 3 methods. We make a conservative decision to use results of the unstabilized run method, because it gives the largest overall estimate for non-linearity error and also because it is the most direct method which makes the fewest assumptions. The systematic error due to PMT nonlinearity is shown in the bottom line of Table 4.5.

\begin{tabular}{|c|c|c|c|c|c|c|c|}
\hline \hline Spectrometer & $\mathrm{B}$ & $\mathrm{B}$ & $\mathrm{B}$ & $\mathrm{A}$ & $\mathrm{A}$ & $\mathrm{A}$ & $\mathrm{A}$ \\
Setup & 3 & 2 & 1 & 1 & 2 & 3 & 2,3 \\
\hline$Q^{2}\left(\mathrm{GeV}^{2} / \mathrm{c}^{2}\right)$ & 0.023 & 0.03 & 0.041 & 0.039 & 0.049 & 0.049 & 0.049 \\
$A_{n}$ & -15.98 & -20.67 & -21.93 & -23.88 & -28.30 & -28.30 & -28.30 \\
\hline Nonlinearity & $-3 \%$ & $2.5 \%$ & -5.3 & $0.9 \%$ & $2.3 \%$ & $-5 \%$ & $-0.24 \%$ \\
$A_{\text {false }}($ Unstab. runs $)$ & 0.5 & -0.5 & 1.1 & -0.22 & -0.65 & 1.4 & 0.06 \\
$A_{\text {false }}($ RMS method $)$ & 0.144 & -0.0532 & 1.25 & 0.102 & 0.154 & -0.274 & 0.005 \\
PMT gain error & 0.380 & 0.130 & 1.100 & 0.170 & - & - & 0.030 \\
(Recalibration $)$ & & & & & & & \\
\hline Nonlinearity Error & 0.50 & 0.50 & 1.10 & 0.20 & - & - & $0.20 *$ \\
\hline \hline
\end{tabular}

Table 4.5: Summary of systematic errors (in ppm) due to non-linearity from various methods of estimation. The values ultimately used were those obtained from the unstabilized runs $(*)$ For $2,3-\mathrm{A}, 0.20 \mathrm{ppm}$ is used instead of $0.06 \mathrm{ppm}$ because this small systematic comes from an approximate cancellation of larger systematics in configurations $2-\mathrm{A}$ and $3-\mathrm{A}$ and is therefore not entirely reliably small. The argument here is that the systematic for combined 2,3-A isn't likely to be smaller than the systematic for 1-A, so the systematic for 1-A is used for configuration $2,3-\mathrm{A}$ 


\subsubsection{Raw Data}

The raw experimental asymmetry Araw was extracted for both spectrometers $\mathrm{A}$ and $\mathrm{B}$ in each $Q^{2}$ setup.

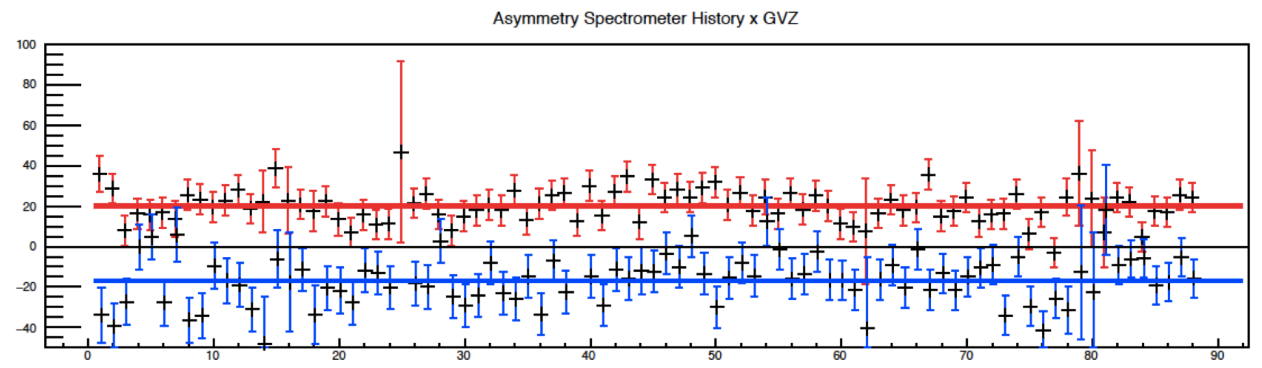

(a) configuration 1: $\operatorname{spA} 23.5^{\circ}\left(Q^{2}=0.039 G e V^{2} / c^{2}\right) \& \operatorname{spB} 20.61^{\circ}\left(Q^{2}=\right.$ $\left.0.041 G e V^{2} / c^{2}\right)$

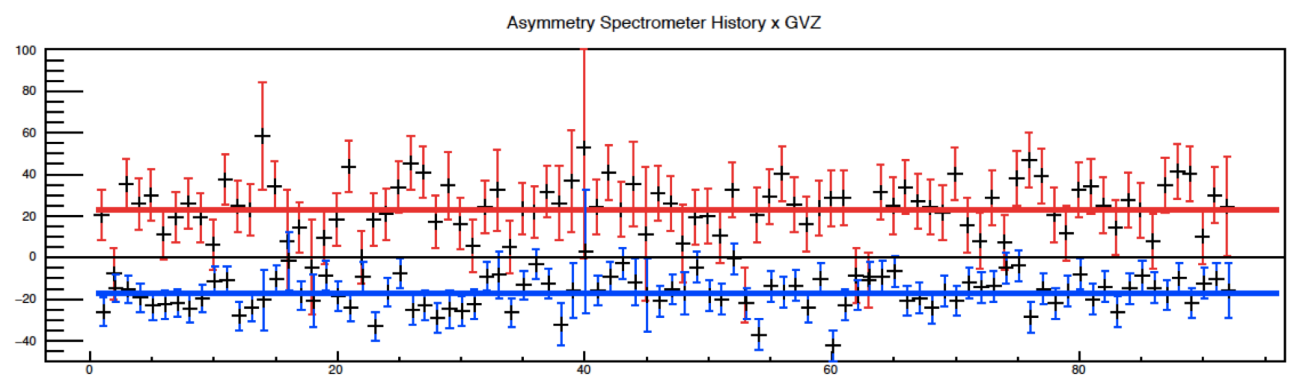

(b) configuration 2: $\operatorname{spA} 25.9^{\circ}\left(Q^{2}=0.049 G e V^{2} / c^{2}\right) \& \operatorname{spB} 17.65^{\circ}\left(Q^{2}=\right.$ $0.03 G e V^{2} / c^{2}$ )

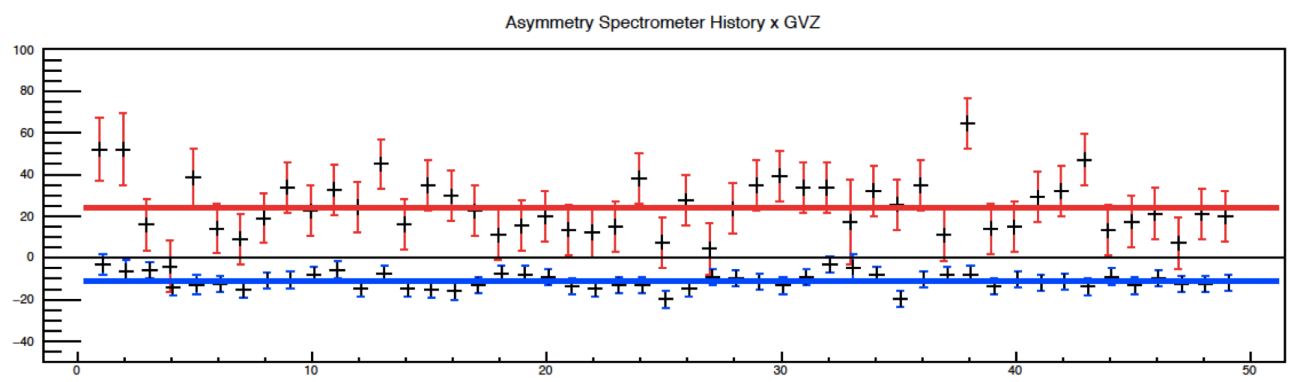

(c) configuration 3: $\operatorname{spA} 25.9^{\circ}\left(Q^{2}=0.049 G e V^{2} / c^{2}\right) \& \operatorname{spB} 15.11^{\circ}\left(Q^{2}=\right.$ $\left.0.023 \mathrm{GeV}^{2} / \mathrm{c}^{2}\right)$

Figure 4-29: Raw summed detector asymmetries (GVZ sign accounted for) for spA (red) and spB (blue) for each spectrometer configuration.

The sign change from periodic insertion of additional $\lambda / 2$-wave plate has been accounted for. Fig. 4-29 shows the sign-corrected Araw in each spectrometer 
for the combined PMT signal in spectrometer A (red points) and spectrometer B (blue points) at various $Q^{2}$, where the momentum range for each setup is also indicated.

Certain runs or events within runs were excluded based on irregularities or sudden large changes in at least one of the following parameters: X21, Y21, X26, Y26, E0, I21, I13, A1 and B1. Most frequently, the irregularity was a sudden shut-off of beam current, a 'beam trip'. Runs with beam trips were not excluded entirely, only the portion of the run without current was discarded.

\subsection{Results 570MeV Data}

Systematic corrections were made to $A_{\text {raw }}$ for beam polarization and polarizationcorrelated fluctuations to obtain the final results on the transverse asymmetry:

$$
A_{n}=\frac{A_{\text {raw }}-A_{I}-A_{G L}-\frac{\partial \sigma}{\partial X} \Delta X-\frac{\partial \sigma}{\partial Y} \Delta Y-\frac{\partial \sigma}{\partial \Theta} \Delta \Theta \ldots}{P_{\perp}}
$$

There is an implementation for the correction of false asymmetries created by: Beam Current (I), beam position (X and Y), beam angles ( $\Phi$ and $\Theta)$, integration gate (GL), and beam polarization. The sensitivities for beam position and angle were calculated and taken from a simulation, while the sensitivities for beam current were measured. Finally, the experimental asymmetry $A_{\text {exp }}$ was normalized to the electron beam polarization to extract the physics asymmetry $A_{n}{ }^{6}$. The uncertainty in the polarization measurement contributes significantly to the systematic error, and in some configurations is the largest contributor. The experimentally determined values for all four $Q^{2}$ kinematics and the corresponding statistical and systematic uncertainties are summarized in Table 4.6.

\footnotetext{
${ }^{6}$ The measurement of polarization $P_{e}$ results in a correction to the physics asymmetry on the order of $A_{n} \approx A_{\text {raw }} / P=A_{\text {raw }} /(1+(P-1)) \approx A_{\text {raw }}(1+(1-P)) \rightarrow A_{\text {false }}=(1-P) A_{\text {raw }} \approx$ $(1-P) * P A_{n}$
} 


\begin{tabular}{|c|c|c|c|c|c|}
\hline \hline Spec. & $\mathrm{B}$ & $\mathrm{B}$ & $\mathrm{B}$ & $\mathrm{A}$ & $\mathrm{A}$ \\
Setup & 3 & 2 & 1 & 1 & 2,3 \\
$Q^{2}\left(\mathrm{GeV}^{2} / \mathrm{c}^{2}\right)$ & 0.023 & 0.03 & 0.041 & 0.039 & 0.049 \\
$A_{n}$ & -15.98 & -20.67 & -21.93 & -23.88 & -28.30 \\
\hline$\delta E$ & $0.00(0.01)$ & $0.00(0.01)$ & $0.00(0.00)$ & $0.00(0.01)$ & $0.00(0.00)$ \\
$A_{I}$ & $-0.68(0.01)$ & $0.86(0.02)$ & $-0.31(0.01)$ & $-0.31(0.01)$ & $0.28(0.01)$ \\
Beam & $-0.02(0.00)$ & $-0.01(0.00)$ & $0.02(0.01)$ & $-0.07(0.02)$ & $-0.001(0.01)$ \\
Gate Length & $-0.01(0.01)$ & $0.00(0.01)$ & $0.01(0.01)$ & $0.01(0.01)$ & $0.00(0.01)$ \\
$P_{e}$ meas. & $-3.42(0.24)$ & $3.18(0.38)$ & $3.67(0.47)$ & $3.99(0.52)$ & $-4.95(0.48)$ \\
PMT gain & $-(0.50)$ & $-(0.50)$ & $-(1.10)$ & $-(0.20)$ & $-(0.20)$ \\
\hline Syst. error & 0.55 & 0.63 & 1.20 & 0.55 & 0.52 \\
Stat. error & 1.06 & 0.96 & 1.52 & 0.97 & 1.37 \\
\hline \hline
\end{tabular}

Table 4.6: Systematic errors are added in quadrature to obtain total systematic error [203]. Values in parenthesis are uncertainties, values not in parenthesis are systematic corrections to measured asymmetries.

The final results on the transverse asymmetry measurement are shown in Fig. 4-30. The black theoretical curve is the model of [210], based on low $Q<<E / c$ approximations in forward scattering. The transverse asymmetry from the interference of the one-photon and two-photon exchange amplitudes can reasonably be described at slightly larger scatting angles by

$$
A_{n} \sim C_{0} \cdot \log \left(\frac{Q^{2}}{m_{e}^{2} c^{2}}\right) \cdot \frac{F_{\text {Compton }}\left(Q^{2}\right)}{F_{c h}\left(Q^{2}\right)}
$$

representing leading $Q^{2}$ behavior and neglecting $\sim Q^{2} / E^{2}$ corrections [208]. $C_{0}$ is calculated from the photoabsorption cross-section for a given target. The ratio of the Compton to charge form factors estimated based on Compton slope parameter 
data in protons and ${ }^{4} \mathrm{He}$, which indicate

$$
\frac{F_{\text {Compton }}\left(Q^{2}\right)}{F_{\text {ch }}\left(Q^{2}\right)} \approx \exp \left(-4 Q^{2} / G e V^{2}\right)
$$

a target independent description. The unknown Compton form factor for ${ }^{12} \mathrm{C}$ was modeled in Fig. 4-30 as [208]

$$
F_{\text {Compton }}\left(Q^{2}\right)=\exp \left(-R_{C h}^{2} Q^{2} / 6\right) \times \exp \left(-4 Q^{2} / G e V\right)
$$

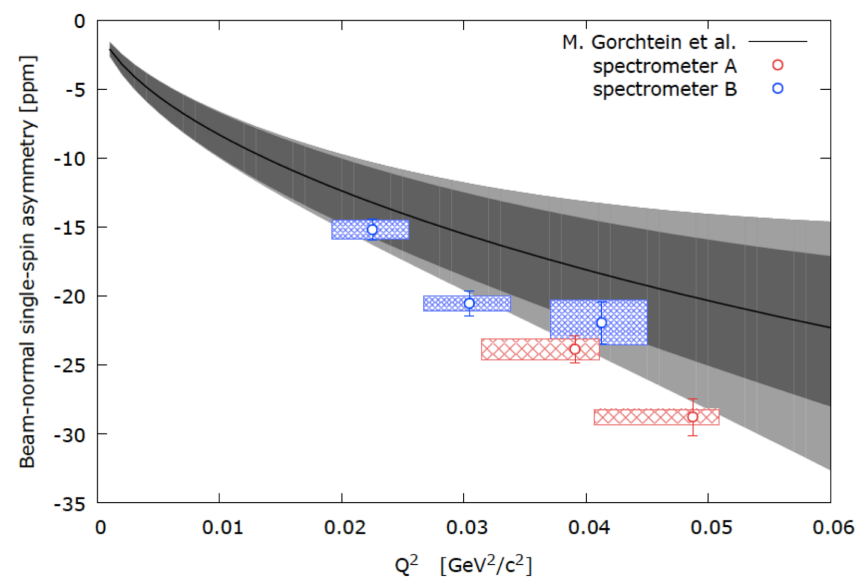

Figure 4-30: Extracted transverse asymmetries $A_{n}$ for the detectors placed in spectrometer A (red points) and spectrometer B (blue points) versus Q2. The width of the given boxes indicates the covered Q2 range. The statistical and systematic uncertainties are given by the error bars and the height of the boxes, respectively. The theoretical calculation of Ref. [210] (black line) is shown for comparison. The given bands belong to the uncertainty of the Compton slope parameter of $10 \%$ (dark grey) and 20\% (light grey) [208]

The grey curves are $10 \%$ and $20 \%$ error bands on the calculation from uncertainty in the Compton slope parameter. The data barely fits in the $20 \%$ uncertainty of the Compton slope parameter, indicating the that ratio of the Compton to charge form factor is not target independent even for a light nucleus like ${ }^{12} \mathrm{C}$ and and heavier nuclei may have an even larger deviations. Notably, similar models failed for 208Pb in PREXI [209]. In PVES experiments, like PREX, these 
two-photon exchange processes provide an important contribution to the systematic uncertainties. Future measurements at MAMI will investigate the transverse asymmetry for heavier nuclei, like $\mathrm{Pb}$, at the same $Q^{2}$ values using similar experimental methodology. 



\section{Chapter 5}

\section{Beam Studies for PREXII and MOLLER}

Monitoring of beam energy, current, position, and angle is critical for both PREXII and MOLLER. Both experiments compare opposite helicity electrons and measure changes in scattering rates. Thus, any change in the polarized beam, including energy/position/intensity changes, correlated with the helicity reversal, is a source of systematic error giving rise to a false asymmetry. Furthermore, beam monitor resolution and noise in the measurement of beam current, energy, and position impacts the statistical uncertainty of the experimental result through the relation $A_{\text {raw }}=A_{\text {det }}-A_{Q}+\alpha \Delta E+\sum_{i} \beta_{i} \Delta x_{i}$, where $A_{\text {raw }}$ is the beam current normalized detector asymmetry, $A_{Q}$ is the beam charge asymmetry, $\Delta E$ is the helicity correlated energy difference, $\Delta x_{i}$ are the helicity correlated position differences, and $\alpha, \beta_{i}$ are the coupling constants. In this chapter we detail the various types of beam monitors to be used for PREXII and MOLLER and show beam study results which demonstrate achievement of the desired resolution for these experiments. 


\subsection{Parity Quality Beam}

Parity Quality Beam (PQB) goals include small helicity correlated beam asymmetry mean values (means), as well as reasonably small amounts of helicity correlated beam noise (widths). In terms of beam monitoring, sufficient resolution in BCM monitors and BPM monitors is desirable. 2015-2016 beam studies evaluated $A_{q}$ intensity asymmetry widths, BCM resolution, BPM widths, and their dependence on current, energy, and frequency.

Helicity correlated beam asymmetries, such as intensity asymmetry $A_{q}$ and position differences $D_{x}$ and $D_{y}$, are evaluated from taking the means of distributions measured by the beam monitor as shown in Fig. 5-1. For PQB, in terms of means, we want to minimize helicity correlated changes: Aq and position differences $\left(<100 \mathrm{ppb},<1 \mathrm{~nm}\right.$, spot size asymmetry $\left.\Delta \sigma / \sigma<10^{-4}\right)$. Widths of the distributions are the RMS fluctuations after parity filtering, which involves integration and taking pair differences, suppressing much of the frequency content of the noise. For PQB, there is a balance to be struck: we want small widths to reduce statistical error on measurements of mean values, but we also want sufficient widths to establish correlations with monitors (i.e. slopes) and perform regression to get corrections (i.e. shifts) to reduce systematic error contributions to asymmetry from helicity correlated beam differences (i.e. means) as shown in Fig. 5-1. This is critical to estimating and subtracting out false asymmetries.

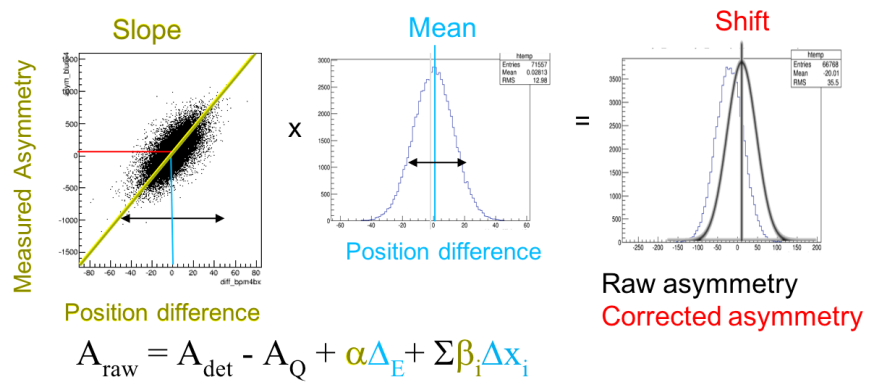

Figure 5-1: Linear regression method for evaluating and correcting false asymmetries from HCBA [183] 


\subsection{Beam Current Monitor}

BCMs are critical to parity experiments not only for systematic beam intensity asymmetry corrections, but also for reaching the desired statistics. Beam current fluctuations contribute significantly to the detector noise in the asymmetry measurement. The current must be normalized out on a helicity-pair to helicity-pair basis to achieve the desired statistical precision during experimental running. The schematic diagram of the HallA BCM system is presented in Fig. 5-2.

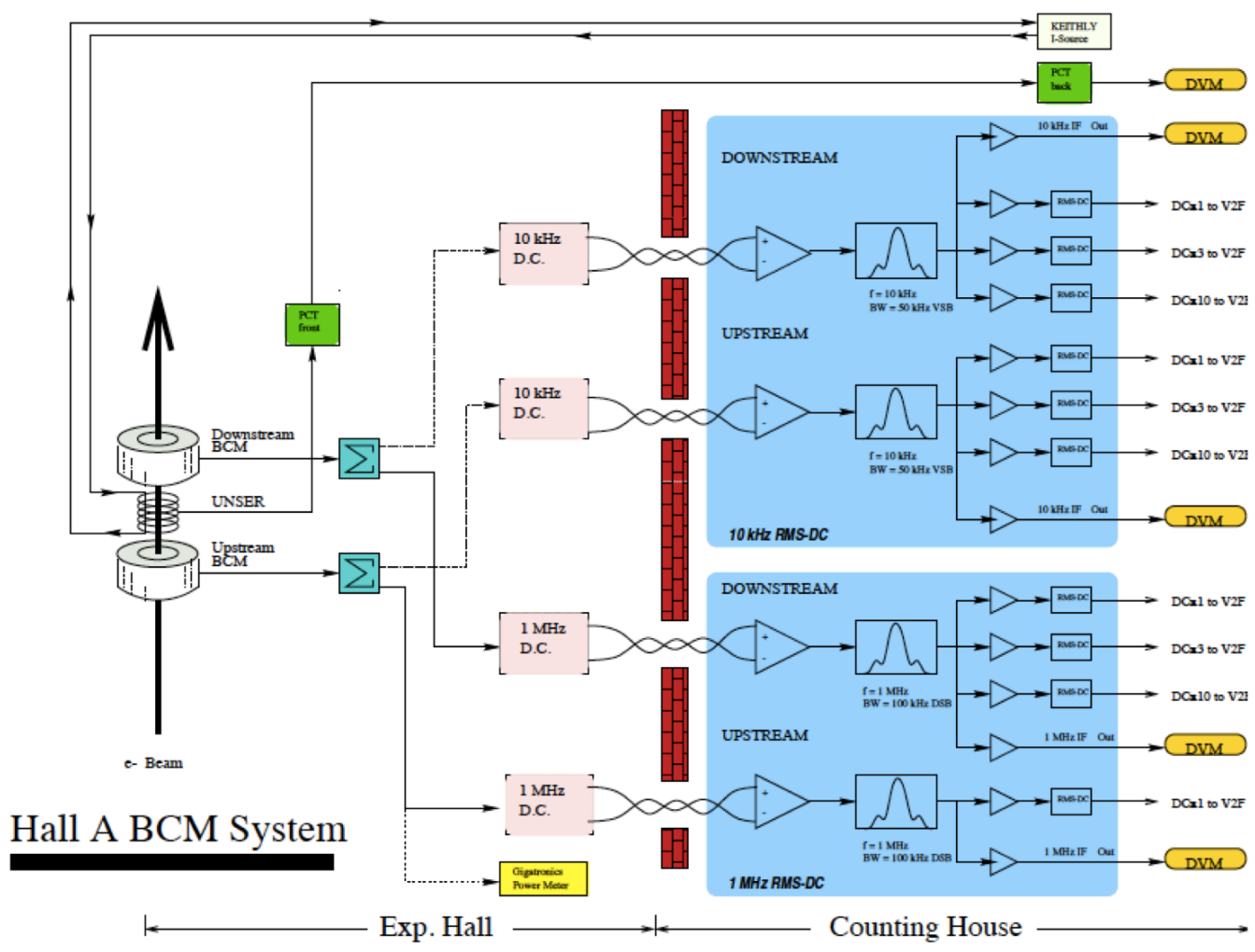

Figure 5-2: The schematic diagram of the BCM system [96]

The parts of the BCM system used in parity experiments are the User and the $1 \mathrm{MHz}$ system. The $1 \mathrm{MHz}$ system consists of two stainless steel pill-box cavities, tuned and critically coupled (Q 1500) to the $1497 \mathrm{MHz}$ component of the beam. The RF signal is frequency down-converted and sent to an RMS-DC converter which provides a DC output proportional to the beam current to be sent to the 
DAQ's.

The $1 \mathrm{MHz}$ BCMs require periodic calibrations against a very linear device. The BCM calibration pedestals are determined using the Unser current monitor, which is designed to be very linear at short time scales (but drifts over several minutes). The Unser is a parametric current transformer (PCT), sensitive to the DC component of the magnetic field generated by the beam current around the beam pipe, whose nominal response to beam current is determined by its transformer [181].

\subsubsection{BCM resolution}

Resolution can be approximately assessed via taking "double differences" between alike beam monitors. $\mathrm{BCM}$ resolution $\sigma_{\text {res }}$ can be assessed from double difference widths $\sigma_{D D}$ of upstream and downstream BCMs as follows. The double difference asymmetry is given by taking the difference between the charge asymmetry as measured by the upstream BCM and the downstream BCM

$$
A_{D D}=A_{U B C M}-A_{D B C M}
$$

This cancels out the real beam noise from intensity fluctuations and leaves the beam monitor electronics noise. Then, the RMS width of the double difference is related to the BCM resolution (assuming no common-mode noise between the two separate monitors)

$$
\sigma_{D D}=\sqrt{\sigma_{r e s, U B C M}^{2}+\sigma_{r e s, D B C M}^{2}}
$$

Assuming, since the two monitors are alike, that they have the same resolution, we obtain

$$
\sigma_{\text {res }}=\sigma_{D D} / \sqrt{2}
$$




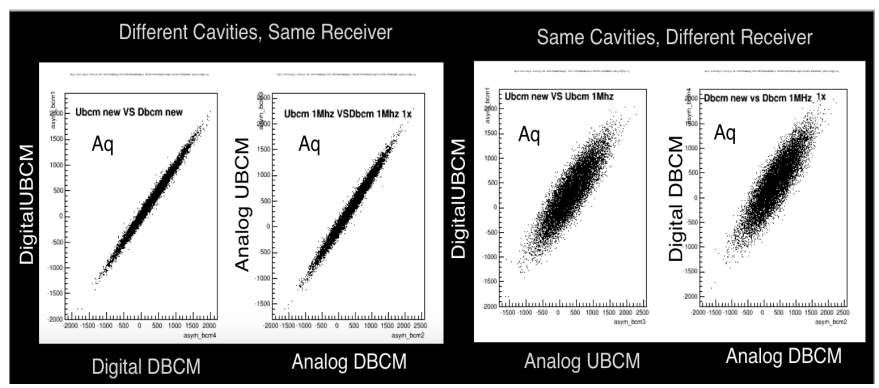

Figure 5-3: Correlations between beam asymmetry as measured by upstream and downstream BCMs for the analog $1 \mathrm{MHz}$ receivers and the digital receivers. Run2333,4.4GeV,12uA [183]

Examining correlations and common mode noise, as in Fig: 5-3, helps establish relationships between monitors and correct measured asymmetries. However, uncorrelated noise (i.e. electronics noise) hurts statistical error via reducing the monitor resolution. For PQB, we want good resolution to minimize our statistical error. We need to know our resolution to determine whether our widths make sense and which monitors are most useful to us during experimental running.

\subsubsection{Current Dependence}

Beam Asymmetry widths as measured in the Hall and in the injector at various currents are shown in Table 5.4 for a $30 \mathrm{~Hz}$ helicity flip rate. Higher currents generally tend to be associated with smaller asymmetry RMS widths. PREXII will run at $70 \mu \mathrm{A}$ and the $30 \mathrm{~Hz}$ widths appear to be reasonable as compared with previous parity experiments.

\begin{tabular}{l|r|r|r|r|} 
Run & Beam Energy & Current & Aq RMS & BCM \\
\hline 2358 & $8.8 \mathrm{GeV}$ & $13.7 \mu \mathrm{A}$ & $310 \mathrm{ppm}$ & $1 \mathrm{MHz}$ HallA \\
2488 & $8.8 \mathrm{GeV}$ & $60 \mu \mathrm{A}$ & $122 \mathrm{ppm}$ & $1 \mathrm{MHz}$ HallA \\
2494 & $8.8 \mathrm{GeV}$ & $45 \mu \mathrm{A}$ & $118 \mathrm{ppm}$ & $1 \mathrm{MHz}$ HallA \\
1905 injector & $8.8 \mathrm{GeV}$ & $60 \mu \mathrm{A}$ & $224 \mathrm{ppm}$ & Injector 0L02
\end{tabular}

Table 5.1: These are small beam charge asymmetry widths (30Hz) [183] 
The current dependence of the BCM resolution (as assessed from double difference widths of upstream and downstream analog $1 \mathrm{MIHz}$ BCMs) is shown in Fig. 5-4. The resolution of the $1 \mathrm{MHz}$ BCM system improves with higher current. For $30 \mathrm{~Hz}$, at $60 \mathrm{uA}$, we have a resolution of $11 \mathrm{ppm}$, which is encouraging for MOLLER. During beam studies, the analog BCMs behaved well most of the time and the resolution looks sufficient for PREXII $(>70 \mathrm{uA}, 120 \mathrm{~Hz})$

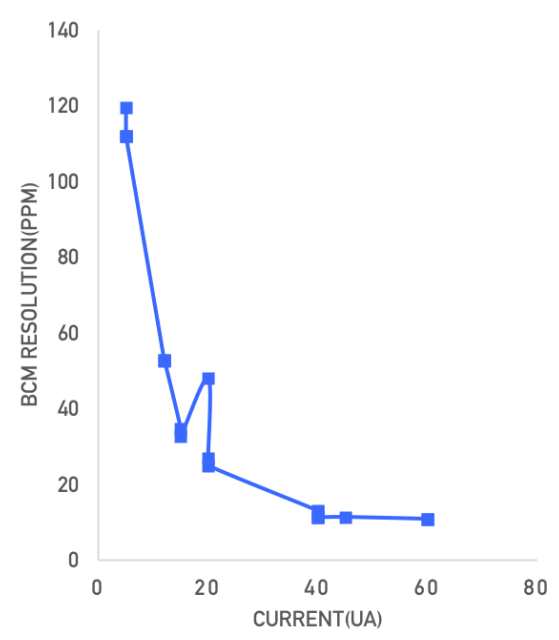

Figure 5-4: BCM resolution vs. current [183]

\subsubsection{Small Angle Monitors}

Small Angle Monitors (SAMs) are 8 quartz detectors with light guides and photo-multiplier tubes placed around the beamline downstream of the pivot/target region as shown in Fig. 5-5. The SAM symmetric design helps disentangle helicity correlated beam position and angle differences. For PREXII, they serve as an important secondary beam monitor for cross-checks of beam charge and position, and potentially beam halo as well. Additionally, the SAMs bear similarity to the detectors which will be used for MOLLER. Their operation during PREXII will serve as an important stepping stone towards towards obtaining a better understanding of beam and detector behavior in preparation for MOLLER. 


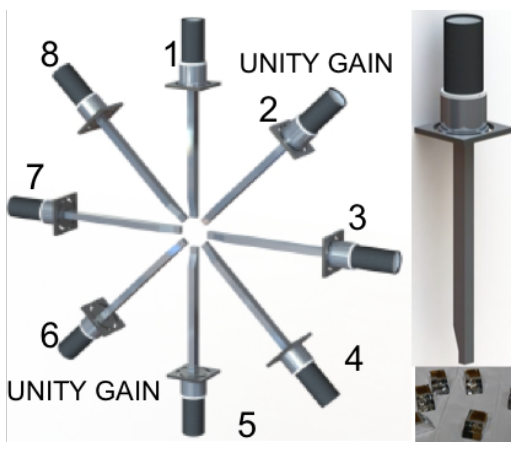

Figure 5-5: SAMs: 8 quartz detectors with light guides placed around beam line. During testing in 2015-2016, only two of the SAMs $(2,6)$ had unity gain bases for the PMT's, while the other 6 had higher gain bases for a large dynamic range during testing. During PREXII, the rates are expected to be very high, so all of the SAMs will have unity gain PMT bases.

SAM's were used in 2015-2016 beam studies to verify the resolution of the $1 \mathrm{MHz}$ BCM system. SAMs are sensitive to beam current fluctuations as shown in Fig. 5-6 and beam position fluctuations as shown in Fig. 5-7. SAM pairs on opposite sides of the beamline tend to have near equal and opposite dependence on beam position motion.
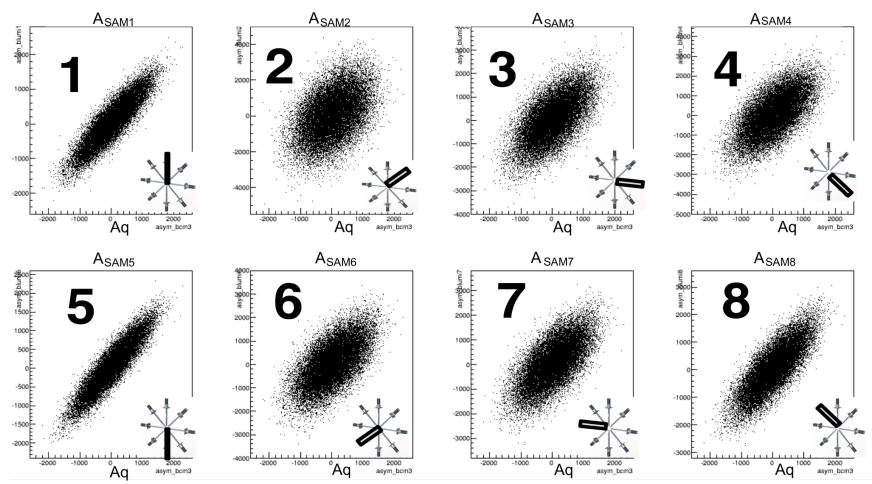

Figure 5-6: SAM Asymmetry vs. BCM Aq. SAMs are normalized to a BCM to eliminate BCM correlated noise. $A_{\text {norm }}=A_{S A M}-A_{q}$. 


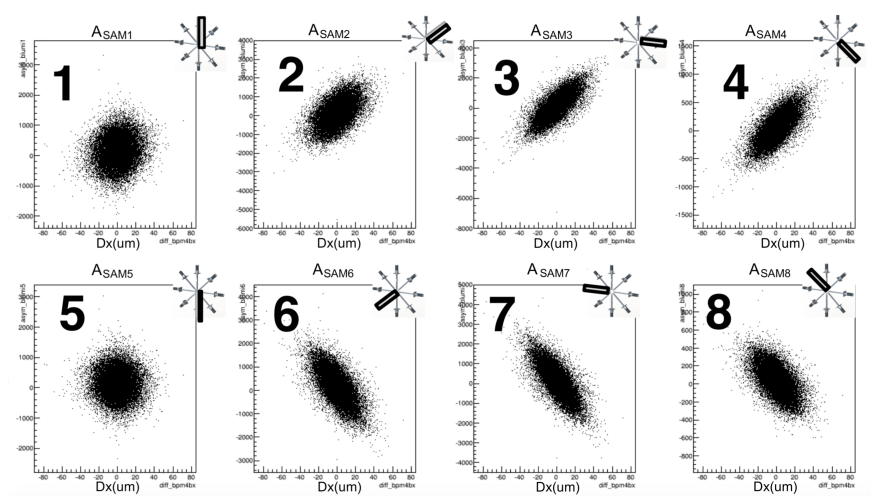

Figure 5-7: SAM Asymmetry vs BPM position difference 4bx. Regress with respect to BPM position differences Dx, Dy to eliminate BPM correlated noise. Combinations of SAM pairs such as SAM1+SAM5 or SAM3+SAM7 also tend to cancel BPM correlated noise.

By examining SAM pairs (SAM1,5 in Fig. 5-5) which are normalized to measured beam current, and regressed with respect to several BPMs to reduce beam position noise, we assess the $\mathrm{BCM}$ resolution in the following way ${ }^{1}$ :

$$
\begin{gathered}
S_{+}=A_{\text {norm }, S A M 1}+A_{\text {norm }, S A M 5}=A_{S A M 1}+A_{S A M 5}-2 A_{B C M} \\
S_{-}=A_{n o r m, S A M 1}-A_{n o r m, S A M 5}=A_{S A M 1}-A_{S A M 5} \\
\sigma_{+}^{2}=\sigma_{1}^{2}+\sigma_{5}^{2}+4 \sigma_{B C M}^{2} \quad \sigma_{-}^{2}=\sigma_{1}^{2}+\sigma_{5}^{2} \\
\sigma_{r e s, B C M}=\frac{1}{2} \sqrt{\sigma_{+}^{2}-\sigma_{-}^{2}} \approx \frac{1}{\sqrt{2}}\left(\sigma_{U B C M}-\sigma_{D B C M}\right)
\end{gathered}
$$

Performing these computations with the SAMs provided independent confirmation of the BCM resolution obtained by taking double-differences. For a particular solid target run, we compare the double-difference implied BCM resolution with

\footnotetext{
${ }^{1}$ Note: while regressing does take out some beam position noise, it introduces some beam position monitor noise. So strictly speaking, for SAMs with greater amounts of beam motion, this method may result erroneously in the sum having a narrower width than the subtraction. Without regression (assuming SAM1,5 have opposite dependence on position): $S_{-}=A_{S A M 1}-$ $A_{S A M 5}+2 \sum_{i} \beta_{i} \Delta X_{i}, \sigma_{-}=\sqrt{\sigma_{1}^{2}+\sigma_{5}^{2}+4 \sum_{i} \beta_{i}^{2} \sigma_{\Delta X_{i}}^{2}}$. With regression: $S_{-}=A_{S A M 1}-$ $A_{S A M 5}+2 \sum_{i}\left(\beta_{i} \Delta X_{i}-\beta_{i}^{r e g} \Delta X_{i}^{B P M}\right)$. If regression obtains the correct slopes $\beta_{i}^{r e g}=\beta_{i}$ and $\Delta X_{i}$ and $\Delta X^{B P M}$ are correlated except for BPM electronics noise $\Delta X_{i}^{B P M}=\Delta X_{i}+\sigma_{B P M}$, then $\sigma_{-}=\sqrt{\sigma_{1}^{2}+\sigma_{5}^{2}+4 \sum_{i} \beta_{i}^{2} \sigma_{B P M}^{2}}$ which may be greater than $\sigma_{+}=\sqrt{\sigma_{1}^{2}+\sigma_{5}^{2}+4 \sigma_{B C M}^{2}}$
} 
that obtained using SAM pairs. Fig. 5-8 shows the distributions containing the RMS values used in the computations of BCM resolution in Table. 5.2. ${ }^{2}$

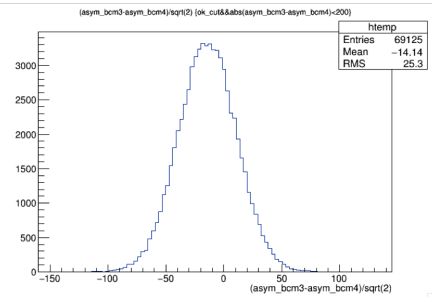

(a)

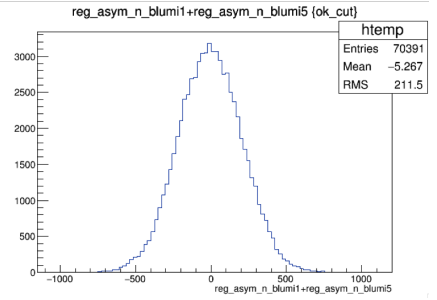

(b)

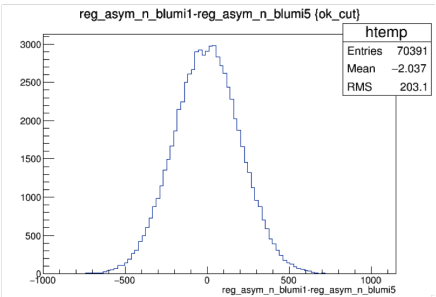

(c)

Figure 5-8: Run 2347: DVCS carbon target, 30Hz, 2.2GeV, $18.6 \mu \mathrm{A}$. (a) Asymmetry double difference between upstream and downstream $1 \mathrm{MHz}$ BCMs $A_{U B C M}-$ $A_{D B C M}$ (b) Sum of normalized SAM1,5 asymmetries $A_{\text {norm }, S A M 1}+A_{\text {norm }, S A M 5}=$ $A_{S A M 1}+A_{S A M 5}-2 A_{B C M}$ (c) Difference between normalized SAM1,5 asymmetries $A_{\text {norm }, S A M 1}-A_{\text {norm }, S A M 5}=A_{S A M 1}+A_{S A M 5}$

\begin{tabular}{|r|r|}
\hline computation & $\mathrm{RMS}$ \\
\hline$\sigma_{B C M}^{D D}:\left(A_{U B C M}-A_{U B C M}\right) / \sqrt{2} \mathrm{RMS}$ & $25.3 \mathrm{ppm}$ \\
$\sigma_{+}: A_{\text {norm,SAM } 1}^{\text {regress }}+A_{\text {norm }, S A M 5}^{\text {regress }} \mathrm{RMS}$ & $211.5 \mathrm{ppm}$ \\
$\sigma_{-}: A_{\text {norm }, \text { regAM } 1}^{\text {regress }}-A_{\text {norm }, S A M 5}^{\text {regress }} \mathrm{RMS}$ & $203.1 \mathrm{ppm}$ \\
$\sigma_{B C M}=\frac{1}{2} \sqrt{\sigma_{+}^{2}-\sigma_{-}^{2}}$ & $29.5 \mathrm{ppm}$ \\
\hline
\end{tabular}

Table 5.2: Verification of BCM resolution obtained by taking double-differences $\sigma_{B C M}^{D D}$ using SAM computations.

The results of Table 5.2 show consistency between the double-difference resolution $\sigma_{B C M}^{D D}$ and the BCM resolution obtained via SAM computations $\sigma_{B C M}$. The 25-30ppm BCM resolution observed is sufficient for PREXII when performed at $120 \mathrm{~Hz}$. In short, SAM's were successfully used in 2015-2016 beam studies to verify the resolution of the $1 \mathrm{MHz} \mathrm{BCM}$ system.

\footnotetext{
${ }^{2}$ Run 2347 , carbon $2.2 \mathrm{GeV}, 18.6 \mathrm{uA}, 30 \mathrm{~Hz}$, regressed with bpms 4a,4b,12 x and y , maxevent 5000
} 


\subsubsection{Energy Dependence}

MOLLER will run at $11 \mathrm{GeV}$ and the energy dependence of the beam asymmetry RMS width is important to assess for statistical reasons. Regarding energy dependence, we map out a variety of measurements of the beam asymmetry RMS width $\sigma_{A q}$ at different energies, currents, and effective frequencies, shown in Fig. 5-9. Higher energies don't appear to bear much relationship to the asymmetry widths observed, while higher currents may generally tend to be associated with smaller widths. PREXII will run at $1 \mathrm{GeV}$, so the energy dependence doesn't matter much for that experiment. However, MOLLER will run at $11 \mathrm{GeV}$ and so the effect of multiple passes in the accelerator on the intensity asymmetry noise was an important parameter to assess. There is no evidence that increasing the number of passes increases the beam asymmetry noise.

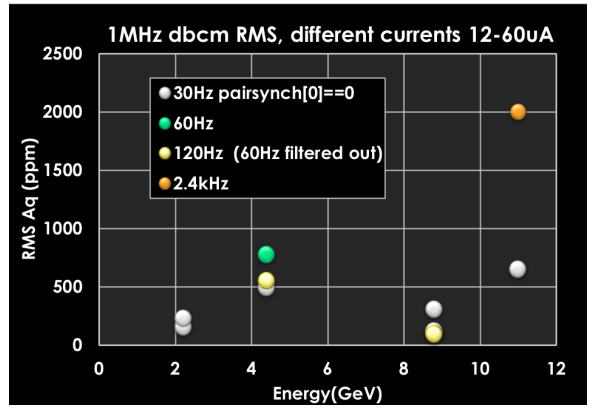

(a)

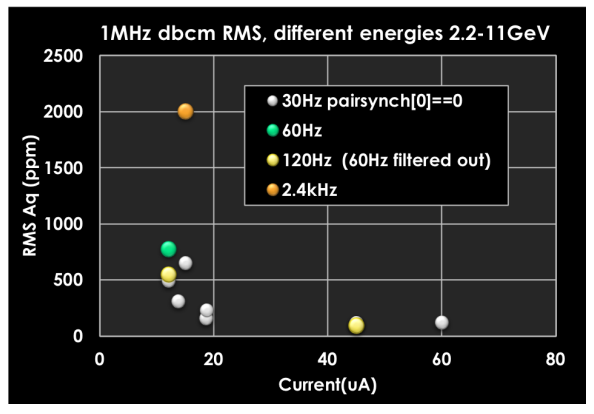

(b)

Figure 5-9: [183]

\subsubsection{Frequency Analysis}

Choice of helicity flip rate can strongly impact the ultimate precision of a parity experiment. MOLLER will run at a $2 \mathrm{kHz}$ flip rate, while PREXII will run at a $120 \mathrm{~Hz}$ or $240 \mathrm{~Hz}$ flip rate. Both the electron beam noise and the monitor resolution depends on the chosen helicity flip rate. Higher frequencies tend to result in smaller widths (scaled to $\sqrt{N}$ counting statistics). Ordinarily, we would perform studies by actually physically changing the helicity flip rate in the machine, but 
this procedure interferes with current running experiments, which had to operate at $30 \mathrm{~Hz}$ during $2015-2016$ beam studies. So, we found a way to 'mimic' higher helicity flip rates by oversampling the data collection rate. The vqwk-ADCs (used during Qweak), by default, oversample by 4. Over the course of a helicity window, instead of integrating the entire window and creating 1 data point, these ADCs integrate over 4 successive separate blocks, $b_{1}, b_{2}, b_{3}, b_{4}$, creates 4 data points as well as evaluating the total $b_{1}+b_{2}+b_{3}+b_{4}$ to obtain the integrated value for the entire helicity window. In data analysis, we can examine higher sample rates by accessing these sub-blocks behind the primary data stream as shown in Fig. 5-10. By taking block-differences between adjacent blocks, we can simulate taking helicity differences at a higher helicity flip rate which would have shorter integration periods. At a $30 \mathrm{~Hz}$ helicity flip rate, with the integration window set to $\sim 33 \mathrm{~ms}$, by examining sub-blocks, we can assess $60 \mathrm{~Hz}$ and $120 \mathrm{~Hz}$ frequencies by examining combinations of sub-blocks corresponding to $\sim 16 \mathrm{~ms}$ and $\sim 8 \mathrm{~ms}$. By examining helicity window sub-blocks, can mimic $60 \mathrm{~Hz}$ and $120 \mathrm{~Hz}$ Pockels Cell flip rates. Generally, if we set the integration time on the ADC to $T_{\text {int }}$, this corresponds to a base helicity flip rate frequency of $1 / T_{\text {int }}$, the vqwk ADC sub-blocks can access $f=1 / T_{\text {int }}, 2 / T_{\text {int }}, 4 / T_{\text {int }}$. By decreasing integration time on the ADC and examining sub-blocks, we can even access $\sim 1 k H z$ frequencies. We note that during 2015-2016 running, the helicity board was set to free clock instead of line synch mode $^{3}$, so frequencies were not exact multiples of or in phase with the $60 \mathrm{~Hz}$ line. The $60 \mathrm{~Hz}$ noise was not filtered out of the data as it normally would be and it manifested as a beat frequency in the $120 \mathrm{~Hz}$ sub-block analysis. By doing a quadlike combination of sub-blocks, we could manually filter out the $60 \mathrm{~Hz}$ beating and mimic a true line-synced $120 \mathrm{~Hz}$ flip rate as shown in Fig. 5-10

\footnotetext{
${ }^{3}$ though in 2019 we discovered linesynch wasn't working and wasn't staying locked all the time
} 


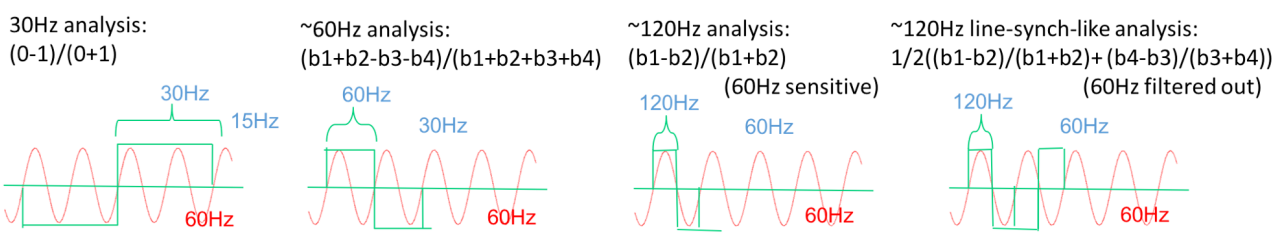

Figure 5-10: Sub-block analysis [183]

\subsubsection{Frequency Dependence}

We studied both the electron beam noise and the monitor resolution dependence on the effective helicity flip rate. The frequency dependence of the beam asymmetry $A q$ RMS width is shown in Table 5.4. When the width is scaled by $\sqrt{N}$ counting statistics, we clearly observe that higher frequencies tend to result in smaller widths. This is also the case for the double-differences widths which assess the BCM resolution as shown in Table 5.4. Double difference widths in $1 \mathrm{MHz}$ BCM system beats $\sqrt{N}$ statistics from the number of samples in the integration time and as we increase the repetition rate, we are 'winning' in that the level of noise at $30 \mathrm{~Hz}$ is more than at $60 \mathrm{~Hz}$, is more than at $120 \mathrm{~Hz}$.

These results are encouraging for PREXII and for MOLLER. For PREXII, based on these results, we will consider running at $120 \mathrm{~Hz}$ or even at $240 \mathrm{~Hz}$, since both the resolution of analog BCM system and the beam asymmetry width improves with higher frequency.

\begin{tabular}{l|r|r|r|r|r}
$\begin{array}{l}\text { Run } \\
\#\end{array}$ & $1 / T_{\text {integrate }}$ & $f$ & $R M S_{A q}$ & sub-block & $R M S_{A q} / \sqrt{f}$ \\
\hline 1905 & $\mathrm{~Hz}$ & $H z$ & $\mathrm{ppm}$ & analysis & $\begin{array}{r}\mathrm{ppm} / \sqrt{H z} \\
1905\end{array}$ \\
30 & 30 & 208.1 & Pairsynch=0 & 38.0 \\
1905 & 30 & 60 & 273.1 & $\frac{\left(b_{1}+b_{2}\right)-\left(b_{3}+b_{4}\right)}{b_{1}+b_{2}+b_{3}+b_{4}}$ & 35.3 \\
1902 & 30 & 120 & 212.7 & $\frac{1}{2}\left(\frac{b_{1}-b_{2}}{\left.b_{1}+b_{2}-\frac{b_{3}-b_{4}}{b_{3}+b_{4}}\right)}\right.$ & 19.42 \\
1902 & 284 & 568 & 643.5 & $\frac{\left(b_{1}+b_{2}\right)-\left(b_{3}+b_{4}\right)}{b_{1}+b_{2}+b_{3}+b_{4}}$ & 27.45 \\
& 284 & 1136 & 531.3 & $\frac{b_{1}-b_{2}}{b_{1}+b_{2}}$ & 15.81
\end{tabular}

Table 5.3: The frequency dependence of the beam asymmetry $A q$ RMS width. 8.8GeV, $60 \mu A$ [183]. $\frac{1}{2}\left(\frac{b_{1}-b_{2}}{b_{1}+b_{2}}-\frac{b_{3}-b_{4}}{b_{3}+b_{4}}\right)$ corresponds to $60 \mathrm{~Hz}$ filtered sub-block analysis. 


\begin{tabular}{l|r|r|r} 
frequency & Double Difference & sub-block & $R M S_{D D} / \sqrt{f}$ \\
$\mathrm{~Hz}$ & $R M S_{A_{U B C M}-A_{D B C M}} \mathrm{ppm}$ & analysis & $\mathrm{ppm} / \sqrt{H z}$ \\
\hline 30 & 75.0 & Nominal & 6.85 \\
60 & 93.5 & $\frac{\left(b_{1}+b_{2}\right)-\left(b_{3}+b_{4}\right)}{b_{1}+b_{2}+b_{3}+b_{4}}$ & 6.04 \\
120 & 85.6 & $\frac{1}{2}\left(\frac{b_{1}-b_{2}}{b_{1}+b_{2}}-\frac{b_{3}-b_{4}}{b_{3}+b_{4}}\right)$ & 3.91
\end{tabular}

Table 5.4: Double difference widths in $1 \mathrm{MHz}$ BCM system, assessing resolution dependence on frequency. 2-pass, multiple frequencies, $4.4 \mathrm{GeV}, 12 \mu \mathrm{A}$, ADC $1 / T_{\text {integrate }}=30 \mathrm{~Hz}$, Run $2333[183]$

\subsection{Digital Receivers}

\subsubsection{Operating Principles}

The following is a summary of John Musson's description of algorithms used in the digital receivers [196].

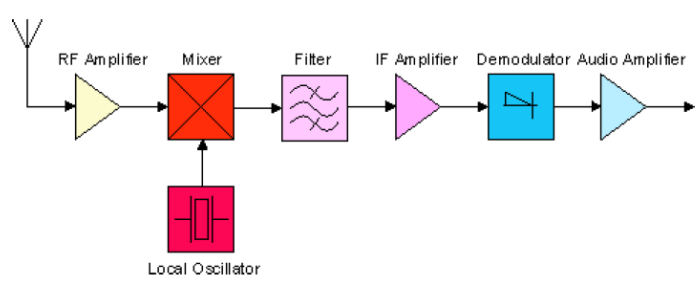

Figure 5-11: Functional block diagram of a basic superhet receiver, describing major subsystems. [196]

In analog superheterodyne (superhet) RF receivers, conventional mixing, phasing, and filtering are used as shown in Fig. 5-11. Critical to the RF receiver is the down-conversion to an intermediate frequency (IF) via a mixer. A mixer combines the desired signal from the antenna with a sinusoid, and subjects the pair to a nonlinear element, producing an upper side band and a lower side band (at the IF)

$$
\sin \left(\phi_{s i g}\right) \sin \left(\phi_{L O}\right)=\frac{1}{2}\left(\sin \left(\phi_{s i g}-\phi_{L O}\right)-\sin \left(\phi_{s i g}+\phi_{L O}\right)\right)
$$

where $\phi_{L O}=2 \pi\left(\omega_{L O} t+\phi_{0, L O}\right)$ is the local oscillator RF signal and $\phi_{s i g}=2 \pi\left(\omega_{\text {sig }} t+\right.$ $\left.\phi_{0, s i g}\right)$ is the incoming signal. As shown in Fig. 5-11, an RF signal is obtained, 
is translated to an intermediate frequency (IF) via a mixer filtered, translated a second time, detected, and demodulated. There are a variety of ways to convert IF AM signals into a DC output (1) use a diode which performs an absolutevalue operation, followed by a lowpass-filter. (b) multiply by a sinusoid of the same frequency and phase (3) if two quadrature sinusoids (i.e. cosine and sine) are used, the requirement of the LO having phase coherence with the transmitted carrier is removed. The LO (local oscillator) need to have minimal amplitude and phase fluctuations (and minimal frequency fluctuations/spectrally narrow).

In digital $\mathrm{RF}$ receivers, there is also downconversion to IF via a mixer as shown in the block diagrams in Fig. 5-15. This is followed by ADC conversion. We consider the sampling frequency $f_{s}$. One option, the most obvious choice, is to simply sample at a $4 \mathrm{X}$ the carrier frequency $f_{s}=4 f_{c}$. During a cycle, this will sample points I, Q, -I, -Q, every 90 degrees, where I and Q represent the In-phase (real) and Quadrature (imaginary) components of the IF signal as shown in Fig. 5-12a. The amplitude and phase can be computed from these data points via

$$
\begin{aligned}
& A=\sqrt{I^{2}+Q^{2}} \\
& \phi=\tan ^{-1}\left(\frac{I}{Q}\right)
\end{aligned}
$$

Another option (which the JLab system employs) is an under-sampling scheme, known as harmonic sampling at a sampling frequency $f_{s}=0.8 f_{c}$, where the sampling is over-rotated by 90 degrees. We still obtain I,Q,-I,-Q data stream at a lower rate of $f_{c}-f_{s}=0.2 f_{c}$ as shown in Fig. 5-12b. Theoretically, any $\left(0.8 f_{c}\right) / n$ is permitted.After the ADC, within the digital processor, the next steps are shown in Fig. 5-13a for the $4 f_{c}$ system and in Fig. 5-13b for the $0.8 f_{c}$ system. First the IF carrier $f_{c}$ is sampled, at the sample frequency $f_{s}$. Then the I,Q,-I,-Q data stream is demultiplexed into I-only, and Q-only components which each have half the original sample rate $f_{s} / 2$ (i.e. decimation of 2 ). Next the I,-I stream and the Q,-Q stream are multiplied by alternating $+/-1$ to make positive stream of I and 
Q. Finally a lowpass filter is applied to obtain the base I and Q signals.

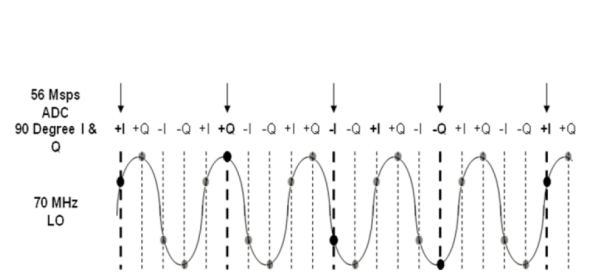

(a)

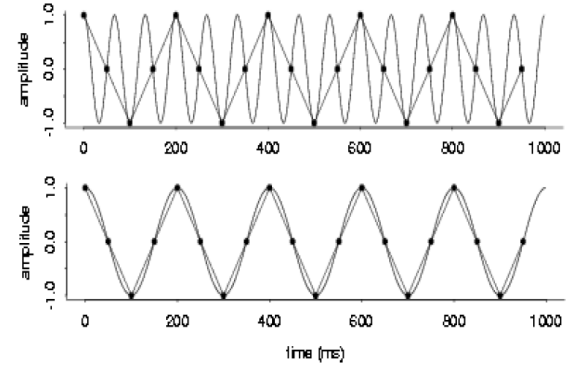

(b)

Figure 5-12: (a) Time-domain description of 4x I/Q sampling process. The output stream contains the rectangular representation of the input signal. (b)Time domain description of harmonic sampling, whereby the input signal is sub-sampled at a rate of $0.8 \mathrm{fc}$, resulting in an aliased signal of $\mathrm{fc}$ - $\mathrm{fs}$ retaining the phase and amplitude features of the original carrier. [196]

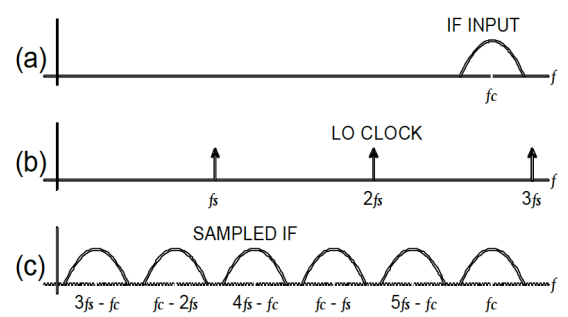

(a) $4 \mathrm{x}$ sampling

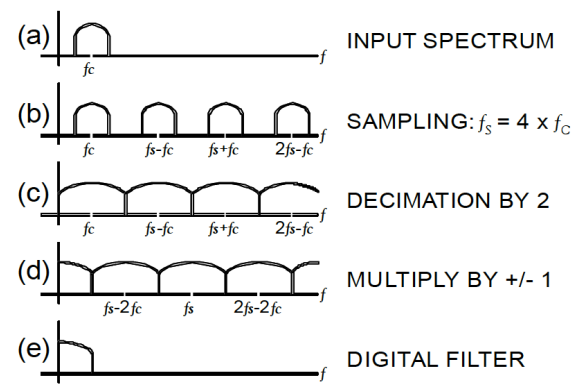

(b) harmonic sampling

Figure 5-13: (4x sampling) Frequency-domain description of the 4x I/Q sampling process. The original carrier (a), with information $\mathrm{BW}=\mathrm{B}$, is translated to baseband (b) by the sampling frequency. Decimation stretches the spectra (c), while reducing the data rate. Finally, multiplying by $+/-1$ sequence and filtering produces a faithful baseband signal.(d) low pass filtering (harmonic sampling)Frequency domain description of harmonic sampling, whereby the input signal is sub-sampled at a rate of $0.8 \mathrm{fc}$, resulting in an aliased signal of $\mathrm{fc}-\mathrm{fs}$ retaining the phase and amplitude features of the original carrier. [196]

\subsubsection{Cavity BPMs}

For low-current running during PREXII/CREX for the Vertical Drift Chamber (VDC) optics tracking calibration process, the cavity BPMs, which can operate 


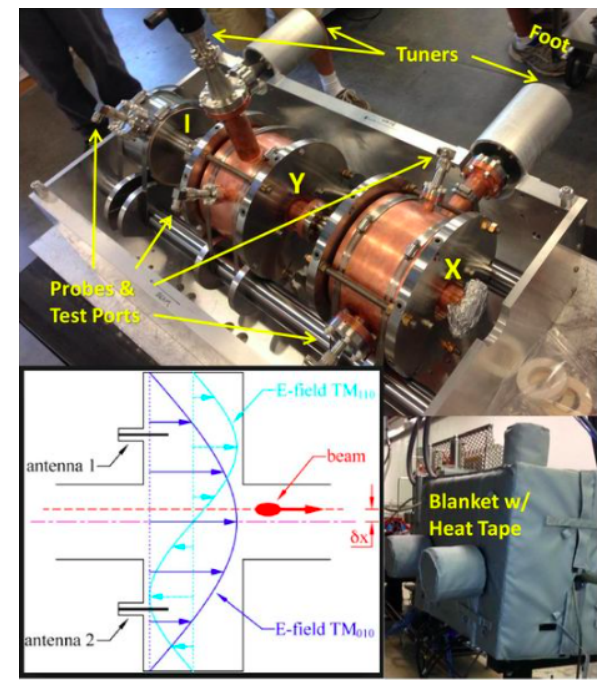

(a)

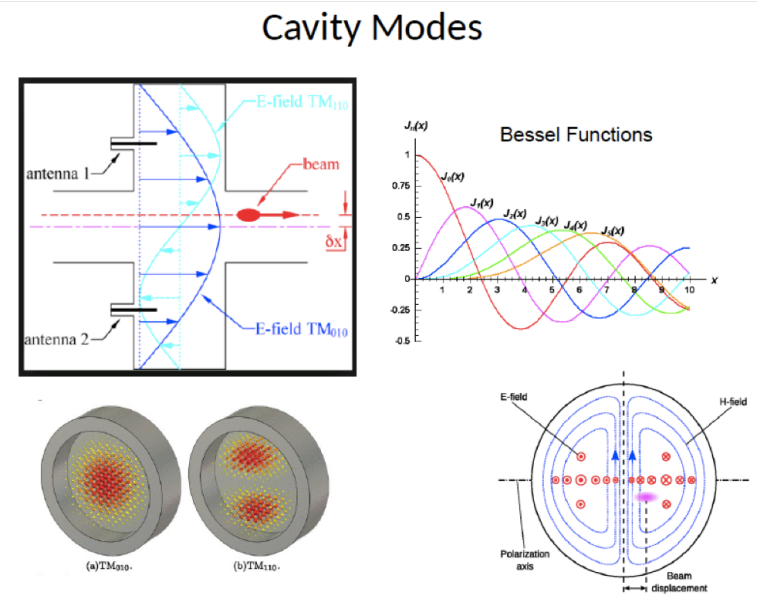

(b)

Figure 5-14: (a) Cavity BPM [199] (b)Cavity Modes [106]

at very low-currents, are required. They are known to achieve $100 \mu \mathrm{m}$ resolution with $120 \mathrm{~Hz}$ bandwidth at 1nA of beam current [199]. A cavity BPM is shown in Fig. 5-14a. The receiver for the cavity BPM is shown in Fig. 5-15c in Sec. 5.3.

\subsubsection{Digital Electronics}

The block diagram for this procedure using digital signal processors (DSP) and field-programmable gate array (FPGA) designs is shown in Fig. 5-15. In the JLab system, we also have the digital systems pass through a D/A converter so that analog DC voltages can be passed to our DAQ system. It is important in parity experiments to have this option of an analog BCM signal coming out of the digital systems, so that we can apply certain filters to better match the detector response and the BCM response. Since the detectors are being normalized with these BCMs, it is important to match them. 


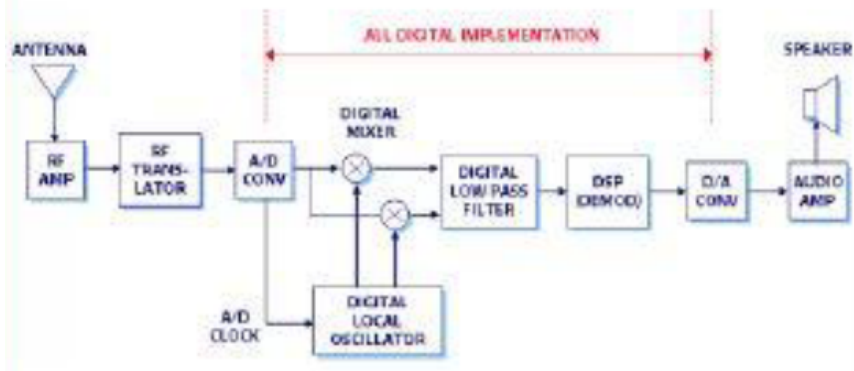

(a)

Stripline BPM Electronics

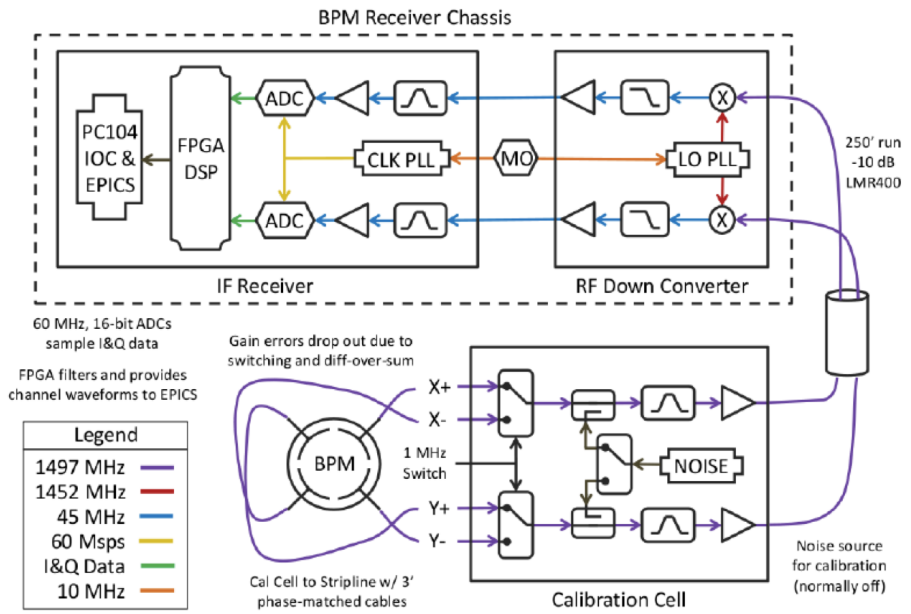

(b)

\section{Cavity BPM Electronics}

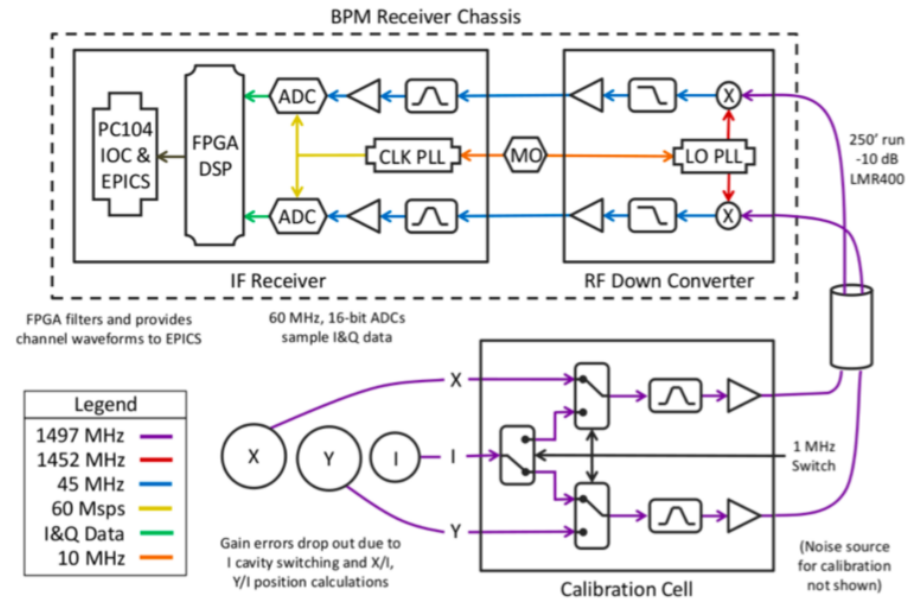

(c)

Figure 5-15: (a)Functional block diagram of a digital receiver system, demonstrating similarity to analog system architecture, but with DSP-specific blocks. All blocks after the ADC are numerically implemented, achieving near-perfect performance [196] (b)Stripline BPM electronics (c)Cavity BPM electronics 


\subsubsection{Digital BCM receiver measurements}

A design challenge in digital systems minimizing the system latency, or processing delay. It takes time to perform operations digitally and apply various digital filters. We observed evidence in 2016 beam study data that the digital $\mathrm{BCM}$ receivers were delayed in time relative to the analog $1 \mathrm{MHz} \mathrm{BCM}$ receivers. The symptoms of this delay are shown in Fig. 5-16a as noise in cross correlations between the BCMs. The proof of the delay came from tune-mode data, where the electron beam was in tune-mode, and a square waveform was detected by the analog BCM, but it was both significantly smoothed and delayed by the digital receivers as shown on a scope in Fig. 5-16b.

The Jlab new digital receiver system has 3 outputs: "fast"/OPS, "adjustable", and "slow"/EPICS. The Digital receiver 'slow'/EPICS output setting has a $5.1 \mathrm{~ms}$ delay(measured with tune beam) due to several low pass filters and additional latency. By changing the output mode to 'fast', removing many of the applied low-pass filters, we can reduce the delay to 16 -18us(relative to the $1 \mathrm{MHz}$ system) and 26-28us total delay relative to actual electron beam. We can further reduce the delay by by-passing several filters in 'straight through' mode, obtaining $11 \mu s($ totaldelay $)=4.5 \mu s($ latency $)+6.5($ risetime $)$. By comparison, the analog $1 \mathrm{MHz}$ system has a small $\sim 10 \mu s($ delay $)=2.5 \mu s($ latency $)+7-8 \mu s($ risetime $)$. This low-latency setting in the digital BCM (and the analog BCM) will work for us in PREXII. We note that in 'straight-through' mode, there was some high frequency noise observable on a scope, which appeared to come from a power supply running near ribbon cables, so this power supply was unplugged from the back panel unused channels to reduce the noise. 


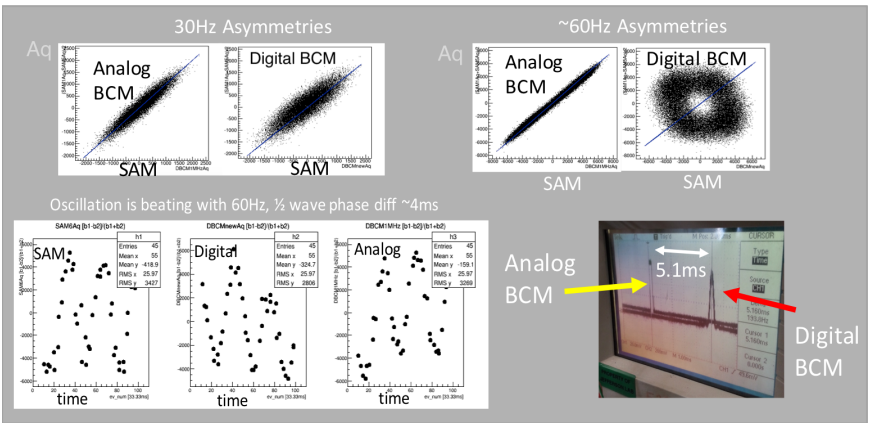

(a)

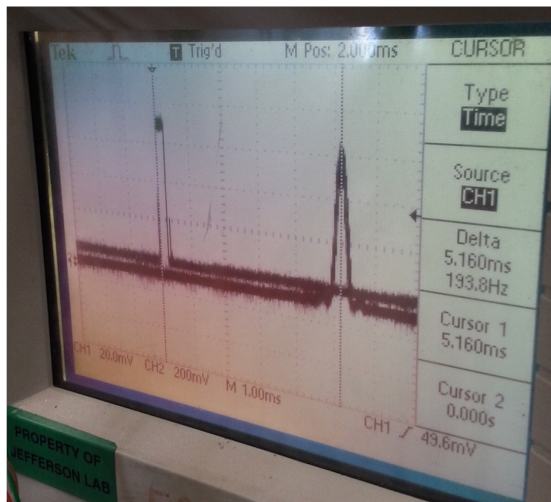

(b)

Figure 5-16: (a)Delay evidence (comparing BCMs with SAM(small angle monitor) quartz/PMT monitors) (b)Digital Latency [183]: Analog dbcm tune beam is the square wave on the left and Digital dbcm tune beam signal is the curved peak on the right

\section{Linearity}

By definition, dynamic range is the ratio of the desired USB or LSB signal and the worst-offending harmonic from the mixing process. Fig. 5-17 is a third-order intercept diagram, since the third-order cross term is usually the most prevalent offending signal. There are many attenuators and gain settings both within the digital receiver system and outside of it. Fig. 5-18 shows an external attenuator as well as an internal attenuator, which can be changed. On the bench, using a '2 tone' measurement, we found attenuators which we thought would achieve $<0.3 \%$ non-linearity at 150uA (the CREX running current) for the 'straight through' mode desirable for PREXII/CREX. It is also desirable to have a large enough $\mathrm{DC}$ analog output signal at the running currents, analogous to the $1 \mathrm{MHz} \mathrm{BCMs}$, which produce $2 \mathrm{~V}$ around $70 \mathrm{uA}$.

We performed a linearity measurement after installing the digital receivers in the Hall A counting house [182]. To study the receiver linearity, we hooked up an RF generator (SRS SG384) down in the hall at 1497MHz and tried putting in $-80 \mathrm{dBm}$ to $8 \mathrm{dBm}$ (where $-40 \mathrm{dBm}$ corresponds to $1 \mathrm{uA}$ in the BCM cavity) for two different internal gain settings in the digital BCM receivers (gain "2" and gain 
Receiver Dynamic Range Parameters

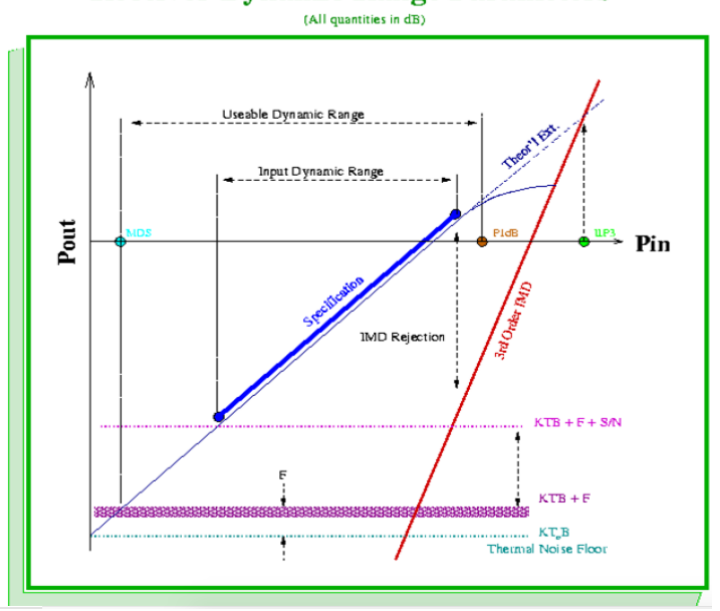

Figure 5-17: Third-order intercept diagram, often used to determine system linearity. The desired system gain is depicted by the blue line, while the red line demonstrates the appearance of undesired third-order signals from the mixing process, and/or other nonlinearities.[196]

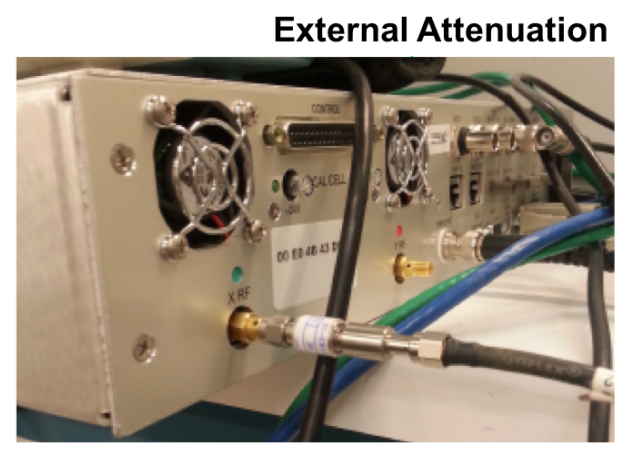

(a)

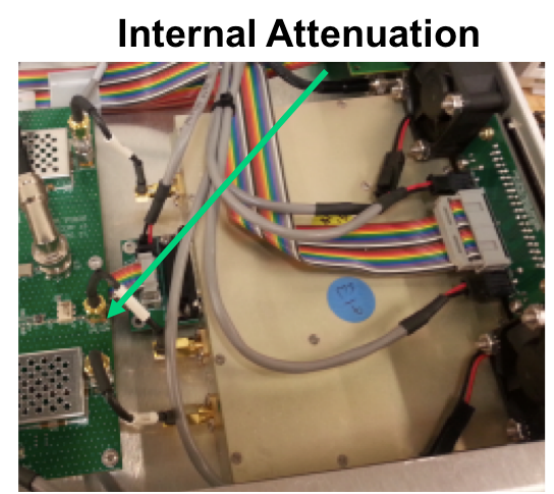

(b)

Figure 5-18: (a)Digital Receiver - External Attenuation (b)Digital Receiver - Internal Attenuation 


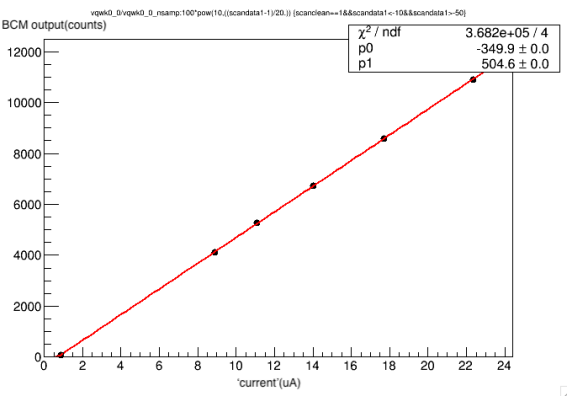

(a) Linearity Test

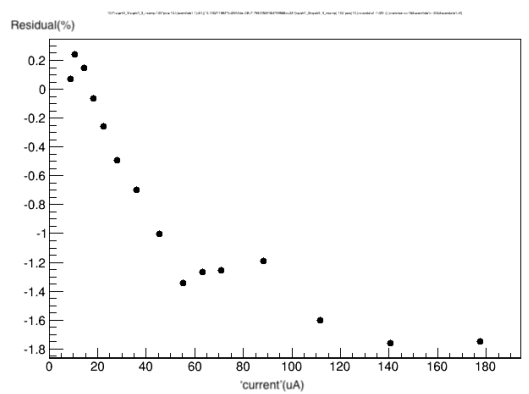

(c) Analog

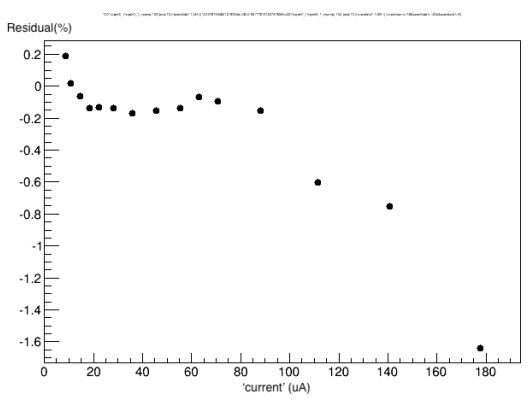

(b) Digital gain 2

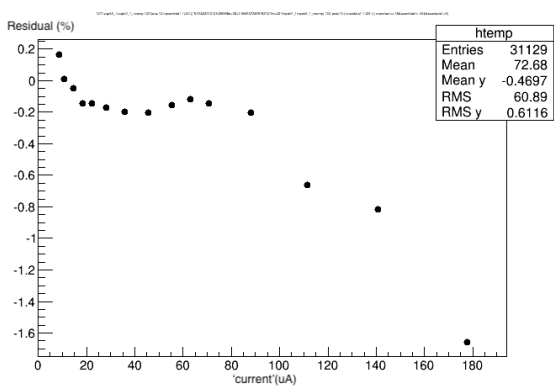

(d) Digital gain 5

Figure 5-19: BCM linearity test (a) Digital BCM signal (channels) vs 'current' (uA) (b) Digital DBCM residual (\%) vs 'current' (uA) for hex gain "2" (c) Analog $1 \mathrm{MHz}$ 1X DBCM residual (\%) vs 'current' (uA) (d) Digital DBCM residual (\%) vs current $(\mathrm{uA})$ for hex gain 5

"5" set with a hex switch). The receivers were located in the counting house and we estimated $-21.7 \mathrm{~dB}$ from the cable transporting the RF signal upstairs. The measurements are shown in Fig. 5-19 for both the digital receiver and the $1 \mathrm{MHz}$ system. We fit to the linear portion of the data (low current), then assessed the deviation from linearity in the residuals.

For the digital receivers, the linearity is comparable to to the analog BCMs. If we properly make use of the digital system settings, it looks quite linear at PREXII current $70 \mu \mathrm{A}$. CREX, however, must run at $150 \mu \mathrm{A}$ and we need an attenuator on the digital BCMs to use them in CREX. We could get better linearity at $150 \mathrm{uA}$ by adding a $3 \mathrm{~dB}$ attenuator outside the receiver after PREXII is over, when we start doing CREX, and that will make the 150uA look like 75uA (near PREX current) to the receiver. We need an attenuator on the digital BCMs to use them during 


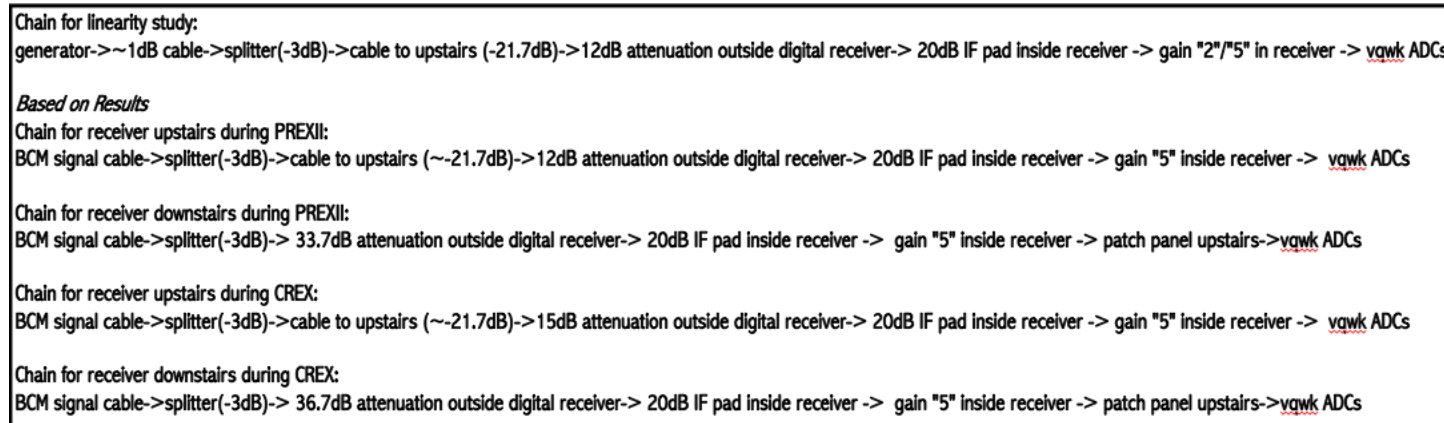

Figure 5-20: Chain from BCM signal to receiver to ADC. Configurations for linearity study, for PREXII, CREX, whether receiver is ultimately located upstairs or downstairs.

CREX. These configurations should be sufficient for PREXII and CREX.

Configuration for PREXII/CREX:The chain from generator to receiver to ADC for the study is shown in Fig. 5-20. Also shown are the chains for PREXII running and CREX running if the receivers are installing in the counting house. However, there is some debate as to whether the receivers should be located upstairs or down in the Hall. Putting the receivers upstairs, protects them from potential radiation damage in the Hall, but there is the potential for slow temperature fluctuations in the cabling. The slow fluctuations are not likely to cause issues for parity experiments. Nevertheless, we show a chain configuration in the case that the receivers are installed in the Hall downstairs, which must account for the fact that the cable length going upstairs acts as an attenuator, so moving the receiver downstairs means adding attenuators to avoid saturation.

This is the cavity BPM digital receiver configuration as of summer 2016 for posterity. 


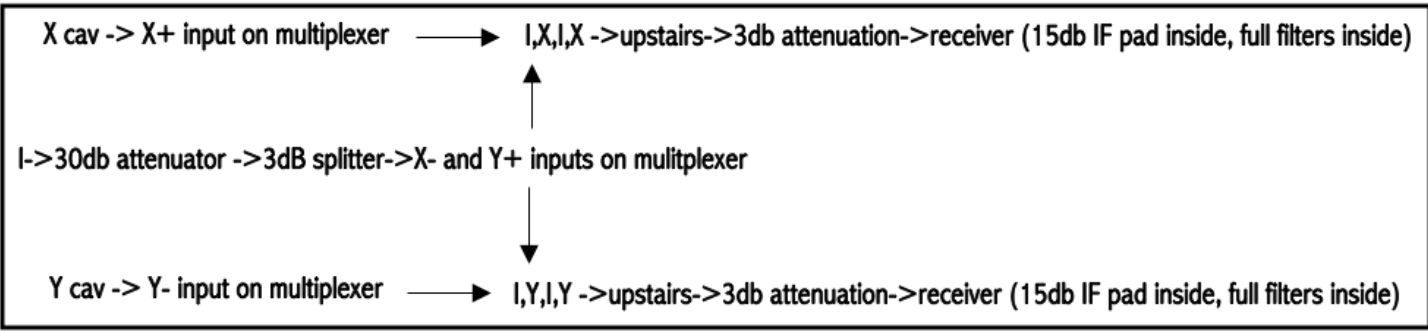

Figure 5-21: BPM cavity receiver configuration in 2016. BPM cavity receivers 1OC1PM1H04B,C,D = =bpm4b,4c,4d in CH upstairs. Outputs set to DAC1,2,3 $=\mathrm{X}, \mathrm{Y}$, and $\mathrm{I}$.

\subsection{Beam Position Monitor}

\subsubsection{Introduction}

There are several types of standard beam position monitors (BPMs) used throughout the accelerator. The BPM types include: antenna/wireline BPMs(types M15 and M20), Stripline BPMs, and Cavity BPMs. The majority of BPMs used in parity experiments are antenna BPMs of type M15 as shown in Fig. 5-22 ${ }^{4}$.

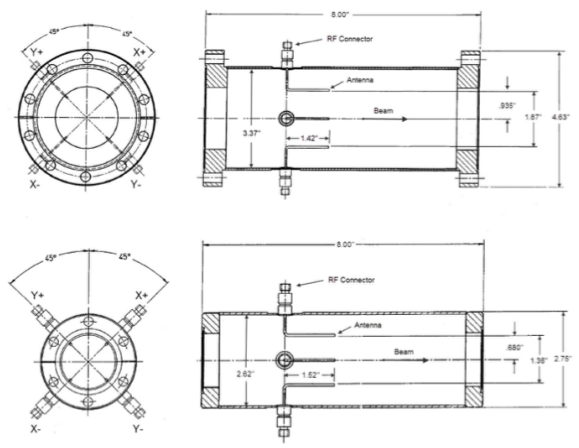

Figure 5-22: Schematic Drawing of an M20 bpm (top) and M15 bpm(bottom) [104] [101] [103]

The stripline beam position monitors $(\mathrm{BPMs})$ consist of four wire antenna which each pickup an RF signal from the pulsed electron beam as it passes near the wires. The four wire channels are denoted $\mathrm{X}+(\mathrm{xm}), \mathrm{X}-(\mathrm{xm}), \mathrm{Y}+(\mathrm{yp})$, and

\footnotetext{
${ }^{4}$ The larger M20 is used in Arc 1, Arc 2, and the Extraction region of the accelerator and the smaller M15 can is used elsewhere [104]
} 
Y- (ym). The signal each wire picks up is proportional to both e-beam intensity and the proximity of the e-beam to the wire in question. The raw signal from these wires is typically autogained, so that the magnitude of the DC converted channel signal is $3 \mathrm{~V}$ and only small, fast deviations can be picked up by the DAQ.

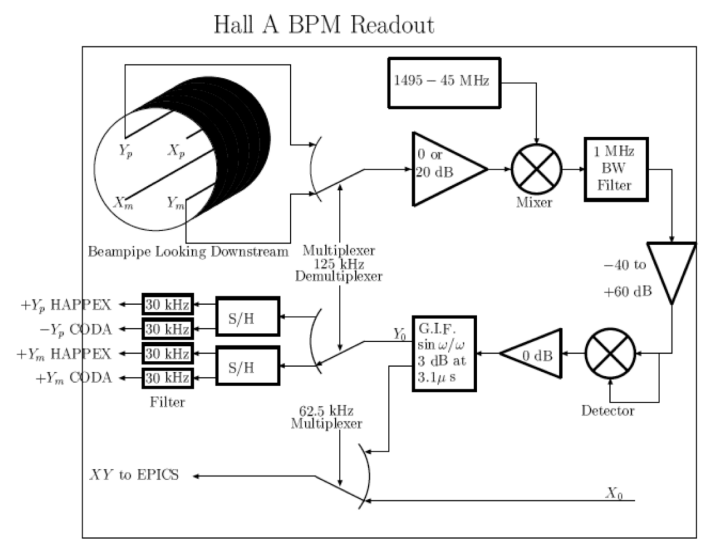

(a)

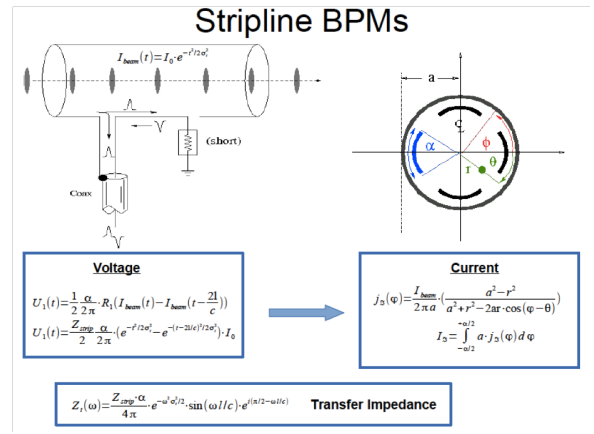

(b)

Figure 5-23: (a) Schematic of the BPM readout electronics [96] (b)Stripline BPMs [106]

In the standard analysis, three combinations of the four wire channels are calculated: bpm x position, bpm y position, and bpm wire sum. The e-beam position is monitored directly by the calculated bpm x,y positions. Due to autogaining, the e-beam current is not directly monitored by the bpm wire sum, which only is used to assess fast, small changes such as charge asymmetry Aq. The mathematical manipulations in the analysis are as follows:

$$
\begin{gathered}
b p m x=\kappa(x p-x m) /(x p+x m) \\
b p m y=\kappa(y p-y m) /(y p+y m) \\
b p m w s=x p+x m+y p+y m
\end{gathered}
$$

Where $\kappa$ is a wireline bpm calibration constant set to $18.76 \mathrm{~mm}$ for M15 antenna BPMs [94] [97]. This equation, used by PREX analysis, an approximation, as 
there can be differences in the individual wires gains and offsets. A more accurate equation used by OPS and Qweak analysis is given by

$$
\begin{gathered}
b p m x=\kappa_{x} \frac{\left(x p-x p_{o f f}\right)-\alpha_{x}\left(x m-x m_{o f f}\right)}{\left(x p-x p_{o f f}\right)+\alpha_{x}\left(x m-x m_{o f f}\right)} \\
b p m y=\kappa_{y} \frac{\left(y p-y p_{o f f}\right)-\alpha_{y}\left(y m-y m_{o f f}\right)}{\left(y p-y p_{o f f}\right)+\alpha_{y}\left(y m-y m_{o f f}\right)} \\
b p m w s=x p+x m+y p+y m
\end{gathered}
$$

where the pedestal offsets are found in calibration runs in Qweak analysis, but OPS uses different values for the offsets.

The antennas are either oriented along the horizontal/vertical axes or they are oriented at $\pm 45^{\circ}$. Those that are oriented along $\pm 45^{\circ}$ are rotated in analysis so that bpmx corresponds to horizontal and bpmy corresponds to vertical. Antenna and Stripline BPMs are rotated to avoid the synchrotron light produced in the magnetic bends [102]. Below is a table 5.5 indicating which bpm types are used where and whether a bpm is rotated or unrotated (note the "M15m" means M15mini, a smaller version of M15 with a difference in sensitivity): 


\begin{tabular}{l|r|r|r|r} 
Region & BPMs & rotated & type & sensitivity \\
\hline Injector $130 \mathrm{keV}$ & $1102,4,6 \& 0 \mathrm{I01}, 1 \mathrm{~A}, 2,2 \mathrm{~A}, 5,7$ & no & M15m & $13.7 \mathrm{~mm}$ \\
\hline Injector $130 \mathrm{keV}$ & 2I01,2I02 & no & M20-mod & $25.67 \mathrm{~mm}$ \\
\hline Injector $6.28 \mathrm{MeV}$ & 0L01-0L04 & yes & M15 & $18.81 \mathrm{~mm}$ \\
\hline Injector $28.8 \mathrm{MeV}$ & $0 \mathrm{~L} 05$ & yes & M15 & $18.81 \mathrm{~mm}$ \\
\hline Injector $123 \mathrm{MeV}$ & 0L06-0L10, 0R01-0R06 & yes & M15 & $18.81 \mathrm{~mm}$ \\
\hline Hall A Compton & 1P01A,1P02A,1P02B & yes & M15 & $18.81 \mathrm{~mm}$ \\
& $1 \mathrm{P} 03 \mathrm{~A}$ & & M20 & $25.67 \mathrm{~mm}$ \\
\hline HallA Arc & $1 \mathrm{C} 01,08,10,12,14$ & yes & M15 & $18.81 \mathrm{~mm}$ \\
\hline HallA & 1H01, 1H04A, 1H04E & yes & M15 & $18.81 \mathrm{~mm}$ \\
& $1 \mathrm{H} 04$ & yes & M15m & $13.7 \mathrm{~mm}$
\end{tabular}

Table 5.5: BPM types in injector and HallA [108]

It should be noted that previous experiments PREXI, and potentially HAPPEX used calibration constants of $18.76 \mathrm{~mm}$ for all bpms. In particular, injector measurements in the $130 \mathrm{keV}$ region had their calibration constants set for M15 bpms, when they are in fact M15-mini bpms. So one should be aware, in examining old plots of injector data, that the position differences in the $130 \mathrm{keV}$ region are actually $13.7 \mathrm{~mm} / 18.8 \mathrm{~mm} 73 \%$ of their reported values. The $0 \mathrm{~L}$ region data was correctly treated as M15 cans. Qweak apparently read the bpm calibration values from OPS, and so was properly using the nominal calibration factors show in the above table for the M15's and M15mini's, so those historical plots can be interpreted normally.

However, the calibration constant used for M15-mini's in the accelerator as of Feb, 2018 is $13.7 \mathrm{~mm}$, but this value is questionable. Measurements performed with a Goubau line scanner [114] indicate the calibration constants are $15.46 \mathrm{~mm} \pm 0.08 \mathrm{~m}$ and $15.52 \mathrm{~mm} \pm 0.08 \mathrm{~mm}$ for $\mathrm{kx}$ and ky respectively, almost $2 \mathrm{~mm}$ different from the value used by the accelerator. Similar measurements performed for M15 bpm 
cans showed calibration constants of $18.4 \mathrm{~mm} \pm 0.12 \mathrm{~mm}$, within $0.4 \mathrm{~mm}$ of the value used in the accelerator. The two new injector bpms 2I01 and 2I02 have calibration constants of, respectively, $26.84 \pm 0.02 \mathrm{~mm}$ and $25.83 \mathrm{mmm} \pm 0.05 \mathrm{~mm}$ (average $26.33 \pm 0.53 \mathrm{~mm})[115][116]$

\subsubsection{Calibration Derivation}

The signal from each wire antenna excited by the beam can be calculated [98] [99] as

$$
\begin{gathered}
S(\phi)=\beta \frac{I_{\text {beam }}}{2 \pi} \frac{a^{2}-r^{2}}{|\vec{a}-\vec{r}|^{2}} \\
=\beta \frac{I_{\text {beam }}}{2 \pi} \frac{a^{2}-r^{2}}{a^{2}+r^{2}-2 a r \cos (\phi-\theta)}
\end{gathered}
$$

where $a$ is the radius of BPM vacuum chamber, $\hat{a}$ is the direction of the wire antenna, $\phi$ specifies the location of the bpm wire at $0, \pi / 4, \pi / 2,3 \pi / 4, \mathrm{r}$ and $\theta$ specify the position of the electron beam, $I_{\text {beam }}$ is the current of the electron beam, and $\beta$ is a geometrical parameter which takes into account the finite thickness of the wire.

To get a sense for the behavior of a the wire response, we evaluate the antenna signal response to the presence of current $\delta S(r, \phi, \theta)=\beta \frac{\delta I_{\text {beam }}}{2 \pi} \frac{a^{2}-r^{2}}{a^{2}+r^{2}-2 a r \cos (\phi-\theta)}$ with respect to $\mathrm{x}$. Evaluating the 1st and 2nd derivatives $\frac{d \delta S(r, \phi, \theta)}{d r}$ and $\frac{d^{2} \delta S(r, \phi, \theta)}{d r^{2}}$ for $\beta \delta I_{\text {beam }}=1, \phi=0$ (examining the $\mathrm{X}+$ wire $), \theta=0$ (moving along $\mathrm{x}$ direction $)$, we obtain the sensitivity of the wire signal to the 0th, 1st and 2nd moments, seeing that each moment decreases by an order of magnitude (Fig. 5-24 ). 

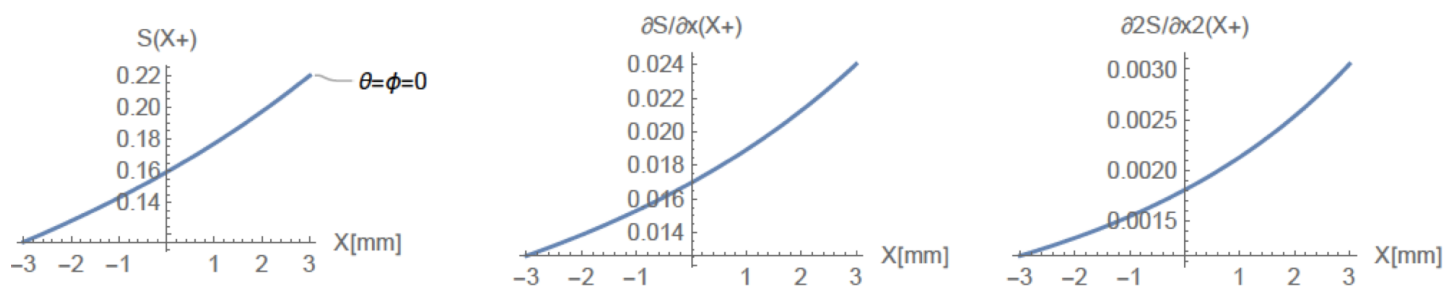

Figure 5-24: Spot Size BPM current Derivatives

The $\mathrm{X}+$ wire response with respect to beam position in the $\mathrm{x}-\mathrm{y}$ plane is illustrated here:

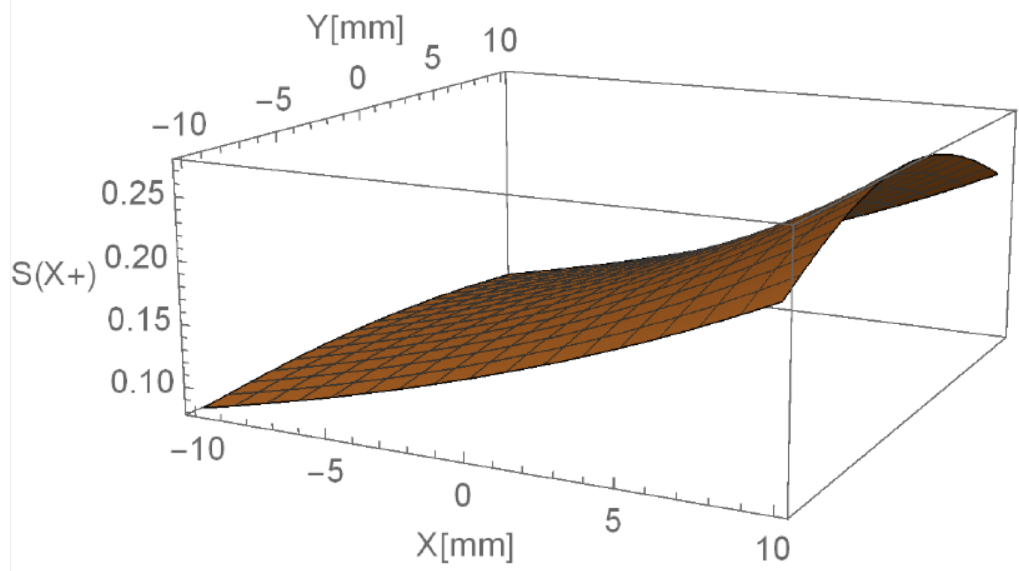

Figure 5-25: $\mathrm{X}+$ wire response vs. beam position in $\mathrm{x}-\mathrm{y}$ plane

From equation 5.2 , using the difference/sum method, we can calculate the position of the beam as

$$
\begin{gathered}
\text { bpmx }=\kappa \frac{S(X+)-S(X-)}{S(X+)+S(X-)}=\kappa \frac{S(\phi=0)-S(\phi=\pi)}{S(\phi=0)+S(\phi=\pi)} \\
=\kappa \frac{2 r \cos (\theta)}{a\left(1+\frac{r^{2}}{a^{2}}\right)}=\kappa \frac{2 x}{a\left(1+\frac{x^{2}+y^{2}}{a^{2}}\right)} \approx \kappa \frac{2 x}{a} \\
\text { bpmx } \approx \frac{a}{2} \frac{S(X+)-S(X-)}{S(X+)+S(X-)}
\end{gathered}
$$

Hence for small $\mathrm{r}$, the calibration constant $\kappa=a / 2$. It should be noted that the difference-over-sum method is an approximation, and assumes that the signal on 
the far side eventually goes to zero (in addition to antennae being represented by very narrow strips) [105]. It is only a decent approximation over a few millimeters.

The e-beam has a finite size which could affect the signals observed. For a gaussian current distribution of the electron beam, the signal for each antenna can be described as

$$
\begin{gathered}
S(\phi)=\int_{0}^{2 \pi} \int_{0}^{a} r^{\prime} d r^{\prime} \beta \frac{j_{\text {beam }}\left(\left|\vec{r}-\overrightarrow{r^{\prime}}\right|\right)}{2 \pi} \frac{a^{2}-r^{\prime 2}}{\left|\vec{a}-\vec{r}^{\prime}\right|^{2}} d \theta^{\prime} \\
=\int_{0}^{2 \pi} \int_{0}^{a} r^{\prime} d r^{\prime} \frac{\beta I_{\text {beam }}}{2 \pi} \frac{e^{\frac{-\left|\vec{r}-r^{\prime}\right|^{2}}{2 \sigma^{2}}}}{2 \pi \sigma^{2}} \frac{a^{2}-r^{\prime 2}}{\left|\vec{a}-\overrightarrow{r^{\prime}}\right|^{2}} d \theta^{\prime}
\end{gathered}
$$

This integral can be evaluated numerically for $\beta=1$ and $I_{\text {beam }}=1$ over the beam pipe area up to radius a, and a linear relationship is confirmed with calibration constant $\kappa \approx a / 2$ (Fig. 5-26).

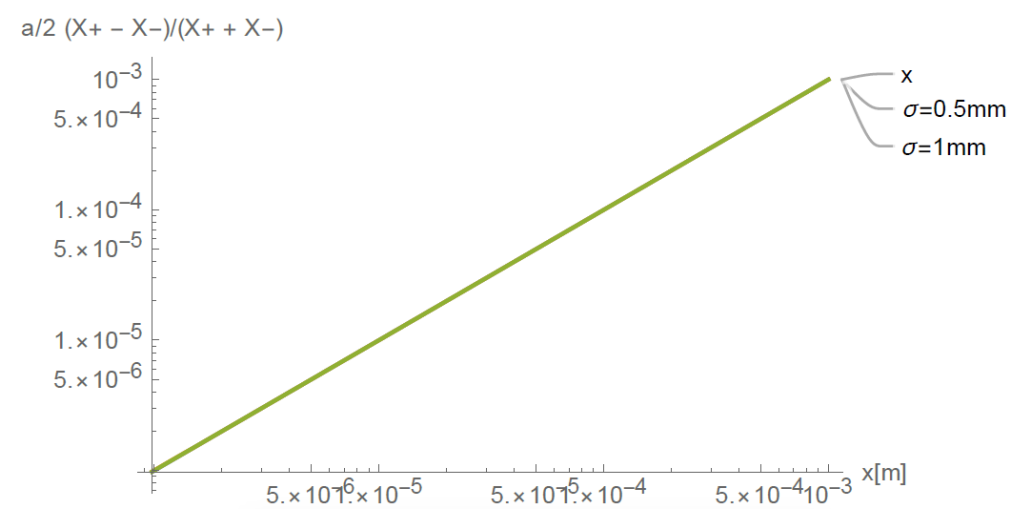

Figure 5-26: Wire Channel Calculation X

Phenomenologically, we observe in the Hall (for type M15 BPMs) that $\kappa \approx$ $18.76 \mathrm{~mm}-18.87 \mathrm{~mm}[100]$ which means $a \approx 37.52 \mathrm{~mm}-37.74 \mathrm{~mm}$. This is approximately consistent with the design drawing (Fig. 5-22 ) which shows an inner radius of $33.3 \mathrm{~mm}$. So, the calibration constants used are within $10 \%$ of their theoretical value. A similar calculation can be done for stripline BPMs, where the only difference is that striplines don't have a thin wire, but rather subtend a large angle $\alpha$ over $\phi(68$ degrees, Fig. 5-27), so their $\kappa$ factor is different. In fact, their 
$\kappa$ factor is obtained using the so-called Goubau Line scanner, and is about $9.9 \mathrm{~mm}$ for most sensors [105].

\section{Stripline BPMs}

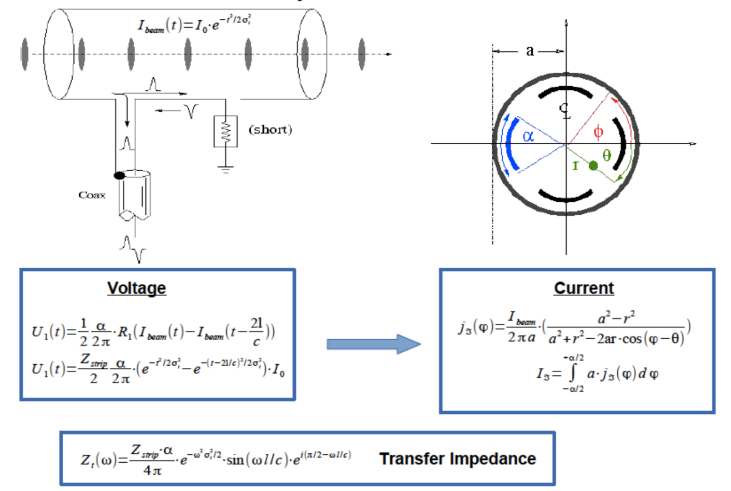

Figure 5-27: Stripline BPMs [106]

\subsubsection{Pedestal Error}

The charge asymmetry $A_{Q}$ can couple to the measured position difference $\Delta X_{M}$ through a pedestal error. We define the pedestal error on each wire a $\Delta P_{p}$ and $\Delta P_{m}$, the symmetric and asymmetric pedestals errors as $\Delta P_{A}=\left(\Delta P_{p}-\Delta P_{m}\right) / 2$ and $\Delta P_{S}=\left(\Delta P_{p}+\Delta P_{m}\right) / 2$, the radial distance to the wire as $\kappa$, and the wire sum as $W_{S}=X_{p}^{A}+X_{m}^{A}=I \alpha \kappa$ proportional to the beam current.

$$
\begin{gathered}
X^{M}=\kappa \frac{X_{p}^{M}-X_{m}^{M}}{X_{p}^{M}+X_{m}^{M}} \\
=\kappa \frac{X_{p}^{A}-X_{m}^{A}+\Delta P_{p}-\Delta P_{m}}{X_{p}^{A}+X_{m}^{A}+\Delta P_{p}+\Delta P_{m}} \\
=\kappa \frac{X_{p}^{A}-X_{m}^{A}+2 \Delta P_{A}}{X_{p}^{A}+X_{m}^{A}+2 \Delta P_{S}} \\
\approx X^{A}+2 \kappa \frac{\Delta P_{A}}{W_{S}}-2 X^{A} \frac{\Delta P_{S}}{W_{S}}
\end{gathered}
$$




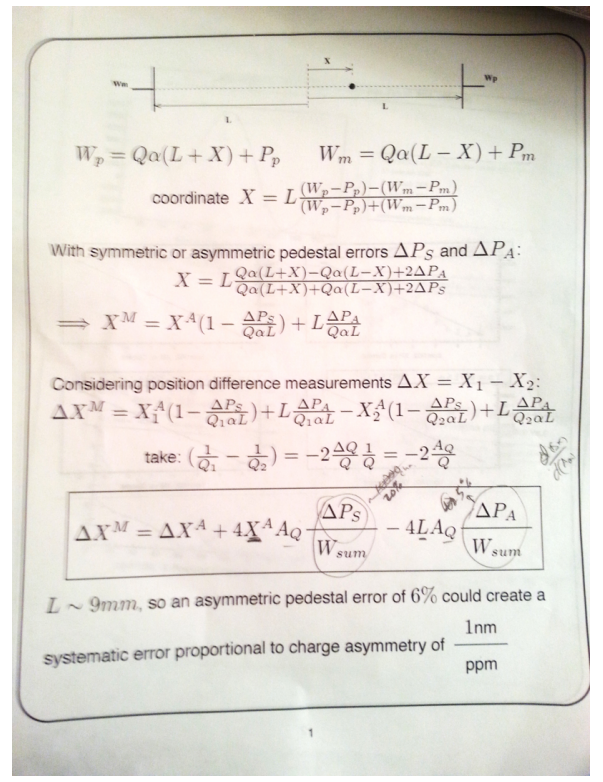

Figure 5-28: Pedestal Error Calculation

The measured position difference can be calculated as

$$
\Delta X=X_{0}-X_{1}=X_{0}^{A}+2 \kappa \frac{\Delta P_{A}}{W_{S 0}}-2 X_{0}^{A} \frac{\Delta P_{S}}{W_{S 0}}-X_{1}^{A}-2 \kappa \frac{\Delta P_{A}}{W_{S 1}}+2 X_{1}^{A} \frac{\Delta P_{S}}{W_{S 1}}
$$

We note that

$$
\frac{1}{W_{S 0}}-\frac{1}{W_{S 1}}=\frac{1}{\alpha}\left(\frac{1}{I+\Delta I}-\frac{1}{I-\Delta I}\right)=\frac{1}{\alpha I}(1-\Delta I / I-1-\Delta I / I) \approx \frac{-2 A_{Q}}{W_{S}}
$$

Hence,

$$
\Delta X^{M}=\Delta X^{A}\left(1+\frac{2 \Delta P_{S}}{W_{S}}\right)+4 X^{A} A_{Q} \frac{\Delta P_{S}}{W_{S}}-4 \kappa A_{Q} \frac{\Delta P_{A}}{W_{S}}
$$

A $5 \%$ asymmetric pedestal error could result in a coupling of $4 \mathrm{~nm} / \mathrm{ppm}$. For nostalgic purposes we also include a transparency astutely created by a certain postdoc [107] in the early 2000's Fig. 5-28 ) 


\subsubsection{BPM widths}

Generally speaking, from $30 \mathrm{~Hz}$ to $1 \mathrm{kHz}$, from $20 \mu \mathrm{A}$ to $100 \mu \mathrm{A}$, BPM position difference widths tend to be $1 \mu m-20 \mu \mathrm{m}$, depending on the BPM. It is a combination or monitor noise as well as noise in the beam position motion. It is more challenging to assess the BPM resolution from a double-difference type of analysis because no two BPMs measure exactly the same quantity, so they must be crosscorrelated and a more complex analysis must be done. Here we simply show that during 2015-2016 studies, the overall position difference widths in the Hall, shown in Fig. 5-30 and in the injector, shown in Fig. 5-29, were reasonable for PREXII. 5 The BPM widths observed are acceptable for PREXII running.

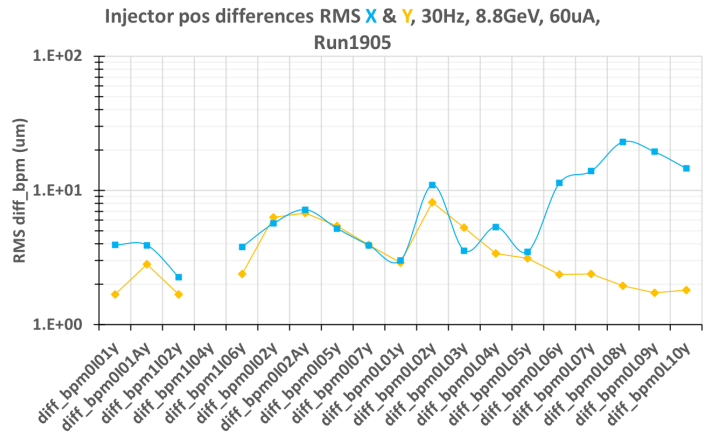

Figure 5-29: Injector BPM position difference widths at 30Hz [183]

\footnotetext{
${ }^{5}$ We note however, that bpm4a in the hall had 'tails' in the distribution tails due to sudden jumps up and down in signal, at the few $\mathrm{mV}$-level, which appeared to be constantly happening in a pattern-like fashion. To filter the tails out in the analysis, we made the cuts : evtbpm4ax $[0]<a$ and evtbpm $4 a x[1]<a$. We note that bpm4b also showed these tails at higher currents.
} 

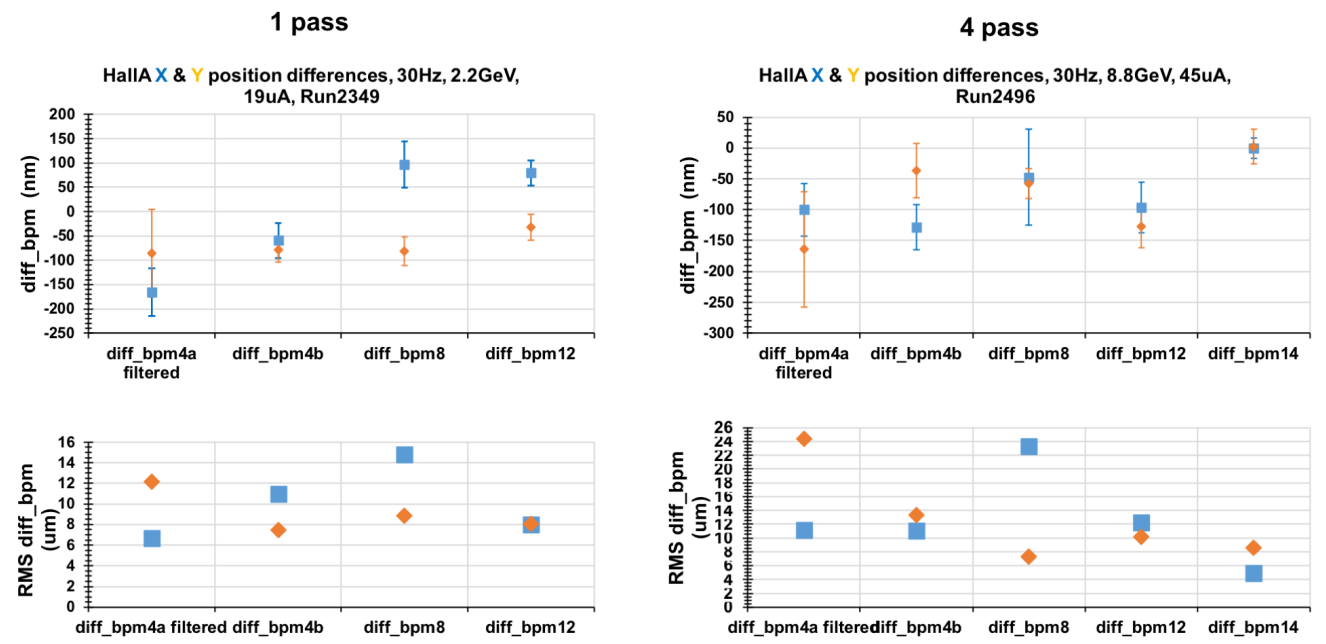

Figure 5-30: HallA BPM position differences and position difference widths at $30 \mathrm{~Hz}[183]$

\subsubsection{Helicity Patern}

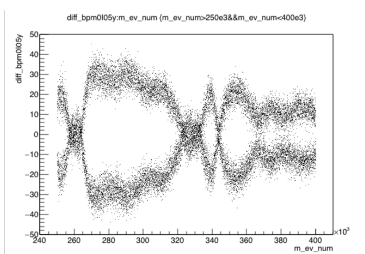

Figure 5-31: 960Hz: large bpm widths observed in the injector due to a beat frequency with the $60 \mathrm{~Hz}$ line

During Qweak, at $960 \mathrm{~Hz}$ helicity flip rate, large bpm widths were observed. During 2017-2018 beam studies, at 960Hz, large bpm widths were also observed in the injector. These large widths are due to a beat frequency with the $60 \mathrm{~Hz}$ line as shown in Fig.5-31 ${ }^{6}$. For MOLLER, it will be important to use an extended multiplet to cancel out the $60 \mathrm{~Hz}$ beat frequency, analogous to using quartets (++ ) for $120 \mathrm{~Hz}$ and octets $(+-+-++-)$ for $240 \mathrm{~Hz}$ flip rates. Octets are insufficient multiplets for $960 \mathrm{~Hz}$, let alone the $2 \mathrm{kHz}$ flip rate required for MOLLER. During

\footnotetext{
${ }^{6}$ Run3330 , 960Hz Mtree, Free clock, 40us Tsettle, 1001.65usec Tstable, Octet, No Delay, $960.02 \mathrm{~Hz}$. Periodicity $>150 \mathrm{e} 3$ events, $\mathrm{T}>156 \mathrm{~s}, \quad<0.0064 \mathrm{~Hz}$ beat and $(960-$ $960.02 \mathrm{~Hz}) / 8=0.0025 \mathrm{~Hz}, \quad$ vqwk SamplesPerBlock $=120, \quad$ GateDelay $=10, \quad$ NumberofBlocks $=4$ (2us/sample)
} 
MOLLER, we will need the helicity control board to be able to generate megamultiplets sufficient to fill a $60 \mathrm{~Hz}$ period with $2 \mathrm{kHz}$ windows (and line-synch at $2 \mathrm{kHz}$ ), in order to cancel out the $60 \mathrm{~Hz}$ beating and getting rid of this extraneous noise.

\subsection{Measuring Spot Size Asymmetries with BPMs}

There are 4 wire channels in a bpm. At present only 3 linear combinations of those channels are assessed to examine beam I,X,Y. There is a 4th linear combination which produces an independent variable which can be examined. This combination is proportional to the elliptical component of the spot size (aka the breathing mode).

\subsubsection{Context}

The electron beam spot-size asymmetry is not directly measured at present, and so our knowledge relies on an implied bound from laser measurements. Slow reversals are necessary, in part because we don't get to measure the spot-size asymmetry on the electron beam directly.

Spot size asymmetries can contribute significant systematic errors to Parity experiments such as PREX/CREX, HAPPEX, Qweak and Moller. In the past, the spot size asymmetry was bounded to be $\Delta \sigma /(2 \sigma)<10^{-4}$ on the laser table. Analytic calculations of how the spot size asymmetry affects the measured physics asymmetry were performed and a bound was placed on the systematic error contribution. For example, in PREXI, it was estimated that a spot size asymmetry of $0.5 \times 10^{-4}$ contributes $2 \mathrm{ppb}$ false asymmetry systematic [110]. For Moller, neglecting effects from synchrotron radiation, the systematic error contribution is estimated to be $<12 \mathrm{ppm} \Delta \sigma / \sigma$ [111], which for $\Delta \sigma / \sigma<10^{-4}$ gives $1 \mathrm{ppb}$. Assuming a factor of 10 suppression from cancellation due to periodic slow helicity reversals, the net contribution will be limited to $0.1 \mathrm{ppb}$. 
Because spot size asymmetry is significant to parity experiments, it is desirable to monitor the spot size asymmetry on the electron beam itself. Ideally, it would be advantageous to measure spot size asymmetry in the Hall, obtain correlations with the detectors, and regress out or estimate systematic errors empirically from real time measurements. At the very least, however, it is desirable to confirm with ebeam the laser limits on the spot-size asymmetry. Here, we present one method of measuring one component of electron beam spot-size asymmetries on the beamline using BPMs (Beam Position Monitors) : the elliptical spot-size asymmetry.

\subsubsection{Measurement with BPM}

The BPMs used throughout the accelerator are described in Sec. 5.4. There are four wire channels in a BPM, denoted $\mathrm{X}+(\mathrm{xm}), \mathrm{X}-(\mathrm{xm}), \mathrm{Y}+(\mathrm{yp})$, and $\mathrm{Y}-(\mathrm{ym})$. Yet, at present only three linear combinations of the four wire channels are calculated to examine beam x-position, $\mathrm{y}$-position, and current via wire-combinations $7:$

$$
b p m x=\kappa \frac{(x p-x m)}{(x p+x m)} \quad \text { bpmy }=\kappa \frac{(y p-y m)}{(y p+y m)} \quad \text { bpmws }=x p+x m+y p+y m
$$

There is a 4th linear combination which produces another independent variable: the elliptical component of the spot size. There are several ways to describe spotsize, but we could model it as having a circular $\sigma_{\text {circ }}$ component and and an elliptical component $\epsilon \approx\left(\sigma_{x}-\sigma_{y}\right) /\left(\sigma_{x}+\sigma_{y}\right)$. The spot size can be described as $\sigma(\theta)=\sigma_{\text {circ }}\left(1+\epsilon \cos \left(2\left(\theta-\theta_{i}\right)\right)\right)$, where $\theta$ is the angle in the plane transverse to the direction of beam propagation. Depending on the orientation of the wire channels and the orientation of the electron beam, a given bpm may be sensitive to $\epsilon$ or insensitive to it. Likewise the spot size asymmetry is modeled as $A_{\sigma}(\theta)=$ $A_{\text {circ }}+A_{\text {elli }} \cos \left(2\left(\theta-\theta_{0}\right)\right)$, where Aelli is the asymmetry due to the elliptical

\footnotetext{
${ }^{7} \kappa$ is a wireline bpm calibration constant set in accordance with the type of BPM described in Table. 5.5
} 
component of the spot size asymmetry, what we refer to as the "breathing mode" of the spot size asymmetry.

$A_{\text {elli }}$ can be related to a difference in $\epsilon$ via Aelli $\approx\left(\epsilon_{0}-\epsilon 1\right) /\left(\sigma_{0}+\sigma 1\right) \approx$ $\Delta \epsilon / 2 \approx\left(\epsilon_{0} / \sigma_{0}-\epsilon_{1} / \sigma_{1}\right) / 2$, where the subscripts 0 and 1 are for two helicity states. $A_{\text {circ }}$ is also an important parameter which we are also sensitive to in a bpm, but it manifests itself similarly to $A_{Q}$ and we cannot distinguish a separate entity (unless perhaps a bpm and bcm are in very close proximity in the beamline). So, in this analysis, we focus on the elliptical term of the spot size asymmetry. Using the bpm wire channels we can obtain information about the elliptical component of the spot size asymmetry $A_{\text {elli }}$.

$$
\begin{gathered}
\text { bpmelli }=\frac{a^{2}(x p+x m-y p-y m)}{8 \sigma^{2}(x p+x m+y p+y m)} \\
\epsilon=\text { bpmelli }- \text { bpmecorr } r=\frac{a^{2}(x p+x m-y p-y m)}{8 \sigma^{2}(x p+x m+y p+y m)}-f \approx \frac{\sigma_{x}-\sigma_{y}}{\sigma_{x}+\sigma_{y}}
\end{gathered}
$$

where $a \approx 2 \kappa=37.52 \mathrm{~mm}$ the radius of M15 BPM vacuum chamber, $\sigma$ is the $e^{-}$beam spot size, and $f$ is a correction term which will be discussed in later sections (Eq. 5.18).

\subsubsection{Analytic Derivation of bpmelli}

The signal induced by the e-beam on the wire with finite thickness can be approximated as [112]

$$
\begin{gathered}
S(\phi) \approx \gamma\left(2 \alpha+4 \frac{\sin (\alpha)}{a}(x \cos (\phi)+y \sin (\phi))\right. \\
+2 \frac{\sin (2 \alpha)}{a^{2}}\left(\left(\sigma_{x}^{2}-\sigma_{y}^{2}+x^{2}-y^{2}\right) \cos (2 \phi)+2 x y \sin (2 \phi)\right)
\end{gathered}
$$

The radius of the BPM aperture is a, the angle subtended by the BPM wire is $\alpha, \mathrm{x}$ and $\mathrm{y}$ give the centroid position of the beam, $\gamma$ is just a scale factor, and $\sigma$ denotes the rms beam width. 
The four bpm wires are located at $\phi=0, \pi / 2, \pi, 3 \pi / 2$. Evaluating the linear combination of wire channels $x p+x m-y p-y m$, treating $\alpha$ as very small, and defining $\sigma_{x}=\sigma+\delta \sigma, \sigma_{y}=\sigma-\delta \sigma$, we obtain to first order is $\delta \sigma$

$$
\frac{x p+x m-y p-y m}{x p+x m+y p+y m} \approx 2 \frac{\left(x^{2}-y^{2}\right)}{a^{2}}+8 \frac{\sigma \delta \sigma}{a^{2}}
$$

Rearranging terms, and noting that $\delta \sigma / \sigma=\left(\sigma_{x}-\sigma_{y}\right) /\left(\sigma_{x}+\sigma_{y}\right)$

$$
\epsilon=\frac{\sigma_{x}-\sigma_{y}}{\sigma_{x}+\sigma_{y}}=\frac{a^{2}}{8 \sigma^{2}} \frac{x p+x m-y p-y m}{x p+x m+y p+y m}-\frac{x^{2}-y^{2}}{4 \sigma^{2}}=\text { bpmelli }- \text { bpmecorr }
$$

where we define bpmelli $=\frac{a^{2}}{8 \sigma^{2}} \frac{x p+x m-y p-y m}{x p+x m+y p+y m}$ and bpmecorr $=\frac{x^{2}-y^{2}}{4 \sigma^{2}}$

\subsubsection{Numeric Derivation of bpmelli}

The equation for the elliptical component of the spot size 5.9 can also be derived from numerical integration. For a gaussian current distribution of the electron beam centered at $\left(x_{0}, y_{0}\right)$ relative to the center of the beamline at $r=0$, the signal for each antenna can be described as

$$
\begin{gathered}
S(\phi)=\int_{0}^{a} d r \beta \int_{0}^{2 \pi} d \theta^{\prime} r I_{\text {beam }} e^{-\frac{\left(r \cos \left(\theta^{\prime}\right)-x_{0}\right)^{2}}{2 \sigma_{x}^{2}}-\frac{\left(r \sin \left(\theta^{\prime}\right)-y_{0}\right)^{2}}{2 \sigma_{y}^{2}}} \\
\times \frac{a^{2}-r^{2}}{4 \pi^{2} \sigma_{x} \sigma_{y}\left(a^{2}+r^{2}-2 a r \cos \left(\theta^{\prime}-\phi\right)\right)} \\
S(X \pm) \approx \iint d x d y \beta I_{\text {beam }} e^{-\frac{\left(x-x_{0}\right)^{2}}{2 \sigma_{x}^{2}}-\frac{\left(y-y_{0}\right)^{2}}{2 \sigma_{y}^{2}}} \frac{a^{2}-x^{2}-y^{2}}{4 \pi^{2} \sigma_{x} \sigma_{y}\left((a \mp x)^{2}+y^{2}\right)} \\
S(Y \pm) \approx \iint d x d y \beta I_{\text {beam }} e^{-\frac{\left(x-x_{0}\right)^{2}}{2 \sigma_{x}^{2}}-\frac{\left(y-y_{0}\right)^{2}}{2 \sigma_{y}^{2}}} \frac{a^{2}-x^{2}-y^{2}}{4 \pi^{2} \sigma_{x} \sigma_{y}\left(x^{2}+(a \mp y)^{2}\right)}
\end{gathered}
$$

For a centered electron beam at $\left(x_{0}, y_{0}\right)=(0,0)$, for each wire location, this integral is evaluated numerically over the circular area centered on the beamline up to radius a, the bpm chamber radius. Forming the linear combination of wire 
channel signals and scaling the result by $a^{2} / \sigma^{2} / 8$, we obtain the elliptical spot size term $\epsilon=\frac{\sigma_{x}-\sigma_{y}}{\sigma_{x}+\sigma_{y}}$ (Fig. 5-32). We note that this normalization is largely to correct $\sigma$ dependence in the numerator $\mathrm{xp}+\mathrm{xm}-\mathrm{yp}-\mathrm{yp}$ as opposed to the denominator $\mathrm{xp}+\mathrm{xp}+\mathrm{yp}+\mathrm{ym}$ which is not very sensitive to $\sigma$ (the wire sum only changes $<0.1 \%$ over the range $200 \mathrm{um}<\sigma<2 \mathrm{~mm}$, whereas the numerator changed by over an OOM).

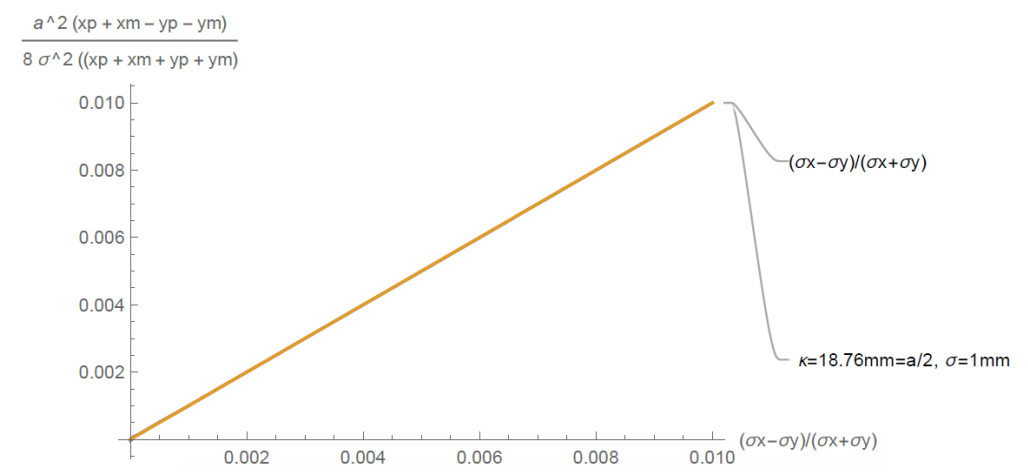

Figure 5-32: Wire Channel Calculation - Spot Size Asymmetry

\subsubsection{Position Dependence of bpmelli}

Regarding the dependence of bpmelli on beam position, this integral is highly dependent on the position of the electron beam $\left(x_{0}, y_{0}\right)$. We set $\epsilon=10^{-4}$ and evaluate bpmelli for several beam positions (Fig. 5-33 ). We observe that there is a large correction term to bpmelli, proportional to the square of the distance from the beamline, approximately $f\left(x_{0}, y_{0}\right)=g 1 \frac{x_{0}^{2}-y_{0}^{2}}{\sigma^{2}}-g 2 \frac{x_{0}^{4}-y_{0}^{4}}{\sigma^{2}}$, where $g 1=0.250014$ and $g 2=2.84739 \mathrm{~m}^{-2}$ when evaluated for $a=2 \kappa, \kappa=18.76 \mathrm{~mm}, \sigma=1 \mathrm{~mm}$. 


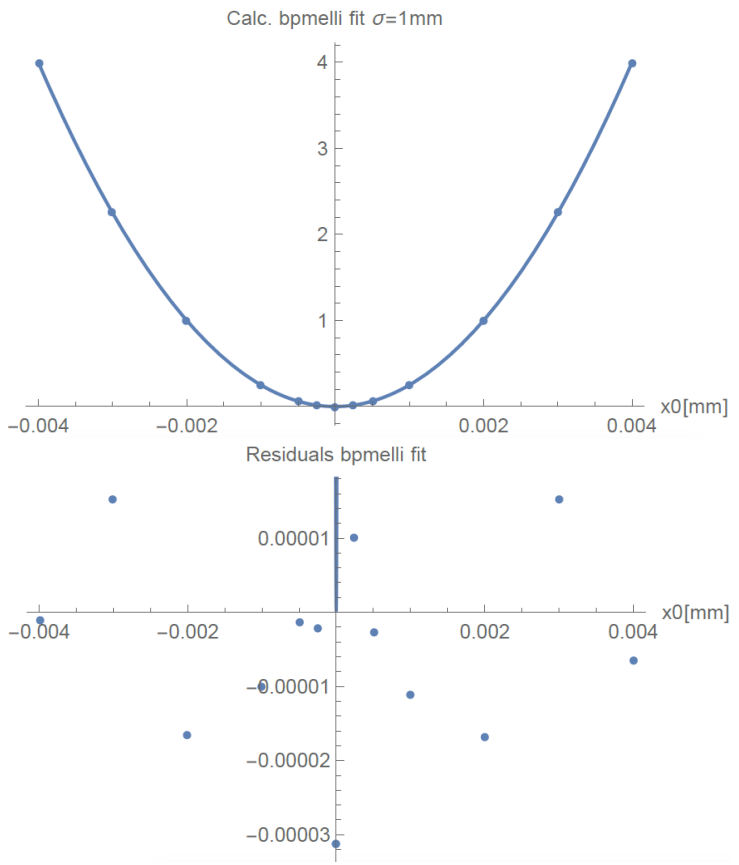

Figure 5-33: Wire Channel Calculation for bpmelli

However, when we evaluate small differences in $\epsilon$, letting $\Delta \epsilon=10^{-4}$, even far from the beamline center, diff_bpmelli is still accurate, whether the beam is a $x_{0}=0$ or at $x_{0}=8 \mathrm{~mm}$ (Fig. 5-34). So, we should expect that the calculated raw ellipticity will vary with beam position, but the pair-wise asymmetry in that ellipticity will still be approximately correct.

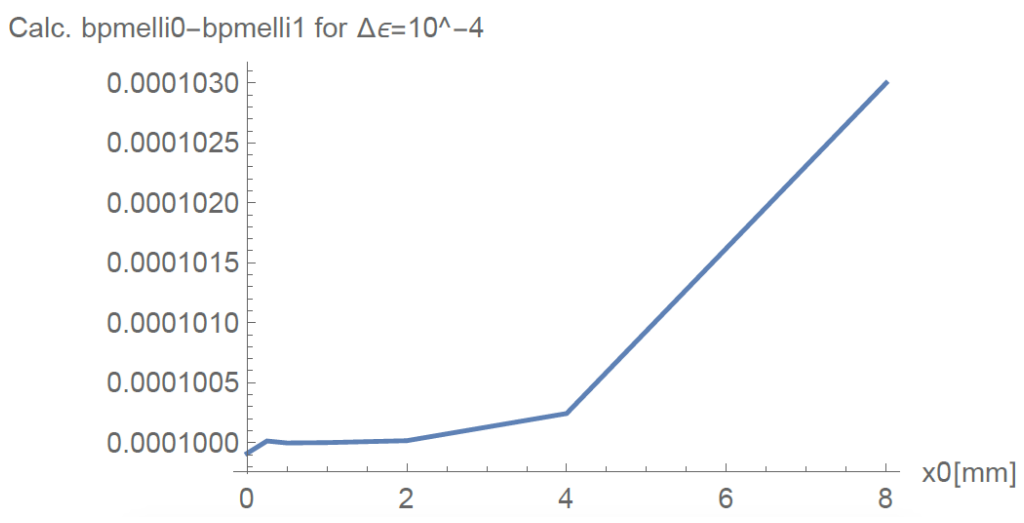

Figure 5-34: Wire Channel Calculation for Aelli 


\subsubsection{Pedestal Error in bpmelli}

The charge asymmetry $A_{Q}$ and the radial spot size asymmetry $A_{\text {circ }}$ (which is indistinguishable from $A_{Q}$ in a bpm) can couple to the measured elliptical spot size asymmetry $A_{\text {elli }}=\Delta \epsilon / 2$ through a pedestal error. We define the pedestal error on the x-wires and y-wires as $\Delta P_{S x}=\left(\Delta P_{x p}+\Delta P_{x m}\right) / 2$ and $\Delta P_{S y}=\left(\Delta P_{y p}+\right.$ $\left.\Delta P_{y m}\right) / 2$, the symmetric and asymmetric pedestals errors as $\Delta P_{A}=\Delta P_{S x}-\Delta P_{S y}$ and $\Delta P_{S}=\Delta P_{S x}+\Delta P_{S y}$, the radial distance to the wire as $a$, and the wire sum as $W_{S}=X_{p}^{A}+X_{m}^{A}+Y_{p}^{A}+Y_{m}^{A}=I \alpha \sigma$ proportional to the beam current and spot-size.

$$
\begin{gathered}
\epsilon^{M}=\frac{a^{2}}{8 \sigma^{2}} \frac{X_{p}^{M}+X_{m}^{M}-Y_{p}^{M}-Y_{m}^{M}}{X_{p}^{M}+X_{m}^{M}+Y_{p}^{M}+Y_{m}^{M}} \\
=\frac{a^{2}}{8 \sigma^{2}} \frac{X_{p}^{A}+X_{m}^{A}-Y_{p}^{A}-Y_{m}^{A}+\Delta P_{x p}+\Delta P_{x m}-\Delta P_{y p}-\Delta P_{y m}}{X_{p}^{A}+X_{m}^{A}+Y_{p}^{A}+Y_{m}^{A}+\Delta P_{x p}+\Delta P_{x m}+\Delta P_{y p}+\Delta P_{y m}} \\
=\frac{a^{2}}{8 \sigma^{2}} \frac{X_{p}^{A}+X_{m}^{A}-Y_{p}^{A}-Y_{m}^{A}+2 \Delta P_{A}}{X_{p}^{A}+X_{m}^{A}+Y_{p}^{A}+Y_{m}^{A}+2 \Delta P_{S}} \\
\approx \epsilon^{A}+2 \frac{a^{2}}{8 \sigma^{2}} \frac{\Delta P_{A}}{W_{S}}-2 \epsilon^{A} \frac{\Delta P_{S}}{W_{S}}
\end{gathered}
$$

The measured elliptical spot-size asymmetry can be calculated as

$$
A_{\text {elli }}^{M}=\frac{1}{2} \Delta \epsilon^{M}=\frac{1}{2}\left(\epsilon_{0}-\epsilon_{1}\right)=\epsilon_{0}^{A} / 2+\frac{a^{2}}{8 \sigma^{2}} \frac{\Delta P_{A}}{W_{S 0}}-\epsilon_{0}^{A} \frac{\Delta P_{S}}{W_{S 0}}-\epsilon_{1}^{A} / 2-\frac{a^{2}}{8 \sigma^{2}} \frac{\Delta P_{A}}{W_{S 1}}+\epsilon_{1}^{A} \frac{\Delta P_{S}}{W_{S 1}}
$$

We note that

$$
\frac{1}{W_{S 0}}-\frac{1}{W_{S 1}}=\frac{1}{\alpha}\left(\frac{1}{I_{0}}-\frac{1}{I_{1}}\right)=\frac{1}{\alpha}\left(\frac{1}{I+\Delta I}-\frac{1}{I-\Delta I}\right) \approx-\frac{2 A_{Q}}{W_{S}}
$$

Hence,

$$
A_{\text {elli }}^{M}=\frac{1}{2} \Delta \epsilon^{M}=A_{\text {elli }}^{A}\left(1+\frac{2 \Delta P_{S}}{W_{S}}\right)+2 \epsilon^{A} A_{Q} \frac{\Delta P_{S}}{W_{S}}-2 \frac{a^{2}}{8 \sigma^{2}} A_{Q} \frac{\Delta P_{A}}{W_{S}}
$$

For $a=2 \kappa, \kappa=13.7 \mathrm{~mm}$ and $\sigma=1.5 \mathrm{~mm}$, for a $5 \%$ asymmetric pedestal error 
the false elliptical spot size asymmetry is $4.2 \times 10^{-} 6 / \mathrm{ppm}$ for $A_{Q}$. To test this equation, we can induce a 5\% asymmetric pedestal error in our analysis. The wire sum signal is $3 \mathrm{e} 4$ channels on the vqwk ADCs. Applying 3000ch to the pedestals of $\mathrm{xp}$ and $\mathrm{xm}$, and -3000ch to yp and ym, we obtain a 5\% asymmetric pedestal error. The slope observed in the first 8 bpms in a PITA scan (Run3338), shows a coupling between $A_{\text {elli }}$ and $A_{Q}$ of $4.05-4.18 \times 10^{-} 6 / \mathrm{ppm}$, consistent with our calculated pedestal error value.

\subsubsection{Position Difference Error in bpmelli}

The position sensitivity correction term in bpmelli, which we now define as bpmecorr, was found to be approximately

$$
\text { bpmecorr }=f(x, y)=g 1 \frac{x^{2}-y^{2}}{\sigma^{2}}-g 2 \frac{x^{4}-y^{4}}{\sigma^{2}}
$$

where $g 1=0.250014$ and $g 2=2.84739 m^{-2}$. We evaluate how this position dependence leads to coupling between position differences and measured $A_{\text {elli }}$.

$$
\begin{gathered}
\Delta \text { bpmecorr }=\Delta f(x, y)=f(x 0, y 0)-f(x 1, y 1) \\
=g 1 \frac{x_{0}^{2}-y_{0}^{2}}{\sigma^{2}}-g 1 \frac{x_{1}^{2}-y_{1}^{2}}{\sigma^{2}}-g 2 \frac{x_{0}^{4}-y_{0}^{4}}{\sigma^{2}}+g 2 \frac{x_{1}^{4}-y_{1}^{4}}{\sigma^{2}} \\
\approx 0.25 \frac{4 x \Delta x-4 y \Delta y}{\sigma^{2}}-2.84 m^{-2} \frac{8 x^{3} \Delta x+8 \Delta x^{3} x}{\sigma^{2}} \approx \frac{x \Delta x-y \Delta y}{\sigma^{2}}
\end{gathered}
$$

We note the $x^{4}$ term is negligible in the mm regime. The coupling between position difference $\Delta x$ and $\Delta$ bpmelli, for $\sigma=x=1 \mathrm{~mm}$, is $10^{-6} / \mathrm{nm}$, which may or may not be significant depending on the size of the position differences and the size of the spot size asymmetry your trying to measure. However, for a more extreme case, $\sigma=0.5 \mathrm{~mm}$ and $x=10 \mathrm{~mm}$, the coupling is $4 e-5 / \mathrm{nm}$, which for $50 \mathrm{~nm}$ position differences gives false $\Delta$ bpmelli of up to $2 \times 10^{-3}$. We can eliminate this dependence approximately in analysis by subtracting the correction 
term bpmecorr from bpmelli : $A_{\text {elli }} \approx \Delta \epsilon / 2 \approx$ diff_bpmelli/2-diff_bpmecorr $/ 2$.

\subsubsection{Spot-Size Scale Factor Error}

It is possible that our estimates of spot-size are different from the actual beam spot size at a given bpm. This can affect both our measured diff_bpmelli, diff_bpmecorr and Aelli. The error is a scale factor given by the square of the ratio of actual beam size to applied beam size.

$$
\begin{aligned}
& \Delta \text { bpmecorr }^{A} \approx \frac{x \Delta x-y \Delta y}{\left(\sigma^{A}\right)^{2}}=\left(\frac{\sigma^{M}}{\sigma^{A}}\right)^{2} \Delta \text { bpmecorr }^{M} \\
& \Delta \text { bpmelli }{ }^{A}=\left(\frac{\sigma^{M}}{\sigma^{A}}\right)^{2} \Delta b p m e l l i^{M} \\
& A_{\text {elli }}^{A}=\frac{1}{2}\left(\Delta \text { bpmelli }^{A}-\Delta \text { bpmecorr }^{A}\right)=\left(\frac{\sigma^{M}}{\sigma^{A}}\right)^{2} A_{\text {elli }}^{M} \\
& \text { scalef }=\left(\frac{\sigma^{M}}{\sigma^{A}}\right)^{2}
\end{aligned}
$$

\subsubsection{Applying bpmelli in Data Analysis}

We analyze our best run of 2017 with the RTP cell, which had 100nm position differences in the injector. We also analyze a subsequent run with an aligned KD*P cell immediately following, which had the same injector setup. These runs are in the $130 \mathrm{keV}$ portion of the injector and cover bpms: 1I02, 1I04, 1I06, 0I01, 0I01A, 0I02, 0I02A, and 0I05.

\section{Determining $\sigma$}

We introduced bpmelli into our standard analysis, pan, treating $a=2 \kappa, \kappa=$ $13.7 \mathrm{~mm}$ for M15-mini bpms, and $\sigma=1.5 \mathrm{~mm}$. The reasoning behind this choice of $\sigma$ includes laser measurements and e-beam optics simulation. The laser spot size 
on the cathode during August 2017 was measured to be $\sigma=0.75 \mathrm{~mm}$. Examining Elegant (Fig. 5-35 ), the beamline simulation software used by the accelerator, if the initial $\sigma$ is $0.25 \mathrm{~mm}$, then for the first $8 \mathrm{bpms}$ in the injector the spot size $\sigma$ is between $0.4 \mathrm{~mm}$ and $0.6 \mathrm{~mm}$, assuming the model in Elegant is correct. If we scale our measured cathode spot size using Elegant, it is reasonable to assume $1.2 \mathrm{~mm}<\sigma<1.8 \mathrm{~mm}$ in the $130 \mathrm{keV}$ injector region. Applying a fixed $\sigma$ is not strictly correct for all bpms since the beam size changes throughout the accelerator, and we expect a $\pm 25 \%$ scale error in the $130 \mathrm{keV}$ region.

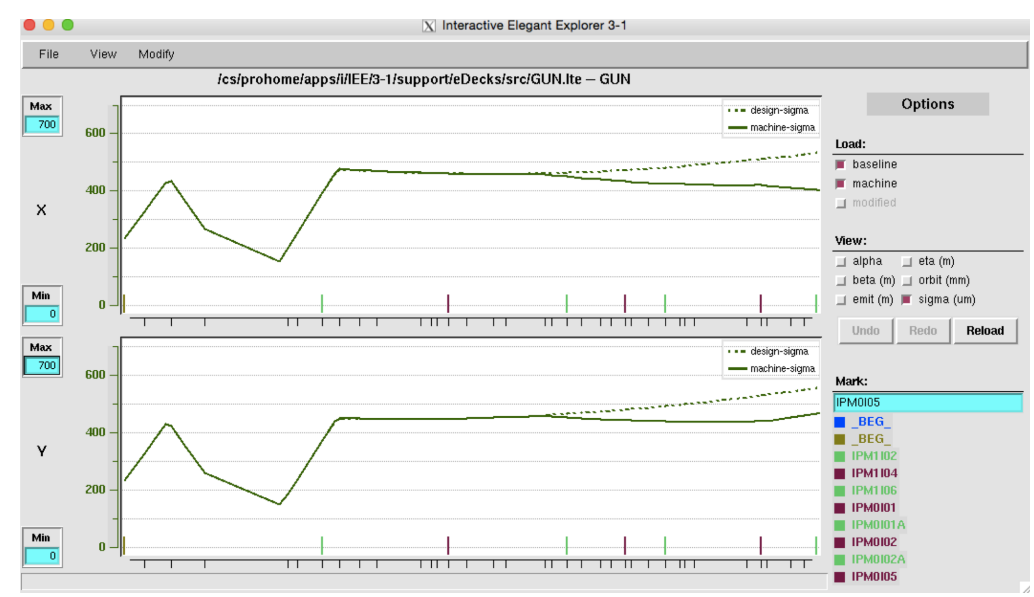

Figure 5-35: ELEGANT spot-size in gun region

Further downstream in the injector, ELEGANT predicts the spot-size changing more significantly. 


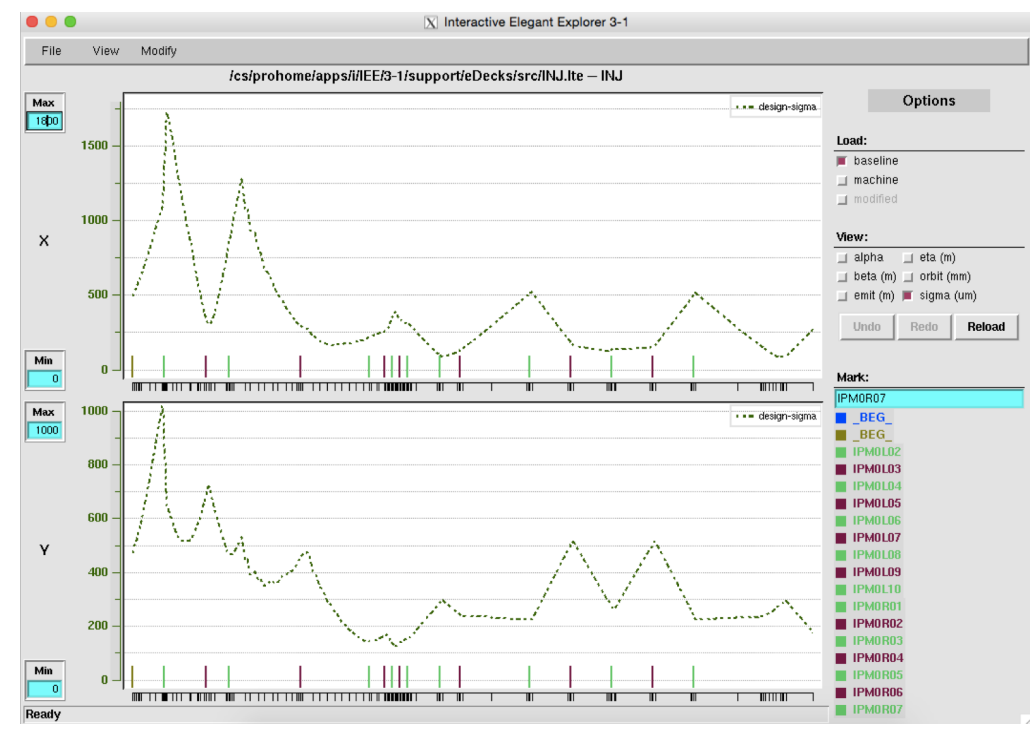

Figure 5-36: ELEGANT spot-sizes

\section{Checking Pedestal Error}

To check the pedestals, we examine a PITA scan in the RTP (Run3338). The couplings between diff_bpmelli and Aq as measured by the PITA scan are small and vary in sign, $\frac{d \Delta b m e l l i}{d A q}$ between $-2.5 \times 10^{-7} / \mathrm{ppm}$ and $1.4 \times 10^{-7} / \mathrm{ppm}$, indicating small pedestal errors. For comparison, when pedestal errors of $5 \%$ are intentionally induced, the slopes are much larger, $8.1 \times 10^{-6} / \mathrm{ppm}-8.25 \times 10^{-6} / \mathrm{ppm}$, and all have the same sign for all 8 bpms. This indicates that our pedestal errors are extremely small.

The same analysis was performed for the PITA scan in the KD*P data (Run3444). The couplings between diff_bpmelli and Aq as measured by this PITA scan are also small and vary in sign, $\frac{d \Delta b p m e l l i}{d A q}$ between $-4 \times 10^{-7} / \mathrm{ppm}$ and $1.5 \times 10^{-8} / \mathrm{ppm}$, indicating small pedestal errors for the $\mathrm{KD}^{*} \mathrm{P}$ data as well.

\section{Checking Couplings to PITAposU/V - RTP}

With the RTP cell, we have the advantage of being able to perform Alpha$\operatorname{pos} \mathrm{U} / \mathrm{V}$ scans which allow us to control the position differences and examine the couplings between diff_bpmelli and diff_bpmecorr and these position differences, 
as well as examine any sensitivity of Aelli to PITA posU/V. The position difference sensitivity of diff_bpmelli and diff_bpmecorr should be very similar, since the purpose of diff_bpmecorr to correct position difference sensitivity when we subtract it out Aelli=(diff_bpmelli-diff_bpmecorr $) / 2$. Any residual correlation of Aelli to AlphaposU/V must be due Aelli actually changing as we vary the voltage.

The position difference sensitivity of diff_bpmecorr is expected to be $x / \sigma^{2}$ where $\mathrm{x}$ is the deviation of the beam from center, which for these runs was $\pm 2 \mathrm{~mm}$ or so, and $\sigma$ is the spot size used, which for this analysis was $1.5 \mathrm{~mm}$. So we expect a position difference sensitivity in the range of $\frac{d \Delta b p m e c o r r}{d \Delta x} \approx \pm 0.9 \times 10^{-7} / \mathrm{nm}$. Examining the tables of the slopes from AlphaposU/V scans below (Fig. 5-37 , Fig. 5-38), that is exactly what we observe in diff_ecorr vs Dx,Dy. To get a sense for the error bars on these slopes, and the range of induced spot-size asymmetries, examine Fig.5-39 and Fig. 5-40 .

\begin{tabular}{|l|r|r|r|r|}
\hline & Aelli vs PITAposU & Delli vs Dx(nm) & Decorr vs Dx(nm) & Aelli vs Dx(nm) \\
\hline 1102 & $-2.5 \mathrm{E}-07$ & $-7.5 \mathrm{E}-07$ & $-8.9 \mathrm{E}-07$ & $6.3 \mathrm{E}-08$ \\
\hline 1104 & $8.7 \mathrm{E}-07$ & $-4.4 \mathrm{E}-08$ & $-3.5 \mathrm{E}-07$ & $1.5 \mathrm{E}-07$ \\
\hline 1106 & $-7.4 \mathrm{E}-07$ & $2.4 \mathrm{E}-06$ & $2.4 \mathrm{E}-07$ & $-1.1 \mathrm{E}-07$ \\
\hline 0101 & $-4.0 \mathrm{E}-07$ & $8.4 \mathrm{E}-09$ & $2.3 \mathrm{E}-07$ & $-1.1 \mathrm{E}-07$ \\
\hline $0101 \mathrm{~A}$ & $3.2 \mathrm{E}-07$ & $-3.6 \mathrm{E}-07$ & $-9.7 \mathrm{E}-07$ & $2.9 \mathrm{E}-07$ \\
\hline 0102 & $-5.4 \mathrm{E}-07$ & $1.6 \mathrm{E}-07$ & $2.6 \mathrm{E}-08$ & $6.9 \mathrm{E}-08$ \\
\hline $0102 \mathrm{~A}$ & $2.1 \mathrm{E}-06$ & $3.6 \mathrm{E}-07$ & $6.8 \mathrm{E}-07$ & $-1.6 \mathrm{E}-07$ \\
\hline 0105 & $6.9 \mathrm{E}-07$ & $-1.8 \mathrm{E}-07$ & $-2.9 \mathrm{E}-07$ & $5.5 \mathrm{E}-08$ \\
\hline
\end{tabular}

Figure 5-37: Run3339 AlphaposU table

\begin{tabular}{|l|r|r|r|r|}
\hline & Aelli vs PITAposV & Delli vs Dy(nm) & Decorr vs Dy(nm) & Aelli vs Dy(nm) \\
\hline 1102 & $1.5 \mathrm{E}-06$ & $8.4 \mathrm{E}-07$ & $1.8 \mathrm{E}-06$ & $-4.7 \mathrm{E}-07$ \\
\hline 1104 & $-2.7 \mathrm{E}-07$ & $2.5 \mathrm{E}-07$ & $3.5 \mathrm{E}-07$ & $-4.8 \mathrm{E}-08$ \\
\hline 1106 & $-1.4 \mathrm{E}-06$ & $3.3 \mathrm{E}-07$ & $8.8 \mathrm{E}-07$ & $-2.8 \mathrm{E}-07$ \\
\hline 0101 & $5.6 \mathrm{E}-08$ & $2.6 \mathrm{E}-07$ & $1.7 \mathrm{E}-07$ & $4.3 \mathrm{E}-08$ \\
\hline $0101 \mathrm{~A}$ & $-5.7 \mathrm{E}-07$ & $1.6 \mathrm{E}-07$ & $-7.0 \mathrm{E}-07$ & $4.3 \mathrm{E}-07$ \\
\hline 0102 & $3.8 \mathrm{E}-08$ & $1.3 \mathrm{E}-07$ & $1.4 \mathrm{E}-07$ & $-4.5 \mathrm{E}-09$ \\
\hline $0102 \mathrm{~A}$ & $-2.6 \mathrm{E}-06$ & $-7.5 \mathrm{E}-07$ & $-1.2 \mathrm{E}-06$ & $2.1 \mathrm{E}-07$ \\
\hline 0105 & $2.2 \mathrm{E}-06$ & $-3.4 \mathrm{E}-07$ & $-7.9 \mathrm{E}-07$ & $2.2 \mathrm{E}-07$ \\
\hline
\end{tabular}

Figure 5-38: Run3339 AlphaposV table

It is also important to know how Aelli is affected by AlphaposU/V. Since we use these voltages to minimize position differences, it is important that in doing so, we are not increasing spot-size asymmetry significantly. The largest slope observed in the $130 \mathrm{keV}$ region is $2 \times 10^{-6} / \mathrm{V}$ in $0 \mathrm{I} 05$ and $0 \mathrm{I} 02 \mathrm{~A}$. That means changing 
AlphaposU/V by 50V may change Aelli by $10^{-4}$ in $0 \mathrm{I} 05$. The position difference control in 0I05 was $13 \mathrm{~nm} / \mathrm{V}$. So, put another way, using AlphaposU/V to correct a position difference of $0.6 \mu \mathrm{m}$ in $0 \mathrm{I} 05$ may change Aelli by $10^{-4}$. These sensitivities can be compared with actual laser table measurements with the linear array, which showed $<4 \times 10^{-7} / \mathrm{V}$, smaller than observed here. We note that the large sensitivity to AlphaposV in 0I05 is opposite in sign to the sensitivity in 0I02A, even though the beam orientation (see next section) is approximately the same for both bpms. It is possible this sensitivity is an artifact of systematic error in subtracting diff_bpmecorr. It should also be noted that our choice of Alpha$\operatorname{pos} \mathrm{U} / \mathrm{V}$ voltages to zero position differences may also come close to the zero of spot-size asymmetries, since even though we applied AlphaposU=12.69V, Alphapos $\mathrm{V}=70.98 \mathrm{~V}$ to get $<80 \mathrm{~nm}$ position differences, as shown in the next section, Aelli was still bounded as $<10^{-4}$.
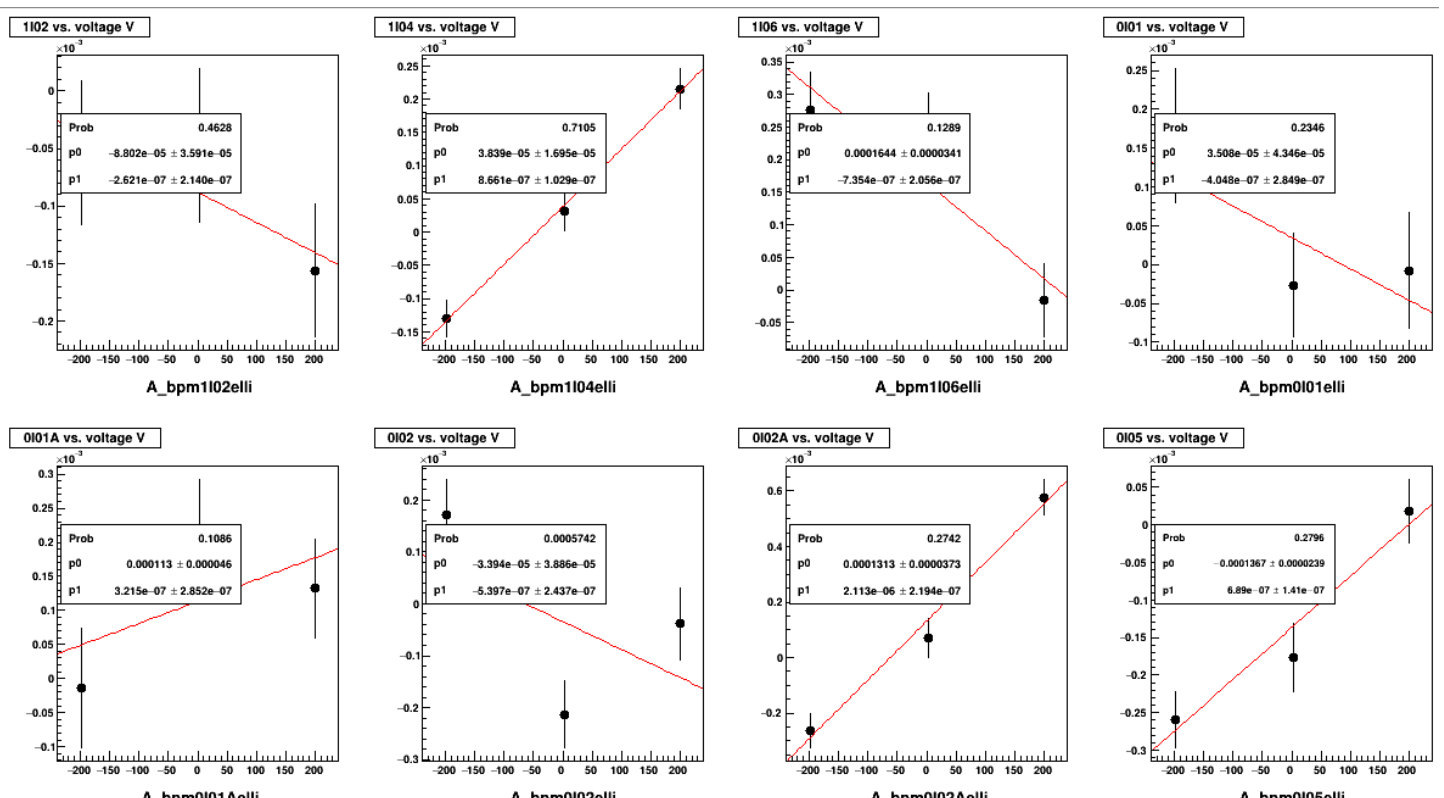

Figure 5-39: Run3339 AlphaposU scan 


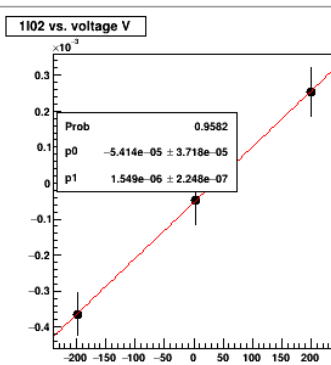

A_bpm 1102elli

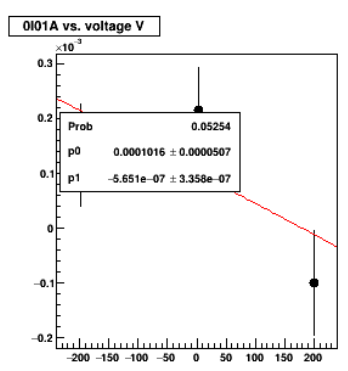

A_bpm0101Aelli

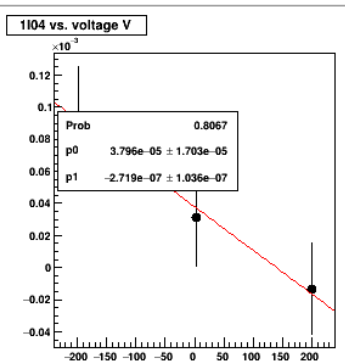

A_bpm1104elli

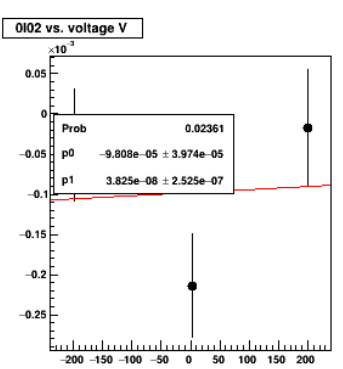

A_bpm0102elli

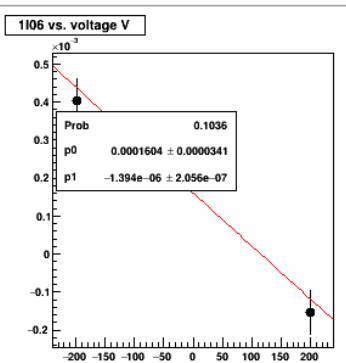

A_bpm1106elli

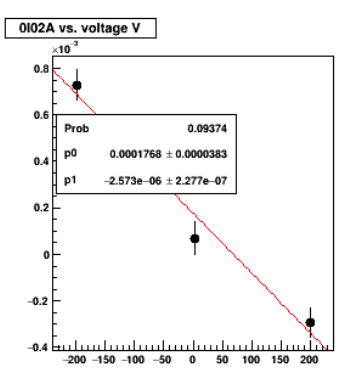

A_bpm0102Aelli

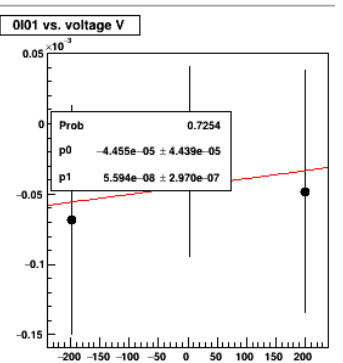

A_bpm0101elli

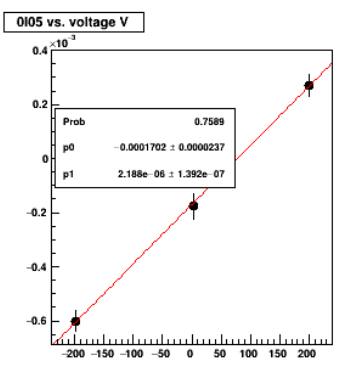

A_bpm0105elli

Figure 5-40: Run3339 AlphaposVscan

\section{e-beam rotation and scale-factor error}

Alphapos U and V scans with the RTP allow us to assess what is happening rotationally to the electron beam, as well as identify any nodes in the propagation. In performing a AlphaposU scan, we make the position differences of a known size along $-45^{\circ}$ on the laser table. By examining the position difference observed in each bpm, we can see if the position difference remains along $-45^{\circ}$ or if it is rotated (i.e. the observed position difference is along only the horizontal, for example). Additionally, if we know we've induced a 1um position difference at the cathode and yet we only observe 100nm at a given bpm, we know that the e-beam is going through a node in the optics of the injector. We can observe that the first bpm in the beamline $1 \mathrm{I} 02$ sees position differences from Alphapos $\mathrm{U}$ along $-48^{\circ}$, so the e-beam at $1 \mathrm{I} 02$ is not rotated relative to the laser table orientation. However, for these injector settings, the subsequent bpms 1I04-0I05 see position differences from AlphaposU mainly along $\approx 0^{\circ}$, the horizontal, indicating the e-beam is rotated by $45^{\circ}$ relative to the laser orientation. That means if we have an elliptical spot 
size asymmetry along the $\mathrm{x} / \mathrm{y}$ on the laser table, we'll see it in bpm1I02 which has it's wire channels along $\mathrm{x} / \mathrm{y}$ and where the e-beam has the same orientation as the laser, but not see it in bpms1I04-0I05. And if we have an elliptical spot size asymmetry along $\mathrm{u} / \mathrm{v}$ on the laser table (i.e. the diagonal), we'll see it in bpms 1I04-0I05 which have their wire channels along $\mathrm{x} / \mathrm{y}$ and where the e-beam is rotated $45^{\circ}$ relative to the laser, but not in $1 \mathrm{I02}$ (for this particular injector setup).

\begin{tabular}{|c|c|c|c|c|c|}
\hline & & \multicolumn{2}{|c|}{$\begin{array}{l}\text { Couplings before solenoid flip } \\
\text { IHWP out, S1, Run3339 }\end{array}$} & \multirow{2}{*}{\begin{tabular}{|l|} 
Est. from $\mathrm{Dr}$ \\
sigma $(\mathrm{mm})$
\end{tabular}} & \multirow{2}{*}{$\frac{(1.5 \mathrm{~mm})^{\wedge} 2 / \text { sigma }^{\wedge} 2}{\text { scale factor error }}$} \\
\hline & & PITAposU (-45deg) & \begin{tabular}{|l|} 
PITAposV (+45deg) \\
\end{tabular} & & \\
\hline $\mathrm{Dr} \sim$ laser & $\mathrm{nm} / \mathrm{V}$ & 7.09 & 5.72 & 0.75 & \\
\hline theta laser & deg & -47.96 & 32.97 & & \\
\hline Dr 1102 & $\mathrm{~nm} / \mathrm{V}$ & 4.56 & 3.82 & 0.49 & 9.33 \\
\hline theta 1102 & deg & 29.20 & -58.56 & & \\
\hline Dr 1104 & $\mathrm{~nm} / \mathrm{V}$ & 5.69 & 5.67 & 0.67 & 5.08 \\
\hline theta 1104 & deg & 7.86 & -85.67 & & \\
\hline Dr 1106 & $\mathrm{~nm} / \mathrm{V}$ & 6.86 & 5.02 & 0.70 & 4.65 \\
\hline theta 1106 & deg & 9.55 & 89.96 & & \\
\hline Dr 0101 & $\mathrm{~nm} / \mathrm{V}$ & 3.65 & 1.34 & 0.29 & 26.39 \\
\hline theta 0101 & deg & 8.44 & 77.63 & & \\
\hline Dr 0I01A & $\mathrm{nm} / \mathrm{V}$ & 1.12 & 1.53 & 0.16 & 93.21 \\
\hline theta $0101 \mathrm{~A}$ & deg & 12.94 & -70.08 & & \\
\hline Dr 0102 & $\mathrm{~nm} / \mathrm{V}$ & 7.75 & 8.80 & 0.97 & 2.40 \\
\hline theta 0102 & deg & 0.91 & -83.86 & & \\
\hline Dr 0102A & $\mathrm{nm} / \mathrm{V}$ & 12.84 & 12.57 & 1.49 & 1.02 \\
\hline theta $0102 \mathrm{~A}$ & deg & 1.34 & -80.78 & & \\
\hline Dr 0105 & $\mathrm{~nm} / \mathrm{V}$ & 12.50 & 9.87 & 1.31 & 1.31 \\
\hline theta 0105 & deg & 6.68 & 89.50 & & \\
\hline
\end{tabular}

Figure 5-41: Couplings Angles 3339

Examining the radial position differences per PITA pos $\mathrm{V}\left(\frac{d D r}{d V}\right)$ in $0 \mathrm{I01}$ and 0I01A, we see only $1-2 \mathrm{~nm} / \mathrm{V}$ at those bpms, when the laser at the cathode was estimated to have $6 \mathrm{~nm} / \mathrm{V}$. This suppression of position differences sensitivity indicated a node in the e-beam optics. Examining 0I02 and 0I05, we see sensitivities like $17 \mathrm{~nm} / \mathrm{V}$ indicating an expansion in the e-beam optics. If the injector were viewed as simply a series of lenses where the beam was centered, from an entirely ray optics perspective, these sensitivities to motion would scale with beam size. In an overly simplified model, the $\frac{d D r_{b p m}}{d D r_{\text {laser }}} \approx \sigma_{b p m} / \sigma_{\text {laser }}$. We can use AlphaposU/V scans to check for errors in the Elegant simulation and errors in our assumption of $\sigma=1.5 \mathrm{~mm}$ at the bpms. We know the laser spot size at the cathode is 
$0.75 \mathrm{~mm}$. Estimating the e-beam spot size from Dr sensitivity, we see spot-sizes in the range $0.2 \mathrm{~mm}-2 \mathrm{~mm}$. Of course, at nodes, the ray-optics model breaks down, so we shouldn't use this model for bpms 0I01 and 0I01A. For the other bpms, we estimate a scale-factor error, discussed in a previous section, which could potentially be applied to Aelli via $A_{\text {elli }}^{A}=$ scale $f A_{\text {elli }}^{M}=\left(\frac{\sigma^{M}}{\sigma^{A}}\right)^{2} A_{\text {elli }}^{M}$. These scale-factor corrections are estimated in this ray-optics model to be anywhere from $0.5 \mathrm{X}-5 \mathrm{X}$. It should be noted that these numbers should be taken lightly, only used for qualitative explanations and not quantitative interpretations. (We note the laser position difference control at the cathode is estimated from the quadphotodiode measurement using throw distance to the cathode $2.014 \mathrm{~m}$ and the throw to the qpd $1.04 \mathrm{~m})$.

\section{RTP and $\mathrm{KD}^{*} \mathrm{P}$ best-case runs}

The analysis of the best case RTP run indicates position differences $<80 \mathrm{~nm}$ and elliptical spot size asymmetries Aelli=(diff_bpmelli-diff_bpmecorr $) / 2$ on the order of $5 \times 10^{-5}$ (Fig. 5-42, in black). diff_bpmelli is also shown in green and the systematic error contribution diff_bpmecorr from the beam not being centered on a given bpm is also shown in red, and they are on a similar order to the spot size asymmetry. The systematic error contribution from Aq $(<5 \mathrm{ppm})$ through pedestal error coupling, found to be $<2 \times 10^{-7} / \mathrm{ppm}$ from the PITA scan, must be $<10^{-6}$ and is therefore negligible. This measurement appears to bound the elliptical spot size asymmetry as being $<5 \times 10^{-} 5$ both along $\mathrm{x} / \mathrm{y}$ (bpm 1I02) and along $\mathrm{u} / \mathrm{v}$ (bpms 1I04-0I05), as discussed in the e-beam rotation section above. 

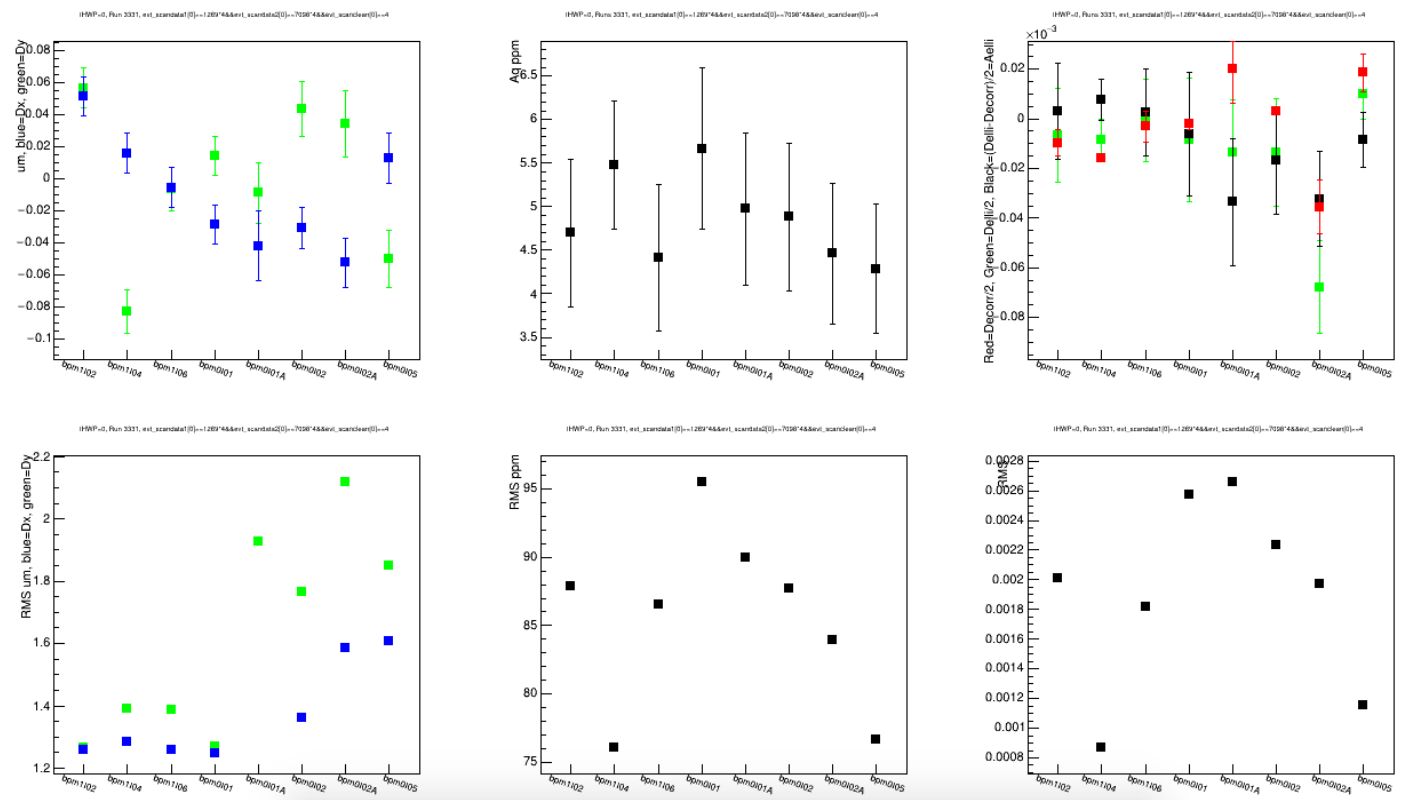

Figure 5-42: RTP cell Run3331 with bpmelli

The KD*P cell shows position differences $<150 \mathrm{~nm}$ and elliptical spot size asymmetries slightly larger than for the RTP, on the order of $10^{-4}$ (Fig. 5-43). The systematic error from diff_bpmecorr is comparable to what it was for the RTP $<5 \times 10^{-5}$ (shown in red). The systematic error contribution from Aq $(<5 \mathrm{ppm})$ through pedestal error coupling is negligible as it was for RTP. 

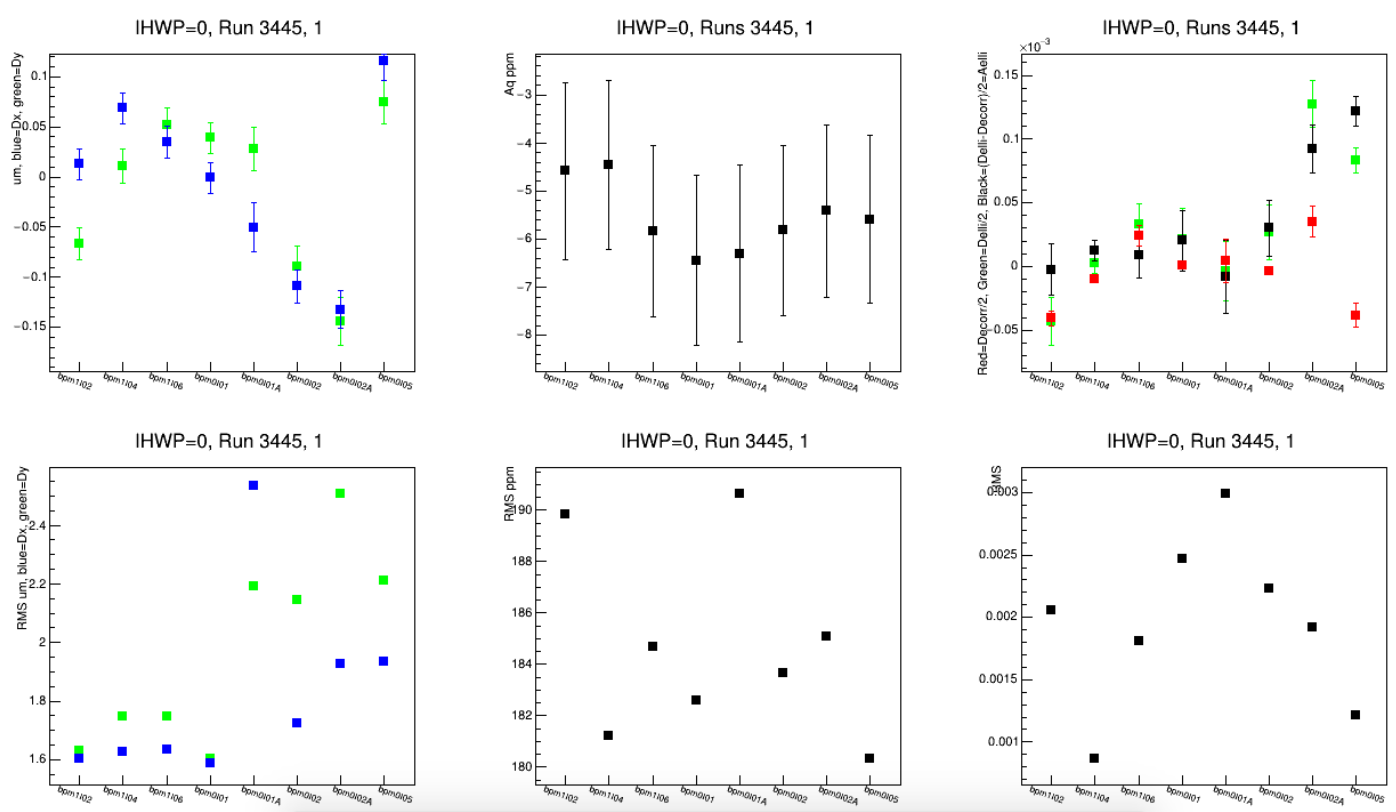

Figure 5-43: KDP Run3445 best-case with bpmelli

This systematic error $\Delta b p m e c o r r$ is from the fact that the e-beam isn't centered on some of the bpms; it is up to $2 \mathrm{~mm}$ off center. The larger the deviation from the beam being centered, the greater the systematic error contribution to $A_{\text {elli }}=\frac{1}{2}(\Delta$ bpmelli $-\Delta$ bpmecorr $)$. We determined from AlphaposU $/ \mathrm{V}$ scans that the contribution for the RTP expected to be $x / \sigma^{2}$ was $<10^{-6} / \mathrm{nm}$. The beam positions $\mathrm{x}, \mathrm{y}$ for each bpm are shown below (Fig. 5-44 a ) and are on the order of $\pm 2 \mathrm{~mm}$ for both the RTP run and $\mathrm{KD} * \mathrm{P}$ run (Fig. 5-44 b ) and look almost identical.

\section{RTP and KD*P runs with scale-factor applied}

We can redo the best-case run plots assuming our $1.5 \mathrm{~mm}$ for spot size in not correct and using the potential scale-factor errors we derived from the PITA pos U/V scans above. Scaling Aelli by these potential scale factor errors produces the following plot for scaled-Aelli in RTP (Fig. 5-45 a ) and KD*P (Fig. 5-45 b ).

The scale-factors for $0 \mathrm{I} 01$ and 0I01A are very large and we discount them because those bpms are at a node where the simplistic ray-optics model these 


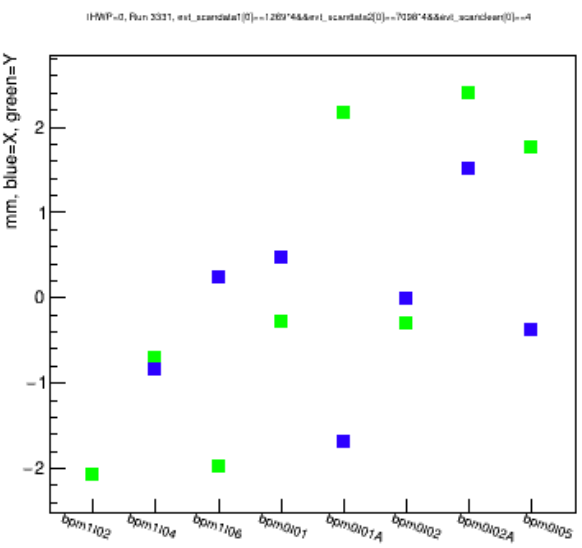

(a) Run3331 RTP XY
$\mathrm{IHWP}=0$, Run 3445,1

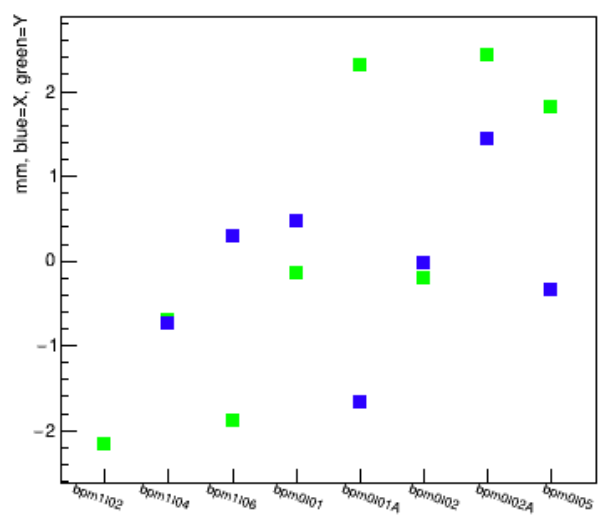

(b) KD*P Run3445 XY

Figure 5-44: beam XY positions along beamline

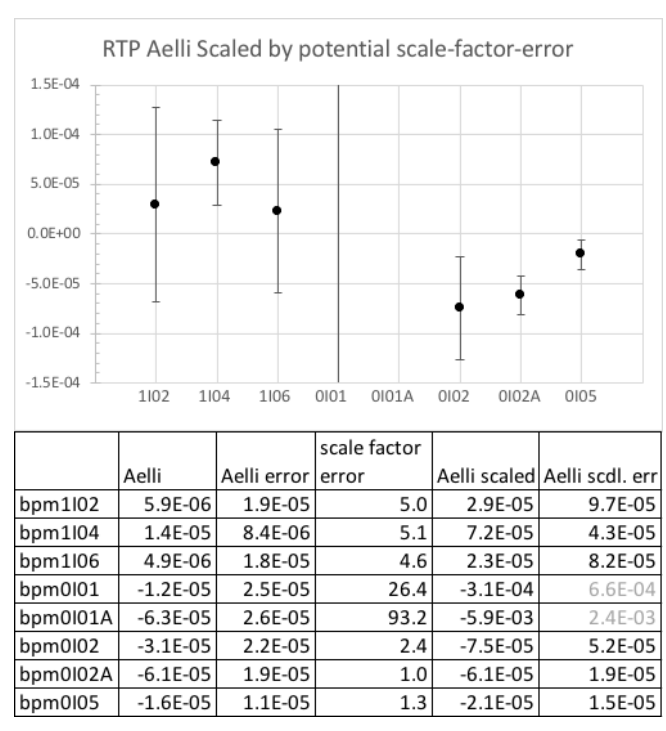

(a) Run3331 RTP Aelli Scaled

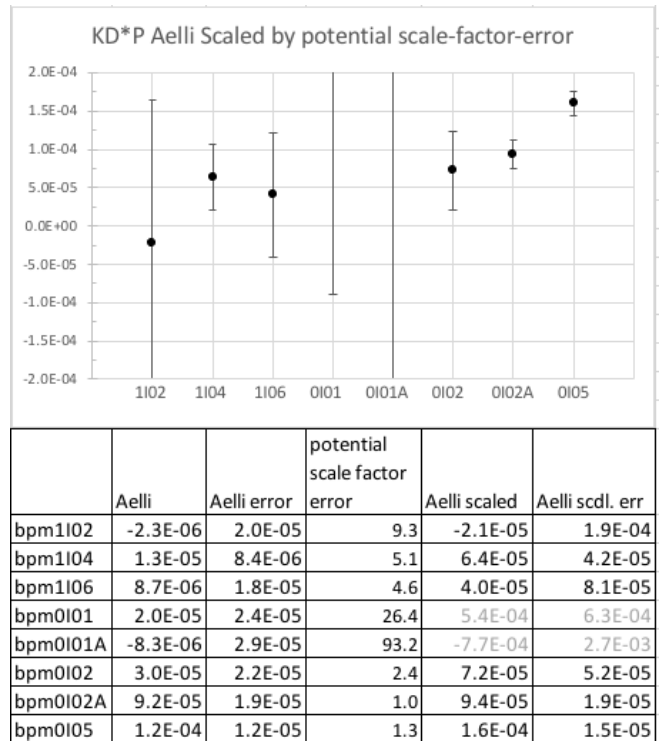

(b) Run3445 KD*P Aelli Scaled

Figure 5-45: Aelli scaled by scale factor error $\left(\sigma^{A} / \sigma^{e s t}\right)^{2}$ estimated from position difference sensitivity to AlphaposU/V scans 
factors were derived from breaks down. Examining bpm1102, which is sensitive to elliptical asymmetries along $\mathrm{x} / \mathrm{y}$ laser table coordinates, Aelli is bounded as $<2 \times 10^{-4}$. Examining bpm0I02A and 0I05, which are sensitive to elliptical asymmetries along $\mathrm{u} / \mathrm{v}\left( \pm 45^{\circ}\right)$ laser table coordinates, Aelli is bounded to be $<$ $5 \times 10^{-5}$ in RTP and $1.5 \times 10^{-4}$ in $\mathrm{KD}^{*} \mathrm{P}$.

\section{Noise}

The best case runs for RTP and KD*P show the RMS of Aelli is $0.8-2.6 \times 10^{-3}$ (Fig. 5-42). These runs were performed at $240 \mathrm{~Hz}$ flip rate, in linesync octet random mode, and analyzed in the multiplet-tree. The integration widow was $3.96 \mathrm{~ms}$ to be precise. So an entire octet consists of the integration of $31.68 \mathrm{~ms}$ of data and so the RMS shown in the multiplet tree is $30 \mathrm{~Hz}-$ like.

$\sqrt{N}$ scaling indicates

$$
\begin{gathered}
m=\text { multiplets } \\
R M S=R M S_{30 H z} \sqrt{(f / m) / 30 H z}
\end{gathered}
$$

The time it takes to reach a particular precision $\mathrm{p}$ on Aelli can be calculated as

$$
\begin{gathered}
N=(f / m) \tau \\
p=R M S / \sqrt{N}=R M S / \sqrt{f \tau / m} \\
\tau(p)=(m / f)(R M S / p)^{2}
\end{gathered}
$$

So for $240 \mathrm{~Hz}$, octet, we are $30 \mathrm{~Hz}$-like and it takes $4-40$ minutes to reach a precision of $\pm 1 \times 10^{-5}$ on Aelli depending on the bpm.

\section{Future Studies}

It would be useful to deliberately create a large Aelli on the laser table, vary it in a predictable way, and observe this large spot size asymmetry in the electron 
beam. This would help test the model, the instrumentation, and the analysis [109]. This could be done by either putting in a $100 \%$ analyzer on the laser table and tilting the RTP cell angle or my installing helicity quad magnets which could induce spot-size asymmetries (along the elliptical mode) which could then be measured with the bpms. It might also be desirable to use the first two solenoids coming off the gun to rotate the e-beam and then just examine the 1st bpm 1I02, analogous to rotating the linear array to examine different spot size asymmetries along different axes.

Two pairs of modified wire BPMs are being installed in 2018, located in the early part of the injector, just after the cathode. They are approximately modified M20 cans. They will be more sensitive to spot-size asymmetry through sensitivity to second moments. The BPMs are coated with a Non-Evaporable Getter (NEG). Second moments from beam models were assessed with Goubau Line (G-Line) scans of the BPMs and they were taken before and after NEG coating by Musson [113]. Results showed that measurements of second moments were possible. Once the BPMs are installed, they will enable monitoring of shape and direction of the beam as it exits the electron gun. Future studies will include these new bpms.

\section{Aelli Conclusions}

We have set bounds on Aelli in the injector with e-beam measurements of $<10^{-4}$. This is consistent with laser table measurements. This is a useful bound for PREX and even for Moller. The other component of spot-size asymmetry, $A_{\text {circ }}$, is only accessible in the e-beam measurements by comparison with a bcm, where Aq is very well known, since $A_{\text {circ }}$ manifests itself in a bpm just like a charge asymmetry. For $A_{\text {circ }}$, laser measurements are probably necessary, but for Aelli e-beam measurements are feasible. 


\subsection{Summary}

We have described the types of beam monitors to be used for PREXII and MOLLER and shown beam study results which demonstrate achievement of the desired resolution for these experiments.

2015-2016 beam studies evaluated $A_{q}$ intensity asymmetry widths, BCM resolution, BPM widths, and their dependence on current, energy, and frequency. SAM's were successfully used in 2015-2016 beam studies to verify that the resolution of the $1 \mathrm{MHz}$ BCM system was 25-30ppm (at $30 \mathrm{~Hz}, 20 \mathrm{uA}$ ) . The resolution of the $1 \mathrm{MHz} \mathrm{BCM}$ system was observed to improve with higher current and appears sufficient for PREXII $(>70 \mathrm{uA}, 120 \mathrm{~Hz})$. Furthermore for $30 \mathrm{~Hz}$, at $60 \mathrm{uA}$, a resolution of $\sim 11 \mathrm{ppm}$ was observed, which is encouraging for MOLLER. We also studied both the electron beam noise and the monitor resolution dependence on the effective helicity flip rate since MOLLER will run at a $2 \mathrm{kHz}$ flip rate, while PREXII will run at a $120 \mathrm{~Hz}$ or $240 \mathrm{~Hz}$ flip rate. Higher frequencies were found to result in smaller widths. The resolution of analog BCM system and the beam asymmetry width improves with higher frequency which is encouraging for PREXII and for MOLLER. Regarding energy dependences, MOLLER will run at $11 \mathrm{GeV}$ and it was important to assess the effect of multiple passes in the accelerator on the intensity asymmetry noise. No evidence was observed that increasing the number of passes increases the beam asymmetry noise.

As regards the digital monitors, we observed evidence in 2016 beam studies data that the digital $\mathrm{BCM}$ receivers were delayed in time relative to the analog $1 \mathrm{MHz}$ BCM receivers. However a low-latency setting was developed in the digital BCM which will work sufficiently well for PREXII. Attenuation configurations were described for PREXII/CREX which will achieve linearity comparable to to the analog $\mathrm{BCMs}$ at $70 \mathrm{uA}$ and $<0.3 \%$ non-linearity at $150 \mathrm{uA}$.

The BPM widths observed from $30 \mathrm{~Hz}$ to $1 \mathrm{kHz}$, from $20 \mu \mathrm{A}$ to $100 \mu \mathrm{A}$, were $1 \mu \mathrm{m}-20 \mu \mathrm{m}$, depending on the BPM and are acceptable for PREXII running. 
However, we note that for MOLLER, at $2 \mathrm{kHz}$ flip rates, large widths are observed due to a beat frequency with the $60 \mathrm{~Hz}$ line. In preparation for MOLLER, the helicity control board must be able to generate mega-multiplets sufficient to fill a $60 \mathrm{~Hz}$ period with $2 \mathrm{kHz}$ windows, in order to cancel out the $60 \mathrm{~Hz}$ beating and get rid of this extraneous noise in BPM signals.

Lastly, we have presented an entirely novel technique of measuring a component of spot-size asymmetry of the electron beam $\left(A_{\text {elli }}\right)$ using a BPM wire combination as yet unexamined in a parity experiment. We have set bounds on Aelli in the injector with e-beam measurements of $<10^{-4}$, consistent with laser table measurements, and a useful bound for PREXII and MOLLER. Aelli's correlation with detectors and SAMs will be examined during PREXII. If correlations are significant, measurement of this parameter could be important for MOLLER which must account for all types of helicity correlated systematic uncertainties more stringently. 


\section{Chapter 6}

\section{RTP Pockels Cell: Parity Quality Beam for MOLLER}

The content of this chapter mirrors an article written by this author which will shortly be submitted for publication [276]. Rubidium Titanyl Phosphate (RTP) has been used in recent years for ultra-fast Pockels cell switches due to its lack of piezo-electric resonances at frequencies up to several hundred MHz. However, crystal non-uniformity in this material leads to poorer extinction ratios than in commonly used $K D^{*} P$ Pockels cells when used in $\lambda / 2$-wave configuration. It leads to voltage dependent beam steering when used in $\lambda / 4$-wave configuration. Here we present an innovative design which uses electric field gradients to counteract crystal non-uniformities and control beam steering down to the nm-level. We demonstrate this RTP Pockels cell design is capable of producing precisely controlled polarized electron beam at Jefferson Laboratory, a national accelerator facility.

\subsection{Introduction}

Pockels cells can be used in a variety of applications including cavity locking in regenerative amplification as well as in electron beam particle accelerators. In electron accelerators, Pockels cells are used to control the spin of the electron beam by 
switching the polarization state of the source laser. In regenerative amplifier laser systems, Pockels cell are often used in a $\lambda / 2$-configuration, switching between horizontal and vertical polarization states, for Q-switching. Non-uniformities in the crystal or electric field lead to poor extinction ratios, cavity leaking and poor amplification. In polarized electron-beam accelerators, Pockels cells are often used in a $\lambda / 4$-wave configuration, switching between right and left circular polarizations, to control the polarization state of light used to produce polarized electron beam from a GaAs photocathode. Pockels cell non-uniformities, when used in in the $\lambda / 4$-wave mode, lead to asymmetries between the right and left circular polarized light states generated and consequently asymmetries in the positive and negative helicity states of the electron beam. In particular, non-uniformities produce helicity-dependent laser beam motion and thus electron-beam position differences. These helicity-correlated position differences can be detrimental to electron beam accelerator parity experiments which make precise comparisons between positive and negative helicity states. Thus, Pockels cell uniformity is of critical importance for both laser amplification and electron beam accelerator applications.

Fast switching capabilities for high repetition rates is also desirable in these Pockels cell applications. Commonly used commercial KD*P Pockels Cells suffer from piezo-electric ringing from acoustic modes when high voltage is suddenly applied to switch polarization states, resulting in a prolonged transition and settle time on the order of 10's of $\mu s$ before the polarization state is fully switched. In regenerative amplifiers for high rep. rate laser systems, a fast-switching Pockels cell with short settle time is desirable. In electron-beam accelerators, when data is taken at a high helicity flip rate, it is also desirable to have a short settle time, to prevent downtime data losses. In $\mathrm{KD}^{*} \mathrm{P}$ cells previously used at the JLab e-beam accelerator (20mm diameter, and the usual 2:1 length:width ratio), this piezo-electric ringing can result in $70-100 \mu$ s of settle time during which period the beam quality is poor and data cannot be taken. The statistical losses due to down time goes as $\sim T_{\text {settle }} f$, where $\mathrm{f}$ is the helicity flip rate. Very high precision 
parity experiments, like the future MOLLER experiment, requiring high $\sim 2 \mathrm{kHz}$ flip rates, depend on the ability to switch helicity state faster and take data at a higher rate than has previously been feasible. ${ }^{1}$ The obvious solution to reducing the settle time of the JLab Pockels cell is to choose a material which suffers less from piezo-electric ringing, even for fast transitions.

RTP(rubidium titanyl phosphate) is a promising material for Pockels cells due to its fast-switching, high repetition rate capabilities. There is no Pockels cell material which can operate as well at high repetition rates as RTP. Unlike commonly used KD*P cells, RTP suffers minimally from piezo-electric ringing artifacts, the resonances being at much higher frequencies, which in high repetition rate pulsed systems can reduce contrast in half-wave mode laser amplification systems and can cost precious transition time in $\lambda / 4$-wave mode electron beam applications. RTP is extremely advantageous in this regard. However, compared with $\mathrm{KD}^{*} \mathrm{P}, \mathrm{RTP}$ 's uniformity is not as good, making for poorer extinction ratios, and in the case of operation in $\lambda / 4$ configuration, producing helicity-dependent laser beam motion. In addition RTP is highly birefringent which makes its uniformity in Pockels cells extremely dependent on the precision of face-cut angles; face cut angles as small as $0.1 \mathrm{mrad}$ can have significant impact on extinction ratios and helicity-correlated position differences.

Here we present a solution which gives us the best of both worlds: fast transition and improved effective uniformity. We have demonstrated $8 \mu$ s transitions in $\lambda / 4$-wave configuration with a large aperture $(12 \mathrm{mmx} 12 \mathrm{~mm})$, transverse, RTP cell (a factor of 10X improvement over the $20 \mathrm{~mm}$ aperture longitudinal $\mathrm{KD}^{*} \mathrm{P}$ previously used at JLab). Furthermore, we present a new RTP Pockels cell design in which crystal intrinsic non-uniformity effects are counteracted with controlled electric-field gradients so that in $\lambda / 4$-wave mode, laser beam helicity correlated

\footnotetext{
${ }^{1}$ Because parity experiments run by flipping helicity states frequently, for the high data rates in the $\sim 2 \mathrm{kHz}$ frequency range required high precision experiments, such as the future MOLLER experiment, this $\sim 100 \mu$ s settle time results data losses on the $10-20 \%$ level, compromising the statistical precision of experiments.
} 
position motion is controllable and kept at the $\sim 10 \mathrm{nrad}, 10 \mathrm{~nm}$ level, while the transition time is kept $<10 \mu s$.

\subsection{Parity Violation Experiments}

In electron beam accelerator facilities like Jefferson Lab, parity-violation experiments are performed which measure asymmetries between reactions with positive(right) and negative(left) helicity states. These experiments measure a parityviolating asymmetry in the differential electron scattering cross section off a target at an angle corresponding to a known energy transfer, and the experimental asymmetry is defined as

$$
A_{e x p}=\frac{d \sigma^{+}-d \sigma^{-}}{d \sigma^{+}+d \sigma^{-}}
$$

where $d \sigma^{+(-)}$refers to the differential cross-section, proportional to the detected rates, for positive and negative electron beam helicity states respectively. The right and left handed longitudinally polarized electrons for such experiments come from right and left circularly polarized light. The Pockels cell controls the spin of the electron beam by switching the polarization state of the laser beam generating it. The Pockels cell is fed a randomized helicity signal which applies either positive or negative high voltages, producing either right or left circularly polarized light, which is incident on a photocathode, producing consecutive windows of right-handed and left-handed electrons. The electrons are accelerated and then sent into the experimental hall where the differential cross-section asymmetries are measured.

To achieve high precision measurements on $A_{\text {exp }}$, the Pockels cell must satisfy both statistical and systematic requirements as regards the electron beam produced. Regarding statistical experimental requirements, in helicity switching, time windows are generated in the electron bunch train at a selected flip rate, 
with the sign of the beam's longitudinal polarization in each window assigned on a pseudo random basis. Frequency selection for helicity flipping affects the noise, measurement widths, and statistical errors significantly. The future MOLLER experiment is a high data rate experiment. So, the RTP Pockels cell which controls the electron beam must switch helicity states very quickly, with minimal dead-time, to obtain sufficient statistical precision at high data rates.

In parity experiments the differential cross-section asymmetries are extremely small. So the symmetry between incident right and left helicity beams is of importance in achieving systematic experimental requirements. As previously stated in Sec. 2.3.7, because this measurement compares right and left handed, opposite helicity, electrons and looks for changes in scattering rates, any change in the polarized beam, correlated with the helicity reversal, can be a potential source for systematic error, or a false asymmetry on $A_{\text {exp }}$ : this includes energy changes, position changes, intensity changes, or spot-size changes. For precise comparisons to be made, the two helicity state beams must be extremely symmetric: their intensity, position, and spot-size must be very nearly identical. As illustrated in Fig. 6-1, an intensity asymmetry in the electron beam can arise from a polarization asymmetry in the laser beam when incident on a polarizing element such as the photocathode. A position difference in the electron beam can arise from a polarization gradient in the Pockels cell, a 1st moment effect producing a shift in central laser beam position. A spot size asymmetry can arise from a 2nd moment in polarization gradient, which can broaden or narrow the beam distribution. 


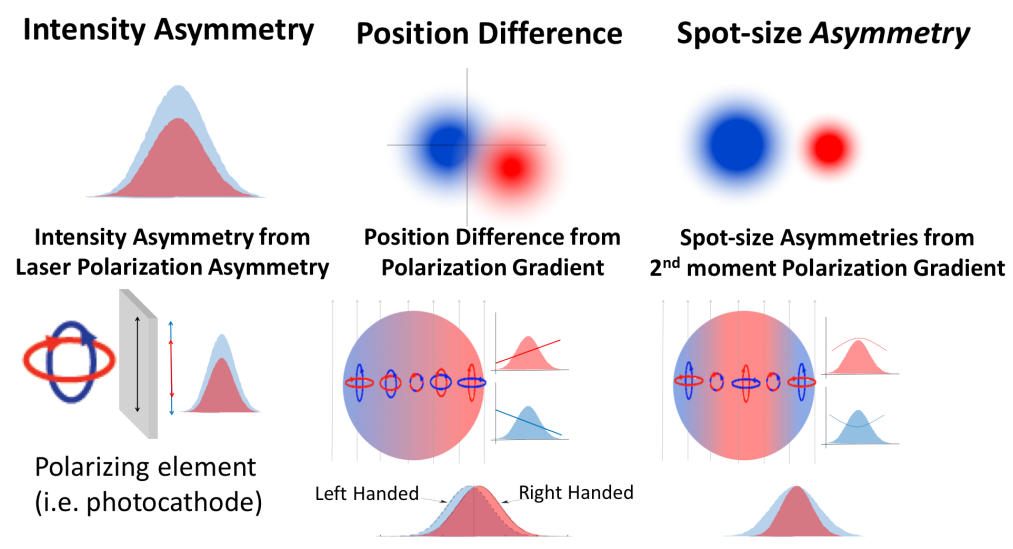

Figure 6-1: Origin of analyzing power dependent beam asymmetries: Here the red and blue ellipses represent polarization ellipses for the opposing right and left circularly polarized states, with residual linear polarization in opposite directions. (a) Coupled to an analyzing power, this can produce an intensity asymmetry (b) If there is a polarization gradient across the beam spot this can produce a position difference (c) If there is a 2nd moment in the polarization gradient across the beam spot this can produce a spot-size asymmetry

Next generation experiment such as MOLLER require electron beam off the cathode with position differences of $<20 \mathrm{~nm}$ and transverse rms spot-size asymmetries of $\sim 10^{-5}$. These requirements motivated the design a new RTP Pockels cell, with many degrees of freedom, to ensure both fast transition times and small helicity-correlated asymmetries.

\subsection{Helicity Correlated Beam Asymmetries}

\subsubsection{Intensity Asymmetry}

A Pockels cell control the polarization state of light passing through it with a voltage induced birefringence via the electro-optic effect. When operated in $\lambda / 2$ configuration, the Pockels cell alternates between acting as a $\lambda / 2$-wave plate, i.e. rotating incident horizontal polarization into vertical polarization, and having nobirefringence, leaving the incident polarization state unchanged. When operated in $\lambda / 4$ wave configuration, the Pockels cell alternates between acting as a quarter- 
wave plate with its fast axis along $+45^{\circ}$ and a quarter wave plate with its fast axis along $-45^{\circ}$, i.e. switching incident linearly polarized light into alternating right and left circular polarization states. Incident horizontally(or vertically) polarized light is exposed to the crystal's fast and slow axes along $\pm 45^{\circ}$ and splits into two beams which propagate with different phases. The phase shifts for the $\pm 45^{\circ}$ components of the incident polarization states along primary fast/slow axes of the crystal are

$$
\phi_{i}^{R(L)}=2 \pi n_{i}^{R(L)} L^{R(L)} / \lambda
$$

where $R(L)$ signify right and left circular polarization states of the outgoing light, i refers to the primary fast and slow axes ( $=\mathrm{y}, \mathrm{z}$ for RTP crystals), $\mathrm{n}$ is the voltage controlled refractive index, and $L^{R(L)}=L_{0}$ is the crystal length which remains fixed (when there is no voltage applied and no piezoelectric effect at play).

In RTP crystals, the voltage dependent refractive indices (along the primary $\mathrm{y}$ and $\mathrm{z}$ axes) are given by [312]

$$
n_{y}^{R(L)}=n_{0, y}-\frac{1}{2} n_{0, y}^{3} r_{23} E_{z}^{R(L)} n_{z}^{R(L)}=n_{0, z}-\frac{1}{2} n_{0, z}^{3} r_{33} E_{z}^{R(L)}
$$

where typically the electrical field $E_{z}=-V / d$ is switched to have opposite sign for right and left polarization and is approximately symmetrically flipped such that $E_{z}^{R} \approx-E_{z}^{L}$ and $V^{R} \approx-V^{L}$ the voltages are nearly equal and opposite.

RTP crystals have a high intrinsic birefringence $\Delta n_{0}=n_{0, z}-n_{0, y} \sim 0.1$ and a single $1 \mathrm{~cm}$ long RTP crystal, standing alone, functions as a $\sim 1000$ th order waveplate at $780 \mathrm{~nm}$. To avoid severe wavelength dependent and temperature dependent effects of a using such a high order waveplate, two crystals are used in RTP Pockels cells with their fast and slow axes in opposite orientations in a socalled 'thermal compensating' design as shown in Fig. 6-2. Such a design causes temperature and wavelength shifts of the first RTP crystal to be canceled by the second RTP crystal $^{2}$. The crystals are cut very precisely to be of equal length such

\footnotetext{
${ }^{2}$ analogous to 0 th order waveplate designs
} 
that $L_{1} \approx L_{2} \equiv L_{0}$ (within $\left.2 \mu \mathrm{m}\right)$ so that the net birefringence is near zero when no voltage is applied. Each of the RTP crystals induces equal and opposite phase shifts such that the Pockels cell acts as a zero-order waveplate when inactive:

$$
\begin{gathered}
\delta_{1}^{R(L)}=2 \pi \Delta n_{1}^{R(L)} L_{0} / \lambda=2 \pi\left(n_{z}^{R(L)}-n_{y}^{R(L)}\right) L_{1} / \lambda \\
\delta_{2}^{R(L)}=2 \pi \Delta n_{2}^{R(L)} L_{0} / \lambda=2 \pi\left(n_{y}^{R(L)}-n_{z}^{R(L)}\right) L_{2} / \lambda \\
\delta_{\text {tot }}^{R(L)}=\delta_{1}^{R(L)}+\delta_{2}^{R(L)}=2 \pi\left(n_{z}^{R(L)}-n_{y}^{R(L)}\right)\left(L_{1}-L_{2}\right) / \lambda \approx 0
\end{gathered}
$$

For two crystals of equal length in succession, the phase shift is $\delta=2 \pi\left(\Delta n_{1}+\right.$ $\left.\Delta n_{2}\right) L_{0} / \lambda$, where $\Delta n_{1}=n_{z}-n_{y}$ is the birefringence of the 1st crystal and $\Delta n_{2}=$ $n_{y}-n_{z}$ is the birefringence of the 2 nd, and so $\delta \approx 0$.

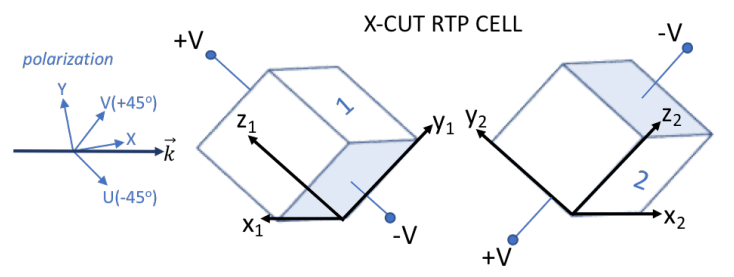

Figure 6-2: RTP thermal compensation design [228]. Beam direction $\hat{k}$ is along the $\hat{x}$ axis in each crystal.

When active in $\lambda / 4$-mode, the two crystals $(1,2)$ are subjected to opposite sign electric fields $E_{z 1} \approx-E_{z 2} \equiv E_{z}$ so that the voltage induced birefringence in both crystals combine additively as follows:

$$
\begin{gathered}
n_{y 1}^{R(L)}=n_{0, y}-\frac{1}{2} n_{0, y}^{3} r_{23} E_{z 1}^{R(L)} n_{z 1}^{R(L)}=n_{0, z}-\frac{1}{2} n_{0, z}^{3} r_{33} E_{z 1}^{R(L)} \\
n_{y 2}^{R(L)}=n_{0, y}-\frac{1}{2} n_{0, y}^{3} r_{23} E_{z 2}^{R(L)} n_{z 2}^{R(L)}=n_{0, z}-\frac{1}{2} n_{0, z}^{3} r_{33} E_{z 2}^{R(L)} \\
\delta_{t o t}^{R(L)}=2 \pi / \lambda\left(\frac{1}{2}\left(n_{0, y}^{3} r_{23}-n_{0, z}^{3} r_{33}\right)\left(E_{z 1}^{R(L)} L_{1}-E_{z 2}^{R(L)} L_{2}\right)+\left(n_{0, z}-n_{0, y}\right)\left(L_{1}-L_{2}\right)\right) \\
\approx 2 \pi L_{0} / \lambda\left(n_{0, y}^{3} r_{23}-n_{0, z}^{3} r_{33}\right) E_{z}^{R(L)}
\end{gathered}
$$


where $E_{z}$ is at the quarter-wave field strength in a two-crystal RTP cell system, approximately given by

$$
\left|E_{\lambda / 4}\right|=\frac{\lambda}{4 L_{0}\left(n_{z 0}^{3} r_{33}-n_{y 0}^{3} r_{23}\right)}
$$

and where the corresponding quarter wave voltage is given by $V=-E_{z} d$

$$
\left|V_{\lambda / 4}\right|=\frac{d \lambda}{4 L_{0}\left(n_{z 0}^{3} r_{33}-n_{y 0}^{3} r_{23}\right)}
$$

where $\mathrm{d}$ is the width of the crystal. For $780 \mathrm{~nm}, \mathrm{~L}=10 \mathrm{~mm}, \mathrm{~d}=12 \mathrm{~mm}$, we have $V_{\lambda / 4}=1491 \mathrm{~V}$ and $E_{\lambda / 4}=124.3 \mathrm{~V} / \mathrm{mm}^{3}$

When operating in $\lambda / 4$-mode, the resultant right and left circular polarization states may not be perfectly circular, having a slight ellipticity, and may not be perfectly symmetric, having different ellipticity for right and left polarization states. This deviation from perfect circular polarization is characterized by the birefringence induced phase shift $\delta$, which each helicity state undergoes.

Each helicity state is nearly circular $(\delta \approx \pm \pi / 2)$ with small deviations from an $\alpha$-phase and a $\Delta$-phase, giving rise to residual linear polarization components as shown in Fig. 6-3. The $\alpha$-phase signifies a component of linear polarization which is symmetric in both polarization states, whereas the $\Delta$-phase signifies an asymmetric component between the polarization states.

$$
\delta^{R(L)}=\mp(\pi / 2+\alpha)-\Delta
$$

\footnotetext{
${ }^{3}$ Empirically observed $V_{\lambda / 4} \sim 1600 \mathrm{~V}$ implies $E_{\lambda / 4}=133.3 \mathrm{~V} / \mathrm{mm}$
} 


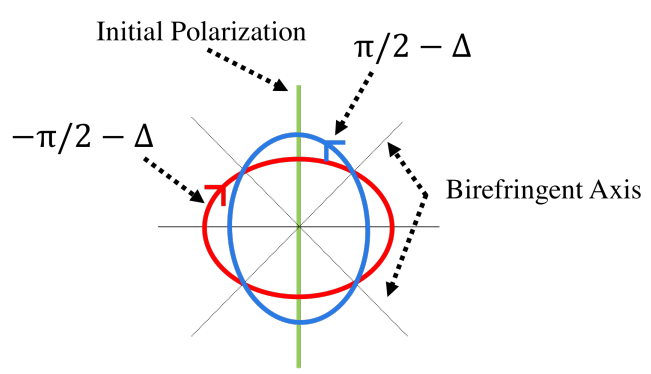

Figure 6-3: $\Delta$-phase is anti-symmetric, resulting in residual linear polarization along complementary axis between the two helicity states light.[229]

In $\lambda / 4$-configuration, it is critical to minimize the asymmetric component of linear polarization. The Pockels cell voltages can control the asymmetric component of linear polarization along the horizontal/vertical axes, defined by "S1" in Stokes parameter terminology (for further discussion see Appendix D.2.1). The Stokes parameters $S_{0}, S_{1}, S_{2}$, and $S_{3}$, respectively define the degree of polarization (DoP), the degree of linear polarization (DoLP) along horizontal/vertical axes, the DoLP along the diagonal $\pm 45^{\circ}$ axes, and the degree of circular polarization (DoCP). We define a $\Delta$-voltage, also called a PITA (Phase Induced Transmission Asymmetry)-voltage [46]), which controls the $\Delta$-phase and an $\alpha$-voltage which controls the $\alpha$-phase as:

$$
V^{R(L)}= \pm\left(\left|V_{\lambda / 4}\right|+V_{\alpha}\right)+V_{\Delta}= \pm V_{0}+V_{\Delta}
$$

where we have defined $V_{0}=\left|V_{\lambda / 4}\right|+V_{\alpha}$. The corresponding electric field in each crystal is $E_{z}=-V / d$

$$
E_{z}^{R(L)}=\mp\left(\left|E_{\lambda / 4}\right|+E_{\alpha}\right)-E_{\Delta}=\mp E_{0}-E_{\Delta}
$$

Such polarization asymmetries lead to intensity asymmetries when exposed to a polarizing element in the beamline. Conversely, intensity asymmetries can be controlled with voltage induced polarization changes when there is a polarizing element. When performing diagnostic tests on the laser table, we use a polarizer 
with $100 \%$ analyzing power as the polarizing element, and measure transmission and intensity asymmetry $A_{I}$ with a photodiode, as shown in Fig 6-4 ${ }^{4}$.

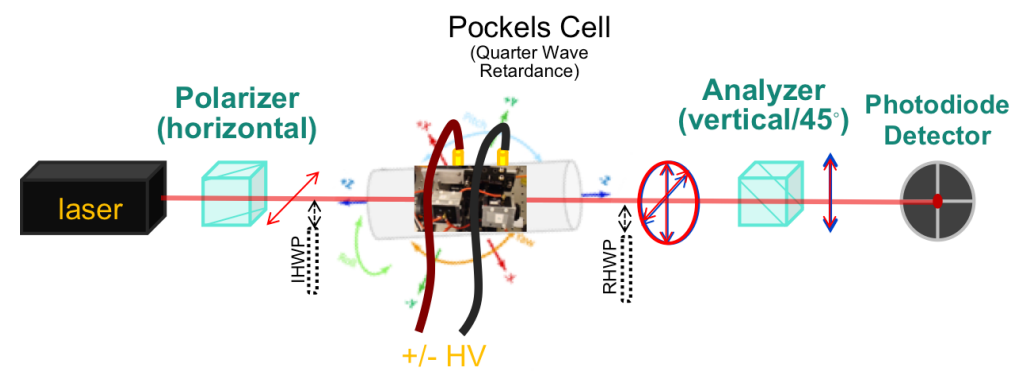

Figure 6-4: This is the cartoon of the typical laser table Pockels cell testing configuration. Horizontally polarized light passes through the Pockels cell which produces L or R circ. Pol light. A second polarizing element is inserted which detects any polarization asymmetry on a photodiode detector angle, translation and voltage adjustments to the Pockels Cell minimize any asymmetry in the degree of linear polarization along S1.

An analyzer is inserted after the Pockels cell with transmission coefficients $T_{x}, T_{y}$ along an axis $\mathrm{x}, \mathrm{y}$ where $\psi$ is the angle subtended between the analyzing direction $\mathrm{x}$ and the initial polarization axis (along $\mathrm{S} 1$, here we assume the horizontal axis). The transmission through a polarizing element for each polarization state is described by:

$$
T^{R(L)}=T \frac{1}{2}\left(1+\epsilon / T \sin (2(\eta-\psi)) \cos \delta^{R(L)}\right)
$$

where $\epsilon=T_{x}-T_{y}, T=\left(T_{x}-T_{y}\right) / 2$ defines the analyzing power of the polarizer(or polarizing element) and $\eta$ is the effective fast-axis of the Pockels cell crystal relative to the horizontal axis.

In the polarized electron source, the left and right circularly polarized light is incident on a photocathode, which acts as a partial polarizer with slight $(<6 \%)$

\footnotetext{
${ }^{4}$ Additionally there is an insertable half-wave-plate IWHP upstream of the PC to convert the H-polarizaton to V-polarization to perform a sign flip to control systematics. There is also a rotating half-wave-plate RHWP downstream of the PC so that the beam polarization can be rotated in accordance with the direction of the analyzing power of the cathode that generates the electron beam
} 
analyzing power. What starts as an asymmetry in laser polarization and would become an asymmetry in light transmission (if the polarizer were optical), instead becomes an asymmetry in the charge of the electron beam. The charge asymmetry $A_{q}$ (also referred to as intensity asymmetry $A_{I}$ for a laser beam), is controlled with the Pockels Cell PITA voltage by inducing $\Delta$-phase polarization changes along S1 (as shown in Fig. 6-3) and the analyzing photocathode.

The intensity asymmetry $A_{I}$ is given by

$$
A_{I}=\frac{T^{R}-T^{L}}{T^{R}+T^{L}} \approx \frac{\epsilon}{T} \sin (2(\eta-\psi)) \frac{1}{2}\left(\cos \delta^{R}-\cos \delta^{L}\right) \approx-\frac{\epsilon}{T} \sin (2(\eta-\psi)) \Delta
$$

where we have used the approximation $\cos \delta^{R}-\cos \delta^{L} \approx \delta^{R}+\delta^{L}=-2 \Delta$. The $\Delta$-phase can be derived by considering the total phase shifts from the two crystals

$$
\begin{gathered}
\Delta=\frac{-1}{2}\left(\delta^{R}+\delta^{L}\right)=\frac{-1}{2}\left(\delta_{t o t}^{R}+\delta_{\text {tot }}^{L}\right)= \\
\pi / \lambda\left(\left(n_{0, y}^{3} r_{23}-n_{0, z}^{3} r_{33}\right)\left(E_{\Delta 1} L_{1}+E_{\Delta 2} L_{2}\right)+\left(n_{0, y}-n_{0, z}\right)\left(L_{1}-L_{2}\right)\right)
\end{gathered}
$$

where for each crystal

$$
\begin{aligned}
& E_{z 1}^{R(L)}=\mp\left(\left|E_{\lambda / 4}\right|+E_{\alpha 1}\right)-E_{\Delta 1}=\mp E_{z 1,0}-E_{\Delta 1} \\
& E_{z 2}^{R(L)}= \pm\left(\left|E_{\lambda / 4}\right|+E_{\alpha 2}\right)+E_{\Delta 2}= \pm E_{z 2,0}+E_{\Delta 2} \\
& V_{z 1}^{R(L)}= \pm\left(\left|V_{\lambda / 4}\right|+V_{\alpha 1}\right)+V_{\Delta 1}= \pm V_{z 1,0}+V_{\Delta 1} \\
& V_{z 2}^{R(L)}=\mp\left(\left|V_{\lambda / 4}\right|+V_{\alpha 2}\right)-V_{\Delta 2}=\mp V_{z 2,0}-V_{\Delta 2}
\end{aligned}
$$

and where $E_{z 1} \approx-E_{z 2} \approx E_{z}$ and these are defined in relation to the overall cell quarter-wave fields as

$$
\Delta \approx \frac{2 \pi L_{0}}{\lambda}\left(n_{0, y}^{3} r_{23}-n_{0, z}^{3} r_{33}\right) E_{\Delta} \approx-\frac{\pi}{2\left|V_{\lambda / 4}\right|} V_{\Delta}
$$




$$
\begin{gathered}
E_{z}^{R(L)}=\frac{E_{z 1}^{R(L)}-E_{z 2}^{R(L)}}{2}=E_{0}-E_{\Delta} \\
E_{0}=\frac{E_{z 1,0}-E_{z 2,0}}{2} \\
E_{\Delta}=\frac{E_{\Delta 1}+E_{\Delta 2}}{2} \\
V_{0}=\frac{V_{z 1,0}-V_{z 2,0}}{2} \\
V_{\Delta}=\frac{V_{\Delta 1}+V_{\Delta 2}}{2}
\end{gathered}
$$

The $\Delta$-phase can have multiple contributions besides electric fields from various sources, including vacuum windows, angular misalignment of the cell, a rotating HWP downstream of the cell, giving rise to $\Delta_{0}$. Hence, the electron beam charge asymmetry $A_{q}$ is given by,

$$
A_{q} \approx \frac{\epsilon}{T}\left(\sin (2(\eta-\psi)) \frac{\pi}{2\left|V_{\lambda / 4}\right|} V_{\Delta}-\Delta_{0}\right)
$$

which when the slow axis of the crystal is along $\eta=45^{\circ}$, reduces to

$$
A_{q} \approx \frac{\epsilon}{T}\left(\cos (2 \psi) \frac{\pi}{2\left|V_{\lambda / 4}\right|} V_{\Delta}-\Delta_{0}\right)
$$

For $100 \%$ analyzer on the laser table (along S1 where $\psi=0^{\circ}, 90^{\circ}=\eta \pm 45^{\circ}$ ) this reduces to

$$
A_{I} \approx-\Delta \approx \frac{\pi}{2\left|V_{\lambda / 4}\right|} V_{\Delta}-\Delta_{0}
$$

The sensitivity of $A_{q}$ to $V_{\Delta}$ is called the PITA-slope $\frac{d A_{I}}{d V_{\Delta}}$, and for our RTP system at $780 \mathrm{~nm}$ is approximately $1053 \mathrm{ppm} / \mathrm{V}^{5}$ for a $\epsilon / T=100 \%$ polarizer along S1 .While the $\Delta$-phase has multiple contributions from various sources giving rise to $\Delta_{0}$, it can be controlled and zeroed out with voltage. Hence, the Pockels cell

\footnotetext{
${ }^{5}$ this depends on the definition of PITA running with the $8 \mathrm{HV}$ system, but we've empirically observed the PITA-individual slope to be $\sim 850 \mathrm{ppm} / V_{\Delta, i n d v}$ where each individual plate is at $+-800 \mathrm{~V}$, which is equivalent to a PITA slope of $\sim 1700 \mathrm{ppm} / V_{\Delta, t o t}$ for $\mathrm{QWV} \sim 1600 \mathrm{~V}$
} 
PITA voltage can control polarization asymmetries along the S1 direction, and can control intensity asymmetries when the polarizing element has a component of analyzing power along the S1 direction (i.e $\epsilon / T \sin (2(\eta-\psi)) \neq 0$ ) .

\section{Angle dependence Intensity asymmetry-S1}

When aligning the Pockels cell in $\lambda / 4$-configuration, angular adjustments are critical for minimizing HCBA (and for maximizing the extinction ratio in $\lambda / 2$ systems). The angle-dependence of the Pockels Cell polarization asymmetry can be derived from the extra birefringence induced when the beam path through the crystal in changed. In general, any passive birefringent element inserted after the Pockels cell, when operated in $\lambda / 4$-configuration, adds a phase shift $\Delta$ to the circular polarization states, producing polarization asymmetry $\Delta$ between right and left states along the direction of birefringence. When analyzed along the asymmetry direction, this gives rise to an intensity asymmetry of magnitude $A_{I}=$ $-\Delta$.

In the case of an X-cut RTP Pockels cell, the beam propagates mainly along the crystal $\mathrm{x}$-axis and the fundamental refractive indices $n_{y}$ and $n_{z}$ which the transverse polarization are exposed to are quite different. At $780 \mathrm{~nm}$, the refractive indices of RTP are shown in Table 6.1.

\begin{tabular}{|l|l|}
\hline$n_{x}$ & 1.7739 \\
\hline$n_{y}$ & 1.7832 \\
\hline$n_{z}$ & 1.8673 \\
\hline
\end{tabular}

Table 6.1: RTP refractive indices at 780nm from Sellmeier's equation [328]

When laser beam propagation is at an slight angle, while this will mix $n_{x}$ into $n_{z}$ or $n_{y}$ slightly, as well as lengthen the beam propagation distance through the crystal. The angle-dependence of the Pockels Cell intensity asymmetry can be derived from the extra birefringence induced phase shift in the beam path 
through the crystal by (1) the extra crystal length when it's tilted at an angle and (2) the effective refractive index mixing with the longitudinal x-axis, which alters the effective birefringence. Both of these mechanisms work together and can be modeled as effectively adding an angle-dependent extra birefringent passive element, which produces an intensity asymmetry

$$
A_{I}\left(\xi_{x 0}, \xi_{y 0}\right)=\frac{\epsilon}{T} \sin (2(\eta-\psi)) \frac{\pi L_{0}}{\lambda}\left(n_{z}+n_{y}\right)\left(\frac{1}{n_{x}^{2}}-\frac{1}{n_{z} n_{y}}\right)\left(2 \xi_{x 0} \xi_{y 0}\right)
$$

where $\xi_{x 0}$ is the yaw tilt of the Pockels cell relative to the crystal's primary x-axis and $\xi_{y 0}$ is the pitch tilt relative to the crystal's primary x-axis. In $\mathrm{S} 1\left(\eta-\psi=45^{\circ}\right)$, the sensitivity to angle is maximal $(\sin (2(\eta-\psi))=1)$. For a $100 \%$ analyzer and $1 \mathrm{~cm}$ long crystals, the angle sensitivity is predicted to be

$$
\frac{d^{2} A_{I}}{d \xi_{x 0} d \xi_{y 0}}=-2 \frac{\pi L_{0}}{\lambda}\left(n_{z}+n_{y}\right)\left(\frac{1}{n_{z} n_{y}}-\frac{1}{n_{x}^{2}}\right)=5137 p p m / m_{r a d}{ }^{2}
$$

For a derivation of the intensity asymmetry angle dependence in RTP see Appendix A.

\subsubsection{Intensity Asymmetry -S2}

In addition to there being an asymmetric degree of linear polarization along the vertical/horizontal axes for the two left and right helicity states, there can also be an asymmetric degree of linear polarization along the diagonal axes. In addition to a polarization asymmetry oriented along the Stokes parameter S1, there can also be an asymmetry along the Stokes parameter S2, signifying the degree of linear polarization along diagonal axes $+45^{\circ}$ and $-45^{\circ}$, as shown in Fig. 6-5.

This $\mathrm{S} 2$ polarization asymmetry can arise in $\mathrm{KD}^{*} \mathrm{P}$ cells from an angular misalignment of the cell, but in RTP cells, due to the large intrinsic birefringence of the crystal, this angular dependence is suppressed in S2 (as can be seen by 
Eq. 6.1). However another kind of misalignment in RTP cells can give rise to this S2 polarization asymmetry: the relative roll angle between the two crystals. In commercial RTP cells, the two crystals are mounted such that they are fixed permanently relative to one another. To obtain the high degree of symmetry required by a parity experiment application, it was necessary to design a cell system with the two crystals mounted independently, allowing for precise control over the relative roll angle between the crystals.

An advantage to this adjustable cell design is that this Pockels cell has control over both S1 polarization and S2 polarization states, which make it a device capable of completely controlling the polarization state of the outgoing light, whereas ordinary Pockels cell designs only have control over S1 polarization. In other accelerator systems, complete control over both S1 and S2 was obtained by the use of two Pockels cells in succession [230]. Whereas, this system uses the two crystals in an RTP cell to obtain the same degree of control with a single system, using relative roll degree of freedom.

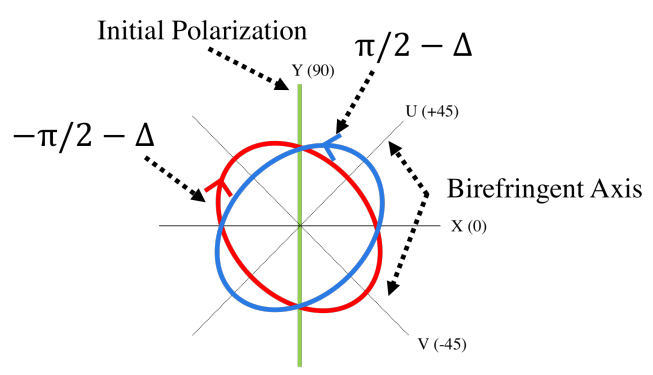

Figure 6-5: Polarization ellipses for asymmetric S2 components on mostly circular polarization states.

\section{Angle dependence Intensity asymmetry-S2}

The S2 polarization asymmetry in RTP does not arise from pitch or yaw angular misalignment. Examining the equation for angle dependence Eq. 6.1, we note that that $A_{I} \approx 0$ in S2 when $\eta=\psi=45^{\circ}$; meaning the dependence on angular adjustments is negligible when analyzing along $\pm 45^{\circ}$. The reason for this lack of 
$\mathrm{S} 2$ angle dependence in RTP, as opposed to in $\mathrm{KD}^{*} \mathrm{P}$ which does have $\mathrm{S} 2$ angle dependence is that RTP is a biaxial crystal and the cell is transverse while $\mathrm{KD}^{*} \mathrm{P}$ is uniaxial and the cell is longitudinal.

Unlike the uniaxial $\mathrm{KD}^{*} \mathrm{P}$, which has nearly equal transverse refractive indices, in RTP the fundamental refractive indices $n_{y}$ and $n_{z}$ which the transverse polarization are exposed to are quite different. The refractive indices of RTP at 780nm are $n_{x}=1.77$ (the beam propagation axis), $n_{y}=1.78, n_{z}=1.87$ (the transverse axes) [328]. By comparison, in $\mathrm{KD}^{*} \mathrm{P}$ the refractive indices are $n_{z} \approx 1.5$ (the beam propagation axis), $n_{x}=n_{y}=1.47$ (the transverse axes). In $\mathrm{KD}^{*} \mathrm{P}$, propagation at a slight tilt angle mixes the longitudinal index $n_{z}$ into the nearly equal transverse indices $n_{x} \approx n_{y}$, altering the direction of the effective transverse fast and slow axes. By contrast, in RTP when the laser beam propagation is at an slight tilt angle, while this will mix $n_{x}$ into $n_{z}$ or $n_{y}$ slightly, it is insufficient to alter the direction of the effective fast and slow axes. The effective fast and slow axis very nearly remain along the original $y, z$ directions of $\pm 45^{\circ}$ regardless of a small tilt angle (because $n_{z}$ and $n_{y}$ are very different to start with). For an RTP crystal, the slow axis $\eta=y=45^{\circ}$ regardless of small $\left(\xi_{x 0}, \xi_{y 0}\right)$ tilt angles.

In the RTP cell, two crystal design, since the primary indices are along $\pm 45^{\circ}$, the effective indices along $0,90^{\circ}$ are identical and hence there can be virtually no birefringence along S2 orientation, and no angle dependent asymmetry. Furthermore, since there is virtually no asymmetry in S2, there are no higher order asymmetry gradients in S2, and hence RTP suffers less less from position differences and spot-size asymmetries than $\mathrm{KD}^{*} \mathrm{P}$ when the analyzer is oriented along $\mathrm{S} 2$.

\section{Roll dependence Intensity asymmetry-S2}

The S2 polarization asymmetry in RTP, while insensitive to angular misalignment, is sensitive to another kind of misalignment specific to transverse, two crystal cell systems: the relative roll angle between the two crystals. The effect of a rel- 
ative roll misalignment on S2 asymmetry can be understood as follows. An extra birefringent element gives rise to a polarization asymmetry, the direction which depends on the orientation of the effective fast and slow axes of the birefringent element. An extraneous birefringence with fast/slow axes along $+45^{\circ} /-45^{\circ}$ and along the RTP crystals $\mathrm{y} / \mathrm{z}$ axes gives rise to an asymmetry along $\mathrm{S} 1$ which can be corrected with Pockels cell PITA voltage. But if there is an extraneous birefringence with a fast/slow axes along $\mathrm{x} / \mathrm{y}$, this gives rise to an asymmetry along S2 which cannot be corrected with Pockels cell voltage. Since there are two crystals, if one crystal has its $\mathrm{y} / \mathrm{z}$ axes slightly rotated relative to the other crystal, it is effectively acting as an extraneous birefringent element which (a) no longer perfectly cancels out the birefringence of the other crystal and (b) has a component of birefringence along $\mathrm{x} / \mathrm{y}$ which can't be corrected with $\mathrm{PC}$ voltage.

The cell system presented here is designed with the two crystals mounted independently, allowing for precise control over the relative roll angle between the crystals. In aligning our RTP cell, we use relative roll between the crystals to minimize the polarization asymmetry along the S2. We predicted by Jones calculus, with two birefringent elements with approximately quarter wave operation, that the S2 polarization dependence on relative roll would result in an intensity asymmetry of $16,200 \pm 580 \mathrm{ppm} / \mathrm{deg}$ [241] when analyzed with a $100 \%$ polarizer along S2 (the error bar is for $\pm 1^{\circ}$ overall cell roll angle). This prediction was confirmed by measurement where the roll angle of one crystal was varied while the roll angle of the other crystal was fixed and obtained $16665 \pm 2 \mathrm{ppm} / \mathrm{deg}$ within the predicted range in Fig. 6-6 [242]. 


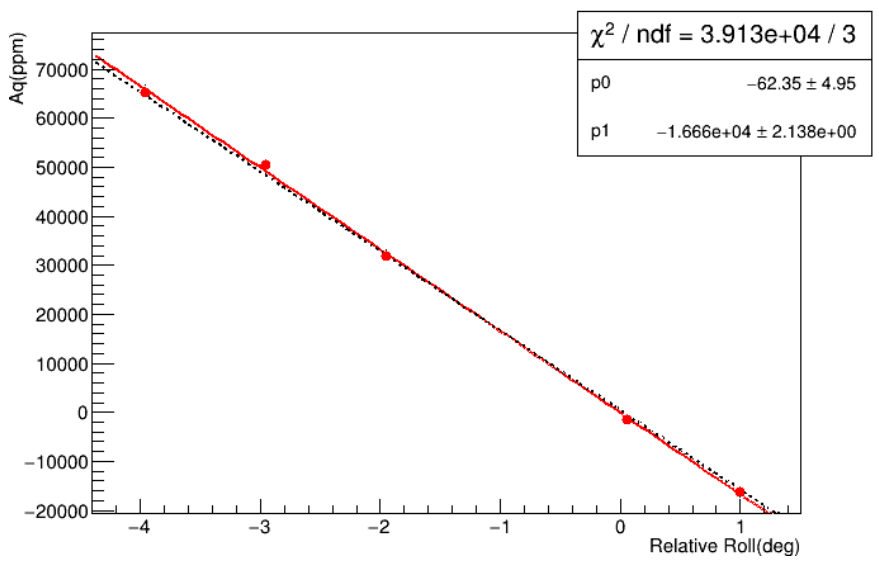

Figure 6-6: Relative Roll and Aq in S2. These measurements were performed by setting rotating the two RTPs relative to eachother, and examining the helicity correlated beam asymmetry when analyzing along S2 (along the crystal's primary axis direction). Red dots are data points, red line is the fit to the data points, black line is the prediction [242]

\subsubsection{Position differences: Analyzing-like}

A gaussian beam with power distribution $P(x)=P_{0} e^{-2 x^{2} / w^{2}}$ which encounters a gradient in transmission $T=\left(T_{0}+\frac{d T}{d x} x\right)$ undergoes a shift in the beam central position characterized by $\langle x\rangle=\frac{\int x P d x}{\int P d x}=\frac{\frac{d T}{d x} w^{2}}{4}$ where the change in first moment is proportional to the transmission gradient. This effect is a shift in central laser beam position. Analogously, as illustrated in Fig. 6-1, a polarization asymmetry gradient in the Pockels cell, when analyzed, gives rise to an intensity asymmetry gradient which in turn produces position differences between right and left polarization states,

A gaussian beam exposed to a helicity dependent gradient $T^{R(L)}=T_{0}\left(1 \pm \frac{d A}{d x} x\right)$ will have helicity dependent power distribution

$$
P^{\prime R(L)}=P_{0} e^{-2 x^{2} / w^{2}}\left(1 \pm \frac{d A}{d x} x\right)
$$


which gives rise to a position difference

$$
D_{x}=x^{R}-x^{L}=\frac{\frac{d A}{d x} w^{2}}{2}=-\frac{\epsilon}{T} \frac{\frac{d \Delta}{d x} w^{2}}{2} \cos (2 \psi)
$$

where $\mathrm{w}$ is the beam waist $(2 \sigma)$ at the Pockels cell and where \pm correspond to right- and left-handed helicity states, $\epsilon / T$ is the analyzing power, and $d \Delta / d x$ is the $\Delta$-phase polarization gradient ${ }^{6}$. We refer to this type of position difference as 'analyzing-like' since the position differences only appear in proportion to the analyzing power. For the electron beam, the spot size of the laser once it reaches the photocathode is important. The position differences scale with spot-size, so at the cathode we take:

$$
D_{x}^{\text {cathode }}=\frac{w_{\text {cathode }}}{w} D_{x}=-\frac{\epsilon}{T} \frac{\frac{d \Delta}{d x} w_{\text {cathode }} w}{2} \cos (2 \psi)
$$

Polarization gradients and the consequential 'analyzing-like' position differences are seen to arise from birefringence gradients in the Pockels cell (other optical elements can also contribute). These birefringence gradients in the Pockels cell have 3 potential sources (1) electric field non-uniformity (2) crystal length variation due to imperfect crystal face cuts (3) intrinsic birefringence gradients in the crystals due crystal imperfections in the growth process or stress in the crystals. These sources of birefringence gradients (that ultimately contribute to asymmetry gradients) can be derived by taking derivatives of the equation describing the birefringence $\Delta$. The asymmetric birefringence component $\Delta$ can be written as:

$$
\Delta=\pi / \lambda\left(\left(n_{0, y}^{3} r_{23}-n_{0, z}^{3} r_{33}\right)\left(E_{\Delta 1} L_{1}+E_{\Delta 2} L_{2}\right)+\left(n_{0, y}-n_{0, z}\right)\left(L_{1}-L_{2}\right)\right)
$$

\footnotetext{
${ }^{6}$ This gradient also gives rise to a helicity correlated asymmetric beam shape as well as a shift in the central position(position difference). Theoretically this beam shape difference could couple in to other HCBAs in beam transport.
} 
where each crystal contributes

$$
\begin{aligned}
& \Delta_{1}=\pi / \lambda\left(\left(n_{0, y}^{3} r_{23}-n_{0, z}^{3} r_{33}\right)\left(E_{\Delta 1} L_{1}\right)+\left(n_{0, y}-n_{0, z}\right) L_{1}\right) \\
& \Delta_{2}=\pi / \lambda\left(\left(n_{0, y}^{3} r_{23}-n_{0, z}^{3} r_{33}\right)\left(E_{\Delta 2} L_{2}\right)-\left(n_{0, y}-n_{0, z}\right) L_{2}\right)
\end{aligned}
$$

The position difference is a vector which can be described by components $D_{x}, D_{y}$ (or by $45^{\circ}$ rotated coordinate system components $D_{u}, D_{v}$ which is more natural, being along the crystals primary axes, where $u=\frac{x-y}{\sqrt{2}}$ and $v=\frac{x+y}{\sqrt{2}}$ ):

$$
\overrightarrow{D_{r}}=D_{x} \hat{x}+D_{y} \hat{y}=D_{u} \hat{u}+D_{v} \hat{v}
$$

where $y_{1}, z_{1}$ are the y,z-axes for crystal 1 and $y_{2}, z_{2}$ are the y,z-axes for crystal 2 as shown in Fig. 6-2. Each crystal contributes to the position difference through its own gradients along primary axes $D_{z 1} \propto \frac{d \Delta_{1}}{d z_{1}}, D_{y 1} \propto \frac{d \Delta_{1}}{d y_{1}}$, where the position differences proportional to the phase-gradient that gives rise to the asymmetry gradient. Each of crystal contributions combine to form the net position difference induced by the Pockels cell:

$$
\begin{gathered}
-D_{u}=D_{z 1}+D_{y 2} \\
D_{v}=D_{y 1}+D_{z 2}
\end{gathered}
$$

Here, for simplicity, we consider just the position difference caused by crystal 1 , and isolate the component only along the crystal's primary axes $z_{1}$ (oriented at $\left.-45^{\circ}\right)$. There exist corresponding expressions for crystal 2 and for the other transverse direction along $-45^{\circ}$. The position difference has three main contributions: field gradients, length gradients, and refractive index gradients which can 
be independently described as $D_{\partial E}{ }^{7}, D_{\partial L}$, and $D_{\partial n}{ }^{8}$ :

$$
\begin{gathered}
D_{z 1} \approx D_{\partial E, z 1}+D_{\partial L, z 1}+D_{\partial n, z 1} \\
D_{\partial E, z 1}=-\frac{w^{2} \pi}{2 \lambda}\left(n_{0, y}^{3} r_{23}-n_{0, z}^{3} r_{33}\right)\left(\frac{d E_{\Delta 1}}{d z_{1}} L_{1}\right) \approx 6-13 n m V^{-1} V_{\delta p o s} \\
D_{\partial L, z 1}=-\frac{w^{2} \pi}{2 \lambda}\left(n_{0, y}-n_{0, z}\right) \theta_{f c, z 1} \approx 1.7-17 u m \\
D_{\partial n, z 1}=-\frac{w^{2} \pi}{2 \lambda} \frac{d\left(n_{0, y}-n_{0, z}\right)}{d z_{1}} L_{1} \approx 20-40 u m
\end{gathered}
$$

where for our RTP cell and laser system, $L_{1}=10 \mathrm{~mm}, d=12 \mathrm{~mm}, w \approx 1 \mathrm{~mm}$, $\lambda=780 \mathrm{~nm}$; we have measured face cut parallelism of $\theta_{f c, z 1}=\frac{d L_{1}}{d z_{1}}=0.01-0.1 \mathrm{mrad}$; we have estimated by simulation the helicity correlated electric field gradient $\frac{d E_{\Delta 1}}{d z_{1}}$ induced by a voltage shift $V_{\delta p o s}$ in our cell design to be $\frac{d E_{\Delta 1}}{d z_{1}} \approx 3100 \pm 1100 m^{-2} V_{\delta p o s}$; and where the intrinsic refractive index gradient in RTP has been measured to be on the order of $\frac{d n_{z}}{d z}=1-2 \times 10^{-5} / \mathrm{cm}>>\frac{d n_{y}}{d z}[239]$ [238].

\section{Reducing analyzing-like Position differences}

We note that the largest birefringence gradients come from the intrinsic refractive index non-uniformity in RTP. While no crystal is perfectly uniform, RTP crystals suffer from greater non-uniformity than $\mathrm{KD}^{*} \mathrm{P}$ crystals which can be more easily grown to extremely large sizes. Unlike in $\mathrm{KD}^{*} \mathrm{P}$ cells, where position differences can be reduced by finding the electric-center in the crystal, in RTP cells,

\footnotetext{
${ }^{7}$ we note that $D_{\partial E}$ is difficult to measure in S1 [248] due to quad-photodiode detector AqDx-Dy coupling

${ }^{8}$ we note there are also additional terms but they are negligible $(<1 \mathrm{~nm})$ for PITA voltages $<$ QWV $1600 \mathrm{~V}$, specifically gradients due to applied PITA voltage $V_{\Delta}$ coupled to the length or birefringence gradient of the Pockels cell.
}

$$
\begin{gathered}
D_{\Delta, \partial L, z 1}=-\frac{w^{2} \pi}{2 \lambda}\left(n_{0, y}^{3} r_{23}-n_{0, z}^{3} r_{33}\right) E_{\Delta 1} \theta_{f c, z 1} \frac{d D_{\Delta, \partial L}}{d V_{\Delta 1}}=0.00026-0.0026 n m / V \\
D_{\Delta, \partial n, z 1}=-\frac{w^{2} \pi}{2 \lambda}\left(3 n_{0, y}^{2} r_{23} \frac{d n_{0, y}}{d z_{1}}-3 n_{0, z}^{2} r_{33} \frac{d n_{0, z}}{d z_{1}}\right)\left(E_{\Delta 1} L_{1}\right)
\end{gathered}
$$


the crystal imperfection is inherent to the system, and tend to compromise extinction ratios in HWV systems and create position differences in QWV systems. Innovation in the Pockels cell design was necessary to overcome these position differences due to non-uniformity. There are several potential solutions to counteract this non-uniformity: (1) reduce the laser spot-size (2) increase the laser divergence and make cell angular alignment adjustments (3) cut the crystal faces with small wedges (4) use a 4 crystal RTP cell system (5) use cell design with ability to control E-field gradients.

\section{Laser spot-size}

We note that having a modest laser beam spot-size in the crystal can help to minimize the gradient experienced by the beam distribution and reduce the resulting position differences. However, when using RTP crystals, we cannot reduce the beam size significantly ( $<1 \mathrm{~mm}$ for $\sim 1$ Watt) or else thermal gradients induced by the laser absorption $(0.75 \% / \mathrm{cm}-4 \% / \mathrm{cm})$ [236] [246] over a small space with high intensity could create additional birefringence non-uniformity leading to position difference drift $(\sim 0.1 \mu m-0.6 \mu \mathrm{m})$ and interfere with Pockels cell performance [245].

Having a modest laser beam spot-size on the photcathode (when used to make electron-beam), can help minimize the analyzing-like position differences in the generated electron beam ${ }^{9}$. Generally, the position differences are linearly proportional to the spot-size on the photocathode.

\section{Angular Dependence of Position Differences}

When a laser beam with slight divergence ( $\sim 1 \mathrm{mrad})$ at the Pockels Cell is used, the asymmetry gradients can be canceled out with Pockels cell angular alignment.

\footnotetext{
${ }^{9}$ During Qweak, the laser spot size on cathode was $0.5 \mathrm{~mm}$ for run1 and $1 \mathrm{~mm}$ for run2 (which implies $4 \sigma=1.7 \mathrm{~mm}$ for run2). Run1 suffered from cathode degradation and polarimetry problems. Run2 was fine in this regard. The $4 \sigma$ spot size for Qweak run2 is a good spot size to aim for $4 \sigma=1.7 \mathrm{~mm}$
} 
This is due to the fact that in addition to Aq gradients with respect to position on the crystal $\frac{d A_{q}}{d X}$, there are also Aq gradients with respect to angle $\frac{d A_{q}}{d \theta}$. An angle dependent gradient in Aq, when combined with a beam divergence, can produce position differences which in principle can cancel the position differences caused by $\frac{d A_{q}}{d X}$.

The angle dependence of Aq with respect to Pockels cell pitch and yaw is a saddle function. If the angle of the Pockels cell is not aligned so it is centered on the saddle-point, there is a 1st order gradient $\frac{d A_{q}}{d \theta}$ which couples via beam divergence $\theta_{d i v}=\frac{d w}{d z}$, through radius and angle coupling, into an effective position difference:

$$
D_{x}=\frac{\frac{d A}{d \theta_{x}} w \theta_{d i v}}{2}=-\frac{\epsilon}{T} \frac{\frac{d \Delta}{d \theta_{x}} \theta_{d i v} w}{2} \cos (2 \psi)
$$

These position differences due to Aq angle dependence are only minimized at the extremum saddle-point, so we refer to centering on the pitch/yaw saddle-point as "angular centering". It is important to align the Pockels cell's angle such that its primary longitudinal axis is parallel to the beam propagation direction, this is where the $d A / d \theta_{x}=d A / d \theta_{y}=0$ and where the position differences are minimized. It is also important that the divergence of the laser beam passing through the crystals be small, otherwise the position differences will be very sensitive to slight angular misalignment and could be quite large.

For a $1 \mathrm{~mm}$ spot size, $1 \mathrm{mrad}$ divergence and $\epsilon / T=100 \%$, with $A q \approx\left(5137 \mathrm{ppm} / \mathrm{mrad}^{2}\right) \theta_{x} \theta_{y}$, we obtain

$$
D_{x} \approx(2.57 \mu \mathrm{m} / \mathrm{mrad}) \theta_{y} \quad D_{y} \approx(2.57 \mu \mathrm{m} / \mathrm{mrad}) \theta_{x}
$$

In order to minimize analyzing-like position differences, we can set the Pockels cell angle such that the position differences caused by the angle dependent gradient in $\mathrm{Aq} \frac{d A_{q}}{d \theta}$ cancel the position differences caused by $\frac{d A_{q}}{d X}$. We refer this optimization, canceling one type of gradient in Aq with another, as "angular alignment". This slight angular adjustment, away from the central saddle-point, on the 
mrad-level, can cancel position differences in a $\lambda / 4$-wave system, and obviously improve extinction ratios in $\lambda / 2$-wave systems.

\section{Crystal wedge cuts}

In principle, one could improve the extinction ratio in $\lambda / 2$-wave configuration and the analyzing like position differences in $\lambda / 4$-wave configuration if the RTP crystals were cut with a $0.1-0.2$ mrad wedge deliberately to cancel out the refractive index gradient along the crystal z-axis. However, such small angle cuts are outside the realm of typical precision.

\section{4 crystal system \& crystal orientations}

The intrinsic refractive index non-uniformity in RTP, predominantly along z, could be counteracted in a 4 crystal system, where each crystal ( 1 and 2$)$ is cut in half to form 4 crystals $(1 \mathrm{a}, 1 \mathrm{~b}, 2 \mathrm{a}, 2 \mathrm{~b})$ and $(\mathrm{a}, \mathrm{b})$ haves are flipped over so the $\mathrm{z}$ and $\mathrm{y}$ axis are in opposite directions (i.e. $z_{1 a}=-z_{1 b}, y_{1 a}=-y_{1 b}$ ), so that the gradients cancel $\left(\frac{d n_{z}}{d z_{1 a}}+\frac{d n_{z}}{d z_{1 b}} \approx 0\right)$, while the opposite voltages are applied to achieve additive active induced birefringence. This arrangement can reduce the net gradient under the assumption that the gradients in each crystal are similar, so they can cancel well when combined ${ }^{10}$. Such a system design is shown in Fig. 6-7.

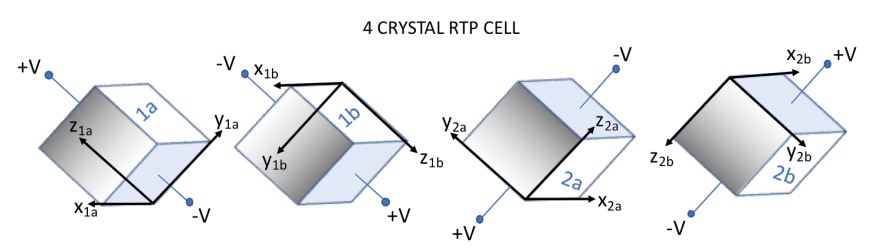

Figure 6-7: 4 crystal RTP cell: $\left.z_{1 a}=-z_{1 b}, y_{1 a}=-y_{1 b}\right)$ so that the gradients cancel $\frac{d n_{z}}{d z_{1 a}}+\frac{d n_{z}}{d z_{1 b}} \approx 0$, while retaining the thermal compensation design

To reduce the combined gradients in our 2 crystal system, we flipped the

\footnotetext{
${ }^{10}$ This seems to be the case for the crystal pair we purchased that were grown from the same batch and cut from the same block. Of course there are other gradients present which may not cancel perfectly, but the bulk could be reduced in this scheme.
} 
orientations of the two crystals relative to one another, in several orientations, to try to cancel out the gradients on one crystal with the gradients of the other crystal as much a possible. While this helped to some extent, empirically we observed an asymmetry gradient along $v \equiv \frac{x+y}{\sqrt{2}}$ due to both crystals combined of $\frac{d A_{q}}{d v} \sim 20,000-50,000 \mathrm{ppm} / \mathrm{mm}$ which would imply, for $w=1 \mathrm{~mm}$, gives position differences of magnitude:

$$
\begin{gathered}
D_{v}=D_{y 1}+D_{z 2}=\frac{\frac{d A_{q}}{d v} w^{2}}{2}=10-25 \mu m \\
D_{x} \sim D_{y} \sim D_{v} / \sqrt{2}=7.1-17.7 \mu m
\end{gathered}
$$

\section{E-field gradient control}

Ultimately we chose to use a cell design with the ability to control the electricfield gradients in the crystals. In order to counteract the crystal intrinsic nonuniformity and make the two helicity states of light passing through the crystals symmetric, we used grounded side panels to induce fringe-electric fields. A cartoon of the basic cell design is shown in Fig. 6-8. 3D electric field modeling informed the finalized design presented in Sec. 6.4.1. Shifting the voltage of the top and bottom plates, while keeping the voltage difference between them at QWV, allows us to induce an electric field gradient $\frac{d E_{z}}{d z}$, while approximately maintaining the appropriate value of the electric field $E_{Q W V}$ near the center of the crystal.

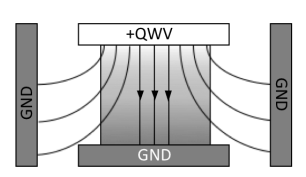

(a) + shift
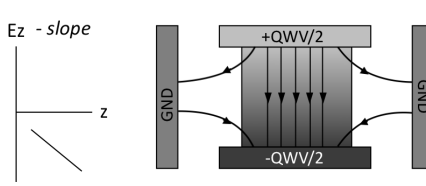

(b) no shift
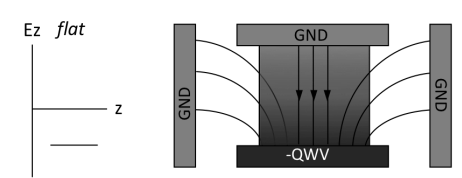

(c) - shift

Figure 6-8: Conceptual Diagram of E-field gradient control in Grounded SidePanel Design: The electric potential is illustrated in grey-scale and electric field lines are drawn conceptually. $+\mathrm{z}$ is up in this diagram.

By controlling the electric field gradients for each helicity state, in both of 
the crystals, the asymmetric position motion of the light can be suppressed. For each helicity state, we can choose to have equal and opposite voltage shifts or have the same voltage shift. Correspondingly, we have the freedom to induce the same electric field gradient for both helicity states $\frac{d E^{R}}{d z}=\frac{d E^{L}}{d z}$, producing a gradient in $\Delta E, \frac{d E_{\Delta}}{d z}$, or we can also induce equal and opposite gradients in each helicity state $\frac{d E^{R}}{d z}=-\frac{d E^{L}}{d z}$, producing a gradient in $E_{0}, \frac{d E_{0}}{d z}$, as defined in Sec. 6.3.1. The gradient in $\Delta E, \frac{d E_{\Delta}}{d z}$, has a small effect on the analyzing-like position differences, expressed in Sec 6.3.3. The gradient in $E_{0}, \frac{d E_{0}}{d z}$, controls another type of position difference, what we refer to as "steering" position differences, through a much stronger, dominant position difference effect, expounded on in Sec. 6.3.4. In operation, rather than adjusting the gradient in $\Delta E, \frac{d E_{\Delta}}{d z}$, we tend to control the gradient in $E_{0}, \frac{d E_{0}}{d z}$, using these "steering" position differences to cancel out the analyzing-like position differences.

\subsubsection{Position differences: Steering}

\section{Describing Steering}

Position differences also arise simply through an angular deviation via GRIN (gradient-index) effects and Snell's Law. This type of position difference is referred to as helicity correlated beam 'steering' an is entirely independent of analyzing power. Steering is a helicity correlated change in angle of the outgoing laser beam after having passed through the Pockels Cell. It produces a position difference between right and left helicity states which increases with throw distance, hence steering is referred to as an 'angle-like' position difference which does not depend on analyzing power. 


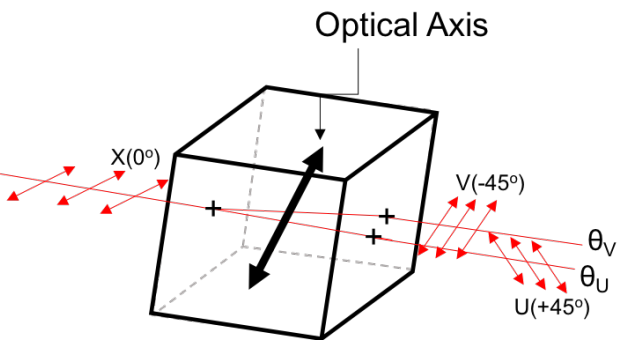

(a)

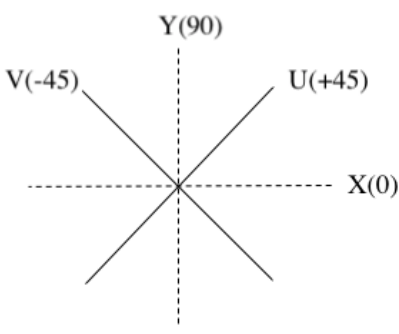

(b)

Figure 6-9: (a)Ray Separation. Horizontally polarized beam splits in the crystal along the primary axes, angles not to scale. [279] (b) Coordinates. Primary crystal axes are along the diagonal with respect to horizontal and vertical polarizations.

Since the crystal in the Pockels cell is birefringent, the beam should be viewed as 2 separate rays: one with a polarization along the diagonal $U\left(45^{\circ}\right)$ and the other along $V\left(-45^{\circ}\right)$, the primary axes of the RTP as shown in Fig. 6-9. These rays can separate via a difference in the refractive index combined with an angled face cut or a difference in the refractive index gradient $\frac{d n_{z}}{d x_{i}}$ and $\frac{d n_{y}}{d x_{i}}$. In RTP, the optoelectric effect $n_{z}=n_{z 0}-\frac{1}{2} r_{33} E_{z} n_{z 0}^{3}$ and $n_{y}=n_{y 0}+\frac{1}{2} r_{23} E_{z} n_{y 0}^{3}$ causes the 2 rays (one for each helicity state) to separate into 4 rays for different primary axes as shown in Fig.6-10 . The difference $\theta^{R}-\theta^{L}$ is the helicity correlated angular steering.

\section{4 rays in RTP crystal}

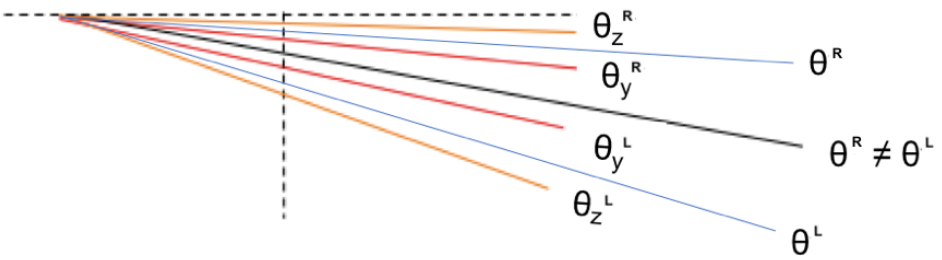

Figure 6-10: 4 Ray Separation: the steering behavior of RTP. Horizontally polarized beam splits in the crystal along the primary axes, and voltage application split the beam further. Angles not to scale, but the relative ordering with grater deviation from $z$ than from $y$ for a given crystal is correct.

Steering can only arise through either a gradient in the average refractive index 
or a length gradient. If the RTP crystal is cut with a slight wedge $\theta_{f c}=\theta_{\text {cut } 1}-\theta_{\text {cut } 2}$, with component $\theta_{f c, x_{i}}$ along $\hat{x}_{i}$, the beam will experience an angular deviation upon exiting the crystal $\theta \sim\left(n_{e f f}-1\right) \theta_{f c, x_{i}}=n_{e f f} \frac{d L}{d x_{i}}$ proportional to the face cut wedge angle or length gradient through Snell's Law. Equivalently, a gradient in the effective refractive index will cause the beam will experience an angular deviation $\theta_{x_{i}}=\frac{d n_{e f f}}{d x_{i}} L$ upon exiting the crystal through GRIN effects. We can generally describe the angle induced from any phase gradient with $\theta_{x_{i}}=\frac{d}{d x_{i}}\left(n_{e f f} L\right)=\frac{\lambda}{2 \pi} \frac{d \phi}{d x}$ . In the case of steering, the laser beam is bent by the crystal in a helicity correlated manner, induced by the voltage applied:

$$
\Delta \theta_{x_{i}}=\theta^{R}-\theta^{L}=\theta_{f c, x_{i}} \Delta n+L \frac{d \Delta n}{d x_{i}}
$$

where $\Delta n=n^{R}-n^{L} \sim \Delta E_{z}^{R}-E_{z}^{L}$

Steering can be modeled as arising from electric field gradients, length gradients/face cuts angles/curvature, and intrinsic refractive index gradients from crystal growth. RTP crystals actually have E-O prism applications where they are intentionally cut with a large wedge and used to control beam position by application of voltage [249] [250]. We have effectively taken this application and incorporated it into our Pockels cell system. As discussed below, we use the electric field gradient to induce steering, providing precise control of the helicity correlated position differences.

Steering is a strongly input polarization dependent effect. Formerly, steering in $\mathrm{KD}^{*} \mathrm{P}$ Pockels cells was thought to be a polarization-independent effect since both horizontal and vertical input polarizations appeared to exhibit the same steering behavior (the "Skew" effect [251]). However, we have since shown that steering is, in fact, quite dependent on input polarization in both $\mathrm{KD}^{*} \mathrm{P}$ and in RTP Pockels cells (for data, see Fig. 6-40 and Appendix D). When the incident beam polarization is along the RTP crystal's primary axes at $\pm 45^{\circ}$ (Fig.6-2), the steering induced for $\mathrm{z}$-axis $+45^{\circ}$ input polarization differs significantly from the 
steering induced for y-axis $-45^{\circ}$ input polarization. Placing an analyzer along S2, along the diagonal $U\left(45^{\circ}\right)$ or along $V\left(-45^{\circ}\right)$, probes the steering for the primary axis polarization states. For a single RTP crystal, analyzing along the diagonal $U\left(45^{\circ}\right)$ isolates $n_{z}$ (or $n_{y}$ ) rays so that $\frac{d n_{z}}{d x_{i}}$ will change for $+E_{z}$ and $-E_{z}$ producing position differences which are angle-like and grow as throw distance increases. S2 steering depends on $\theta_{z}$ as illustrated in Fig. 6-10.

When the input polarization is horizontal or vertical, upon entering the Xcut RTP crystal, the light is split into 2 rays, one with polarization along the $\mathrm{z}$ axis, and the other with polarization along the $\mathrm{y}$ axis. For RTP, the incident $\mathrm{H} / \mathrm{V}$ polarized light propagates along the crystal $\mathrm{x}$-axis direction with nearly equal components along the $n_{z}, n_{y}$ directions which are the crystals primary axes. In general, for a birefringent material, the refractive indexes mix to form an effective index and $\mathrm{n}$ is more generally described as $\mathrm{n}(\theta, \phi)$, where $(\theta, \phi)$ describe the polarization direction, and can be computed through the index ellipsoid of the crystal, which is defined by the surface $\frac{x_{1}^{2}}{n_{1}^{2}}+\frac{x_{2}^{2}}{n_{2}^{2}}+\frac{x_{3}^{2}}{n_{3}^{2}}=1$. We calculate $\mathrm{n}(\theta, \phi)$ by noting $x_{1}=n(\theta, \phi) \sin (\theta) \cos (\phi), x_{2}=n(\theta, \phi) \sin (\theta) \sin (\phi), x_{3}=n(\theta, \phi) \cos (\theta)$. Hence, $\frac{1}{n^{2}(\theta, \phi)}=\frac{\sin ^{2}(\theta) \cos ^{2}(\phi)}{n_{1}^{2}}+\frac{\sin ^{2}(\theta) \sin ^{2}(\phi)}{n_{2}^{2}}+\frac{\cos ^{2}(\theta)}{n_{3}^{2}}$. This effective refractive index in the RTP crystal is approximately given by the average of $n_{z}$ and $n_{y}$ when the incident polarization state is horizontal or vertical. Consequently, the steering for $\mathrm{H}$ and $\mathrm{V}$ input polarizations is simply the average of the steering along the $\pm 45^{\circ}$, y and z primary crystal axes. With no analyzer present (or with the analyzer along the horizontal), there is steering given by the average gradients $\frac{d}{d x_{i}} \frac{n_{z}+n_{y}}{2}=\frac{d}{d x_{i}} \frac{n_{z 0}-\frac{1}{2} r_{33} E_{z} n_{z 0}^{3}+n_{y 0}+\frac{1}{2} r_{23} E_{z} n_{y 0}^{3}}{2}$ which does not cancel and has a dependence on the sign of the E-field. There is helicity-correlated beam steering steering for $\mathrm{H}$ and $\mathrm{V}$ polarization in $\mathrm{S} 1$ as well as for no analyzer. Fig. 6-10 reflects the conclusion that no-analyzer steering in RTP does not cancel and depends on the difference between $\frac{\theta_{z, 0}+\theta_{y, 0}}{2}-\frac{\theta_{z, 1}+\theta_{y, 1}}{2}$. 


\section{Derivation}

We examine the helicity correlated phase gradients for each input polarization state component. These polarization components undergo phase shifts

$$
\phi_{i}^{R(L)}=2 \pi n_{i}^{R(L)} L^{R(L)} / \lambda
$$

where $\mathrm{R}(\mathrm{L})$ indicates right and left circular polarization states determined by the sign of the voltage applied to the Pockels Cell as controlled by the helicity signal.

The phase shift in each of the two crystals, for each $\pm 45^{\circ}$ polarization component, is given by (see Fig.6-2)

$$
\begin{aligned}
& \phi_{45^{\circ}, 1}^{R(L)}=2 \pi n_{z 1}^{R(L)} L_{1} / \lambda \quad \phi_{-45^{\circ}, 1}^{R(L)}=2 \pi n_{y 1}^{R(L)} L_{1} / \lambda \\
& \phi_{45^{\circ}, 2}^{R(L)}=2 \pi n_{y 2}^{R(L)} L_{2} / \lambda \quad \phi_{-45^{\circ}, 2}^{R(L)}=2 \pi n_{z 2}^{R(L)} L_{2} / \lambda
\end{aligned}
$$

The total phase shift for the two crystals combined, for each $\pm 45^{\circ}$ polarization component, is given by:

$$
\begin{gathered}
\phi_{45^{\circ}, t o t}^{R(L)}=2 \pi\left(n_{z 1}^{R(L)} L_{1}+n_{y 2}^{R(L)} L_{2}\right) / \lambda=2 \pi / \lambda\left(n_{0, z} L_{1}+n_{0, y} L_{2}-\frac{1}{2} n_{0, z}^{3} r_{33} E_{z 1}^{R(L)} L_{1}\right. \\
\left.-\frac{1}{2} n_{0, y}^{3} r_{23} E_{z 2}^{R(L)} L_{2}\right) \\
\phi_{-45^{\circ}, t o t}^{R(L)}=2 \pi\left(n_{y 1}^{R(L)} L_{1}+n_{z 2}^{R(L)} L_{2}\right) / \lambda=2 \pi / \lambda\left(n_{0, y} L_{1}+n_{0, z} L_{2}-\frac{1}{2} n_{0, y}^{3} r_{23} E_{z 1}^{R(L)} L_{1}\right. \\
\left.-\frac{1}{2} n_{0, z}^{3} r_{33} E_{z 2}^{R(L)} L_{2}\right)
\end{gathered}
$$

Averaging the phase shifts for polarization components along $\pm 45^{\circ}$, we obtain a general equation for the overall phase shift:

$$
\phi_{\text {avg }}^{R(L)}=\left(\phi_{45^{\circ}, t o t}^{R(L)}+\phi_{-45^{\circ}, t o t}^{R(L)}\right) / 2=\pi / \lambda\left(\left(n_{0, z}+n_{0, y}\right)\left(L_{1}+L_{2}\right)\right.
$$




$$
\left.-\frac{1}{2}\left(n_{0, z}^{3} r_{33}+n_{0, y}^{3} r_{23}\right)\left(E_{z 1}^{R(L)} L_{1}+E_{z 2}^{R(L)} L_{2}\right)\right)
$$

As stated above, since in one crystal the electric field $E_{z}$ is along the primary crystal axis $+\hat{z}$, and in the other crystal the electrics field $E_{z}$ is opposite the primary crystal axis $-\hat{z}$, the effect of the electric field will increase the refractive indices $n_{y}, n_{z}$ in one crystal and decrease the refractive indices $n_{y}, n_{z}$ in the other crystal. So the total average phase shift is, largely, the same for both helicity states. However the gradient of the average phase shift is not the same for both helicity states as we will show.

The opto-electric effect in RTP results in a change in refractive index to the two primary axes $\mathrm{y}$ and $\mathrm{z}$ of

$$
\Delta n_{z}=-n_{z 0}^{3} r_{33}\left(E_{z}^{R}-E_{z}^{L}\right) ; \Delta n_{y}=-n_{y 0}^{3} r_{23}\left(E_{z}^{R}-E_{z}^{L}\right)
$$

with $\left(E_{z}^{R}-E_{z}^{L}\right)=2 E_{z 0}$. The helicity correlated refractive index gradient is then given by

$$
\frac{d \Delta n_{j}}{d x_{i}}=-3 n_{j 0}^{2} r_{j 3} \frac{d n_{j 0}}{d x_{i}} E_{z 0}-n_{j 0}^{3} r_{j 3} \frac{d E_{z 0}}{d x_{i}}
$$

where $j=2,3(\mathrm{y}, \mathrm{z})^{11}$ refers to the input polarization state of the beam along $\pm 45^{\circ}$, the primary fast and slow axes of the RTP crystal. A gradient in the electric field or intrinsic refractive indices in each crystal gives rise to a voltage dependent phase gradient which steers the beam as a wedge does, producing an angle-difference (and hence position difference for throw distance D) [240]

$$
\theta_{a i r, d e f l}=\theta_{f c, x_{i}}(n-1)+L \frac{d n}{d x_{i}}
$$

The steering due to these gradients in a single RTP crystal is given by

$$
\left.\Delta \theta_{x_{i}}\right|_{j}=-n_{j 0}^{3} r_{j 3}\left(\left(\theta_{f c, x_{i}}+3 \frac{L}{n_{j 0}} \frac{d n_{j 0}}{d x} r_{j 3}\right) E_{z 0}+\frac{d E_{z 0}}{d x_{i}} L\right)
$$

\footnotetext{
${ }^{11} r_{23}$ is the electro-optic coupling coefficient between $n_{y}$ and $E_{z}$ and $r_{33}$ is the electro-optic coupling coefficient between $n_{z}$ and $E_{z}$
} 


$$
\left.D_{x}\right|_{i}=\left.\Delta_{x} \theta\right|_{i} D=-n_{j 0}^{3} r_{j 3} D\left(\left(\theta_{f c, x_{i}}+3 \frac{L}{n_{j 0}} \frac{d n_{j 0}}{d x} r_{j 3}\right) E_{z 0}+\frac{d E_{z 0}}{d x_{i}} L\right)
$$

We simply take the average of the steering along $\pm 45^{\circ}$, the $\mathrm{y}$ and $\mathrm{z}$ primary crystal axes, to obtain the steering for $\mathrm{H}$ and $\mathrm{V}$ input polarizations:

$$
\left.D_{x_{i}}\right|_{H, V}=\frac{1}{2}\left(\left.D_{x_{i}}\right|_{y}+\left.D_{x_{i}}\right|_{z}\right)=D_{x_{i}, \text { fixed }}-\frac{1}{2}\left(n_{y 0}^{3} r_{23}+n_{z 0}^{3} r_{33}\right) D \frac{d E_{z 0}}{d x_{i}} L
$$

where $D_{x_{i}, \text { fixed }}$ is the unchangeable intrinsic steering position difference from 1 crystal given by

$$
D_{x, \text { fixed }}=-n_{y 0}^{3} r_{23} D\left(\left(\theta_{f c}+3 \frac{L}{n_{y 0}} \frac{d n_{y 0}}{d x} r_{23}\right) E_{z 0}-n_{z 0}^{3} r_{33} D\left(\left(\theta_{f c}+3 \frac{L}{n_{z 0}} \frac{d n_{z 0}}{d x} r_{33}\right) E_{z 0}\right.\right.
$$

The steering due to each of the two RTP crystals combines additively, resulting in a total angle difference averaged over y and $\mathrm{z}$ polarizations and summed over each crystal.

\section{Simulation}

We simulated the induced electric field gradients along the z-axis of each crystal in our RTP cell design. Fig. 6-11 shows the electric field $E_{z}$ for three different voltage configurations with the same voltage difference between the top and bottom plate but differing in the voltage offset, $V_{\text {apos }}$ of the plates relative to the grounded side panels. We refer to these states as 'grounded' configuration with a positive voltage shift, 'balanced' configuration with no voltage shift, and 'reverse grounded' configuration with a negative voltage shift. Fig. 6-11a shows the electric field of the 'grounded' configuration with a positive voltage shift $V_{\alpha p o s}=+V_{\lambda / 4} / 2$, when the bottom plate is grounded at the same voltage as the side panels, inducing a maximal gradient in the $+\mathrm{z}$ direction. Fig. 6-11b shows the electric field of the 'balanced' configuration, with no voltage shift $V_{\text {opos }}=0$, when the top and bottom plates have equal and opposite voltages relative to the grounded the side panels, inducing a symmetric field with zero first order term at the crystal center. 
Fig. 6-11c shows the electric field of the 'reverse grounded' configuration, with a negative voltage shift $V_{\alpha p o s}=-V_{\lambda / 4} / 2$, when the top plate is set the same voltage as the side panels, inducing a maximal gradient in the $-\mathrm{z}$ direction. By keeping the voltage difference between the plates constant, and changing the voltage shift relative to the grounded side panels, we control the electric field gradient without changing the electric field $E_{z}$ magnitude, and hence control the position differences without changing the $\delta$-phase much. The voltages are set independently for each helicity state such that when the polarity of the electric field is switched, the sign of the induced electric field gradient reverses as well.

Fig. 6-12a shows the electric field gradient $\frac{d E_{z}}{d z}$ for each helicity state as a function of voltage shift $V_{\alpha p o s}$, corresponding to scanning from the grounded configuration, across the balanced configuration $\left(V_{\alpha p o s}=0\right)$, to the reverse grounded configuration. The red line with pink uncertainties corresponds to positive helicity state, and the blue line with green uncertainties corresponds to the negative helicity state. The uncertainty in $\frac{d E_{z}}{d z}$ is determined by the resolution of the mesh in the $3 \mathrm{D}$ simulation; also included in the $\frac{d E_{z}}{d z}$ error bar is the uncertainty from centering the laser position on the crystal within $\pm 1 \mathrm{~mm}$ of center. Fig. 6-12b corresponds to the helicity correlated change in the electric field gradient $\Delta \frac{d E_{z}}{d z}=\frac{d E_{z}^{R}}{d z}-\frac{d E_{z}^{L}}{d z}=2 E_{z, 0}$. We find $\frac{d E_{z, 0}}{d z}=\left(3100 \pm 1100 \mathrm{~V} / \mathrm{m} 2 / V_{\text {opos }}\right) V_{\text {opos }}$.

Examining the steering due to the first RTP crystal along the primary axis $z_{1}$, for $\lambda=780 \mathrm{~nm}, L=10 \mathrm{~mm}, r_{33}=35 \mathrm{pm} / \mathrm{V}, r_{23}=12.5 \mathrm{pm} / \mathrm{V}, n_{y}=1.7832$, $n_{z}=1.8673$, for horizontal input polarization

$$
\Delta \theta_{z 1}=D_{z 1} / D=-\frac{1}{2}\left(n_{y 0}^{3} r_{23}+n_{z 0}^{3} r_{33}\right) L \frac{d E_{z 1,0}}{d z_{1}}
$$

for $\frac{d E_{z 1,0}}{d z_{1}}=\left(3100 \pm 1100 \mathrm{~V} / \mathrm{m} 2 / V_{\alpha p o s}\right) V_{\alpha p o s}$, we obtain the steering sensitivity to voltage

$$
\frac{d \Delta \theta_{z 1}}{d V_{\alpha p o s}}=-\frac{1}{2}\left(n_{y 0}^{3} r_{23}+n_{z 0}^{3} r_{33}\right) L \frac{d E_{z 1,0}}{d z_{1}}=-4.6 \pm 1.7 \mathrm{nrad} / \mathrm{V}
$$


(a) Grounded

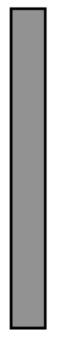

(b) Balanced

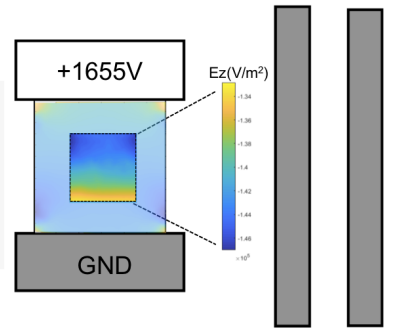

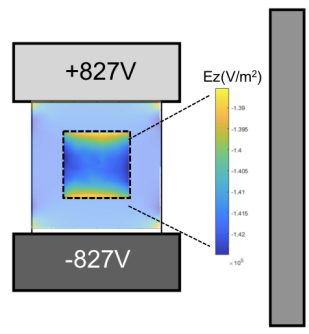

(c) Reverse Grounded
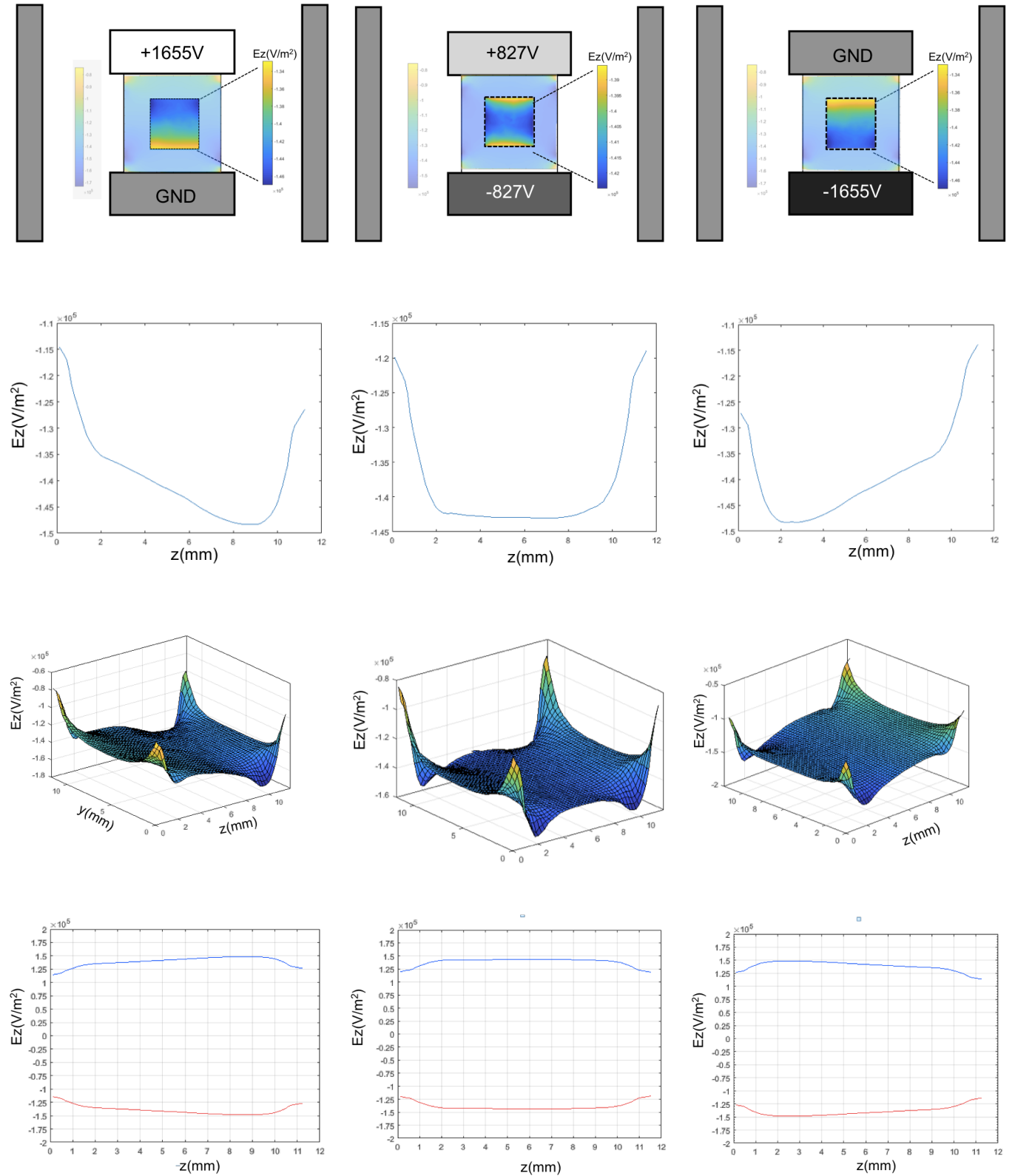

Figure 6-11: Electric field calculation for RTP cell design (a) Grounded configuration: in which one plate is grounded for both helicity states. (b) Balanced configuration: in which voltage is applied symmetrically to the top and bottom plates (c) Reverse grounded configuration: in which the opposite plate is grounded. For each configuration, the electric fields are shown for each helicity state as red and blue curves. 


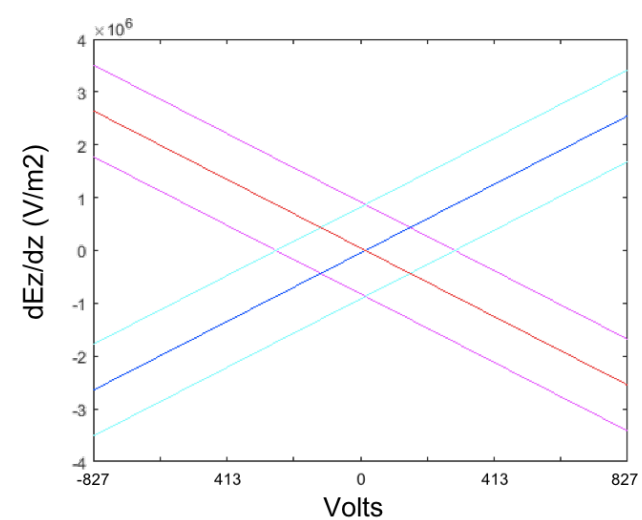

(a)

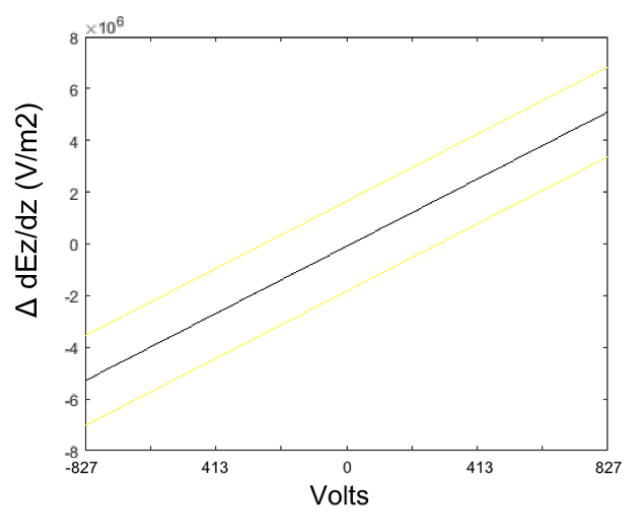

(b)

Figure 6-12: Simulations of Steering gradient $d\left(d E_{z} / d z\right) / d V_{\alpha p o s}=-3100 \pm$ $1100 \mathrm{~V} / \mathrm{m} 2 / V_{\text {opos }}$

where each polarization component along $z_{1}$ and $y_{1}$ was steered by:

$$
\begin{aligned}
& \left.\frac{d \Delta \theta_{z 1}}{d V_{\alpha p o s}}\right|_{z 1}=-n_{z 0}^{3} r_{33} L \frac{d E_{z 1,0}}{d z_{1}}=-7.1 \pm 2.5 \mathrm{nrad} / \mathrm{V} \\
& \left.\frac{d \Delta \theta_{z 1}}{d V_{\text {opos }}}\right|_{y 1}=-n_{z 0}^{3} r_{33} L \frac{d E_{z 1,0}}{d z_{1}}=-2.2 \pm 0.8 \mathrm{nrad} / \mathrm{V}
\end{aligned}
$$

\subsubsection{RHWP scans}

To assess the Pockels Cell alignment, we examine the asymmetry between polarization states using a rotating-HWP (RHWP). Analyzing power dependent HCBA's are caused by asymmetric linear polarizations. A RHWP changes the orientation of this asymmetric linear polarization. The transmission through an analyzer, measured with respect to RHWP angle determines the degree and orientation of asymmetric linear polarization. The RHWP is inserted downstream of the Pockels cell, before subsequent elements in the beamline. For laser beam studies, subsequent elements include a $100 \%$ analyzer (Glan-Taylor polarizer), followed by mirrors and a photodiode detector. For the electron beam, subsequent elements include the vacuum windows (with some birefringence of their own) and photocathode with slight analyzing power. The electron beam is then character- 
ized by beam current and position monitors. RHWP scans assess the level of alignment of the Pockels cell. If the RHWP and an additional retardation plate downstream of the RHWP (such as the vacuum windows) is inserted between the PC and the analyzer (in this case the photocathode), the HCBA becomes [163]

$A_{q}=-\frac{\epsilon}{T}\left[\beta \sin (2 \rho-2 \psi)+\gamma \sin (2 \theta-2 \psi)+\Delta_{S 1} \cos (4 \theta-2 \psi)+\Delta_{S 2} \sin (4 \theta-2 \psi)\right]$

where $\theta$ is the RHWP angle, $\epsilon / T$ is the analyzing power, $\psi$ is the analyzing direction, $\beta$ is the phase shift from and $\rho$ is the orientation of the additional retardation plate (vacuum windows), $\gamma$ is due to the RHWP's deviation from being a perfect $\lambda / 2$-plate, and $\Delta$ is anti-symmetric phase shift described previously which can arise either in S1 or S2. In aligning the Pockels Cell, minimizing the $4 \theta$ terms is desirable. We can also minimize the impact of the vacuum windows on the offset term by rotating the photocathode direction such that its analyzing power matches the vacuum window birefringence such that $\psi=\rho$. The $2 \theta$ term corresponds to an imperfect RHWP and can be reduced by using a RHWP very well matched to the laser wavelength.

RHWP scans are also used to assess the position differences and steering. The position differences $D_{x}, D_{y}$ with respect to RHWP angle can be described by

$$
\begin{aligned}
& D_{x i}=-\frac{\epsilon}{T}\left[\partial_{\beta i} \sin (2 \rho-2 \psi)+\partial_{\gamma_{i}} \sin (2 \theta-2 \psi)\right. \\
& \left.\quad+D_{x i}^{S 1} \cos (4 \theta-2 \psi)+D_{x i}^{S 2} \sin (4 \theta-2 \psi)\right]+D_{x i}^{\text {steer }}
\end{aligned}
$$

where $\partial_{\beta i}$ corresponds to the birefringence gradient in the vacuum windows, $\partial_{\beta i}$ is the birefringence gradient in the RHWP which is typically negligible, $D_{x i}^{S 1}$ is the $4 \theta$ term from $\mathrm{S} 1$ analyzing-like position differences ${ }^{12}$. A comprehensive $4 \theta$ term amplitude is given by $D_{x i}^{4 \theta}=\sqrt{\left(D_{x i}^{S 1}\right)^{2}+\left(D_{x i}^{S 2}\right)^{2}}$. The $4 \theta$ terms can be inferred from

\footnotetext{
${ }^{12}$ We note that in RTP RHWP we have observed the $4 \theta$ position difference term to be in phase with the Aq $4 \theta$ term, indicating $\left.D_{x i}^{S 2}\right)^{2}$ is small compared with $D_{x i}^{S 1}$ as in Fig. 6-14b
} 
the individual measured position differences $D_{x i}^{\theta}$ where the analyzer is probing the polarization along $\theta$ and corresponding position difference:

$$
D_{x i}^{S 1}=\frac{D_{x i}^{0^{\circ}}-D_{x i}^{90^{\circ}}}{2} \quad D_{x i}^{S 2}=\frac{D_{x i}^{45^{\circ}}-D_{x i}^{-45^{\circ}}}{2} \quad D_{x i}^{s t e e r}=\frac{D_{x i}^{45^{\circ}}+D_{x i}^{-45^{\circ}}}{2}
$$

Each crystal 1,2 contributes individually to position differences $D_{x i}^{p o l, 1}$ and $D_{x i}^{p o l, 2}$. We note that in RTP, due to the values of the opto-electric coefficients, typically $D_{x i}^{45^{\circ}, 1} \approx 3 D_{x i}^{-45^{\circ}, 1}$ and $D_{x i}^{-45^{\circ}, 2} \approx 3 D_{x i}^{45^{\circ}, 2}$. An illustration of an RHWP scan and how to interpret it is shown in Fig. 6-13.

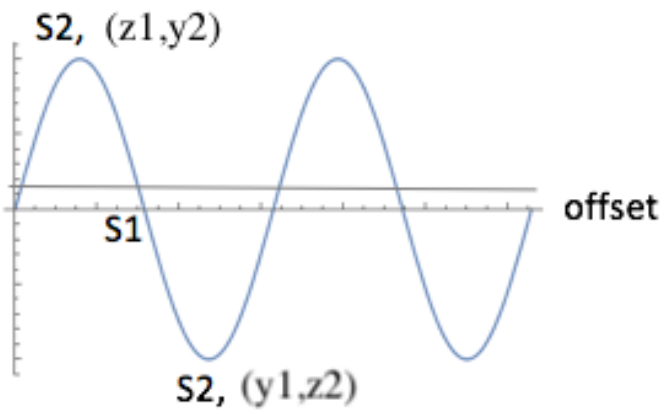

Figure 6-13: RHWP scan example isolating how S1 and S2 contribute to RHWP scans. Contributions from crystals: $z_{1}$ refers to the z-axis of crystal $1, z_{2}$ refers to the z-axis of crystal $2, y_{1}$ refers to the y-axis of crystal $1, y_{2}$ refers to the y-axis of crystal 2.

Fig. 6-14 shows RHWP scans taken on the laser table with the RTP cell for different steering conditions. One crystal has had its electric field gradient altered in order to shift the steering-like position difference along the "V" $\left(-45^{\circ}\right)$ direction. The steering term was changed by about $1.2 \mu \mathrm{m}$ for $\mathrm{a} \sim 1 \mathrm{~m}$ throw distance by using voltages ${ }^{13}$.

\footnotetext{
${ }^{13}$ The Aq $4 \theta$ term changed a bit for two reasons: (1) it takes time to perform a RHWP scan and due to temperature fluctuations, the ideal PITA voltage can change (2) Depending on the location of the laser on the crystal, there can be some coupling between the Aq and the electric field gradient inducing voltage.
} 


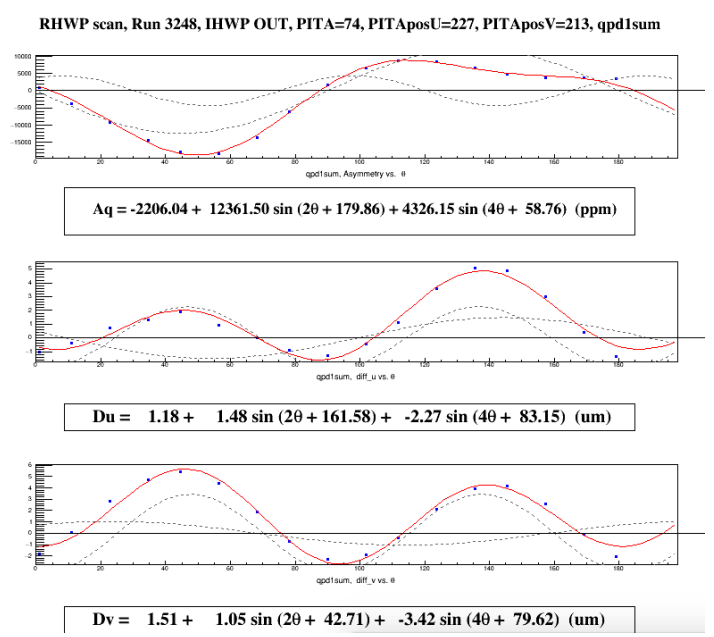

(a)

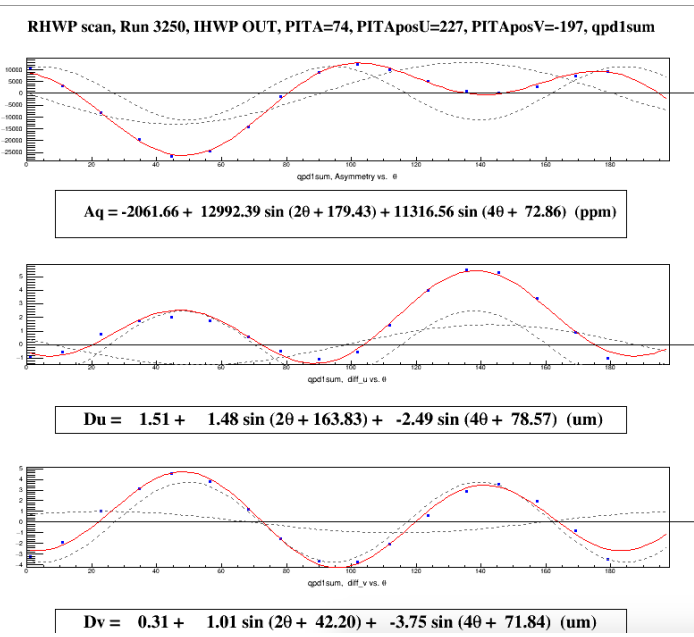

(b)

Figure 6-14: RHWP scans RTP: (a) RHWP scan performed with larger Dv steering offset (b) RHWP scan performed with smaller Dv steering offset, where PITAposV has been used to alter the electric field gradient in one of the RTP crystals. S1 is near $\theta=0^{\circ}$ [280].

We note that the above equations predict that when the steering term is changed, the $4 \theta$ term can also change (for the $100 \%$ analyzer scan). If one of the crystals, say crystal 1 , has its steering term changed as $D_{\text {steer }}^{\text {new }}=D_{\text {steer }}+\Delta_{\text {steer }}$, then the position difference for polarization state $\mathrm{U}\left(45^{\circ}\right)$ is given by $D^{45^{\circ}, 1}+\Delta_{\text {steer }} / 2$, the position difference for polarization state $\mathrm{V}\left(-45^{\circ}\right)$ is given by $D^{-45^{\circ}, 1}+3 \Delta_{\text {steer }} / 2$, and the $4 \theta$ term will be given by $D^{4 \theta}=\sqrt{\left(D^{S 1}\right)^{2}+\left(D^{S 2}-\Delta_{\text {steer }}\right)^{2}}$. This is discussed further in the subsequent section (Sec. 6.3.6).

\subsubsection{Analyzing/Angle-like Position Differences}

There are essentially two separate beams within the birefringent crystal, one linearly polarized along $+45^{\circ}$ and one along $-45^{\circ}$. Considering this model, steering/anglelike position differences, when analyzed by the cathode, actually create analyzinglike position differences. These analyzing-like position differences arise from the same mechanism as steering, are also angle-like, and increase proportionally with throw distance from the Pockels cell. We refer to them as steering induced 
analyzing/angle-like position differences.

Since steering is polarization dependent, if there is steering, then there are analyzing/angle-like position differences. This will show up in an RHWP scan as a $4 \theta$ term in $\mathrm{S} 2$. There is a throw-distance dependent $4 \theta$ term in S2. At a longer throw distance, the steering is larger and the S2 position differences are also larger. In terms of minimizing position differences, we have two knobs: the voltage induced electric-field gradient to control the steering offset and S2 $4 \theta$ term, and PC angle to control S1 $4 \theta$ term. As stated previously, The S1 $4 \theta$ terms can be zeroed out with PC angle, if there is a small divergence of the laser in the Pockels cell as explained in Sec. 6.3.3. Assuming that the S2 $4 \theta$ term and the steering offset have the same origin these degrees of freedom should be sufficient. It seems not unlikely that zeroing out steering for no-analyzer could have the effect of zeroing $4 \theta$ terms in $\mathrm{S} 2$.

How do we choose the RHWP angle? Inducing steering, to say, cancel out S1 position differences, can, in principle, also create larger S2 $4 \theta$ position differences. Is there an RHWP angle where it is more difficult to zero out the position difference with steering because, while canceling the $\mathrm{S} 1$ position difference, we actually create an S2 position difference? In RTP, the electro-optic coefficient $r_{33}$ is 3 times larger than $r_{23}$. This means that for a single RTP, if the $45^{\circ} \mathrm{S} 2$ position difference were to shift by $\mathrm{X}$ the $-45^{\circ} \mathrm{S} 2$ position difference will shift by $3 \mathrm{X}$, and the noanalyzer/steering position difference, given by the average of the two, is $2 \mathrm{X} 14$

$$
D_{x i}^{-45^{\circ}}=1 X \quad D_{x i}^{s t e e r}=2 X \quad D_{x i}^{45^{\circ}}=3 X \quad D_{x i}^{S 2}=\frac{D_{x i}^{45^{\circ}}-D_{x i}^{-45^{\circ}}}{2}=1 X
$$

Assuming no other terms ${ }^{15}$, the constraint for steering cancellation of $4 \theta$ compo-

\footnotetext{
${ }^{14}$ In $\mathrm{KD} * \mathrm{P}$, the $45^{\circ}$ pos difference will shift by $\alpha+\beta$ and the $-45^{\circ}$ position difference will shift by $\alpha-\beta$, while the steering is $\alpha$.

${ }^{15}$ which is a textitmajor assumption
} 
nents is given by

$$
\begin{gathered}
0=\left(D_{x i}^{S 1} \cos (4 \theta)+D_{x i}^{S 2} \sin (4 \theta)\right) \epsilon / T+D_{x i}^{\text {steer }} \\
D_{x i}^{\text {steer }}=-D_{x i}^{S 1} \cos (4 \theta) /(\sin (4 \theta) / 2+T / \epsilon)
\end{gathered}
$$

A plot of the optimal $D_{x}^{\text {steer }} / D_{x i}^{S 1}$ vs $\theta$ is illustrated in Fig. 6-15 based on Eq. 6.2 for various analyzing powers, from $100 \%$ to $10 \%$ (typical for a photocathode is 3-6\%). There are RHWP angles for which it would a greater amount of steering to zero out the position differences. For $100 \%$ analyzer, this solution has maxima and minima at $\theta \approx 55^{\circ}, 145^{\circ}$ and $\theta \approx 84^{\circ}, 174$, these are between $\mathrm{S} 1\left(0^{\circ}, 45^{\circ}\right)$ and $\mathrm{S} 2\left(22.5^{\circ}, 67.5^{\circ}\right)$, like placing an analyzer between $45^{\circ}$ and $90^{\circ}$. For smaller analyzing powers, the $\theta$ requiring the largest steering to cancel the position differences gradually gets closer to an S1 angle, until for very small analyzing powers, setting the RHWP angle near S1 results in the largest required steering to cancel out, however as the analyzing power is small, not much is required.

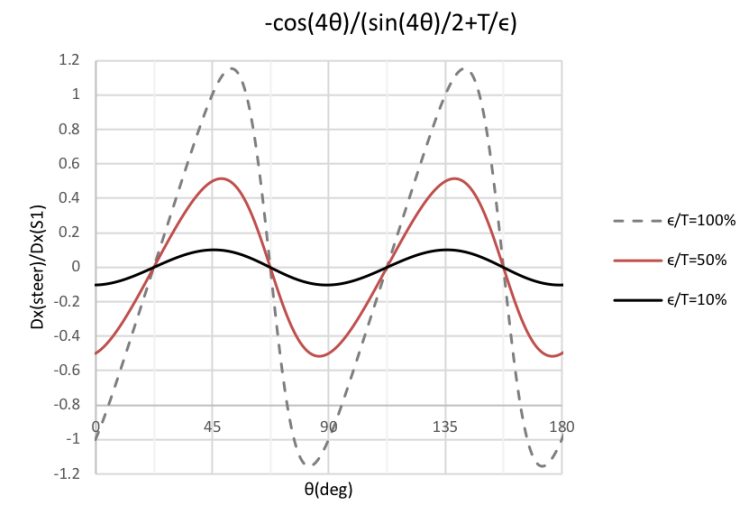

Figure 6-15: RHWP angle and analyzing/angle-like position differences

Fig. 6-16 shows an example of two RHWP scans with electron beam in the injector. In these scans, voltage offsets were used to induce a steering offset ${ }^{16}$. The

\footnotetext{
${ }^{16}$ Effective throw to the cathode was $\sim 2 m$ with a $2 \mathrm{~m}$ steering lens. Cathode spot size was $4 \sigma=2.9 \mathrm{~mm}$ (Horiz), $3.1 \mathrm{~mm}$ (Vert). Spot size at the Pockels cell was $2 \sigma=$
} 
photocathode was measured to have an analyzing power of $6 \%$ and we observe that in this case, because of the small analyzing power, inducing steering with voltage has a major effect on the offset terms and very little effect on the $4 \theta$ terms. The $D_{x}$ offset changed by $230 \mathrm{~nm}$ and the $D_{y}$ offset changed by $930 \mathrm{~nm}$, but nothing else significantly changed.

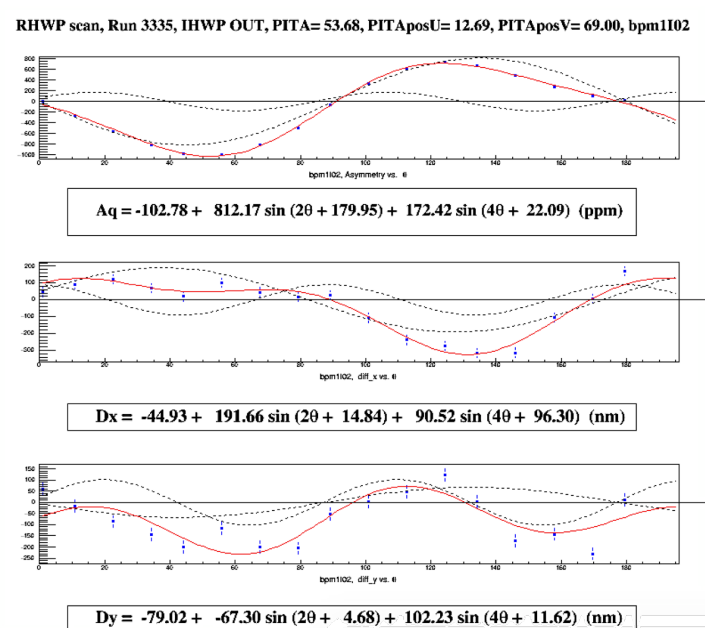

(a)

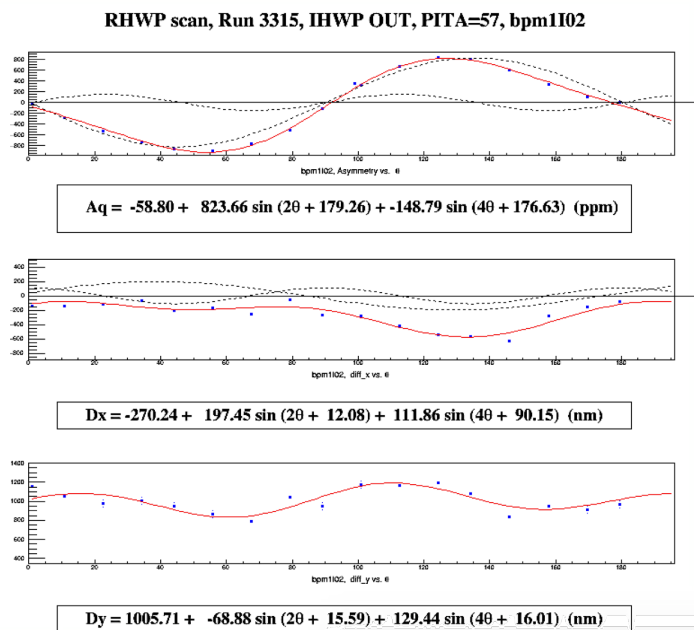

(b)

Figure 6-16: RTP RHWP scans e-beam: (a) RHWP scan performed with PITAposU $=13 \mathrm{~V}, \mathrm{PITApos} \mathrm{V}=69 \mathrm{~V}$ with large steering offsets (b) RHWP scan performed with PITAposU $=-24 \mathrm{~V}$, PITAposV $=-125 \mathrm{~V}$ where the steering offsets have been reduced. S1 is near $\theta=0^{\circ}$. [281]

\subsubsection{Spot size asymmetries}

Just as there are two kinds of position differences, there are also two kinds of spot-size asymmetries. The analyzing-like spot-size asymmetries arise from the 2nd moment of the intensity asymmetry as shown in Fig. 6-1. If a helicity correlated intensity asymmetry contains a 2 nd order term with respect to position on the crystal face or angle, a spot-size asymmetry is induced. This type of spotsize asymmetry is proportional to the power of the analyzing element and depends on its orientation.

$\bar{w}=0.825 \mathrm{~mm}($ Horizontal $), 0.94 \mathrm{~mm}($ Vertical $)$. Divergence at $\mathrm{PC}$ center was $d w / d z=$ $0.51 \mathrm{mrad}$ (horizontal), $0.66 \mathrm{mrad}$ (vertical). 
The second type of spot size asymmetry is related to and originates from the same effects as steering, only it's a 2nd order effect instead of first order. The crystal acts like a GRIN (gradient-index) lens, in a helicity correlated manner.

Spot-size asymmetries must be described in 2D. We refer the spot size asymmetry in general as $A_{\sigma}$ and along a particular axis as $A_{\sigma, x_{i}}$. There are several ways to describe spot-size, but we could model it as having a circular $\sigma_{\text {circ }}$ component and and an elliptical component $\epsilon_{\sigma} \approx\left(\sigma_{x}-\sigma_{y}\right) /\left(\sigma_{x}+\sigma_{y}\right)$ along some particular axis x. The spot size can be described as $\sigma(\theta)=\sigma_{\text {circ }}\left(1+\epsilon_{\sigma} \cos \left(2\left(\theta-\theta_{i}\right)\right)\right)$, where $\theta$ is the angle in the plane transverse to the direction of beam propagation. Likewise we can describe the spot size asymmetry as $A_{\sigma}(\theta)=A_{\text {circ }}+A_{\text {elli }} \cos \left(2\left(\theta-\theta_{0}\right)\right)$, where Aelli is the asymmetry due to the elliptical component of the spot size asymmetry, what we refer to as the "breathing mode" of the spot size asymmetry. $A_{\text {elli }}$ can be related to a difference in $\epsilon_{\sigma}$ via $A_{\text {elli }} \approx\left(\epsilon_{\sigma 0}-\epsilon_{\sigma 1}\right) /\left(\sigma_{0}+\sigma_{1}\right) \approx \Delta \epsilon / 2 \approx\left(\epsilon_{\sigma 0} / \sigma_{0}-\epsilon_{\sigma 1} / \sigma_{1}\right) / 2$, where the subscripts 0 and 1 are for two helicity states.

In the following sections we analytically describe the dependence of the spotsize asymmetry on various 2nd order terms in the RTP Pockels Cell.

\section{Spot size asymmetries: analyzing-like}

Analyzing-like spot-size asymmetries arise only if the beam encounters a polarizing element and are proportional to the analyzing power $\epsilon / T$. There are two kinds of mechanisms through which analyzing-like spot size asymmetries can occur: (1) asymmetry moments with respect to translation $\frac{d^{2} A}{d^{2} x_{i}}$ in the Pockels cell (2) asymmetry gradients with respect to angle $\frac{d^{2} A}{d^{2} \xi_{i}}$ in the Pockels cell. The spot-size asymmetries arising from these mechanisms can be reduced with beam parameters: either reducing the laser spot-size or the laser divergence in the cell. In the case of translational moments, $\frac{d^{2} A}{d^{2} x_{i}}$, the spot-size asymmetries can be reduced by using a smaller beam waist. In the case of angular moments, $\frac{d^{2} A}{d^{2} \xi_{i}}$, the spot-size asymmetries can be reduced by using a smaller beam divergence. 


\section{Translational Dependence}

A gaussian beam has power distribution $P(x)=P_{0} e^{-2 x^{2} / w^{2}}$. If it's exposed to a helicity dependent asymmetry gradient in the crystal, the power distribution becomes $P^{\prime}=P_{0} e^{-2 x^{2} / w^{2}}\left(1 \pm \frac{d A}{d x} x \pm \frac{1}{2} \frac{d^{2} A}{d x^{2}} x^{2}\right)$. Considering the expectation value of the position and its square to calculate the RMS,

$$
\langle x\rangle=\frac{\int x P^{\prime} d x}{\int P^{\prime} d x}= \pm \frac{\frac{d A}{d x} w^{2}}{\left(4 \pm \frac{1}{2} \frac{d^{2} A}{d x^{2}} w^{2}\right)} \quad\left\langle x^{2}\right\rangle=\frac{\int x^{2} P^{\prime} d x}{\int P^{\prime} d x}=\frac{w^{2}\left(4 \pm \frac{3}{2} \frac{d^{2} A}{d x^{2}} w^{2}\right)}{4\left(4 \pm \frac{1}{2} \frac{d^{2} A}{d x^{2}} w^{2}\right)}
$$

we obtains

$$
\begin{gathered}
\sigma=\sqrt{\left\langle x^{2}\right\rangle-\langle x\rangle^{2}} \approx \frac{w}{2} \pm \frac{w^{3} \frac{d^{2} A}{d x^{2}}}{16}-\frac{w^{3} \frac{d A^{2}}{d x}}{16} \pm \frac{3}{128} \frac{d^{2} A}{d x^{2}} \frac{d A^{2}}{d x} w^{5} \\
A_{\sigma}=\frac{\sigma_{R}-\sigma_{L}}{\sigma_{R}+\sigma_{L}} \approx \frac{\frac{d^{2} A}{d x^{2}} w^{2}}{8}+\frac{\frac{d^{2} A}{d x^{2}}\left(\frac{d A}{d x}\right)^{2} w^{4}}{16}
\end{gathered}
$$

Factoring in the condition that we may not have $100 \%$ analyzing power and noting the difference between spot-sizes at the cathode and Pockels cell ${ }^{17}$, we obtain for the spot-size difference

$$
\Delta \sigma=\sigma_{+}-\sigma-\approx \frac{\epsilon}{T} w_{\text {cathode }}\left(\frac{\frac{d^{2} A}{d x^{2}} w_{P C}^{2}}{4}+\frac{\frac{d^{2} A}{d x^{2}}\left(\frac{d A}{d x}\right)^{2} w_{P C}^{4}}{8}\right)
$$

\section{Angular Dependence}

Spot size asymmetry is a 2 nd order effect and can be related to the 2 nd derivative of charge asymmetry Aq with respect to position or angle. Angular dependence in the transmission can couple into an effective position dependence because of the laser beam divergence. A significant portion of the spot-size asymmetry comes from a crystal angle-dependence in conjunction with beam divergence which couples angle to position $r \approx R \theta \approx\left(w / \theta_{w}\right) \theta$ (as derived in Sec. in the Appendices). A gaussian beam divergence couples angle to position and so an

\footnotetext{
${ }^{17} w_{\text {cathode }}=\left(1-D_{2} / f+D_{\text {eff }} \theta / w\right) w$, see Sec. D.2.3 in Appendices
} 
angle-dependent gradient couples into a position-dependent gradient giving rise to spot-size asymmetries.

In terms of angle, a gaussian beam has power distribution $P(x)=P_{0} e^{-2 \theta^{2} / \theta_{w}^{2}}$ and if it's exposed to a helicity dependent asymmetry gradient, then $P^{\prime}=P_{0} e^{-2 \theta^{2} / \theta_{w}^{2}}(1 \pm$ $\left.\frac{d A}{d \theta} \theta \pm \frac{1}{2} \frac{d^{2} A}{d \theta^{2}} \theta^{2}\right)$. Considering the expectation value of the angle and its square to calculate the RMS,

$$
\begin{gathered}
\langle\theta\rangle=\frac{\int \theta P^{\prime} d \theta}{\int P^{\prime} d \theta}=\frac{ \pm \frac{d A}{d \theta} \theta_{w}^{2}}{\left(4 \pm \frac{1}{2} \frac{d^{2} A}{d \theta^{2}} \theta_{w}^{2}\right)} \quad\left\langle\theta^{2}\right\rangle=\frac{\int \theta^{2} P^{\prime} d x}{\int P^{\prime} d \theta}=\frac{\theta_{w}^{2}\left(4 \pm \frac{3}{2} \frac{d^{2} A}{d \theta^{2}} \theta^{2}\right)}{4\left(4 \pm \frac{1}{2} \frac{d^{2} A}{d \theta^{2}} \theta_{w}^{2}\right)} \\
\theta_{\sigma}=\sqrt{\left\langle\theta^{2}\right\rangle-\langle\theta\rangle^{2}} \approx \frac{\theta_{w}}{2} \pm \frac{\theta_{w}^{3} \frac{d^{2} A}{d \theta^{2}}}{16}-\frac{\theta_{w}^{3} \frac{d A^{2}}{1 \theta}}{16} \pm \frac{3}{128} \frac{d^{2} A}{d \theta^{2}} \frac{d A^{2}}{d \theta} \theta_{w}^{5}
\end{gathered}
$$

where $\theta_{\sigma}$ is the $1 / 2$ angle divergence after the Pockels cell, and $\theta_{w}$ is the full angle divergence. Taking into account coupling between position and angle $x(\theta) \approx$ $\theta\left(w / \theta_{w}\right)$, implies $\sigma \approx \theta_{\sigma}\left(w /\left(\theta_{w}\right)\right)$,

$$
\begin{gathered}
\sigma \approx \frac{w}{\theta_{w}}\left(\frac{\theta_{w}}{2} \pm \frac{\theta_{w}^{3} \frac{d^{2} A}{d \theta^{2}}}{16}-\frac{\theta_{w}^{3} \frac{d A^{2}}{d \theta}}{16} \pm \frac{3}{128} \frac{d^{2} A}{d \theta^{2}} \frac{d A^{2}}{d \theta} \theta_{w}^{5}\right) \\
A_{\sigma}=\left(\sigma_{+}-\sigma_{-}\right) /\left(\sigma_{+}+\sigma_{-}\right) \approx \frac{\frac{d^{2} A}{d \theta^{2}} \theta_{w}^{2}}{8}+\frac{\frac{d^{2} A}{d \theta^{2}}\left(\frac{d A}{d \theta}\right)^{2} \theta_{w}^{4}}{16}
\end{gathered}
$$

Factoring in the condition that we may not have $100 \%$ analyzing power and noting the difference between spot-sizes at the cathode and Pockels cell, we obtain for the spot size difference

$$
\Delta \sigma=\sigma_{+}-\sigma-\approx \frac{\epsilon}{T} w_{\text {cathode }}\left(\frac{\frac{d^{2} A}{d \theta^{2}} \theta_{P C}^{2}}{4}+\frac{\frac{d^{2} A}{d \theta^{2}}\left(\frac{d A}{d \theta}\right)^{2} \theta_{P C}^{4}}{8}\right)
$$

The asymmetry angle dependence in RTP was derived as (Equation 6.1)

$$
A_{I}\left(\xi_{x 0}, \xi_{y 0}\right)=\frac{\epsilon}{T} \sin (2(\eta-\psi)) \frac{\pi L_{0}}{\lambda}\left(n_{z}+n_{y}\right)\left(\frac{1}{n_{x}^{2}}-\frac{1}{n_{z} n_{y}}\right)\left(2 \xi_{x 0} \xi_{y 0}\right)
$$




$$
=\frac{\epsilon}{T} \sin (2(\eta-\psi)) \frac{\pi L_{0}}{\lambda}\left(n_{z}+n_{y}\right)\left(\frac{1}{n_{x}^{2}}-\frac{1}{n_{z} n_{y}}\left(\xi_{u 0}^{2}-\xi_{v 0}^{2}\right)\right)
$$

denoting $u=\frac{x+y}{\sqrt{2}}$ and $v=\frac{x-y}{\sqrt{2}}$. In the thermal compensation design of the RTP cell, the birefringence cancellation along S2 $(\eta=\psi)$ implies no angle dependent birefringence gradient, so RTP should suffer from less spot size asymmetry than $\mathrm{KD}^{*} \mathrm{P}$ when analyzing along $\mathrm{S} 2$. The analyzing-like spot size asymmetry in S2 for $\mathrm{RTP}$ is $A_{\sigma}^{S 2}=0$.

The angle dependence of Aq combined with beam divergence position-angle coupling gives rise to angle dependent spot size asymmetries in S1. We obtain an elliptical analyzing-like spot size asymmetry for RTP:

$$
\begin{array}{cc}
\frac{d A^{2}(\theta)}{d \xi_{x}^{2}}=0 & A_{\sigma x}=0 \quad \frac{d A^{2}(\theta)}{d \xi_{y}^{2}}=0 \quad A_{\sigma y}=0 \\
\frac{d A^{2}(\theta)}{d \xi_{u}^{2}}=+2 k & A_{\sigma u}=\frac{\epsilon}{T} \theta_{P C}^{2} \frac{k}{4}\left(1+2\left(k \xi_{u 0}\right)^{2} \theta_{P C}^{2}\right) \\
\frac{d A^{2}(\theta)}{d \xi_{v}^{2}}=-2 k & A_{\sigma v}=-\frac{\epsilon}{T} \theta_{P C}^{2} \frac{k}{4}\left(1+2\left(k \xi_{v 0}\right)^{2} \theta_{P C}^{2}\right)
\end{array}
$$

where

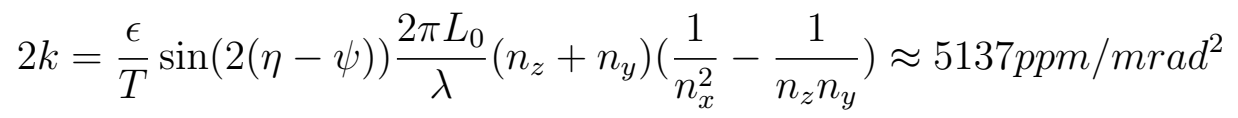

Near alignment where $\frac{d A}{d \xi} \approx 0$ with the beam is sitting on the Aq saddle point in angle dependence, for a $0.5 \mathrm{mrad}$ beam divergence at the Pockels cell and a $6 \%$ analyzing power oriented directly along S1, the spot size asymmetry along the diagonal $u=+45^{\circ}, v=-45^{\circ}$ axes will be $A_{\sigma u, 45^{\circ}}=1 \times 10^{-5}$ and $A_{\sigma v,-45^{\circ}}=-1 \times 10^{-5}$.

\section{Spot size asymmetries: angle-like}

The same mechanism which gives rise to steering and angle-like position differences can also give rise to angle-like spot size asymmetries. The Pockels cell 
can induce a helicity-correlated divergence difference $D_{\theta}$. For a throw distance $D$, the corresponding spot size asymmetry is given by $A \sigma=\frac{D}{2 w_{\text {cathode }}} D_{\theta}$.

These angle-like spot size asymmetries can be either be (1) independent of analyzing power, just like the steering described in Sec. 6.3.4 (2) or they can be dependent on the analyzing power, analogous to the analyzing/angle-like position differences described in Sec.6.3.6.

The divergence of the beam coming out of the Pockels cell depends on the divergence of the beam coming in, the curvature of the crystal face cuts ${ }^{18}$, the curvature of the field ${ }^{19}$, and any 2 nd moments in the refractive index gradients (acting as a GRIN lens):

$$
\theta_{d i v} \sim \frac{\theta_{\text {air }, d e f l}}{d x_{i}}=\frac{d^{L}}{d x_{i}^{2}}(n-1)+2 \frac{d n}{d x_{i}} \frac{d L}{d x_{i}}+\frac{d^{2} n}{d x_{i}^{2}} L
$$

The helicity correlated refractive index 2 nd moment is given by

$$
\frac{d^{2} \Delta n_{j}}{d x_{i}^{2}}=-6 n_{j 0} r_{j 3}\left(\frac{d n_{j 0}}{d x_{i}}\right)^{2} E_{z 0}-3 n_{j 0}^{2} r_{j 3} \frac{d^{2} n_{j 0}}{d x_{i}^{2}} E_{z 0}-3 n_{j 0}^{2} r_{j 3} \frac{d n_{j 0}}{d x_{i}} \frac{d E_{z 0}}{d x_{i}}-n_{j 0}^{3} r_{j 3} \frac{d 2 E_{z 0}}{d x_{i}^{2}}
$$

The helicity-correlated divergence differences along $x_{i}$ for polarization $\mathrm{j}$ due to these 2nd moments in one crystal is given by

$$
\begin{gathered}
\left.D_{\theta i}\right|_{j} \sim \frac{d^{L}}{d x_{i}^{2}}(\Delta n)+2 \frac{d \Delta n}{d x_{i}} \frac{d L}{d x_{i}}+\frac{d^{2} \Delta n}{d x_{i}^{2}} L \\
\sim-r_{j 3} n_{j 0}^{3}\left(\left(2 \frac{d^{L}}{d x_{i}^{2}}+6 \frac{1}{n_{j 0}} \frac{d n_{j 0}}{d x_{i}} \frac{d L}{d x_{i}}+6 \frac{1}{n_{j 0}^{2}}\left(\frac{d n_{j 0}}{d x_{i}}\right)^{2} L+3 \frac{1}{n_{j 0}} \frac{d^{2} n_{j 0}}{d x_{i}^{2}} L\right) E_{z 0}\right. \\
\left.+\left(2 \frac{d L}{d x_{i}}+3 \frac{1}{n_{j 0}} \frac{d n_{j 0}}{d x_{i}} L\right) \frac{d E_{z 0}}{d x_{i}}+L \frac{d^{2} E_{z 0}}{d x_{i}^{2}}\right)
\end{gathered}
$$

Most of this helicity-correlated divergence is intrinsic to the cell, the rest is due

\footnotetext{
${ }^{18}$ The curvature of the face cuts and the 2 nd moments in the refractive indices were measured [282] and found to be $<2 \mu \mathrm{rad} / \mathrm{mm}$ angle change in curvature vs transverse distance

${ }^{19}$ In fact, we have an idea to split the side panels in half and add 4 more HV's connected to the side panels to induce a helicity correlated E-field 2nd moment to induce spot-size asymmetries in the beam so that spot-size sensitivities can be measured in experiments
} 
to electric field gradients which can be adjusted.

$$
\left.D_{\theta i}\right|_{j} \equiv-r_{j 3} n_{j 0}^{3}\left(\partial_{\text {intrinsic }}+\partial E_{z 0}\right)
$$

For no analyzer, the helicity-correlated divergence from a single RTP (one of two), will be an average of the components along the polarization primary axes y and $\mathrm{z}$

$$
\left.D_{\theta i}\right|_{\text {no anal }} \equiv-\left(r_{33} n_{z 0}^{3}+r_{23} n_{y 0}^{3}\right) / 2\left(\partial_{\text {intrinsic }}+\partial E_{z 0}\right)
$$

For a throw distance $D$, the corresponding spot size asymmetry is given by

$$
\left.A_{\sigma i}\right|_{\text {no anal }}=\frac{D}{2 w_{\text {cathode }}} D_{\theta i}=-\frac{D\left(r_{33} n_{z 0}^{3}+r_{23} n_{y 0}^{3}\right)}{4 w_{\text {cathode }}}\left(\partial_{\text {intrinsic }}+\partial E_{z 0}\right)
$$

For the analyzer along $\mathrm{S} 2$ in the $\pm 45^{\circ}$ directions,

$$
\begin{aligned}
& \left.D_{\theta i}\right|_{-45^{\circ}} \equiv-\left(r_{23} n_{y 0}^{3}\right)\left(\partial_{\text {intrinsic }}+\partial E_{z 0}\right) \\
& \left.D_{\theta i}\right|_{+45^{\circ}} \equiv-\left(r_{33} n_{z 0}^{3}\right)\left(\partial_{\text {intrinsic }}+\partial E_{z 0}\right)
\end{aligned}
$$

with corresponding spot size asymmetries of

$$
\begin{aligned}
& \left.A_{\sigma i}\right|_{-45^{\circ}}=-\frac{\epsilon}{T} \frac{D r_{23} n_{y 0}^{3}}{2 w_{\text {cathode }}}\left(\partial_{\text {intrinsic }}+\partial E_{z 0}\right) \\
& \left.A_{\sigma i}\right|_{+45^{\circ}}=-\frac{\epsilon}{T} \frac{D r_{33} n_{z 0}^{3}}{2 w_{\text {cathode }}}\left(\partial_{\text {intrinsic }}+\partial E_{z 0}\right)
\end{aligned}
$$

We make the observation that since $r_{33} n_{z 0}^{3} \approx 3 r_{23} n_{y 0}^{3}$, if the spot size asymmetry that is steering-like and independent of analyzer is $\left.A_{\sigma i}\right|_{\text {no anal }}=2 X$, then the analyzing/angle -like spot size asymmetries are $\left.A_{\sigma i}\right|_{-45^{\circ}}=1 X,\left.A_{\sigma i}\right|_{+45^{\circ}}=3 X$. 


\subsubsection{Interferometric-Etalon Effect}

Using a narrow-band CW laser (10MHz, LD785-SEV300 , 785WOPK04.C05), we observed an etalon problem in RTP crystals. The RTP crystals are polished so well, with parallelism $<10$ arcsec, that the reflections of the front and back faces of the crystal interfere with one another. The crystal acts as an interferometer. The etalon effect is an interference between the back reflections of the front face and the back face of a crystal. The reflectivity and thus the transmission depends on the phase shift between the two back-reflections. In a running Pockels cell, the phase shift is being altered by application of voltage. Hence when we apply a voltage to the Pockels Cell, the transmission changes. This etalon effect in RTP produces a helicity correlated charge asymmetry. The magnitude of the produced charge asymmetry is determined by the reflection coefficient of the crystal faces $A_{q}=2 R \cos \phi^{20}$. It is a problem because it leads to a helicity dependent reflection coefficient, hence transmission coefficient, hence charge asymmetry. The etalon effect can interfere with transmission and produce helicity correlated asymmetries and gradients. The charge asymmetry produced will be the same order as the reflection coefficient of a single face $0.2 \%$. Here we derive the effect on the transmitted asymmetry for each polarization state along primary y,z axis for one crystal.

The reflection and transmission is given by:

$$
\begin{gathered}
R(\phi)=2 R(1+\cos \phi) \\
T=1-R=1-2 R(1+\cos \phi)
\end{gathered}
$$

\footnotetext{
${ }^{20} \phi$ is the phase difference between the two arms of the interferometer, in this case, the difference in phase between the reflected beam off the front face of the crystal and the reflected beam off the back face of the crystal, which experiences a phase shift from passing through crystal twice, once going forwards and once upon reflecting back
} 


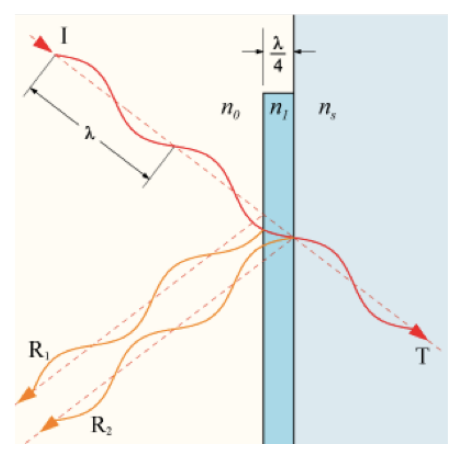

Figure 6-17: Reflection interference in a quarter-wave anti-reflection coating [252]

For the $\mathrm{z}$ axis on one crystal:

$$
\begin{gathered}
\phi_{z}=2 \pi n_{z} L_{0} / \lambda \\
n_{z}^{R(L)}=n_{0, z}-\frac{1}{2} n_{0, z}^{3} r_{33} E_{z}^{R(L)} \\
E_{z}=V / d
\end{gathered}
$$

The transmitted asymmetry due to this interference effect is

$$
\begin{gathered}
A_{T}=\frac{T^{R}-T^{L}}{T^{R}+T^{L}}=R \frac{-\cos \phi^{R}+\cos \phi^{L}}{1-R\left(1+\cos \phi^{R}+1+\cos \phi^{L}\right)} \\
\approx R\left(-\cos \phi^{R}+\cos \phi^{L}\right) \\
\approx R\left(-\cos \left(2 \pi n_{z}^{R} L_{0} / \lambda\right)+\cos \left(2 \pi n_{z}^{L} L_{0} / \lambda\right)\right) \\
\approx R\left(-\cos \left(2 \pi\left(n_{0, z}-\frac{1}{2} n_{0, z}^{3} r_{33} E_{z}^{R}\right) L_{0} / \lambda\right)+\cos \left(2 \pi\left(n_{0, z}-\frac{1}{2} n_{0, z}^{3} r_{33} E_{z}^{L}\right) L_{0} / \lambda\right)\right) \\
\approx R\left(-\cos \left(2 \pi\left(n_{0, z}-\frac{1}{2} n_{0, z}^{3} r_{33} V^{R} / d\right) L_{0} / \lambda\right)+\cos \left(2 \pi\left(n_{0, z}-\frac{1}{2} n_{0, z}^{3} r_{33} V^{L} / d\right) L_{0} / \lambda\right)\right.
\end{gathered}
$$

Similarly for the y axis on one crystal:

$$
\begin{gathered}
\phi_{y}=2 \pi n_{y} L_{0} / \lambda \\
n_{y}^{R(L)}=n_{0, y}-\frac{1}{2} n_{0, y}^{3} r_{23} E_{z}^{R(L)}
\end{gathered}
$$


$A_{T}=R\left(-\cos \left(2 \pi\left(n_{0, y}-\frac{1}{2} n_{0, y}^{3} r_{23} V^{R} / d\right) L_{0} / \lambda\right)+\cos \left(2 \pi\left(n_{0, y}-\frac{1}{2} n_{0, y}^{3} r_{23} V^{L} / d\right) L_{0} / \lambda\right.\right.$

We have observed that our crystal faces have reflection coefficients of $0.2-0.5 \%$ and can produce charge asymmetries 3000-10,000ppm as shown in the Fig. 6-38.

There only real solution to fix the etalon effect is to make the RTP not an etalon. The requirements for an etalon are (1) non-negligible reflection coefficients (2) parallel faces (3) narrow-bandwidth (4) temporal overlap of reflected pulses (5) spatial overlap and coherence of reflected pulses. At least one of these 5 requirements must be violated to eliminate the etalon effect in the RTP. Suppressing this interferometric-etalon effect is discussed in further detail in $\mathrm{C}$

\subsection{Design}

\subsubsection{8-HV Design}

All crystals suffer from some degree of non-uniformity. In order to counteract this non-uniformity and minimize helicity correlated beam asymmetries, an innovation in the design of the RTP Pockels Cell was required. We used grounded plates to induce fringe-electric fields. By controlling the electric field gradients, the helicity correlated position differences could be suppressed.

In commercial designs, the 2 crystals have a common grounded plate and two HV plates on the top of each crystal as shown in Fig. 6-18a and a common HV voltage is applied to the top plates of both crystals. We gain the ability to control the electric field gradients by a simple design change. We separated the two crystals' ground plates, so each crystal electric field is independently controlled, added grounded side-panels near the sides of each crystal, and added more several $\mathrm{HV}$ power supplies so that the voltage on each of the $4 \mathrm{HV}$ plates can be independently set to different values as illustrated in Fig.6-18b and Fig.6-19. There are 8 independent voltages in total, for each of the $4 \mathrm{HV}$ plates on the two crystals for both helicity states. We also designed the cell mount with additional degrees of 
freedom to have control over the relative pitch, yaw, roll, horizontal and vertical translation between the two crystals as well as the overall pitch, yaw, roll, horizontal and vertical translation degrees of freedom. The Pockels cell is designed with two RTP crystals (Raicol 12x12x10mm, AR coated), with full angular and translational control of each crystal, and grounded side-panels in addition to $4 \mathrm{HV}$ plates. 8 high voltages are applied to the cell, driven with a opto-diode switch. The laser polarization states are switched within $12 \mu \mathrm{s}$, while controlling the direction and intensity of the laser beam to keep it extremely symmetric (at nm-level and ppm-level).

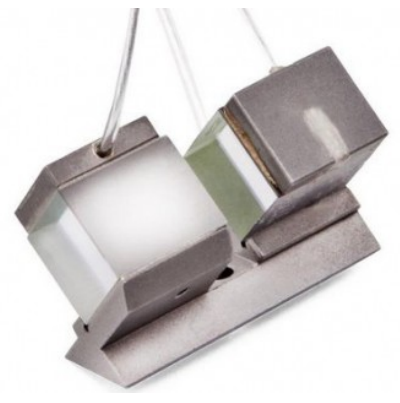

(a) Raicol Design [253]

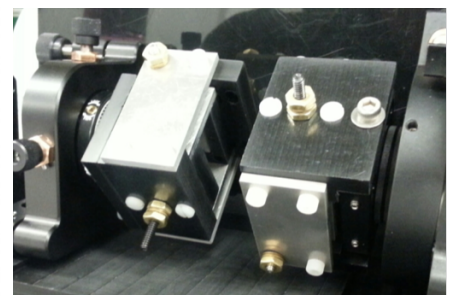

(b) Side Panel Design

This design allows for a straightforward control over the electric field gradient along the z-axis of each crystal without changing the magnitude of the E-field very much. Since the voltage setting controls the electric field gradient along the $\mathrm{z}$-axis of each crystal, it controls the steering along the z-axis for each crystal. The first RTP crystal's electric field gradient controls and the steering along $-45^{\circ}$ and the second crystal's electric field gradient controls the steering along $+45^{\circ}$ as shown by the orientations in Fig. 6-19.

We have the ability, with our 8 independent voltages, to control the delta-phase, the alpha-phase, and to create a delta-phase gradient or alpha-phase gradient. While position differences in S1 are caused by a delta-phase gradient, steering is controlled by an alpha-phase gradient. The voltage shift in each crystal which induces steering we refer to as $\alpha$-position voltage, because it controls the alpha- 
phase gradient. ${ }^{21}$ The first crystal with its z-axis along $U$ controls the steering along the U-direction with -position-U voltage, and the second crystal with z-axis along $\mathrm{V}$ controls the steering along the $\mathrm{V}$-direction with PITA-position-V voltage. corresponding to the $\pm 45^{\circ}$ directions denoted ' $\mathrm{U}$ ' and ' $\mathrm{V}$ ' $V_{\alpha p o s, U}$ and $V_{\alpha p o s, V}$

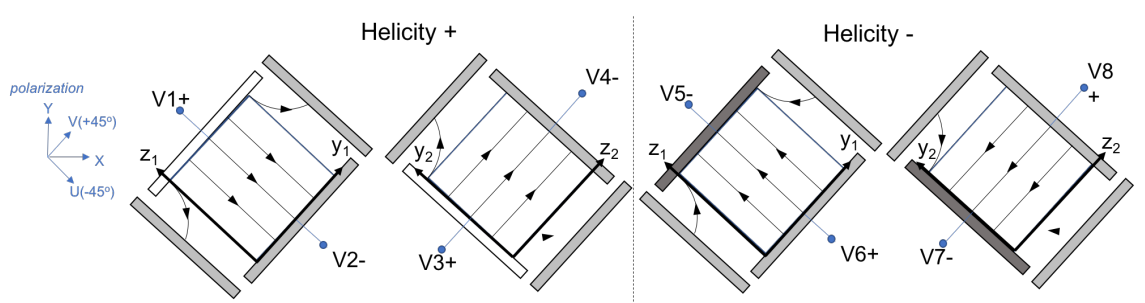

Figure 6-19: Defining axes of RTP cell and configuration of 8HV's. Figure illustrates case of $V_{\alpha p o s, U}=V_{\alpha p o s, V}=V_{\lambda / 4}>0$ with grounded side panels.

The equations describing how the voltage settings are used to induce steeringlike position differences via $V_{\alpha p o s, U / V}$ are shown in Table 6.2 .

\begin{tabular}{|r|r|r|r|r|r|}
\hline$H V$ & hel. & crystal & pol. & orien.,dir. & equation \\
\hline 1 & + & 1 & + & $+z 1,+U$ & $V_{1}=V_{\lambda / 4}+V_{\alpha, 1}+V_{\Delta, 1}+V_{\alpha p o s, U}+V_{\delta p o s, U}$ \\
\hline 2 & + & 1 & - & $-z 1,-U$ & $V_{2}=-V_{\lambda / 4}-V_{\alpha, 1}-V_{\Delta, 1}+V_{\alpha p o s, U}+V_{\delta p o s, U}$ \\
\hline 3 & + & 2 & + & $-z 2,-V$ & $V_{3}=V_{\lambda / 4}+V_{\alpha, 2}+V_{\Delta, 2}+V_{\alpha p o s, V}+V_{\delta p o s, V}$ \\
\hline 4 & + & 2 & - & $+z 2,+V$ & $V_{4}=-V_{\lambda / 4}-V_{\alpha, 2}-V_{\Delta, 2}+V_{\alpha p o s, V}+V_{\delta p o s, V}$ \\
\hline 5 & - & 1 & - & $+z 1,+U$ & $V_{5}=-V_{\lambda / 4}-V_{\alpha, 1}+V_{\Delta, 1}-V_{\alpha p o s, U}+V_{\delta p o s, U}$ \\
\hline 6 & - & 1 & + & $-z 1,-U$ & $V_{6}=V_{\lambda / 4}+V_{\alpha, 1}-V_{\Delta, 1}-V_{\alpha p o s, U}+V_{\delta p o s, U}$ \\
\hline 7 & - & 2 & - & $-z 2,-V$ & $V_{7}=-V_{\lambda / 4}-V_{\alpha, 2}+V_{\Delta, 2}-V_{\alpha p o s, V}+V_{\delta p o s, V}$ \\
\hline 8 & - & 2 & + & $+z 2,+V$ & $V_{8}=V_{\lambda / 4}+V_{\alpha, 2}-V_{\Delta, 2}-V_{\alpha p o s, V}+V_{\delta p o s, V}$ \\
\hline
\end{tabular}

Table 6.2: HV\#, helicity, crystal, HV polarity, orientation, steering direction, equation

\footnotetext{
${ }^{21}$ referred to as 'PITA'-position voltage in elogs. The name "PITA-position-voltage" is a misnomer and we should actually call it "alpha-position-voltage" because it induces an alphaphase gradient and it is that which causes steering.
} 
The voltages relate to the quarter-wave voltage via

$$
V_{\lambda / 4}=\left(V_{1}-V_{2}+V_{3}-V_{4}-V_{5}+V_{6}-V_{7}+V_{8}\right) / 8-\left(V_{\alpha, 1}+V_{\alpha, 2}\right) / 2
$$

Our 8 degrees of freedom translate into (1) an alpha-phase for crystal 1 and (2) an alpha-phase for crystal 2 (where we tend to keep these equal, and combine them into to one parameter $V_{\alpha}=V_{\alpha, 1}=V_{\alpha, 2}$ )

$$
V_{\alpha, 1}=\left(V_{1}-V_{2}-V_{5}+V_{6}\right) / 4-V_{\lambda / 4} \quad V_{\alpha, 2}=\left(V_{3}-V_{4}-V_{7}+V_{8}\right) / 4-V_{\lambda / 4}
$$

(3) a delta-phase for crystal 1 and (4) a delta-phase for crystal 2 (where we tend to keep these equal, and combine them into to one parameter $V_{\Delta}=V_{\Delta, 1}=V_{\Delta, 2}$ )

$$
V_{\Delta, 1}=\left(V_{1}-V_{2}+V_{5}-V_{6}\right) / 4 \quad V_{\Delta, 2}=\left(V_{3}-V_{4}+V_{7}-V_{8}\right) / 4
$$

(5) an alpha-phase-gradient for crystal 1, along its z axis, used for steering along $+45^{\circ}$ and (6) an alpha-phase-gradient for crystal 2 along its z axis, used for steering along $-45^{\circ}$

$$
V_{\alpha p o s, U}=\left(V_{1}+V_{2}-V_{5}-V_{6}\right) / 4 \quad V_{\alpha p o s, V}=\left(V_{3}+V_{4}-V_{7}-V_{8}\right) / 4
$$

(7) a delta-phase-gradient for crystal 1 , along its $\mathrm{z}$ axis $+45^{\circ}$ and finally (8) a delta-phase-gradient for crystal 2 , along its $\mathrm{z}$ axis $-45^{\circ}$, though we tend to not use the delta phase gradient and keep $V_{\delta p o s, V}=V_{\delta p o s, U}=0 .{ }^{22}$

$$
V_{\delta p o s, U}=\left(V_{1}+V_{2}+V_{5}+V_{6}\right) / 4 \quad V_{\delta p o s, V}=\left(V_{3}+V_{4}+V_{7}+V_{8}\right) / 4
$$

In table 6.2, helicity "+" corresponds to the Pockels cell acting as a QWP

\footnotetext{
${ }^{22}$ In principle we could also induce a delta-phase gradient, but we do not currently do this. It would alter the Aq gradient in S1, and for no analyzer ,it would have the effect of shifting the average position of the beam, but not induce position differences.
} 
with fast axis along $+45^{\circ}$ relative to the horizontal ${ }^{23}$, such that horizontal input polarization $\vec{H}=\left[\begin{array}{l}1 \\ 0\end{array}\right]$ (IHWP out) becomes right-circularly polarized $\vec{R}=\frac{1}{\sqrt{2}}\left[\begin{array}{c}1 \\ -i\end{array}\right]$, carrying spin-angular momentum $-\hbar$. Helicity "-" corresponds to the Pockels cell acting as a QWP with fast axis along $-45^{\circ}$ relative to the horizontal, such that horizontal input polarization $\vec{H}=\left[\begin{array}{l}1 \\ 0\end{array}\right]$ (IHWP out) becomes left-circularly polarized $\vec{R}=\frac{1}{\sqrt{2}}\left[\begin{array}{l}1 \\ i\end{array}\right]$, carrying spin-angular momentum $+\hbar$. In the photocathode, upon core-level excitation by circularly polarized light, the angular momentum of the light, or helicity, is transferred to the emitted photoelectron. The angular momentum of the emitted photoelectron is the sum of the helicity and the orbital magnetic quantum number of the initial state [271]. ${ }^{24}$

The HV driver is composed of an optocoupler system. Each of the 8 HV's are driven by two optodiodes in parallel ${ }^{25}$. Each of the two optodiodes is opened by one of two 1W LED's in series, pulsed at the beginning of each helicity cycle for $\sim 20 \mu s-40 \mu s$, powered by $8 \mathrm{~V}-9 \mathrm{~V}$ of DC voltage $(4-4.5 \mathrm{~V}$ on each LED). The circuit diagram is shown in Fig. 6-20.

\footnotetext{
${ }^{23} \mathrm{X}$ in a right-handed $\mathrm{XYZ}$ coordinate system where $\mathrm{Z}$ is the beam propagation axis

${ }^{24} \mathrm{~A}$ Mott measurement (on the HallB laser) determined the sign of the beam polarization to be $-86.6 \%$ for IHWPout and for $87.2 \%$ for IHWPin [272]

${ }^{25} \mathrm{An}$ optodiode is a high-voltage diode which switches states upon exposure to IR light and can be controlled using LEDs. The optodiodes are made by a Voltage Multipliers Inc. (VMI) part number OZ100SG. Our design has two optodiodes in parallel to allow for more current to flow to the Pockels cell for a faster transition
} 


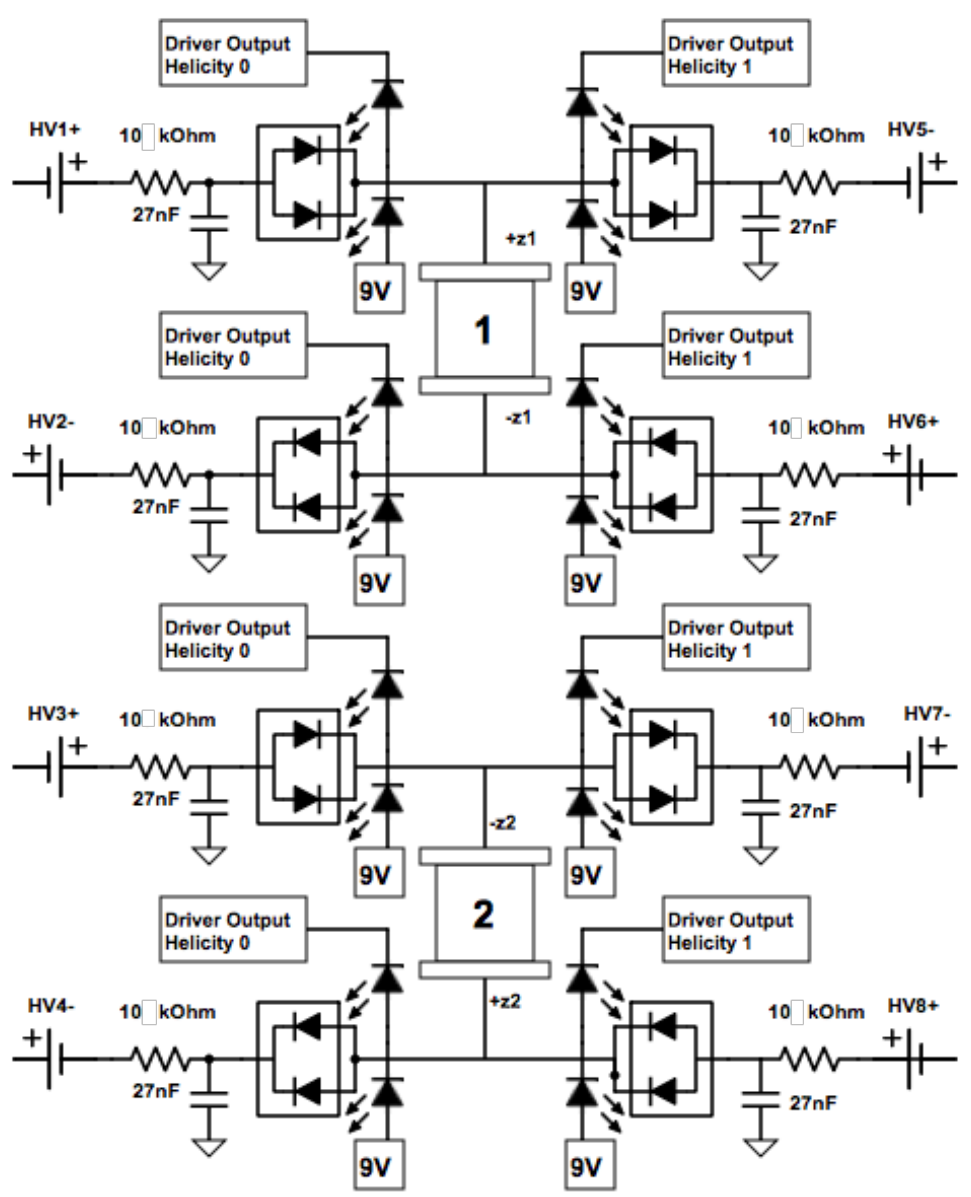

Figure 6-20: Diagram of the $8 \mathrm{HV}$ driver configuration with optodriver

Fig. 6-21 and Fig.6-22b show all the components of the cell system and the physically assembled cell. The two crystals have individual Al plates, Al side panels, and are held together by a delrin sandwich design, each mounted individually to a rotation stage and a 6axis optics mount. The whole assembly is mounted on a 4axis angle mount which adjusts pitch and yaw, a rotation stage which adjusts roll, and two translation stages for $\mathrm{X}$ and $\mathrm{Y}$ control. A full list of components required is found in [273], the CAD file can be found in [274] and Raicol crystals detail are located in [275] ${ }^{26}$. Several spares may need to be made when the ${ }^{26}$ The crystals are Raicol RTP Matched Pair $2 \mathrm{x}(12 \mathrm{x} 12 \mathrm{x} 10 \mathrm{~mm})$ at $780 \mathrm{~nm}, 12 \mathrm{x} 12 \mathrm{~mm}$ aper-
ture, $10 \mathrm{~mm}$ length crystals, with length mismatch between crystal pair $<2 \mu \mathrm{m}$, with extinction
ratio $>23 \mathrm{~dB}$ in clear aperture $10 \mathrm{~mm}$, face cut parallelism $<10$ arcsec, AR coated at $780 \mathrm{~nm}$,
$\mathrm{R}<0.2 \%$, and capacitance $4-6 \mathrm{pF}$. The power supplies are IGES $4 \mathrm{~W} 2 \mathrm{kV}$ BPS series. The LED's 
system is installed at JLab, especially since Moller will runs for such a long time.

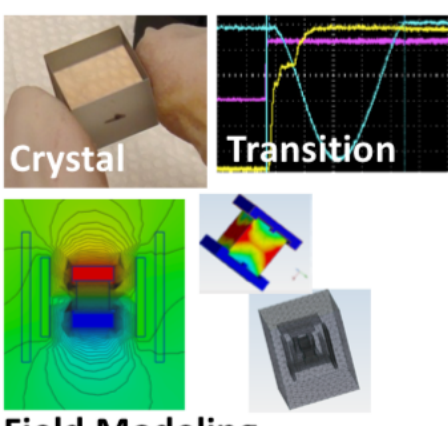

Field Modeling

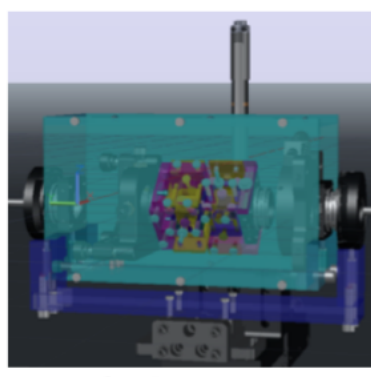

CAD design

Figure 6-21: RTP cell design

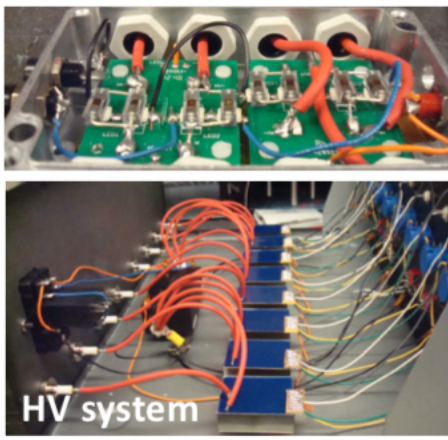

HV̄ system

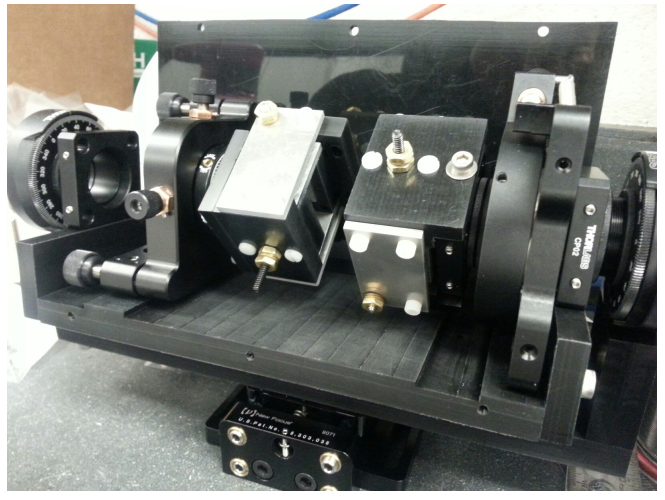

(a)

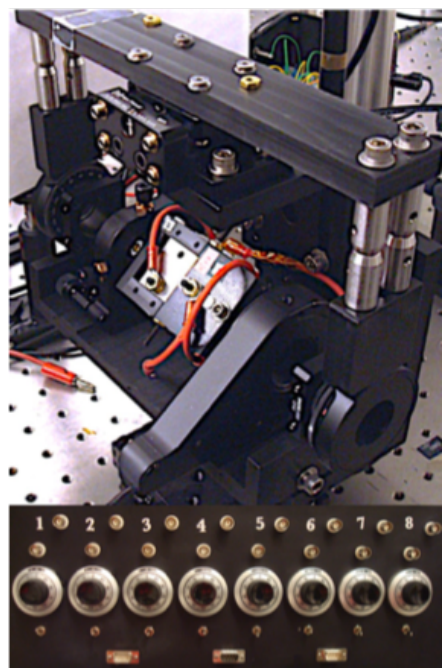

(b)

Figure 6-22: Assembled RTP mount

\subsubsection{Opto-Driver}

Fig. 6-24 shows the LED driver circuit ${ }^{27}$. It produces a 3-step square waveform of current through the LEDs as shown in Fig.6-23a.

are OSRAM-Opto-Semiconductors Platinum Dragon. The optocouplers are VMI OZ100HG. The mount includes thorlabs PT1-Z8 translation stages, Newport 9071 4-axis mount, thorlabs PRM1Z7 motorized rotation mount, thorlabs K6XS 6axis mirror mount, and an Edmund optics $3 \times 3$ right angle bracket.

${ }^{27}$ John Hansknecht (hansknec@jlab.org) also built a spare driver (with very similar design, though only 2 steps, powered by $8 \mathrm{~V}$, and $60-70 \mu$ s long LED pulse) that is fed a helicity signal from fiber optic light source and converts it to a TTL signal [290]. 
The 1st step of the 3-step waveform begins at the start of the helicity window, the 2nd step begins at a time which is adjustable, both the 1st and 2nd steps end together at a time which is adjustable, and the 3rd step is a constant low current which is permitted to flow throughout the entire helicity window [283]. Upon switching to the opposite helicity state, the current flow through one set of LEDs ceases and the current flow through another set begins. The resistors in the circuit are potentiometers that allow for adjustment of the LED current waveform shown in Fig.6-23a ${ }^{28}$. The adjustable parameters are the start time of the 2nd step in current, the stop time of the 1st and 2nd steps, and the magnitude of the currents for both the 1st and 2nd steps. To drive the RTP, the magnitudes of the 1st and 2nd steps are set to maximal current, they remain on for $20-40 \mu \mathrm{s}$, and the start time of the 2nd step is set as early as possible at the beginning of the window, in effect driving the RTP as fast as possible.

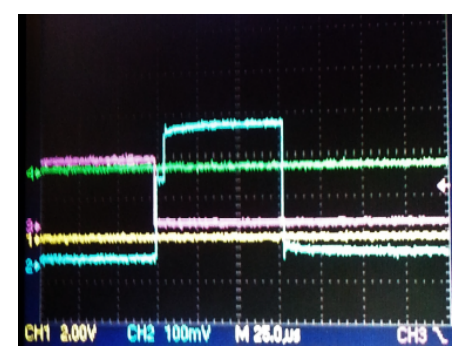

(a)

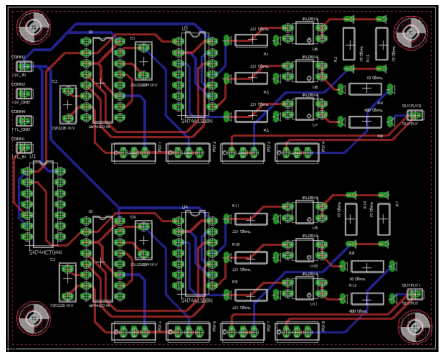

(b)

Figure 6-23: (a)LED pulse - 3 steps, $\mathrm{I} 1+\mathrm{I} 3, \mathrm{I} 1+\mathrm{I} 2+\mathrm{I} 3$,I3. Blue trace is LED pulse as detected by a photodiode. Pink trace is the TTL helicity trigger signal. [283] (b)PCB driver [283]

\footnotetext{
${ }^{28}$ Note that resistor values and capacitor values are variable. The shown $225 \mathrm{nF}$ capacitor has been replaced with $2.2 \mathrm{nF}$ capacitor, the shown $2.2 \mathrm{nF}$ capacitor has been replaced with a $2.5 \mathrm{nF}$ capacitor, and an old version of the circuit had just $100 \mathrm{nF}$ capacitors [307]. The potentiometers are $50 \mathrm{kOhm}$ and $50 \mathrm{Ohm}$ range.
} 


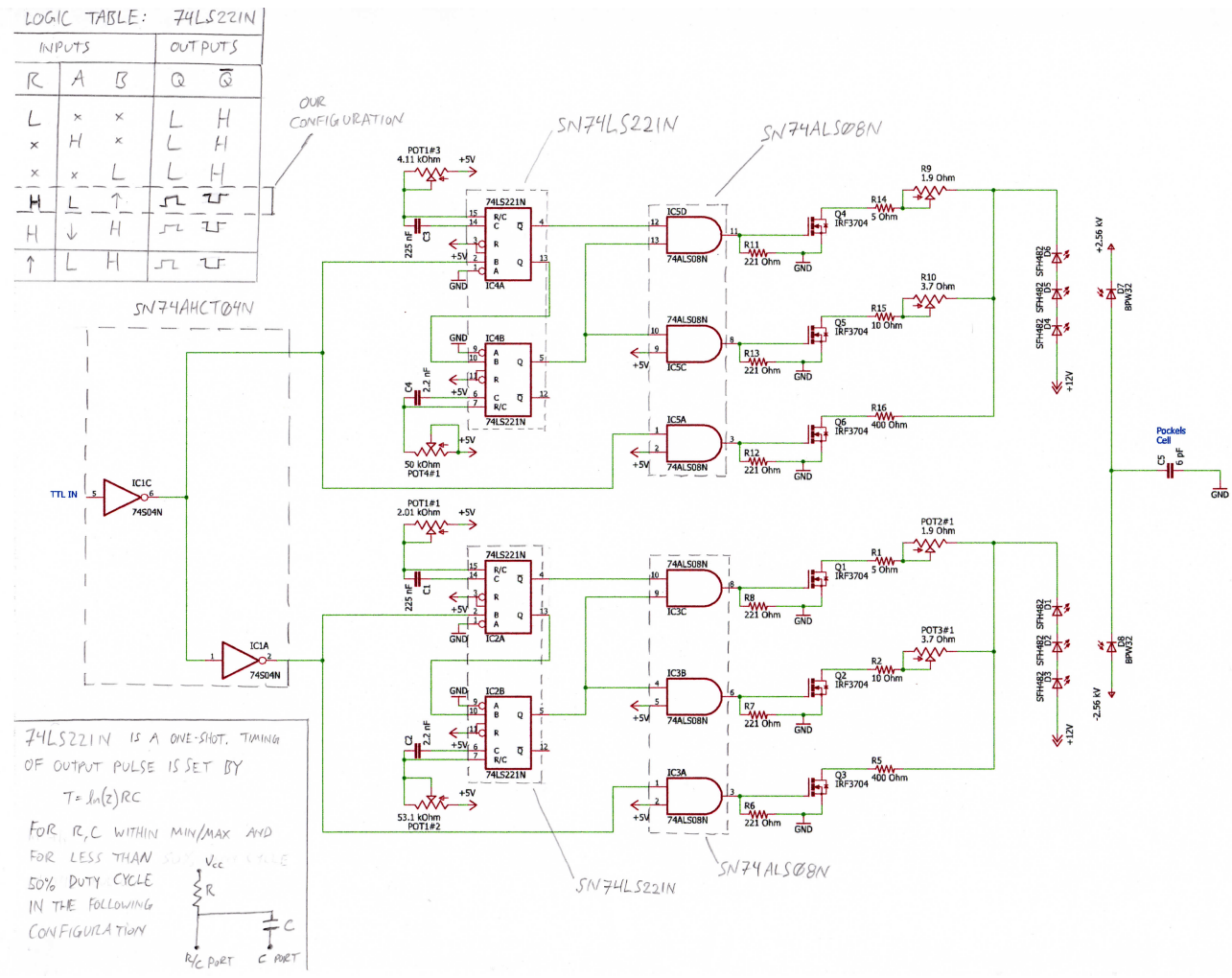

Figure 6-24: Annotated Pockels Cell Opto-driver Circuit Diagram [284]

The 8-output HV driver consists of 4 of the circuits shown in Fig. 6-24, allowing for individual adjustment of the driver settings for each of the 8 HV's. The reason for this control of individual voltages is that the voltage settings of the $8 \mathrm{HV}$ system can affect the transition time ithrough weighting different optocouplers and LED's. As different voltages are increased, their optodriver/LED's become the greater determiner of the transition times and they can be weighted more heavily. The placement of the LED's near the optodiodes as well as the age of the optodiode can vary the transition times, so we made a flexible 8 output driver to compensate for any differences in the various LED's/optodiodes. The potentiometer settings are adjusted for each of the 8 switches to match to the same transition time for each of the $8 \mathrm{HV}$ 's individually, compensating for differences in optocoupler/LEDs. 


\subsubsection{Considerations for Fast Switching}

The predominant reason behind trying to use an RTP Pockels Cell is for the sake of fast switching. The RTP Pockels cell scheme will allow for faster helicity switching during MOLLER. Due to slow switching and piezo-electric ringing in $\mathrm{KD}^{*} \mathrm{P}$, using the JLab KD*P cell for MOLLER could result in $20 \%$ loss of data. At $2 \mathrm{kHz}$ helicity switching, there is $100 \mu \mathrm{s}$ of deadtime in $\mathrm{KD}^{*} \mathrm{P}$ some of which is due to ringing at $4 \%$ amplitude as shown in Fig 6-25. However in RTP, which has low piezo-electric coefficients, there is virtually no ringing $(<0.2 \%$ amplitude) when the transition time is reduced to $12 \mu \mathrm{s}$ in a commercial $4 \mathrm{x} 4 \mathrm{~mm}$ RTP cell as shown in Fig. 6-25 on the right. Switching from KD*P to RTP reduces the deadtime by $10 \mathrm{X}$.

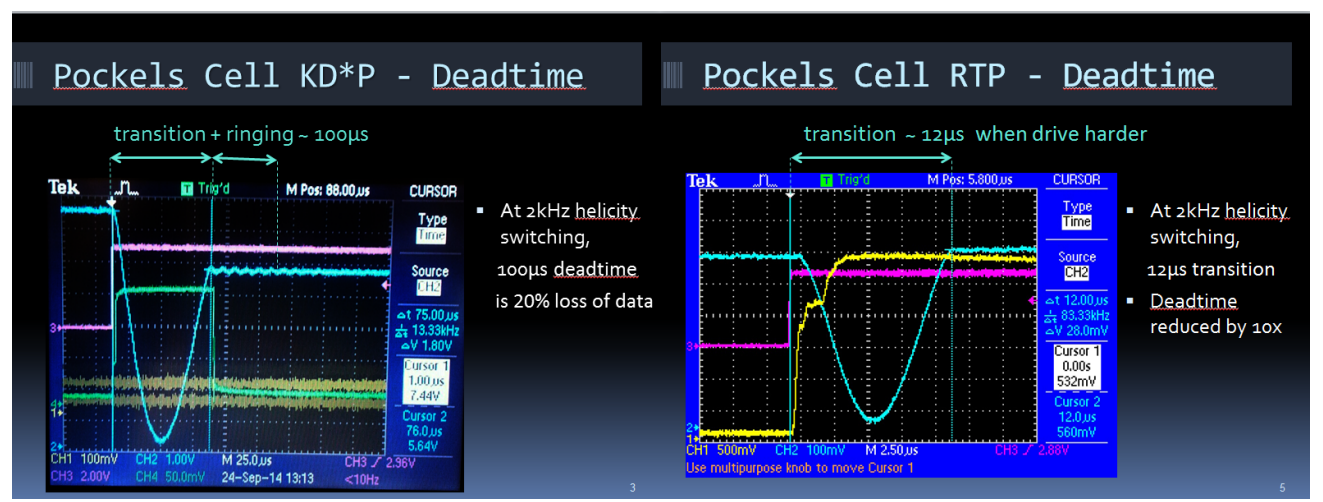

Figure 6-25: Transition Time: These measurements were performed by driving the Pockels Cells near QWV and examining the transmission through an analyzer oriented along $\mathrm{S} 1$, the birefringence axis. $\mathrm{KD}^{*} \mathrm{P}$ ringing has $10 \mu$ s period. Left: blue trace is transmission through $\mathrm{KD}^{*} \mathrm{P}$ cell, pink trace is helicity trigger, green trace is the LED pulse detected by a photodiode. Right: blue trace is transmission through RTP cell, pink trace is helicity trigger, yellow trace is the LED pulse detected by a photodiode.

The transition time is determined by many factors including the capacitance of the system, the QWV of the cell, the current limit through the optocoupler, and the piezoelectric resonances. The current limit dictates that the transition time is proportional to $t=\beta C V$ where $\beta$ is determined by $I_{\text {lim }}$. Systems with larger a QWV, a higher capacitive load, or a lower current limit take longer to 
transition, placing an upper bound on the transition time. Piezo-electric ringing can be driven by very fast transitions, so this places a lower bound on how fast we can drive the Pockels cell. Fig. 6-26 shows our 12x12x10mm RTP crystals being driven at QWV within $12 \mu s$ (with $<1 \%$ ringing).

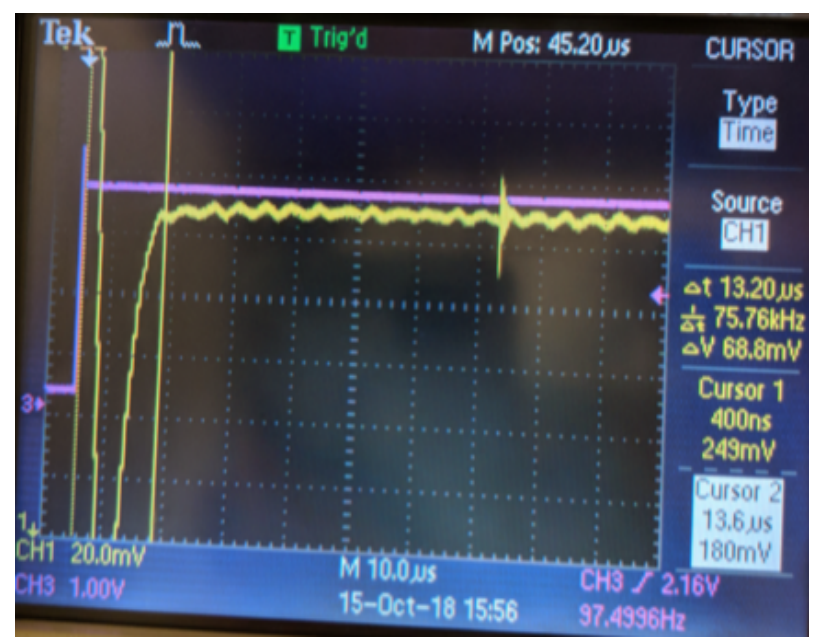

Figure 6-26: $13 \mu$ s transition time with $<1 \%$ ringing [290]

If a larger RTP crystal is used, or larger plate electrodes are used, the capacitance of the RTP cells ( $2-3 \mathrm{pF}$ ) does not change when the crystal aperture is increased so long as the plate geometry remains constant. Making the plates oversized increases the capacitance only slightly since the dielectric constant of RTP is quite large $\left(13 \epsilon_{0}\right)$. The main change in transition time comes from an increase in the necessary QWV for larger crystals. The QWV scales with the aperture size, so doubling the aperture width doubles the QWV which doubles the transition time. This scaling of transition time with aperture size should be considered when using a larger RTP cell. Lengthening the RTP crystals reduces the QWV, but increases the cell capacitance.

The fact that our Pockels cell design has grounded side panels, and isn't just 2 plates on either side of a crystal, but rather 4 plates, changes the capacitance of the system. The side-panels used in our RTP design for steering change the effective capacitance of the system for different voltage settings. Adding side panels to the 
RTP mount (for steering control with voltage) changes transition time a little due to need to accumulate a different amount of charge on the plates to achieve the same voltage. Changing the voltage applied to the plates relative to the grounded side-panels (shifting ground) also changes the transition time a little for the same reason. The expected transition time can change by 10-30\% [302], across the full range of possible HV settings. For MOLLER, it is important to be aware that the side panels do affect the transition times for different $V_{\alpha p o s, U / V}$ settings, and that changing $V_{\alpha p o s, U / V}$ significantly can affect the transition times a little bit. When using the opto-driver, the transition time is limited in RTP by the current limit through the optocoupler driving the transition and the capacitance of the Pockels cell. This current limit can always be improved by using more optocouplers, turning up the voltage on the LED's, aligning the optocouplers with the LED's better, or replacing old optocouplers with fresh ones. Longer transition times can always be reduced increasing the number of optocouplers used in parallel or by using a different driver. Longevity for the opto-driver remains an issue and it is probably desirable to design a solid-state switch instead.

John Hansknecht ${ }^{29}$ built a solid-state driver for the RTP cell based on the driver circuit for the RTP tune-mode generators on the JLab injector table [291]. The limitation in the solid state driver the piezo-electric ringing in RTP. RTP has a reputation for having little to no ringing, but KTP is a similar crystal to RTP, and its piezo-electric resonance is simply at a higher frequency for a given geometry than for most other Pockels cell materials [288]. Ringing has been observed in these $12 \times 12 \times 10 \mathrm{~mm}$ RTP crystals when driven in under $7-8 \mu \mathrm{s}$, and significant ringing has been observed when using the solid-state driver at maximal current as shown in Fig. 6-27. Fig. 6-27a shows an impulse response measurement in the frequency domain revealing piezo-electric resonances of the X-cut RTP Pockels cell with $12 \times 12 \times 10 \mathrm{~mm}$ crystals. The lowest frequency resonance is at $200-250 \mathrm{kHz}$, corresponding to a period of $\sim 4-5 \mu s$. The next high amplitude resonance is at

\footnotetext{
${ }^{29}$ hansknec@jlab.org
} 
$350 \mathrm{kHz}$, corresponding to a period of $3 \mu \mathrm{s}$. [286]. The resonances depends on the crystal geometry, but generally larger crystals leads to lower excitation frequencies which can be more easily excited by short transition times.

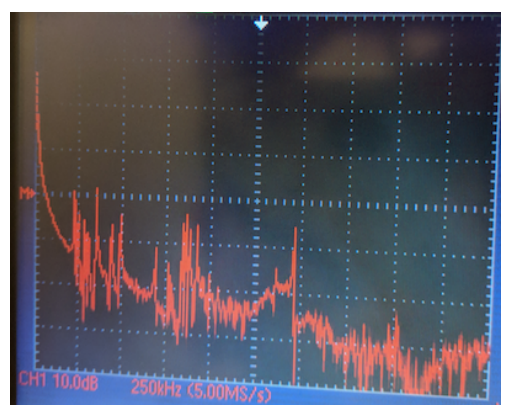

(a)

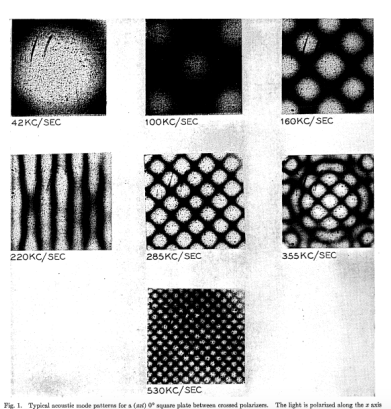

(b)

Figure 6-27: (a) Impulse response measurement in frequency domain showing piezo-electric resonances of the X-cut RTP Pockels cell with $12 \times 12 \times 10 \mathrm{~mm}$ crystals. The lowest frequency resonance is at $200-250 \mathrm{kHz}$, corresponding to a period of $\sim$ $4-5 \mu \mathrm{s}$. The next high amplitude resonance is at $350 \mathrm{kHz}$, corresponding to a period of $3 \mu \mathrm{s}$. [286] (b) Acoustic Resonances in $\mathrm{KD}^{*} \mathrm{P}$ [289]. Shown for visualization purposes.

The generic rule of thumb relating the bandwidth of signal to its $90 \%$ rise time is given as $B W \times t_{90 \%}=0.35$ [292]. In a similar fashion, we derived a rule of thumb for transition time to maintain small ringing amplitude for a given resonance frequency. Modeling the impulse to the crystal as gaussian, which integrates to a soft square wave with a well defined rise-time t(10-90\%), the gaussian impulse was Fourier transformed, which produces a gaussian in frequency domain which falls off from its $\mathrm{DC} 0 \mathrm{~Hz}$ value. We define suppression to mean a factor of $1 / 100 \mathrm{X}$ in amplitude at the resonance frequency $\mathrm{f}$ compared with the $\mathrm{DC} 0 \mathrm{~Hz}$ amplitude in the F.T. curve. This should correspond to $1 \%$ ringing. We found that the rule of thumb to maintain $<1 \%$ ringing was [293]

$$
\begin{gathered}
f_{1 \% \text { amplitude }} \times t_{10-90 \%}=2 \\
t_{\text {measured }}<2 T / 0.8
\end{gathered}
$$


where $\mathrm{f}=1 / \mathrm{T}$ is the resonance frequency, $\mathrm{T}$ is the resonance period, $t_{10-90 \%}$ is the 10-90\% transition time. Empirically, we measure the full transition time in the lab so $t_{10-90 \%} \sim 0.8 t_{\text {measured }}$. For the RTP resonance observed $T=4-5 \mu s$, the transition time $t_{\text {measured }}>10-12.5 \mu \mathrm{s}$ to maintain $<1 \%$ ringing. For faster transition times such a $4 \mu s$, we observed greater ringing of $5-10 \%$ as shown in Fig. 6-28. Ringing even up to $10-15 \%$ was observed when using the solid state driver without resistors to soften the transition as shown in Fig. 6-29.

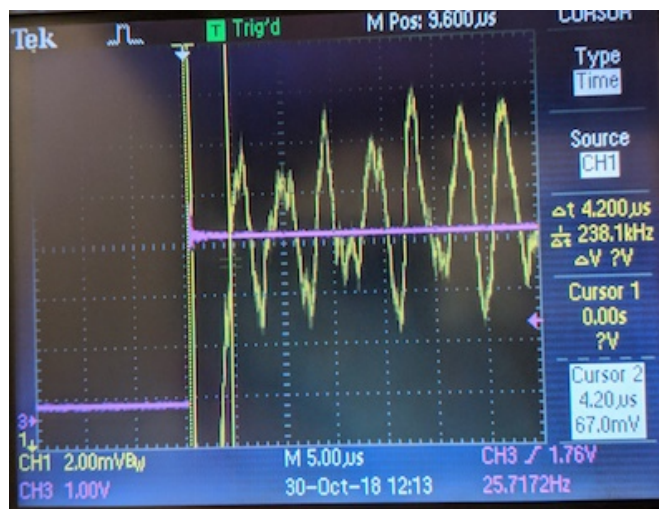

(a)

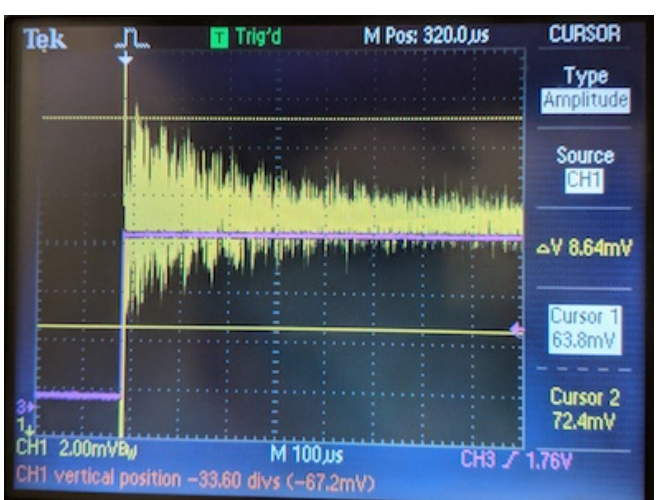

(b)

Figure 6-28: Solid state switch with 50kOhm resistors on the HV lines to soften the transition down to $4 \mu \mathrm{s}$. Shows $8.6 \%$ ringing. [286]

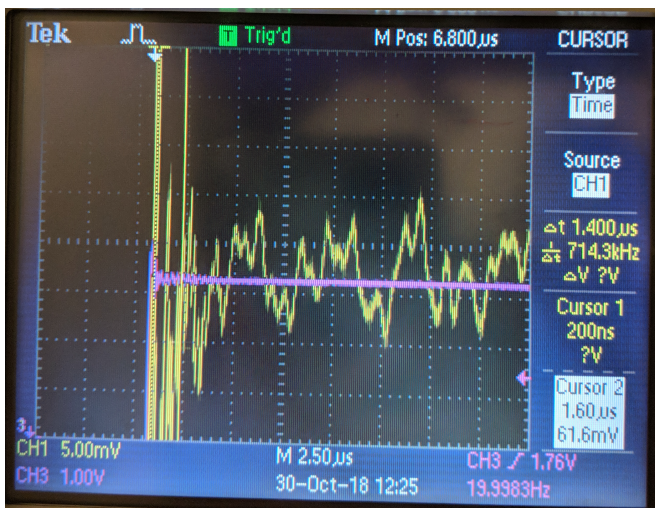

(a)

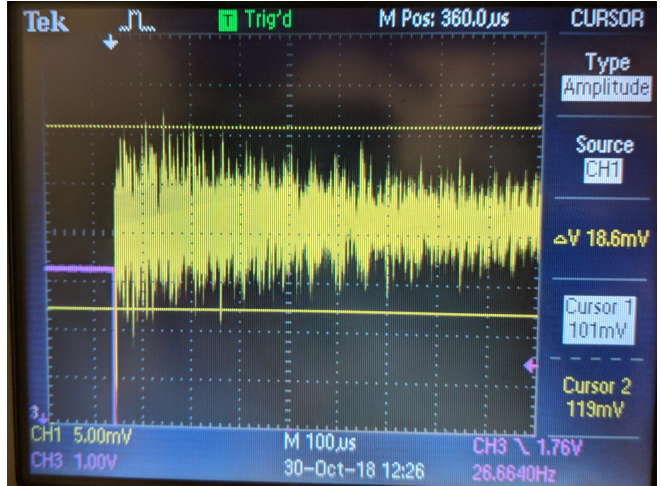

(b)

Figure 6-29: Solid state switch with no resistors. Shows $10 \%$ ringing for this $<2 \mu \mathrm{s}$ transition time . [287] 


\subsubsection{PC-history effect/4-peak effect}

A Pockels cell crystal functions mostly as a capacitor, but it has a slight conductivity, and therefore also functions slightly as a resistor. A capacitor doesn't have a uniform electric field, it suffers from edge effects, but the electric field inside a resistor is uniform. [295] It obeys Laplace's equation. For steady currents and uniform conductivity $\nabla \cdot E=\frac{1}{\sigma} \nabla \cdot J=0$, the charge density is zero and any unbalanced charge resides on the surface. Laplace's equation holds within a homogeneous ohmic material carrying a steady current. The boundary conditions are the potentials at the $\mathrm{HV}$ plates is constant on the surface $J \cdot \hat{n}=0$, else the charge would leak into the surrounding space, therefore $E \cdot \hat{n}=0$ and $\partial V / \partial n=0$. The solution to Laplace's equation is $V(z)=V_{0} z / d$ with uniform electric field $E=-\nabla V=-V_{0} / d \hat{z}$.

A Pockels cell crystal, upon switching states, starts off functioning as a capacitor before current starts to flow, but over time ( $\mu$ s-ms scale) it starts to function more like a resistor. Initially, the electric field takes the form it would take if the crystal were only a capacitor, with no leakage current flowing. But as current flow sets in, the crystal becomes more resistor-like, and the electric field changes, becoming more uniform over time. The fringe fields straighten and the electric field becomes increasingly uniform over the helicity window. It is intuitive to think of the Pockels cell as an RC circuit with its own intrinsic characteristic time constant ${ }^{30}$, determined by is very small $\mathrm{pF}$-level capacitance, and very large $T \Omega$-level resistance. The resistance of each of our crystals was estimated by rough measurement to be $730 G \Omega$ [296] and capacitance $3.3 p F$ [297], so the RC leakage current time constant is interpreted as $\tau=R C=2.4 \mathrm{sec}$. The multi-peak structure characteristic of the Pockels Cell history effect (observed at JLab in previous years) is due to this intrinsic settling time from the conductivity of the crystal..

\footnotetext{
${ }^{30}$ Lisa Kaufmann noted in her thesis [298] "Early in our studies we understood that there was a time constant associated with the PC "remembering" the helicity from the window immediately preceding a pair, but further investigation revealed that the PC retardation remembers the helicity over a long time period."
} 
The PC-history effect strongly depends on how non-uniform the electric field is initially. In order for conductivity to matter, there has to be a transverse component to the field lines near the edge of the crystal initially. The greater the edge effects, the more impact conductivity has. The conductivity will tend to cause accumulation of surface charge to straighten the field lines. If the field is initially quite uniform and if the field lines are already straight, the conductivity doesn't affect it significantly. The greater the field distortion initially, the larger impact the resistivity will have because ultimately that means more surface charge accumulated to straighten out the field.

Fig. 6-30a shows the asymmetry $A_{q}$ for each helicity pattern, where the preceding helicity pair is annotated. We observe the cell has a 'memory' of previous helicity windows, the Aq depends on the history of the helicity pattern preceding. The multi-peak structure is dependent on the helicity sequence pattern, and the separation between peaks is dependent on the crystal resistivity, the initial fringe fields determined by the cell geometry, and the helicity flip rates. Figure 6-30a shows the multipeak structure for three possible helicity sequence patterns [298]: (1) pair-toggle(RLRLRL) which produces only one peak all asymmetry pairs have the same history, (2) quad-random helicity pattern (random sequences of RLLR or LRRL) which provides four distinct history possibilities (R-RL, L-LR, L-RL, or R-LR) with the R-RL and L-LR switches having no voltage change before the first window of the pair and occurring with greater frequency and (3) pair-random (blue, RL or LR) which has the same four history possibilities as quad-random, but with none having greater frequency than others.

We have modeled the PC-history effect in 2D (using Quickfield). As input parameters, we measured the resistance of the KD*P and RTP Pockels cells [299] and inferred a bulk conductivity for the crystals: $\sigma_{K D * P}=2.6259 * 10^{-9} \mathrm{~S} / \mathrm{m} \pm 3.2 \%$ and $\sigma_{R T P}<=6.06 * 10^{-11} \mathrm{~S} / \mathrm{m}$ (with the assumption that the majority of the conductance was in the crystal volume, not on the surface). The $2 \mathrm{D}$ model for $\mathrm{KD}^{*} \mathrm{P}$ does surprisingly well at predicting the behavior of the $\mathrm{PC}$-history effect as 
shown in Fig. 6-31.

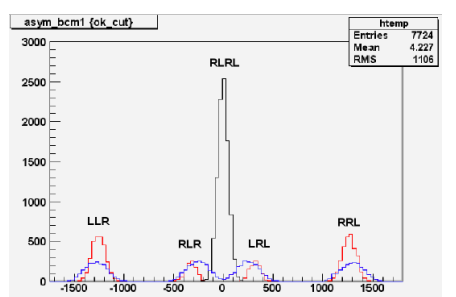

(a)

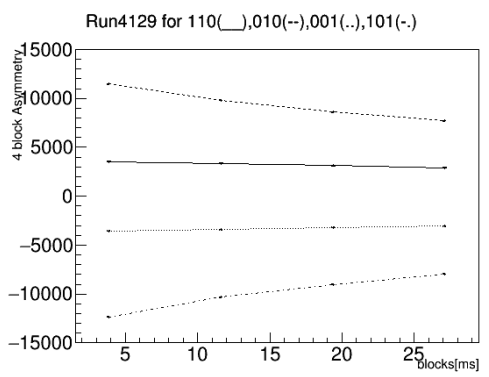

(c)

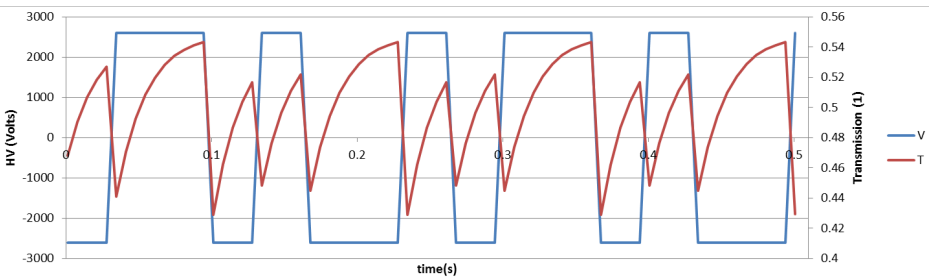

(b)

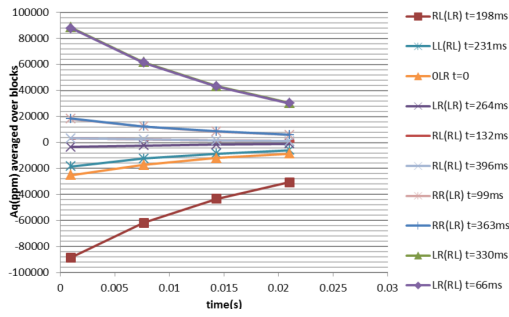

(d)

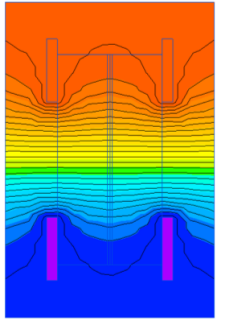

(e)

Figure 6-30: KD*P PC-history effect: (a) The multi-peak structure of AQ is shown for the quad-random (red), pair random (blue), and pair-toggle (black) helicity patterns. The asymmetry is plotted in units of ppm. [298] (b) 2D model of transmission in S1 vs. time for quad-random pattern at 30Hz [300] (c) measured Aq for pair-random helicity pattern at $30 \mathrm{~Hz}$ [300] (d) 2D model of Aq over subblocks of a helicity window at $30 \mathrm{~Hz}$ [300] (e) geometry used in 2D model [300]

We have also measured and modeled the PC-history effect in RTP. While the 2D model (with the assumption of no surface currents) doesn't predict the amplitude of the decay during a window very well, (Fig. 6-31 0.03\%predicted instead of $0.3 \%$ ), it does predict the asymmetry distribution quite well as shown in Fig. 6-32. 


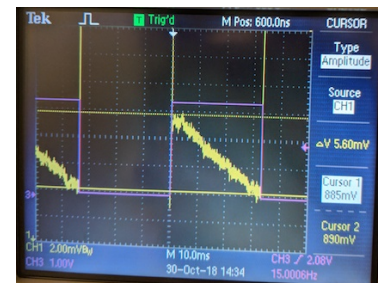

(a)

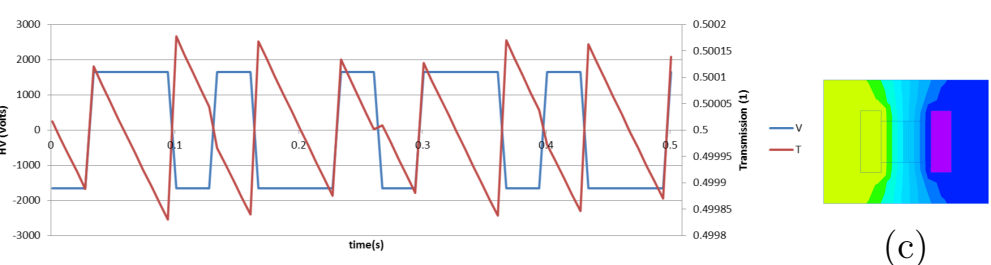

(b)

Figure 6-31: RTP PC-history effect: (a) Measurement of transmission in S1 vs. time for pair-random pattern observed $0.3 \%$ over $30 \mathrm{~Hz}$ window [285] (b) 2D model of transmission in S1 vs. time for quad-random pattern calculated $0.03 \%$ over $30 \mathrm{~Hz}$ windows for conductivity $R T P=6.06 * 10^{-11} \mathrm{~S} / \mathrm{m}$ [300] (b) RTP model geometry [300]

We observe that for higher helicity flip rates, the PC-history effect affects the asymmetry profile less significantly. The PC-history effect is more extreme for longer helicity windows and lower flip rates; at higher flip rates, matters equilibrate. This is predicted in our model as shown for RTP comparing $30 \mathrm{~Hz}$ with $120 \mathrm{~Hz}$ in Fig. 6-32 as well as by observation in $\mathrm{KD}^{*} \mathrm{P}$ [300]. For $2 \mathrm{kHz}$ flip rates in MOLLER, the four-peak effect in RTP will be greatly diminished, and certainly less significant that it would be for $\mathrm{KD}^{*} \mathrm{P}$.

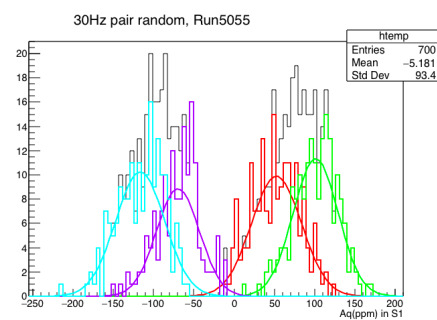

(a)

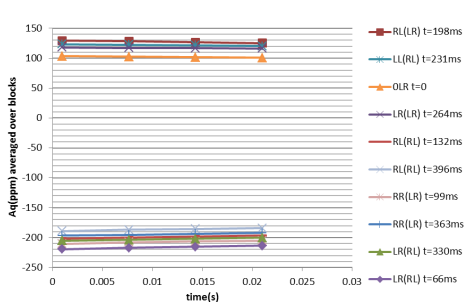

(b)

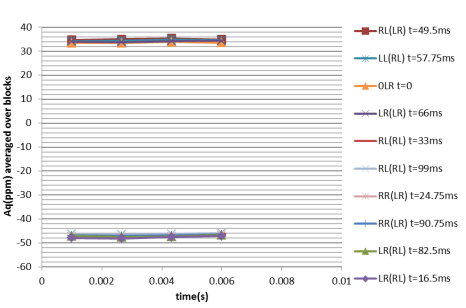

(c)

Figure 6-32: RTP 4-peak effect: (a) measured Aq for pair-random helicity pattern at $30 \mathrm{~Hz}$ [301] (b) 2D model of Aq over sub-blocks of a helicity window at $30 \mathrm{~Hz}$ [300] (c) 2D model of Aq over sub-blocks of a helicity window at $120 \mathrm{~Hz}$ [300]

\subsubsection{Lifetime - Mode of Failure}

JLab observed crystal degradation in commercial cell (with metal cement) which had the symptom of decreased responsiveness to voltage, ultimately failure 
to hold voltage, and crystal graying and transmission reduction as shown in Fig. 6-33. This occurred when DC voltage was (on average, periodically) applied for a long time. A possible explanation is ion migration from silver oxide across the crystal face [294] from the silver cement. Our cell has no cement, and an improvement to the cell design could be using gold sputter coating for the electric plates on the crystal.

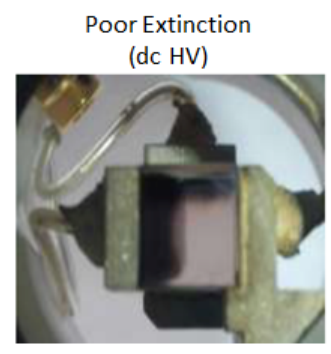

Figure 6-33: Tune Mode Generators Degradation: Fast RTP electro-optics crystals used to shutter beam (Pulsed Mode). A simpler design was implemented in $\mathrm{Su} 2016$ where DC voltage was applied all the time. Contrary to vendor claim, the RTP cell suffer from ion migration from electrode to RTP. (from talk by Joe Grames, photo from John Hansknecht)

Mostly of the degradation we observe is due to the driver - not the crystals. Over several months time using the optodriver, the transitions aren't as sharp, despite driving as hard as possible with this setup. This is likely due to dragon LED's dying and emitting less light and/or the optocouplers degrading and responding less well to light (they become brownish in appearance after some time), so the transition slows down. Driver degradation mechanisms observed include: LED reduction in brightness, optodiode reduced responsiveness to light (and brownish discoloration), mosfet breaking, resistor burn-out in RC buffer for $\mathrm{HV}$ power supplies [286]. 


\subsection{Characterization}

The RTP cell was fully characterized as regards the intensity asymmetry, position differences, and spot-size asymmetry dependence on position, angle, roll and voltage.

Polarized light was incident on the RTP Pockels cell, which was set to flip between \pm QWV, alternating helicity states, and the transmitted light was detected downstream of the Pockels cell (as in Fig. 6-4). A quad-photodiode (Fig. 6-34) detected the transmission and beam position for each helicity state, our data acquisition system (DAQ) integrated over each helicity window, and our data analysis code formed an asymmetry (or pair difference) between the signals for right and left helicity states. Measured parameters were formed by taking difference between successive states of opposite helicity. The intensity asymmetry $A_{I}$, position difference in x $D_{x}$, and position difference in y $D_{y}$, were defined as:

$$
A_{I}=\frac{I^{R}-I^{L}}{I^{R}+I^{L}} \quad D_{x}=x^{R}-x^{L} \quad D_{y}=y^{R}-y^{L}
$$

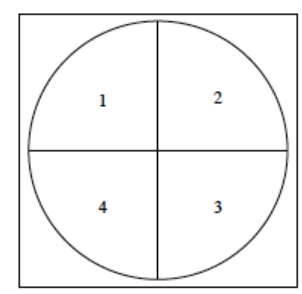

Figure 6-34: Quad-photodiode layout [259]

The intensity of the transmitted beam is proportional to the sum of the photodiode pad signals and the horizontal and vertical positions of the beam are computed through taking differences between the pad signals as follows:

$$
\begin{gathered}
I_{\text {sum }}=I_{1}+I_{2}+I_{3}+I_{4} \\
x=(x-\text { const }) \frac{\left(I_{2}+I_{3}\right)-\left(I_{1}+I_{4}\right)}{I_{1}+I_{2}+I_{3}+I_{4}} ; \quad y=(y-\text { const }) \frac{\left(I_{1}+I_{2}\right)-\left(I_{3}+I_{4}\right)}{I_{1}+I_{2}+I_{3}+I_{4}}
\end{gathered}
$$


where $I_{1}, I_{2}, I_{3}$ and $I_{4}$ are the responses of the pads $1,2,3$ and 4 respectively to the incident light and $\mathrm{x}$-const, $\mathrm{y}$-const are the calibrated proportionality factors. The calibration procedure [259] determined the relative gain and pedestal(accounting for non-linearity in response) for each pad: $I_{i}=\operatorname{gain}_{i} *\left(S_{i}-\right.$ $\left.\operatorname{ped}_{i}\right)$.

Multiple lasers were used in the Pockels cell characterization, as described in subsequent sections. These laser table studies were done at several helicity flip rates including $30 \mathrm{~Hz}$, with $\sim 33 \mathrm{~ms}$ integration windows, and at $240 \mathrm{~Hz}$, with $\sim 4 m s$ integration windows. To minimize $60 \mathrm{~Hz}$ noise, the helicity flip rate was line synced to $60 \mathrm{~Hz}$ and quartet patterns +--+ (or octet patterns +--+-++- ) were used to cancel out $60 \mathrm{~Hz}$ noise [263].

\subsubsection{Alignment Procedure}

When aligning the cell, we first check that the laser beam at the cell is has waist $2 \sigma \sim 1 \mathrm{~mm}$, and the divergence is $<1 \mathrm{mrad}$, and we check that the effective throw distance to the cathode (accounting for the steering lens) is $>50 \mathrm{~cm}$ and the spot size on the spiricon-cathode is $4 \sigma<3 \mathrm{~mm}$ (preferably $1.7 \mathrm{~mm}$, but not smaller because cathode degrades more quickly). We roughly center the Pockels cell aperture on the laser beam using a lens cleaning tissue in front of the cell to disperse the light and examine transmitted beam on an IR viewer. We align the back-reflections $50 \mathrm{~cm}$ to $1 \mathrm{~m}$ upstream of the cell so they overlap the incoming beam as closely as possible. We use a spinning linear polarizer to approximately minimize the degree of linear polarization for both helicity states (to within 5\%), using PITA voltage, overall roll, and relative roll. More precisely, with a GlanTaylor polarizer, we minimize Aq in S1 with PITA voltage then minimize Aq in $\mathrm{S} 2$ with relative roll ${ }^{31}$. We zero out the steering effects (measured with qpd) with

\footnotetext{
${ }^{31}$ We can more precisely set the relative roll using the RHWP (or an analyzer with fine angle control) by finding the RHWP angle at which the PITA slope for RTP\#1 ( PIT $A_{1}$ or $V_{\Delta, 1}$ ) is minimum, then finding the RHWP angle at the PITA slope for RTP\#2 $\left(P I T A_{2}\right.$ or $\left.V_{\Delta, 2}\right)$ is minimum, then rotating the relative roll to make the minima occur at the same RHWP angle
} 
no analyzer using alpha-phase gradients. Finally, we attempt to reduce the birefringence gradient effects in S1, the analyzing-like position differences (measured with qpd), using the Pockels cell pitch and yaw angle ${ }^{32}$. With electron beam, at RHWP angle set to S1, we minimize Aq in S1 with PITA voltage. Then, choosing an RHWP angle near S2 (i.e. where PITA slope is small), we examine $\mathrm{Aq}^{33}$ and perform Aq PITA-voltage feedback if possible, otherwise setting the PITA voltages to approximately minimize Aq. We set alpha-position- $\mathrm{U}$ and alpha-position- $\mathrm{V}$ to reduce/minimize position differences in the injector either manually or using a position difference feedback mechanism. Examine the position differences further down in the injector beam-line, past the chopper, into the $5 \mathrm{MeV}$ region, making sure the position differences remain small and there is no significant clipping on apertures.

\subsubsection{Translation scan}

The Pockels cell was mounted on motorized stages which allowed for horizontal and vertical position control. The cell sensitivity to position was measured by translation the cell with the Thorlabs stages. A LD785-SED30 diode CW laser was used $\left(18.10^{\circ} \mathrm{C}, 180 \mathrm{~mA}, 75 \mathrm{~mW}\right)$ and sent through a single mode fiber and GlanTaylor polarizer, producing $4.3 \mathrm{~mW}$ of horizontally polarized $785 \mathrm{~nm}$ light. The 785nm horizontally polarized light was incident on the RTP Pockels cell, which was set to flip between \pm QWV , and the transmitted light analyzed with a vertical polarizer downstream of the Pockels cell (as in Fig. 6-4), and detected on a quadphotodiode. The cell transverse position was scanned over an $\mathrm{X} / \mathrm{Y}$ grid pattern and the intensity asymmetry dependence on cell position was measured.

The translation scan, Fig. 6-35a, shows the intensity asymmetry $A_{I}$ (in S1)

\footnotetext{
${ }^{32}$ This is because in $\mathrm{S} 1$, we are measuring position differences both from Aq gradients and steering, so we'd need the steering to be near zeroed out first, to know we are actually zeroing out Aq gradients.

${ }^{33}$ If the Aq is very large in S2, even when it is minimized in S1, such that very large PITA-V'S would have to be used to correct it, instead use relative roll (remotely) to minimize Aq in S2 on e-beam
} 
as a function of Pockels cell transverse position in the $\mathrm{X} / \mathrm{Y}$ place perpendicular to the beam propagation axis. This measurement of $A_{I}$ with respect to position shows the birefringence gradient inherent in the system. Empirically we observed an asymmetry gradient along $v \equiv \frac{x+y}{\sqrt{2}}$ due to both crystals combined of $\frac{d A_{q}}{d v} \sim$ $20,000-50,000 \mathrm{ppm} / \mathrm{mm}$, which in theory, for $w=1 \mathrm{~mm}$, gives position differences of magnitude:

$$
\begin{gathered}
D_{v}=D_{y 1}+D_{z 2}=\frac{\frac{d A_{q}}{d v} w^{2}}{2}=10-25 \mu \mathrm{m} \\
D_{x} \sim D_{y} \sim D_{v} / \sqrt{2}=7.1-17.7 \mu \mathrm{m}
\end{gathered}
$$

The parallelism of the crystals' face cuts, the electric field non-uniformity, the variation in intrinsic refractive index along the crystals growth axis, stress gradients in the crystal, all affect the birefringence gradient. This translation scan motivated the new cell design: because these gradients are intrinsic to the RTP system, and give rise to analyzing-like position differences which cannot be minimized by translational alignment, it became necessary redesign the cell to zero out position differences using voltage induced steering.

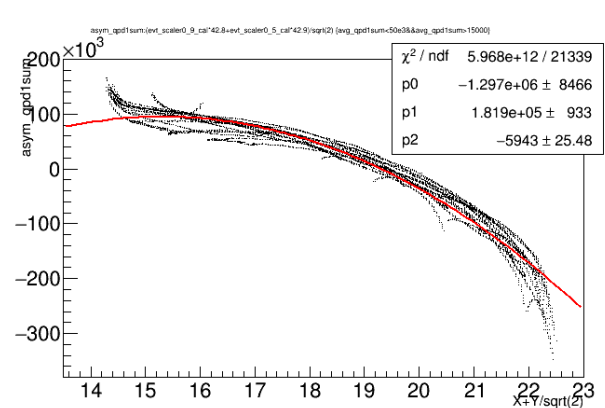

(a) Run 4340 [232]

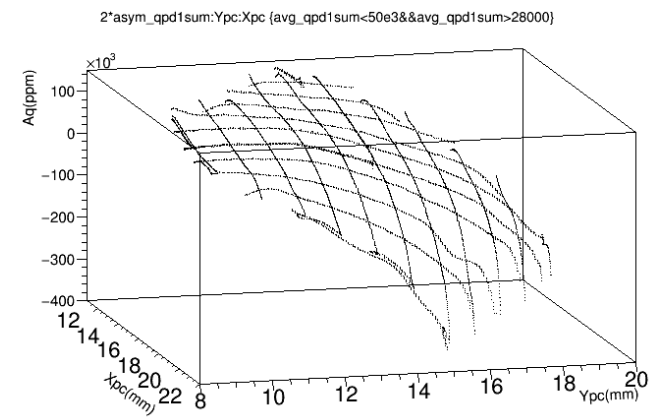

(b) Run 4340 (approximate reproduction in 3D) [232]

Figure 6-35: Translation scan

We note that in an RTP cell system, it is very challenging to obtain a flat asymmetry across the cell face, especially since the crystal is so highly bire- 
fringent $(\delta n \sim 0.1)$, and there is an intrinsic refractive index gradient along the z-axis growth direction. Even a slight non-parallelism in face cuts (a slight length gradient) can lead to a significant gradient in the asymmetry. We have also measured face cut parallelism of each crystal by examining the back reflections of the front and back crystal faces, and obtained for the 1st crystal $\theta_{f c, z 1}=\frac{d L}{d z_{1}}=-0.079 \pm 0.01 \mathrm{mrad}, \theta_{f c, y 1}=\frac{d L}{d y_{1}}=0.024 \pm 0.01 \mathrm{mrad}$, and for the 2nd crystal $\theta_{f c, z 2}=\frac{d L}{d z_{2}}=-0.055 \pm 0.01 \mathrm{mrad}, \theta_{f c, y 2}=\frac{d L}{d y_{2}}=0.015 \pm 0.01 \mathrm{mrad}$ [264]. The bound on the parallelism $\theta_{f c}=\frac{d L}{d x_{i}} \approx 0.01-0.1 \mathrm{mrad}$ in these RTP crystals indicates for a $1 \mathrm{~mm}$ beam, the induces position differences would be

$$
D_{\partial L, x_{i}}=-\frac{w^{2} \pi}{2 \lambda}\left(n_{0, y}-n_{0, z}\right) \theta_{f c} \approx 1.7-17 u m
$$

The results indicate the major contribution to the birefringence gradient comes more from the intrinsic refractive index gradient, than from face cut non-parallelism.

\subsubsection{Angle scans}

The cell sensitivity to angle was measured by using a Newport 9071 Four-Axis Tilt Aligner, to control the pitch and yaw of the Pockels cell at the mrad-level ${ }^{34}$. For this measurement, performed at UVa, a picosecond pulsed laser (Edinburgh Instruments EPL-785) was used, with central wavelength 783.0nm, 66.5ps pulse width, $3.4 \mathrm{~nm}$ bandwidth, and $20 \mathrm{MHz}$ pulse repetition rate. The laser was coupled through a single mode fiber the output of which was $\sim 10.3 \mu \mathrm{W}$, refocused to have $\sim 1 \mathrm{mrad}$ divergence, and cleanup with a Glan-Tayler polarized which transmitted $\sim 6 \mu W$ of horizontally polarized light to the cell. The helicity flip rate for this measurement was $240 \mathrm{~Hz}$, with randomized helicity pair pattern. The light transmitted through the Pockels cell was analyzed with a vertical polarizer (S1),

\footnotetext{
${ }^{34}$ In the past, in our group's work, it was assumed this mount has the same pitch and yaw sensitivity in terms of mrads/turn. This is not the case. Additionally there was a set screw left in which was supposed to be removed, but which remained in previously. For these reasons, previous year's measurements of angle sensitivity should be called into question.
} 
and detected on a fast photodiode. The cell angle scanned over several pitch/yaw positions in $\mathrm{a} \sim 8 x 8 \mathrm{mrad}^{2}$ grid. The yaw control on this mount is $8 \mathrm{mrad} /$ turn and pitch control is $4.6 \mathrm{mrad} / \mathrm{turn}$. The intensity asymmetry $A_{I}$ dependence on cell angle was measured and is shown is Fig. 6-36. A saddle function was fit to the data of functional form $A_{I}=k\left(\right.$ pitch $\left.-p_{0}\right)\left(y a w-y_{0}\right)+A_{0}$ and the measured angle sensitivity was obtained: $k^{\text {meas }}=4527 \pm 942 \mathrm{ppm} / \mathrm{mrad}^{2}$ with $20.8 \%$ error . The dominant errors are from angle measurement calibration and Aq measurement calibration. The predicted angle sensitivity is $k=5137 \mathrm{ppm} / \mathrm{mrad}^{2}$ (Sec. 6.3.1) which is consistent with the measured sensitivity.

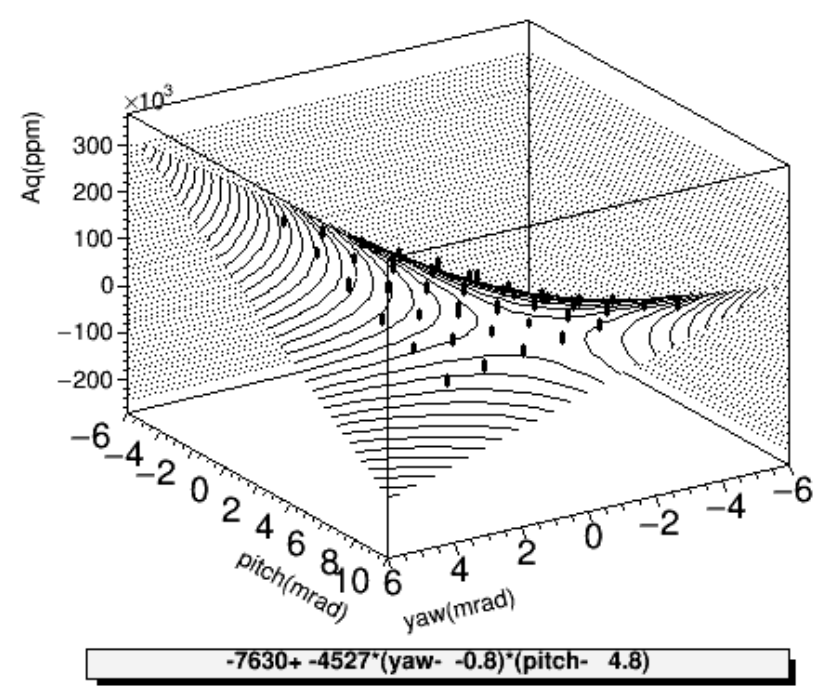

Figure 6-36: Run5202 RTP analyzer S1 pitch-yaw scan [244]

An additional study on the angle dependence of analyzing-like position differences was performed with the qpd detector. This measurement is, in theory (Sec. 6.3.3), very dependent on the laser beam size and divergence at the cell. The beam conditions were measured to be [248] $\theta_{w x}=1.40+-0.05 \mathrm{mrad}$, $\theta_{w y}=1.30+-0.05 \mathrm{mrad}, w_{P C x}=1.29+-0.03 \mathrm{~mm}, w_{P C y}=1.45+-0.11 \mathrm{~mm}$, where $w=2 \sigma$ and $\theta_{w}=\frac{d w}{d z}$. These measured beam parameters imply a prediction on the angle dependence of $\left|D_{x}\right|=(4.6+-0.2 u m / m r a d) p i t c h,\left|D_{y}\right|=$ $(4.8+-0.4 u m / m r a d) y a w$ (see Sec. 6.3.3). The results of scanning pitch and yaw 
are show in Fig. 6-37. As predicted based on the saddle function in $A_{I}$, yaw couples predominantly to the position difference in $\mathrm{x}$ and pitch couples predominantly to the position difference in $\mathrm{y}$. The angle dependence was measured to be $D_{x}=(-5.36 \pm 0.91 \mu \mathrm{m} / \mathrm{mrad})$ pitch,$D_{y}=(-7.37 \pm 1.25 \mu \mathrm{m} / \mathrm{mrad})$ yaw, are from angle measurement calibration ( $8 \%$ on the scale factor of $\mathrm{mrad} / \mathrm{turn}$ [244]) and qpd measurement calibration $\left(15 \%{ }^{35}\right)$. The measurement is consistent with the calculated value within $1 \sigma$ for pitch sensitivity and within $2 \sigma$ for yaw sensitivity. This measurement demonstrates that indeed angle adjustments to the Pockels cell can counteract the analyzing-like position differences caused by birefringence gradients with a modest beam divergence of $\sim 1 \mathrm{mrad}$ for a $1 \mathrm{~mm}$ beam spot size.

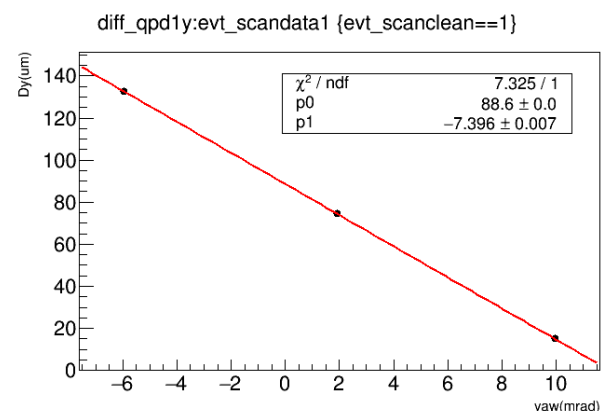

(a) Run5226 RTP S1 Dy vs yaw

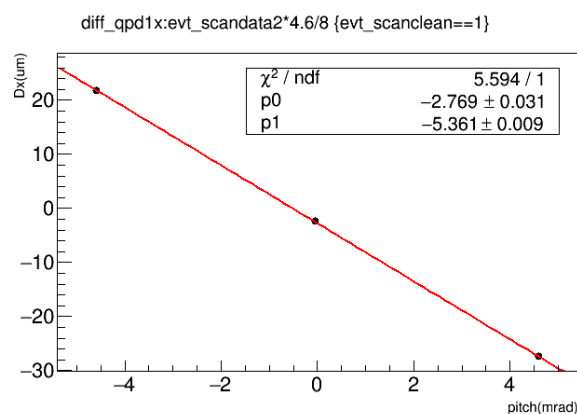

(b) Run5227 RTP S1 $D_{x}$ vs pitch

Figure 6-37: $D_{x}, D_{y}$ angle dependence in $\mathrm{S} 1$ [248]

\subsubsection{Etalon scans}

As discussed in Sec. 6.3.8, the etalon effect is due to an interference of the back and front face reflections off the crystal and is caused by the extreme precision with which the RTP crystals are cut. The etalon effect manifests itself as a charge asymmetry dependence the back reflection coefficient R, crystal length, and delta phase. Both transmission and reflection can undergo oscillations $\sim R \cos \phi$ due to this interference. In operation, we successfully suppressed the etalon effect by

\footnotetext{
${ }^{35} 40 \mathrm{mV}$ signal on each pad with $20 \mathrm{mV}$ pedestal, so an error of TR $20 \mathrm{mV} /(40 \mathrm{mV}+20 \mathrm{mV}) \sim$ $30 \%$ is hypothetically possible on each pad which translated into a calibration error on x-const of $\sim 15 \%$
} 
using a broadband, pulsed laser. However, we took advantage of the etalon effect and used a narrow-band CW laser to perform studies which helped characterize the electro-optic coefficients $r_{33}$ and $r_{23}$. .

For these etalon scans an LD785-SEV300 Thorlabs diode laser (785nm central wavelength, $10 \mathrm{MHz}$ linewidth [265]) was used. The etalon measurements were performed by setting input polarization along the fast axis or the slow axis $\left( \pm 45^{\circ}\right)$ with a HWP, scanning the applied voltage for one helicity state $V^{L}\left(V^{R}\right)=0-$ $5000 \mathrm{~V}$ while keeping the voltage for the opposite helicity $V^{R}\left(V^{L}\right)=$ const. fixed , and examining the transmission without an analyzer. As calculated in Sec. 6.3.8, the predicted functional form of the transmitted asymmetry $A_{T}$ is proportional to the back reflection coefficient $\mathrm{R}$ and the cosine of the scanned voltage, the frequency of which scales with the electro-optics coefficients as follows:

for $V^{R}=V^{+}=4500 \mathrm{~V}$, the etalon asymmetry in transmission for polarization states along the $\mathrm{z}$ and $\mathrm{y}$ primary axes are given by

$$
\begin{aligned}
& A_{T}^{z}\left(V^{R}=4500 V\right)=R\left(-\cos \left(C_{4500 V}^{z}\right)+\cos \left(2 \pi\left(n_{0, z}-\frac{1}{2} n_{0, z}^{3} r_{33} V^{L}\right) L_{0} /(\lambda d)\right)\right. \\
& A_{T}^{y}\left(V^{R}=4500 V\right)=R\left(-\cos \left(C_{4500 V}^{y}\right)+\cos \left(2 \pi\left(n_{0, y}-\frac{1}{2} n_{0, y}^{3} r_{23} V^{L}\right) L_{0} /(\lambda d)\right)\right.
\end{aligned}
$$

and for $V^{L}=V^{-}=-100 \mathrm{~V}$, the etalon asymmetry in transmission for polarization states along the $\mathrm{z}$ and $\mathrm{y}$ primary axes are given by

$$
\begin{aligned}
& A_{T}^{z}\left(V^{L}=-100 V\right)=R\left(-\cos \left(C_{-100 V}^{z}\right)+\cos \left(2 \pi\left(n_{0, z}-\frac{1}{2} n_{0, z}^{3} r_{33} V^{R}\right) L_{0} /(\lambda d)\right)\right. \\
& A_{T}^{y}\left(V^{L}=-100 V\right)=R\left(-\cos \left(C_{-100 V}^{z}\right)+\cos \left(2 \pi\left(n_{0, z}-\frac{1}{2} n_{0, z}^{3} r_{33} V^{R}\right) L_{0} /(\lambda d)\right)\right.
\end{aligned}
$$

We performed such measurements multiple times, scanning $V^{L}(R)$ with $V^{R}(L)$ fixed, and fit the results with a cosine function to obtain a value for the cycle frequency $\left.f_{z}=2 \pi \frac{1}{2} n_{0, z}^{3} r_{33}\right) L_{0} /(\lambda d)$ ) for polarization along the crystal z-axis and $\left.\left.f_{y}=2 \pi \frac{1}{2} n_{0, y}^{3} r_{23}\right) L_{0} /(\lambda d)\right)$ for polarization along the crystal y-axis. This was done 
for polarization states along the crystal's z-axis and y-axis separately. We performed these measurements on each of the two crystals. The laser suffered from mode-hopping so this accounted for some deviations from the fits. It was easier to get a more accurate value for measurements along the z-axis since $r_{33}$ is larger than $r_{23}$ which allowed for more cycles within our limited voltage range of $0-5000 \mathrm{~V}$. An example of one of our measurements for the z-axis and y-axis is shown in Fig. $6-38$.
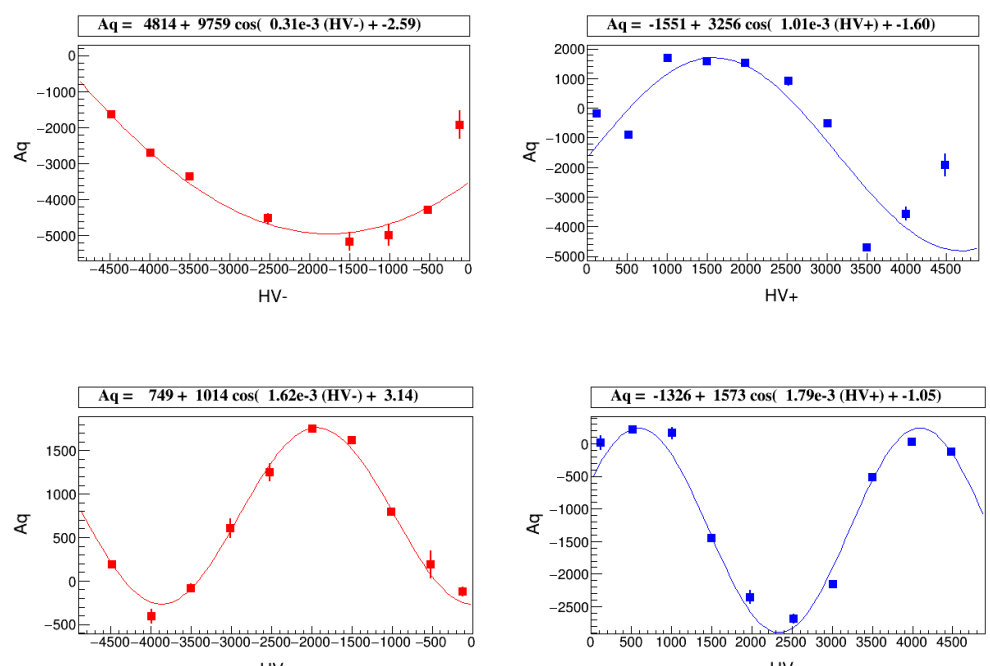

HV-

Figure 6-38: Etalon scans [247]. Etalon effect in RTPs. These measurements were performed by setting input polarization along the fast axis (bottom) or the slow axis(top) at $\pm 45^{\circ}$ with a HWP, scanning the applied voltage, and examining the transmission without an analyzer

We used 10 such measurements of the z-axis and 7 such measurements of the yaxis to obtain our resulting average cycle frequency of $\bar{f}_{z}=1.46 \pm 0.21(R M S 0.65) m V^{-1}$ and $\bar{f}_{y}=0.86 \pm 0.09(R M S 0.25) m V^{-1}$. Using the values for $n_{y}=1.7832, n_{z}=$ 1.8673 (from Sellmeier's equation [328]), $L_{0}=10 \mathrm{~mm}, \lambda=785 \mathrm{~nm}$ ([235]), and $d=$ $12 \mathrm{~mm}$, we obtain the measured electro-optic coefficients $r_{33}^{M}=67 \pm 10(R M S 30) \mathrm{pm} / \mathrm{V}$ , $r_{23}^{M}=45 \pm 5(R M S 13) \mathrm{pm} / \mathrm{V}, r_{33}^{M}-r_{23}^{M}=22 \pm 11(R M S 33) \mathrm{pm} / \mathrm{V}$ whereas reported values at $1064 \mathrm{~nm}$ are $r_{33}=35 \mathrm{pm} / \mathrm{V}, r_{23}=12.5 \mathrm{pm} / \mathrm{V}$, and $r_{33}-r_{23}=$ $22.5 \mathrm{pm} / \mathrm{V}[236]$ and at $633 \mathrm{~nm} r_{33}=35.1 \mathrm{pm} / \mathrm{V}, r_{23}=12.6 \mathrm{pm} / \mathrm{V}$, and $r_{33}-r_{23}=$ 
22.5pm/V[237]. While this particular measurement isn't very precise, this method is an interesting one, and if a more narrowband CW laser were used on an uncoated RTP crystal with higher reflection coefficients, a more accurate interferometric measurement of the electro-optic coefficients could be made at various wavelengths.

\subsubsection{Steering control}

Steering is an angle difference between right and left helicity states, a helicity correlated change in angle of the outgoing laser beam after having passed through the Pockels Cell. The RTP cell design was intended to allow for straightforward control over helicity correlated beam steering via the the electric field gradient along the z-axis of each crystal. The first crystal has its z-axis along $U\left(-45^{\circ}\right.$ direction) and it controls the steering along the U-direction with alpha-position- $U$ voltage $V_{\alpha p o s, U}$. The second crystal has its z-axis along $\mathrm{V}\left(+45^{\circ}\right.$ direction $)$ and it controls the steering along the $\mathrm{V}$-direction with alpha-position- $\mathrm{V}$ voltage $V_{\alpha p o s, V}$.

For the characterization of the RTP cell's steering control, we used JLab's HallA pulsed diode laser which is a frequency doubled with a PPLN crystal (focused before the crystal with an lens $\mathrm{f}=40 \mathrm{~mm}$, and refocused after the PPLN with an $\mathrm{f}=35 \mathrm{~mm}$ lens), has a $30-50 \mathrm{ps}$ pulse duration, $500 \mathrm{MHz}$ repetition rate, central wavelength of $776.5 \mathrm{~nm}$, and $0.2 \mathrm{~nm}$ bandwidth [231]. The study was performed with horizontal input polarization before the cell, no analyzer downstream of the cell, and qpd detector to measure the beam position differences. Because steering produces a position difference which increases with throw distance, and steering is referred to as an 'angle-like' position difference which does not depend on analyzing power, no analyzer was used in the study and the throw distance from the Pockels Cell center the the qpd was measured to be $140 \mathrm{~cm}$.

As predicted in Sec. 6.3.4, for horizontal input polarization, there is a linear dependence between $V_{\alpha p o s, U}$ applied to the first crystal, inducing a field gradient 
$\frac{d E_{z 1,0}}{d z_{1}}$, and the steering angle $\Delta \theta_{U}$ along $U=-45^{\circ}=\frac{x-y}{\sqrt{2}}$. The steering control of position differences with voltage was simulated to be approximately:

$$
\left|\Delta \theta_{U}\right|=\left|\frac{1}{2}\left(n_{y 0}^{3} r_{23}+n_{z 0}^{3} r_{33}\right) L \frac{d E_{z 1,0}}{d z_{1}} V_{\alpha p o s}\right|=4.6 \pm 1.7 \mathrm{nrad} / V V_{\alpha p o s U}
$$

Fig. 6-39 shows the results of a scan of alpha-position-U voltage $V_{\alpha p o s U}$ at JLab. The results show a position difference sensitivity to voltage of $\frac{D_{u}}{d V_{\alpha p o s}}=$ $3.68 \pm 0.118 \mathrm{~nm} / \mathrm{V}$ at a throw distance of $140 \mathrm{~cm}$, corresponding to a measured steering control of $\frac{d \Delta \theta_{U}}{d V_{\alpha p o s}}=2.63 \pm 0.084 \mathrm{nrad} / \mathrm{V}$. The laser steering dependence on applied voltage was found to be linear as predicted, and while on the low end of simulated $4.6 \pm 1.7 \mathrm{nrad} / \mathrm{V}$, more than sufficient to control and zero out any position differences intrinsic to the crystal system. ${ }^{36}$

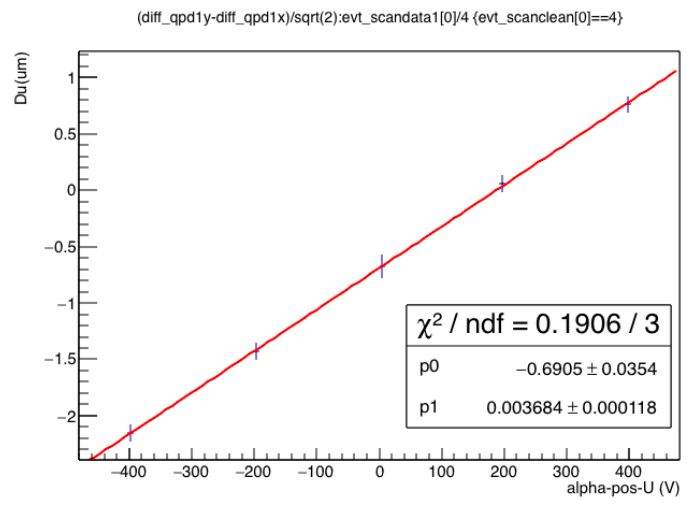

Figure 6-39: Alpha-phase-U Voltage Scan: Run3218, IHWPout, no analyzer, qpd at $140 \mathrm{~cm}[256]$

An additional study was performed in which the steering sensitivity to voltage was amplified by placing small copper strips on the side of the crystals, thereby maximizing the steering control. By placing a grounded conductor (copper strips) directly on the side of the crystals, we could induce larger field gradients. Then a

\footnotetext{
${ }^{36}$ The range of control is $-800 \mathrm{~V}<V_{\alpha p o s U}<800 \mathrm{~V},-800 \mathrm{~V}<V_{\alpha p o s V}<800 \mathrm{~V}$ which corresponds to $D_{u}= \pm 2.1 \mu \mathrm{m}, D_{v}= \pm 2.1 \mu \mathrm{m}, D_{r}=\sqrt{D_{u}^{2}+D_{v}^{2}}=3.0 \mu \mathrm{m}$ at a $1 \mathrm{~m}$ throw distance. When used in conjunction with a $<7 \%$ analyzing element, such as a photocathode, the S1 analyzing-like position differences for a $1 \mathrm{~mm}$ beam are at most $7 \%$ on $10-25 \mu \mathrm{m}$ which is $0.7-1.75 \mu \mathrm{m}$, well within the range of the steering control.
} 
more accurate comparison of the steering effect for $n_{y}$ and in $n_{z}$ could be made, and further information concerning the electro-optic coefficients $r_{33}$ and $r_{23}$ could be obtained. In these alpha-position-voltage scans, the crystal was oriented with its primary axes z \& y along the horizontal \& vertical, respectively, so that input polarization $(\mathrm{H} / \mathrm{V})$ was along the primary crystal axes and alpha-position-voltage induced steering along $D_{x}$ and $D_{y}$.
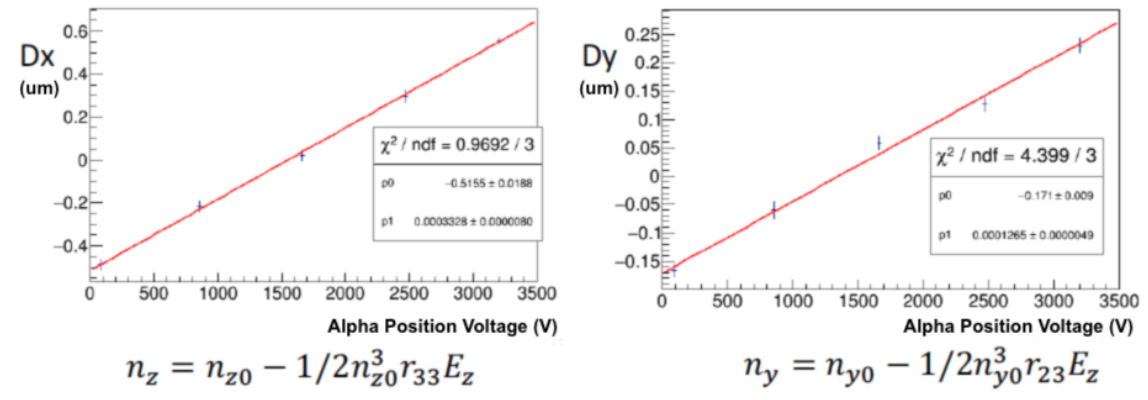

Figure 6-40: Alpha-Position Voltage Scans, Copper strips. Left: Run3143, input polarization $\mathrm{H}, \mathrm{nz}$, qpd $70 \mathrm{~cm}$. Right: Run3148, input polarization V, ny, qpd $70 \mathrm{~cm}$

Fig.6-40 shows the results of these alpha-position-voltage scans. We note that the steering for input polarization along $\mathrm{nz}$ and is approximately $3 \mathrm{X}$ larger than the steering for input polarization along ny. Taking the ratio of the slopes gives a measure of $\frac{n_{z}^{3} r_{33}}{n_{y}^{3} r_{23}}=(0.3328 \pm 0.008) /(0.1265 \pm 0.0049)=2.63 \pm 0.17$, $3 \sigma$ from the predicted value of 3.21. Assuming $n z$ and $n y$ are known from Sellmeir's equation, we obtain a measured value for the ratio of the electrooptic coupling coefficients of $r_{33}^{M} / r_{23}^{M}=2.20 \pm 0.1$, whereas the predicted value is $r_{33} / r_{23}=(35 \mathrm{pm} / \mathrm{V}) /(12.5 \mathrm{pm} / \mathrm{V})=2.8$. We can combine our etalon effect measurement of $r_{33}^{M}-r_{23}^{M}=22 \pm 11(\mathrm{RMS} 33) \mathrm{pm} / \mathrm{V}$ and our steering measurement of $r_{33}^{M} / r_{23}^{M}=2.2 \pm 0.1$ to obtain measurements of the electro-optic coefficients:

$$
r_{33}=\left(r_{33}-r_{23}\right) /\left(1-r_{23} / r_{33}\right)=40.3 \pm 20.2 p m / V
$$




$$
r_{23}=\left(r_{23}-r_{33}\right) /\left(1-r_{33} / r_{23}\right)=18.3 \pm 9.3 p m / V
$$

\subsubsection{RHWP scans}

The results of RHWP scans performed with the quad-photodiode are shown in Fig. 6-41 ${ }^{37}$. To minimize the steering offset terms in these RHWP scans, the steering voltages alpha-pos- $\mathrm{U}$ and alpha-pos- $\mathrm{V}$ were optimized with independent values for IHWPout and IHWPin states. To minimize the position difference $4 \theta$ terms, position differences in S1 were reduced with Pockels cell pitch and yaw. The RHWP scans show steering terms of $<70 \mathrm{~nm}$ at a $1.5 \mathrm{~m}$ throw distance and $4 \theta$ terms of $<1 \mu \mathrm{m}$ for a $4 \sigma=2.6 \mathrm{~mm}$ spot size ${ }^{38}$.

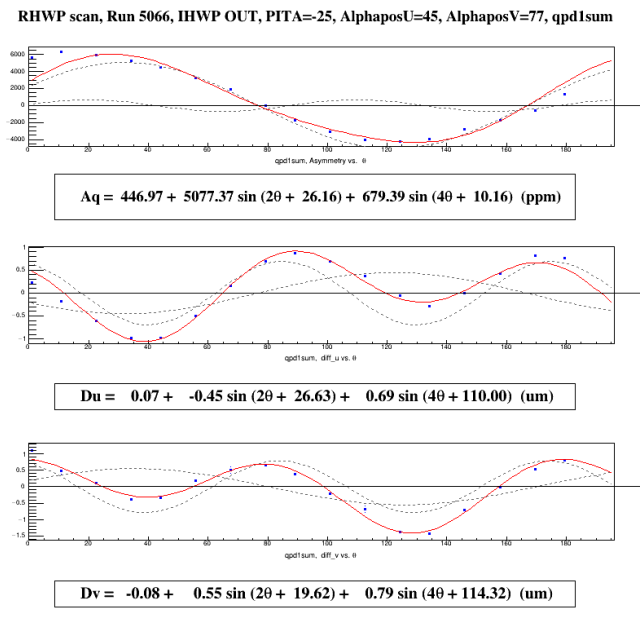

(a) IHWP out

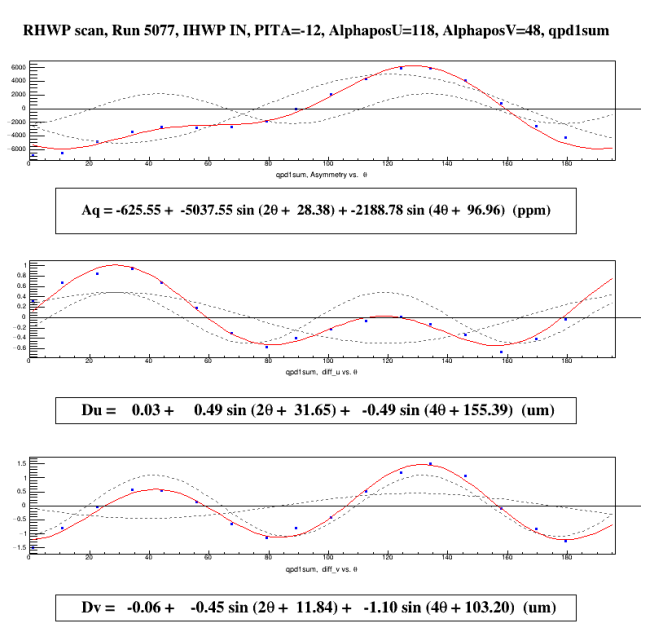

(b) IHWP in

Figure 6-41: RHWP scans: (a) IWHP out, PITA voltage set to minimize Aq in S1, Run5066 (b) IHWP in, PITA voltage set to minimize Aq in S1, Run5077 [255]

\footnotetext{
${ }^{37}$ For a detailed description of RHWP scans, see [163]

${ }^{38}$ Conditions: Captain Expando lens $(f \sim 1 m$, named by John Hansknecht $)$ inserted 46inches upstream of cell. Spot size at PC center $2 \sigma=w=0.60 \mathrm{~mm}$ (Horizontal), $0.65 \mathrm{~mm}$ (Vertical), divergences at $\mathrm{PC}$ center $d w / d z=-0.39 \mathrm{mrad}$. Effective throw from PC to qpd $1.5 \mathrm{~m}$. At qpd, spot size $4 \sigma X / Y=1.32 / 1.29 \mathrm{~mm}$ with calibration constants $x-$ const $=0.442 \mathrm{~mm}$, $y-$ const $=0.445 \mathrm{~mm}$. Path to cathode: $2 \mathrm{~m}$ steering lens, cathode spot size $2 \sigma=$ $1.2 \mathrm{~mm}$ (Horiz), $1.3 \mathrm{~mm}$ (Vert), distance to cathode $\sim 3.1 \mathrm{~m}$, distance to steering lens $\sim 1.067 \mathrm{~m}$, effective throw from PC to cathode $\sim 2.015 \mathrm{~m}$, cathode analyzing power $\sim 5 \%[261]$
} 


\subsubsection{Spot size asymmetries - linear array measurements}

The linear-array photodiode (LAPD) detector ${ }^{39}$ is an array of 16 photodiode pads, each with an active area of $1.22 \times 1.84 \mathrm{~mm}$, separated $0.25 \mathrm{~mm}$ apart [260] as shown in Fig. 6-42. Typically 6-8 pads are used to perform measurements of the spot-size asymmetry of the laser after passing through the Pockels cell.

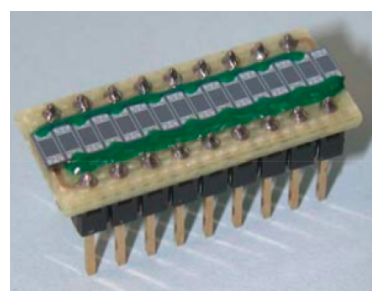

Figure 6-42: Linear-array photodiode (LAPD) detector [260]

The linear array measures the spot-size of the laser via either arithmetic and gaussian methods (as described in [260]). In the arithmetic method, the mean beam position $\bar{x}$ and the beam spot size $\sigma$ are computed from weighted sums of the intensities of the individual pads as:

$$
\begin{gathered}
\bar{x}=\frac{\sum_{i} I\left(x_{i}\right) x_{i}}{\sum_{i} I\left(x_{i}\right)} \\
\sigma=\frac{\sum_{i} I\left(x_{i}\right)\left(x_{i}-\bar{x}\right)^{2}}{\sum_{i} I\left(x_{i}\right)}
\end{gathered}
$$

where where $x_{i}$ and $I\left(x_{i}\right)$ are the beam position and intensity on the $i^{\text {th }}$ element of the array. The gaussian method takes the beam intensity across all the pads and a gaussian fit is performed to extract the beam position and spot-size.

To estimate the electron beam spot-size asymmetry $A \sigma$ off the photocathode, we must use laser table measurements of $A \sigma$ and infer the electron beam asymmetry from that of the laser beam. The laser is focused with a fish-eye lens system onto the narrow axis of the linear array, while also being spread out across 6-pads

\footnotetext{
${ }^{39}$ Advanced Photonic Inc's (API) Blue Enhanced Linear Array Silicon Photodiode (PDBC216).
} 
of the linear array. We collected a series of measurements with the array oriented along $\mathrm{X}, \mathrm{Y},+45^{\circ},-45^{\circ}$ for no analyzer, analyzer 90deg, analyzer 0deg ${ }^{40}$. This full set of 12 measurements (for S1,S2, no-analyzer along X,Y, $+45^{\circ},-45^{\circ}$ ) must be performed as tabulated in Fig. 6-43 to fully characterize the spot size asymmetry.

\begin{tabular}{|c|c|c|c|c|}
\hline & $\begin{array}{l}\text { Extracted } \\
\text { A } \sigma \text { at qpd } \\
1 \mathrm{~m} \text { throw } 100 \% \\
\text { analyzer }\end{array}$ & & $\begin{array}{l}\text { Extracted } \\
\text { A } \sigma \text { at cathode } \\
2.015 \mathrm{~m} \text { throw } \\
6 \% \text { analyzer }\end{array}$ & \\
\hline & Arms & Armsg & Arms & Armsg \\
\hline $\mathrm{X}$ no-analyzer & $-5.32 \mathrm{E}-05$ & \begin{tabular}{|c|}
$-5.99335 E-05$ \\
\end{tabular} & $-1.07 \mathrm{E}-04$ & $-1.21 \mathrm{E}-04$ \\
\hline $\mathrm{XS2}$ & $-6.09 E-06$ & $1.57 \mathrm{E}-05$ & $-3.65 \mathrm{E}-07$ & 9.39E-07 \\
\hline X S1 & $1.58 \mathrm{E}-04$ & $1.12 \mathrm{E}-04$ & $9.48 \mathrm{E}-06$ & $6.71 \mathrm{E}-06$ \\
\hline Y no-analyzer & $-2.03 E-06$ & $4.68616 \mathrm{E}-06$ & $-4.08 \mathrm{E}-06$ & $9.44 \mathrm{E}-06$ \\
\hline Y S2 & $-2.15 \mathrm{E}-05$ & $-3.74 \mathrm{E}-05$ & $-1.29 \mathrm{E}-06$ & $-2.25 \mathrm{E}-06$ \\
\hline Y S1 & $2.43 \mathrm{E}-05$ & $2.83 \mathrm{E}-05$ & $1.46 \mathrm{E}-06$ & $1.70 \mathrm{E}-06$ \\
\hline 45 no-analyzer & $-8.49 \mathrm{E}-05$ & $-1.09 E-4$ & $-1.71 \mathrm{E}-04$ & $-2.20 \mathrm{E}-04$ \\
\hline $45 \mathrm{~S} 2$ & $-2.66 \mathrm{E}-05$ & $1.02 \mathrm{E}-05$ & $-1.60 \mathrm{E}-06$ & $6.12 \mathrm{E}-07$ \\
\hline $45 \mathrm{~S} 1$ & $5.83 \mathrm{E}-04$ & 7.19E-04 & $3.50 \mathrm{E}-05$ & 4.31E-05 \\
\hline-45 no-analyzer & $4.91 \mathrm{E}-06$ & $2.78309 \mathrm{E}-05$ & $9.89 \mathrm{E}-06$ & 5.61E-05 \\
\hline-45 S2 & $-2.02 \mathrm{E}-04$ & $-3.28 \mathrm{E}-04$ & $-1.21 \mathrm{E}-05$ & $-1.97 \mathrm{E}-05$ \\
\hline-45 S1 & $1.79 \mathrm{E}-05$ & $1.29 \mathrm{E}-04$ & $1.07 \mathrm{E}-06$ & $7.72 \mathrm{E}-06$ \\
\hline
\end{tabular}

Figure 6-43: Spot-size asymmetry laser measurements [319]: Shows RTP 2017 linear array measurements for $\mathrm{S} 1, \mathrm{~S} 2$, no-analyzer along $\mathrm{X}, \mathrm{Y},+45^{\circ},-45^{\circ}$ using both arithmetic and gaussian methods as well as inferred spot-size asymmetries off the photocathode.

Ideally, the measurements would be performed at a throw distance comparable to the effective throw distance to the cathode or performed a second time for a second throw distance. This is because some spot-size asymmetries could be analyzing-like, angle-like, or analyzing/angle-like as described in Sec. 6.3.7. In this analysis, we assume $A_{\sigma}^{n o-a n a l}$ is angle-like and that $A_{\sigma}^{S 1}$ and $A_{\sigma}^{S 2}$ are analyzing-like. To obtain $A_{\sigma}^{S 1 / S 2}$ we first subtract out $A_{\sigma}^{\text {no-anal }}$ from the raw S1/S2 measurements. To infer the spot-size asymmetry at the cathode, we scale $A_{\sigma}^{S 1 / S 2}$ down by the analyzing power of the cathode $\epsilon / T \approx 6 \%$ and scale $A_{\sigma}^{\text {no-anal }}$ by the ratio of the throw distances $D_{\text {cathode }} / D_{q p d}$.

\footnotetext{
${ }^{40}$ Conditions- spot size at PC center $2 \sigma=w=0.825 \mathrm{~mm}$ (Horizontal), $0.94 \mathrm{~mm}$ (Vertical), divergences at $\mathrm{PC}$ center $d w / d z=0.51 \mathrm{mrad}$ (horizontal), $0.66 \mathrm{mrad}($ vertical $), 2 \mathrm{~m}$ steering lens, cathode spot size $4 \sigma=2.9 \mathrm{~mm}$ (Horiz), $3.1 \mathrm{~mm}$ (Vert), distance to cathode $\sim 3.1 \mathrm{~m}$, distance to steering lens $\sim 1.067 \mathrm{~m}$, effective throw from $\mathrm{PC}$ to cathode $\sim 2.015 \mathrm{~m}$, cathode analyzing power $\sim 6 \%$
} 

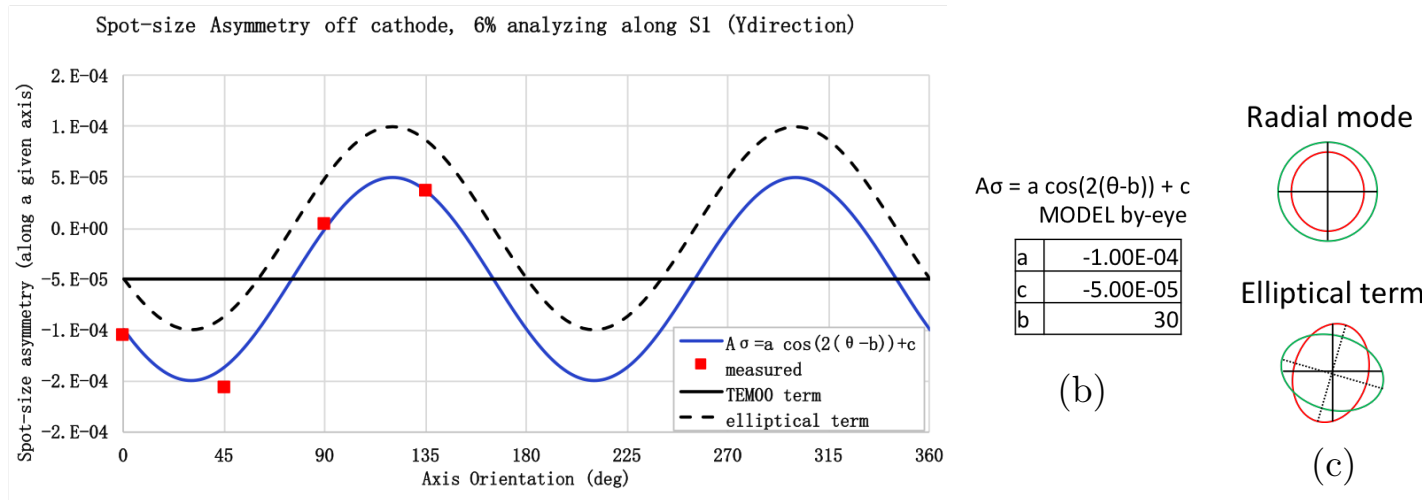

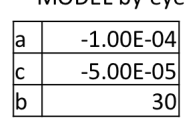

(b)
Elliptical term

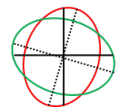

(c)

(a)

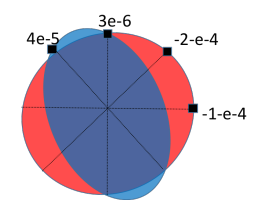

(d)

Figure 6-44: [319] e-beam spot-size asymmetry estimates : Shows inferred e-beam spot-size asymmetries for cathode analyzing power along S1. Based off of RTP 2017 linear array measurements [319] for S1 and no-analyzer along X,Y, $+45^{\circ},-45^{\circ}$ using arithmetic methods)

The inferred the electron beam spot-size asymmetries off the cathode are shown in Fig. 6-44 ${ }^{41}$. In the calculation to make this plot, we assumed an RHWP angle such that the cathode had its $6 \%$ analyzing power oriented along predominantly along S1, and summed the no-analyzer and S1 contributions as befitting the cathode analyzing direction. The fit results indicate an elliptical spot-size asymmetry component of $\sim 10^{-4}$ along $30^{\circ}$ and a radial spot-size asymmetry component of $\sim 5 \times 10^{-5}$. In Sec. 6.3.7, we predicted that with a beam divergence of $0.5 \mathrm{mrad}$ for $6 \%$ analyzer along S1, at a minimum the RTP spot-size asymmetries from angledependence in Aq would be $1 \times 10^{-5}$ along $45^{\circ}$. This predicted minimum value is an order of magnitude smaller than with what is observed here, so it is likely that either (1) spot-size asymmetry could be much improved by angular alignment or (2) intrinsic curvature in the crystals face cuts, 2 nd moments in the refractive indices, or electric field gradients contribute more strongly to the ultimate spot-size

\footnotetext{
${ }^{41}$ using the algebraic method measurement results
} 
asymmetries than angle dependences. It is possible that the spot-size asymmetries could be improved, in particular by changing the cathode effective throw distance (determined by the steering lens) or by choice of RHWP angle.

For another beam setup in Jan 2019, with a smaller spot size through the crystal, RHWP scans were performed using the linear array to characterize the spot size asymmetry. The results are shown in Fig. 6-45 and Fig. 6-46 with the linear array oriented vertically and horizontally for an effective throw distance of $\sim 6 \mathrm{~m}$. The scans indicate, for electron off a cathode with $5 \%$ analyzer power, an estimated max contribution of $5 \times 10^{-6}$ to $A_{r m s}$ from $4 \theta$ terms and a max contribution of $2 \times 10^{-5}$ from non-analyzing terms ${ }^{42}$. Putting this result in perspective, this spot-size asymmetry is likely good enough for MOLLER.

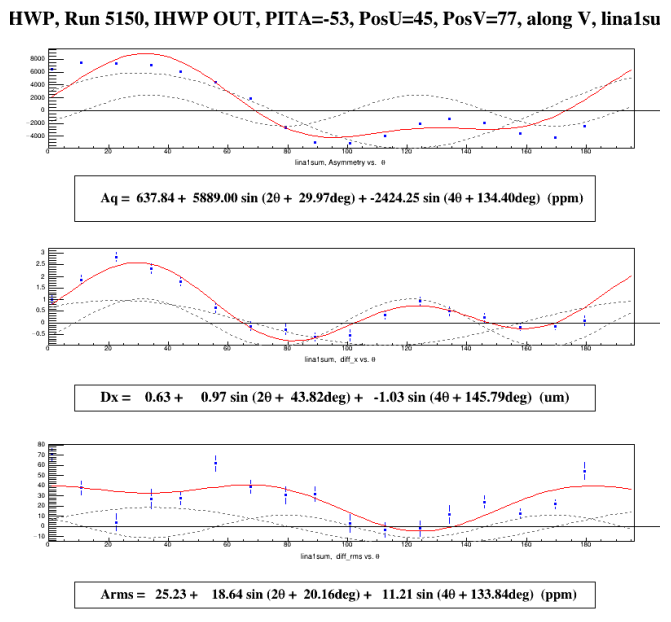

(a) Vertical, IHWPout

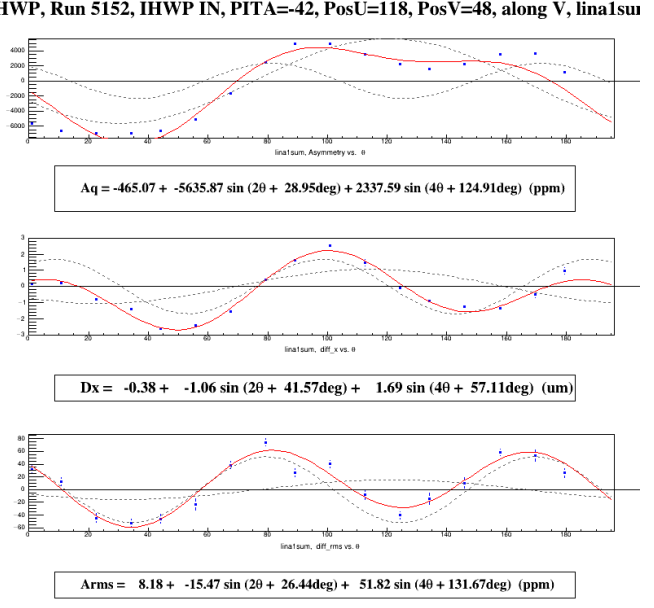

(b) Vertical, IHWPin

Figure 6-45: Linear Array RHWP scans: (a) Vertical orientation, IHWPout (b) Vertical orientation, IHWPin [262]

\footnotetext{
${ }^{42}$ Conditions- Captain Expando lens $(f \sim 1 m)$ inserted 46in upstream of cell. Spot size at PC center $2 \sigma=w=0.60 \mathrm{~mm}$ (Horizontal), $0.65 \mathrm{~mm}$ (Vertical), divergences at $\mathrm{PC}$ center $d w / d z=$ $-0.39 \mathrm{mrad}$. Effective throw from PC to linear array $5.93 \mathrm{~m}$. Pre-linear array focusing optics are: a diverging lens $(-75 \mathrm{~mm}, 0.28 \mathrm{~m}$ upstream of linear array) and a fish eye lens $5 \mathrm{~cm}$ upstream of the linear array. Spot size RMS on linear array was $1.6 \mathrm{~mm}$ and 6 -pads were used. Path to cathode: $2 \mathrm{~m}$ steering lens, cathode spot size $2 \sigma=1.2 \mathrm{~mm}$ (Horiz), $1.3 \mathrm{~mm}($ Vert $)$, distance to cathode $\sim 3.1 \mathrm{~m}$, distance to steering lens $\sim 1.067 \mathrm{~m}$, effective throw from PC to cathode $\sim 2.015 m$, cathode analyzing power $\sim 5 \%[261]$
} 


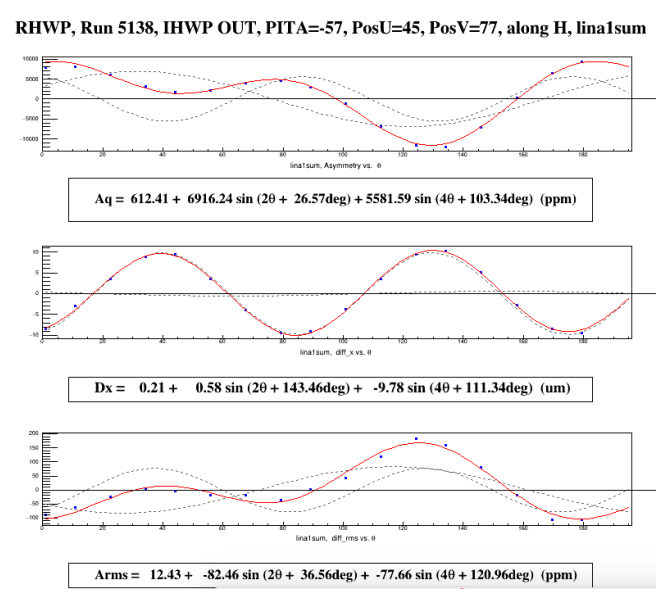

(a) Horizontal, IHWPout

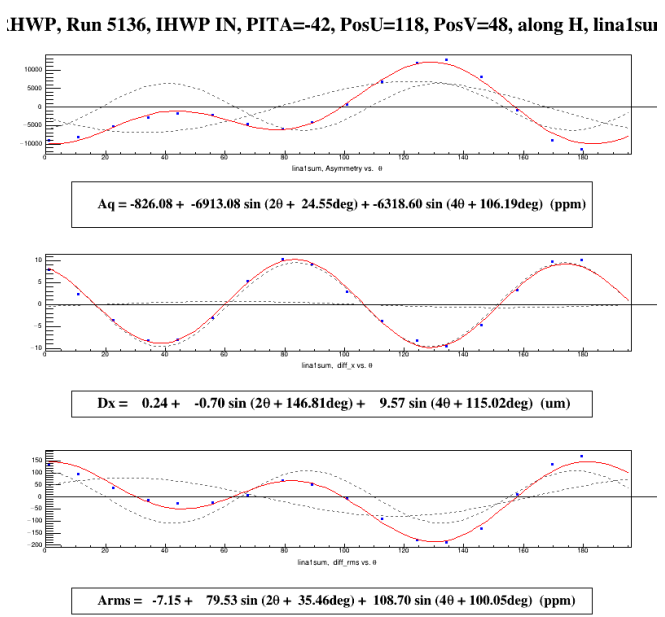

(b) Horizontal, IHWPin

Figure 6-46: Linear Array RHWP scans: (a) Horizontal orientation, IHWP out (b) Horizontal orientation, IHWPin [262]

\subsubsection{Temperature Sensitivity}

In the thermal compensation design, any change in the refractive indices due to temperature affects both crystals equally. Because the crystals are aligned with opposing $y, z$ axis, the net birefringence remains near zero, even when the overall temperature of both crystals changes. While the thermal compensation design does a great deal to mitigate temperature effects on the Pockels Cell performance, some small thermal fluctuations are still empirically observed as shown in Fig. $6-47$.

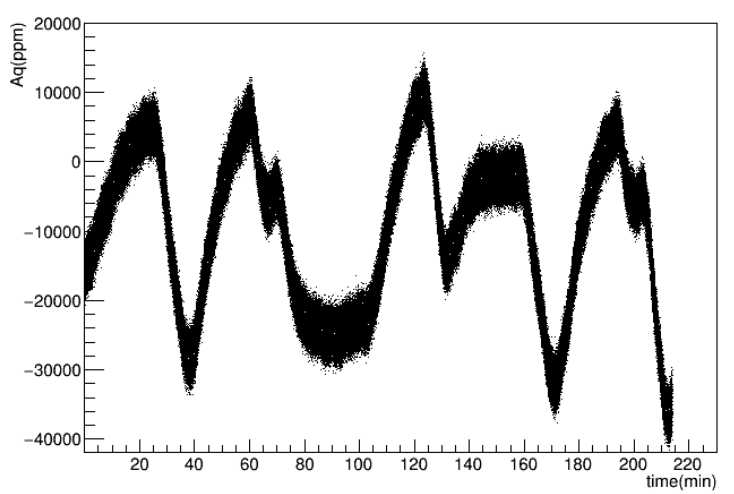

Figure 6-47: Aq fluctuation (Run4883 3hours) 
Fluctuates in the intensity asymmetry $A_{I}$ through a $100 \%$ analyzer are approximately $\pm 20,000 \mathrm{ppm}$ over the course of several hours. The wavelength stability of the laser precluded the possibility that this fluctuation was due to wavelength changes, leaving temperature fluctuations as the main potential contributor to instability. Theoretically, we expect the overall temperature to have very little affect because of the thermal compensation design ${ }^{43}$, especially since the two crystals are very nearly equal in length to within $2 \mu \mathrm{m}$ according to specifications. However, the effect of a temperature difference, in particular, between the two RTP crystals is not cancelled out by the thermal compensation design. The dominant effect is the temperature difference between the crystals. When crystal 1 has a higher temperature than crystal 2 , the temperature difference $T_{1}-T_{2}$ gives rise to a net temperature induced birefringence $\Delta \phi_{T 12} \sim T 1-T 2$, which in QWV operation gives (and 100\% analyzing power) rise to an intensity asymmetry $A_{I, T 12} \approx \Delta \phi_{T 12}$

We calculated the sensitivity to a temperature differences [267] based on the refractive index temperature dependence [268]. At $25^{\circ} \mathrm{C}, d n_{z} / d T \sim 1.22 \times 10^{-5} /{ }^{\circ} \mathrm{C}$ and $d n_{y} / d T \sim 3.88 \times 10^{-6} /{ }^{\circ} \mathrm{C}$ (at $25^{\circ} \mathrm{C}$ ) implies a birefringence temperature sensitivity $d \Delta n / d T \sim 0.831 \times 10^{-5} /{ }^{\circ} \mathrm{C}$, which gives rise to an asymmetry $A q=\Delta \phi=$ $2 \pi \Delta n L / \lambda$ such that $d A_{q} / d T \sim 0.67 /{ }^{\circ} \mathrm{C} \sim 6.65 e 5 \mathrm{ppm} /{ }^{\circ} \mathrm{C}$.

The temperature dependence the the RTP cell was measured between $70-80^{\circ} \mathrm{F}$. Resistive heaters $(\sim 2.7$ Watts) were attached to the delrin housing on the outside of each of the two crystal mounts along with two thermocouples to monitor the temperature of each mount. The heater on the first crystal's mount was switched on, allowing one crystal to heat up more than the other, and then switched off, allowing the crystals to cool down together at different rates. The thermocouple readings for the two mounts were periodically recorded while the intensity asymmetry was continuously measured. A few measurements of temperature de-

\footnotetext{
${ }^{43}$ If the crystals are mismatched in length, by $\mathrm{dL}$, we have $6.65 \mathrm{e} 4 \mathrm{ppm} /{ }^{\circ} \mathrm{C} / \mathrm{mm}$. So a mismatch of $\mathrm{dL}=10 \mathrm{~mm}$, returns us back $6.65 \times 10^{5} \mathrm{ppm} /{ }^{\circ} \mathrm{C}$ for one crystal above. A mismatch of $2 \mathrm{um}$ gives us $133 \mathrm{ppm} /{ }^{\circ} \mathrm{C}$ where the temperature is the absolute temperature of both crystals. $20 \mathrm{um}$ mismatch gives $1330 \mathrm{ppm} /{ }^{\circ} \mathrm{C}$.
} 
pendence were taken as shown in Fig. 6-48. Taken together, the measurement results give $\frac{d A_{q}{ }^{M}}{d T}=6.75 \times 10^{5} \pm 2.18 \times 10^{5} \mathrm{ppm} /{ }^{\circ} \mathrm{C}$ for the RTP cell sensitivity to temperature difference between the crystals, consistent with the calculated value of $\sim 6.65 e 5 \mathrm{ppm} /{ }^{\circ} \mathrm{C}$.

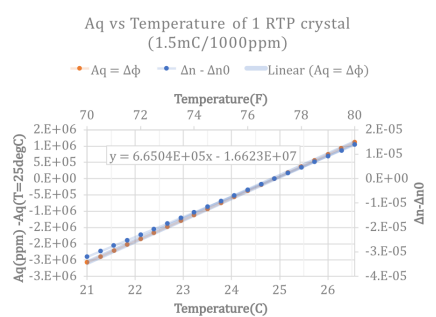

(a) Calculated [267]

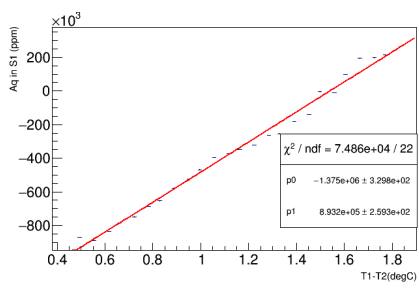

(b) Measured [266]

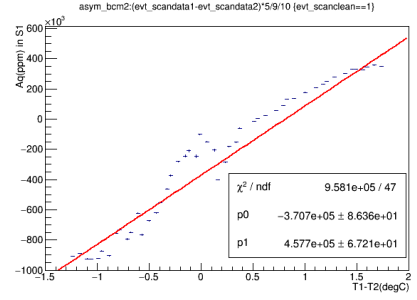

(c) Measured [266]

Figure 6-48: Aq vs T1-T2 (temperature difference between crystals). Measured: Run 5081 \& Run5082, RTP1 heated and RTP2 heat off.

This confirmed temperature sensitivity indicates the observed $\pm 20,000 \mathrm{ppm}$ fluctuations correspond to a fluctuation of $\sim \pm 30 \mathrm{mK}$ temperature difference between the crystal pair. It is hard to control the temperature difference between the crystals at the milli-Kelvin level, but the intensity asymmetry can easily be corrected with voltage, since $20 \mathrm{kppm}$ can be zeroed out with a small $\sim 20 \mathrm{~V}$ PITA-voltage adjustment. The temperature induced birefringence is well within PITA-voltage induced birefringence adjustment range.So, during operation, we simply correct temperature fluctuation with a PITA-voltage feedback loop, rather than trying to force two crystals to maintain milli-Kelvin temperature differences.

This temperature difference sensitivity is one reason why it is important to not focus the laser down tightly for high powers in RTP cells. If one crystal absorbs slightly more power than the other, this leads to a temperature difference in the shape of the beam. The smaller the beam, the greater the gradients induced. This can lead to degradation of performance. At JLab, with 4 laser beams on one crystal, it could lead to thermal effects from one Hall's laser interfering with the performance of the Pockels cell on another Hall's beam, as well as a sensitivity of HCBA to whether or not some Hall's beam have tripped, are in use, and changes to 
other Hall's currents. When using RTP crystals, we cannot reduce the beam size significantly $(<1 \mathrm{~mm}$ for $\sim 1$ Watt) or else thermal gradients induced by the laser absorption $(0.75 \% / \mathrm{cm}-4 \% / \mathrm{cm})$ [236] [246] over a small space with high intensity could create additional position differences $(\sim 0.1 \mu \mathrm{m}-0.6 \mu \mathrm{m})$ and interfere with Pockels cell performance [245].

For further discussion on temperature sensitivity fluctuations and feedback see Appendix B.

\subsection{Results: Electron beam measurements}

\subsubsection{Charge Asymmetries and Position Differences}

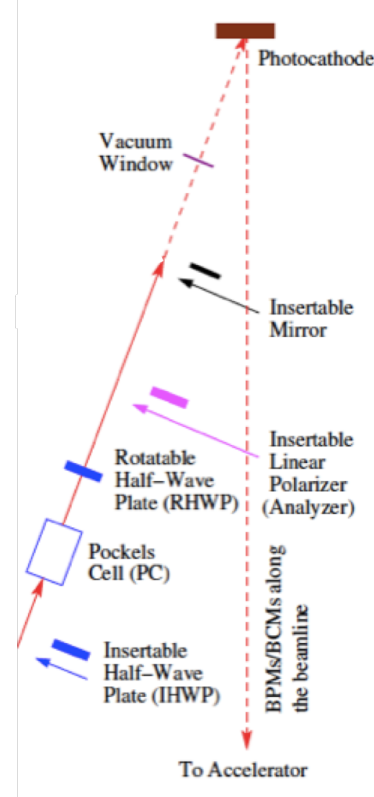

Figure 6-49: JLab table layout [270]

The RTP cell was installed in the polarized injector source at Jefferson Laboratory. Polarized electron beam was produced with the JLab HallA laser described in Sec. $6.5 .5^{44}$. The reduce the laser spot size, a $50 \mathrm{~cm}$ lens was inserted $1 \mathrm{~m}$

${ }^{44}$ paragraph 2 
upstream of the cell. The divergence at the Pockels cell center was measured to be $\theta_{w, x}=d w / d z=0.51 \mathrm{mrad}, \theta_{w, y}=0.66 \mathrm{mrad}$ and the laser spot size $(2 \sigma)$ at the cell was measured to be $w_{P C x}=0.825 \mathrm{~mm}, w_{P C y}=0.94 \mathrm{~mm}$. Downstream of the cell, in-between the cell and the photocathode, there is a rotatable halfwave-plate (RHWP) for rotating the polarization state relative to the analyzing direction of the photocathode, a 'steering' lens (1.067m downstream of the cell) to refocus the laser beam onto the cathode ( $3.1 \mathrm{~m}$ downstream of the cell), and vacuum windows which have slight birefringence gradients. The cathode steering lens is typically an $\mathrm{f}=2 \mathrm{~m}$ lens, and the effective throw distance from the cell to the cathode is $\sim 2.015 m$ (given by $D_{\text {eff }}=D_{\text {tot }}-\left(D_{\text {tot }}-D_{\text {lens }}\right) * D_{\text {lens }} / f$ ). However, under these conditions the spot-size on the cathode was quite large, $w_{x}=1.45 \mathrm{~mm}$, $w_{y}=1.505 \mathrm{~mm}$, compared with spot-size at the cathode during previous parity experiments at JLab. For example, during Qweak, the spot-size at the cathode was $w \sim 0.85 \mathrm{~mm}$ (and $w \sim 0.425 \mathrm{~mm}$ during qweak Run1 ${ }^{45}$ ). So, for this study, to mimic experimental conditions, the steering lens was changed to a $\mathrm{f}=75 \mathrm{~cm}$ lens which reduced the spot-size at the cathode to $w_{x}=1.135 \mathrm{~mm}, w_{y}=1.055$, and changed the effective throw distance from the cell to the cathode to $D_{\text {eff }} \sim 20 \mathrm{~cm}$.

The cathode used during this study was measured to have quantum efficiency $Q E \sim 0.69 \%$ [269], as shown in Fig. 6-50, and an analyzing power of 7\%, producing $\sim 90 \%$ polarized electron beam.

\footnotetext{
${ }^{45}$ this spot size was too small at the high $>100 \mu \mathrm{A}$ currents and lead to issues with the QE degradation on the cathode which correlated to degradation in the degree of polarization and the need for multiple polarimetry measurements
} 


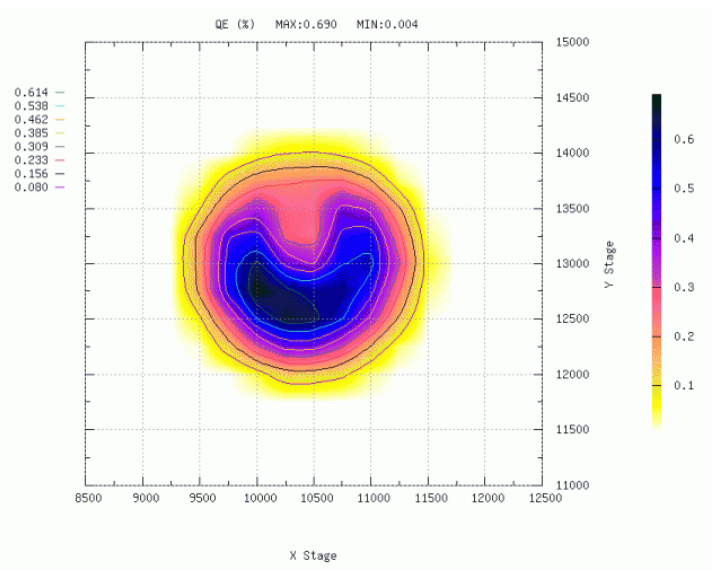

Figure 6-50: QE scan showing 0.69\% [269]

Just after the cathode, the beam position of the $130 \mathrm{keV}$ electron beam was detected by multiple beam position monitors (BPMs) in its transport along the beamline. Each BPM consists of four RF antenna, detecting $500 \mathrm{MHz}$ repetition rate e-beam pulses, and the beam position is computed by comparing the magnitude of the four wire-channel responses (denoted $X^{+}, X^{-}, Y^{+}, Y^{-}$). Analogous to the quad-photodiode measurement, the BPM measurement of beam position in $\mathrm{x}$ and y consists of wire-channel signal differences and sums:

$$
b p m x=\kappa\left(X^{+}-X^{-}\right) /\left(X^{+}+X^{-}\right) \quad b p m y=\kappa\left(Y^{+}-Y^{-}\right) /\left(Y^{+}+Y^{-}\right)
$$

where $\kappa$ is a proportionality factor $(13.7 \mathrm{~mm}$ in this region of the accelerator) based on the geometry of the BPM.

Measurements were taken at a helicity flip rate of $204 \mathrm{~Hz}(\sim 4 m s$ helicity windows) to more quickly converge to nm-level statistical precision on electron beam position monitors. To suppress $60 \mathrm{~Hz}$ noise, the helicity flip rate was line synced and pseudo-random octet helicity patterns $(+--+-++-$ or -++$+--+)$ were used to cancel $60 \mathrm{~Hz}$ noise. The helicity signal reporting to the DAQ was delayed by 16 helicity windows to prevent pickup [263].

Position difference feedback was performed, using the RTP steering control voltages $V_{\alpha p o s, U}$ and $V_{\alpha p o s, V}$, to minimize the measured position differences on a 
BPM (1I04) in the $130 \mathrm{keV}$ region of the injector, shortly after the cathode, before acceleration. Feedback was performed every 2 minutes, after sufficient precision was obtained on the position difference measurement to make the correction meaningful at the level of $20 \mathrm{~nm}$ during each interval. The feedback coefficients used were on the order of $\sim 2 n m / V$. The $\mathrm{x}$ and y position differences, $D_{x}$ and $D_{y}$, accumulated over time are shown for each feedback interval in Fig. 6-51. After approximately 30 minutes, the accumulated average horizontal position difference $D_{x}$ converged to $<5 \mathrm{~nm}$ and the accumulated average horizontal position difference $D_{y}$ converged to $<1 \mathrm{~nm}$. This RTP Pockels cell successfully controlled position differences in the electron beam with nm-level precision. The steering control voltages $V_{\alpha p o s, U}$ and $V_{\alpha p o s, V}$ ultimately used for position difference corrections were small $<50-170 \mathrm{~V}$ out of the $\pm 800 \mathrm{~V}$ range.
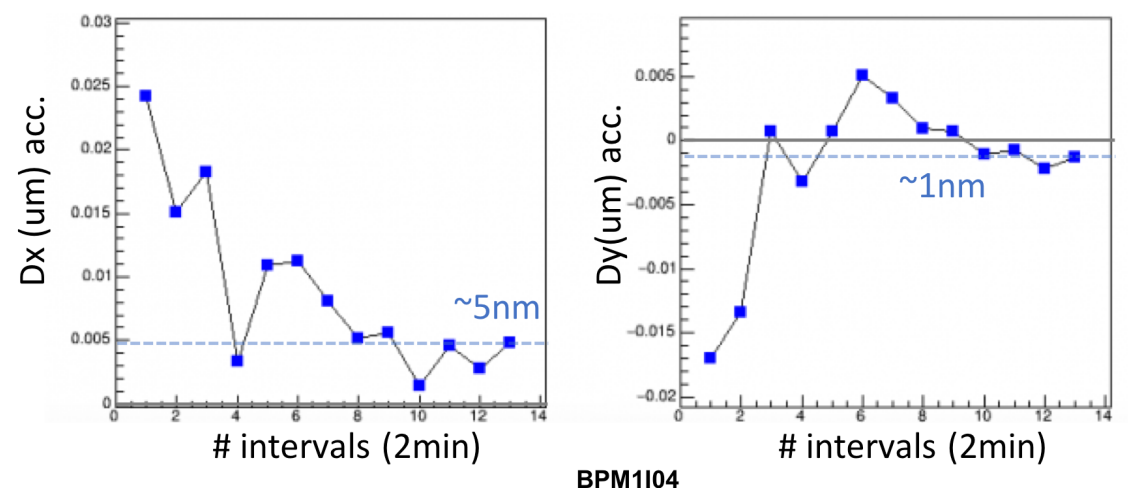

Figure 6-51: Using PC voltages, obtained 1-5nm convergence within 30min [257]

After feedback was performed on one BPM in the injector (BPM 1I04), the position differences at other BPMs ${ }^{46}$ throughout the beamline were measured as shown in Fig.6-52. We use multiple BPMs in the beamline because the e-beam varies in size and has nodes as well as undergoing rotation, and the BPMs have different sensitivities to beam position/angle. The RTP Pockels Cell successfully

\footnotetext{
${ }^{46}$ These BPMs include 2 newly installed bpms just after the cathode: 2I01, 2I02. These are modified M-20 cans which have calibration coefficients $\kappa=25.67 \mathrm{~mm}$. The other BPMs in this plot are M15-mini cans with $\kappa=13.7 \mathrm{~mm}$, or according to the Goubau line scanner $\kappa=15.59 \mathrm{~mm}$. Further down in the injector beamline, the bpms are M15 cans with $\kappa=18.76-18.81 \mathrm{~mm}$ or according to the Goubau line scanner $\kappa=18.4 \mathrm{~mm}$
} 
produced e- beam which achieved $<30 \mathrm{~nm}$ position differences in the $1^{\text {st }} 10$ beam position monitors in the $130 \mathrm{keV}$ region of the injector at JLab. This measurement shows the smallest position differences that have historically ever been observed in this region of the JLab accelerator. The RTP Pockels cell demonstrated the best ever control of position differences in this region.

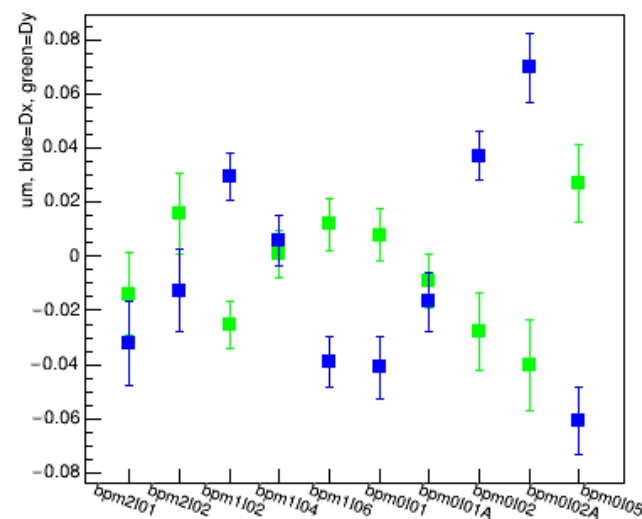

(a) $D_{x}, D_{y}<70 \mathrm{~nm}$

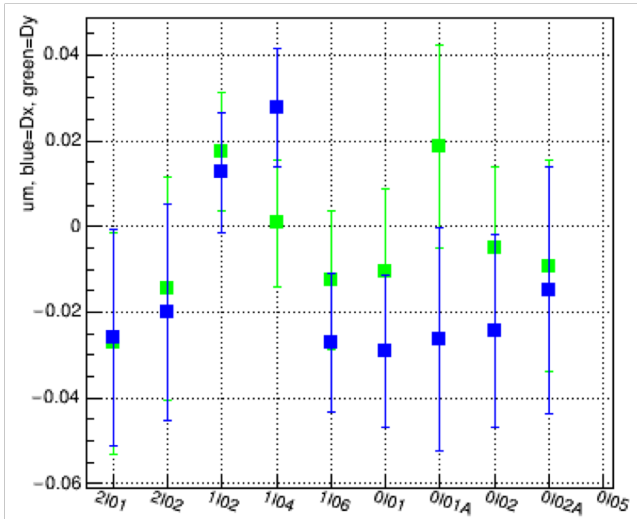

(b) $D_{x}, D_{y}<30 \mathrm{~nm}$

Figure 6-52: (a) Position differences $<70 \mathrm{~nm}$ in $130 \mathrm{keV}$ region, Run4017 [257] (b) Position differences $<30 \mathrm{~nm}$ in $130 \mathrm{keV}$ region, Run 1141 [258]

We compare this measurement with that of the position differences measured during Qweak [56]. Despite the spot-size on the cathode being a factor of 2X larger in our recent measurement, the position differences during Qweak were $<200 \mathrm{~nm}$ in the $130 \mathrm{keV}$ region, whereas the RTP achieved $<70 \mathrm{~nm}$ in the same region.

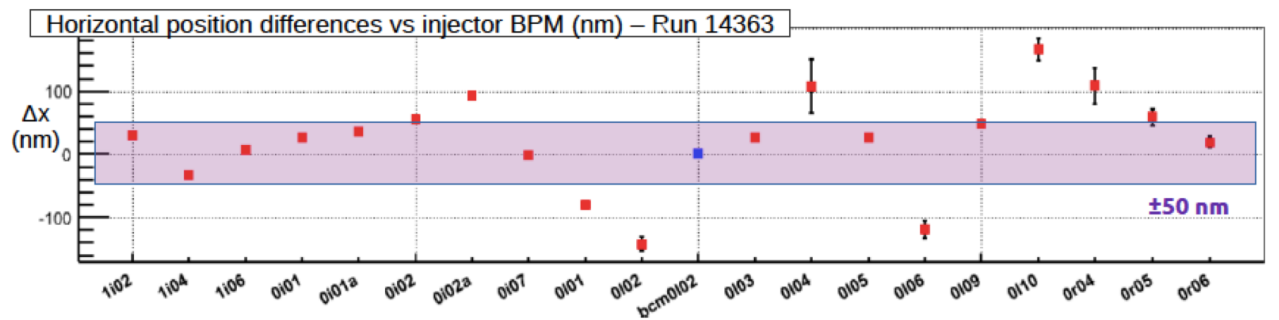

Figure 6-53: Qweak horizontal position differences. These are defined as $\frac{1}{2}\left(x^{R}-\right.$ $x^{L}$ ) and so to compare to our measurements, they must be multiplied by $2 \mathrm{X}$. [56] 
formed feedback on charge asymmetry $A_{q}$ with Pockels cell PITA voltage. As previously stated in Sec. 6.5.8, the RTP suffers from some slow drifts due to fluctuation in the temperature difference between the two RTP crystals of $\sim 30 \mathrm{mK}$. This is correctable with PITA voltage feedback, we can adjust PITA voltage to keep intensity asymmetry minimized

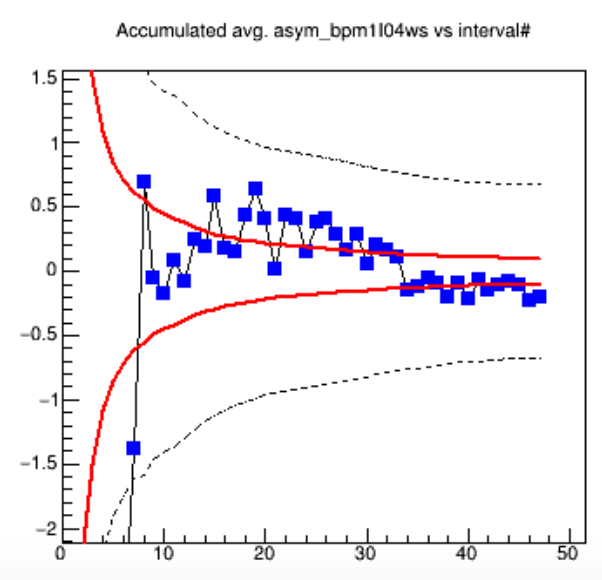

(a) Aq feedback S1

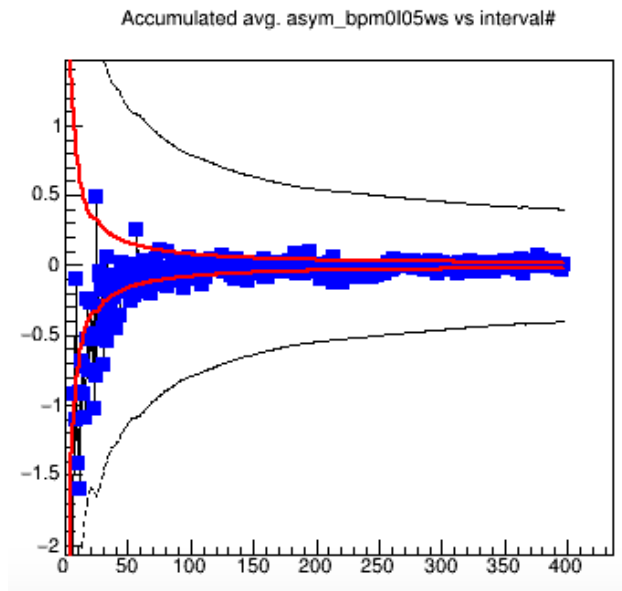

(b) Aq feedback $\sim \mathrm{S} 2$

Figure 6-54: Aq feedback: (a) S1, Run4109, 1I04, RHWP 67.5 (b) near S2, Run3972, 0I05, RHWP $45^{\circ}$

$A_{q}$ feedback was performed, using the RTP PITA voltages to minimize the measured charge asymmetry on a BPM in the $130 \mathrm{keV}$ region of the injector. Feedback was performed in 7.5second intervals at two different RHWP settings. One RHWP angle $\left(67.5^{\circ}\right)$ was set to align the Pockels cell S1 polarization direction along the analyzing axis of the photocathode, maximizing the sensitivity of $A_{q}$ to PITA voltage and temperature fluctuation. The other RHWP angle $\left(45^{\circ}\right)$ reduced the sensitivity to PITA voltage and temperature fluctuations by a factor of $10 \mathrm{X}$. The charge asymmetries, accumulated over time in both case are shown for each feedback interval in Fig. 6-54. After approximately 10 minutes (Fig. 6-54b), the accumulated $A_{q}$ in bpm $1 \mathrm{I} 04$ converged to $<1 \mathrm{ppm}$ (for RHWP angle set to S1 at $67.5^{\circ}$ ). After approximately 2hours (Fig. 6-54b), the accumulated $A_{q}$ in bpm $0 \mathrm{I} 05$ converged to $<0.1 \mathrm{ppm}$ (for RHWP angle set to near S2 at $45^{\circ}$ ). Statistically, the 
theoretical limit for the rate of convergence $A_{q}$ is between $R M S / \sqrt{N_{\text {interval }}}$ and $R M S / N_{\text {interval }}$ depending on the type of noise being measured. In both measurements, the charge asymmetry converged faster than $R M S / \sqrt{N_{\text {interval }}}$ (shown as the black dotted line), and nearly as fast as $R M S / N_{\text {interval }}$. Despite slow drifts from temperature fluctuation, the RTP Pockels cell successfully controlled charge asymmetry in the electron beam as well as the previously used KD*P cell.

\section{4 hall running \& Polarimetry}

When using the RTP cell in HallA parity experiments, it's absolutely necessary to run PITA-voltage feedback on charge asymmetry drifts in Aq. The polarization asymmetry will drift in RTP if not corrected by PITA-voltage. During 4 Hall running, during a parity experiment, one of the Halls must chosen to monitor the asymmetry Aq which will be minimized with PITA-voltage. This Hall's Aq is treated as the definitive measure of polarization asymmetry ${ }^{47}$. When running all 4 halls, it will be necessary to run PITA-voltage feedback using just one hall (i.e. HallA), and use the IA cells for charge asymmetry feedback on the other halls.

For non-parity experiments, 4 hall operation is simple. We will set the RHWP such that the cathode analyzing power is mostly along S2, so the fluctuations of Aq, which are 20-30k ppm for 100\% analyzer in S1, are first suppressed by the cathode being a 6-7\% analyzer down to Aq fluctuations of 1500-2000ppm in S1, then rotating the RHWP close to S2 (within $\sim 10 \%$ of $\mathrm{S} 2$ ), there will only be 150-200ppm Aq fluctuations, which is fine for non-parity experiments.

The electron beam degree of longitudinal polarization is not a concern in terms of variations from the Pockels Cell ${ }^{48}$. If there is an increase in the degree of linear polarization (DoLP) of $\Delta \approx 2-3 \%$, then there is only a tiny reduction in degree of circular polarization(DoCP) on the order of $\sim \Delta^{2} / 2 \approx 0.02-0.05 \%$. The small

\footnotetext{
${ }^{47}$ Subsequently, clipping and non-polarization like charge asymmetries may incidentally get corrected with polarization asymmetries

${ }^{48}$ The longitudinal polarization of the electron beam is largely determined from the cathode and the Wien angle
} 
fluctuations in the DoLP do not translate into any significant changes to the DoCP and DoP of the laser or to the e-beam degree of longitudinal polarization.

For invasive polarimetry measurements (the Moller polarimeter), it may be necessary to perform PITA-voltage feedback on charge asymmetry drifts in Aq. Since the measurements are invasive, the nominal Hall BCM will likely be unavailable as a charge asymmetry monitor for feedback during Moller polarimetry measurements. So, an alternative monitor must be used for feedback instead. An upstream beam position monitor in the Hall could be used, and must be calibrated and cabled to the parity DAQ. An injector BCM could also be used, as long as the feedback mechanism allows for a non-zero Aq set-point, so long as the beam current of the other Halls remains the same, and there is a mechanism for dealing with beam trips in other halls.

\subsubsection{Position Differences Transport: Adiabatic Damping \& Apertures}

The new RTP Pockels cell system can provide fast flipping and suitable control of position differences in the early, $130 \mathrm{keV}$ region of the injector. However, besides minimizing the position differences coming off the cathode, the accelerator beam transport is also important in maintaining the small HCBA's we achieve with RTP as the beam propagates through the accelerator. If beam optics deviate from design, significant correlations can develop which are detrimental to maintaining small position differences.

Parity quality beam for MOLLER must be both achieved with RTP and maintained throughout the accelerator. Two important considerations for optimal electron beam transport are: clean apertures and adiabatic damping.

An example of beam transport issues is shown in Fig. 6-55a. For unknown reasons, large position differences $\left(D_{x} \sim 0.6 \mu \mathrm{m}, D_{y} \sim 0.4 \mu \mathrm{m}\right)$ were observed to appear further downstream in the injector, after apertures and RF elements (past 
the prebuncher, wien, and chopper), and were very large as compared with the tiny $<60 \mathrm{~nm}$ position differences upstream region in the injector. One possible explanation is that if the beam spot-size deviated from design and became very large, due to focusing magnets not working optimally for instance, position differences would become correspondingly large. An alternative explanation is another degree of freedom, not seen by BPMs early in the injector, such a longitudinal asymmetries as described in Sec. 6.6.4.

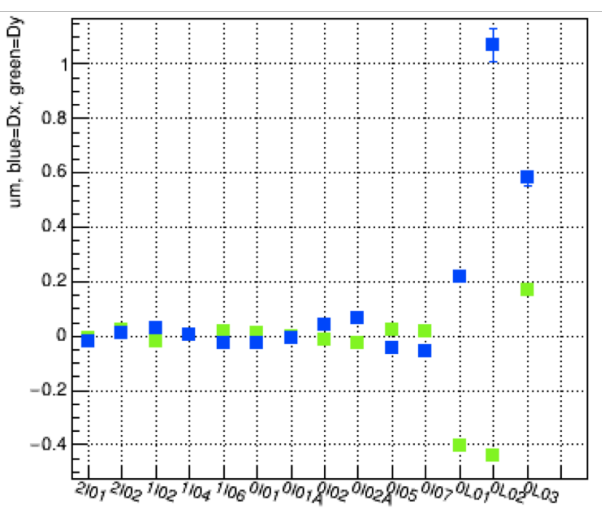

(a)

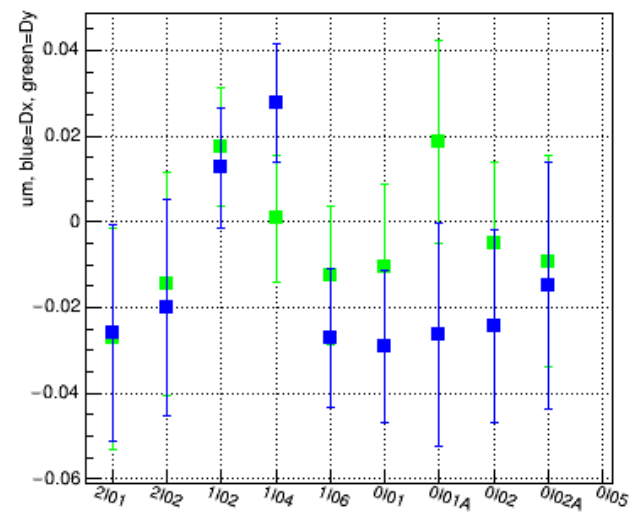

(b)

Figure 6-55: (a) Extreme Case of appearance of large position differences in transport: RTP cell, Run4017 to FC2 [257] (b) Mild case of position difference growth in beam transport, Run1146 [258]

\section{Apertures}

Beam losses due to clipping on apertures in the injector is an important factor to consider in achieving parity quality beam. Apertures tend to couple position differences into charge asymmetries and couple spot-size asymmetries into position differences. This can be understood by simply considering what would happen to the beam current when it is both clipped on one side and suffering from position differences between helicity states: the beam motion causes changes to current and the position differences causes charge asymmetries. So, if there are large position differences $D_{x}, D_{y}$ and significant losses on apertures, there will be significant Aq variations along the beamline. If there are large spot-size asymmetries and signif- 
icant losses on apertures, there will be significant enlarging of position difference along the beamline.

Table 6.3 shows an estimate of how apertures losses couple position differences to Aq and couple spot-size asymmetries to position differences. The calculation is performed assuming a gaussian beam with spot-size $\sigma \sim 1 \mathrm{~mm}$ and a sharp edge aperture [304] (as shown in Fig. 6-56). The table shows that charge asymmetry variations of $>50 \mathrm{ppm}$ can be created by a $5 \%$ aperture loss and $1 \mu \mathrm{m}$ position differences. Aperture losses of 2-5\% are typical [305] and such variations in Aq have been observed in the past along the beamline in the injector (particularly after the chopper). Furthermore, position differences of $>70 \mathrm{~nm}$ can be created by $\sim 5 \%$ aperture losses in a beam with moderate $2 \times 10^{-4}$ spot-size asymmetries and a $\sigma 1 \mathrm{~mm}$ spot-size . Even for a quite small aperture loss of $1 \%,>30 \mathrm{~nm}$ position differences get created for such a beam (assuming a pure gaussian). Of course, the values in Table 6.3 depend on spot-size: a smaller spot-size will lead to larger charge asymmetries for given position differences, and a larger spot-size will lead to larger position differences for given spot-size asymmetries ${ }^{49}$.

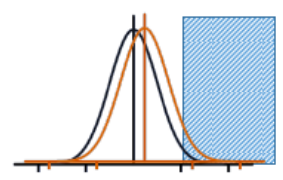

Figure 6-56: Aperture loss calculation

\footnotetext{
${ }^{49}$ The electron beam spot size is typically smaller in the early injector $\sigma \approx 0.3-0.5 \mathrm{~mm}$ and considerably smaller in the late injector and after acceleration $\sigma \approx 0.1-0.2 \mathrm{~mm}$
} 


\begin{tabular}{|r|r|r|r|}
\hline aperture loss & initial HCBA & spot-size & HCBA created by aperture \\
\hline \hline $5 \%$ & $D_{x}=1 \mathrm{um}$ & $\sigma=1 \mathrm{~mm}$ & $A q=54 \mathrm{ppm}$ \\
\hline $10 \%$ & $D_{x}=1 \mathrm{um}$ & $\sigma=1 \mathrm{~mm}$ & $A q=94 \mathrm{ppm}$ \\
\hline $20 \%$ & $D_{x}=1 \mathrm{um}$ & $\sigma=1 \mathrm{~mm}$ & $A q=174 \mathrm{ppm}$ \\
\hline $1 \%$ & $A_{\sigma}=2 \times 10^{-4}$ & $\sigma=1 \mathrm{~mm}$ & $D_{x}=34 \mathrm{~nm}$ \\
\hline $5 \%$ & $A_{\sigma}=2 \times 10^{-4}$ & $\sigma=1 \mathrm{~mm}$ & $D_{x}=76 \mathrm{~nm}$ \\
\hline $10 \%$ & $A_{\sigma}=2 \times 10^{-4}$ & $\sigma=1 \mathrm{~mm}$ & $D_{x}=92 \mathrm{~nm}$ \\
\hline $20 \%$ & $A_{\sigma}=2 \times 10^{-4}$ & $\sigma=1 \mathrm{~mm}$ & $D_{x}=95.8 \mathrm{~nm}$ \\
\hline
\end{tabular}

Table 6.3: Aperture losses of varying degrees coupling HCBAs for $\sigma=1 \mathrm{~mm}$ spot size e-beam [304].

\section{Adiabatic Damping}

Couplings are important in beam transport of position differences. Good optical transport throughout the injector and accelerator is crucial. Laser beam position differences on the photocathode produce electron beam position differences in the injector before acceleration. If the electron beam is aligned well, acceleration can reduce position differences from $500 \mathrm{~nm}$ in the injector to $50 \mathrm{~nm}$ in the Hall, as shown in Fig. 6-57, for example ${ }^{50}$.

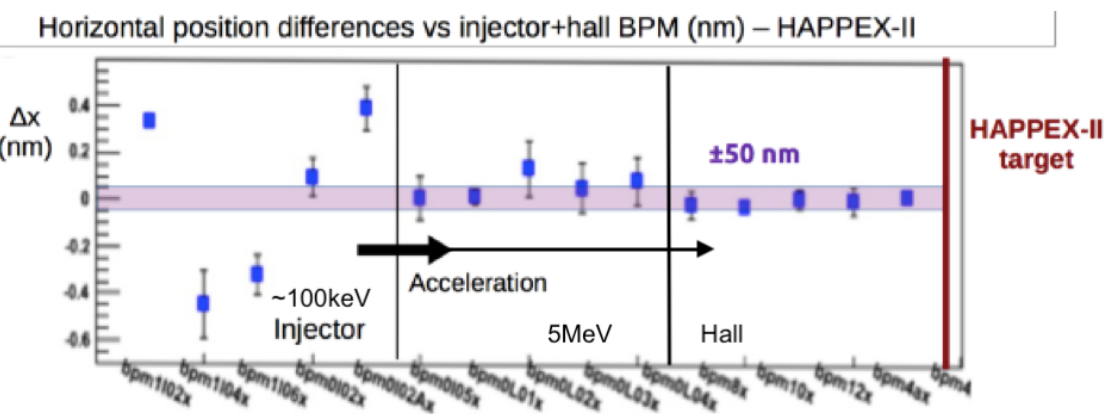

Figure 6-57: Adiabatic Damping during Happex II Experiment - 100X

\footnotetext{
${ }^{50}$ It is important to note that empirically observed adiabatic damping of 100X (from HAPPEX) is not strictly correct due to a calibration detail (since "M15-mini" instead of M15 BPMs are actually used in first part of the injector) so this 100X should be scaled down to $\sim 70 X$ (see Sec. 5.4)
} 
This position difference suppression is achieved through adiabatic damping, essentially from a relativistic boost where the transverse degrees of freedom matter less. The occupied phase space is limited and the position difference suppression scales as $1 /$ p (Fig. 6-58).
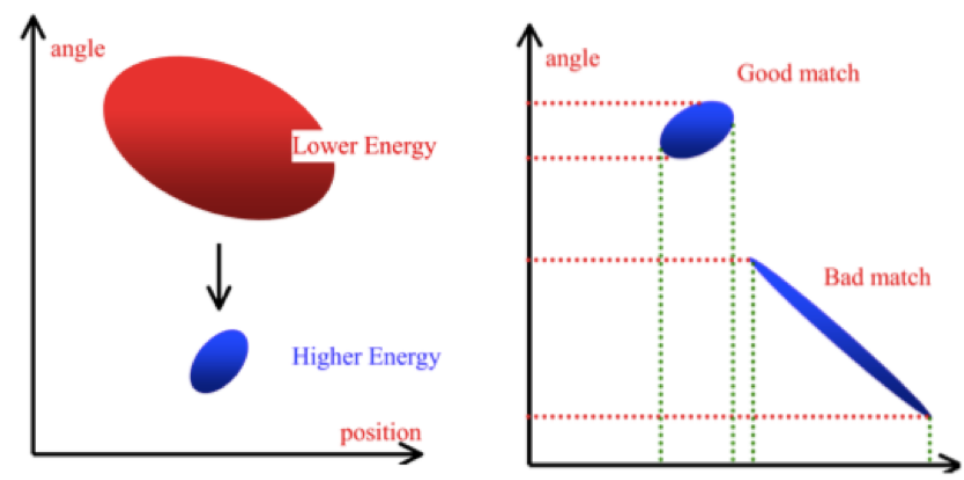

Figure 6-58: Adiabatic Damping - Good optical transport VS poor optical transport [303]

The area of the beam distribution in phase space (emittance) is inversely proportional to the momentum. Adiabatic damping is how we will get from $20 \mathrm{~nm}$ $100 \mathrm{~nm}$ position difference in the injector to $1 \mathrm{~nm}$ in the hall on target. This estimated via:

$$
\text { Damping } \approx \sqrt{\frac{p_{\text {Hall }}}{p_{\text {injector }}}} \approx \sqrt{\frac{11 G e V}{130 k e V}} \approx 290
$$

If beam optics deviate from design, significant correlations can develop. Coupling in transverse phase space spreads the emittance out. For example, During Qweak, the position differences in the hall were actually larger than they were in the injector ${ }^{51}$. For Moller, Good optical transport throughout the injector and accelerator is critical.Theoretically for Moller, the adiabatic damping could be as high as $>200 \mathrm{X}$, though in practice the adiabatic damping observed during former experiments did not reach theoretical limits and a conservative estimate of the achievable suppression for Moller is 30X.

\footnotetext{
${ }^{51}$ and some helicity magnets were used to suppress hall position differences, instead of just improving the beam optics to reduce the correlations
} 


\subsubsection{Spot Size asymmetries}

Spot size asymmetries contribute systematic errors to Parity experiments such as PREX/CREX, HAPPEX, Qweak and Moller. A desirable goal for MOLLER would be to suppress the systematic error contribution from spot-size asymmetries to $<0.1 \mathrm{ppb}$ (as described in Sec. 2.3.7). The effect of a helicity-correlated difference in the beam spot-size for the proposed spectrometer/collimator geometry, has been simulated to give rise to systematics on the level of $12 p p m \times \delta \sigma / \sigma$ [1] 52 . With RTP, we have observed laser spot size asymmetries and inferred electron beam spot-size asymmetries off the cathode of $A_{\sigma}=\delta \sigma /(2 \sigma) \sim 0.5-2 \times 10^{-4}$ in the injector (Sec. 6.5.7), which would contribute $\sim 1.2-4.8 p p b$ to systematics given no other mechanisms of suppression. To reach MOLLER goals, a factor of 10X-40X suppression of spot-size asymmetries is required from a combination of three sources: (1) reduction in the laser beam spot size asymmetry $A_{\sigma}(2)$ cancellation due to periodic slow helicity reversals (3) synchrotron light emittance growth suppressing the spot size asymmetry. Previously, slow helicity reversals have been observed in the past to lend a factor of 10X suppression. Furthermore, suppression between spot size asymmetry in the injector and spot-size asymmetry contribution in the Hall, due to synchrotron light emittance growth, should greatly suppress the spot size asymmetry by a factor of $\sim 25 X-100 X$ as described in the following section. Even if the RTP spot-size asymmetries are not reduced at all, the total suppression from slow reversals and emittance growth should be more than enough to achieve the desired $<1$ ppb systematic error contributions.

\section{Emittance Growth and Spot Size}

Emittance growth is a stochastic process in accelerator which increases the electron beam spot size [62]. Emittance growth at $11 \mathrm{GeV}$ is shown for both vertical and horizontal dimensions in Fig. 6-59. For both vertical and horizontal, the

\footnotetext{
${ }^{52}$ Since $A_{\sigma}=\delta \sigma /(2 \sigma)$, this equation translates into a systematic contribution of $24 p p m \times A_{\sigma}$
} 
spot size experiences growth by $5-10 \mathrm{X}$. The spot-size helicity dependence in the hall is diluted by this synchrotron emittance growth contribution. Synchrotron light emittance dilution should greatly suppress the spot size asymmetry [62]. This suppression only works for stochastic noise - truly incoherent emittance growth; coherent beam size changes (damping, raster, focusing) don't matter. Quantitatively, if $\sigma_{(i n j) R, L}$ is the helicity dependent spot size in the injector and if $\sigma_{\text {synch }}$ is the spot size "noise" from synchrotron incoherent growth, then the total width is given by the quadrature sum $\sigma_{R, L}^{2}=\sigma_{\text {synch }}^{2}+\sigma_{(i n j) R, L}^{2}$, which was observed to grow by $5-10 \mathrm{X}$.
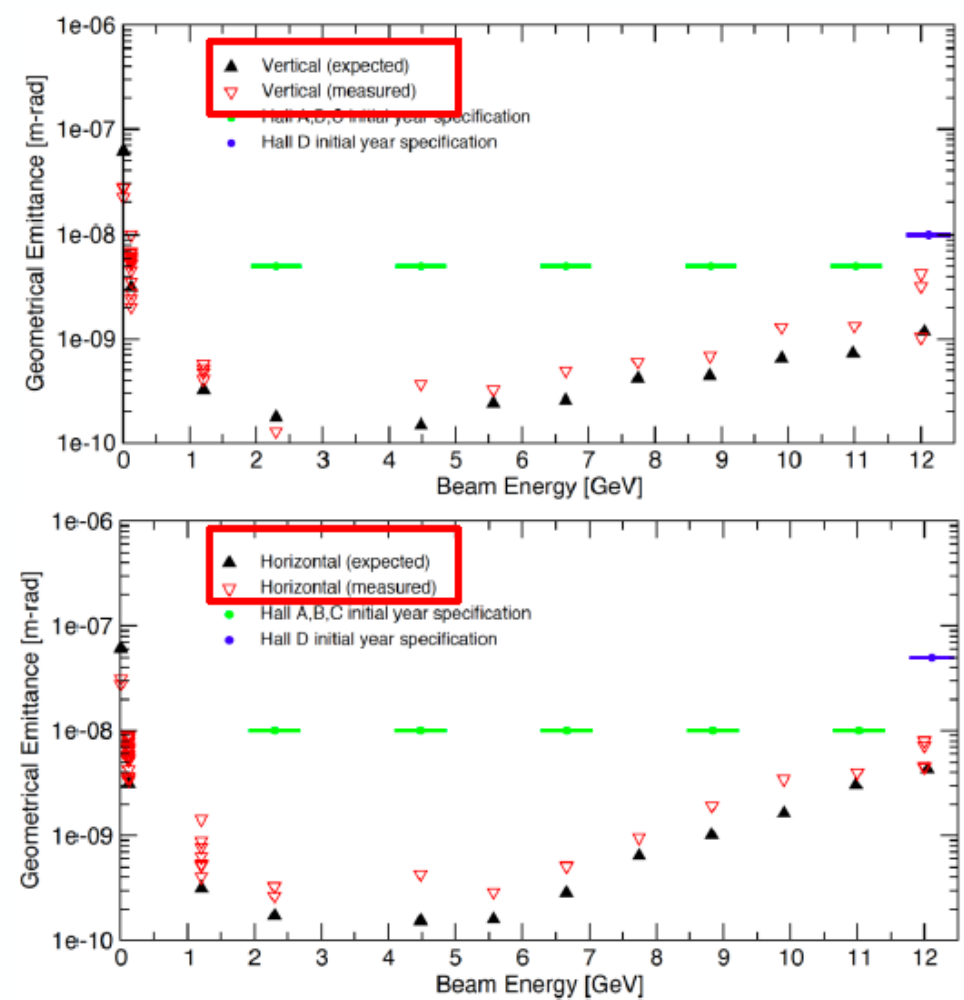

Figure 6-59: Emittance growth at $11 \mathrm{GeV}$ : factor of 5-10X [62]

The suppression of intrinsic spot-size asymmetry $\delta \sigma_{i} / \sigma_{i}$, where $\sigma_{i}$ is the intrin- 
sic spot size, with emittance growth can be derived as [306]:

$$
\frac{\delta \sigma}{\sigma}=\frac{\sigma_{i}^{2}}{\sigma_{\text {synch }}^{2}+\sigma_{i}^{2}} \frac{\delta \sigma_{i}}{\sigma_{i}}
$$

with the spot-size asymmetry decreasing as $\sigma_{\text {synch }}^{2}$ in the approximation $\sigma_{\text {synch }}>>$ $\sigma_{i}$. Hence for the observed emittance growth $\sigma / \sigma_{i} \sim 5-10 X$ the intrinsic spot size asymmetry is suppressed by a factor of $A_{\sigma} / A_{\sigma, i} \sim 25-100 X$, much smaller than the original asymmetry value. This suppression from emittance growth should be more than enough to achieve the desired $<1 \mathrm{ppb}$ systematic error contributions from spot-size asymmetries for MOLLER.

A new consideration for MOLLER spot-size asymmetries is the effect of a longitudinal spot size asymmetry (described in Sec. 6.6.4). How a longitudinal spot-size asymmetry/bunch-length asymmetry is affected by the stochastic process of emittance growth and how it might couple to transverse spot-size asymmetries after acceleration are important considerations to be explored further.

\section{Measuring Elliptical Spot-size Asymmetry on Electron Beam}

As stated in Sec. 5.5, the BPM wire channels can be used in a new way to obtain information about the elliptical component of the spot size asymmetry $A_{\text {elli }}$. Reiterating significant equations from Sec. 5.5 to explain the results in Fig. 6-60, we have:

$$
\begin{gathered}
\text { bpmelli }=\frac{a^{2}(x p+x m-y p-y m)}{8 \sigma^{2}(x p+x m+y p+y m)} \\
\epsilon=\text { bpmelli-bpmecorr }=\frac{a^{2}(x p+x m-y p-y m)}{8 \sigma^{2}(x p+x m+y p+y m)}-f(x, y) \approx \frac{\sigma_{x}-\sigma_{y}}{\sigma_{x}+\sigma_{y}} \\
\text { Aelli }=(\text { diff_bpmelli }- \text { diff_bpmecorr }) / 2
\end{gathered}
$$

where $a \approx 2 \kappa$ is approximately the radius of the BPM vacuum chamber, $\kappa$ is a wireline bpm calibration constant set in accordance with the type of BPM de- 
scribed in Table. $5.5^{53}, \sigma$ is the $e^{-}$beam spot size, and $f(x, y)$ is a correction term. The position sensitivity correction term in bpmelli, which we now define as bpmecorr, is approximately

$$
\text { bpmecorr }=f(x, y)=g 1 \frac{x^{2}-y^{2}}{\sigma^{2}}-g 2 \frac{x^{4}-y^{4}}{\sigma^{2}}
$$

$\Delta$ bpmecorr $=\approx 0.25 \frac{4 x \Delta x-4 y \Delta y}{\sigma^{2}}-2.84 m^{-2} \frac{8 x^{3} \Delta x+8 \Delta x^{3} x}{\sigma^{2}} \approx \frac{x \Delta x-y \Delta y}{\sigma^{2}}$ where $g 1=0.250014, g 2=2.84739 m^{-2}$.

We show the Aelli results of of our best run of 2017 with the RTP cell, which had 100nm position differences in the injector ${ }^{54}$ (for further analysis details, see Sec. 5.5).

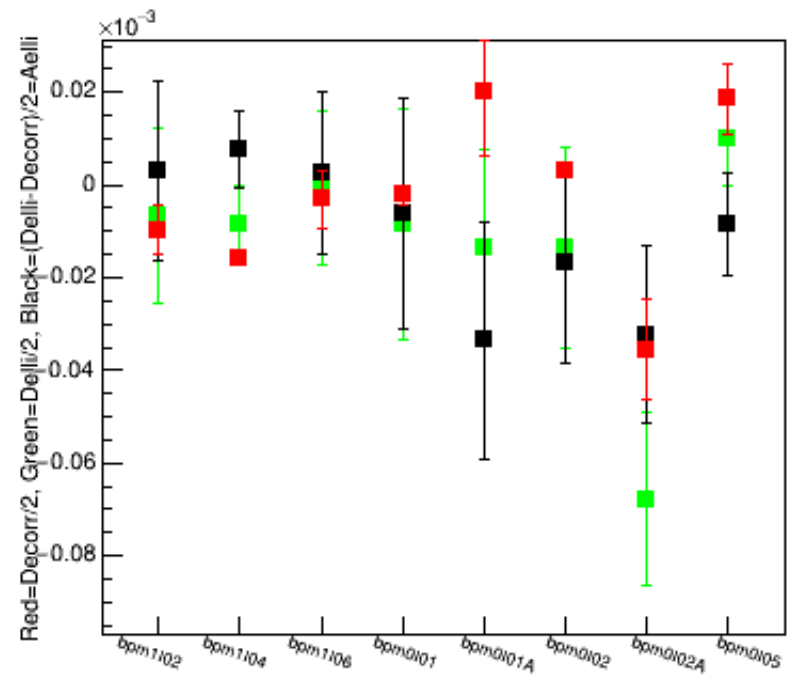

Figure 6-60: RTP cell: elliptical spot-size asymmetries in the injector (Run3331)

Using e-beam measurements in the injector, we have set bounds of $<2 \times 10^{-4}$ on $A_{\text {elli }}$, which are consistent with laser table measurements. We note that while R\&D methods ${ }^{55}$ for measuring $A_{\sigma}$ in the e-beam line are helpful, the MOLLER

\footnotetext{
${ }^{53} \kappa=18.76 \mathrm{~mm}$ and $a \approx 2 \kappa=37.52 \mathrm{~mm}$ for M15 antenna BPMs [94] [97]

${ }^{54} 130 \mathrm{keV}$ region, bpms: 1I02,1I04,1I06,0I01,0I01A,0I02,0I02A, and 0I05

${ }^{55}$ Possibly new RF monitors, BPM $A_{\text {elli }}$ measurements (Sec. 6.6.3), modified harp scans, halo monitors, or Compton apertures might be used to infer spot-size asymmetries on the beamline
} 
experiment should not rely on the ability to make such measurements in the beamline.

\subsubsection{Longitudinal Asymmetries}

Besides clean apertures and adiabatic damping, there may be other degrees of freedom, such as longitudinal asymmetries (discussed in Sec. 6.6.4) which may be coupled into transverse asymmetries through beam transport. The example of beam transport issues shown in Fig. 6-55a, in which position differences became suddenly larger downstream in the injector, suggests some degree of freedom, not seen by BPMs early in the injector, where beam asymmetries can "hide" and emerge later in the machine [62]. Such a longitudinal asymmetries could couple to transverse asymmetries that are important for parity experiments. It is apparent that attention must be paid to these longitudinal couplings.

\section{Temporal RF bunch studies - KD*P Empirical Observations}

Interesting recent studies of temporal bunch properties show the importance of accelerator beam transport in maintaining small HCBA's. The temporal RF bunch study of the e-beam produced with the KD*P Pockels cell was done with a "chopper scan", as shown in Fig. 6-61. The chopper stretches the longitudinal pulse into an arc on a circle. A slit aperture can be narrowed down cutting out a small time slice of the RF bunch. In a chopper scan, the RF phase is scanned and the current I, charge asymmetry Aq, and beam position X,Y, is measured as a function of time in an approximately 50ps electron bunch. This type of scan provides information regarding the pulse intensity distribution, charge asymmetry distribution, and bunch position over the RF pulse (the pulse shape). 


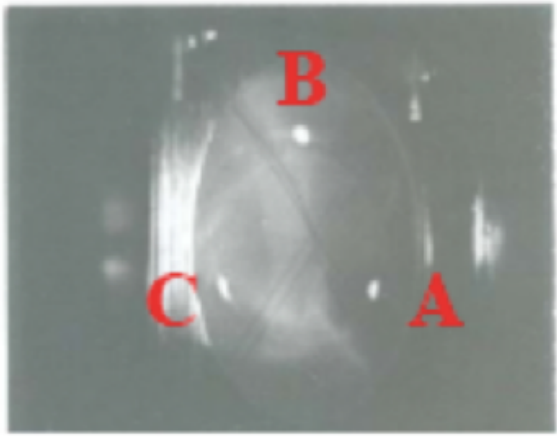

(a)

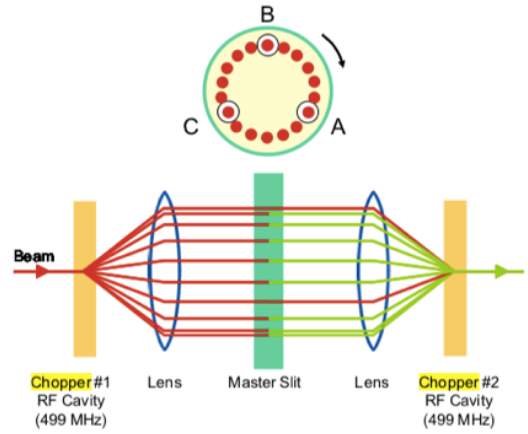

(b)

Figure 6-61: Chopper

In an attempt to study "beam halo", during Qweak, chopper scans were performed. The purpose was to examine the longitudinal beam tails and the front and back ends of the beam and look for large asymmetries. A typical chopper scan is shown in Fig. 6-62.

Asymmetry vs. bunch position

Narrow slit, scanning chopper phase to measure portions of each bunch
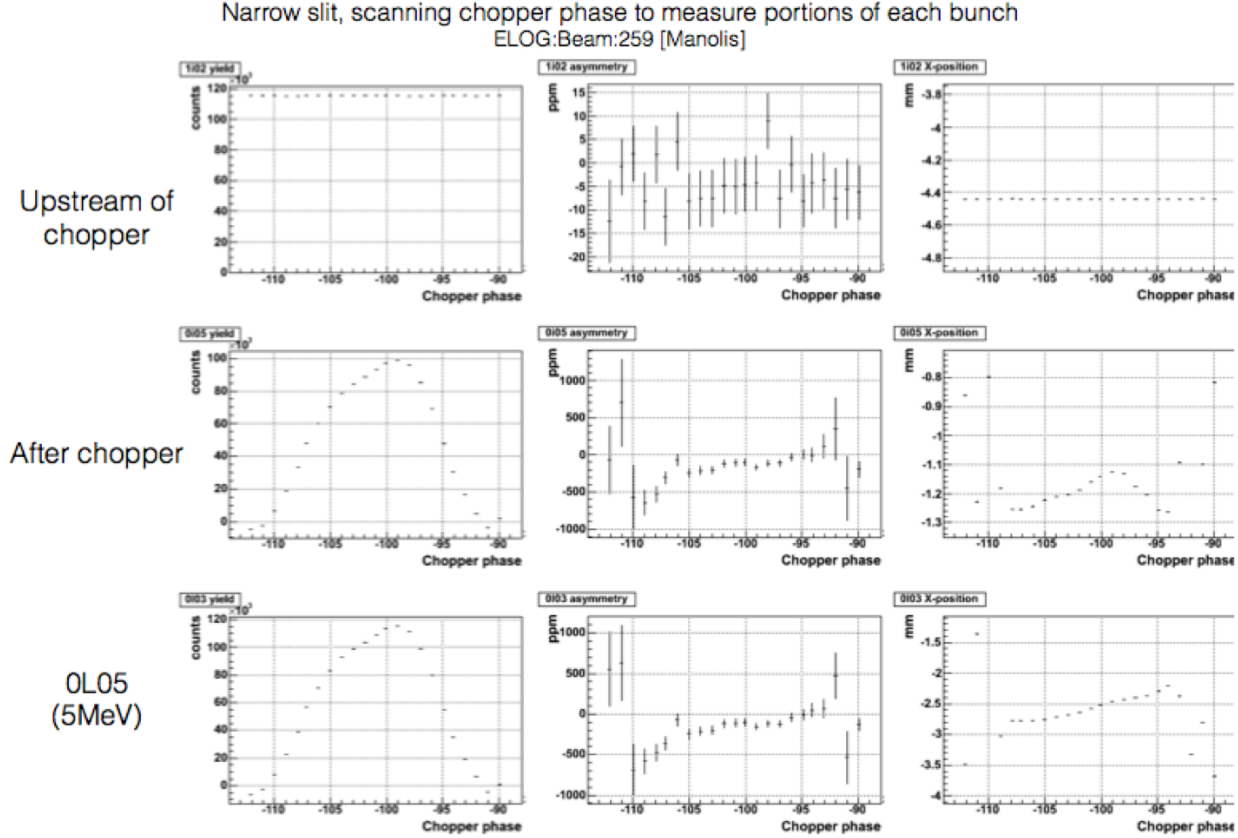

http://qweak.jlab.org/elog/Beam/259

Figure 6-62: Qweak chopper scan

More recently, chopper scans were performed with $\mathrm{KD}^{*} \mathrm{P}$ with the new gun as 
shown in Fig. 6-63. Fig. 6-63 a pulse length/pulse duration of $5.4^{\circ}$ at $500 \mathrm{MHz}$ (or $30 \mathrm{fsec}, \sim 9 \mathrm{~mm})$. We observe slightly larger asymmetries in the tails of the beam pulse in the plot of Aq ("asymbpm0I05ws") vs chopper phase. Longitudinal charge asymmetry in the beam tails can be characteristic of a longitudinal bunch length asymmetry/spot size asymmetry. A bunch length asymmetry could develop via space charge effects. For example, a beam with more charge density will have a tendency to blow-up faster with greater expansion rate in both transverse and longitudinal dimensions as is travels through the beamline early in the injector. Longtudinal bunch length asymmetries could develop further downstream in the injector, during or after acceleration, and is challenging to measure.

We also observe the edges of the beam are spatial separated from the core of the beam in plots of bpmx, bpmy vs chopper phase. There is a transverse coupling of $\frac{d X}{d \phi}=-0.09 \mathrm{~mm} / \mathrm{deg}, \frac{d Y}{d \phi}=-0.13 \mathrm{~mm} / \mathrm{deg}$. The transverse temporal structure in $\mathrm{X}$ indicates a "C" shape and the structure in $\mathrm{Y}$ indicates an "S" shape. The $1^{\text {st }}$ moment coupling term $\frac{d X}{d \phi}$ could be from a relative tilt in the beam propagation axis with respect to the bpm's measurement axis (either a crooked beam or a crooked bpm). $2^{\text {nd }}$ moment curvature in $X(\phi), \frac{d^{X}}{d \phi^{2}}$ indicates a " $\mathrm{C}$ "shape. 3rd moment curvature in $X(\phi), \frac{d^{3} X}{d \phi^{3}}$ indicates an "S" shape. Similarly, $1^{\text {st }}$ moment $\frac{d D_{x}}{d \phi}$ could be from a helicity correlated relative tilt in the beam propagation axis, like an angle-difference. A $2^{\text {nd }}$ moment curvature $\frac{d^{2} D_{x}}{d \phi^{2}}$ indicates a helicity correlated "C" shape deviation. A $3 r d$ moment curvature $\frac{d^{3} D_{x}}{d \phi^{3}}$ indicates a helicity correlated "S" shape deviation. 


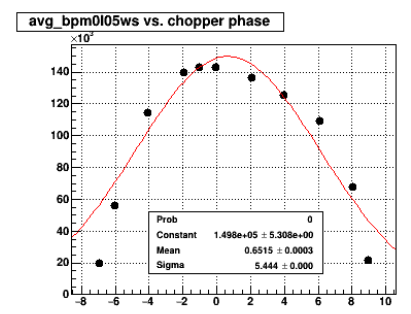

phase(deg)

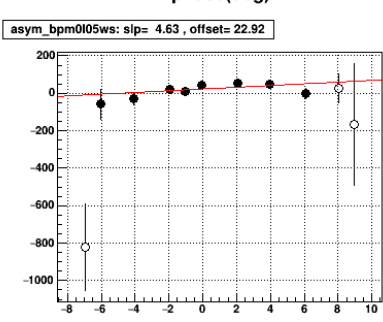

chopper phase(deg)

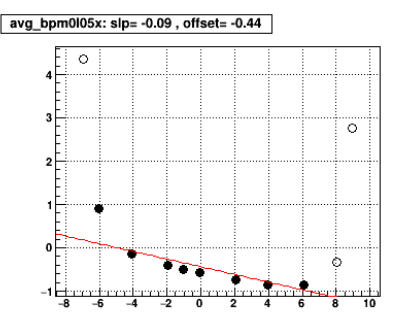

chopper phase(deg)

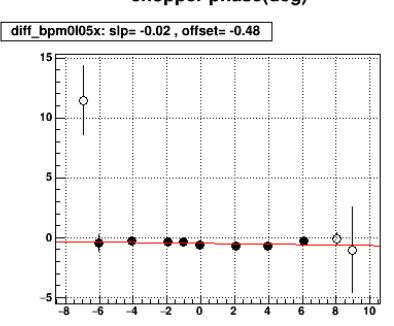

chopper phase(deg)

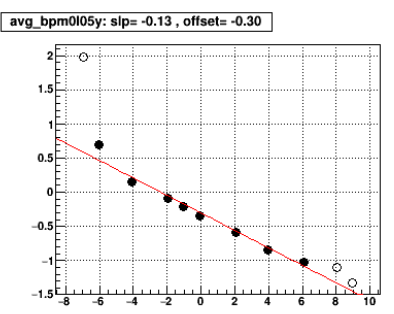

chopper phase(deg)

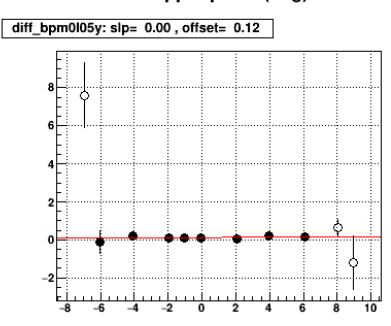

chopper phase(deg)

Figure 6-63: KD*P Chopper Scan 2018, S2 Run4202 bpm0I05 [257]

\section{Formulation}
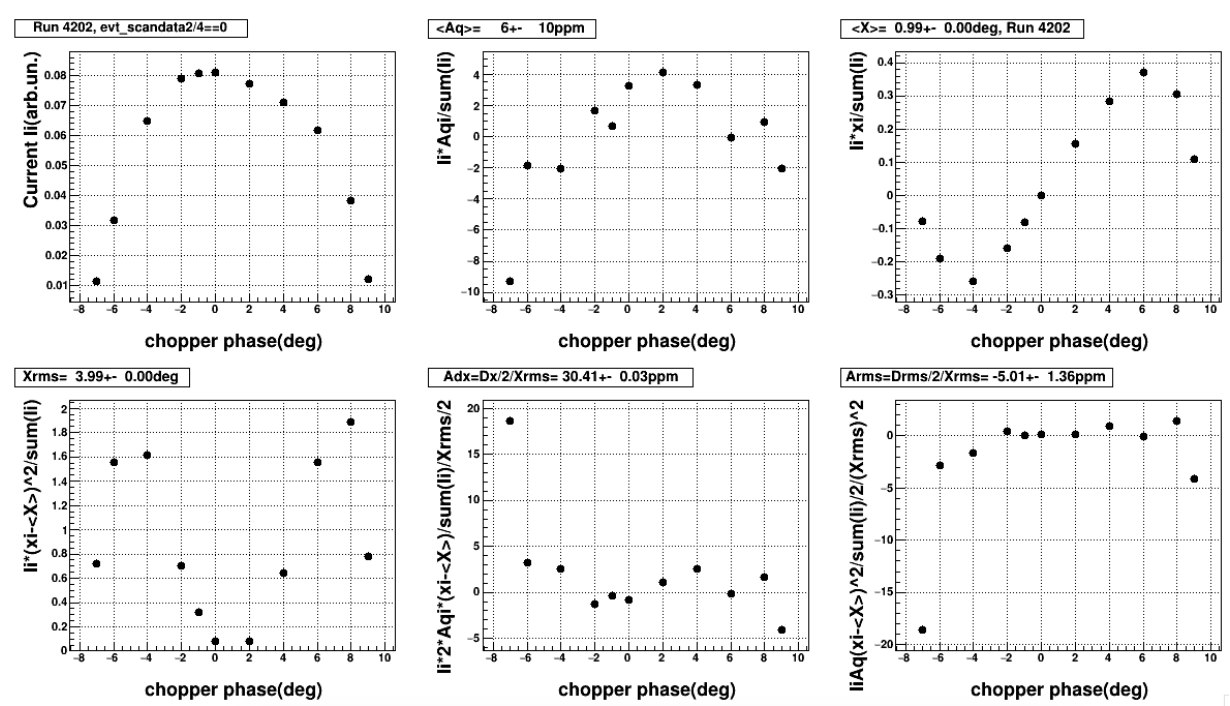

Figure 6-64: Computations for KD*P Chopper Scan 2018, S2 Run4202 bpm0I05 [257]

The 4-D electron beam pulse can be parametrized in $\mathrm{z}$ as:

$$
I(x, y, z, t): \quad t=\frac{\phi}{2 \pi f} \quad z=v_{z} t \quad I(z)=\iint I(x, y, z) d x d y
$$


Aq various points along the chopper phase $\phi_{i}$, we measure the beam current $I_{i}$ that passes through the chopper slit, charge asymmetry $A_{q}$, transverse beam position bpmx,y and position differences $D_{x}, D_{y}$. Using $I_{i}$ and $A_{q}$ in particular, we can determine the longitudinal beam properties including the pulse duration/length $\sigma_{\phi}$, the bunch-length asymmetry $A_{\sigma_{\phi}}$, mean longitudinal phase $\langle\phi\rangle$, and the longitudinal phase difference/longitudinal position difference $\left\langle D_{\phi}\right\rangle$.

$$
\begin{gathered}
\left\langle A_{q}\right\rangle=\frac{\sum_{i} \delta \phi_{i} A_{q_{i}} I_{i}}{\sum_{i} \delta \phi_{i} I_{i}} \quad\langle\phi\rangle=\frac{\sum_{i} \delta \phi_{i} \phi_{i} I_{i}}{\sum_{i} \delta \phi_{i} I_{i}} \quad \sigma_{\phi}=\sqrt{\frac{\sum_{i} \delta \phi_{i}\left(\phi_{i}-\langle\phi\rangle\right)^{2} I_{i}}{\sum_{i} \delta \phi_{i} I_{i}}} \\
\left\langle D_{\phi}\right\rangle=\frac{\sum_{i} \delta \phi_{i} 2 I_{i} A_{q_{i}}\left(\phi_{i}-\langle\phi\rangle\right)}{\sum_{i} \delta \phi_{i} I_{i}} \\
\left\langle D_{\sigma_{\phi}}\right\rangle=\sqrt{\frac{\sum_{i} \delta \phi_{i} 2 I_{i} A_{q_{i}}\left(\phi_{i}-\langle\phi\rangle\right)^{2}}{\sum_{i} \delta \phi_{i} I_{i}}} \quad\left\langle A_{\sigma_{\phi}}\right\rangle=\frac{\left\langle D_{\phi}\right\rangle}{2 \sigma_{\phi}}
\end{gathered}
$$

A longitudinal phase difference could arise from a variety of sources (including possibly a large frequency chirp on laser pulse). These longitudinal phase differences/position differences can couple transversely through $\frac{d X}{d \phi}$ as

$$
D_{x}=\frac{d X}{d \phi} A_{D \phi} 2 \sigma_{x}=\left\langle D_{\phi}\right\rangle \frac{d X}{d \phi} \frac{\sigma_{x}}{\sigma_{\phi}}
$$

where we define spot size $\sigma_{x}=X_{r m s}$.

The longitudinal bunch length asymmetry, if it arises from space charge effects, could feasibly indicate comparable transverse spot-size asymmetries from the same mechanism

$$
A_{\sigma} \approx A_{\sigma_{\phi}}
$$

The computations shown in Fig. 6-64 indicate a bunch-length asymmetry of $A_{\sigma_{\phi}}=-5 \times 10^{-} 6$, a longitudinal position asymmetry of $A_{D \phi}=30 \mathrm{ppm}$ at $500 \mathrm{MHz}$, a longitudinal position difference of $D_{\phi}=2.4 \times 10^{-4} \mathrm{deg}$ at $500 \mathrm{MHz}$ (or $1.3 \mathrm{f} \mathrm{sec}$, $\sim 400 \mathrm{~nm}$ ), and a transverse coupling of $\frac{d X}{d \phi}=-0.09 \mathrm{~mm} / \mathrm{deg}, \frac{d Y}{d \phi}=-0.13 \mathrm{~mm} / \mathrm{deg}$ 
which indicates potential induced transverse position differences of $D_{x}=-22 \mathrm{~nm}$, $D_{y}=-31 \mathrm{~nm}$ on a $\sigma=0.5 \mathrm{~mm}$ beam. We examine a more extreme case in the following sections.

\section{Longitudinal Position Differences}

When minimizing e-beam position differences with the RTP cell, we noticed that further downstream, after the chopper, there was a change in the magnitude of the position differences: they became larger (see Fig. 6-55a). This sudden change suggests beam transport issues and could have been due to beam spot-size blowup or it could suggest a "hidden" beam parameter that only becomes manifest in bpms further downstream in the injector. Longitudinal position differences could potentially be this "hidden" beam parameter.

In the chopper scan of Fig. 6-64, with ordinary running conditions, the potential induced transverse position differences were small $(<30 \mathrm{~nm})$. Here we examine another chopper scan in a more extreme case. We set the charge asymmetry to $A_{q}=1500 \mathrm{ppm}$ on a bpm before the chopper (bpm1I04) and then performed the chopper scan shown in Fig.6-65.

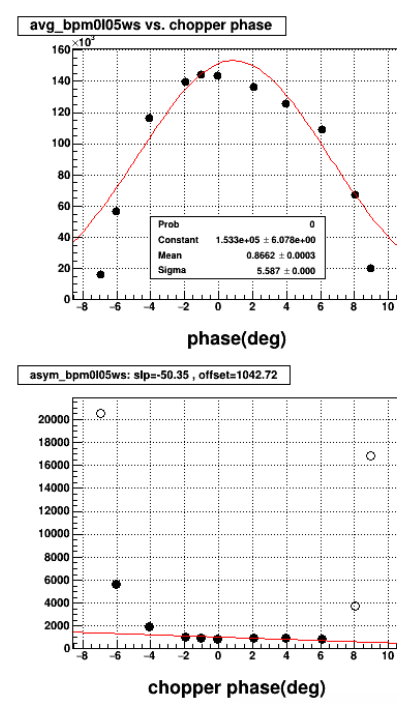

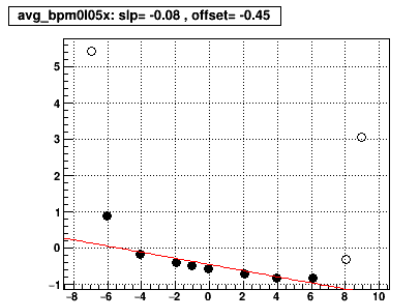

chopper phase(deg)

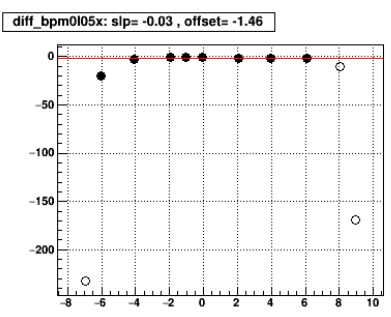

chopper phase(deg)

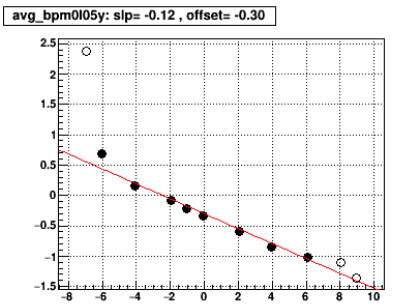

chopper phase(deg)

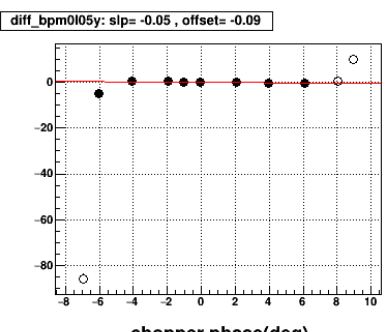

chopper phase(deg)

Figure 6-65: Chopper Scan: S2, bpm0I05, KD*P, Run4202, [257] 

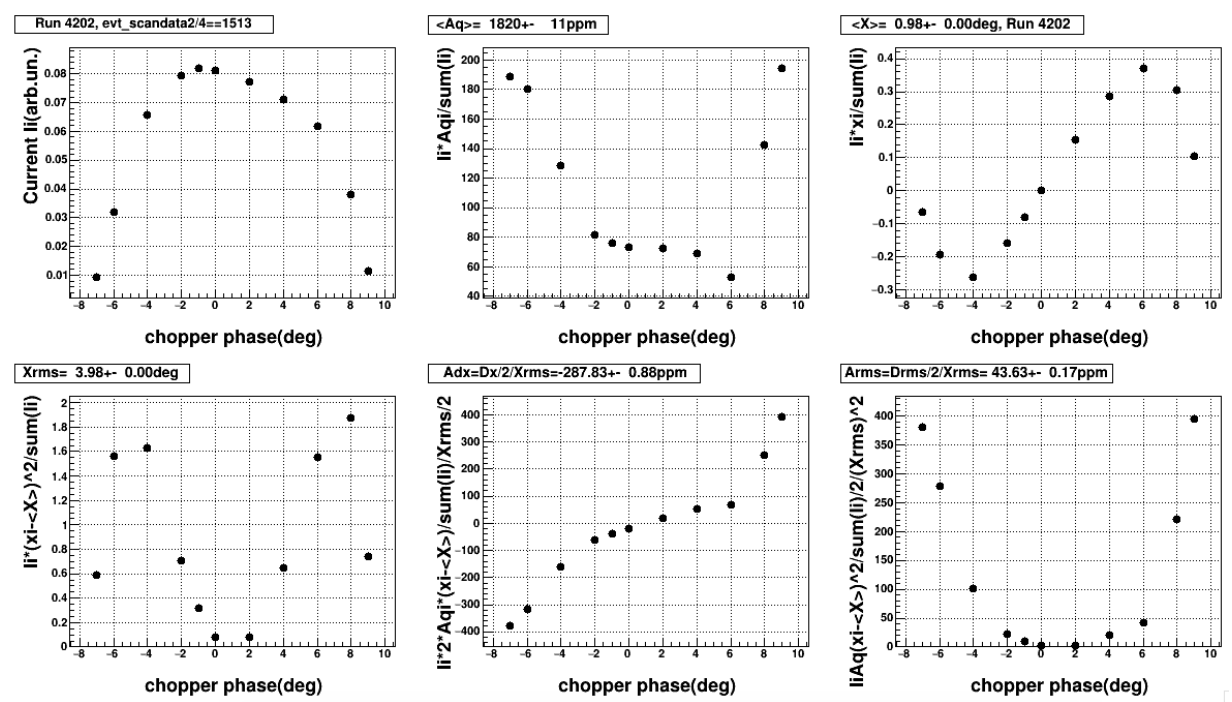

Figure 6-66: Chopper Scan Computations: S2, bpm0I05, KD*P, Run4202, [257]

The computations shown in Fig. 6-66 indicate a longitudinal position asymmetry of $A_{D \phi}=-288 \mathrm{ppm}$ at $500 \mathrm{MHz}$, a longitudinal position difference of $D_{\phi}=$ $-2.3 \times 10^{-3} \mathrm{deg}$ at $500 \mathrm{MHz}$ ( or $12.8 \mathrm{fsec}, \sim 3.8 \mu \mathrm{m}$ ), and a transverse coupling of $\frac{d X}{d \phi}=-0.08 \mathrm{~mm} / \mathrm{deg}, \frac{d Y}{d \phi}=-0.12 \mathrm{~mm} / \mathrm{deg}$ from which we infer potential induced transverse position differences of $D_{x}=184 \mathrm{~nm}, D_{y}=276 \mathrm{~nm}$ on a $\sigma=0.5 \mathrm{~mm}$ beam. The transverse coupling structure in X shows a "C" shape and Y shows an "S" shape. The position difference structure of $D_{x}$ shows a "C" shape while Dy shows an "S" shape.

It is possible that some of the longitudinal-transverse coupling $\frac{d X}{d \phi}$ could be simply an artifact of performing the chopper scan, moving the overall beam position with a changing chopper phase. To make sure the transverse coupling was indeed due to the front and back ends of the electron beam pulse actually being at different transverse positions in space, we performed a chopper scan with the slit fully open. With the slit fully open, the overall beam position only changed with a slope of $\frac{d X}{d \phi}<0.03$ with respect to chopper phase. In contrast, with the slit closed, the longitudinal-transverse coupling was $\frac{d X}{d \phi}=-0.2$. So $<10 \%$ of the observed coupling was due to an overall beam motion artifact of performing the 
chopper scan.
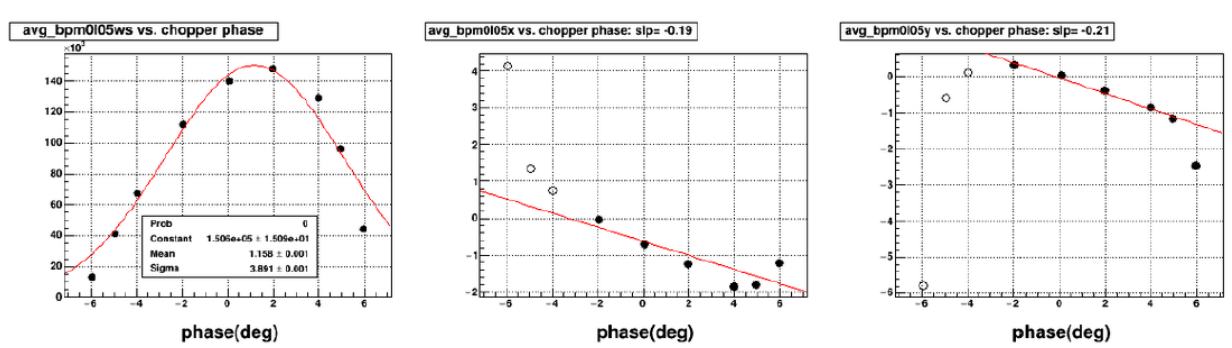

Figure 6-67: KD*P Chopper scan with slit closed [62]

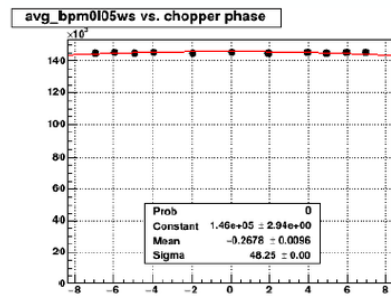

phase(deg)

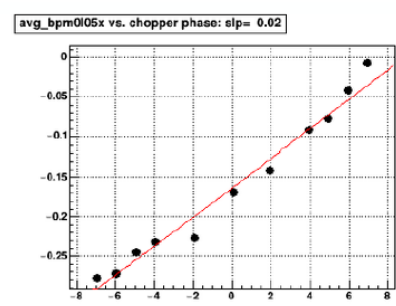

phase(deg)

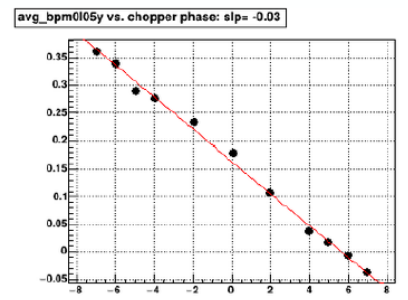

phase(deg)

Figure 6-68: $\mathrm{KD}^{*} \mathrm{P}$ Chopper scan with slit open [62]

\section{Longitudinal Bunch Length Asymmetries}

Observe that the bottom left panel of Fig. 6-65 shows that when there is an overall charge asymmetry $A_{q}=1500 \mathrm{ppm}$ coming off the cathode, by the time the e-beam reaches the chopper, the tails of the beam pulse have developed very large charge asymmetries, more so than in the main part of the beam pulse. With non-zero average charge asymmetry, the beam "blows up" with large asymmetries in tail, which are not as obvious when the average Aq is small. This suggests a bunch-length asymmetry, where the right-handed e-beam with more charge has spread out more than the the left-handed e-beam with less charge. Space charge effects could be a mechanism that would cause this bunch-length asymmetry.

The computations shown in Fig. 6-66 indicate a bunch-length asymmetry of $A_{\sigma_{\phi}}=4.4 \times 10^{-} 5$ on a pulse length of $3.98^{\circ}$ at $500 \mathrm{MHz}$ (or $22.1 \mathrm{fsec}, 6.63 \mathrm{~mm}$ ) from which we infer a potential induced transverse spot-size asymmetry of $A_{\sigma} \sim$ $A_{\sigma_{\phi}}=4.4 \times 10^{-} 5$ assuming it arises from the same space charge effect mechanism. 
For Moller, it is important to consider these longitudinal bunch length asymmetries and they mean in terms of spot-size asymmetries in the experimental Hall. This longitudinal bunch-length asymmetry, unlike transverse spot size asymmetries, are not necessarily suppressed in acceleration by synchrotron stochastic processes. If this longitudinal asymmetry winds up coupled to the transverse dimension, it translates into a transverse spot-size asymmetry in the Hall.

\section{Longitudinal Structure Origins - Calculations}

The aforementioned chopper studies were performed with KD*P. Further chopper studies should be performed with RTP in the future in case there is any intrinsic difference in how the two types of cell affect the longitudinal beam structure. Here we analytically verify that the longitudinal structure does not intrinsically arise from simply using an RTP Pockels cell instead of a KD*P cell. We note that empirical measurements should also be performed with RTP.

\section{Aside: Helicity correlated phase shift}

We note the helicity correlated average phase shift difference is fairly small. This can be reasoned out from the thermal compensation design. Considering the birefringence corresponding to a difference in refractive indices $n_{z}-n_{y}$ ), the helicity-dependent part adds between the two crystals, while the helicity-independent part cancels. Conversely, it must be that the the phase shift corresponding to the average of the refractive indices $n_{y}+n_{z}$, the helicity-dependent part cancels between the crystals, while the helicity-independent part adds. We can see this by plugging in equations for the two crystals' fields $E_{z 1}^{R(L)}=E_{z}^{R}(L)=\mp E_{z 1,0}-E_{\Delta 1}=$ $\mp\left(\left|E_{\lambda / 4}\right|+E_{\alpha_{1}}\right)-E_{\Delta}$ and $E_{z 2}^{R(L)}= \pm E_{0}+E_{\Delta 2}= \pm\left(\left|E_{\lambda / 4}\right|+E_{\alpha_{2}}\right)+E_{\Delta}$. We define the difference in crystal length via $L_{1}=L_{0}+\delta L_{12} / 2$ and $L_{2}=L_{0}-\delta L_{12} / 2$. We obtain for the average phase shifts

$$
\phi_{\text {avg }}^{R(L)}=\pi / \lambda\left(\left(n_{0, z}+n_{0, y}\right)\left(L_{1}+L_{2}\right)\right.
$$




$$
\left.-\frac{1}{2}\left(n_{0, z}^{3} r_{33}+n_{0, y}^{3} r_{23}\right)\left(\left(\mp\left(\left|E_{\lambda / 4}\right|+E_{\alpha_{1}}\right)-E_{\Delta}\right) L_{1}+\left( \pm\left(\left|E_{\lambda / 4}\right|+E_{\alpha_{2}}\right)+E_{\Delta}\right) L_{2}\right)\right)
$$

We obtain for the helicity correlated average phase shift difference

$$
\phi_{\text {avg }}^{R}-\phi_{\text {avg }}^{L}=\pi / \lambda\left(n_{0, z}^{3} r_{33}+n_{0, y}^{3} r_{23}\right)\left(\left|E_{\lambda / 4}\right|+\frac{E_{\alpha_{1}}+E_{\alpha_{2}}}{2}\right) \delta L_{12}+L_{0}\left(E_{\alpha_{1}}-E_{\alpha_{2}}\right)
$$

For $\lambda=780 \mathrm{~nm}, n_{0, z}=1.867, n_{0, y}=1.783, r_{23}=12.5 \mathrm{pm} / \mathrm{V}, r_{33}=35 \mathrm{pm} / \mathrm{V}$, $L_{0}=10 \mathrm{~mm}$, we note $\left(n_{0, z}^{3} r_{33}+n_{0, y}^{3} r_{23}=156.9 \mathrm{pm} / \mathrm{V}\right.$. For a quarter-wave-field of $\left|E_{\lambda / 4}\right|=\frac{\lambda}{4 L_{0}\left(n_{z 0}^{3} r_{33}-n_{y 0}^{3} r_{23}\right)}=124.3 \mathrm{~V} / \mathrm{mm}$ and a length difference of $\delta L_{1,2}=2 \mu \mathrm{m}$, and for $E_{\alpha_{1}}=E_{\alpha_{2}}=0$ we obtain

$$
\phi_{\text {avg }}^{R}-\phi_{\text {avg }}^{L}=\pi / \lambda(156.9 \mathrm{pm} / \mathrm{V})(124.3 \mathrm{~V} / \mathrm{mm}) 2 \mu \mathrm{m}=122 \mathrm{pm} / \lambda
$$

This helicity correlated phase shift difference would, in a pulsed laser, correspond to a longitudinal position difference along the propagation axis $D_{z}$ (as opposed to $D_{x}$ and $\left.D_{y}\right)$, but it would be a very tiny effect. We note that whatever helicity correlated phase shift difference is caused by the difference in crystal length can be corrected by weighting one of the crystals with higher voltages, such that

$$
\frac{E_{\alpha_{1}}-E_{\alpha_{2}}}{\left|E_{\lambda / 4}\right|+\frac{E_{\alpha_{1}}+E_{\alpha_{2}}}{2}}=\frac{\delta L_{12}}{L_{0}}
$$

having the shorter crystal proportionately do more work essentially, via applying a difference in the crystals $\alpha$-phase voltages. We typically keep these $\alpha$-voltages the same for both crystals, but in principle, we could change them slightly without affecting cell operation significantly. If $E_{\alpha_{1}}$ and $E_{\alpha_{2}}$ differed by $10 \%$ of $E_{\lambda / 4} \mid$, this could make a helicity correlated phase shift difference

$$
\phi_{\text {avg }}^{R}-\phi_{\text {avg }}^{L} \approx \pi / \lambda(156.9 \mathrm{pm} / \mathrm{V})(10 \mathrm{~mm})(0.1 \times 124.3 \mathrm{~V} / \mathrm{mm})=61 \mathrm{~nm} / \lambda
$$


corresponding to a longitudinal position difference along the propagation axis $D_{z}=61 \mathrm{~nm}$ (aka helicity correlated time difference $D_{t}=0.2 \mathrm{fs}$ at $v=c$ ). So we have more then enough range in the voltages to correct a helicity correlated phase longitudinal position difference on the order of $D_{z} \sim 100 \mathrm{~nm}$, only changing the $\alpha$-voltages by $10 \%$ of the $Q W V$.

\subsection{Conclusion}

We have presented an innovative ultra-fast switch RTP cell design which uses electric field gradients to counteract crystal non-uniformities, leading to improved extinction ratios (in $\lambda / 2$-wave configuration) and minimization of voltage dependent beam steering (in $\lambda / 4$-wave configuration). This RTP Pockels cell design has been demonstrated to be capable of producing precisely controlled polarized electron beam at Jefferson Laboratory and control beam steering down to the nm-level with voltage feedback. Additionally, the cell has an increased number of degrees of freedom and can control both S1 and S2 polarizations. The precision reached with the RTP cell offers sufficient control over and minimization of intensity asymmetry, position differences, and spot-size asymmetry to perform PREX II, an upcoming parity violation electron scattering experiment at JLab. The position differences achieved with electron beam were $<30 \mathrm{~nm}$. The RTP Pockels cell system will provide fast flipping and suitable control of position differences and parity quality beam for the future MOLLER experiment, providing an unprecedented precision on the electron weak charge and electroweak mixing angle. Future studies will explore the importance of accelerator beam transport in maintaining small helicity correlated beam asymmetries achieved by the Pockels Cell. 


\section{Chapter 7}

\section{Conclusion and Summary}

MOLLER will measure $\sin ^{2} \theta_{W}$ at low momentum transfer $\left(Q^{2} \approx 0.0056 \mathrm{GeV}^{2}\right)$ by examining the parity-violating asymmetry $A_{P V}$ to $0.8 \mathrm{ppb}$ precision in the scattering of a longitudinally polarized electron beam off electrons in a liquid hydrogen target; thereby measuring the weak charge of the electron $Q_{W}^{e}$, proportional to the product of the electron's vector and axial-vector couplings to the $Z_{0}$ boson, to $2.4 \%$ precision. We have presented a number of the experimental techniques which will be employed to to reach the experimental precision goals: $2.1 \%$ statistical and $1.1 \%$ systematic. These include achieving the requirements on the properties fo the polarized electron beam, a high luminosity liquid hydrogen target with extremely small pulse-to-pulse density fluctuations, and the requirement of a high signal-to-background ratio [46]. In particular, we have new Pockels cell for the polarized source, using RTP (Rubidium Titanyl Phosphate) crystals, designed to meet MOLLER's statistical and systematic experimental precision goals.

We have presented an RTP Pockels cell design that provides the best of both worlds for MOLLER: fast transition and improved effective uniformity. Using RTP was incredibly successful in terms of reducing transition time, and allowing for higher flip rate and data taking. We have demonstrated $8 \mu$ s transitions with a large aperture $(12 \mathrm{mmx} 12 \mathrm{~mm})$, transverse, 2 crystal RTP cell (a factor of 10X improvement over the longitudinal KD*P previously used at JLab). Furthermore, we 
have shown the new RTP Pockels cell is capable of self-correcting intrinsic crystal non-uniformity effects. These effects are counteracted with controlled electric-field gradients so that laser beam helicity correlated position motion is controllable and kept on the order of the $\sim 10 \mathrm{nrad}, 10 \mathrm{~nm}$ level, while the transition time is kept below $10 \mu \mathrm{s}$. The electron beam position differences in the $130 \mathrm{keV}$ region of the JLab injector were kept at the sub-30nm level.The RTP Pockels cell system has been demonstrated to be capable of producing precisely controlled polarized electron beam at Jefferson Laboratory and control beam steering down to the $1 \mathrm{~nm}$-level with voltage feedback. The system will provide fast flipping and suitable control of position differences and parity quality beam for the future MOLLER experiment, providing an unprecedented precision on the electron weak charge and electroweak mixing angle.

The precision reached with the RTP cell offers sufficient control over and minimization of intensity asymmetry, position differences, and spot-size asymmetry to be used in the upcoming PREX II (Lead Radius Experiment) which will measure the weak skin thickness of the ${ }^{208} \mathrm{~Pb}$ nucleus in 2019. PREX-I measured an asymmetry of $0.6 \mathrm{ppm}$, and inferred a neutron skin thickness of $R_{n}-R_{p}=0.33_{-0.18}^{+0.16}$ fm, interestingly inconsistent with measured neutron star properties and existing models (though it is within sigma). PREX-II is expected to achieve a factor of 3 improvement on the precision of the neutron skin thickness than for PREX-I, which was statistics limited.

We have described the experimental design of PREX-II, which will measure the parity violating asymmetry $A_{P V}$ for $1 \mathrm{GeV}$ electrons scattering from ${ }^{208} \mathrm{~Pb}$ at five degrees, and should be sensitive to the neutron radius of ${ }^{208} \mathrm{~Pb}$ to $1 \%( \pm 0.05 \mathrm{fm})$ precision [164]. We have described several ways in which PREX-II precision will be improved over PREX-I including greater statistics, reduced beam asymmetries, and multiple transverse asymmetry measurements for ${ }^{12} \mathrm{C}$ and ${ }^{208} \mathrm{~Pb}$ both from Mainz and PREX-II. PREX-I did not achieve its $A_{P V}$ statistical precision goal of $3 \%$ due to equipment failure as a result of high radiation [171]. We have 
presented extensive work on PREX-II radiation shielding, ensuring the upcoming experiment will be radiation-hard and the desired statistics will be obtained. While the systematics were well under control in PREX-I, experience suggests that by using the new RTP cell, the beam systematics could be controlled even better than they were during PREX-I.

Lastly, we have presented the results of the ${ }^{12} \mathrm{C}$ transverse asymmetry measurement performed at MAMI at various $Q^{2}$ at constant $570 \mathrm{MeV}$ beam energy. The PREX-II targets are composed of both ${ }^{208} \mathrm{~Pb}$ and ${ }^{12} \mathrm{C}$ in the form of diamond used for its thermal conductivity. Measuring the transverse asymmetry $A_{n}$ in ${ }^{208} \mathrm{~Pb}$ is important for precision scattering experiments like PREX because the parity-violating asymmetry $A_{P V}$ has a systematic uncertainty corresponding to a false measured asymmetry $A_{n}^{m}$ which comes from a non-zero transverse component of the beam polarization $A_{n}^{m}=A_{n} \vec{P}_{e} \cdot \hat{k}$. For heavy nuclei, like ${ }^{208} \mathrm{~Pb},{ }^{48} \mathrm{Ca}$ accounting for the transverse asymmetry remains a theoretical challenge and twophoton exchange calculations, which account for dispersion corrections but neglect Coulomb distortions, fail for the heavier ${ }^{208} \mathrm{~Pb}$ nucleus [209]. So, it is important that measurements of $A_{n}$ in scattering off heavy nuclei are performed for a range of $\mathrm{Z}$ at various beam energies. The MAMI data indicates the that ratio of the Compton to charge form factor is not target independent even for a light nucleus like ${ }^{12} \mathrm{C}$ and that and heavier nuclei may have an even larger deviations. Notably, similar models failed for ${ }^{208} \mathrm{~Pb}$ in PREX-I [209]. In PVES experiments, like PREX, these two-photon exchange processes provide an important contribution to the systematic uncertainties. Future measurements at MAMI will investigate the transverse asymmetry for heavier nuclei, like ${ }^{208} \mathrm{~Pb}$, at the same $Q^{2}$ values using similar experimental methodology.

We have shown the methods by which PREX-II will provide a clean and precise measurement of $R_{N}$, the RMS radius of neutrons in a heavy nucleus, and further constrain the equation of state (EOS) of highly dense matter. The results of the upcoming experiment will will have impact on both nuclear physics 
and astrophysics including atomic parity violation experiments, heavy ion collisions, neutron star structure, and the nuclear EOS for neutron stars which impacts gravitational waveforms of neutron star collisions as measured by LIGO. 


\section{Appendix A}

\section{Derivation: Angle Dependence in RTP and KD*P}

\section{A.1 Angle Deflection Within Crystal: Inside vs Outside}

Typically in an angle scan measurement, the crystal is rotated in pitch and yaw by a known angle and the laser remains fixed. In calculation, however, the laser angle within the crystal as compared to it's primary axes is used instead. Converting between these two scenarios is not as straightforward as a simple change of reference frame because of Snell's law.

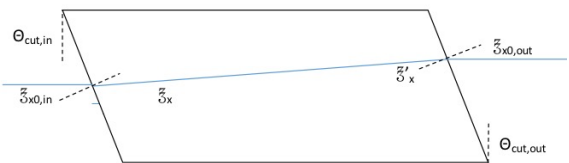

(a)

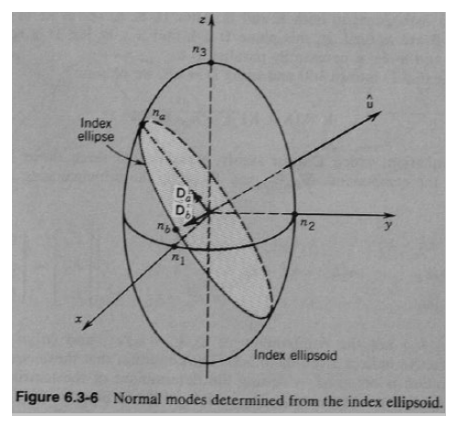

(b)

Figure A-1: (a) Angular deviations (b) index ellipsoid 
Making small angle approximations and treating the refractive index outside the crystal as 1 ,

$$
\begin{gathered}
\xi_{x_{0}, \text { out }}=\xi_{x_{0}, \text { in }}+\left(\theta_{\text {cut }, \text { out }}-\theta_{\text {cut }, \text { in }}\right) n, \quad \xi_{x}=\xi_{x_{0}, \text { in }} / n+\theta_{\text {cut }, \text { in }} \\
\xi_{x_{0}, \text { out }}=\left(\xi_{x}-\theta_{\text {cut }, \text { out }}\right) n
\end{gathered}
$$

We have taken into account the imperfect angle cuts on the crystal faces in these equations.

In general, for a birefringent material, the index of refraction is also angle dependent due to the components of the light polarization having components along different primary crystal axes, mixing the refractive indexes. $\mathrm{n}$ is more generally described as $\mathrm{n}(\theta, \phi)$ as can be computed through the index ellipsoid of the crystal, which is defined by the surface $\frac{x_{1}^{2}}{n_{1}^{2}}+\frac{x_{2}^{2}}{n_{2}^{2}}+\frac{x_{3}^{2}}{n_{3}^{2}}=1$.

We calculate $\mathrm{n}(\theta, \phi)$ by noting $x_{1}=n(\theta, \phi) \sin (\theta) \cos (\phi), x_{2}=n(\theta, \phi) \sin (\theta) \sin (\phi), x_{3}=$ $n(\theta, \phi) \cos (\theta)$. Hence,

$$
\frac{1}{n^{2}(\theta, \phi)}=\frac{\sin ^{2}(\theta) \cos ^{2}(\phi)}{n_{1}^{2}}+\frac{\sin ^{2}(\theta) \sin ^{2}(\phi)}{n_{2}^{2}}+\frac{\cos ^{2}(\theta)}{n_{3}^{2}}
$$

For polarization mainly along a primary axis $n_{i}$ with small angles deviations $\xi$, the perturbation on the refractive index will take the quadratic form

$$
n(\xi) \approx n_{i}\left(1+b \xi^{2}\right)
$$

where b is small. Snell's Law in a birefringent material is rewritten as a phasematching condition

$$
\begin{gathered}
n(\xi) \sin (\xi)=n_{0} \sin \left(\xi_{0}\right) \\
n(\xi) \xi \approx n_{0} \xi_{0}
\end{gathered}
$$

where $\xi$ is the propagation angle inside the crystal, and $\xi_{0}$ is the propagation 
angle outside the crystal. Putting these equations together, we obtain

$$
n_{i} \xi+n_{i} b \xi^{3}-n_{0} \xi_{0}=0
$$

Since $\mathrm{b} \ll 1$, the solution is close to $n_{i} \xi \approx n_{0} \xi_{0}$. Plugging this in, we obtain

$$
\xi \approx \frac{n_{0}}{n_{i}} \xi_{0}+\frac{n_{0}^{3}}{n_{i}^{3}} b \xi_{0}^{3}, n(\xi) \approx n_{i}\left(1+b \frac{n_{0}^{2}}{n_{i}^{2}} \xi_{0}^{2}\right)
$$

Taking into account the face cuts on the crystal, and treating the refractive index outside the crystal as 1 , we obtain equation of the form

$$
\begin{gathered}
\xi_{0, \text { out }}=\xi_{0, \text { in }}+\left(\theta_{\text {cut }, \text { out }}-\theta_{\text {cut }, \text { in }}\right) n(\xi) \approx \xi_{0, \text { in }}+\left(\theta_{\text {cut }, \text { out }}-\theta_{\text {cut }, \text { in }}\right)\left(n_{i}\left(1+b \frac{1}{n_{i}^{2}} \xi_{0, \text { in }}^{2}\right)\right) \\
\xi=\xi_{0, \text { in }} / n(\xi)+\theta_{\text {cut }, \text { in }} \approx \frac{1}{n_{i}} \xi_{0, \text { in }}+\frac{1}{n_{i}^{3}} b \xi_{0}^{3}+\theta_{\text {cut }, \text { in }} \\
\xi_{0, \text { out }}=\left(\xi-\theta_{\text {cut }, \text { out }}\right) n(\xi) \approx\left(\xi-\theta_{\text {cut }, \text { out }}\right)\left(n_{i}\left(1+b \frac{1}{n_{i}^{2}} \xi_{0, \text { in }}^{2}\right)\right)
\end{gathered}
$$

\section{A.2 Fast-axis direction angle dependence in $\mathrm{KD}^{*} \mathrm{P}$}

The orientation of the effective fast axis of the crystal is different when the crystal is tilted. For $\mathrm{KD}^{*} \mathrm{P}$, the fundamental refractive indices for the $\mathrm{x}$ ' and $\mathrm{y}$ ' (along $\pm 45^{\circ}$ ) axes are approximately the same $n_{x^{\prime}} \approx n_{y^{\prime}} \approx n_{o}$, so the effective fast and slow axes are determined by the direction beam propagation within the crystal $\left(\xi_{x^{\prime}}, \xi_{y^{\prime}}\right) \approx \frac{1}{n_{o}}\left(\xi_{x^{\prime} 0}, \xi_{y^{\prime} 0}\right)$ due to the crystal tilt, which mixes the ordinary index $n_{o}$ with the extraordinary index $n_{e}$. We note that the effective fast axis direction relative to the horizontal ( $\mathrm{x}$-axis) is simply given by

$$
\sin (\eta)=\xi_{x} / \sqrt{\xi_{x}^{2}+\xi_{y}^{2}}
$$


where $\xi_{x}=\frac{\xi_{x^{\prime}}-\xi_{y^{\prime}}}{\sqrt{2}}$ is along the horizontal axis and $\xi_{y}=\frac{\xi_{x^{\prime}}+\xi_{y^{\prime}}}{\sqrt{2}}$ is along the vertical axis. We can define the effective slow axis as $\tau=\eta+\pi / 2=$ and

$$
\sin (\tau)=\sin (\eta+\pi / 2)=\cos (\eta)=\xi_{y} / \sqrt{\xi_{x}^{2}+\xi_{y}^{2}}
$$

we refer to the fast axis as a (along angle $\eta$ ) and refer to the slow axis as b (along angle $\tau$ ). We note that in $\mathrm{KD}^{*} \mathrm{P}$, the effective slow axis $\mathrm{b}$ is determined by the incoming beam's transverse component direction $\vec{\xi}_{b}=\left(\xi_{x}, \xi_{y}\right)$ upon entering the crystal. The fast-axis a (along $\eta$ ) is simply transverse to that.

\section{A.3 Asymmetry from extraneous birefringent ele- ment}

When the Pockels cell flips between right and left circular polarization states, it can be described as inducing a phase shift $\delta$

$$
\delta^{R(L)}=\mp(\pi / 2+\alpha)-\Delta
$$

where $\alpha$ and $\Delta$ refer to symmetric and antisymmetric deviation from quarter wave circular polarization.

If an analyzer is inserted after the Pockels cell with transmission coefficients $T_{x}, T_{y}$ along an axis $\mathrm{x}, \mathrm{y}$ where $\psi$ is the angle subtended between the analyzing direction $\mathrm{x}$ and the horizontal axis. The transmission through a polarizing element for each polarization state is described by:

$$
\begin{aligned}
& T^{R(L)}=T \frac{1}{2}\left(1+\epsilon / T \cos (2(\psi-\eta)) \cos \delta^{R(L)}\right) \\
& T^{R(L)}=T \frac{1}{2}\left(1+\epsilon / T \sin (2(\eta-\psi)) \cos \delta^{R(L)}\right)
\end{aligned}
$$

where $\epsilon=T_{x}-T_{y}, T=\left(T_{x}-T_{y}\right) / 2$ defines the analyzing power of the polarizer(or 
polarizing element) and $\eta$ is the effective fast-axis of the Pockels cell crystal relative to the horizontal axis. The intensity asymmetry $I_{A}$ is given by

$$
\begin{aligned}
& A_{q}=\frac{T^{R}-T^{L}}{T^{R}+T^{L}} \approx \frac{\epsilon}{T} \cos (2(\psi-\eta)) \frac{1}{2}\left(\cos \delta^{R}-\cos \delta^{L}\right) \approx-\frac{\epsilon}{T} \cos (2(\psi-\eta)) \Delta \\
& A_{q}=\frac{T^{R}-T^{L}}{T^{R}+T^{L}} \approx \frac{\epsilon}{T} \sin (2(\eta-\psi)) \frac{1}{2}\left(\cos \delta^{R}-\cos \delta^{L}\right) \approx-\frac{\epsilon}{T} \sin (2(\eta-\psi)) \Delta
\end{aligned}
$$

where we have used the approximation $\cos \delta^{R}-\cos \delta^{L} \approx \delta^{R}+\delta^{L}=-2 \Delta$.

The asymmetry arising from an extraneous birefringent element contributing a delta-phase shift $\Delta=\zeta=2 \pi \delta n / L$ is given by

$$
A_{q}=-\frac{\epsilon}{T} \zeta \sin (2(\eta-\psi))
$$

\section{A.4 Refractive Index angle dependence in $\mathrm{KD}^{*} \mathrm{P}$}

Specifically, for $\mathrm{KD}^{*} \mathrm{P}$, the light propagates along the crystal z-axis direction, with incident polarization horizontal, with nearly equal components along the $n_{x}^{\prime}$, $n_{y}^{\prime}$ directions $\left( \pm 45^{\circ}\right)$ which are the crystals new primary axes when under the influence of an electric field. $\hat{r} \approx(0,0,1)$ and $\hat{p} \approx\left(\frac{1}{\sqrt{2}}, \frac{1}{\sqrt{2}}, 0\right)$.

Upon entering the crystal, the light will split into 2 rays, ray1 with polarization along the effective fast axis $a-z$ plane, and ray2 with polarization along the slow axis $b-z$ plane where $\mathrm{a}$ and $\mathrm{b}$ axis are perpendicular to one another.

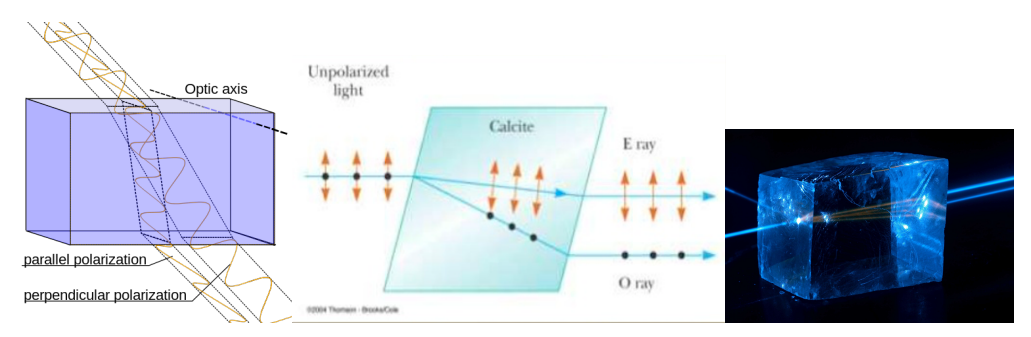

Figure A-2: Light refracting upon entering a birefringent material such as calcite [310] 


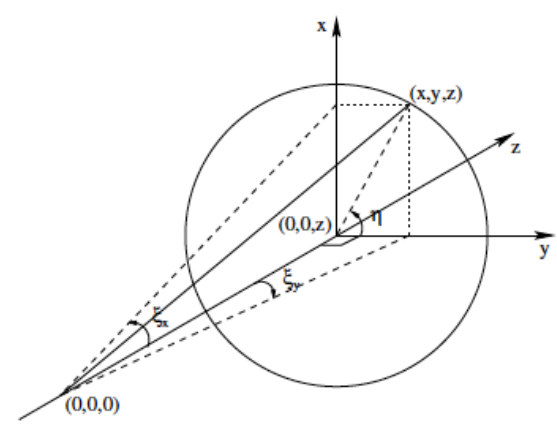

Figure A-3: The ray of light incident on an optical material at an angle $\xi$. $\eta$ is the orientation of the material's fast-axis from y-axis. [163]

The input ray propagating in the direction $\hat{r} \approx\left(\xi_{x 0}, \xi_{y 0}, 1-\xi_{x 0}^{2} / 2-\xi_{y 0}^{2} / 2\right)$ in the lab $\mathrm{x}-\mathrm{y}-\mathrm{z}$ coordinate system is expressed as $\hat{r} \approx\left(\xi_{a 0}, \xi_{b 0}, 1-\xi_{a 0}^{2} / 2-\right.$ $\left.\xi_{b 0}^{2} / 2\right)$ in the a-b-z coordinate system. The ray can be divided into two polarization components $\hat{p_{01}}=\left(\cos \left(\xi_{a 0}\right), 0,-\sin \left(\xi_{a 0}\right)\right) \approx\left(1-\xi_{a 0}^{2}, 0,-\xi_{a 0}\right)$ and $\hat{p_{02}}=$ $\left(0, \cos \left(\xi_{b 0}\right),-\sin \left(\xi_{b 0}\right)\right) \approx\left(0,1-\xi_{b 0}^{2},-\xi_{b 0}\right)$.

For the component of input polarization along the az plane, an angle $\xi_{a 1}$ within the crystal both lengthens the propagation distance of ray1 by $\frac{L_{0} \xi_{a 1}^{2}}{2}$ in the crystal and mixes refractive indices $n_{a}, n_{z}: \hat{r_{1}}=\left(\sin \left(\xi_{a 1}\right), 0, \cos \left(\xi_{a 1}\right)\right) \approx\left(\xi_{a 1}, 0,1-\xi_{a 1}^{2} / 2\right)$ and $\hat{p_{1}}=\left(\cos \left(\xi_{a 1}\right), 0,-\sin \left(\xi_{a 1}\right) \approx\left(1-\xi_{a 1}^{2}, 0,-\xi_{a 1}\right)\right.$. An angle $\xi_{b 1}$ has no effect on the refractive index along a, only the distance of propagation is lengthened by $\frac{L_{0} \xi_{b 1}^{2}}{2}$. Putting $\xi_{a 1}$ and $\xi_{b 1}$ together, we obtain

$$
\hat{r_{1}} \approx\left(\xi_{a 1}, \xi_{b 1}, 1-\xi_{a 1}^{2} / 2-\xi_{b 1}^{2} / 2\right), \hat{p_{1}} \approx\left(1-\xi_{a 1}^{2} / 2,0,-\xi_{a 1}\right), L_{1}=L_{0}\left(1+\xi_{a 1}^{2} / 2+\right.
$$
$\left.\xi_{b 1}^{2} / 2\right)$

For this polarization direction, the index ellipsoid equation yields

$$
\begin{gathered}
\frac{1}{n_{1}^{2}\left(\xi_{a 1}\right)} \approx \frac{\left(1-\xi_{a 1}^{2}\right)^{2}}{n_{a 1}^{2}}+\frac{\xi_{a 1}^{2}}{n_{z}^{2}} \\
n_{1}\left(\xi_{a 1}\right) \approx\left(\frac{\left(1-\xi_{a 1}^{2}\right)^{2}}{n_{a 1}^{2}}+\frac{\xi_{a 1}^{2}}{n_{z}^{2}}\right)^{-}(1 / 2) \approx n_{a}\left(1+\frac{n_{z}^{2}-n_{a}^{2}}{2 n_{z}^{2}} \xi_{a 1}^{2}\right)
\end{gathered}
$$

Snell's law can be approximated as $n_{0} \xi_{a 0}=n_{1}\left(\xi_{a 1}\right) \xi_{a 1} \approx n_{a}\left(1+\frac{n_{z}^{2}-n_{a}^{2}}{2 n_{z}^{2}} \xi_{a 1}^{2}\right) \xi_{a 1} \approx$ $n_{a} \xi_{a 1}$. 
Putting these equations together, we obtain

$$
n_{a} \xi_{a 1}+n_{a} \frac{n_{z}^{2}-n_{a}^{2}}{2 n_{z}^{2}} \xi_{a 1}^{3}-n_{0} \xi_{a 0}=0
$$

Since $\frac{n_{z}^{2}-n_{a}^{2}}{2 n_{z}^{2}} \ll 1$, the solution is close to $n_{a} \xi_{a 1} \approx n_{0} \xi_{a 0}$. Plugging this in, we obtain

$$
\xi_{a 1} \approx \frac{n_{0}}{n_{a}} \xi_{a 0}-\frac{n_{0}^{3}}{n_{a}^{3}} \frac{n_{z}^{2}-n_{a}^{2}}{2 n_{z}^{2}} \xi_{a 0}^{3} \approx \frac{n_{0}}{n_{a}} \xi_{a 0}, n_{1}\left(\xi_{a 1}\right) \approx n_{a}\left(1+\frac{n_{z}^{2}-n_{a}^{2}}{2 n_{z}^{2}} \frac{n_{0}^{2}}{n_{a}^{2}} \xi_{a 0}^{2}\right) \approx n_{a}
$$

Similarly for $\xi_{b 1}$, Snell's law becomes $n_{1}\left(\xi_{a 1}\right) \xi_{b 1} \approx n_{0} \xi_{b 0}$, yielding

$$
\xi_{b 1} \approx \frac{\xi_{b 0} n_{0}}{n_{a}}\left(1-\frac{n_{z}^{2}-n_{a}^{2}}{2 n_{z}^{2}} \frac{n_{0}^{2}}{n_{a}^{2}} \xi_{a 0}^{2}\right) \approx \frac{n_{0}}{n_{a}} \xi_{b 0}
$$

Generalizing to include effects of face cuts on the crystal and calculating the direction of the outgoing beam upon exiting the crystal

$$
\xi_{x 1, \text { out }}=\xi_{x 0, \text { in }}+\left(\theta_{x c u t, \text { out }}-\theta_{x c u t, i n}\right) n_{1}\left(\xi_{a 1}\right) \quad, \quad \xi_{y 1, \text { out }}=\xi_{y 0, \text { in }}+\left(\theta_{y c u t, \text { out }}-\theta_{y c u t, \text { in }}\right) n_{1}\left(\xi_{a 1}\right)
$$

We perform this analysis again for the 2nd ray. For the component of input polarization along the yz plane, an angle $\xi_{b 2}$ within the crystal both lengthens the propagation distance of ray 2 by $\frac{\xi_{b 2}^{2}}{2}$ in the crystal and mixes refractive indices $n_{b}, n_{z}: \hat{r_{2}}=\left(0, \sin \left(\xi_{b 2}\right), \cos \left(\xi_{b 2}\right)\right) \operatorname{approx}\left(0, \xi_{b 2}, 1-\xi_{b 2}^{2} / 2\right)$ and $\hat{p_{2}}=$ $\left(0, \cos \left(\xi_{b 2}\right),-\sin \left(\xi_{b 2}\right) \approx\left(0,1-\xi_{b 2}^{2},-\xi_{b 2}\right)\right.$. An angle $\xi_{a 2}$ has no effect on the refractive index, only the distance of propagation is lengthened by $\frac{L_{0} \xi_{a 2}^{2}}{2}$. Putting $\xi_{a} 2$ and $\xi_{b} 2$ together, we obtain

$$
\hat{r_{2}} \approx\left(\xi_{a 2}, \xi_{b 2}, 1-\xi_{a 2}^{2} / 2-\xi_{b 2}^{2} / 2\right), \hat{p_{2}} \approx\left(0,1-\xi_{b 2}^{2} / 2,-\xi_{b 2}\right), L_{2}=L_{0}\left(1+\xi_{a 2}^{2} / 2+\right.
$$
$\left.\xi_{b 2}^{2} / 2\right)$

For this polarization direction, the index ellipsoid equation and Snell's law 
yield

$$
\begin{gathered}
n_{2}\left(\xi_{b 2}\right) \approx n_{b}\left(1+\frac{n_{z}^{2}-n_{b}^{2}}{2 n_{z}^{2}} \frac{n_{0}^{2}}{n_{b}^{2}} \xi_{b 0}^{2}\right) \approx n_{b} \\
\xi_{b 2} \approx \frac{n_{0}}{n_{b}} \xi_{b 0}-\frac{n_{0}^{3}}{n_{b}^{3}} \frac{n_{z}^{2}-n_{b}^{2}}{2 n_{z}^{2}} \xi_{b 0}^{3} \approx \frac{n_{0}}{n_{b}} \xi_{b 0} \quad, \quad \xi_{a 2} \approx \frac{\xi_{a 0} n_{0}}{n_{b}}\left(1-\frac{n_{z}^{2}-n_{b}^{2}}{2 n_{z}^{2}} \frac{n_{0}^{2}}{n_{b}^{2}} \xi_{b 0}^{2}\right) \approx \frac{n_{0}}{n_{b}} \xi_{a 0}
\end{gathered}
$$

Generalizing to include effects of face cuts on the crystal and calculating the direction of the outgoing beam upon exiting the crystal

$\xi_{x 2, \text { out }}=\xi_{x 0, \text { in }}+\left(\theta_{x c u t, \text { out }}-\theta_{x c u t, \text { in }}\right) n_{2}\left(\xi_{b 2}\right) \quad, \quad \xi_{y 2, \text { out }}=\xi_{y 0, \text { in }}+\left(\theta_{y c u t, \text { out }}-\theta_{y c u t, \text { in }}\right) n_{2}\left(\xi_{b 2}\right)$

For $\mathrm{KD}^{* \mathrm{P}}, n_{0}=n_{\text {air }} \approx 1, n_{a} \approx n_{b} \approx n_{o}, n_{z}=n_{e}$ and the electro-optic coupling yields refractive indices along the $\pm 45^{\circ}$ directions

$$
n_{x^{\prime}}=n_{o}-\frac{1}{2} n_{o}^{3} r_{63} E_{z}, n_{y^{\prime}}=n_{o}+\frac{1}{2} n_{o}^{3} r_{63} E_{z}
$$

where the helicity correlated index differences $\Delta n_{x^{\prime}}=-\frac{1}{2} n_{o}^{3} r_{63} \Delta E_{z}$ and $\Delta n_{y^{\prime}}=$ $+\frac{1}{2} n_{o}^{3} r_{63} \Delta E_{z}$ and $\mathrm{r} 63=23.6 \mathrm{pm} / \mathrm{V}$ [308]

We consider the terms in birefringence $\delta n\left(\xi_{a 0}, \xi_{b 0}\right)=n_{1}-n_{2}$ which will contribute to a $\Delta$-phase through a phase shift $\Delta \zeta\left(\xi_{a 0}, \xi_{b 0}\right)$. We consider helicity averaged terms which contribute to both helicity states phases $\zeta^{R(L)}$ with the same sign, as the $\Delta$-phase is defined to do. The helicity averaged terms are simply:

$$
\begin{gathered}
\zeta=2 \pi \delta(n L) / \lambda=\frac{2 \pi}{\lambda}\left(n_{1} L_{1}-n_{2} L_{2}\right) \\
\xi_{a}=\xi_{a 1} \approx \xi_{a 2} \approx \frac{1}{n_{o}} \xi_{a 0}, \xi_{b}=\xi_{b 1} \approx \xi_{b 1} \approx \frac{1}{n_{o}} \xi_{b 0} \\
n_{1} \approx n_{o}+\frac{n_{e}^{2}-n_{o}^{2}}{2 n_{e}^{2} n_{o}} \xi_{a 0}^{2}, n_{2} \approx n_{o}+\frac{n_{e}^{2}-n_{o}^{2}}{2 n_{e}^{2} n_{o}} \xi_{b 0}^{2} \\
\delta n=n_{1}-n_{2}=\frac{n_{e}^{2}-n_{o}^{2}}{2 n_{e}^{2} n_{o}}\left(\xi_{a 0}^{2}-\xi_{b 0}^{2}\right), L=L_{1} \approx L_{2} \approx L_{0}\left(1+\left(\frac{1}{n_{o}^{2}}\right)\left(\xi_{a 0}^{2}+\xi_{b 0}^{2}\right)\right)
\end{gathered}
$$


The overall phase shift $\zeta$ is given by

$$
\zeta=\frac{2 \pi}{\lambda} \frac{n_{e}^{2}-n_{o}^{2}}{2 n_{e}^{2} n_{o}}\left(\xi_{a 0}^{2}-\xi_{b 0}^{2}\right)\left(1+\frac{1}{n_{o}^{2}}\left(\xi_{a 0}^{2}+\xi_{b 0}^{2}\right)\right) L_{0}
$$

We note that in $\mathrm{KD}^{*} \mathrm{P}$, the slow axis $\mathrm{b}$ is determined by the incoming beam transverse component direction $\vec{\xi}_{b}=\left(\xi_{x}, \xi_{y}\right)$. The fast-axis a (along $\eta$ is simply the direction for which the beam has no component. Hence, the magnitude of $\xi_{b}$ is given by $\left|\xi_{b}\right|=\sqrt{\xi_{x}^{2}+\xi_{y}^{2}}$ and the magnitude of $\xi_{a}=0$. The phase shift can then be rewritten in terms of $\left(\xi_{x 0}, \xi_{y 0}\right)$ as

$$
\begin{gathered}
\zeta\left(\xi_{x_{0}}, \xi_{y_{0}}\right)=-\frac{2 \pi}{\lambda} \frac{n_{e}^{2}-n_{o}^{2}}{2 n_{e}^{2} n_{o}}\left(\xi_{x 0}^{2}+\xi_{y 0}^{2}\right)\left(1+\frac{1}{n_{o}^{2}}\left(\xi_{x 0}^{2}+\xi_{y 0}^{2}\right)\right) L_{0} \\
\approx \frac{\pi}{\lambda} \frac{n_{o}^{2}-n_{e}^{2}}{n_{e}^{2} n_{o}}\left(\xi_{x 0}^{2}+\xi_{y 0}^{2}\right) L_{0}
\end{gathered}
$$

\section{A.5 Asymmetry angle dependence in $\mathrm{KD}^{*} \mathrm{P}$}

Given the above information regarding the angle dependence of $\zeta\left(\xi_{a 0}, \xi_{b 0}\right)$ and $\eta\left(\xi_{x 0}, \xi_{y 0}\right)$, we can derive the angle dependence of the asymmetry $A_{q}\left(\xi_{x 0}, \xi_{y 0}\right)=$ $-\frac{\epsilon}{T} \zeta \sin \left(2\left(\eta\left(\xi_{x 0}, \xi_{y 0}\right)-\psi\right)\right)$. Note that

$$
\begin{aligned}
& \sin (2(\eta-\psi))=\sin (2 \eta) \cos (2 \psi)-\sin (2 \psi) \cos (2 \eta) \\
& =2 \sin \eta \cos \eta \cos (2 \psi)-\left(1-2 \sin ^{2} \eta\right) \sin (2 \psi) \\
& =2 \sin \eta \sqrt{1-\sin ^{2} \eta} \cos (2 \psi)-\left(1-\frac{2 \xi_{x}^{2}}{\xi_{x}^{2}+\xi_{y}^{2}}\right) \sin (2 \psi) \\
& =\frac{2 \xi_{x} \xi_{y}}{\xi_{x}^{2}+\xi_{y}^{2}} \cos (2 \psi)+\frac{\xi_{x}^{2}-\xi_{y}^{2}}{\xi_{x}^{2}+\xi_{y}^{2}} \sin (2 \psi)
\end{aligned}
$$

Hence the angle dependence of Aq can be rewritten in terms of the beam 
incident angle in air $\left(\xi_{x 0}, \xi_{y 0}\right)=\frac{1}{n_{o}}\left(\xi_{x}, \xi_{y}\right)$ as

$$
\begin{gathered}
A_{q}\left(\xi_{x_{0}}, \xi_{y_{0}}\right)=-\frac{\epsilon}{T} \zeta\left(\xi_{x_{0}}, \xi_{y_{0}}\right)\left(\frac{2 \xi_{x 0} \xi_{y 0}}{\xi_{x 0}^{2}+\xi_{y 0}^{2}} \cos (2 \psi)+\frac{\xi_{x 0}^{2}-\xi_{y 0}^{2}}{\xi_{x^{0}} 2+\xi_{y 0}^{2}} \sin (2 \psi)\right) \\
=-\frac{\epsilon}{T} \frac{\pi L_{0}}{\lambda} \frac{n_{o}^{2}-n_{e}^{2}}{n_{e}^{2} n_{o}}\left(2 \xi_{x 0} \xi_{y 0} \cos 2 \psi+\left(\xi_{x 0}^{2}-\xi_{y 0}^{2}\right) \sin 2 \psi\right)
\end{gathered}
$$

In $\mathrm{KD}^{*} \mathrm{P}$, at $780 \mathrm{~nm} n_{o} 1.4988, n_{e} 1.4617$ [308]. The Pockels cell is $40 \mathrm{~mm}$ in length so theoretically, the angle dependence of the $\mathrm{KD}^{*} \mathrm{P}$ for a $100 \%$ analyzer should be

$$
\frac{d^{2} A_{q}}{d \xi_{0}^{2}}=2 \frac{\pi L_{0}}{\lambda} \frac{n_{o}^{2}-n_{e}^{2}}{n_{e}^{2} n_{o}}=11,052 \mathrm{ppm} / \mathrm{mrad}^{2}
$$

Empirically, we observe a smaller angle dependence than predicted.

\section{A.6 Fast-axis direction angle dependence in RTP}

In the case of an X-cut RTP pockels cell, the beam propagates mainly along the crystal x-axis and the fundamental refractive indices $n_{y}$ and $n_{z}$ which the transverse polarization are exposed to are quite different. At $780 \mathrm{~nm}$, the refractive indices of RTP are $n_{x}=1.7739, n_{y}=1.7832, n_{z}=1.8673$ (from Sellmeier' $\mathrm{s}$ equation [328]). When laser beam propagation is at an slight angle, while this will mix $n_{x}$ into $n_{z}$ or $n_{y}$ slightly, it is insufficient to alter the direction of the effective fast and slow axes. The effective fast and slow axis very nearly remain along the original $y, z$ directions regardless of a small tilt angle. For a single RTP crystal , $\eta=y=45^{\circ}$. 


\section{A.7 Asymmetry angle dependence model in RTP}

The asymmetry arising from an extraneous birefringent element contributing a delta-phase shift $\Delta=\zeta=2 \pi \delta n / L$ is given by

$$
A_{q}=-\frac{\epsilon}{T} \zeta \sin (2(\eta-\psi))
$$

Because the RTP Pockels has so many internal crystal axes to keep track of, we define the lab coordinate system in the transverse plane as $0^{\circ}$ (horizontal ), $90^{\circ}$ (vertical), $+45^{\circ}$, and $-45^{\circ}$ (diagonal).

We can model the RTP asymmetry angle dependence on the KD*P asymmetry angle dependence as having an S1 term and an S2 term

$$
A_{q}\left(\xi_{x}, \xi_{y}\right)=-\frac{\epsilon}{T}\left(\zeta_{S 1} \cos 2 \psi+\zeta_{S 2} \sin 2 \psi\right)
$$

We can can derive individually (1) the birefringence induced phase-shift $\zeta_{S 1}$ for an analyzer along S1 (x-axis $0^{\circ}$ ) which is sensitive to birefringence $\delta n=n_{45^{\circ}}-$ $n_{135^{\circ}}$ along the diagonals and (2) the birefringence induced phase-shift $\zeta_{S 2}$ for an analyzer along S2 $\left(45^{\circ}\right)$ which is sensitive to birefringence $\delta n=n_{0^{\circ}}-n_{90^{\circ}}$ along the vertical and horizontal.

\section{A.8 Refractive Index angle dependence in RTP}

For the first crystal, the fast axis a (in this case corresponding to crystal's $\mathrm{y}$-axis) is along $45^{\circ}$ and slow axis b (in this case corresponding to crystal's z-axis) is along $135^{\circ}$. For ray 1 (the fast ray) in RTP1,

$$
\begin{gathered}
\xi_{45^{\circ}, 1} \approx \frac{1}{n_{y}} \xi_{45^{\circ}, 0}, \quad \xi_{135^{\circ}, 1} \approx \frac{1}{n_{y}} \xi_{135^{\circ}, 0} \\
n_{1}^{(1)}\left(\xi_{45^{\circ}, 1}\right) \approx n_{y}\left(1+\frac{n_{x}^{2}-n_{y}^{2}}{2 n_{x}^{2}} \frac{1}{n_{y}^{2}} \xi_{45^{\circ}, 0}^{2}\right), L_{1}^{(1)}=L_{0}\left(1+\frac{1}{2 n_{y}^{2}}\left(\xi_{45^{\circ}, 0}^{2}+\xi_{135^{\circ}, 1}^{2}\right)\right)
\end{gathered}
$$


For ray 2 (the slow ray) in RTP1,

$$
\begin{gathered}
\xi_{135^{\circ}, 2} \approx \frac{1}{n_{z}} \xi_{135^{\circ}, 0}, \quad \xi_{45^{\circ}, 2} \approx \frac{1}{n_{z}} \xi_{45^{\circ}, 0} \\
n_{2}^{(1)}\left(\xi_{135^{\circ}, 2}\right) \approx n_{z}\left(1+\frac{n_{x}^{2}-n_{z}^{2}}{2 n_{x}^{2}} \frac{1}{n_{z}^{2}} \xi_{135^{\circ}, 0}^{2}\right), L_{2}^{(1)}=L_{0}\left(1+\frac{1}{2 n_{z}^{2}}\left(\xi_{45^{\circ}, 0}^{2}+\xi_{135^{\circ}, 1}^{2}\right)\right)
\end{gathered}
$$

For the 2nd crystal, the fast axis a (in this case corresponding to crystal's yaxis) is along $135^{\circ}$ and slow axis b (in this case corresponding to crystal's z-axis) is along $45^{\circ}$. For ray 1 along $45^{\circ}$ (the slow ray) in RTP2,

$$
\begin{gathered}
\xi_{45^{\circ}, 1} \approx \frac{1}{n_{z}} \xi_{45^{\circ}, 0}, \quad \xi_{135^{\circ}, 1} \approx \frac{1}{n_{z}} \xi_{135^{\circ}, 0} \\
n_{1}^{(2)}\left(\xi_{45^{\circ}, 1}\right) \approx n_{z}\left(1+\frac{n_{x}^{2}-n_{z}^{2}}{2 n_{x}^{2}} \frac{1}{n_{z}^{2}} \xi_{45^{\circ}, 0}^{2}\right), L_{1}^{(2)}=L_{0}\left(1+\frac{1}{2 n_{z}^{2}}\left(\xi_{45^{\circ}, 0}^{2}+\xi_{135^{\circ}, 1}^{2}\right)\right)
\end{gathered}
$$

For ray 2 along $135^{\circ}$ (the fast ray) in RTP2,

$$
\begin{gathered}
\xi_{135^{\circ}, 2} \approx \frac{1}{n_{y}} \xi_{135^{\circ}, 0}, \quad \xi_{45^{\circ}, 2} \approx \frac{1}{n_{y}} \xi_{45^{\circ}, 0} \\
n_{2}^{(2)}\left(\xi_{135^{\circ}, 2}\right) \approx n_{y}\left(1+\frac{n_{x}^{2}-n_{y}^{2}}{2 n_{x}^{2}} \frac{1}{n_{y}^{2}} \xi_{135^{\circ}, 0}^{2}\right), L_{2}^{(2)}=L_{0}\left(1+\frac{1}{2 n_{y}^{2}}\left(\xi_{45^{\circ}, 0}^{2}+\xi_{135^{\circ}, 1}^{2}\right)\right)
\end{gathered}
$$

where we have assumed that the crystal face cuts are small enough that the angle at which the beam enters the first crystal is the same upon exiting and entering the second crystal.

We obtain a phase shift in S1 due to birefringence along $\pm 45^{\circ}$ for the first crystal $\zeta^{(1)}$ and the 2 nd crystal $\zeta^{(2)}$ and combine them to obtain the total phase shift $\zeta_{S 1}=\zeta^{(1)}+\zeta^{(2)}$

$$
\begin{gathered}
\zeta^{(1)}=\frac{2 \pi}{\lambda}\left(n_{1}^{(1)} L_{1}^{(1)}-n_{2}^{(1)} L_{2}^{(1)}\right), \quad \zeta^{(2)}=\frac{2 \pi}{\lambda}\left(n_{1}^{(2)} L_{1}^{(2)}-n_{2}^{(2)} L_{2}^{(2)}\right) \\
\zeta_{S 1}=\frac{\pi L_{0}}{\lambda}\left(n_{z}+n_{y}\right)\left(\frac{1}{n_{z} n_{y}}-\frac{1}{n_{x}^{2}}\right)\left(\xi_{45^{\circ}, 0}^{2}-\xi_{135^{\circ}, 0}^{2}\right)
\end{gathered}
$$


We note that since the fast/slow axes for RTP is along $\pm 45^{\circ}$ regardless of the the small angle deviation of the incoming beam from longitudinal propagation. So the birefringence can only contribute to an S1 phase shift. The birefringence along $0^{\circ}$ and $90^{\circ}$ will always be 0 for an individual crystal and is also 0 for two crystals combined (so long as their axes are aligned properly). This is obvious from the fast that $n_{0^{\circ}}=n_{90^{\circ}}=\frac{n_{1} n_{2}}{\sqrt{\left(n_{1}^{2}+n_{2}^{2}\right) / 2}}$ so $\delta n=n_{0^{\circ}}-n_{90^{\circ}}=0$. Hence

$$
\zeta_{S 2}=0
$$

\section{A.9 Asymmetry angle dependence in RTP}

Putting the S1 and S2 behavior together, we obtain the asymmetry angle dependence for two RTP crystals in succession

$$
A_{q}\left(\xi_{45^{\circ}, 0}, \xi_{135^{\circ}, 0}\right)=\frac{\epsilon}{T} \cos 2 \psi \frac{\pi L_{0}}{\lambda}\left(n_{z}+n_{y}\right)\left(\frac{1}{n_{z} n_{y}}-\frac{1}{n_{x}^{2}}\right)\left(\xi_{135^{\circ}, 0}^{2}-\xi_{45^{\circ}, 0}^{2}\right)
$$

This equation can be rewritten in terms of the lab coordinates $\mathrm{x}$ (horizontal) and $\mathrm{y}($ vertical $)$, where $\xi_{45^{\circ}, 0}=\left(\xi_{x 0}+\xi_{y 0}\right) / \sqrt{2}$ and $\xi_{135^{\circ}, 0}=\left(-\xi_{x 0}+\xi_{y 0}\right) / \sqrt{2}$

$$
A_{q}\left(\xi_{x 0}, \xi_{y 0}\right)=\frac{\epsilon}{T} \cos 2 \psi \frac{\pi L_{0}}{\lambda}\left(n_{z}+n_{y}\right)\left(\frac{1}{n_{x}^{2}}-\frac{1}{n_{z} n_{y}}\right)\left(2 \xi_{x 0} \xi_{y 0}\right)
$$

Note that $A_{q}$ is zero in $\mathrm{S} 2(\psi=\pi / 4)$, meaning its dependence on pitch/yaw angular adjustments is negligible when analyzing along $\pm 45^{\circ}$.

At $780 \mathrm{~nm}$, the refractive indices of RTP are $n_{x}=1.7739, n_{y}=1.7832, n_{z}=$ 1.8673 (from Sellmeier's equation [328]). The length of each crystal is $10 \mathrm{~mm}$, so for a $100 \%$ analyzer in $\mathrm{S} 1$ the angle sensitivity is predicted to be

$$
\frac{d A_{q}}{d \xi_{0}}=-2 \frac{\pi L_{0}}{\lambda}\left(n_{z}+n_{y}\right)\left(\frac{1}{n_{z} n_{y}}-\frac{1}{n_{x}^{2}}\right)=5,137 p p m / m_{r a d}
$$





\section{Appendix B}

\section{Tech Note: RTP Temperature Dependence and Aq Feedback}

\section{B.1 Thermal Compensation}

Unlike $\mathrm{KD}^{*} \mathrm{P}$ crystals, which are uniaxial and allow for a longitudinal cell design, RTP crystals are biaxial crystals. This means they have unique refractive indices along each of the 3 primary crystal axes. RTP crystals are highly birefringent $(\Delta n \approx 0.1)$ to such an extent that a $1 \mathrm{~cm}$ crystal functions like a 1000 order waveplate and is therefore extremely temperature dependent and wavelength dependent. This large intrinsic birefringence is what necessitates the use of the socalled "thermal compensation design" in which two RTP crystals are used together with the fast axis of one crystal perpendicular to the fast axis of the other, so as to cancel the birefringence. This thermal compensation design used by all RTP cell manufacturers is exactly the same as the design of a zero-order waveplate and it is done for precisely the same reason: multi-order waveplates are sensitive to temperature and wavelength. They are sensitive to temperature because refractive indices $n_{e}$ and $n_{o}$, and birefringence $\Delta n=n_{e}-n_{o}$, change with temperature $\frac{d \Delta n}{d T}$. They are sensitive to wavelength directly via the induced phase shift 
$\frac{d \Delta \phi}{d \lambda}=\frac{d}{d \lambda} 2 \pi \Delta n L / \lambda=-2 \pi \Delta n L \Delta \lambda / \lambda^{2}$. Most commercial multi-order waveplates are order 10. RTPs of the nominal $1 \mathrm{~cm}$ length are order 1000 at $780 \mathrm{~nm}$. Zeroorder waveplates consist of 2 multi-order waveplates with fast axes perpendicular to each other. RTP cells consist of 2 RTP crystals with fast axes perpendicular to each other. The design between zero-order waveplates and RTP cells is completely analogous.

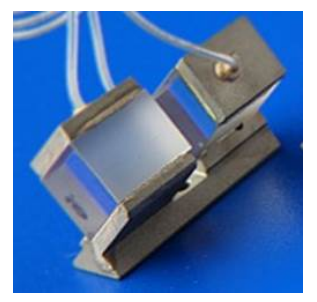

Figure B-1: Thermal Compensation Design. RTP crystals in typical mount. Photograph by Raicol.

\section{B.2 Temperature Differences}

The thermal compensation design only works if both birefringent elements are at the same temperature and of equal length. The change in phase shift of the first element $\delta \Delta \phi_{1}$ must be canceled by the change in phase shift of the second element $\delta \Delta \phi_{2}$.

$$
\begin{gathered}
0=\delta \Delta \phi_{1}+\delta \Delta \phi_{2}=\frac{d \Delta \phi_{1}}{d T} \delta T_{1}+\frac{d \Delta \phi_{2}}{d T} \delta T_{2}=\frac{d\left(2 \pi \Delta n_{1} L / \lambda\right)}{d T} \delta T_{1}+\frac{d\left(2 \pi \Delta n_{2} L / \lambda\right)}{d T} \delta T_{2} \\
\frac{d 2 \pi\left(n_{o}-n_{e}\right) L / \lambda}{d T} \delta T_{1}+\frac{d 2 \pi\left(n_{e}-n_{o}\right) L / \lambda}{d T} \delta T_{2}=2 \pi L / \lambda \frac{d\left(n_{o}-n_{e}\right)}{d T}\left(\delta T_{1}-\delta T_{2}\right) \\
=2 \pi L / \lambda \frac{d\left(n_{o}-n_{e}\right)}{d T} \delta T_{12}
\end{gathered}
$$

When there is a temperature difference between the two crystals, it leads to a net phase shift, and when running the Pockels cell at QWV and analyzing, it leads to a charge asymmetry Aq. The Aq arising from a retardation plate that 
induces a phase shift $\Delta \phi$, and has its fast-axis at an angle $\eta$ from the horizontal can be expressed as [316]

$$
A q=-\frac{\epsilon}{T} \Delta \phi \sin (2 \eta-2 \psi)
$$

where $\epsilon / T$ is the analyzing power, $\psi$ is the angle subtended by one of the analyzing axes relative to the horizontal. For a 100\% analyzer oriented along $\mathrm{S} 1$ (vertical), where the crystal primary axes $\mathrm{y}-\mathrm{z}$ are along $\mathrm{S} 2\left(45^{\circ}\right)$, and there is a temperature difference $\Delta T_{12}$ between the crystals, we can expect a charge asymmetry of

$$
A q=\eta=2 \pi L / \lambda \frac{d\left(n_{z}-n_{y}\right)}{d T} \Delta T_{12}
$$

Sellmeier's equation [328] gives $n_{x 0}=1.774, n_{y 0}=1.783, n_{z 0}=1.867$ at $780 \mathrm{~nm}$. The length of each crystal is $\mathrm{L}=10 \mathrm{~mm}$, with a negligible length difference tolerance of $2 \mathrm{um} \mathrm{[317]} \mathrm{.} \mathrm{The} \mathrm{temperature} \mathrm{dependence} \mathrm{of} \mathrm{the} \mathrm{RTP} \mathrm{refractive} \mathrm{indices} \mathrm{is}$ $d n_{z} / d T=1.22 \times 10^{-} 5 K^{-1}, d n_{y} / d T=3.88 \times 10^{-} 5 K^{-1}$ at $25^{\circ} C$ [268], and hence the birefringence temperature dependence is $8.31 \times 10^{-6} \mathrm{~K}^{-1}$. There is some slight non-linearity with respect to temperature in the $20-30^{\circ} \mathrm{C}$ temperature range, but it is quite small. For our RTP crystals at $780 \mathrm{~nm}$, we estimate the charge asymmetry from a temperature difference between the crystals to be $d A q / d T_{12}=$ $6.7 \times 10^{5} \mathrm{ppm} /{ }^{\circ} \mathrm{C}=670 \mathrm{ppm} / \mathrm{mK}$.

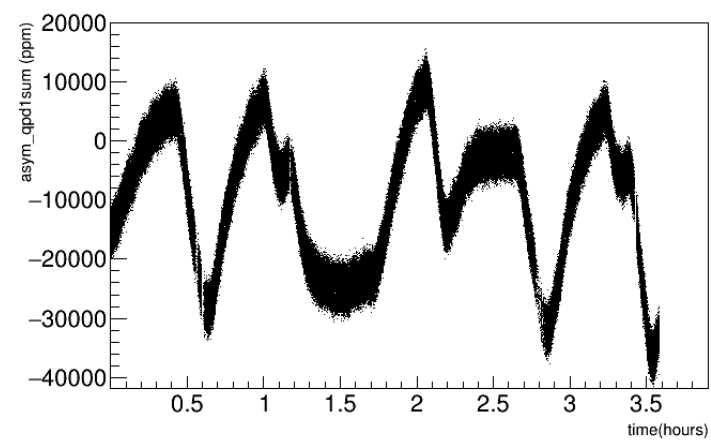

Figure B-2: Run4883, RTP cell, analyzed in S1 [321] 
Both in the lab at UVa and at JLab, we observe slow fluctuation in Aq by 30,000ppm over time (Fig. B-2 ) and we believe this to be related to a fluctuation in the temperature difference between the crystals. We don't believe this is due to wavelength fluctuations because the wavelength stability of the JLab 1064nm pump laser for the PPLN, prior to wavelength doubling to $780 \mathrm{~nm}$, was measured to be $1553.625 \mathrm{~nm}$ over 10 minutes with no change in peak wavelength to $0.001 \mathrm{~nm}$ precision[318]. For a crystal length difference of $\delta L_{12}=2 \mu m$ we'd expect $A q=$ $-2 \pi \Delta n \delta L_{12} / \lambda \Delta \lambda / \lambda=1735 \mathrm{ppm} / \mathrm{nm}$ and for $0.001 \mathrm{~nm}$, that would only be $<2 \mathrm{ppm}$ for the observe wavelength stability. The temperature difference between the crystals only need by $45 \mathrm{mKelvin}$ to induce a $30,000 \mathrm{ppm}$ charge asymmetry, so it is the more likely culprit of the observe fluctuations. We note that the fluctuations were observed for both a commercial RTP cell and our UVa cell. The commercial RTP cell has $6 \times 6 \mathrm{~mm}$ crystals silver cemented to the same base electrode, separated by a few millimeters, and enclosed in a window sealed mount. Our UVa RTP cell with larger $12 \times 12 \mathrm{~mm}$ crystals in an open mount design separated by 1-2" with independent electrodes. At Jlab, the commercial cell Aq drifted by $10 \mathrm{k} \mathrm{ppm}$ in 30min, while the UVa mount drifted by 14,000ppm over 20min in S1 [319]. This implies that a mount redesign in favor of a more traditional design will not do much to alleviate the temperature difference fluctuations.

We induced a temperature difference between the crystal mounts by slowly heating one of the mounts with resistive heaters and measuring the temperature of both mounts with thermocouples while observing Aq in S1 [266]. Both crystals increased in temperature, one crystal by $\sim 4^{\circ} \mathrm{F}$ and the other by $\sim 1^{o} \mathrm{~F}$, with the temperature difference range $\sim 0-3^{\circ} \mathrm{F}, 0 \sim-1.5^{\circ} \mathrm{C}$. We observed in Fig. B-3a that $d A q / d T_{12} \sim 8.9 \times 10^{5} \mathrm{ppm} /{ }^{\circ} \mathrm{C} \sim 890 \mathrm{ppm} / \mathrm{mK}$ which is within $30 \%$ of our theoretically predicted value. It should be noted that the temperature of the mounts does not necessarily correspond to the temperature of the crystals and other studies involving heating/cooling the mounts and measuring the temperature of the mount did not all show such consistent results, so this measurement is very 
approximate and should only be notes on the OOM level.

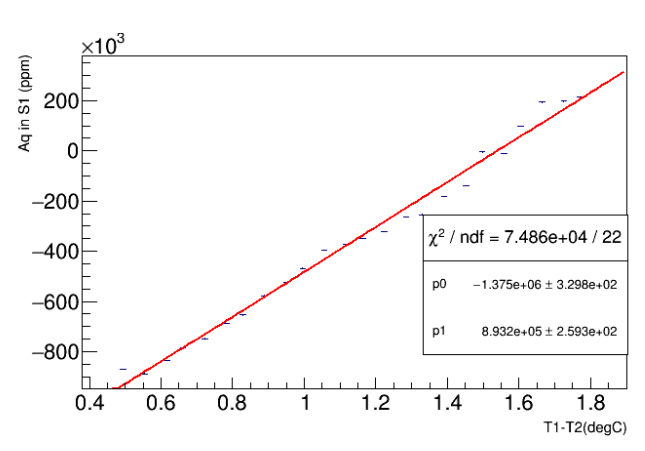

(a) Measurement

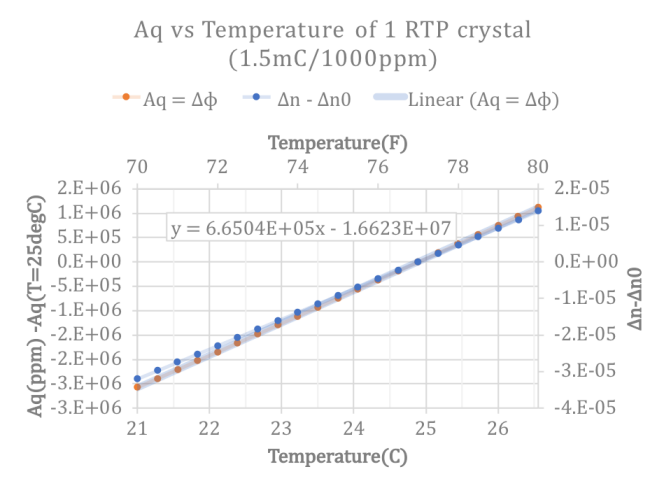

(b) Calculation

Figure B-3: dAq/dT calculation compared to measurement. (a) Run 5081 UVa Aq vs temperature difference between crystal mounts T1-T2 with resistive heaters on one crystal mount[266] (b) Calculation of Aq vs $\mathrm{T}$ [268] where one crystal is $25^{\circ} \mathrm{C}$ and $\mathrm{T}$ is the temperature of the $2 \mathrm{nd}$ crystal

\section{B.3 Temperature Gradient}

Just as a net temperature difference between the two crystals can result in a charge asymmetry, a temperature gradient can result in a position difference or a spot-size asymmetry. Position differences and spot-size asymmetries could arise in more than one way. A position difference could arise from a birefringence gradient when analyzing in $\mathrm{S} 1$, proportional to $\sim d A q / d X$. A position difference could also arise for no analyzer or in S1 from a gradient in the refractive index along a primary axis. Similarly thermal gradients could give rise to spot size asymmetries in $\mathrm{S} 1 \sim d^{2} A q / d X^{2}$, or spot size asymmetries without analyzing through another mechanism. It has been found through calculation [322] that the predominant contribution to position differences and spot-size asymmetries is likely to come through birefringence gradients in S1. Even for very small analyzing powers, this is the dominant effect for the RTP.

It is important to note that it is not an overall temperature gradient that matters, rather a difference in thermal gradient between the two crystals. The 
thermal compensation design will cancel any birefringence, birefringence gradient, or birefringence 2 nd moment that is seen by both crystals equally. It is only when one crystal has a thermal gradient not seen by the other crystal that gives rise to position differences and spot-size asymmetries.

\section{B.3.1 Gradient Calculation}

The position difference and spot size asymmetry arising from 1st and 2nd moments of Aq in $\mathrm{S} 1$ are given by

$$
\begin{gathered}
D_{x}=\frac{w_{P C} w_{q p d}}{2} \frac{d A q}{d x}=\frac{w_{P C} w_{q p d} \pi L}{\lambda} \frac{d\left(n_{z}-n_{y}\right)}{d T} \frac{d \Delta T_{12}}{d x} \\
A_{\sigma}=\frac{w_{P C}^{2}}{4} \frac{d^{2} A q}{d x^{2}}=\frac{w_{P C}^{2} \pi L}{2 \lambda} \frac{d\left(n_{z}-n_{y}\right)}{d T} \frac{d^{2} \Delta T_{12}}{d x^{2}}
\end{gathered}
$$

where $w_{P C}$ refers to the beam $2 \sigma$ waist at the Pockels Cell, $w_{q p d}$ is the beam waist at the location of measurement, in this case at the quad-photodiode, and $\Delta T_{12}$ is a temperature difference between the crystals.

\section{Numerical Estimate of Gradient Effect}

We can make an educated guess on the temperature gradient within one of the crystals, and using equations B.3 and B.4, estimate the effect we might observe after analyzing with a polarizer or the cathode. Importantly this calculation is for a gradient seen by only one of the crystals and not the other. If the gradient is experienced by both crystals, there is no net effect.

We assume that the temperature difference across two sides of a single crystal cannot exceed the temperature difference observed between the two separate crystals, as the crystals are separated by inches and one crystal is only $12 \mathrm{~mm}$ across. We observed that the two crystals temperature difference could fluctuate by possibly $\pm 45 \mathrm{mK}$ (Sec. B.2), so we set the temperature gradient to be $\sim 100 \mathrm{mK} /$ crystal width $\approx 100 \mathrm{mK} / 12 \mathrm{~mm} \approx 8.3 \mathrm{mK} / \mathrm{mm}$. For the second moment, we made a sim- 
ilar assumption that $d^{2} T / d x^{2} \sim 100 m K /(12 m m)^{2}$ so that the temperature varies no more than $50 \mathrm{mK}$ over $6 \mathrm{~mm}$ across the crystal.

The temperature gradient gives rise to a refractive index gradient and birefringence gradient as seen in B-4.

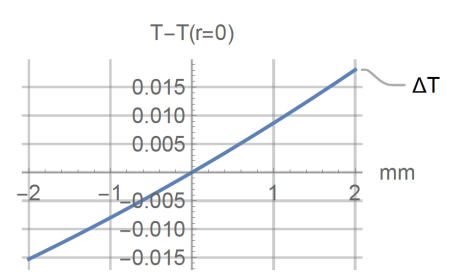

(a)

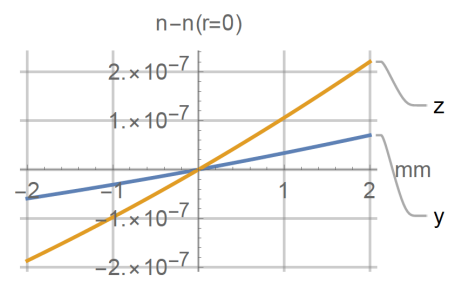

(b)

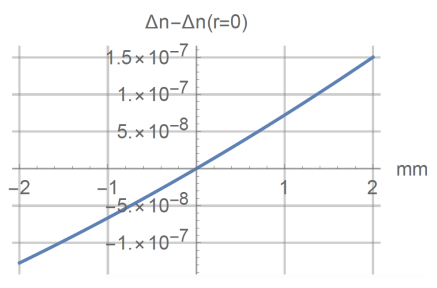

(c)

Figure B-4: Thermal Gradient Calculation (a) Educated guess for the type of temperature gradient (with 2nd moment) one of the crystals might experience (b) temperature change experienced by the refractive indices $n_{z}$ and $n_{y}$ relative to $n_{z}$ and $n_{y}$ at the crystal center (c) temperature induced birefringence

At QWV, any extra birefringence experienced by the nearly circularly polarized light will induce a charge asymmetry Aq. So the birefringence gradient, by Eq. B.3, gives rise to a charge asymmetry gradient when the beam is analyzed. Fig. B-5 shows the induced Aq gradient for a 100\% analyzer along S1.

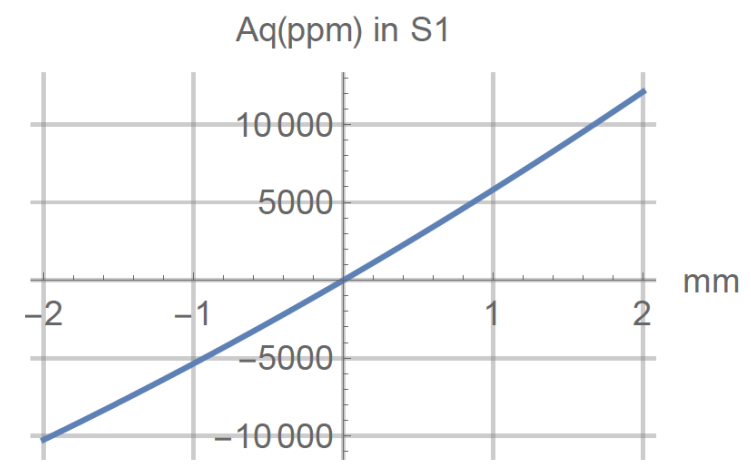

Figure B-5: Thermal induced Aq gradient for a 100\% analyzer along S1

1st and 2nd moments in Aq give rise to position differences and spot size asymmetries in S1. Fig. B-6 shows the position differences and spot-size asymmetries for a $100 \%$ analyzer and a $0.88 \mathrm{~mm}$ beam. The position difference observed at the 
crystal center is 2 um and the maximum spot size asymmetry is $10^{-4}$. So if our educated guess for the magnitude of thermal gradient is correct, we should expect thermal fluctuations in Dx on the order of $2 u m$ and fluctuations in $A \sigma$ on the order of $10^{-4}$ when analyzing on the laser table in S1.
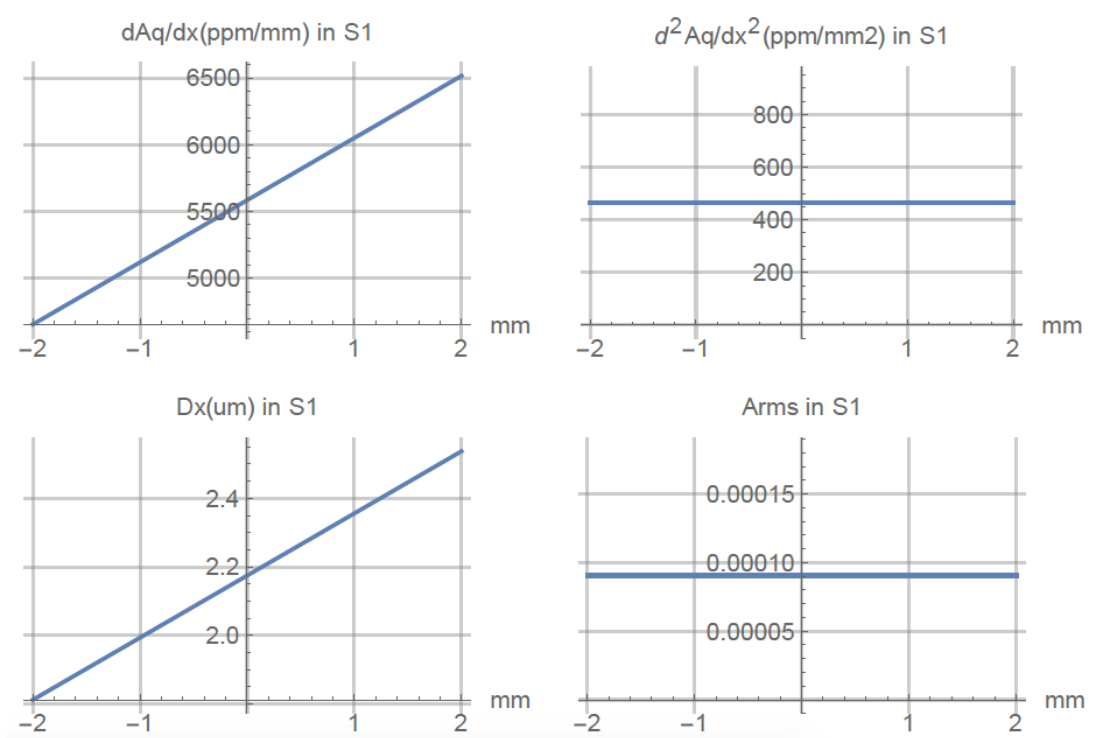

Figure B-6: Thermal induced Aq 1st and 2nd moments and the corresponding Dx and spot-size asymmetry for a $0.88 \mathrm{~mm}$ beam and $100 \%$ analyzer along S1

The laser spot size at the Pockels Cell was measured to be $0.825 \mathrm{~mm}$ horizontally and $0.94 \mathrm{~mm}$ vertically [329], so for this calculation we assumed the average $w_{P C}=0.8825 \mathrm{~mm}$. For simplicity, assumed that the spot size at the position difference measurement location is the same as the spot size at the Pockels cell $w_{q p d}=w_{P C}$. Regarding Dx it's important to note that the value of Dx will be proportional to the beam size at the location of the measurement device, so if the quad-photodiode sees a larger beam spot or if a bpm see a larger beam spot, these calculated values for Dx should be scaled up accordingly. The analyzing power is also important. The cathode has an analyzing power of $<6 \%$ [330], so the position differences and spot size asymmetries seen in the injector for a $0.88 \mathrm{~mm}$ beam due to thermal gradients might be $\sim 120 \mathrm{~nm}$ and $\sim 6 \times 10^{-6}$. If we were to rotate the RHWP so that the cathode was analyzing along $50^{\circ}$, near S2, instead of along $90^{\circ}$ 
along S1, the analyzing power along S1 would only be $0.525 \%$ and there would be further suppression of the thermally induced position differences drifts down to $11 \mathrm{~nm}$ and spot size asymmetries down to $5.3 \times 10^{-7}$, at a manageable level for Moller.

\section{B.3.2 Observed Temperature Gradient Fluctuations}

We attempted to examine the position difference stability over time on the laser table, using the quad-photodiode and analyzing in S1 (Fig. B-7 ). We observed fluctuations on the order of $2 \mathrm{um}$, although most of the fluctuations were suspiciously correlated with fluctuations in Aq, shown in Fig. B-2. Small pedestal errors can cause instrumental coupling between measured $D_{x}, D_{y}$ and Aq, which we estimated with a PITA scan, which nominally doesn't change position differences much, mostly just Aq. The PITA scan showed potential instrumental coupling between Aq and Dx,Dy in the qpd $\sim 1-5 \times 10^{-5} \mu \mathrm{m} / \mathrm{ppm}$. The potential instrumental coupling could account for most of the observed fluctuations of Dx,Dy as shown by the green curves in Fig. B-7. However separate from the possible Aq coupling contribution, Dx appears to drift over 3.5 hours by $2 \mathrm{um}$. So it is possible that the temperature gradient might be as large as $10 \mathrm{mK} / \mathrm{mm}$ as was postulated in Sec. B.3.1. 

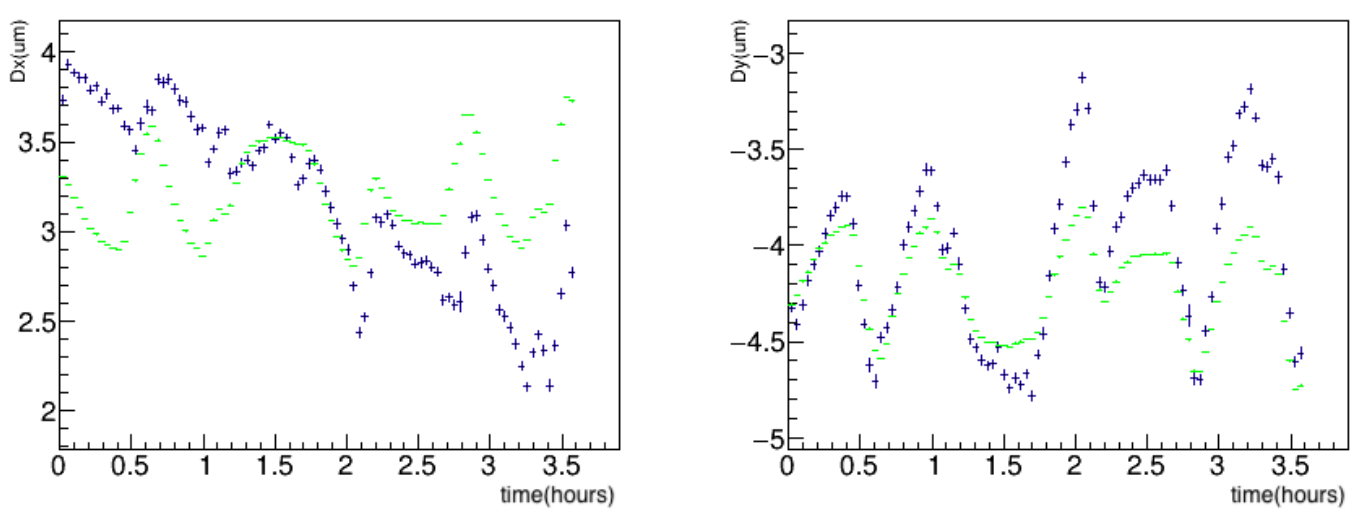

Figure B-7: Run4883 [321] where Dx,Dy are measured with the qpd in S1 are shown in black and an OOM estimate of potential contribution from Aq instrumental coupling to measured $D x, y=$ coupling $\times A q$ is shown in green, where the coupling constant $\mp 2.1 \times 10^{-5} \mu \mathrm{m} / \mathrm{ppm}$ (obtained from a PITA scan [323] ) and Aq from Fig. B-2 was used.

\section{Observed Fluctuations for no analyzer and S2}

\section{B.4 Laser Heating}

The power of the laser beam incident on the Pockels Cell can be up to $\sim 300 \mathrm{~mW}$ depending on what e-beam current the Hall is running at. There are 4 lasers, one for each Hall, which might be turned on and off, might be aligned on top of one another or not. It is conceivable that with a $1 \mathrm{~mm}$ spot-size area, $1 \mathrm{~W}$ of light could be hitting the RTP cell. So, it is a relevant question: what kind of thermal gradients might be caused by a small laser beam $(1 \mathrm{~mm})$ of high-ish $(1 \mathrm{~W})$ power.

As stated perviously, any thermal gradient seen by both crystals equally will give rise to equal and opposite birefringence phase shifts because of the thermal compensating design, and thus cancel each other out. A laser beam will pass through both crystals, get absorbed slightly, and give rise to nearly the same gradients in both crystals since both crystals see nearly the same size of beam with nearly the same wattage. Most of the temperature gradient effects in the first crystal will be canceled by the second. The residual left over comes from the 
slight difference in power seen by the second crystal due to some power having been absorbed by and reflected by the first crystal.

The greater the power absorbed in the crystal, the greater the thermal gradient and the difference in thermal gradient between the crystals. The values for the reported absorption for RTP vary considerably. The crystal growth company Cristal Laser reports that absorption depends on the crystal batch, but typical values at $1064 \mathrm{~nm}$ are $\alpha=0.01-0.02 \% / \mathrm{cm}$ [328]. Raicol, the company which manufactured the UVa RTP crystals, reports a Transmission of $>98.5 \%$ for a $2 \mathrm{~cm} 2$ crystal cell. Neglecting contributions of reflection coefficients, that's an upper limit on absorption of $<0.75 \% / \mathrm{cm}$. Raicol reported to LIGO [326] that absorption at $1064 \mathrm{~nm}$ for their crystals was $<0.01 \% / \mathrm{cm}$ and at $532 \mathrm{~nm}$ the absorption increases to 0.2 $0.4 \% / \mathrm{cm}$. Redoptrinics.com [327] reports an absorption coefficient of $4 \% / \mathrm{cm}$ at $532 \mathrm{~nm}$ and $0.05 \% / \mathrm{cm}$ at $1064 \mathrm{~nm}$. Measurements at UVa with $780 \mathrm{~nm}$ showed the transmission of the 1cm long RTP crystal "Galadriel" was 98.75\% [324] and the reflection coefficient was $\sim 0.2-0.7 \%$ depending on crystal and polarization axis [325], indicating the upper limit on absorption at 780nm was $\alpha<0.55 \%-1 \% / \mathrm{cm}$.

The greater the thermal conductivity of the crystal, the more difficult it is to create a thermal gradient. The thermal conductivity of KTA, KTP, and RTA are $\mathrm{k}=18 \mathrm{~mW} / \mathrm{cm} / \mathrm{K}, \mathrm{k}=30 \mathrm{~mW} / \mathrm{cm} / \mathrm{K}$, and $\mathrm{k}=16 \mathrm{~mW} / \mathrm{cm} / \mathrm{K}$, respectively [268]. The thermal conductivity of RTP is unknown to this author. As KTP is the crystal most closely related to $\mathrm{RTP}$, we use $\mathrm{k}=30 \mathrm{~mW} / \mathrm{cm} / \mathrm{K}$

This calculation uses the measured value for the RTP absorption coefficient at UVa which is $0.75 \% / \mathrm{cm}$, the measured laser spot-size at the Pockels Cell at $\mathrm{JLab} \mathrm{w}=0.88 \mathrm{~mm}$ [329], thermal conductivity for $\mathrm{KTP} \mathrm{k}=30 \mathrm{~mW} / \mathrm{cm} / \mathrm{K}$, and $1 \mathrm{~W}$ of power.

The thermal gradient in each crystal from a gaussian beam of power $P_{\text {tot }}$ and $2 \sigma$ width $w=F W H M / \sqrt{2 \ln 2}$ can be found using the heat equation:

$$
\nabla^{2} T(r)+q(r) / k_{t h}=0
$$




$$
\begin{gathered}
q(r)=\alpha I=\alpha I_{0} e^{-2 r^{2} / w^{2}} \\
P_{t o t}=\frac{1}{2} \pi I_{0} w^{2} \\
\frac{1}{s} \frac{\partial T}{\partial s}+\frac{\partial^{2} T}{\partial x^{2}}+\frac{2 \alpha P_{t o t}}{\pi k_{t h} w^{2}}=0
\end{gathered}
$$

where $\mathrm{T}(\mathrm{r})$ is the temperature, $\mathrm{q}(\mathrm{r})$ is the heat source, $k_{t h}$ is the thermal conductivity of the RTP material, and $\alpha$ is the absorption coefficient per unit length. We neglect crystal edge effects in this calculation and assume the steady state heat flow is radial. For a gaussian laser beam, giving rise to a gaussian heat source $q(r)$, the following solution can be obtained for the radial temperature profile [331]

$$
T=T_{0}+\frac{\alpha P_{t o t}}{4 k_{t h} \pi} \sum_{0}^{\infty} \frac{(-1)^{n}\left(\frac{2 r^{2}}{w^{2}}\right)^{n}}{(n)(n !)}
$$

where $\alpha$ is the absorption coefficient per unit length, $\mathrm{P}$ is the incident laser power, $\mathrm{r}$ is the radial coordinate and $\mathrm{w}$ is the beam radius.

We both numerically solved the heat equation and performed the above sum up to the first 100 terms and obtained consistency to the level of $10^{-} 8^{\circ} \mathrm{C}$. The result for the temperature gradient in one of the two crystals is shown in Fig. B-8

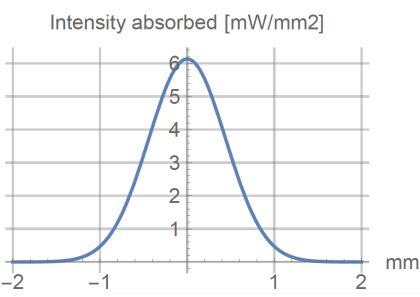

(a)

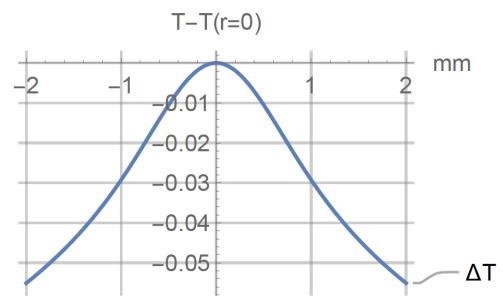

(b)

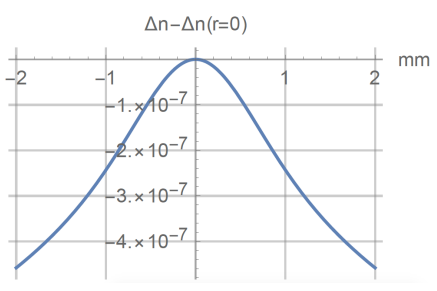

(c)

Figure B-8: Laser induced thermal gradient for RTP with absorption coefficient $0.75 \% / \mathrm{cm}$, laser spot-size $\mathrm{w}=0.88 \mathrm{~mm}$, thermal conductivity $\mathrm{k}=30 \mathrm{~mW} / \mathrm{cm} / \mathrm{K}$ (from KTP crystal), and 1W of laser power. (a) Power absorbed by the first (of 2) $10 \mathrm{~mm}$ length RTP crystal (b) calculated temperature gradient within crystal in ${ }^{\circ} \mathrm{C}(\mathrm{c})$ temperature induced birefringence in the first RTP crystal

However, the relevant quantity here is the difference in temperature between 
the two crystals (Fig. B-9 ) after a $0.75 \%$ absorption of power in the first crystal. This temperature gradient difference between the crystals gives rise to a net phase gradient, a charge asymmetry Aq (Fig. B-10) with 1st and 2nd moments (Fig. B-11 ), and position differences and spot-size asymmetries when analyzing in S1 (Fig. B-11).

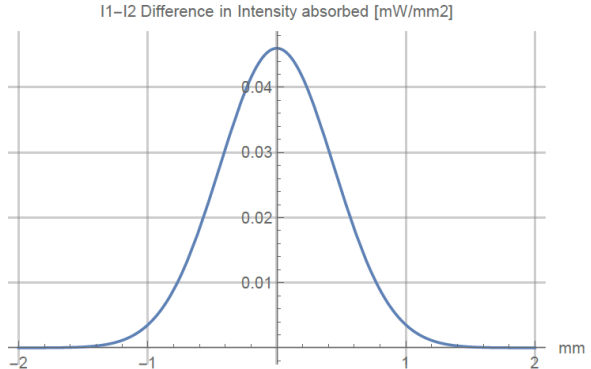

(a)

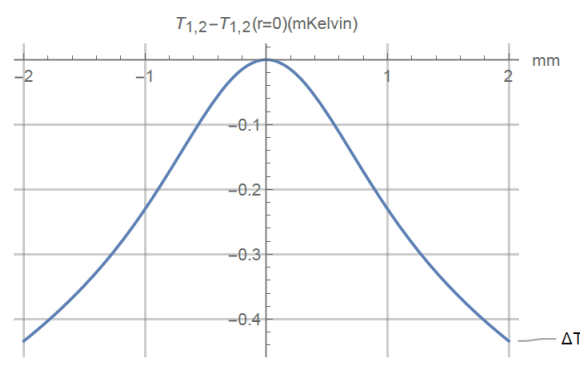

(b)

Figure B-9: Laser induced difference in thermal gradient between the 2 RTP crystals with absorption coefficient $0.75 \% / \mathrm{cm}$, laser spot-size $\mathrm{w}=0.88 \mathrm{~mm}$, thermal conductivity $\mathrm{k}=30 \mathrm{~mW} / \mathrm{cm} / \mathrm{K}$ (from $\mathrm{KTP}$ crystal), and $1 \mathrm{~W}$ of laser power. (a) Difference in power absorbed by the first and second $10 \mathrm{~mm}$ length RTP crystals (b) Difference in calculated temperature gradient within the 2 crystals in mKelvin

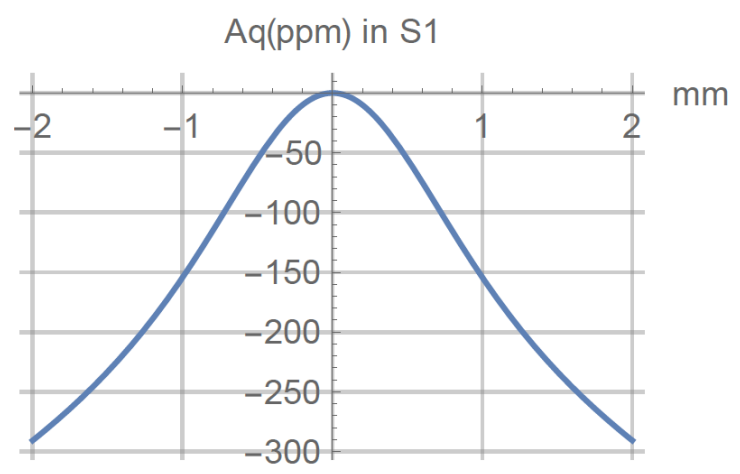

Figure B-10: Laser heating induced Aq for a 100\% analyzer along S1 

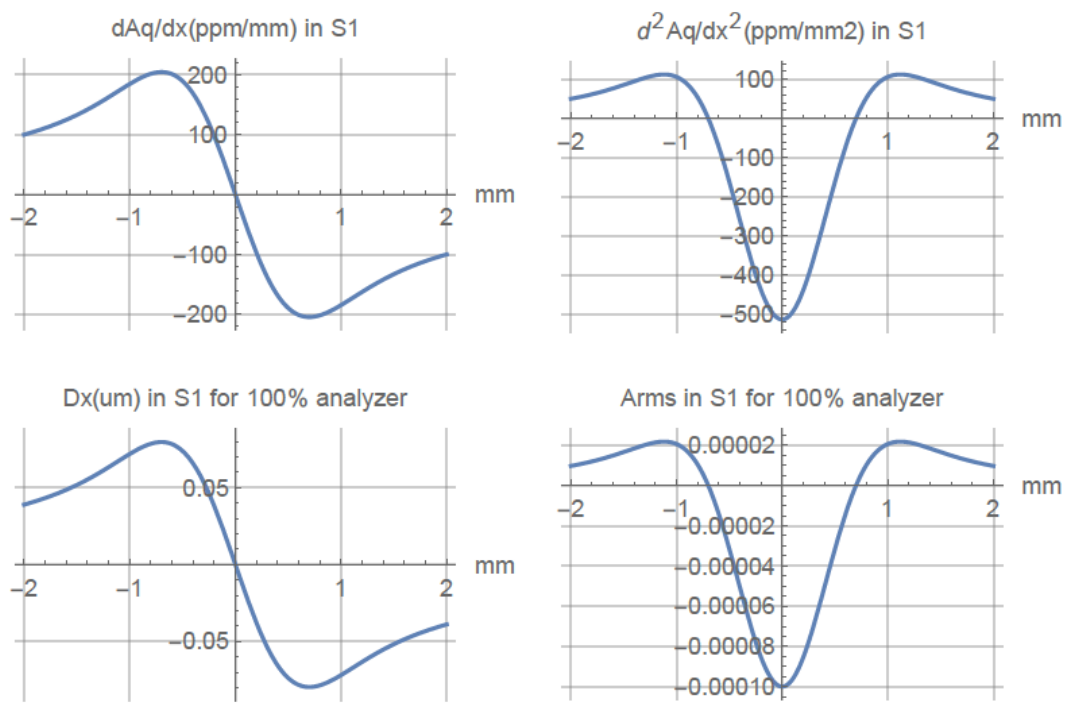

Figure B-11: Laser heating induced Aq 1st and 2nd moments and the corresponding Dx and spot-size asymmetry for a $0.88 \mathrm{~mm}$ beam and $100 \%$ analyzer along S1

The maximal laser induced position difference is $0.8 \mathrm{um}$ when analyzing at $100 \%$ in S1. The cathode only has an analyzing power of $<6 \%$. Furthermore, the RHWP may be rotated so the cathode's analyzing axis is not along S1(near $90^{\circ}$ ), but rather closer to $\mathrm{S} 2$ at $50^{\circ}$ for example, producing only a $0.525 \%$ residual analyzing component along S1. This reduced analyzing power serves to suppress the potential laser heating induced position differences in the e-beam, reducing them to $48 \mathrm{~nm}$ in the case of the cathode analyzing along $90^{\circ}(\mathrm{S} 1)$ and further reducing them to $4.2 \mathrm{~nm}$ in the case of using a RHWP rotation so the cathode analyzes along $50^{\circ}$ (near S2) for a $0.88 \mathrm{~mm}$ e-beam spot size. The same argument applies for spot-size asymmetry $A_{\sigma}$ which we observe to have a maximal laser induced value of $10^{-4}$ when analyzing at $100 \%$ in S1. $A_{\sigma}$ is reduced by the cathode down to $6 \times 10^{-6}$ in the case of the cathode analyzing along $90^{\circ}(\mathrm{S} 1)$ and further reduced down to $5.25 \times 10^{-7}$ in the case of using a RHWP rotation so the cathode analyzes along $50^{\circ}$ (near S2). 


\section{B.5 Suppressing Effect of Temperature Drifts on e-beam}

There are many ways in which the laser could experience a deviation in phase shift from quarter-wave when passing through the Pockels Cell. The laser interacts strictly with the optical coefficients of the crystal and those have a variety of couplings: electro-optic coefficients, thermo-optic coefficients, and stress-optic coefficients. A phase shift could come from wavelength drift, temperature drift via temperature induced birefringence, voltage change via voltage induced birefringence, or stress change via stress induced birefringence. We can control the phase shift experienced by the laser in many ways: we could measure temperature and control temperature with heating/cooling; we could measure birefringence and control it with heating/cooling; we could measure Aq and control it by putting stress on the crystal; we could measure wavelength and control voltage to compensate for it. But in a Pockels Cell the most straightforward method is to measure the polarization asymmetry and use voltage to control the birefringence. After all, controlling birefringence with voltage precisely what a pockels cell is designed to do in the first place. With a Pockels Cell, we can use voltage to change the birefringence and compensate for all other physics effects which couple to optical coefficients.

\section{B.5.1 Feedback}

To 0th order, the Pockels cell is aligned and the PITA voltages set on the laser table to zero out the polarization asymmetry. However, in the case of RTP, due to thermal drifts in $\Delta$-phase, it is necessary to feedback on the Pockels Cell PITA voltage to correct the polarization asymmetry. On the laser table, we directly measure the polarization asymmetry by inserting a $100 \%$ polarizer which analyzes along $90^{\circ}(\mathrm{S} 1)$, thereby converting a polarization asymmetry into an in- 


\section{B.5. SUPPRESSING EFFECT OF TEMPERATURE DRIFTS ON E-BEAM394}

tensity asymmetry measurable by a photodiode. When electron beam is being generated, however, another method of monitoring the polarization asymmetry is employed. The photo-cathode serves as a partial polarizer with a small analyzing power (4-6\%) which analyzes along an axis determined by the RHWP, thereby partially converting a polarization asymmetry into a charge asymmetry measurable by BCMs and BPMs. During an experiment, the charge asymmetry is measured by the BCMs in the Hall. During testing of the RTP cell and initial alignment, the charge asymmetry is monitored by either a BPM or a BPM wire sum in the injector. The measured charge asymmetry can be corrected by feedback on the PITA voltage of the Pockels Cell, feedback on the IA cell, or a combination of the two. For the RTP cell, as it is known that thermal drifts incur polarization asymmetries, it is desirable to correct the birefringence drift with feedback on the PITA voltage.

In order for feedback to work well, three settings must be appropriately selected (1) RHWP angle (2) feedback interval (3) monitor. With the KD*P cell, RHWP angle was selected based on constraints in minimizing position differences. With the RTP cell, we have the freedom to choose any RHWP angle we wish since position differences can always be zeroed out with PITAposU/V voltages and any charge asymmetry offset term or $2 \theta$ term in RHWP scans can be nearly zeroed out by RTP relative roll. We are free to choose any RHWP angle. There are two combating considerations in selecting RHWP angle. On the one hand, setting the RHWP angle so the cathode analyzes along S2 would give essentially zero analyzing power along S1, zero coupling between polarization asymmetry drifts and charge asymmetry drifts Aq. The measured charge asymmetry Aq would remain more stable, near zero, and only vary due to downstream helicity-correlated clipping on apertures which could be corrected with the IA cell. On the other hand, we don't want to use the IA cell, we'd rather use PC voltages, and we want to be able to measure and control the polarization asymmetry. In order to use PC voltages to correct Aq, we have to have some degrees of analyzing power along S1 


\section{B.5. SUPPRESSING EFFECT OF TEMPERATURE DRIFTS ON E-BEAM395}

to get a significant enough PITA slope. Further, in order to measure and control the drifting polarization asymmetry, we have to turn it into a charge asymmetry and measure it with a $\mathrm{BCM}$ downstream. These considerations dictate that we ought to select a RHWP angle far enough from S1 so that Aq doesn't drift too much due to thermal effects, but far enough from S2 that we are still able to measure the polarization asymmetry drift and still able to correct other sources of Aq, such as helicity correlated clipping, with reasonably low PITA voltages.

The feedback interval is simply the length of time over which the charge asymmetry is measured before applying a correction with PITA voltage. In selecting the feedback interval and the monitor, we must take into account the electronic noise of the monitor in the selection of the interval. The feedback interval must be long enough to obtain decent statistical accuracy on Aq, otherwise we'd just be falsely correcting electronics noise as if it were polarization asymmetry and introducing noise into the beam, which is undesirable. One the other hand, the feedback interval must be short enough that the central value of Aq doesn't change too much over the course of the interval, otherwise you allow Aq to drift faster than you correct it and feedback will fail to converge.

If feedback settings have been chosen properly, the charge asymmetry Aq should converge fairly quickly as RMS/N where $\mathrm{N}$ is the number of feedback intervals. During feedback, whatever is measured in one interval is cancelled by the correction-induced asymmetry in the next interval. After many intervals, you find noise has gone down by $1 / \mathrm{N}$, rather $1 / \operatorname{sqrt} N$. If drift is slow relative to feedback cycle speed, it acts like a constant, and is taken out. If drift is fast relative to feedback speed, it acts like the random noise that gives you the RMS, so is just just part of the RMS that falls as $1 / \mathrm{N}$. As the feedback cycle is made faster, the drift is effectively smaller, as drift approaches random noise, then you get closer to the case where the drift is tracked well by feedback and removed.

During HAPPEX-III, typical feedback the helicity reversal rate was $29.6 \mathrm{~Hz}$ (each helicity window was $33.83 \mathrm{~ms}$ long), the intervals were 3550 events (1775 
pairs) collected during a two minute interval cycle, for which the typical statistical uncertainty was $18 \mathrm{ppm}$ for the HallA BCM while the Aq drifted between 0-50 ppm [333]. The KD*P PITA slope was for $100 \%$ analyzing 634ppm/V [334] and the analyzing power of the cathode was $4 \%$ [335] with the RHWP set to $50^{\circ}$ [336]. $\mathrm{S} 1$ was near $62^{\circ}$ so the cathode was analyzing along an axis $21^{\circ}$ away from $\mathrm{S} 1$ and an effective analyzing power of $1.62 \%$ along S1. Hence, the effective PITA slope used in feedback was $\sim 15 \mathrm{ppm} / \mathrm{V}$ out of typical QWV of $2.4 \mathrm{kV}$.

During Qweak, the helicity flip rate was $\sim 1 \mathrm{kHz}$, the feedback intervals was 40ms in Run2 and $80 \mathrm{~ms}$ in Run1 [338]. The charge feedback is generally limited to $1 \%$ by the accuracy of the AQ measurement due to $\mathrm{BCM}$ resolution or a non-linearity in the experimental apparatus. The cathode analyzing power was 3-4\% [338] and the RHWP was set such that the effective PITA slope was $0.808 \mathrm{ppm} /$ count $\left(2^{16}\right.$ counts $\left./ 4000 \mathrm{~V}\right)=16.3 \mathrm{ppm} / \mathrm{V}$ [339] , implying an effective analyzing power of $\sim 2.5 \%$ along S1. A plot [337] of the RMS/N convergence of Aq during Qweak is shown in Fig. B-12 .

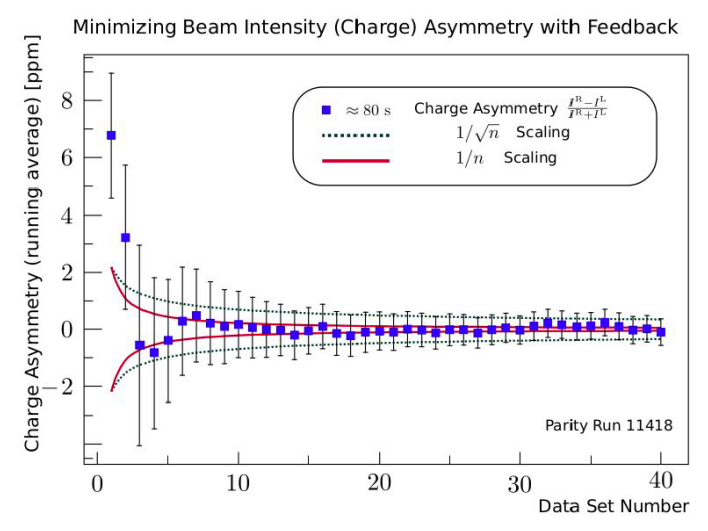

Figure B-12: Active charge feedback. Each Aq data point is a running average of data up to that point. The horizontal axis is the number of 80 s intervals. RMS/N statistical convergence are the dotted curves. RMS $/ \sqrt{N}$ statistical convergence are the red curves.

Using laser table measurements of the RTP polarization asymmetry drift and e-beam measurements with $240 \mathrm{~Hz}$ octets, we calculated what the Aq drift and noise might look like in the injector for various RHWP settings. We took the laser 
table run shown in Fig. B-2 and scaled it down by the effective analyzing power of the cathode. The Aq RMS observed in the injector for $20 \mathrm{uA}$ beam was $90 \mathrm{ppm}$ (for $30 \mathrm{~Hz}$-like $240 \mathrm{~Hz}$-octets), so artificial noise was introduced in our calculation to keep the RMS near 90ppm. Then we modeled what feedback would do to the Aq signal for different feedback intervals. Aq appeared to be well controlled for 3 second interval cycles and for an effective cathode analyzing power of $0.525 \%$, achieved by rotating the RHWP so the photocathode( $6 \%$ analyzer $)$ is $\sim 5^{\circ}$ from S2. The results are shown in Fig. B-13 . 

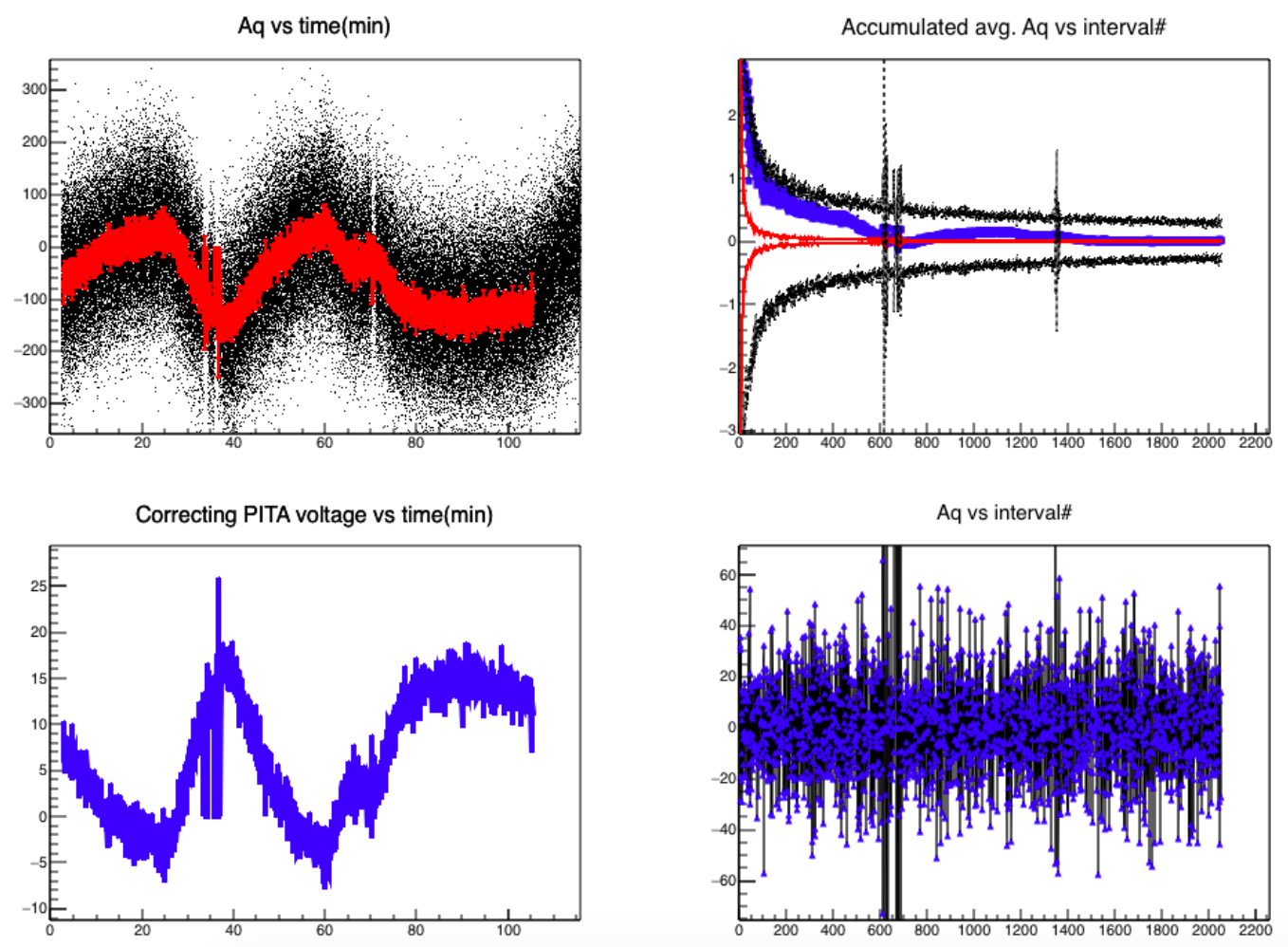

Figure B-13: Modeling charge feedback on RTP cell. (top-left) The expected noise and drift in Aq without feedback, black dots are individual events, red points are mean Aq for each interval with bars showing the RMS (bottom-left) PITA voltages which would be used for correcting Aq drift for feedback on (top-center) Feedback convergence. Each Aq data point is a running average of data up to that point. The horizontal axis is the number of 3 s intervals. RMS/N statistical convergence are the dotted curves. RMS $/ \sqrt{N}$ statistical convergence are the red curves. (bottomcenter) same as top-center only without RMS error bars (top-right). The expected drift in Aq with feedback on where bars signify the RMS (bottom-right) same as top-right without RMS bars.

The expected noise and drift without feedback is shown on the top left of Fig. B-13 . On the laser table, Aq drift is $\pm 30,000$ ppm over several hours. Here, with the small $0.525 \%$ analyzing power, the drift in Aq is $150 \mathrm{ppm}$ over several hours, as shown by the central values of the red data points on the top-left plot of Fig. B-13, where the error bars just correspond to the 90ppm RMS. The PITA voltages used for correcting 150ppm are constrained to be $<15 \mathrm{~V}$ (out of $800 \mathrm{~V}$ QWV in $8 \mathrm{HV}$ configuration) where the effective PITA slope is $\sim 10 \mathrm{ppm} / \mathrm{V}$ 
$($ PITA slope $=1698 \mathrm{ppm} / \mathrm{V}$ for $100 \%$ analyzer $)$. Feedback serves to suppress Aq fluctuations down to 50ppm, a factor of $2 \mathrm{X}$ reduction from 90ppm raw $30 \mathrm{~Hz}$ RMS, and suppress slow fluctuations well below the noise, considerably smaller than for the unstabilized drift, as shown in the top-right and bottom-right plots of Fig. B-13 . This 3sec interval setting also leads to a nice convergence rate between RMS $/ \mathrm{N}$ and RMS $/ \sqrt{N}$ of the accumulated average Aq (accumulated up to that point in time) shown by the bottom-center plot of Fig. B-13.

Other settings were tested with $0.25 \%, 0.5 \%, 1 \%, 2 \%$ analyzing powers with $0.5 \mathrm{sec}-2 \mathrm{sec}$ feedback intervals. They converged at a rate in-between RMS $/ \mathrm{N}$ and RMS $/ \sqrt{N}$ convergence. The PITA voltages used to correct the Aq temperature induced slow drifts from are the same regardless of analyzing power and were below $20 \mathrm{~V}$. 


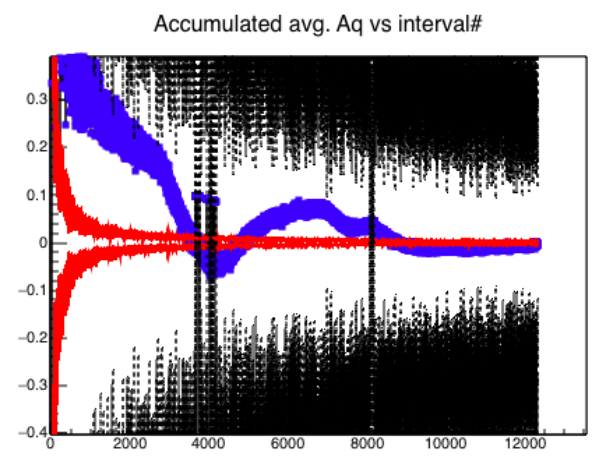

(a) $2 \%$ analyzer, $0.5 \mathrm{~s}$ intervals Accumulated avg. Aq vs interval\#

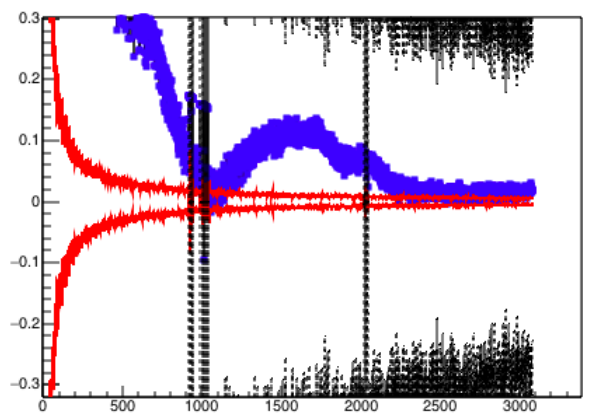

(c) $0.5 \%$ analyzer, $2 \mathrm{~s}$ intervals

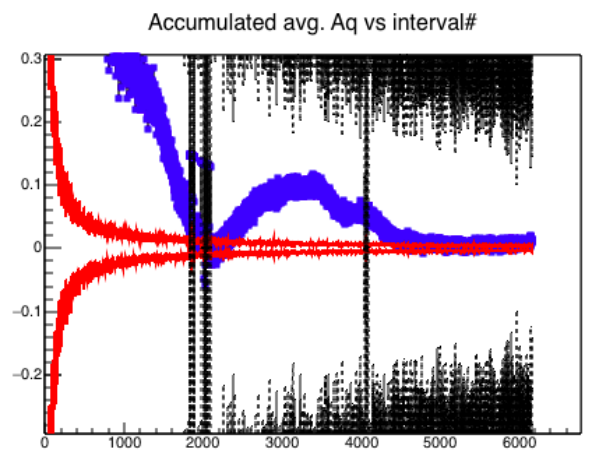

(b) $1 \%$ analyzer, 1 s intervals Accumulated avg. Aq vs interval\#

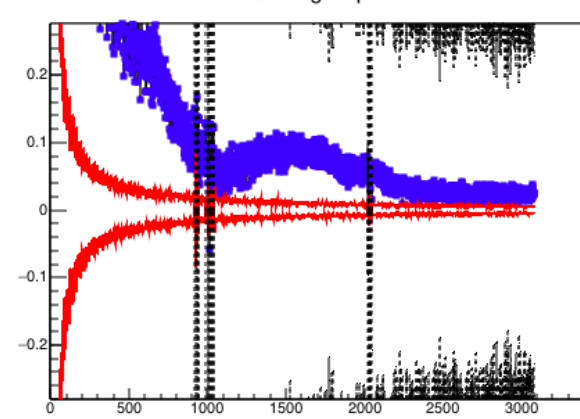

(d) $0.25 \%$ analyzer, $2 \mathrm{~s}$ intervals

Figure B-14: Modeling different settings for charge feedback on RTP cell. Each Aq data point is a running average of data up to that point. The horizontal axis is the number of feedback intervals. RMS/N statistical convergence are the dotted curves. RMS $/ \sqrt{N}$ statistical convergence are the red curves.

We also tried $0.5 \mathrm{sec}$ intervals for the small $0.525 \%$ analyzing power and it appeared to work quite well, converging near RMS/N, keeping Aq small. However, reducing the interval time might come at a slight cost. If the interval is too short, much of noise being corrected in real beam may be simply electronics noise as explained in Sec. B.5.2. 

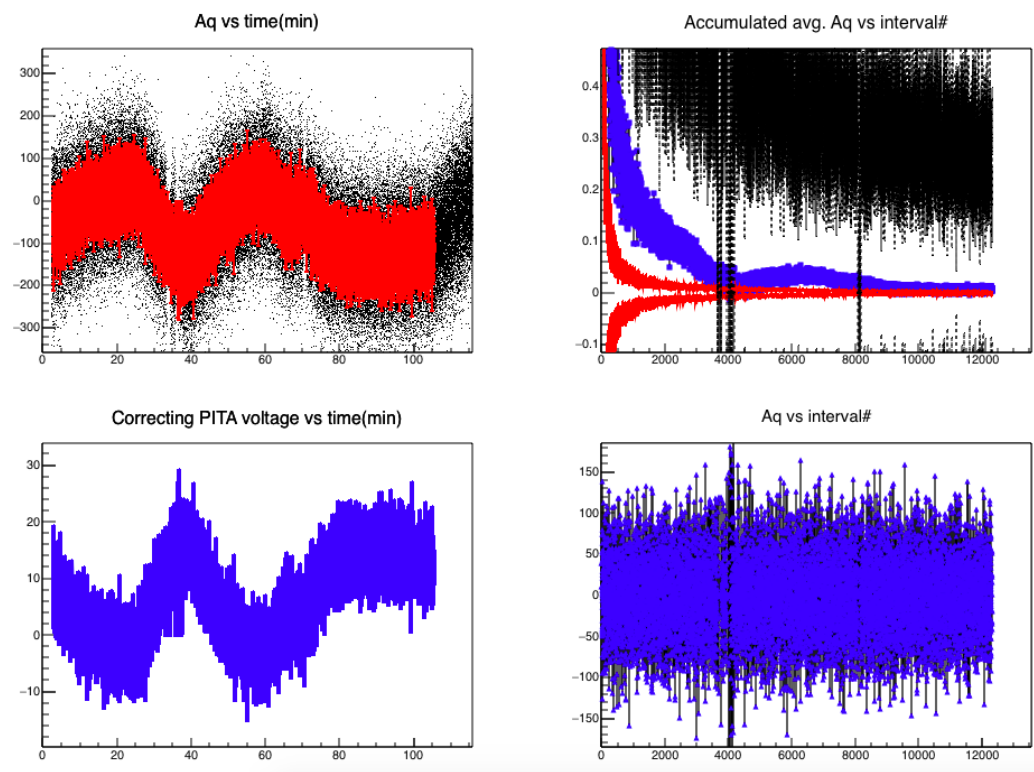

Figure B-15: Modeling charge feedback on RTP cell for $0.525 \%$ analyzer, $0.5 \mathrm{~s}$ intervals. (left) Each Aq data point is a running average of data up to that point. The horizontal axis is the number of intervals. RMS/N statistical convergence are the dotted curves. RMS $/ \sqrt{N}$ statistical convergence are the red curves. (right) Aq for each individual interval with feedback running.

\section{B.5.2 Depolarization}

Depending on how feedback on PITA voltage is done, one could effectively depolarize the e-beam to some extent. There are 3 obvious sources of fluctuations in measured Aq in the hall: (1) polarization asymmetry (2) non-polarization-like intensity asymmetry (3) electronics noise. Polarization asymmetry is controlled by PITA voltage, which changes the polarization state of the beam, the beam is then analyzed partially by the cathode, which translates polarization asymmetry into a charge asymmetry Aq you can measure with a bcm. Non-polarizationlike intensity asymmetry, or pure intensity asymmetry, could be thought of as encompassing the portion of the charge asymmetry which has nothing to do with the polarization state and is just an intensity asymmetry. For example, intensity noise in the Hall could come from the beam clipping on apertures in transport and that would have nothing to do with polarization. A pure intensity asymmetry 


\section{B.5. SUPPRESSING EFFECT OF TEMPERATURE DRIFTS ON E-BEAM402}

should, in principle, be corrected with something like the IA cell, not the Pockels Cell PITA voltage. Electronics noise in the BCM in the Hall obviously contributes to fluctuations in the measured Aq.

It's important to choose feedback integration intervals wisely based on actual beam polarization drift/noise and other noise sources. If the interval is too short, much of noise being corrected may be simply electronics noise. If feedback corrects electronics noise instead of actual polarization changes, then it's creating polarization noise, aka depolarizing the beam. It's also important that transport into the hall is good and transmission is high, because otherwise a significant part of the Aq noise may be due to clipping on apertures. Clipping on apertures would have nothing to do with polarization, and yet you'd be "correcting" it with PITA voltage, introducing quasi-depolarization by changing the polarization to fix an intensity fluctuation. The potential for creating quasi-depolarization becomes more significant when Aq is less sensitive to PITA voltage, i.e. the PITA slope is smaller, the cathode analyzing power is very small or the RHWP is rotated for for small PITA slope. This is because a correction to Aq requires a larger PITA voltage, a larger polarization change, in order to couple into a BCM measurable charge asymmetry. So, when selecting a RHWP angle, it's important to consider how much of what is measured by the BCM used for feedback is a pure polarization asymmetry and how much is from pure intensity noise and electronics noise. The PITA slope should not be made too small.

The degree of polarization of a beam can be defined as

$$
D o P=\sqrt{S_{1}^{2}+S_{2}^{2}+S_{3}^{2}} / S_{0}
$$

where $S_{0}$ signifies a normalization to total power, $S_{1}$ relates to the degree of linear polarization along the $\mathrm{H} / \mathrm{V}$ axes, $S_{2}$ relates to the degree of linear polarization along the $\pm 45^{\circ}$ axes, and $S_{3}$ relates to the degree of circular polarization. These quantities may be measured for a laser with a polarizer and a QWP. The 
are defined as follows:

$$
\begin{gathered}
S_{0}=I_{0}^{o}+I_{90^{\circ}}=I_{45^{\circ}}+I_{-45^{\circ}} \\
S_{1}=I_{0^{\circ}}-I_{90^{\circ}} \\
S_{2}=2 I_{45^{\circ}}-S_{0}=I_{45^{\circ}}-I_{-45^{\circ}} \\
S_{3}=S_{0}-2 I_{45^{\circ}, Q W P}
\end{gathered}
$$

where $I_{\theta}$ refers to the power transmitted through a polarizer oriented at angle $\theta$ and S3 is measured by first inserting a quarter-wave-plate before the polarizer.

A nearly circularly polarized beam with slight linear polarization along $\mathrm{H} / \mathrm{V}$ can be described as $S_{1} / S_{0}=A_{p}, S_{2} / S_{0}=0, S_{3} / S_{0}=\sqrt{1-A_{p}^{2}} \approx 1-A_{p}^{2} / 2$. Let's say that Ap, the polarization asymmetry, fluctuates over time with RMS $A_{p, r m s}$. The degree of linear polarization is given by the expectation value $D o L P=$ $\sqrt{\left.<S_{1}^{2}\right\rangle_{t}} / S 0=A_{p, r m s}$. The degree of circular polarization is given by the expectation value

$$
\begin{gathered}
D o C P=\sqrt{<S_{3}^{2}>_{t}} / S_{0} \approx<\sqrt{\left(1-A_{p}^{2} / 2\right)^{2}}>_{t} \approx \sqrt{1-<A_{p}^{2}>+<A_{p}^{4}>/ 4} \\
\approx \sqrt{1-A_{p, r m s}^{2}+A_{p, r m s}^{4} / 4} \approx 1-A_{p, r m s}^{2} / 2
\end{gathered}
$$

The degree of polarization is then $D o P=\sqrt{\left.\left.<S_{1}^{2}\right\rangle_{t}+<S_{3}^{2}\right\rangle_{t}} / S 0 \approx 1$.

When applying PITA voltages in feedback we are attempting to reduce the fluctuations in polarization asymmetry. However the physical measurable is the charge asymmetry Aq, not Ap, and Aq can be plagued by noise which does not relate to polarization asymmetry drifts. When the noise in measured Aq, call it $A_{\text {noise }}$, is falsely corrected with PITA voltage, we introduce a quasi-"depolarization" of the beam from $A_{\text {noise }}$. To state it more accurately, we don't strictly depolarize the beam, i.e. reduce the beam DoP, but we do reduce the degree of circular polarization DoCP and we increase the DoLP. 


\section{B.5. SUPPRESSING EFFECT OF TEMPERATURE DRIFTS ON E-BEAM404}

A small PITA slope for a small cathode analyzing power $\epsilon$ exacerbates the effect. If $A q=\epsilon A p$, then when Aq is corrected by PITA, $A p=A q / \epsilon$ and $D o L P=$ $A_{p, r m s}=A_{q, r m s} / \epsilon=A_{\text {noise }} / \epsilon$. The reduction in DoCP is given by $\left(A_{\text {noise }} / \epsilon\right)^{2} / 2$

The feedback interval must be selected to ensure that the beam doesn't suffer too great a reduction in degree of circular polarization(DoCP). Critically in feedback the electronics noise in the monitor is reduced by extending the length of the feedback cycle. $A_{\text {noise }} \sim R M S / \sqrt{n}$, where $\mathrm{n}$ is the number of helicity pairs in a feedback interval. For an analyzing power $\epsilon, D o L P=\frac{R M S}{\epsilon \sqrt{n}}$ and $1-D o C P=\frac{R M S^{2}}{2 \epsilon^{2} n}$. For example, if a BCM has electronics noise of $\mathrm{RMS}=100 \mathrm{ppm}$, the cathode analyzing power along $\mathrm{S} 1$ is $0.5 \%$, and the feedback interval is $30 \mathrm{sec}$ long at $30 \mathrm{~Hz}$, $\mathrm{n}=450$ pairs, then the potential reduction in DoCP is $0.004 \%$ and the potential increase in DoLP is $0.094 \%$. For Moller, it is stated that the polarization of the beam must be known to within $0.4 \%$ [11] . So, it is important to avoid altering the polarization too much, ensure the feedback interval is long enough and ensure the analyzing power of the cathode is large enough.

\section{B.5.3 Pickoff Design}

Sometimes feedback is done with both PITA voltage and IA cells. It would be nice to distinguish polarization noise from intensity noise; to be able to tell what was a polarization fluctuation and fix it with the PITA voltage, and tell what was an intensity fluctuation and fix it with the IA cell. Otherwise you could either (a) fail to fix a polarization fluctuation (allow depolarization to remain in the case of just IA) or (b) introduce a depolarization which wasn't necessarily there in the case of just using PITA voltage for feedback. Currently, this distinction is not a possibility. However there is a possible configuration which could allow for such a distinction.

A separate polarization-asymmetry feedback loop might involve the following design. A small high-transmission pickoff could be inserted after the Pockels cell 


\section{B.5. SUPPRESSING EFFECT OF TEMPERATURE DRIFTS ON E-BEAM405}

and remain in place during e-beam running. The pickoff's reflected beam would be passed through a vertical polarizer and monitored by a photodiode constantly. We note the reflected and transmitted beams would experience slightly differing amounts of birefringence, which means zeroing out the electron beam Aq would result in a non-zero laser beam Aq as measured by this pickoff-photodiode. The goal would be to stabilize the measured laser Aq on the pickoff-photodiode and keep it at the same value. The pickoff-photodiode would be used as a independent monitor of polarization asymmetry. PITA feedback would only be used to stabilize the actual laser polarization asymmetry.

In the case of multiple Hall running, if multiple Hall lasers were on, the pickoff beam could be passed through a small commercial Pockels Cell, with ultra fast flipping ability, hooked up to the HallA 499MHz control signal and passed through a vertical polarizer. So, the pickoff-photodiode would only examine the HallA laser beam and the $499 \mathrm{MHz}$ flipping would block out the other three Hall lasers.

Separately, another feedback loop, perhaps with an improved IA cell, would control charge asymmetry Aq in the Hall as measured by the BCMs. This feedback loop would only control the residual, leftover asymmetries not corrected by the polarization-asymmetry feedback loop. It would only be focused on correcting nonpolarization effects like transmission issues and aperture clipping with intensity.

Thus, two independent effects would be properly controlled by two independent feedback loops monitoring two separate quantities: Aq in the Hall and $\mathrm{Aq}$ (polarization asymmetry) on the laser table.

\section{B.5.4 Temperature Feedback}

Instead of using PITA voltages, one could employ temperature control on a temperature sensitive birefringent element. The crystals are too temperature sensitive to easily feedback on, but a multi-order waveplate is temperature sensitive to a much lesser extent. For example the Thorlabs WPMH05M-780 - 1/2" Mounted 
Multi-Order Half-Wave Plate, 1" Mount, $780 \mathrm{~nm}$ made from $1.00 \mathrm{~mm}$ thick highquality crystalline quartz could be inserted after the Pockels cell with its fast axes along S2 so that changes in its birefringence contribute to S1 asymmetries. The temperature dependence of the refractive indices of crystal quartz/fused silica as for the ordinary axis $d n_{o} / d T \approx-7.5 p p m K^{-1}$ and for the extraordinary axis $d n_{e} / d T \approx-9 p p m K^{-1}$ at $780 \mathrm{~nm}$ [332]. The temperature dependence of such a waveplate is:

$$
A q=2 \pi L / \lambda \frac{d\left(n_{o}-n_{e}\right)}{d T} \Delta T \approx 12,083 p p m / K \Delta T
$$

If we really wanted to do temperature feedback on Aq instead of PITA voltage feedback, heating and cooling a multi-order waveplate by $3{ }^{\circ} \mathrm{C}$ would be sufficient to control the RTP temperature induced fluctuations of 30,000ppm. 


\section{Appendix C}

\section{Tech Note: Interferometric/Etalon Effect}

Here we discuss ways to suppress the etalon effect including: (1) non-negligible reflection coefficients (2) parallel faces (3) narrow-bandwidth (4) temporal overlap of reflected pulses (5) spatial overlap and coherence of reflected pulses.

\section{C.1 Temporal overlap}

This is not only potentially detrimental to precision $\lambda / 4$-wave applications, but in cavity locking applications it's possible the etalon effect could compromise extinction ratios. However, this etalon effect can be mitigated by using a pulsed laser, with short enough pulse duration, or by using longer RTP crystals $(10 \mathrm{~mm}$ length typical). Then the etalon effect can be mitigated by the fact that there is a temporal delay between the reflection off the front face of the crystal and the reflection off the back face of the crystal. Since, a shorter pulse duration or a longer crystal can help reduce the etalon effect, both the laser pulse duration and the crystal length must be considered in Pockels cell design. The temporal structure of the laser is important when using RTP Pockels cell, and it's important not to use very long pulse durations. At JLab, the pulse duration is 30-50ps and the 
crystal length of our RTP's are 10mm. These conditions were sufficient to mitigate the etalon effect for the HallA laser in 2016 (etalon measurement constrained to $<1 \mathrm{ppm}$ ). In 2019, we observed an etalon effect of 300ppm for a $0.6 \mathrm{~mm}$ FWHM spot size and an etalon of 80ppm for a 2mm FWHM spot size for the HallA laser at $500 \mathrm{MHz}$. The HallB laser (at $250 \mathrm{MHz}$ ) showed a larger etalon of $\sim 1000 \mathrm{ppm}$ for a $0.6 \mathrm{~mm}$ FWHM spot size and an etalon of 200ppm for a 2mm FWHM spot size. So, shorter pulse tails or longer crystals may be desirable in the future.

\section{Etalon Suppression}

A gaussian beam $I(t)=\sqrt{\frac{2}{\pi \tau^{2}}} e^{-2 t^{2} / \tau^{2}}$ with pulse width $\tau=t_{F W H M} / \sqrt{2 \ln 2}$ incident on a crystal with reflection coefficient $R=r^{2}$, will undergo an interference between the two reflected pulses off the front and back face of the crystal, and the transmitted light is given by

$$
T=1-R=1-2 r^{2}\left(1+e^{-\frac{\Delta t^{2}}{2 \tau^{2}}} \cos \omega \Delta t\right)
$$

where $\Delta t=2 \mathrm{Ln} / \mathrm{c}$ is the delay between the two back reflections, the time it takes for light to traverse the crystal twice. We refer to the term $\frac{1}{\gamma}=e^{-\frac{\Delta t^{2}}{2 \tau^{2}}}$ as the etalon suppression factor because $\gamma=1$ for a CW beam and as the pulse duration becomes shorter and the beam overlap less, they interfere less, and the etalon interference amplitude is suppressed by a larger factor $\gamma>>1$. For reference, in a $10 \mathrm{~mm}$ crystal, the delay between the two pulses is $115-130 \mathrm{ps}$ and for a typical pulse, the FWHM is around 55ps, so the overlap is in the beam tails.

Figure C-1a shows the etalon suppression factor with respect to FWHM pulse duration for various crystal lengths and for both $n_{y}$ and $n_{z}$ primary crystal axes. Because the JLab beam in reality is not a pure gaussian, we also show the same plot of suppression factor with respect to the $4 \tau$ pulse duration (similar to the $4 \sigma$ spot size) in Fig. C-1b which encompasses the entire pulse to it's full-width$1 / e^{8} 1 / 3000-\max$. Since it is the beam tails that matter in this case, this $4 \tau$ plot 
should be helpful in chopper scans when examining the tails of the e-beam and predicting how it might affect the etalon amplitude: find where the intensity drops off to $1 / 3000$ the max, use that full width value in Fig. C-1b to predict how bad the etalon will be. A zoomed in plot just for $\mathrm{L}=10 \mathrm{~mm}$ is shown in Fig. C-1c for reference. We note that the suppression factor falls off extremely quickly, even on a $\log$ scale, and it is the beam tails overlapping that is important, so after-pulses in the laser could be quite detrimental. Adjusting the pulse shape with the seed laser at JLab and iterating etalon scans and chopper scans may be an important procedure to go through. 


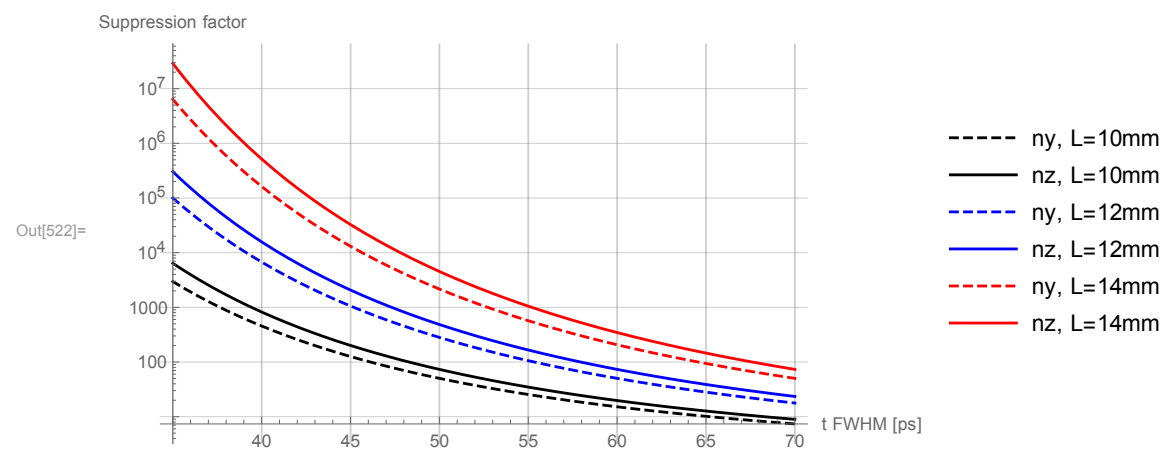

(a) $\gamma$ vs. FWHM pulse duration

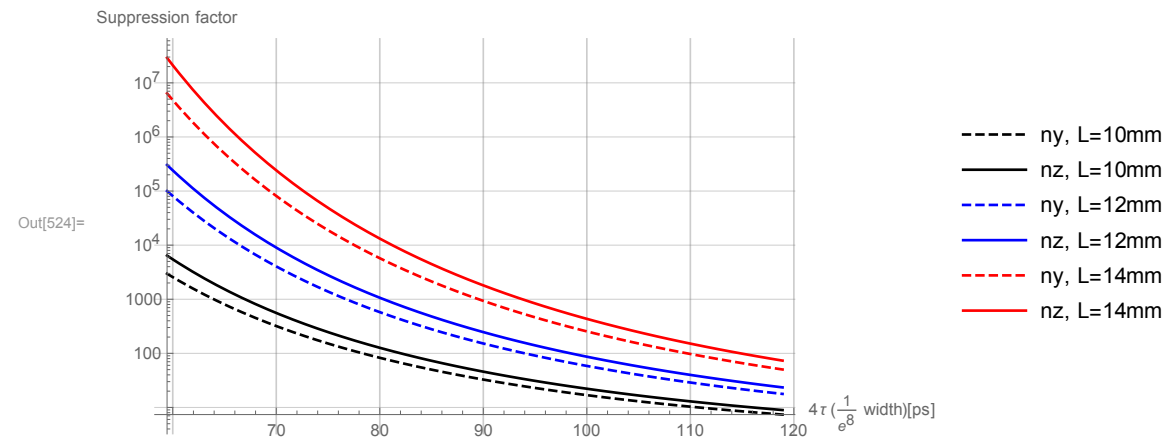

(b) $\gamma$ vs. $4 \tau$ pulse duration

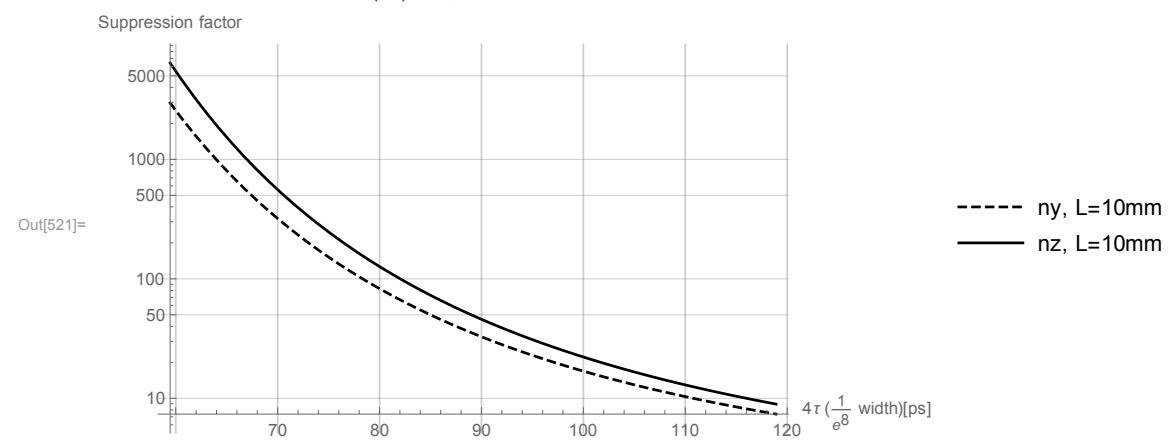

(c) $\gamma$ vs. $4 \tau$ pulse duration $(L=10 \mathrm{~mm})$

Figure C-1: Etalon suppression factor: For a pure gaussian temporal distribution, for $L=10,12,14 \mathrm{~mm}$ crystals, for both RTP primary axes $n_{z}=1.867, n_{y}=1.783$

To suppress a 5000ppm etalon from a reflection coefficient of $0.5 \%$, a suppressionfactor of $\gamma \sim 1000$ is desirable for the Hall A laser to maintain an etalon induced $A q<5 p p m$. For the other Halls, the requirements may be less stringent, for example Hall B maintaining an etalon induced $A q<100 p p m$ would only require $\gamma \sim 50$. Of course, buying longer crystals helps significantly, by orders of magnitude, and as long as the piezo-electric resonance isn't too low-frequency, you could 
still flip helicity states quickly with short transition times and minimal ringing. There are additional ways to reduce etalon besides crystal length, however, as described below.

\section{C.2 Reflection Coefficients}

Regarding reducing reflection coefficients, we cannot afford to have even a very small charge asymmetry, so attempting to reduce reflectivity down to the ppm level is unfeasible. Even the best AR coatings would be unlikely to eliminate charge asymmetry sufficiently, though improving the AR coating would certainly help improve the current performance. It is worth noting that Reflection curves sent by manufacturers are not accurate as most testing of AR coatings in done on glass substrates instead on on the crystal material, and since the refractive index of RTP can be very high $\left(n_{x}=1.78, n_{y}=1.789, n_{z}=1.877\right.$ at $780 \mathrm{~nm}$, calculated from Sellmeier equation, Optical Materials 22 (2003) 155-162 ) compared to glass (n 1.5), the AR coating performance can be compromised. For example, our RTPs reflection curves from Raicol indicate $0.05 \%$ reflection, but we observe effects that indicate $0.2-0.5 \%$ reflection.

\section{C.3 Non-Parallel Face Cuts}

Regarding non-parallel faces, how parallel is too parallel? This possible solution requires further research into the fundamentals of etalons. However, in the best case scenario, using the least stringent argument, we could say the required face cut angle(scaled by refractive index due to Snell's Law) must be larger than the beam divergence in order for the back reflecting beams not to overlap at some finite distance upstream $\theta>\theta_{\text {divergence }} / n=\lambda / n \pi \omega_{0}$. For a $1 \mathrm{~mm}$ beam, this face cut angle is at least $0.3 \mathrm{mrad}^{1}$, whereas currently the face cut angles are $0.05 \mathrm{mrad}$.

\footnotetext{
${ }^{1}$ typical divergence of JLab beam at cell is $\sim<0.5 \mathrm{mrad}$
} 
At the very least, this solution requires either (a) new crystals or (b) recutting of crystals to spec by manufacturer. It is very much worth pointing there are other more stringent arguments (i.e. the back-face reflected beam must be displaced by its FWHM of $1 \mathrm{~mm}$ relative to the the front-face reflection) which would require face cuts $100 \mathrm{mrad}$ to avoid etalon effects.

Are non-parallel face cuts a problem? Not for delta phase or analyzing, but it can be a problem for alpha-phase. As long as the face cuts of the two crystals are matched to each other, non-parallel face cuts are not a problem for delta-phase. As long as the path length of a given part of the beam through each crystal is the same, the delta phase is approximately constant as long as no PITA voltage is applied. We can see this by taking the face cuts of the two crystals at angle $\theta$ such that they compliment each other, neglecting all other small order terms,

$$
A_{q}(X) \approx \frac{2 \pi \theta X E_{P I T A}}{L E_{Q W V}}
$$

For $\mathrm{L}=10 \mathrm{~mm}, \mathrm{X}=1 \mathrm{~mm}, \mathrm{PITA} / \mathrm{QWV} 0.2, \theta=100 \mathrm{mrad}$, we get that Aq changes by $12,000 \mathrm{ppm} / \mathrm{mm}$ which is about $50 \%$ of the gradient we currently observe, so even a 100mrad cut does not raise asymmetry gradients to unacceptable levels.

As for analyzing power, the RTP crystals are inherently birefringent and already have their own natural analyzing power proportional to the reflection coefficient. Fortunately, since the two crystals fast axes are oriented perpendicular to each other, the analyzing power in the interface between the two crystals cancels and the only analyzing left over is the analyzing power of the exit face. Any analyzing power will be along the crystal's primary axes, along S2, and most of it will not be helicity correlated and only produce a small alpha-phase along S2, making the beam slightly linearly polarized along S2. The magnitude of this alpha-phase linear polarization is proportional to the reflection coefficient which is $<0.5 \%$, and since the analyzing power by definition power $=\epsilon / T=\frac{T_{1}-T_{2}}{\frac{1}{2}\left(T_{1}+T_{2}\right)}=\frac{R_{2}-R_{1}}{1-\left(R_{1}+R_{2}\right) / 2} \approx$ $R_{2}-R_{1}<R$ must be less than the reflectivity, the induced linear polarization is 
$<0.5 \%$ along S2. As a comparison, typical degree of linear polarization we were able to achieve with $\mathrm{KD}^{*} \mathrm{P}$ on the lab bench was $5 \%$, so this effect is relatively small.

The alpha-phase term could potentially be a problem for non-parallel face cuts. If the face cut is large enough, it can cause the beam to go through a significant transition from circular to linear polarization across the cell face, in other words the out going beam would not have HCBA but it would be comprised of portions of circular and some linear polarization, which could be a highly unusual running condition to say the least. It is important to ensure the face cuts are not so large that they create any significant alpha-phase across the diameter of the beam. Using the equation for alpha phase in RTPs, taking the face cuts of the two crystals at angle $\theta$ such that they compliment each other and neglecting all other small order terms, we arrive at

$$
\alpha \approx \frac{\pi \theta X}{2 L}
$$

For $\mathrm{L}=10 \mathrm{~mm}, \mathrm{X}=1 \mathrm{~mm}, \mathrm{PITA} / \mathrm{QWV} 0.2, \theta=100 \mathrm{mrad}$, we get that $\alpha$ changes by $0.015 \mathrm{rad} / \mathrm{mm}$. Further using $D O L P \approx \frac{\alpha}{1+(\alpha / 2)^{2}}$, we see that across the face the crystal, the DOLP would increase by $1.5 \% / \mathrm{mm}$. The typical DOLP we are able to achieve with $\mathrm{KD}^{*} \mathrm{P}$ is $5 \%$, so this cut does not raise DOLP gradients to unacceptable levels by comparison. A smaller face cut angle of $0.5 \mathrm{mrad}$ would only increase the DOLP by $0.08 \%$ at the edges of a $1 \mathrm{~mm}$ spot size, which a negligible amount.

Regarding analyzing-like position differences, as described in Sec. 6.5.2, a face cut non-parallelism of $\theta_{f c}=\frac{d L}{d x_{i}} \approx 0.01-0.05 \mathrm{mrad}$ (the Raicol crystal specs) induces position differences $D_{L g r a d, x_{i}}=-\frac{w^{2} \pi}{2 \lambda}\left(n_{0, y}-n_{0, z}\right) \theta_{f c} \approx 1.7-8.5 u m$ for a $1 \mathrm{~mm}$ spot size. If an intentional face cut of $0.5 \mathrm{mrad}$ is made to reduce the etalon effect, the induced position differences would be $85 \mu \mathrm{m}$ for a $1 \mathrm{~mm}$ spot size and $21 \mu \mathrm{m}$ for a $0.5 \mathrm{~mm}$ spot size. To avoid creating large position difference from large birefringence gradients, the face cuts could be done on each RTP crystal in 
opposing directions: for example a $0.5 \mathrm{mrad}$ face cut on $\mathrm{X}-\mathrm{Z}$ place on RTP \#1 and $-0.5 \mathrm{mrad}$ on $\mathrm{X}-\mathrm{Y}$ plane of $\mathrm{RTP} \# 2$ which is rotated by $90^{\circ}$ as illustrated in Fig. C-2. By making equal and opposing face cuts on both crystals, these induced position differences can be entirely avoided.

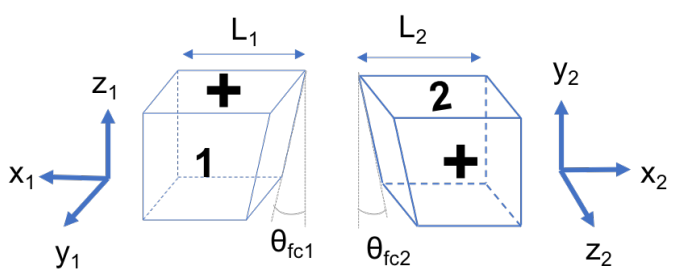

Figure C-2: Opposing face cuts: Face cuts $\theta_{f_{c 1}}=\theta_{f c 2} \approx 0.5 \mathrm{mrad}$ within a desired tolerance of $\left|\theta_{f c 1}-\theta_{f c 2}\right|<0.05 \mathrm{mrad}$ and tolerance of length difference $\left|L_{1}-L_{2}\right|<2 \mu m$ (typical)

Regarding steering position differences, as described in Sec. 6.3.4, a face cut non-parallelism of $\theta_{f_{c}} \approx 0.01-0.05 \mathrm{mrad}$, induces fixed (unchangeable/intrinsic) steering position differences $D_{x_{i}, \text { fixed }}$ (the steering position difference of a single crystal) is given by $\left|\left(n_{y 0}^{3} r_{23}+n_{z 0}^{3} r_{33}\right) E_{z 0} \theta_{f c} D\right| \approx 1-4 n m$ at a throw distance $D=2 m^{2}$. This quantity is negligible. Even if an intentional face cut of $0.5 \mathrm{mrad}$ were made to reduce the etalon effect, the induced position differences would only be $40 \mathrm{~nm}$.

If etalon effect elimination required face cuts of $0.5 \mathrm{mrads}$, the typical $0.5-1 \mathrm{~mm}$ beam would likely function properly. In order to accommodate large face cuts, designing focusing optics to reduce the spot size at the Pockels cell is a possibility, but it is important that small beam divergence be maintained, since it could not only greatly alter the HCBA behavior the crystal but could also make the etalon effect worse. Face cuts are only a feasible solution if they are on the order of $<0.5 \mathrm{mrad}$ or opposing (equal and opposite) face cuts are made on both crystals, do not cause unmanageably large position differences, and do not cause the alphaphase to change so much that the edges of a $1 \mathrm{~mm}$ beam are significantly linearly

\footnotetext{
${ }^{2}$ At JLab, the typical effective cathode throw distance with a $2 \mathrm{~m}$ lens is $\mathrm{D}=2 \mathrm{~m}$
} 
polarized.

\section{C.4 Laser Bandwidth}

Regarding the laser bandwidth, it is entirely possible to use a laser on the lab-bench with the right properties which eliminate the etalon effect in the RTP crystals. However, what actually matters is the properties of the laser at JLab which will be used in conjunction with these RTP cells. What are the requirements on bandwidth? There are two bandwidth requirements (1) the bandwidth of the laser must be at least as large as the etalon free-spectral-range (i.e. wavelength changes are causing phase shifts large enough to go through at least one interference fringe) $F S R=\frac{c}{2 n L}[H z]=\frac{\lambda^{2}}{2 n L}[n m]$ (2) the wavelength must be changing on a time scale faster than the helicity switching (otherwise the helicity window will not be averaging over the interference fringes and a helicity correlated charge asymmetry will appear and at the very least cause non-Poisson-like Aq distributions). The FSR of our RTP crystal $1 \mathrm{~cm}$ in length with refractive index 1.8 near $780 \mathrm{~nm}$, is $0.02 \mathrm{~nm}$. We intend to use RTPs when switching helicity states at a rate of $2 \mathrm{kHz}$. To be one the safe side, say we need 10 fringes averaged over 10 cycles in a window, then we require $0.2 \mathrm{~nm}$ bandwidth oscillating at at least $10 \mathrm{kHz}$. In other words, the noisier the laser, the better for eliminating the etalon effect.

\section{Self Analyzing}

It is worth noting that due to the biaxial nature of RTP and its large intrinsic birefringence, the surface of the RTP will have a natural analyzing power simply by Fresnel's equations. The power will scale down with reflectivity, so if the AR coating are very good, it will bring down the analyzing power of the crystal faces. However, no AR coating can match both transverse refractive indices, so there will always be some small analyzing power along the direction of the crystal axis (S2). It will give rise to a small alpha phase. The etalon effect also can lead to 
self analyzing along the crystal axes, along S2. This is also largely an alpha-phase effect. 


\section{Appendix D}

\section{Tech Note: KD*P Steering and General Pockels Cell Matters}

\section{D.1 KD*P}

\section{D.1.1 Steering in $\mathrm{KD}^{*} \mathrm{P}$}

Steering is a helicity correlated change in angle of the outgoing laser beam after having passed through the Pockels Cell. It produces a position difference between right and left helicity states which increases with throw distance, hence steering is referred to as an 'angle-like' position difference. Previously, steering (aka the 'Skew/Paschke' effect) in $\mathrm{KD}^{*} \mathrm{P}$ was thought to be a non-polarization-dependent effect since both $\mathrm{H}$ and $\mathrm{V}$ input polarizations appeared to exhibit the same steering behavior. However, we have since shown that steering is, in fact, quite polarization dependent. When the input polarizations are along the crystal's primary axes at $\pm 45^{\circ}$, steering for $+45^{\circ}$ input polarization was observed to differ significantly from the steering for $-45^{\circ}$ input polarization, indicating polarization dependance. Furthermore, it was found that the steering for $\mathrm{H}$ and $\mathrm{V}$ input polarizations was approximately the average of the steering along the $\pm 45^{\circ}$ primary crystal axes. So, our model for steering in $\mathrm{KD}^{*} \mathrm{P}$ has to include in polarization dependent effects. 


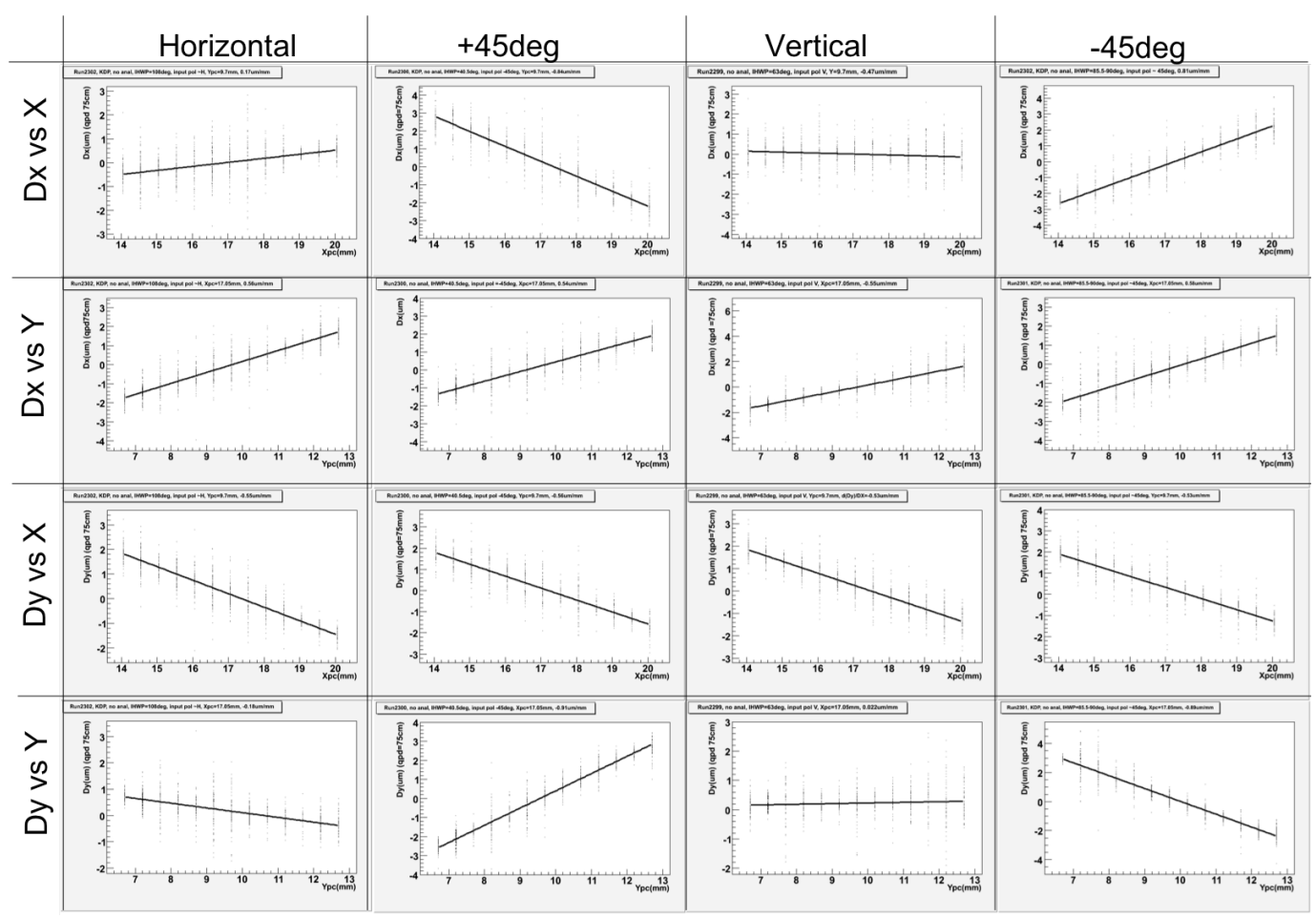

Figure D-1: Steering observed with different input polarization states, no analyzer, in translation scan for 'Princeton' Cell (commercial Fastpulse 10mm 1040 $\mathrm{KD}^{*} \mathrm{P}$ [313]) [314]. Note that the sign of Dx,y on the qpd is arbitrary compared with the sign of the translation stage Xpc, Ypc.

The model we use is phenomenological and it is postulated based on several robust empirical observations of $\mathrm{KD}^{*} \mathrm{P}$ cells. The model must satisfy the following 4 requirements :

- steering for $\mathrm{H} / \mathrm{V}$ input polarization of the form

$$
\left.\theta_{x}^{s t}\right|_{H}=\left.\theta_{x}^{s t}\right|_{V}=\left.\beta y \quad \theta_{y}^{s t}\right|_{H}=\left.\theta_{y}^{s t}\right|_{V}=\beta x
$$

- steering for $\pm 45^{\circ}$ input polarizations along primary axes $\mathrm{x}^{\prime}, \mathrm{y}^{\prime}$ of the form

$$
\begin{gathered}
\left.\theta_{x}^{s t}\right|_{x^{\prime}}=2 \alpha x+\left.\beta y \quad \theta_{y}^{s t}\right|_{x^{\prime}}=2 \alpha y+\beta x \\
\left.\theta_{x}^{s t}\right|_{y^{\prime}}=-2 \alpha x+\left.\beta y \quad \theta_{y}^{s t}\right|_{y^{\prime}}=-2 \alpha y+\beta x
\end{gathered}
$$


where $\alpha \approx \beta$.

- Aq in S1 exhibiting a 'saddle' in the $\mathrm{x} / \mathrm{y}$ plane: translational dependence observed

$$
A_{q}=\gamma x y
$$

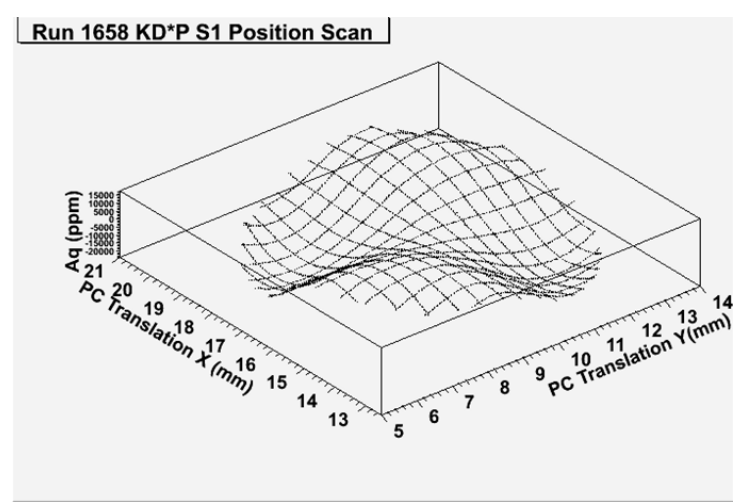

Figure D-2: Intensity asymmetry in Translation scan [315], analyzing in S1, for 'Princeton' Cell (commercial Fastpulse 10mm 1040 KD*P [313])

- A transmission 'hole' when analyzing in S1: the average transmission has a radial dependence as the cell is translated in the $\mathrm{x} / \mathrm{y}$ plane

$$
T=T_{0}\left(1-\eta\left(x^{2}+y^{2}\right)\right)
$$
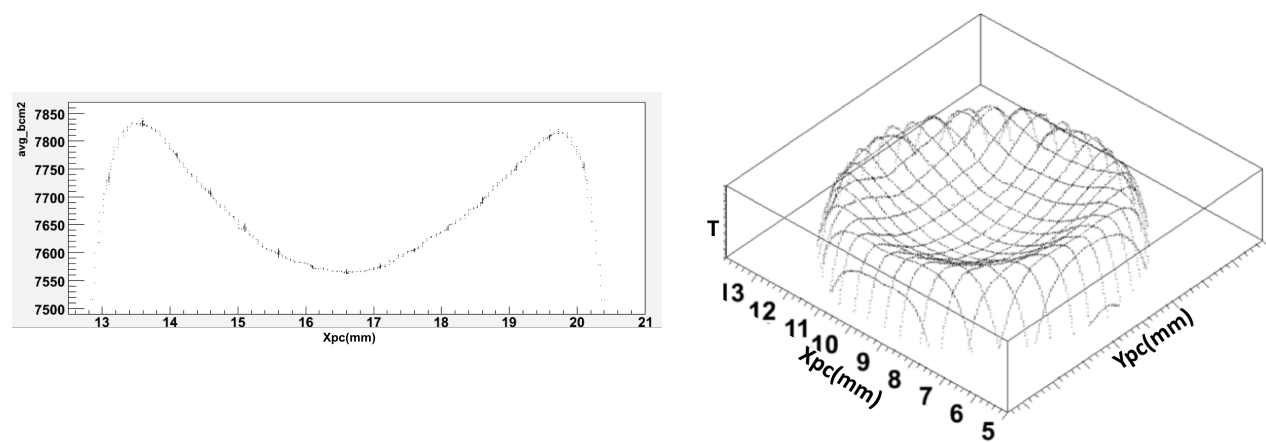

Figure D-3: Transmission in Translation scan [315], analyzing in S1, for 'Princeton' Cell (commercial Fastpulse 10mm 1040 KD*P [313]) 


\section{Model}

Our model reproduces all 4 of these observations. We can take our phenomenological model as having two main components:

1. A radial electric field gradient that changes sign with helicity. Within the parametrization $\left.E_{z}^{R(L)}=\mp\left(E_{z, 0}+E_{\alpha}\right)-E_{\Delta}\right)$, we let

$$
E_{\alpha}=\beta_{e}\left(x^{2}+y^{2}\right)
$$

2. A piezo-electric helicity correlated length change (or an effect which mimics this). Within the parametrization $L^{R(L)}=L_{0} \pm L_{a}+L_{d}$, we let

$$
L_{a}=\beta_{l} x y
$$

With just these two assumptions, by tracking wave-phase-fronts for the two primary polarization axes, we can reproduce all four $\mathrm{KD}^{*} \mathrm{P}$ observed behaviors. A $\mathrm{H} / \mathrm{V}$ input polarization is split upon entering the $\mathrm{KD} * \mathrm{P}$ crystal into two components along the $\mathrm{i}=\mathrm{x}^{\prime}, \mathrm{y}^{\prime}\left( \pm 45^{\circ}\right)$ primary axes. These polarization components undergo phase shifts

$$
\phi_{i}^{R(L)}=2 \pi n_{i}^{R(L)} L^{R(L)} / \lambda
$$

where $\mathrm{R}(\mathrm{L})$ indicates right and left circular polarization states determined by the sign of the voltage applied to the Pockels Cell as controlled by the helicity signal.

The opto-electric effect in $\mathrm{KD}^{*} \mathrm{P}$ results in a equal and opposite change in refractive index to the two primary axes

$$
n_{x^{\prime}}^{R(L)}=n_{o}-\frac{1}{2} n_{o}^{3} r_{63} E_{z}^{R(L)} n_{y^{\prime}}^{R(L)}=n_{o}+\frac{1}{2} n_{o}^{3} r_{63} E_{z}^{R(L)}
$$

The assumptions of our model postulate

$$
\left.E_{z}^{R(L)}=\mp\left(E_{z, 0}+E_{\alpha}\right)-E_{\Delta}\right)=\mp\left(E_{z, 0}+\beta_{e}\left(x^{2}+y^{2}\right)-E_{\Delta}\right)
$$




$$
L^{R(L)}=L_{0} \pm L_{a}+L_{d}=L_{0} \pm \beta_{l} x y+L_{d}
$$

From the above information regarding the phase shifts, we deduce all the observed effects in $\mathrm{KD}^{*} \mathrm{P}$. The electric field variation produces polarization dependent steering, i.e. steering which is of opposite sign for input polarizations along the diagonal $\pm 45^{\circ}$ primary axes. The length variation produces polarization independent steering, i.e. steering which has the same sign for both $\pm 45^{\circ}$ primary axes and $\mathrm{H}$ and $\mathrm{V}$ polarizations.

We should note that an alternative model to the length gradient would involve the average refractive index gradient taking the following form:

$$
\begin{gathered}
n_{x^{\prime}}^{R(L)}=n_{o}-\frac{1}{2} n_{o}^{3} r_{63} E_{z}^{R(L)}+n_{a}^{R(L)} n_{y^{\prime}}^{R(L)}=n_{o}+\frac{1}{2} n_{o}^{3} r_{63} E_{z}^{R(L)}+n_{a}^{R(L)} \\
n_{a}^{R(L)}= \pm n_{a}= \pm \beta^{\prime} x y \\
n_{H / V}^{R(L)} \approx n_{a v g}=\frac{1}{2}\left(n_{x^{\prime}}+n_{y^{\prime}}\right)=\frac{1}{2}\left(n_{o}+n_{a}^{R(L)}-\frac{1}{2} n_{o}^{3} r_{63} E_{z}+n_{o}+n_{a}^{R(L)}+\frac{1}{2} n_{o}^{3} r_{63} E_{z}\right) \\
\approx n_{o}+n_{a}^{R(L)} \approx n_{o} \pm n_{a} \approx n_{o} \pm \beta^{\prime} x y
\end{gathered}
$$

Which would have the same effect as a length gradient of the form $L_{a}=\beta x y$

A more precise calculation for the effective refractive index for the horizontal and vertical polarization states is given by

$$
\begin{gathered}
\frac{1}{n_{H / V}^{2}}=\frac{1}{2 n_{y^{\prime}}^{2}}+\frac{1}{2 n_{x^{\prime}}^{2}}=\frac{1}{2\left(n_{\text {avg }}+\epsilon\right)^{2}}+\frac{1}{2\left(n_{\text {avg }}-\epsilon\right)^{2}} \\
=\frac{1}{2\left(n_{\text {avg }}^{2}+2 \epsilon n_{\text {avg }}+\epsilon^{2}\right)}+\frac{1}{2\left(n_{\text {avg }}^{2}-2 \epsilon n_{\text {avg }}+\epsilon^{2}\right)} \approx \frac{1}{\left(n_{\text {avg }}^{2}\right.}-\epsilon^{2} \\
n_{H / V}^{R(L)} \approx\left|n_{\text {avg }}\right|\left(1+n_{\text {avg }}^{2} \epsilon^{2}\right)^{-1 / 2} \approx\left|n_{\text {avg }}\right|\left(1-\frac{1}{2} n_{\text {avg }}^{2} \epsilon^{2}\right)
\end{gathered}
$$

where $\epsilon=\frac{1}{2}\left(n_{y}^{\prime}-n_{x^{\prime}}\right)=\frac{1}{2} n_{o}^{3} r_{63} E_{z}^{R(L)}, n_{y^{\prime}}^{R(L)}=n_{\text {avg }}+\epsilon$, and $n_{x^{\prime}}^{R(L)}=n_{\text {avg }}-\epsilon$. Hence,

$$
n_{H / V}^{R(L)} \approx\left|n_{o} \pm n_{a}\right|\left(1-\frac{1}{2}\left(\left(n_{o} \pm n_{a}\right) \frac{1}{2} n_{o}^{3} r_{63} E_{z}^{R(L)}\right)^{2}\right)
$$


It is possible that this higher order term contributes some to steering, but it is likely to be much smaller than the 1st order effects.

\section{Aq Saddle}

To deduce the charge asymmetry, we examine the delta-phase

$$
\begin{gathered}
\delta^{R(L)}=\mp(\pi / 2+\alpha)-\Delta=\phi_{x^{\prime}}^{R(L)}-\phi_{y^{\prime}}^{R(L)}=-\frac{2 \pi n_{o}^{3} r_{63}}{\lambda} E_{z}^{R(L)} L^{R(L)} \\
A_{q}=\frac{1}{2}\left(\cos \delta^{R}-\cos \delta^{L}\right) \approx-\Delta=\frac{1}{2}\left(\delta^{R}+\delta^{L}\right)=\frac{2 \pi n_{o}^{3} r_{63}}{\lambda}\left(E_{\Delta} L_{0}+\left(E_{0}+E_{\alpha}\right) L_{a}+E_{\delta} L_{d}\right) \\
=\frac{2 \pi n_{o}^{3} r_{63}}{\lambda}\left(E_{\Delta} L_{0}+\left(E_{0}+E_{\alpha}\right) L_{a}+E_{\delta} L_{d}\right) \approx \frac{2 \pi n_{o}^{3} r_{63}}{\lambda}\left(E_{\Delta} L_{0}+E_{0} \beta_{l} x y\right)=k V_{P I T A}+\gamma x y
\end{gathered}
$$

Hence, there is a 'saddle' in Aq through the function xy.

\section{Transmission Hole}

To deduce the Transmission through a vertical polarizer, we examine the alphaphase

$$
\begin{aligned}
& T=1+\frac{1}{2}\left(\cos \delta^{R}+\cos \delta^{L}\right) \approx 1+\frac{1}{2}\left(\delta^{R}-\delta^{L}+\pi\right)=1-\alpha \\
& =\pi / 2+\frac{2 \pi n_{o}^{3} r_{63}}{\lambda}\left(\left(E_{0}+E_{\alpha}\right) L_{0}+L_{a} E_{\Delta}+\left(E_{0}+E_{\alpha}\right) L_{d}\right) \\
& \approx \pi / 2+\frac{2 \pi n_{o}^{3} r_{63}}{\lambda}\left(E_{0} L_{0}+L_{0} \beta_{e}\left(x^{2}+y^{2}\right)\right) \sim\left(1-\eta\left(x^{2}+y^{2}\right)\right.
\end{aligned}
$$

Hence there is a 'hole' in the transmission through a vertical polarizer with radial dependence $x^{2}+y^{2}$.

\section{Polarization Dependent Steering}

To deduce steering, we first define the helicity correlated phase shift for each polarization state

$$
\Delta \phi_{x^{\prime}}=\phi_{x^{\prime}}^{R}-\phi_{x^{\prime}}^{L}=(2 \pi / \lambda) \Delta\left(n_{x^{\prime}} L\right)=(2 \pi / \lambda)\left(n_{x^{\prime}} \Delta L+\Delta n_{x^{\prime}} L\right)
$$




$$
\Delta \phi_{y^{\prime}}=\phi_{y^{\prime}}^{R}-\phi_{y^{\prime}}^{L}=(2 \pi / \lambda) \Delta\left(n_{y^{\prime}} L\right)=(2 \pi / \lambda)\left(n_{x^{\prime}} \Delta L+\Delta n_{y^{\prime}} L\right)
$$

In accordance with our model the phase shifts for the two polarization state components will be

$$
\begin{gathered}
\Delta \phi_{x^{\prime}}=\frac{4 \pi}{\lambda}\left(n_{o} L_{a}+n_{o}^{3} r_{63}\left(\left(E_{0}+E_{\alpha}\right)\left(L_{0}+L_{d}\right)+L_{a} E_{\Delta}\right)\right) \\
\cdots \approx \frac{4 \pi}{\lambda}\left(n_{o} L_{a}+n_{o}^{3} r_{63}\left(E_{0}+E_{\alpha}\right) L_{0}\right) \approx \frac{4 \pi}{\lambda}\left(n_{o} \beta_{l} x y+n_{o}^{3} r_{63}\left(E_{0}+\beta_{e}\left(x^{2}+y^{2}\right)\right) L_{0}\right)
\end{gathered}
$$

Likewise,

$$
\begin{aligned}
\Delta \phi_{y^{\prime}} & =\frac{4 \pi}{\lambda}\left(n_{o} L_{a}-n_{o}^{3} r_{63}\left(\left(E_{0}+E_{\alpha}\right)\left(L_{0}+L_{d}\right)+L_{a} E_{\Delta}\right)\right) \\
& \approx \frac{4 \pi}{\lambda}\left(n_{o} \beta_{l} x y-n_{o}^{3} r_{63}\left(E_{0}+\beta_{e}\left(x^{2}+y^{2}\right)\right) L_{0}\right)
\end{aligned}
$$

where $\beta_{e}$ is the coupling to a helicity correlated change that is similar to an E-field change, $\beta_{l}$ is the coupling to a helicity correlated change that is similar to a length change.

The phase shift experienced by $\mathrm{H}$ and $\mathrm{V}$ input polarizations is computed by averaging the phase shifts for $x^{\prime}, y^{\prime}$ polarizations

$$
\Delta \phi_{H, V}=\frac{1}{2}\left(\Delta \phi_{x^{\prime}}+\Delta \phi_{y^{\prime}}\right) \approx \frac{4 \pi n_{o} L_{a}}{\lambda} \approx \frac{4 \pi n_{o}}{\lambda} \beta_{l} x y
$$

We deduce the steering using Snell's law. A face cut angle in the crystal is a length gradient $\theta_{f c}=\frac{d}{d x} L$ which causes the light to bend upon exiting the crystal by an angle $n_{a i r} \theta=n \theta_{f c}=n \frac{d}{d x} L$. Equivalently, a refractive index gradient would do the same thing. We can generally describe the angle induced from any phase gradient with $\theta=\frac{d}{d x}(n L)=\frac{\lambda}{2 \pi} \frac{d \phi}{d x}$. In the case of steering, we examine the helicity correlated gradients in the phase difference for each polarization state i

$$
\left.\theta_{x}^{s t}\right|_{i}=\frac{\lambda}{2 \pi} \frac{d}{d x} \Delta \phi_{i}
$$




$$
\left.\theta_{y}^{s t}\right|_{i}=\frac{\lambda}{2 \pi} \frac{d}{d y} \Delta \phi_{i}
$$

Plugging in our phase shifts for input polarizations $x^{\prime}, y^{\prime}$ and $H, V$ we obtain

$$
\begin{gathered}
\left.\theta_{x}^{s t}\right|_{x^{\prime}}=2 n_{o} \beta_{l} y+4 n_{o}^{3} r_{63} \beta_{e} L_{0} x=2 \alpha x+\beta y \\
\left.\theta_{y}^{s t}\right|_{x^{\prime}}=2 n_{o} \beta_{l} x+4 n_{o}^{3} r_{63} \beta_{e} L_{0} y=2 \alpha y+\beta x \\
\left.\theta_{x}^{s t}\right|_{y^{\prime}}=2 n_{o} \beta_{l} y-4 n_{o}^{3} r_{63} \beta_{e} L_{0} x=-2 \alpha x+\beta y \\
\left.\theta_{y}^{s t}\right|_{y^{\prime}}=2 n_{o} \beta_{l} x-4 n_{o}^{3} r_{63} \beta_{e} L_{0} y=-2 \alpha y+\beta x \\
\left.\theta_{x}^{s t}\right|_{H, V}=2 n_{o} \beta_{l} y=\left.\beta y \quad \theta_{y}^{s t}\right|_{H, V}=2 n_{o} \beta_{l} x=\beta x
\end{gathered}
$$

Hence our model reproduces the steering behavior observed for all possible input polarization states.

It is important to observe that the steering observed for $\mathrm{H} / \mathrm{V}$ input polarizations does not arise from the electric field gradient in this model. Electric field variations produce steering which is of EQUAL and opposite sign for input polarizations along the diagonal $\pm 45^{\circ}$ primary axes. This comes from the assumption that the refractive indices couple to the electric fields in an equal and opposite way: $n_{x^{\prime} / y^{\prime}}=n_{o} \mp \frac{1}{2} n_{o}^{3} r_{63} E_{z}$. When the steering for $\pm 45^{\circ}$ polarizations is averaged to obtain horizontal and vertical polarizations, the behavior is cancelled. This cancellation can be understood easily when the average refractive index seen by horizontal polarization is computed: $n_{H / V} \approx \frac{1}{2}\left(n_{x^{\prime}}+n_{y^{\prime}}\right)=$ $\frac{1}{2}\left(n_{o}-\frac{1}{2} n_{o}^{3} r_{63} E_{z}+n_{o}+\frac{1}{2} n_{o}^{3} r_{63} E_{z}\right) \approx n_{o}$ (although there is a higher order term $n_{H / V} \approx n_{o}\left(1+\frac{1}{2} n_{o}^{2}\left(\frac{1}{2} n_{o}^{3} r_{63} E_{z}\right)^{2}\right)$. Steering can only arise through Snell's law either from a gradient in the average refractive index or through a length gradient. Here, the average refractive index for horizontal and vertical input polarizations sees no dependence on electric field gradients. So, if the effect of the electric field is equal and opposite for $\pm 45^{\circ}$ polarizations, there is no steering from electric fields for $\mathrm{H} / \mathrm{V}$ polarizations. The only other ways steering can arise through Snell's law is 
(1) if the refractive indices for $\pm 45^{\circ}$ polarizations aren't perfect in their cancellation (2) from a length gradient, which is the necessary second component of our model

\section{D.1.2 The Etalon effect in $\mathrm{KD}^{*} \mathrm{P}$}

Why isn't the etalon effect a problem with KD*P? Firstly, KD*P's are uniaxial crystals with no intrinsic birefringence and hence can be cut with a large nonparallelism without compromising their performance. Secondly, even if the face cuts were quite parallel, and an etalon effect was observed, the phase shift of the fast axis component of the back reflection would be precisely equal and opposite to the phase shift of the slow axis component of the back reflection, and the two on average would cancel leading to no helicity correlated charge asymmetry. This is for two reasons (1) because the $\mathrm{KD}^{*} \mathrm{P}$ is uniaxial and the Pockels effect induces a symmetrical shift in fast/slow refractive indices $n_{x} \approx n_{0}-\frac{1}{2} n_{0}^{3} r_{63} E_{z}$ and $n_{y} \approx n_{0}+\frac{1}{2} n_{0}^{3} r_{63} E_{z}$ and (2) because the $\mathrm{KD}^{*} \mathrm{P}$ cell is running at QWV $\Delta n L=\pi / 2$, so the phase shift the back-face reflection undergoes in going $2 \mathrm{~L}$ back and forth round trip is $+\pi / 2$ for the fast axis and $-\pi / 2$ for the slow axis and $A_{q}=R\left(\cos \phi_{f}+\right.$ $\left.\cos \phi_{s}\right)=R\left(\cos \left(\phi_{0}+\pi / 2\right)+\cos \left(\phi_{0}-\pi / 2\right)\right)=0$. The KD*P crystals are coated with Sol-gel which is broadband with a reflectance of $0.05-0.1 \%$ and if the face cuts were parallel we would be able to examine the etalon effect for the individual fast and slow axes and observe an Aq 1000ppm. We we able to examine the behavior of $\mathrm{KD}^{*} \mathrm{P}$ cell and observed no etalon effect to within $100 \mathrm{ppm}$. Therefore the face cuts of our KD*P cell must be more non-parallel than the RTP crystals (i.e. $>0.05 \mathrm{mrad}$ ). 

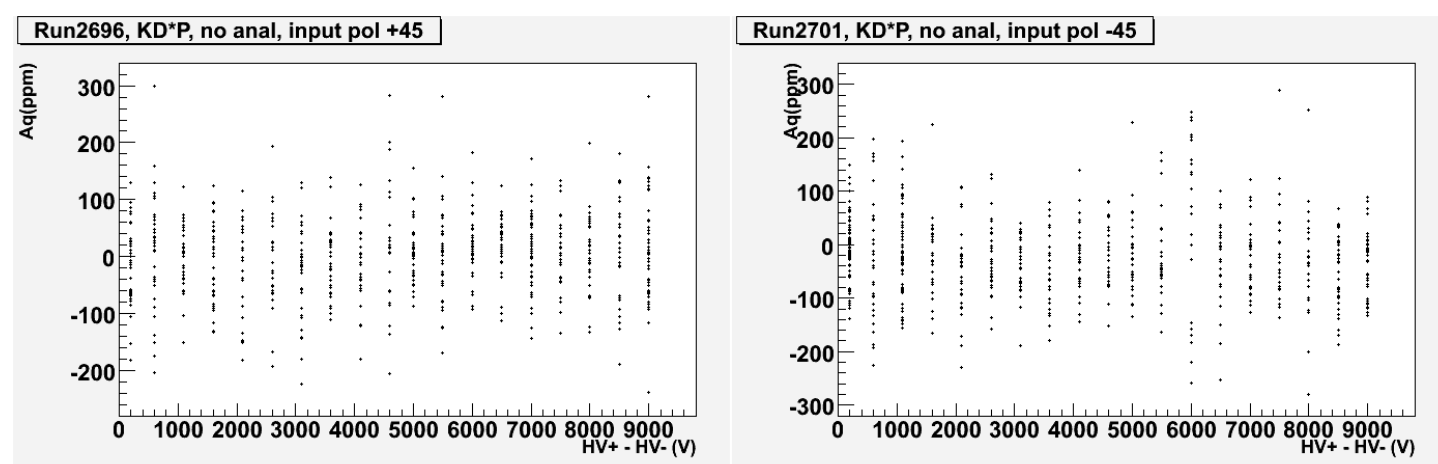

Figure D-4: Etalon effect in KDP. These measurements were performed by setting input polarization along the fast axis or the slow axis (+-45deg) with a HWP, scanning the applied voltage, and examining the transmission without an analyzer

\section{D.1.3 Intensity Asymmetry Angle Dependence in KD*P}

This result is presented in Appendix A and is different form Rupesh's thesis [163]. We note that the Newport 4-axis mount calibration, due to the presence of a set set and differing calibration factor between pitch and yaw mar/turn, caused some confusion in connecting calculation to actual measurements. Note that $\mathrm{KD}^{*} \mathrm{P}$ has angle dependence in $\mathrm{S} 1$ and $\mathrm{S} 2$, but RTP just has angle dependence in $\mathrm{S} 1$.

\section{D.2 General Pockels Cell Considerations}

\section{D.2.1 Formulation}

Here we summarize the formalized we used in describing Pockels cell behaviors. Phase shifts for polarization along primary fast/slow axes $\mathrm{i}\left(=\mathrm{x}^{\prime}, \mathrm{y}^{\prime}\right.$ for $\mathrm{KD} \mathrm{D}^{*} \mathrm{P},=\mathrm{y}, \mathrm{z}$ for RTP)

$$
\phi_{i}=2 \pi n_{i} L / \lambda
$$


Helicity correlated phase shifts for components of polarization along primary axes $\mathrm{i}, \mathrm{j}$

$$
\phi_{i}^{R(L)}=2 \pi n_{i}^{R(L)} L^{R(L)} / \lambda
$$

where $L^{R(L)}=L_{0}$ if there is not piezoelectric effect at play, and

$$
n_{i}^{R(L)}=n_{0, i}-\frac{1}{2} n_{0, i}^{3} r_{i} E_{z}
$$

for $\mathrm{KD}^{*} \mathrm{P}$ this becomes

$$
n_{x^{\prime}}^{R(L)}=n_{o}-\frac{1}{2} n_{o}^{3} r_{63} E_{z}^{R(L)} n_{y^{\prime}}^{R(L)}=n_{o}+\frac{1}{2} n_{o}^{3} r_{63} E_{z}^{R(L)}
$$

for RTP this becomes [312]

$$
n_{y}^{R(L)}=n_{0, y}-\frac{1}{2} n_{0, y}^{3} r_{23} E_{z}^{R(L)} n_{z}^{R(L)}=n_{0, z}-\frac{1}{2} n_{0, z}^{3} r_{33} E_{z}^{R(L)}
$$

where and typically the electrical field $E_{z}=V / d$ is symmetrically flipped $E_{z}^{R} \approx$ $-E_{z}^{L}$ and $V^{R} \approx-V^{L}$ the voltages are nearly the same. We define a PITA voltage and alpha voltage as

$$
\begin{gathered}
\left.E_{z}^{R(L)}=\mp\left(E_{z, 0}+E_{\alpha}\right)-E_{\Delta}\right) \\
\left.V^{R(L)}=\mp\left(V_{0}+V_{\alpha}\right)-V_{\text {PITA }}\right)
\end{gathered}
$$

Defining $\delta$-phase

$$
\delta^{R(L)}=\mp(\pi / 2+\alpha)-\Delta=\phi_{x^{\prime}}^{R(L)}-\phi_{y^{\prime}}^{R(L)}
$$

Transmission. Insert an analyzer after the Pockels cell with transmission coefficients $T_{x}, T_{y}$ along an axis $\mathrm{x}$, where $\psi$ is the angle subtended between $\mathrm{x}$ and the horizontal axis. The transmission through the analyzer is given by:

$$
T^{R(L)}=T \frac{1}{2}\left(1+\epsilon / T \cos (2 \psi) \cos \delta^{R(L)}\right)
$$


where $\epsilon=T_{x}-T_{y}, T=\left(T_{x}-T_{y}\right) / 2$ defines the analyzing power of the polarizer(or polarizing element).

Approximation

$$
\begin{gathered}
\cos \delta^{R(L)}=\cos (\mp(\pi / 2+\alpha)-\Delta) \approx 1-\alpha \mp \Delta \\
\cos \delta^{R}-\cos \delta^{L} \approx \delta^{R}+\delta^{L}=-2 \Delta \\
\cos \delta^{R}+\cos \delta^{L} \approx \delta^{R}-\delta^{L}+\pi=-2 \alpha
\end{gathered}
$$

Intensity asymmetry

$$
A_{q}=\frac{T^{R}-T^{L}}{T^{R}+T^{L}} \approx \frac{\epsilon}{T} \cos (2 \psi) \frac{1}{2}\left(\cos \delta^{R}-\cos \delta^{L}\right) \approx-\frac{\epsilon}{T} \cos (2 \psi) \Delta
$$

for $100 \%$ analyzer

$$
A_{q} \approx-\Delta
$$

Transmission for $100 \%$ analyzer

$$
T=\frac{T^{R}+T^{L}}{2}=1+\frac{1}{2}\left(\cos \delta^{R}+\cos \delta^{L}\right) \approx 1+\frac{1}{2}\left(\delta^{R}-\delta^{L}+\pi\right)=1-\alpha
$$

\section{Stokes parameters}

The Stokes parameters are defined (and measured) as [277] [278]:

$$
\begin{gathered}
S_{0}=T_{x}+T_{y}>=\sqrt{S_{1}^{2}+S_{2}^{2}+S_{3}^{2}} \quad S_{1}=T_{x}-T_{y} \quad S_{2}=T_{45^{\circ}}-T_{135^{\circ}}=2 \times T_{45^{\circ}}-S_{0} \\
S_{3}=S_{0}-2 \times T_{45^{\circ}}^{Q W P} \text { in }=\text { Lossfactor } \times\left(T_{45^{\circ}}^{Q W P i n}-T_{135^{\circ}}^{Q W P i n}\right) \\
\text { Lossfactor }=T \text { (before analyzer, QWP out }) / T(\text { before analyzer, } Q W P \text { in })
\end{gathered}
$$

where S3 is found by inserting a QWP in with its fast axis along the horizontal and measuring the transmission with the polarizer along $45^{\circ}, 135^{\circ}$ and taking into 
account the loss-factor due to imperfect transmission through the waveplate. The degree of polarization is $D_{o} P=\sqrt{S_{1}^{2}+S_{2}^{2}+S_{3}^{2}} / S_{0}$ and and the Stokes sets are

$$
S_{P P}=\left[S_{0}, S_{1}, S_{2}, S_{3}\right]_{P P}=(1-P) *\left[S_{0}^{\prime}, 0,0,0\right]_{U N P}+P *\left[S_{0}^{\prime}, S_{1}^{\prime}, S_{2}^{\prime}, S_{3}^{\prime}\right]_{E L P}
$$

$S_{E L P}=\left[S_{0}^{\prime}, S_{1}^{\prime}, S_{2}^{\prime}, S_{3}^{\prime}\right]_{E L P}=(1 / P) *\left[S_{0}, S_{1}, S_{2}, S_{3}\right]_{P P}-((1-P) / P) *\left[S_{0}, 0,0,0\right]_{U N P}$

where we define $\mathrm{PP}=$ partially polarized, $\mathrm{ELP}=$ elliptically polarized, $\mathrm{UNP}=$ unpolarized. Note that $S_{0}^{\prime}=S_{0}$ and may be normalized to 1 . In normalized form,

$$
s_{P P}=\left[1, s_{1}, s_{2}, s_{3}\right]_{P P}=\left[1, S_{1} / S_{0}, S_{2} / S_{0}, S_{3} / S_{0}\right]_{P P}
$$

$s_{E L P}=\left[1, s_{1}^{\prime}, s_{2}^{\prime}, s_{3}^{\prime}\right]_{E L P}=(1 / P) *\left(1 / S_{0}\right) *\left[1, S_{1}, S_{2}, S_{3}\right]_{P P}-((1-P) / P) *[1,0,0,0]_{U N P}$

\section{D.2.2 Angle-like Steering in $\mathrm{KD}^{*} \mathrm{P}$}

\section{Ray Optics View}

The operation of a $\mathrm{KD}^{*} \mathrm{P}$ crystal in a Pockels cell incurs voltage dependent phase shifts on the beam $\phi=2 \pi n L / \lambda$. There can be gradients $\frac{d n}{d X}, \frac{d L}{d X}, \frac{d n}{d \theta}, \frac{d n}{d \theta}$. These gradients are evident in PC translation scans.

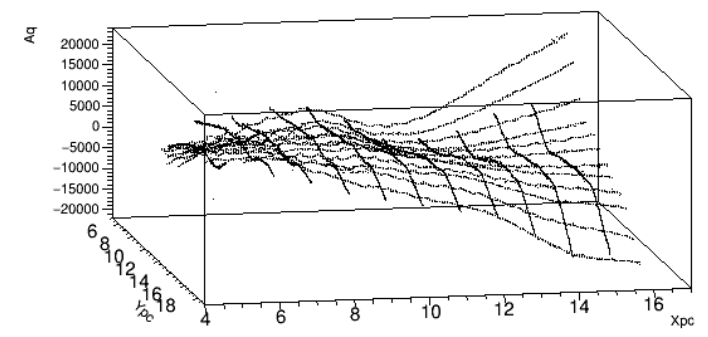

Figure D-5: Charge asymmetry gradient for active $\mathrm{KD}^{*} \mathrm{P}$ with a vertical analyzer after cell("Gandalf",Run2815, elog 656)

A $\frac{d n}{d X}$ gradient can lead to an angle change $\Delta \theta$ just as $\frac{d L}{d X}$ can by Snell's Law. (Secondarily, it should be noted, this angle change $\Delta \theta$ could via $\frac{d n}{d \theta}, \frac{d n}{d \theta}$ create 
a phase shift associated with the crystal's angle-dependence, thereby connecting position gradients with angular dependence gradients).

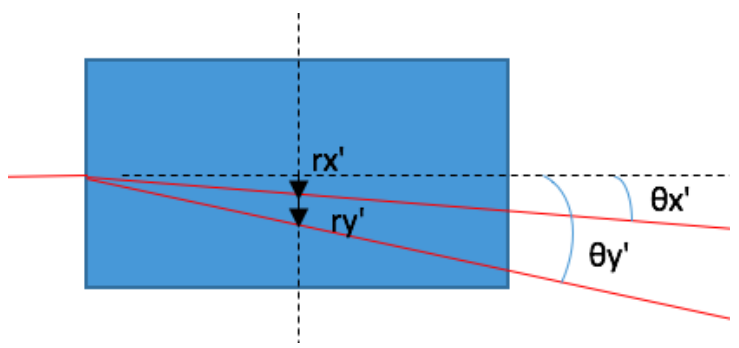

Figure D-6: Ray Separation. Horizontally polarized beam splits in the crystal along the primary axes, angles not to scale.

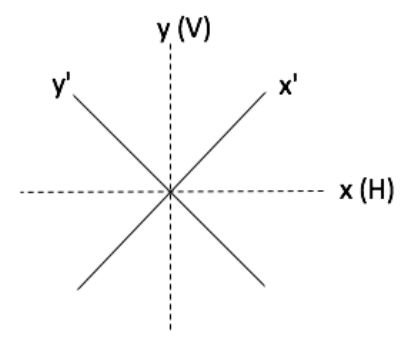

Figure D-7: Coordinates. Primary crystal axes are along the diagonal with respect to horizontal and vertical polarizations.

Since the crystal in the Pockels cell is birefringent, the beam should be viewed as 2 separate rays: one with a polarization along the diagonal x' and the other along $\mathrm{y}^{\prime}$, the primary axes of the $\mathrm{KD}^{*} \mathrm{P}$. These rays separate via a difference in $\frac{d n_{x^{\prime}}}{d x}$ and $\frac{d n_{y^{\prime}}}{d x}$. In KD*P, $n_{x^{\prime}}=n_{0}-\frac{1}{2} r_{33} E_{z} n_{0}^{3}$ and $n_{y^{\prime}}=n_{0}+\frac{1}{2} r_{33} E_{z} n_{0}^{3}$ and the 2 rays separate into 4 rays for difference helicity states and different primary axes. The gradients on average $\frac{d}{d x} \frac{n_{x^{\prime}}+n_{y^{\prime}}}{2}=\frac{d}{d x} \frac{n_{0}-\frac{1}{2} r_{33} E_{z} n_{0}^{3}+n_{0}+\frac{1}{2} r_{33} E_{z} n_{0}^{3}}{2}=\frac{d n_{0}}{d x}$ have zero dependence on E-field, so in this model, there is no mean helicity correlated beam shift to first order for $\mathrm{H}$ and $\mathrm{V}$ polarization; there is no helicity-correlated beam steering steering for $\mathrm{H}$ and $\mathrm{V}$ polarization to first order in $\mathrm{S} 1$ and for no analyzer.

In $\mathrm{S} 2$, however, analyzing isolates $n_{x^{\prime}}$ and $n_{y^{\prime}}$ rays so that $\frac{d n_{x^{\prime}}}{d x}$ will change for $+\mathrm{Ez}$ and $-\mathrm{Ez}$ producing position differences. In $\mathrm{S} 2$, there will be a position 
difference $\frac{d n_{x^{\prime}}}{d x}$ which is angle-like and grows as Leff increases and depends on how far away you place the detector from the pockels cell.

\section{Cancelling}

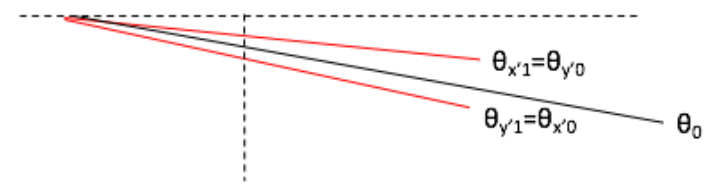

Not cancelling

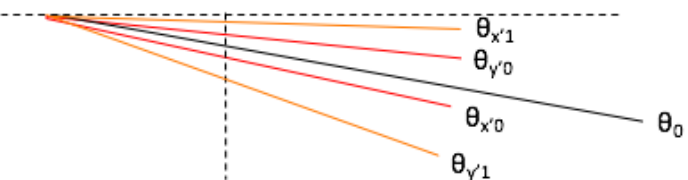

Figure D-8: 4 Ray Separation. Horizontally polarized beam splits in the crystal along the primary axes, and voltage application split the beam further, angles not to scale.

Since we do see angle-like steering empirically without an analyzer, it must be that the $n_{x^{\prime}}$ and $n_{y^{\prime}}$ don't cancel out so nicely and give rise to steering for no analyzer and for S1. This is the "normal" steering known about for quite some time and mentioned in previous theses. There are several mechanisms which could give rise to a non-cancellation in the electric field dependent terms in the refractive indices : contributions from transverse field components Ex and Ey in the cell, 2nd order Kerr contributions, stress-electric field coupling terms, terms which rotate the primary axes of the crystal, intrinsic birefringence $\left(n_{0 x} \neq n_{0 y}\right)$, asymmetric electro-optic coupling terms $\left(r_{33}\right.$ etc. $)$. Any term which (1) couples to electric field (2) breaks x,y equal-and-opposite symmetry gives rise to angle-like steering in $\mathrm{KD}^{*} \mathrm{P}$.

S2 steering just depends on $\theta_{x}^{\prime}$. S1 and no-analyzer steering depends on the difference between $\frac{\theta_{x^{\prime}, 0}+\theta_{y^{\prime}, 0}}{2}-\frac{\theta_{x^{\prime}, 1}+\theta_{y^{\prime}, 1}}{2}$ and it makes no difference whether you are analyzing or not, the angle-like position differences will be the same.

In S1, the "normal" steering term will be the same as when no analyzer is in. In $\mathrm{S} 2$, this transforms and $\Delta<\theta_{x^{\prime}}+\theta_{y^{\prime}}>$ isn't critical, only $\theta_{x^{\prime} 0}-\theta_{x^{\prime} 1}$ matters, which is predictable from $n_{x^{\prime}}=n_{0}-\frac{1}{2} r_{33} E_{z} n_{0}^{3}$ and a gradient in either $\mathrm{E}$ or $\mathrm{n}$. Since this term is more predictable to first order, it is likely to dominate over the "normal" steering for no-analyzer, it is likely to be balanced for S2 and S2+90 
$\left(n_{x^{\prime}} \& n_{y^{\prime}}\right)$, i.e. equal and opposite so the "normal" steering will produce and offset in an RHWP scan in S1. And S2 steering will cause large position differences to be located at S2 RHWP angles.

$$
\begin{gathered}
\theta_{x^{\prime} 0}-\theta_{x^{\prime} 1}=R H W P @ S 2\left(45^{\circ}\right) \\
\theta_{y^{\prime} 0}-\theta_{y^{\prime} 1}=R H W P @ S 2+90^{\circ}\left(135^{\circ}\right)=4 \theta \text { Amplitude } \\
\frac{\theta_{x^{\prime}, 0}+\theta_{y^{\prime}, 0}}{2}-\frac{\theta_{x^{\prime}, 1}+\theta_{y^{\prime}, 1}}{2}=R H W P @ S 1\left(0^{\circ} \& 90^{\circ}\right) \\
=\frac{R H W P @ S 2}{2}+\frac{R H W P @ S 2+90^{\circ}}{2}=\text { offset }
\end{gathered}
$$

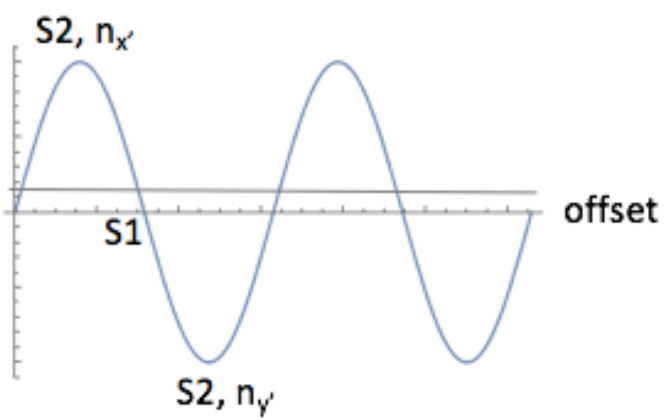

Figure D-9: RHWP scan. Isolating how angle-like steering in S1 and S2 contribute to position differences in RHWP scans.

It should be noted that a more accurate model would consist of 4 gaussian beams each with polarization along the two primary axes of the crystal, 2 for each helicity state which make up approximately circular polarization. 


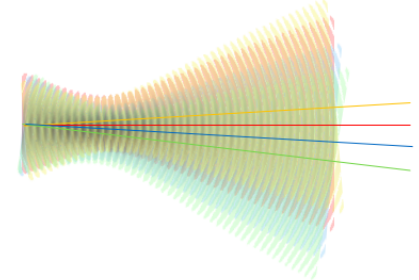

Figure D-10: 4 Gaussians. Horizontally polarized beam splits in the crystal along the primary axes, and voltage application split the beam further, making 4 gaussian beam, 2 for each helicity state.

\section{Does angle-like steering depend on input angle or position? Beam size or divergence? No.}

Phenomenologically , "normal" no-analyzer steering depends on position, which is how the offset term in RHWP scans is controlled via Pockels Cell translation. For no-analyzer and for S1 $D_{x} \approx a Y L_{e f f}, D_{y} \approx b X L_{e f f}$. For 2 input beam positions separated by $\Delta w$, the output $\mathrm{HCB}$ angle will be $b \Delta x$ and position difference $b \Delta x L_{e f f}$. For $-\Delta w$, the position difference is $-b \Delta x L_{e f f}$. A beam of diameter $2 w$ will have an average position difference change from its spot-size of $\frac{b \Delta x L_{e f f}-b \Delta x L_{e f f}}{2}=0$. Therefore, spot-size doesn't change "normal" steering in this linear model.

What about S2 angle-like steering? Phenomenologically, position differences in S2 have been observed to be linear with $\mathrm{X}, \mathrm{Y}$ translation. Hence, just as for no-analyzer, the spot-size will not affect S2 angle-like steering.

What about divergence? "Normal" steering depends on PC angle in measurements. Again, it's a linear dependence and $+\Delta \theta$ and $-\Delta \theta$ will cancel out on average. Beam divergence does not affect steering in S2.

In summary, since PC angle affects position differences linearly in S1 and S2, the beam divergence does not affect the angle-like steering terms in S1,S2.

\section{Angle-like Steering Terms Equations}

S1 \& no analyzer: 
$D_{x}=L_{e f f} \frac{d \theta_{\text {steeringx }}}{d y} \Delta y, D_{y}=L_{e f f} \frac{d \theta_{\text {steeringy }}}{d x} \Delta y$, where $\Delta y=y-y_{0}$ is the position change of the Pockels Cell from "center" y0 and $\Delta x=x-x_{0}$ is the position change of the Pockels Cell from "center" $\mathrm{x} 0$ where the steering is 0 .

S2:

$D_{x}=\epsilon L_{e f f} \beta\left(\frac{d^{2}\left(n_{x^{\prime}} L\right)}{d x^{2}} \Delta x+\frac{d^{2}\left(n_{x^{\prime}} L\right)}{d y d x} \Delta y\right), D_{y}=\epsilon L_{e f f} \beta\left(\frac{d^{2}\left(n_{x^{\prime}} L\right)}{d y^{2}} \Delta y+\frac{d^{2}\left(n_{x^{\prime}} L\right)}{d x d y} \Delta x\right)$, where this quantity is measurable with the QPD and it is related to $n_{x^{\prime}}=n_{0}-$ $\frac{1}{2} r_{33} E_{z} n_{0}^{3}$ field gradients, index gradients, and possibly length gradients. $\epsilon$ is the analyzing power of the photocathode.

These terms do not change with spot size or with divergence, but depend on $L_{e f f}$ an depend on the analyzing power for the term in S2.

We can rewrite them in terms of measurable quantities. For a $100 \%$ analyzer,

$\frac{d D_{x}}{d x}$ (angle-like,translation-dep, no-analyzer $)=L_{q p d} \frac{d \theta_{\text {steering }, x}}{d y}$

$\frac{d D_{x}}{d x}$ (angle-like,translation-dep, S2) $=L_{q p d} \beta \frac{d^{2}\left(n_{x^{\prime}} L\right)}{d x^{2}}$

$\frac{d D_{x}}{d y}\left(\right.$ angle-like,translation-dep, S2) $=L_{q p d} \beta \frac{d^{2}\left(n_{x^{\prime}} L\right)}{d y d x}$

\section{No Analyzer Angle-Dependent Steering}

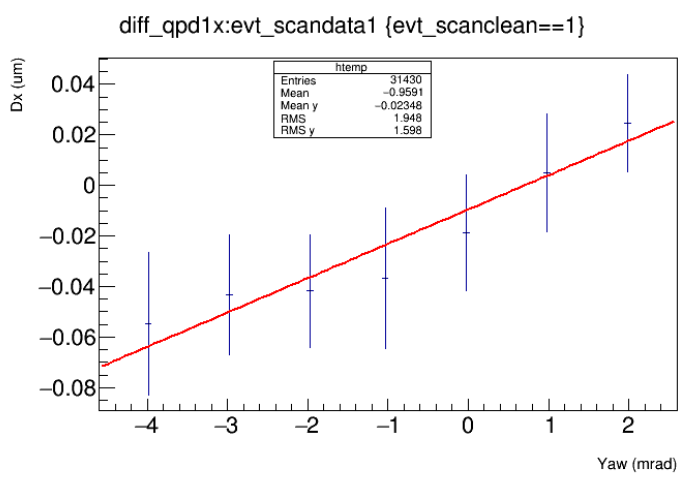

Figure D-11: Yaw and Steering. Even with no analyzer in, the PC angle can cause further steering in the beam (Run2861)

We observe that even without an analyzer, the position differences change with pockels cell angle (observed for input polarization vertical and horizontal in elog666, Run2861, 40mm length KD*P Gandalf). There are some additional lin- 
ear terms with respect to angle of the pockels cell observed for no analyzer with input polarization $\mathrm{H}$ or $\mathrm{V}$ of $<20 \mathrm{~nm} / \mathrm{mrad} 84 \mathrm{~cm}$ away. That's $<23 \mathrm{nrad} / \mathrm{mrad}$ of $\mathrm{PC}$ angle and for an Leff of $1.6 \mathrm{~m}$ (for a $2 \mathrm{~m}$ steering lens), that contributes $38 \mathrm{~nm}$ position difference for a $1 \mathrm{mrad}$ misalignment of PC angle. To compare with translational steering, for Horizontal input polarization, we observe $42 \mathrm{~nm} /(1 / 10 \mathrm{~mm})$ in $\mathrm{PC}$ translation at $84 \mathrm{~cm}$ away. That's $50 \mathrm{nrad} /(1 / 10 \mathrm{~mm})$ of $\mathrm{PC}$ translation. When aligning the Pockels Cell and finding optimal position, if the angle is off by $0.5 \mathrm{mrad}$, the zeroed out steering translation position could be off by $1 / 10 \mathrm{~mm}$, so we should account for that shift .

If we assume the observed PC angle dependent terms are angle-like and not position-like steering, they can account for as follows:

S1, S2 \& no analyzer:

$$
D_{x}=L_{e f f}\left(\frac{d \theta_{\text {steeringx }}}{d \theta_{x}} \theta_{x}+\frac{d \theta_{\text {steeringx }}}{d \theta_{y}} \theta_{y}\right), D_{y}=L_{e f f}\left(\frac{d \theta_{\text {steeringy }}}{d \theta_{y}} \theta_{y}+\frac{d \theta_{\text {steeringy }}}{d \theta_{x}} \theta_{x}\right)
$$

\section{D.2.3 Position-like Steering in $\mathrm{KD}^{*} \mathrm{P}$}

\section{Gradients Discussion}

Linear gradients in the asymmetry Aq with respect to pockels cell position $\mathrm{X}$ in $\mathrm{S} 1$, produce position differences Dx. 2nd moment terms in asymmetry Aq with respect to pockels cell position $\mathrm{X}$ produce $D x \sim X$ terms. In $\mathrm{S} 1, \mathrm{Aq}$ is determined by to $\phi$ in an expansion of $\operatorname{Cos} \phi$ near QWV where $\phi$ is the birefringence $\phi_{x^{\prime}}-\phi_{y^{\prime}}=2 \pi\left(n_{x^{\prime}}-n_{y^{\prime}}\right) L / \lambda$ and the Aq profile is determined the gradients in this quantity. There are linear, quadratic and higher order terms in the S1 Aq profile arising from birefringence gradients and crystal length gradients.

For $\mathrm{S} 2$, there is to first order, no net birefringence since $\mathrm{S} 2$ is defined along a primary axis of the crystal, so it can't have any translation dependence. In practice, phenomenologically, this may not be the case. There is a linear relationship observed $D x \sim a X, D x \sim-a Y, D y \sim a X, D y \sim-a Y$ or $D x \sim-a X, D x \sim a Y$ , $D y \sim a X, D y \sim-a Y$ depending on the sign for Xpc and Xqpd. However, it is 
possible these observed terms simply come from the mechanism of angle-like S2 steering via Snell's Law described above, in which case the phenomenon observed depends on Leff and is angle-like and not position-like.

The fact is there are Aq variations observed in S2 and translational dependencies in position differences observed in $\mathrm{S} 2$. The Aq variations may contribute some to the observed position differences via $D_{x} \sim \frac{d A q}{d x}$ and $\frac{d D_{x}}{d x} \sim \frac{d^{2} A q}{d x^{2}}$ just as they do in S1. These are "position-like" position differences which will not depend on Leff, but which will certainly depend on the spot-size.

Whether in $\mathrm{S} 1, \mathrm{~S} 2$, or no-analyzer, if there is an $\mathrm{Aq}, \frac{d A}{d x}$, or $\frac{d^{2} A}{d x^{2}}$ it will give rise to position difference offsets and translational dependencies.

Several contributing factors to "position-like" position differences exist:

$\frac{d A}{d x}$ - Dx offsets, $\frac{d A}{d \theta}$ - zero for "good" choice of angle but otherwise produces Dx offsets, $\frac{d^{2} A}{d x^{2}} \Delta x$ - Dx translational dependence, $\frac{d^{2} A}{d \theta^{2}} \Delta \theta$ - Dx angle dependence, $\frac{d^{2} A}{d x^{2}} \& w_{\text {spot }}$ - Aq offsets which must be corrected with PC angle, $\frac{d^{2} A}{d \theta^{2}} \& \theta_{d i v}-\mathrm{Aq}$ offsets which must be corrected with PC angle and which in turn makes a position difference.

\section{Radius and Angle Coupling}

For a gaussian beam, $\mathrm{r}$ and $\theta$ are coupled in the ray-optics view. How are they coupled?

Figure D-12: Wavefront. Propagation of wavefront of a gaussian beam. 


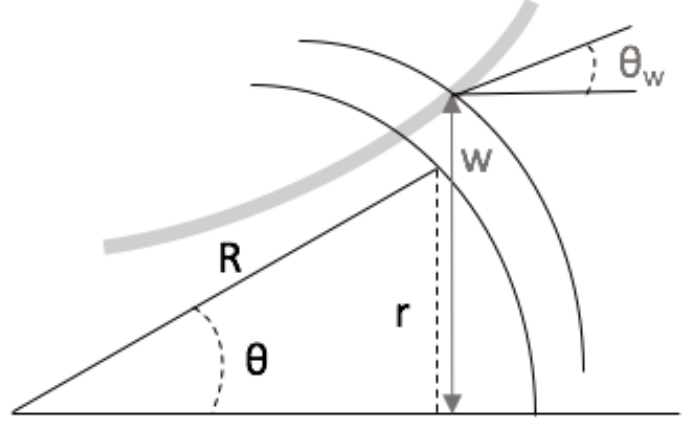

Figure D-13: Radius-Angle Coupling. Wavefront determines Radius of Curvature which couples beam radius and direction.

Let $\theta_{w}$ denote the actual half-angle divergence at position $\mathrm{z}$ with $1 / e^{2}$ radius W.

$$
\begin{gathered}
r=R \operatorname{Sin}(\theta) \approx R \theta \\
w(z)=w_{q} \sqrt{1+\frac{z^{2}}{z_{R}^{2}}} \\
z_{R}=\pi w_{0}^{2} / \lambda \\
R(z)=z\left(1+\left(z_{R} / z\right)^{2}\right) \\
\theta_{w}=\frac{d w}{d z}=\frac{\lambda}{\pi w_{0}} \sqrt{\frac{1}{z_{R}^{2} / z^{2}+1}}
\end{gathered}
$$

Derivation:

$$
\begin{gathered}
w_{0}=\frac{\lambda}{\pi w_{0}} \frac{\pi w_{0}^{2}}{\lambda}=\frac{\lambda}{\pi w_{0}} z_{R} \\
\left(w_{0} / z_{R}\right) \sqrt{z_{R}^{2}+z^{2}}=\frac{\lambda}{\pi w_{0}} \sqrt{z_{R}^{2}+z^{2}} \\
w_{0} \sqrt{1+z^{2} / z_{R}^{2}}=\frac{\lambda}{\pi w_{0}} z\left(1+z_{R}^{2} / z^{2}\right) \sqrt{\frac{1}{z_{R}^{2} / z^{2}+1}} \\
w(z)=R(z) \theta_{w}(z) \\
r \approx R \theta \approx\left(w / \theta_{w}\right) \theta
\end{gathered}
$$


If $P(x)=P_{0} e^{-2 x^{2} / w^{2}}$ describes a gaussian beam, we may then also write $P(\theta)$ as well. For small angles, there's an approximately linear relationship $\theta(r)=r\left(\theta_{w} / w\right)$ and $r(\theta)=\theta\left(w / \theta_{w}\right)$ where $\theta_{w}=\frac{\lambda}{\pi w_{0}} \sqrt{\frac{1}{z_{R}^{2} / z^{2}+1}}, w^{2}=w_{0}^{2}+w_{0}^{2} \frac{z^{2} \lambda^{2}}{\pi w_{0}^{4}}$. For $\mathrm{r}=0, \theta=0$ and for $\mathrm{r}=\mathrm{w}, \theta=\theta_{w}$, the divergence. Hence $P(\theta)=P_{0} e^{-2 \theta^{2} / \theta_{w}^{2}}$.

In terms of ray-optics if the steering lens is L1 from the Pockels Cell and the cathode is L2 from the steering lens,

$$
\begin{aligned}
& {\left[\begin{array}{c}
r_{f} \\
\theta_{f}
\end{array}\right]=\left[\begin{array}{cc}
1-L_{2} / f & L_{1}+L_{2}-L_{2} L_{1} \\
-1 / f & 1-L_{1} / f
\end{array}\right]\left[\begin{array}{l}
r \\
\theta
\end{array}\right]} \\
& r_{f}=\left(1-L_{2} / f\right) r+\left(L_{1}+L_{2}-L_{2} L_{1} / f\right) \theta
\end{aligned}
$$

Using $L_{e f f}=\left(L_{1}+L_{2}-L_{2} L_{1} / f\right)$ and $\theta=\left(\theta_{w} / w\right) r$, we obtain

$$
r_{f}=\left(1-L_{2} / f+L_{e f f} \theta_{w} / w\right) r
$$

Hence

$$
w_{\text {cathode }}=\left(1-L_{2} / f+L_{\text {eff }} \theta_{P C} / w_{P C}\right) w_{P C}
$$

which in turn makes a position difference. Note that if the beam goes through a a focus after the Pockels cell, $w_{\text {cathode }}$ is allowed to be negative to take into account the sign flip.

\section{1st moment terms}

A gaussian beam has power distribution $P(x)=P_{0} e^{-2 x^{2} / w^{2}}$ and it's exposed to a helicity dependent gradient $P^{\prime}=P_{0} e^{-2 x^{2} / w^{2}}\left(1 \pm \frac{d A}{d x} x\right)$. Considering the expectation value of the position,

$$
<x>=\frac{\int x P^{\prime} d x}{\int P^{\prime} d x}=\frac{\frac{d A}{d x} w^{2}}{4}
$$


For helicity 0: $r_{0}=\frac{d A}{d x} w^{2} / 4=<x_{0}>$. For helicity 1: $r_{1}=-\frac{d A}{d x} w^{2} / 4=<x_{1}>$. Taking into account coupling between position and angle $\theta(x) \approx x\left(\theta_{w} / w\right), \theta_{0}=$ $\frac{d A}{d x} w \theta_{w} / 4$ and $\theta_{1}=-\frac{d A}{d x} w \theta_{w} / 4$.

In terms of ray optics, $r_{f}=\left(1-L_{2} / f\right) r+\left(L_{1}+L_{2}-L_{2} L_{1} / f\right) \theta$. Generalizing to take into account analyzing power and coupling in the gaussian beam between position and angle $\theta(x) \approx x\left(\theta_{w} / w\right)$,

$$
\begin{gathered}
\Delta x_{f}=\epsilon\left(<x_{0}>-<x_{1}>\right) \\
=\epsilon\left(\left(1-L_{2} / f\right)\left(r_{0}-r_{1}\right)+\left(L_{1}+L_{2}-L_{2} L_{1} / f\right)\left(\theta_{0}-\theta_{1}\right)\right. \\
=\epsilon\left(\frac{d A}{d x} w^{2} / 2\right)\left(1-L_{2} / f+L_{e f f} \theta_{w} / w\right)
\end{gathered}
$$

Applying the relation between spot-sizes at the cathode and pockels cell $w_{\text {cathode }}=$ $\left(1-L_{2} / f+L_{e f f} \theta_{P C} / w_{P C}\right) w_{P C}$, we obtain

$$
\Delta x_{f}=\epsilon w_{P C} \frac{d A}{d x} \frac{w_{\text {cathode }}}{2}
$$

Likewise, in terms of angle $P^{\prime}=P_{0} e^{-2 \theta^{2} / \theta_{w}^{2}}\left(1 \pm \frac{d A}{d \theta} \theta\right)$. And its expectation value

$$
<\theta>=\frac{\int \theta P^{\prime} d \theta}{\int P^{\prime} d \theta} \approx \frac{\frac{d A}{d \theta} \theta_{w}^{2}}{4}
$$

For helicity 0: $\theta_{0}=\frac{d A}{d \theta} \theta_{w}^{2} / 4=<\theta_{0}>$. For helicity 1: $\theta_{1}=-\frac{d A}{d \theta} \theta_{w}^{2} / 4=<\theta_{1}>$. Taking into account coupling between position and angle $x(\theta) \approx \theta\left(w / \theta_{w}\right), x_{0}=$ $\frac{d A}{d \theta} \theta_{w} w / 4$ and $x_{1}=-\frac{d A}{d \theta} \theta_{w} w / 4$. In terms of ray optics,

$\Delta x_{f}=\epsilon\left(<x_{0}>-<x_{1}>\right)=\epsilon\left(\left(1-L_{2} / f\right)\left(r_{0}-r_{1}\right)+\left(L_{1}+L_{2}-L_{2} L_{1} / f\right)\left(\theta_{0}-\right.\right.$ $\left.\theta_{1}\right)=\epsilon\left(\frac{w}{\theta_{w}}\right)\left(\frac{d A}{d x} \theta_{w}^{2} / 2\right)\left(1-L_{2} / f+L_{e f f} \theta_{w} / w\right)$

Applying the relation between spot-sizes at the cathode and Pockels cell $w_{\text {cathode }}=$ $\left(1-L_{2} / f+L_{e f f} \theta_{P C} / w_{P C}\right) w_{P C}$, we obtain

$$
\Delta x_{f}=\epsilon w_{\text {cathode }} \frac{d A}{d \theta} \frac{\theta_{P C}}{2}
$$




\section{Including 2nd moment terms}

There are 2 nd order terms $\frac{d^{2} A}{d \theta^{2}}, \frac{d^{2} A}{d x^{2}}$ which lead to a linear dependence of Dx on $\mathrm{X}, \theta$ alignment: $\frac{d^{2} A}{d x^{2}} x, \frac{d^{2} A}{d \theta_{x}^{2}} \theta_{x}, \frac{d^{2} A}{d y d x} y, \frac{d^{2} A}{d \theta_{y} d \theta_{x}} \theta_{y}$. These terms act as corrections to the 1st moment terms.

$$
\begin{gathered}
\Delta x_{f, \text { translation }}=\epsilon w_{P C}\left(\frac{d A}{d x}+\frac{d^{2} A}{d x^{2}} x+\frac{d^{2} A}{d y d x} y\right) \frac{w_{\text {cathode }}}{2} \\
\Delta x_{f, \text { angle }}=\epsilon w_{\text {cathode }}\left(\frac{d A}{d \theta}+\frac{d^{2} A}{d \theta_{x}^{2}} \theta_{x}+\frac{d^{2} A}{d \theta_{y} d \theta_{x}} \theta_{y}\right) \frac{\theta_{P C}}{2}
\end{gathered}
$$

We can rewrite the coefficients of these terms as a function of measurable quantities. For a $100 \%$ analyzer at the QPD position,

$D_{x}($ position-like, translation-dep $)=\frac{w_{P C} w_{q p d}}{2} \frac{d A}{d x}$

$\frac{d D_{x}}{d x}\left(\right.$ position-like, translation-dep) $=\frac{w_{P C} w_{q p d}}{2} \frac{d^{2} A}{d x^{2}}$

$\frac{d D_{x}}{d y}$ (position-like, translation-dep) $=\frac{w_{P C} w_{q p d}}{2} \frac{d^{2} A}{d y d x}$

$D_{x}($ position-like, angle-dep $)=\frac{\theta_{P C} w_{q p d}}{2} \frac{d A}{d \theta}$

$\frac{d D_{x}}{d \theta_{x}}$ (position-like, angle-dep) $=\frac{\theta_{P C} w_{q p d}}{2} \frac{d^{2} A}{d \theta_{x}^{2}}$

$\frac{d D_{x}}{d \theta_{y}}\left(\right.$ position-like, angle-dep) $=\frac{\theta_{P C} w_{q p d}}{2} \frac{d^{2} A}{d \theta_{y} d \theta_{x}}$

\section{Asymmetry Offset Terms}

There are Aq offset terms which cannot be corrected with PITA voltage in S2 and which must be corrected either by translation or angle. Usually, we do this with angle. Offset terms can arise from spot size $\mathrm{w}$ and divergence $\theta_{\text {div }}$ combined with 2nd order terms of Aq: $\frac{d^{2} A}{d x^{2}}, \frac{d^{2} A}{d \theta^{2}}$.

In $\mathrm{KD}^{*} \mathrm{P}$, the asymmetry angle dependence can be approximated to secondorder as

$$
A(\theta)=-\frac{\epsilon}{T} \frac{\pi D}{\lambda} n_{o} \frac{\left(n_{o}^{2}-n_{e}^{2}\right)}{n e^{2}}\left[2 \xi_{x} \xi_{y} \cos (2 \psi)+\left(\xi_{x}^{2}-\xi_{y}^{2}\right) \sin (2 \psi)\right]=
$$




$$
=-k\left[2 \xi_{x} \xi_{y} \cos (2 \psi)+\left(\xi_{x}^{2}-\xi_{y}^{2}\right) \sin (2 \psi)\right]
$$

This is an expansion of $\sin \left(\xi_{x}\right)$ and $\sin \left(\xi_{y}\right)$ terms and higher order terms do exist and could potentially manifest themselves in large deviations from the Aq saddlepoint or in position differences and spot size asymmetries. We only examine the 2nd order terms here.

For a gaussian beam, $P(\theta)=P_{0} e^{-2 \theta^{2} / \theta_{w}^{2}}$. Examining the averaged values of the asymmetry over the beam in $\mathrm{S} 2$,

$$
\begin{gathered}
<A\left(\theta+\delta \theta_{x}\right)>\approx \frac{\int A\left(\theta+\delta \theta_{x}\right) P(\theta) d \theta}{\int P d \theta} \approx \frac{\int-k\left(\theta+\delta \theta_{x}\right)^{2} e^{-2 \theta^{2} / \theta_{w}^{2}} d \theta}{\int e^{-2 \theta^{2} / \theta_{w}^{2} d \theta} d \theta} \approx-k \delta \theta_{x}^{2}-k / 4 \theta_{w_{x}}^{2} \\
<A\left(\theta+\delta \theta_{y}\right)>\approx+k \delta \theta_{y}^{2}+k / 4 \theta_{w_{y}}^{2}
\end{gathered}
$$

An astigmatic/elliptical divergence of the laser shifts the asymmetry such that the zero of $\mathrm{Aq}$ is not at $\theta_{x}=\theta_{y}=0$. For $1 \mathrm{mrad}$ half-angle divergence, $k=$ $2.4 x 10^{4} \mathrm{ppm} / \mathrm{mrad}^{2}$ (theoretically, experimentally this is $10 \mathrm{X}$ smaller), this shifts by 6000 ppm meaning to zero out $\mathrm{Aq}$ in $\mathrm{S} 2$, we have to pick an angle not centered on the saddle point and off by $0.5 \mathrm{mrad}$, leading to a position difference.

In $\mathrm{S} 1, A=-k 2 \xi_{x} \xi_{y}$ and the divergence along the diagonal x', $\mathrm{y}^{\prime}$ is $\theta_{x^{\prime}}=\frac{\theta_{w_{x}}+\theta_{w_{y}}}{\sqrt{2}}$ and $\left\langle A\left(\theta+\delta \theta_{x^{\prime}}\right)>\approx-k \frac{\delta \theta_{x}+\delta \theta_{y}}{\sqrt{2}}-\frac{k}{4} \frac{\theta_{w_{x}}+\theta_{w_{y}}}{\sqrt{2}}\right.$.

All together,

$$
\begin{gathered}
<A(\theta)>=-\frac{\epsilon}{T} \frac{\pi D}{\lambda} n_{o} \frac{\left(n_{o}^{2}-n_{e}^{2}\right)}{n e^{2}} \\
\times\left[\left(2 \xi_{x} \xi_{y}+\theta_{w_{x^{\prime}}}^{2} / 4-\theta_{w_{y^{\prime}}}^{2} / 4\right) \cos (2 \psi)+\left(\xi_{x}^{2}-\xi_{y}^{2}+\theta_{w_{x}}^{2} / 4-\theta_{w_{y}}^{2} / 4\right) \sin (2 \psi)\right]
\end{gathered}
$$

where x' and y' are $45^{\circ}$ relative to $\mathrm{x}, \mathrm{y}$. Either S1 or S2 could have and Aq offset if the divergence for $\mathrm{x}, \mathrm{y}$ or $\mathrm{x}^{\prime}, \mathrm{y}^{\prime}$ differ. But if the beam is elliptical along 
$\mathrm{x} / \mathrm{y}$ then $\mathrm{S} 2$ has the offset term.

2nd order terms in translational dependence combined with a spot-size can also result in Aq offsets. For a quadratic $A(x) \sim x^{2}$ or $2 x y$ or $y^{2}$,

$$
A_{\text {offset }}=\frac{\epsilon}{T}\left(\frac{w_{x}^{2}}{4} \frac{d^{2} A}{d x^{2}}+\frac{w_{y}^{2}}{4} \frac{d^{2} A}{d y^{2}}+\frac{w_{x} w_{y}}{4}\left(\frac{d^{2} A}{d x d y}+\frac{d^{2} A}{d y d x}\right)\right)
$$

The total offset in S2 is important because it cannot be corrected with voltage so it must be corrected with $\mathrm{PC}$ angle misalignment. The total offset can be written as the sum of the spot-size induced and divergence induced offsets.

$$
\begin{aligned}
& \left.A_{o f f s e t, t o t a l}\right|_{S_{1}}=A_{o f f s e t, \text { size }}+A_{o f f \text { set,divergence }} \\
& =\frac{\epsilon}{T}\left(\left.\frac{w_{x}^{2}}{4} \frac{d^{2} A}{d x^{2}}\right|_{S_{1}}+\left.\frac{w_{y}^{2}}{4} \frac{d^{2} A}{d y^{2}}\right|_{S_{1}}+\frac{w_{x} w_{y}}{4}\left(\left.\frac{d^{2} A}{d x d y}\right|_{S_{1}}+\left.\frac{d^{2} A}{d y d x}\right|_{S_{1}}\right)\right) \\
& -\frac{\epsilon}{T} \frac{\pi D}{\lambda} n_{o} \frac{\left(n_{o}^{2}-n_{e}^{2}\right)}{n e^{2}}\left[\theta_{w_{x^{\prime}}}^{2} / 4-\theta_{w_{y^{\prime}}}^{2} / 4\right] \\
& \left.A_{\text {offset,total }}\right|_{S_{2}}=\frac{\epsilon}{T}\left(\left.\frac{w_{x}^{2}}{4} \frac{d^{2} A}{d x^{2}}\right|_{S_{2}}+\left.\frac{w_{y}^{2}}{4} \frac{d^{2} A}{d y^{2}}\right|_{S_{2}}+\frac{w_{x} w_{y}}{4}\left(\left.\frac{d^{2} A}{d x d y}\right|_{S_{2}}+\left.\frac{d^{2} A}{d y d x}\right|_{S_{2}}\right)\right) \\
& -\frac{\epsilon}{T} \frac{\pi D}{\lambda} n_{o} \frac{\left(n_{o}^{2}-n_{e}^{2}\right)}{n e^{2}}\left[\theta_{w_{x}}^{2} / 4-\theta_{w_{y}}^{2} / 4\right] \approx \epsilon\left(\frac{w^{2}}{4}\right)\left(\left.\frac{d^{2} A}{d x^{2}}\right|_{S_{2}}+\left.\frac{d^{2} A}{d y^{2}}\right|_{S_{2}}+\left(\left.\frac{d^{2} A}{d x d y}\right|_{S_{2}}+\left.\frac{d^{2} A}{d y d x}\right|_{S_{2}}\right)\right) \\
& -\frac{\epsilon}{T} \frac{\pi D}{\lambda} n_{o} \frac{\left(n_{o}^{2}-n_{e}^{2}\right)}{n e^{2}}\left[\theta_{w_{x}}^{2} / 4-\theta_{w_{y}}^{2} / 4\right]
\end{aligned}
$$

The astigmatism/ellipticity in the divergence of the beam and the spot size on the PC both affect the Aq offset in S2 which in turn affects the angle at which the PC must be set.

Alignment of the $\mathrm{PC}$ usually involves setting $\mathrm{Aq}=0$ in $\mathrm{S} 2$ via angle adjustment and setting no-analyzer position differences to zero using translation adjustments on the QPD with $100 \%$ analyzing power. If there's no offset in S2, then we sit on the saddlepoint and $\frac{d A}{d \theta}=0$. If there is an offset, then $\theta_{0}$ is chosen so 
$\mathrm{A}=0$ and $\frac{d A}{d \theta}=\frac{d^{2} A}{d \theta^{2}} \theta_{0}$. Suppose there is a positive Aq offset in S2, we must decrease the asymmetry by setting $\theta_{x 0}$ to a non-zero value, while $\theta_{y 0}=0$. We solve $\left.A_{\text {offset,size }}\right|_{S_{2}}+\left.A_{\text {offset,divergence }}\right|_{S_{2}}+A\left(\theta_{x 0}\right)=0$ and obtain

$$
\begin{gathered}
\theta_{x 0}=\sqrt{\frac{1}{k}\left(\left.A_{\text {offset }, \text { total }}\right|_{S_{2}}\right)} \\
=\sqrt{\frac{\theta_{w_{y}}^{2}}{4}-\frac{\theta_{w_{x}}^{2}}{4}-\left(\left.\frac{w_{x}^{2}}{4} \frac{d^{2} A}{d x^{2}}\right|_{S_{2}}+\left.\frac{w_{y}^{2}}{4} \frac{d^{2} A}{d y^{2}}\right|_{S_{2}}+\frac{w_{x} w_{y}}{4}\left(\left.\frac{d^{2} A}{d x d y}\right|_{S_{2}}+\left.\frac{d^{2} A}{d y d x}\right|_{S_{2}}\right)\right)}
\end{gathered}
$$

Or if the offset in Aq is negative, we obtain

$$
\begin{gathered}
\theta_{y 0}=\sqrt{\frac{-1}{k}\left(\left.A_{\text {offset,total }}\right|_{S_{2}}\right)} \\
=\sqrt{\frac{\theta_{w_{x}}^{2}}{4}-\frac{\theta_{w_{y}}^{2}}{4}+\left(\left.\frac{w_{x}^{2}}{4} \frac{d^{2} A}{d x^{2}}\right|_{S_{2}}+\left.\frac{w_{y}^{2}}{4} \frac{d^{2} A}{d y^{2}}\right|_{S_{2}}+\frac{w_{x} w_{y}}{4}\left(\left.\frac{d^{2} A}{d x d y}\right|_{S_{2}}+\left.\frac{d^{2} A}{d y d x}\right|_{S_{2}}\right)\right)}
\end{gathered}
$$

These Aq offset angle corrections will give rise to the presence of position differences both in S1 and S2 via

$$
\Delta x_{f, \text { angle }}=\epsilon w_{\text {cathode }}\left(\frac{d A}{d \theta_{x}}+\frac{d^{2} A}{d \theta_{x}^{2}} \theta_{x}+\frac{d^{2} A}{d \theta_{y} d \theta_{x}} \theta_{y}\right) \frac{\theta_{P C}}{2}
$$

\section{Angle Asymmetry Gradients}

The angle dependence of Aq combined with beam divergence position-angle coupling gives rise to angle dependent position differences in both S1 and S2. An astigmatism/ellipticity in the laser which forces the $\mathrm{KD}^{*} \mathrm{P}$ angle to be offset from the angle-saddle-point will cause the beam to sit on an asymmetry gradient with respect to angle. The laser divergence will then couple this gradient with respect to angle to a gradient with respect to position and hence, a position difference.

$$
\begin{gathered}
A(\theta)=-k\left[2 \theta_{x} \theta_{y} \cos (2 \psi)+\left(\theta_{x}^{2}-\theta_{y}^{2}\right) \sin (2 \psi)\right] \\
\frac{d A(\theta)}{d \theta_{x}}=-2 k\left[\theta_{y} \cos (2 \psi)+\theta_{x} \sin (2 \psi)\right]
\end{gathered}
$$




$$
\frac{d A(\theta)}{d \theta_{y}}=-2 k\left[\theta_{x} \cos (2 \psi)-\theta_{y} \sin (2 \psi)\right]
$$

For small divergence, there's an approximately linear relationship coupling angle and position $\theta(r)=r\left(\theta_{w} / w\right)$ and $r(\theta)=\theta\left(w / \theta_{w}\right)$ w. At the location of the Pockels Cell,

$$
\begin{aligned}
& \frac{d A(\theta)}{d x}=\frac{d A(\theta)}{d \theta_{x}} \frac{d \theta_{x}}{d x}=-2 k \frac{\theta_{w_{x}}}{w_{x}}\left[\theta_{y} \cos (2 \psi)+\theta_{x} \sin (2 \psi)\right] \\
& \frac{d A(\theta)}{d y}=\frac{d A(\theta)}{d \theta_{y}} \frac{d \theta_{y}}{d y}=-2 k \frac{\theta_{w_{y}}}{w_{y}}\left[\theta_{x} \cos (2 \psi)-\theta_{y} \sin (2 \psi)\right]
\end{aligned}
$$

These gradients will give rise to position differences at the qpd:

$$
\begin{aligned}
D_{x}=\frac{\theta_{w_{x}} w_{q p d_{x}}}{2} \frac{d A}{d \theta_{x}} & =-k \theta_{w_{x}} w_{q p d_{x}}\left[\theta_{y} \cos (2 \psi)+\theta_{x} \sin (2 \psi)\right] \\
D_{y}=\frac{\theta_{w_{y}} w_{q p d_{y}}}{2} \frac{d A}{d \theta_{y}} & =-k \theta_{w_{y}} w_{q p d_{y}}\left[\theta_{x} \cos (2 \psi)-\theta_{y} \sin (2 \psi)\right] \\
k & =\frac{\epsilon}{T} \frac{\pi D}{\lambda} n_{o} \frac{\left(n_{o}^{2}-n_{e}^{2}\right)}{n e^{2}}
\end{aligned}
$$

This effect means that small beam divergence through the Pockels cell is critical for minimizing position differences. Typically, the divergence is $<0.5 \mathrm{mrad}$ going through the Pockels cell. In the past, attempts have been made to tightly focus the laser through the Pockels cell with a lens in order to reduce spot-size and position differences. It was observed that rather than reducing the position differences, this tight focus within the Pockels cell amplified position differences very strongly. The equations above explain this effect. Focusing the laser tightly tends to increase the divergence outside the focus, which increases the position differences, even though the beam spots-size is small. When the Raleigh range is short, it's hard to keep the whole length of the $40 \mathrm{~mm}$ long crystal in a where the divergence is $<0.5 \mathrm{mrad}$. If the focus of the laser is not exactly in the center of the crystal, or if there is an astigmatism such that the horizontal and vertical foci occur in different locations, the average divergence will be large and contribute significantly 
to position differences. This when attempting to reduce position differences, it is important to reduce both spot-size and divergence within the $\mathrm{KD}^{*} \mathrm{P}$ crystal.

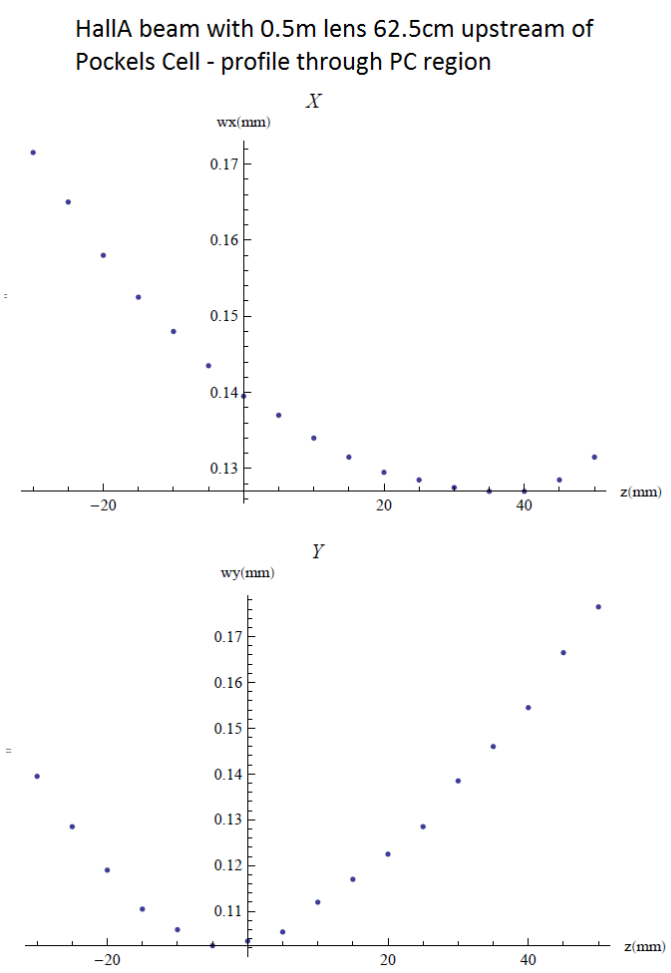

Figure D-14: Beam Profile. A 50cm lens is placed upstream of the Pockels cell. Due to laser astigmatism, the $\mathrm{x} / \mathrm{y}$ foci occur at different locations and the average divergence is not small.

\section{Non-Gaussian Tails}

For a gaussian beam, $P(\theta)=P_{0} e^{-2 \theta^{2} / \theta_{w}^{2}}$ the asymmetry gradients as a function of PC angle don't change with laser divergence, at least when the function form of asymmetry only contains second order terms in angle. But a non-symmetric beam can cause the asymmetry gradients to change in measurement.

Suppose we model a tail as just a flat-top distribution with a mean $\theta_{t 0}$ and with $\theta_{t w}$ and Intensity $P_{t}$ so that the new distribution in angle is $P(\theta)=P_{0} e^{-2 \theta^{2} / \theta_{w}^{2}}+P_{t}$ for $\theta_{t 0}-\theta_{t w}<\theta<\theta+\theta_{t w}$ and $P(\theta)=P_{0} e^{-2 \theta^{2} / \theta_{w}^{2}}$ otherwise. We assume that the peak power of the tail is much smaller than the peak power of the gaussian in our 
approximation below

$$
\begin{gathered}
<A\left(\theta+\delta \theta_{x}\right)>\approx \frac{\int A\left(\theta+\delta \theta_{x}\right) P(\theta) d \theta}{\int P d \theta} \\
\approx-k \delta \theta_{x}^{2}-k / 4 \theta_{w_{x}}^{2}+\frac{P_{t} k \sqrt{2 / \pi} \theta_{t w x}}{P_{o}\left|\theta_{w x}\right|}\left(-4 \delta \theta_{x} \theta_{t 0 x}-2 \theta_{t 0 x}^{2}+\theta_{w x}^{2} / 2-2 \theta_{t w x}^{2} / 3\right) \\
<A\left(\theta+\delta \theta_{y}\right)>\approx \frac{\int A\left(\theta+\delta \theta_{y}\right) P(\theta) d \theta}{\int P d \theta} \\
\approx+k \delta \theta_{y}^{2}+k / 4 \theta_{w_{y}}^{2}-\frac{P_{t} k \sqrt{2 / \pi} \theta_{t w y}}{P_{o}\left|\theta_{w y}\right|}\left(-4 \delta \theta_{y} \theta_{t 0 y}-2 \theta_{t 0 y}^{2}+\theta_{w y}^{2} / 2-2 \theta_{t w y}^{2} / 3\right) \\
\frac{d<A\left(\theta+\delta \theta_{x}\right)>}{d \delta \theta_{x}} \approx-2 k \delta \theta_{x}-4 \theta_{t 0 x} \frac{P_{t} k \sqrt{2 / \pi} \theta_{t w x}}{P_{o}\left|\theta_{w x}\right|} \\
\frac{d<A\left(\theta+\delta \theta_{y}\right)>}{d \delta \theta_{y}} \approx+2 k \delta \theta_{y}+4 \theta_{t 0 y} \frac{P_{t} k \sqrt{2 / \pi} \theta_{t w y}}{P_{o}\left|\theta_{w y}\right|}
\end{gathered}
$$

Non-Gaussian tails can give rise to position difference offsets, charge asymmetry offsets and linear terms in Aq with respect to angle.

\section{Photocathode Analyzing Power}

The strength an direction of the photocathode's analyzing power is a significant parameter. Let the analyzing direction be $\psi_{\text {cathode }}$ relative to the vertical. Define positive $\epsilon$ such that polarization along $\psi_{\text {cathode }}$ has a higher transmission than polarization along $\psi_{\text {cathode }}+90^{\circ}$. If we rotate a waveplate such that the input polarization is along $\theta_{\text {pol }}$ the analyzing power in that direction will be

$$
\begin{gathered}
\epsilon\left(\theta_{\text {pol }}\right)=\epsilon_{0} \operatorname{Cos}\left[2\left(\theta_{\text {pol }}-\psi_{\text {cathode }}\right)\right] \\
\epsilon\left(S_{1}, V\right)=\epsilon_{0} \operatorname{Cos}\left[2 \psi_{\text {cathode }}\right] \\
\epsilon\left(S_{1}+90^{\circ}, H\right)=\epsilon_{0} \operatorname{Cos}\left[\pi-2 \psi_{\text {cathode }}\right] \\
\epsilon\left(S_{2},+45^{\circ}\right)=\epsilon_{0} \operatorname{Cos}\left[\pi / 2-2 \psi_{\text {cathode }}\right]
\end{gathered}
$$




$$
\epsilon\left(S_{2},-45^{\circ}\right)=\epsilon_{0} \operatorname{Cos}\left[-\pi / 2-2 \psi_{\text {cathode }}\right]
$$

It should be noted that the 2 theta RHWP terms wind up dominating once the cathode analyzing power is rotated.

It should be noted that to predict behavior in the injector, it is necessary to know the focusing and electron beam propagation post-pockels cell. The position differences observed in beam BPM will be proportional to the spot-size of the electron beam. The electron beam coming off the cathode will be the same size as the laser beam incident upon it assuming no quadratic terms in QE.

The quantum efficiency also affects the position differences. There can be linear and quadratic terms in QE and analyzing power $\frac{d Q E}{d x}, \frac{d \epsilon_{0}}{d x}, \frac{d^{2} Q E}{d x^{2}}, \frac{d^{2} \epsilon_{0}}{d x^{2}}$.

Suppose the gaussian spot over time degrades the QE proportional to the intensity of light. Then there will be a "hole" in the QE, gaussian in shape, which will result in a smaller efficiency near the laser peak and a relatively larger efficiency near the laser spot wings, resulting in a larger electron beam effective spot size than the laser beam incident upon it. The larger electron beam spot size will then result in larger position differences as the QE hole deepens. Numerically, if the average photocathode QE drops by some fraction $\mathrm{D}$ due to a gaussian laser spot $P_{0} e^{-2 x^{2} / w^{2}}$, the QE profile would conform to the function $\left(1-\sqrt{2} D e^{-2 x^{2} / w^{2}}\right)$ and the electron beam would come off the cathode as $\left(1-\sqrt{2} D e^{-2 x^{2} / w^{2}}\right) e^{-2 x^{2} / w^{2}}$ which to first order has a spot size modified by a factor $w_{e-}=w_{\text {laser }}\left(1+\frac{\left(1-1 / e^{2}\right) D}{2 \sqrt{2}}\right)$

If we a have a position difference in a gaussian laser beam which is then exposed to a gaussian QE form, what does that do to the position difference? we can compute the expectation value of the position to first order $<x>\approx \frac{\int Q E(x) P(x+\Delta x) d x}{\int Q E(x) P(x+\Delta x) d x} \approx$ $\frac{\int x\left(1-\sqrt{2} D e^{-2 x^{2} / w^{2}}\right) e^{-2(x+\Delta x)^{2} / w^{2}} d x}{\int\left(1-\sqrt{2} D e^{-2 x^{2} / w^{2}}\right) e^{-2(x+\Delta x)^{2} / w^{2}} d x} \approx-\Delta x(1+D / 2)$. Therefore, the position difference increases as the QE hole deepens. $D_{x, e-}=<x_{0}>-<x_{1}>=\left(x_{0}-x_{1}\right)(1+D / 2)=$ $D_{x, l a s e r}(1+D / 2)$

This equation was demonstrated empirically at JLab by moving the laser out 
of a QE hole D-15 and comping overall position differences in the injector D-26 .

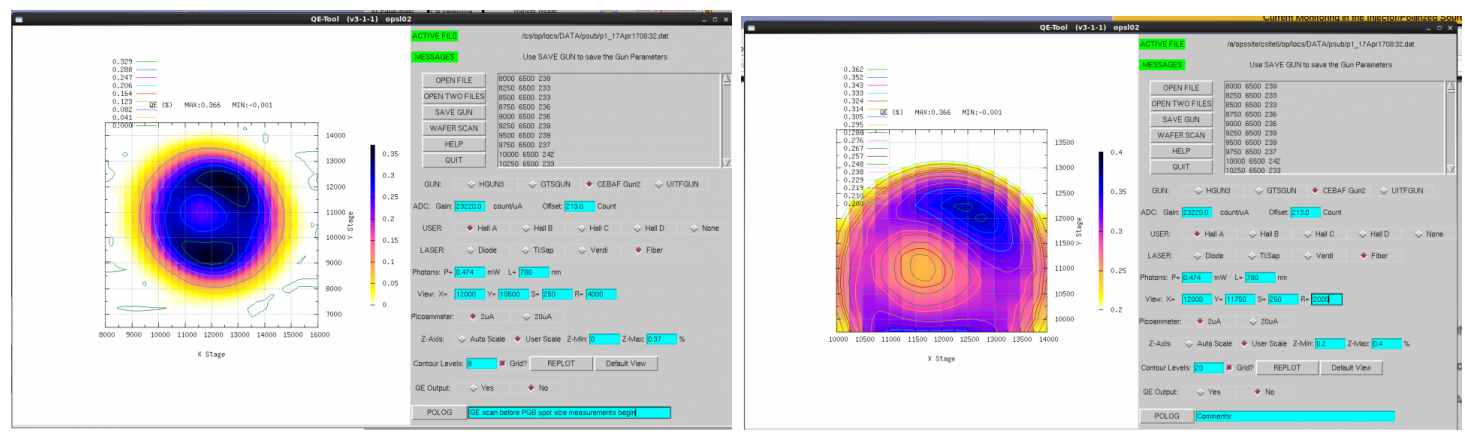

Figure D-15: QE scans of photocathode showing a QE hole where the laser had been

The hole-equation predicts when comparing a flat region of $0.507 \% \mathrm{QE}(0.352 \%$ gun off) with a hole region with $0.3674 \% \mathrm{QE}(0.25 \%$ gun off), if the laser is about the size of the hole, the position differences will scale by a factor of $0.73 \mathrm{X}$, so $38 \%$ decrease in position differences outside the hole. Average position differences in the injector showed a factor of $0.77 \mathrm{X}$ decrease in y position differences and $0.64 \mathrm{X}$ in $\mathrm{x}$ position differences and a $0.71 \mathrm{X}$ decrease overall. The observed effect of the QE hole was very similar to what what predicted by simply assuming the hole was the same size as the laser beam.

Regarding any 1st order slopes in the QE, this will shift the overall position by $\frac{1}{4} \frac{d Q E}{d x}$, but not the position differences.

There can be a gradient in the analyzing power. The analyzing power of the the photocathode was measured by rotating the RHWP with the PC HV off for three locations :

- (1) in a high QE plateau uniform region: analyzing power $=5.36 \% \pm 0.37 \%$, $\mathrm{QE}($ gun off $) \approx 0.352 \%, \mathrm{QE}($ gun on $) \approx 0.5067 \%$ (Polog 3470276)

- $(2)$ on a QE gradient: analyzing power $=5.62 \pm 0.37 \%, \mathrm{QE}$ (gun off $) \approx 0.324 \%, \mathrm{QE}$ (gun on) $\approx 0.4886 \%$

- $(3)$ in a QE hole: analyzing power $=5.97 \% \pm 0.37 \%$, QE(gun off $) \approx 0.25 \%$, 
$\mathrm{QE}($ gun on $) \approx 0.3674 \%$

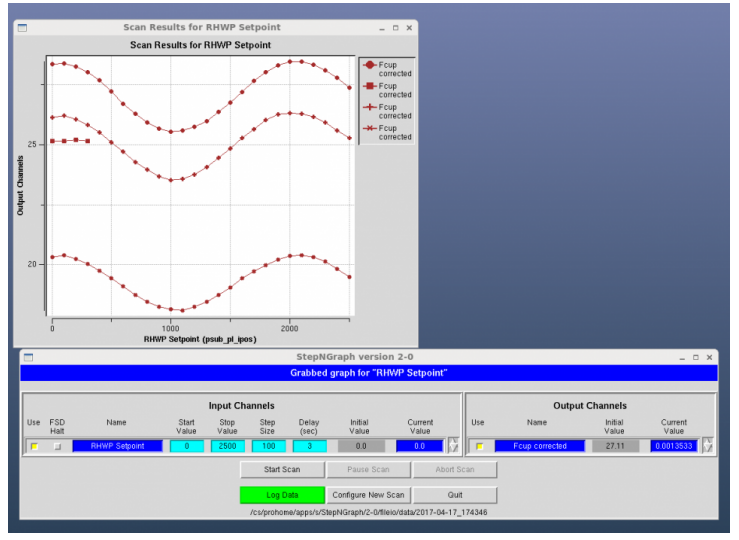

Figure D-16: Cathode analyzing power at three locations (1) in a QE hole (2) on a QE gradient (3) in a high QE plateau uniform region. Polog 3470271

If there is a slope in the analyzing power, it will make no difference to position differences which are not polarization dependent, such as the angle-like steering terms present with no analyzer. However, if there is, for example, a charge asymmetry in S1 and a gradient in the analyzing power over the face of the cathode, then there will be a position difference proportional to the size of the beam spot, the gradient, and the asymmetry.

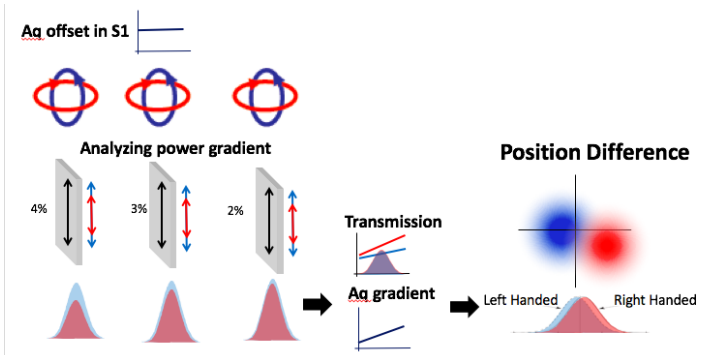

Figure D-17: Gradient in Analyzing Power on the photocathode combined with a linear polarization asymmetry leads to a position difference

Quantitatively, if a circularly polarized gaussian beam has power distribution $P(x)=P_{0} e^{-2 x^{2} / w^{2}}$, with an S1 asymmetry in polarization Aq, and it's exposed to an analyzing power, the resulting distribution will be $P^{\prime}=P_{0} e^{-2 x^{2} / w^{2}} \frac{1}{2}(1 \pm$ 
$\epsilon A q)$. If there's a gradient in the analyzing power, the distribution will be $P^{\prime}=$ $P_{0} e^{-2 x^{2} / w^{2}} \frac{1}{2}\left(1 \pm\left(\epsilon+\frac{d \epsilon}{d x} x\right) A q\right)$. Considering the expectation value of the position,

$$
<x>=\frac{\int x P^{\prime} d x}{\int P^{\prime} d x}= \pm \frac{A_{q, S 1} \frac{d \epsilon}{d x} w^{2}}{4}
$$

Hence the position difference induced by an analyzing power gradient on the photocathode is

$$
D_{x}=\frac{A_{q} \frac{d \epsilon}{d x} w_{c a t h o d e}^{2}}{2}
$$

\section{Measurable Quantities}

Many translation scans and angle scans were performed at UVa on a Goose and Housego KD*P with $20 \mathrm{mmm}$ aperture ("Gandalf") for input polarization $\mathrm{H}$, $\mathrm{V},+45^{\circ},-45^{\circ}$, and for no analyzer after the PC, analyzer oriented along V (S1), and analyzer oriented along $\mathrm{H}(\mathrm{S} 2)$. These studies were done with qpd at a couple different distances from the PC to distinguish angle-like and position like steering terms.
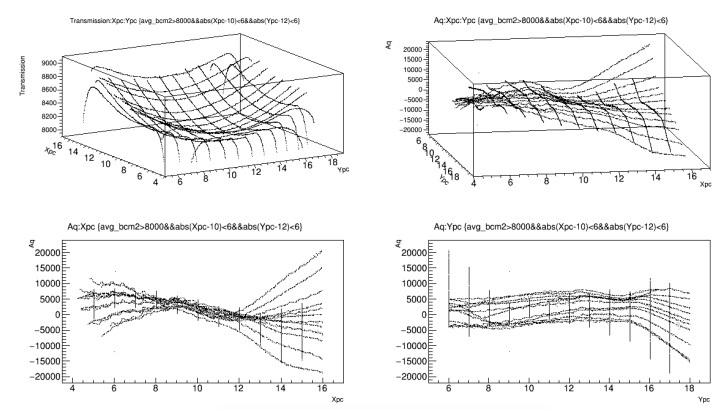

Figure D-18: Charge asymmetry gradient for active $\mathrm{KD}^{*} \mathrm{P}$ with a vertical analyzer after cell("Gandalf", Run2815, elog 656) 

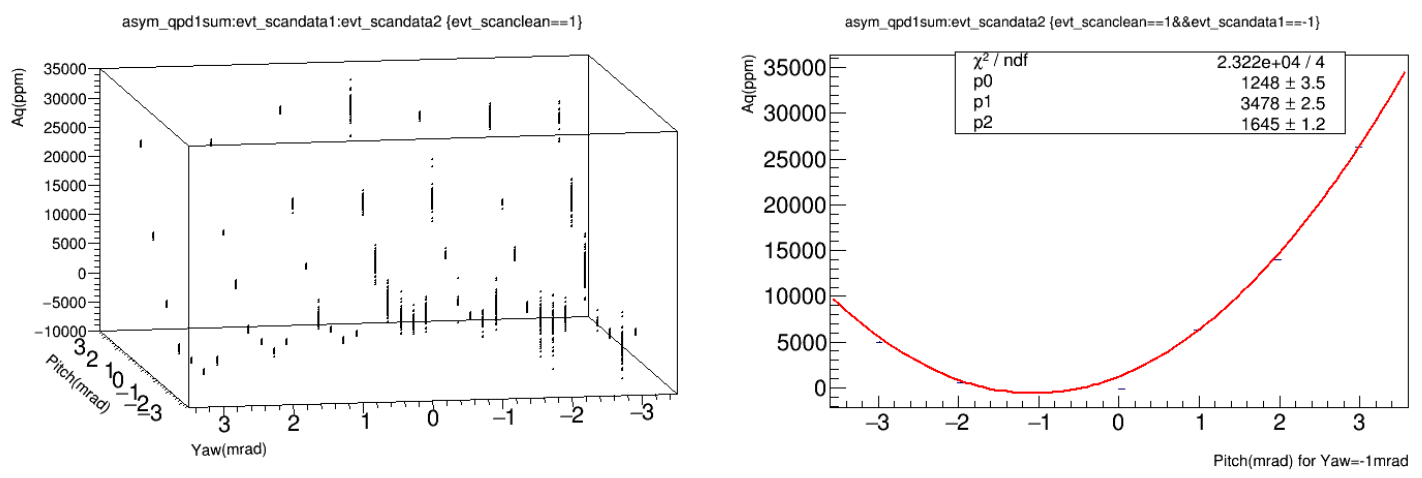

Figure D-19: Charge asymmetry gradient for active $\mathrm{KD}^{*} \mathrm{P}$ with a vertical analyzer after cell("Gandalf", Run2857, elog 665)

In the table below, na=no-analyzer and $\mathrm{H}, \mathrm{x}^{\prime}, \mathrm{y}^{\prime}$ indicates the input polarization direction. All units are Dx[um], Dy[um],x[mm],y[mm], $\theta[\mathrm{mrad}], \mathrm{A}[\mathrm{ppm}], \mathrm{L}[\mathrm{m}], \mathrm{w}[\mathrm{mm}], \theta_{\text {div }}[\mathrm{mrad}]$ are evaluated near Xpc 8-9.2mm, Ypc 10.8-11.5mm. Values obtained for KD*P cell Gandalf, $20 \mathrm{~mm}$ aperture, $40 \mathrm{~mm}$ length with an $800 \mathrm{~nm}$ laser with qpd $84 \mathrm{~cm}$ from cell center (see elog 718). A simulation of KD*P RHWP scans is done in $\operatorname{elog} 719$.

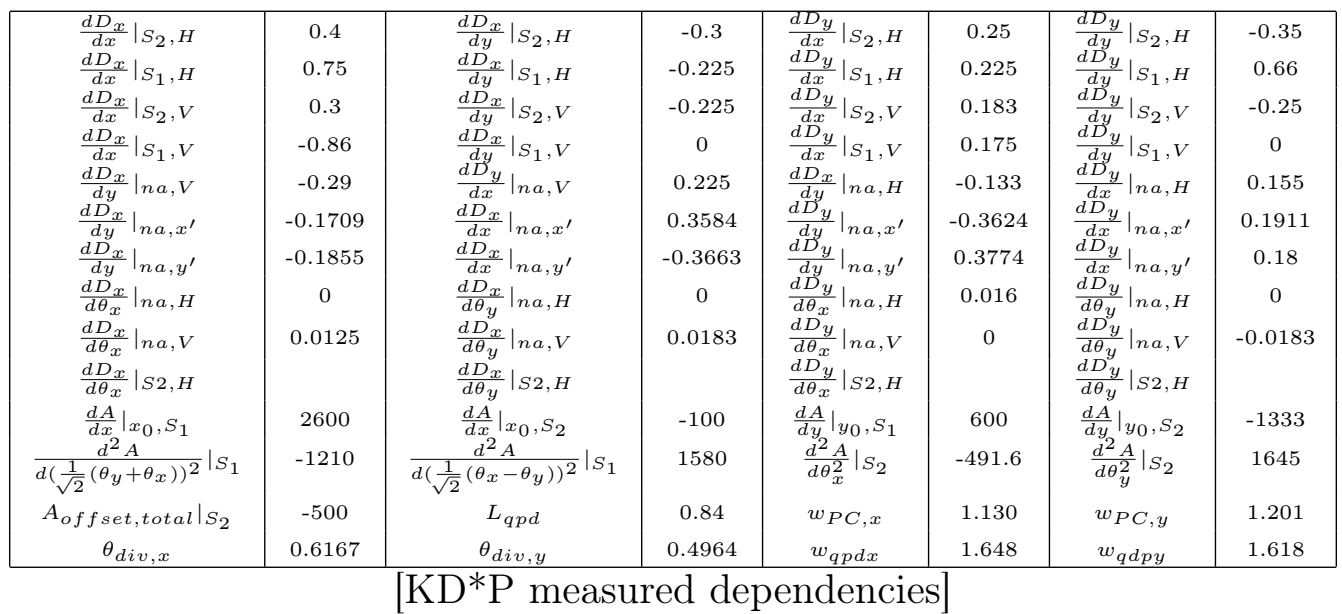

Table D.1: Data from runs: 2841, 2838, 2850, 2848, 2847 and 2826, 2827, 2828, 2860, 2861, 2857, 2841, 2859 and 2857, 2857 elog714, elog714 (in order of each row)

In converting measurables to the characterization parameters, we can average $\mathrm{H}$ and $\mathrm{V}$ polarization measurements and use the following equations to aid us: 
$\left.\frac{d D_{x}}{d x}\right|_{S_{2}}=\left.\frac{d D_{x}}{d x}\right|_{S_{2}}($ position-like $)+\left.\frac{d D_{x}}{d x}\right|_{S_{2}}($ angle-like $)=\left.\frac{w_{P C} w_{q p d}}{2} \frac{d^{2} A}{d x^{2}}\right|_{S_{2}}+\left.L_{q p d} \beta \frac{d^{2}\left(n_{x^{\prime}} L\right)}{d x^{2}}\right|_{S_{2}}$ $\left.\frac{d D_{x}}{d y}\right|_{S_{2}}=\left.\frac{d D_{x}}{d y}\right|_{S_{2}}($ position-like $)+\left.\frac{d D_{x}}{d y}\right|_{S_{2}}($ angle-like $)=\left.\frac{w_{P C} w_{q p d}}{2} \frac{d^{2} A}{d y d x}\right|_{S_{2}}+\left.L_{q p d} \beta \frac{d^{2}\left(n_{x^{\prime}} L\right)}{d y d x}\right|_{S_{2}}$ $\left.\frac{d D_{x}}{d x}\right|_{S_{1}}=\left.\frac{d D_{x}}{d x}\right|_{S_{1}}$ (position-like) $+\left.\frac{d D_{x}}{d x}\right|_{S_{1}}$ (angle-like) $=\left.\frac{w_{P C} w_{q p d}}{2} \frac{d^{2} A}{d x^{2}}\right|_{S_{1}}$ $\left.\frac{d D_{x}}{d y}\right|_{S_{1}}=\left.\frac{d D_{x}}{d y}\right|_{S_{1}}\left(\right.$ position-like) $+\left.\frac{d D_{x}}{d y}\right|_{S_{1}}($ angle-like $)=\left.\frac{w_{P C} w_{q p d}}{2} \frac{d^{2} A}{d y d x}\right|_{S_{1}}+\left.L_{q p d} \frac{d \theta_{\text {steering }, x}}{d y}\right|_{S_{1}}$ $\left.\frac{d D_{x}}{d y}\right|_{n o-a n a l, H p o l}=\left.\frac{d D_{x}}{d y}\right|_{n o-a n a l, H p o l}($ angle-like $)=\left.L_{q p d} \frac{d \theta_{\text {steering, } x}}{d y}\right|_{n o-a n a l, H p o l}$ $\left.\frac{d D_{x}}{d y}\right|_{n o-a n a l, x^{\prime} p o l}=\left.\frac{d D_{x}}{d y}\right|_{n o-a n a l, x^{\prime} p o l}($ angle-like $)=\left.L_{q p d} \beta \frac{d^{2}\left(n_{x^{\prime}} L\right)}{d y d x}\right|_{n o-a n a l, x^{\prime} p o l}$ $\left.\frac{d D_{x}}{d x}\right|_{n o-a n a l, x^{\prime} p o l}=\left.\frac{d D_{x}}{d x}\right|_{n o-a n a l, x^{\prime} p o l}($ angle-like $)=\left.L_{q p d} \beta \frac{d^{2}\left(n_{x^{\prime}} L\right)}{d x^{2}}\right|_{n o-a n a l, x^{\prime} p o l}$ $\left.\frac{d D_{x}}{d y}\right|_{n o-a n a l, y^{\prime} p o l}=\left.\frac{d D_{x}}{d y}\right|_{n o-a n a l, y^{\prime} p o l}($ angle-like $)=\left.L_{q p d} \beta \frac{d^{2}\left(n_{y^{\prime}} L\right)}{d y d x}\right|_{n o-a n a l, y^{\prime} p o l}$ $\left.\frac{d D_{x}}{d x}\right|_{n o-a n a l, y^{\prime} p o l}=\left.\frac{d D_{x}}{d x}\right|_{n o-a n a l, y^{\prime} p o l}($ angle-like $)=\left.L_{q p d} \beta \frac{d^{2}\left(n_{y^{\prime}} L\right)}{d x^{2}}\right|_{n o-a n a l, y^{\prime} p o l}$

\begin{tabular}{|c|c|c|c|c|c|c|c|}
\hline $\begin{array}{c}\frac{d \theta_{s t, y}}{d x} \\
\frac{d \theta_{s t, y}}{d \theta_{x}} \\
\left.\frac{d A}{d x}\right|_{x_{0}, S_{1}} \\
\left.\frac{d^{2} A}{d y^{2}}\right|_{y_{0}, S_{1}} \\
\left.\frac{d^{2} A}{d y d x}\right|_{x_{0}, y_{0}, S_{2}} \\
\left.\frac{d^{2} A}{d \theta_{y} d \theta_{x}}\right|_{\theta_{0}} \\
\beta \frac{d^{2}\left(n_{x^{\prime}} L\right)}{d x^{2}} \\
\beta \frac{d^{2}\left(n_{y^{\prime}} L\right)}{d x^{2}}\end{array}$ & $\begin{array}{c}0.226 \frac{\mathrm{urad}}{\mathrm{mm}} \\
9.52 \frac{\mathrm{nrad}}{\mathrm{mrad}} \\
1284 \frac{\mathrm{ppm}}{\mathrm{mm}} \\
340 \frac{\mathrm{ppm}}{\mathrm{mm}} \\
-88 \frac{\mathrm{ppm}}{\mathrm{mm}^{2}} \\
-1395 \frac{\mathrm{ppm}}{\mathrm{mrad}} \\
0.4266 \frac{\mathrm{urad}}{\mathrm{mm}} \\
-0.4361 \frac{\mathrm{urad}}{\mathrm{mm}}\end{array}$ & $\begin{array}{c}\frac{d \theta_{s t, x}}{d y} \\
\frac{d \theta_{s t, x}}{d \theta_{y}} \\
\left.\frac{d^{2} A}{d x^{2}}\right|_{x_{0}, S_{1}} \\
\left.\frac{d^{2} A}{d x d y}\right|_{x_{0}, y_{0}, S_{1}} \\
\left.\frac{d A}{d y}\right|_{y_{0}, S_{2}} \\
\left.\frac{d^{2} A}{d \theta_{x} d \theta_{y}}\right|_{\theta_{0}} \\
\beta \frac{d^{2}\left(n_{x^{\prime}} L\right)}{d y^{2}} \\
\beta \frac{d^{2}\left(n_{y^{\prime}} L\right)}{d y^{2}}\end{array}$ & $\begin{array}{c}-0.252 \frac{\mathrm{urad}}{\mathrm{mm}} \\
-10.89 \frac{\mathrm{nrad}}{\mathrm{mrad}} \\
-60 \frac{\mathrm{ppm}}{\mathrm{mm} \mathrm{m}^{2}} \\
104 \frac{\mathrm{ppm}}{\mathrm{mm}} \\
-147.7 \frac{\mathrm{ppm}}{\mathrm{mm}} \\
-1395 \frac{\mathrm{ppm}}{\mathrm{mrad}} \\
-0.4341 \frac{\mathrm{urad}}{\mathrm{mm}} \\
0.4492 \frac{\mathrm{urad}}{\mathrm{mm}}\end{array}$ & $\begin{array}{c}\frac{d \theta_{s t, x}}{d \theta_{x}} \\
\frac{d \theta_{s t, x}}{d \theta_{x}} \\
\left.\frac{d^{2} A}{d y d x}\right|_{x_{0}, y_{0}, S_{1}} \\
\left.\frac{d A}{d x}\right|_{x_{0}, S_{2}} \\
\left.\frac{d^{2} A}{d y^{2}}\right|_{y_{0}, S_{2}} \\
\left.\frac{d^{2} A}{d \theta_{x}^{2}}\right|_{\theta_{0}} \\
\beta \frac{d^{2}\left(n_{x^{\prime}} L\right)}{d x d y} \\
\beta \frac{d^{2}\left(n_{y^{\prime}} L\right)}{d x d y}\end{array}$ & $\begin{array}{c}7.44 \frac{\mathrm{nrad}}{\mathrm{mrad}} \\
0 \\
15 \frac{\mathrm{ppm}}{\mathrm{mm}} \\
-167.2 \frac{\mathrm{ppm}}{\mathrm{mm}} \\
72 \frac{\mathrm{ppm}}{\mathrm{mm}} \\
-491.6 \frac{\mathrm{ppm}}{\mathrm{mrad}} \\
0.2275 \frac{\mathrm{urad}}{\mathrm{mm}} \\
0.2142 \frac{\mathrm{urad}}{\mathrm{mm}}\end{array}$ & $\begin{array}{c}\frac{d \theta_{s t, x}}{d \theta_{y}} \\
\frac{d \theta_{s t, y}}{d \theta_{y}} \\
\left.\frac{d A}{d y}\right|_{y_{0}, S_{1}} \\
\left.\frac{d^{2} A}{d x^{2}}\right|_{x_{0}, S_{2}} \\
\left.\frac{d^{2} A}{d x d y}\right|_{x_{0}, y_{0}, S_{2}} \\
\left.\frac{d^{2} A}{d \theta_{y}^{2}}\right|_{\theta_{0}} \\
\beta \frac{d^{2}\left(n_{x^{\prime}} L\right)}{d y d x} \\
\beta \frac{d^{2}\left(n_{y^{\prime}} L\right)}{d y d x}\end{array}$ & $\begin{array}{c}10.89 \frac{\mathrm{nrad}}{\mathrm{mrad}} \\
0 \\
261 \frac{\mathrm{ppm}}{\mathrm{mm}} \\
-13 \frac{\mathrm{ppm}}{\mathrm{mm}^{2}} \\
33 \frac{\mathrm{ppm}}{\mathrm{mm}^{2}} \\
1645 \frac{\mathrm{ppm}}{\mathrm{mrad}} \\
-0.2035 \frac{\mathrm{urad}}{\mathrm{mm}} \\
-0.2208 \frac{\mathrm{urad}}{\mathrm{mm}}\end{array}$ \\
\hline
\end{tabular}

Empirically,

$$
\left.\frac{d D_{x}}{d y}\right|_{n o-a n a l, H p o l}=\frac{1}{2}\left(\left.\frac{d D_{x}}{d y}\right|_{n o-a n a l, x^{\prime} p o l}+\left.\frac{d D_{x}}{d y}\right|_{n o-a n a l, y^{\prime} p o l}\right)
$$

implies

$$
\begin{aligned}
& \frac{d \theta_{\text {steering, } x}}{d y}=\beta\left(\frac{d^{2}\left(n_{x^{\prime}} L\right)}{d y d x}+\frac{d^{2}\left(n_{y^{\prime}} L\right)}{d y d x}\right) \\
& \frac{d \theta_{\text {steering,y }}}{d x}=\beta\left(\frac{d^{2}\left(n_{x^{\prime}} L\right)}{d x d y}+\frac{d^{2}\left(n_{y^{\prime}} L\right)}{d x d y}\right)
\end{aligned}
$$

And we know from experience,

$0=\frac{d \theta_{\text {steering }, x}}{d x}=\beta\left(\frac{d^{2}\left(n_{x^{\prime}} L\right)}{d x^{2}}+\frac{d^{2}\left(n_{y^{\prime}} L\right)}{d x^{2}}\right)=\frac{d \theta_{\text {steering,y }}}{d y}=\beta\left(\frac{d^{2}\left(n_{x^{\prime}} L\right)}{d y^{2}}+\frac{d^{2}\left(n_{y^{\prime}} L\right)}{d y^{2}}\right)$

which implies

$$
\frac{d^{2}\left(n_{x^{\prime}} L\right)}{d x^{2}}=-\frac{d^{2}\left(n_{y^{\prime}} L\right)}{d x^{2}}
$$




$$
\frac{d^{2}\left(n_{x^{\prime}} L\right)}{d y^{2}}=-\frac{d^{2}\left(n_{y^{\prime}} L\right)}{d y^{2}}
$$

Theoretically, for a $40 \mathrm{~mm} \mathrm{KD*P}$ cell, at $800 \mathrm{~nm}$ wavelength we'd predict(ref: elog 662) a value 10X larger than what we observe in the lab:

$$
\begin{gathered}
k=\frac{\epsilon}{T} \frac{\pi D}{\lambda} n_{o} \frac{\left(n_{o}^{2}-n_{e}^{2}\right)}{n e^{2}}=12,102 \frac{p p m}{m_{r a d}^{2}}=\frac{-1}{2} \frac{d^{2} A}{d \theta_{y} d \theta_{x}} \\
=\frac{-1}{2} \frac{d^{2} A}{d \theta_{x} d \theta_{y}}=\frac{-1}{2} \frac{d^{2} A}{d \theta_{x}^{2}}=\frac{1}{2} \frac{d^{2} A}{d \theta_{y}^{2}}
\end{gathered}
$$

By entering these values into our equations, we can predict 4 data points in the RHWP scans at $\mathrm{S} 1, \mathrm{~S} 2, \mathrm{~S} 1+90, \mathrm{~S} 2+90$. We are able to predict the $4 \theta$ terms to within a factor of 2 in the case of the UVa Pockels cell with a $100 \%$ analyzer (Gandalf $\mathrm{KD}^{*} \mathrm{P}$, elog )
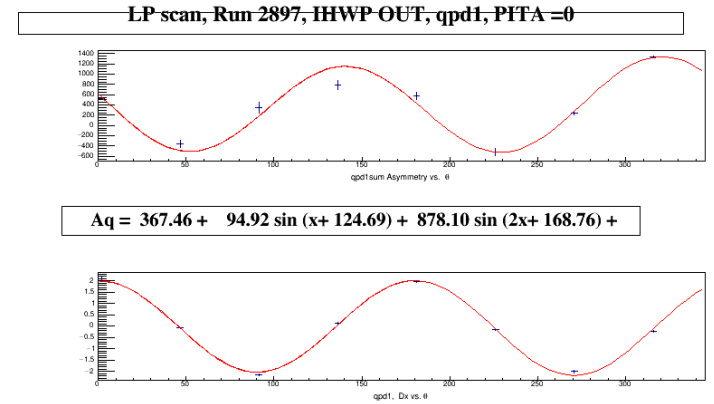

$D x=-0.05+-0.07 \sin (x+168.49)+2.05 \sin (2 x+88.83)+$

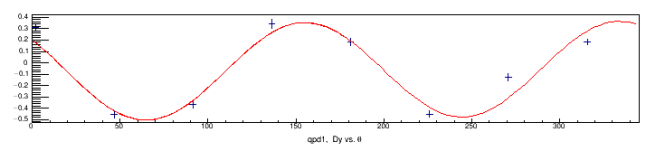

$\mathrm{Dy}=-0.07+\quad-0.02 \sin (\mathrm{x}+6.88)+\quad 0.42 \sin (2 \mathrm{x}+140.89)+$

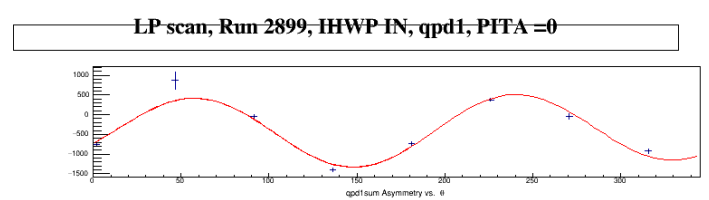

$A q=-389.59+100.38 \sin (x+149.15)+-850.60 \sin (2 x+152.06)+$

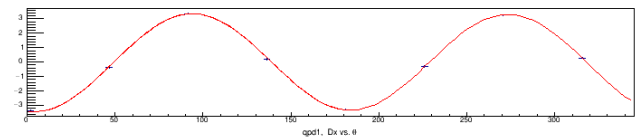

$D x=-0.02+-0.06 \sin (x+109.43)+-3.34 \sin (2 x+82.46)+$

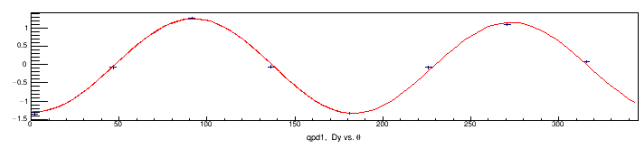

Dy $=-0.05+0.06 \sin (x+21.77)+-1.25 \sin (2 x+84.76)+$

Figure D-20: Rotation of the cube polarizer analyzer scan (equivalent to RHWP scan) for IHWP in and IHWP out. UVa KD*P Cell Gandalf. 


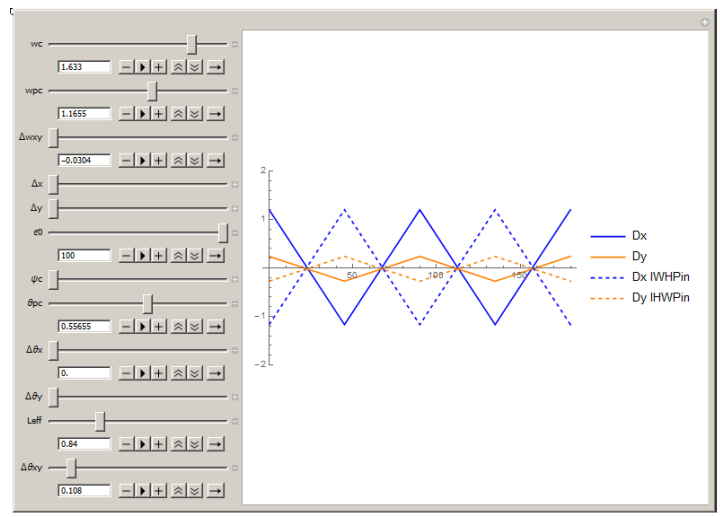

Figure D-21: Predictions of RHWP scan for UVa KD*P Cell Gandalf.

We can also attempt to predict JLab RHWP scans, though we don't know the focusing after the photocathode on the various BPMs in the beamline. However, examining the first bpm 0I01 after the photocathode a RHWP observed is shown from Haplog 3288, Run2219 and Run2220. This demonstrates that to within a factor 2 or so, we can estimate the behavior of the position differences in the injector with this model of the $\mathrm{KD}^{*} \mathrm{P}$ characterization.

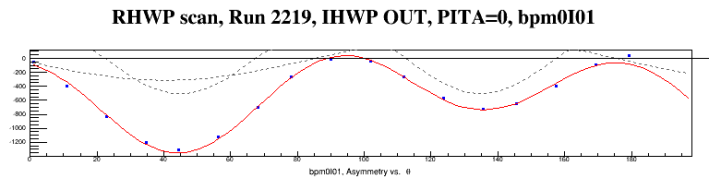

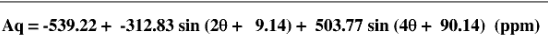

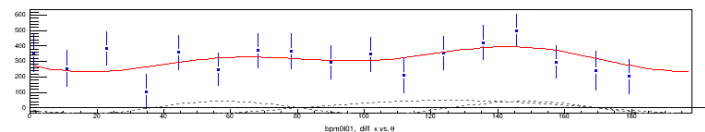

$\mathrm{Dx}=315.61+-49.00 \sin (2 \theta+19.69)+-42.72 \sin (4 \theta+37.24)(\mathrm{nm})$

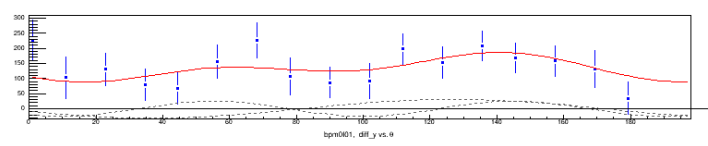

$\mathrm{Dy}=134.92+-31.01 \sin (2 \theta+16.23)+\cdot-25.29 \sin (4 \theta+49.20)(\mathrm{nm})$

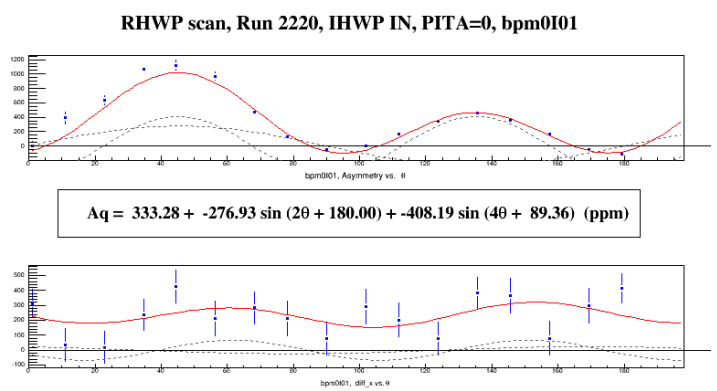

$D x=234.86+23.72 \sin (2 \theta+111.30)+-67.12 \sin (4 \theta+23.71)(n m)$

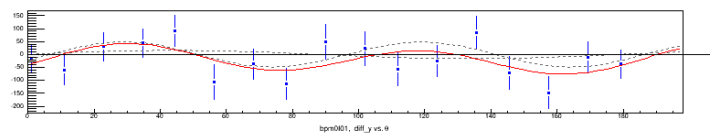

$\mathrm{Dy}=\cdot .19 .60+15.96 \sin (2 \theta+3.08)+-48.14 \sin (4 \theta+153.89)(\mathrm{nm})$

Figure D-22: RHWP scan for IHWP in and IHWP out. JLab KD*P cell.

\section{Spot-size Studies}

At UVa and at JLab, spot-size studies were performed which demonstrated the power of spot-size reduction in minimizing position differences. 
The studies at UVa were done with a well collimated $\leq 0.1-0.2 \mathrm{mrad}$ divergence beam, insertion of $1 \mathrm{~m}$ lens upstream of the cell, and insertion of a $-75 \mathrm{~cm}$ lens downstream of the cell before the quad-photodiode.

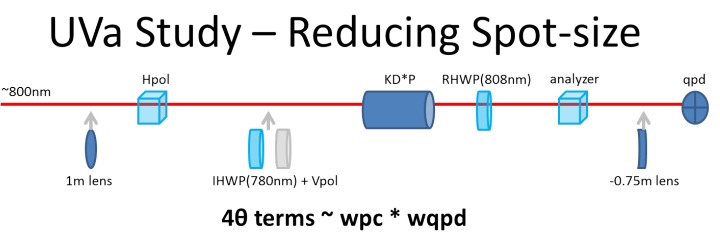

Figure D-23: UVa Spot Size Study Table Layout.

The UVa spot size studies (Lab26 elog 750) demonstrate the power of spot-size reduction in minimizing position differences. The $4 \theta$ terms in RHWP scans quantify the analyzing-like, position-like position differences, arising from asymmetry gradients. The table below of the UVa results show that the position differences are approximately proportional to the spot-size at the qpd and in the Pockels cell. In the upstream lens insertion, the divergence of the beam at the Pockels cell remained small so that divergence dependence played less of a role.

\begin{tabular}{|c|c|c|c|c|c|c|c|c|}
\hline & $\begin{array}{c}w_{P C x} \\
\mathrm{~mm}\end{array}$ & $\begin{array}{c}w_{P C y} \\
\mathrm{~mm}\end{array}$ & $\begin{array}{c}w_{q p d x} \\
\mathrm{~mm}\end{array}$ & $\begin{array}{c}w_{q p d x} \\
\mathrm{~mm}\end{array}$ & $\begin{array}{c}4 \theta \text { term Dx } \\
\mu \mathrm{m}\end{array}$ & $\begin{array}{c}4 \theta \text { term Dy } \\
\mu \mathrm{m}\end{array}$ & $\begin{array}{c}\text { Predicted Reduction } \\
\frac{w_{p c} w_{q p d}}{w_{p c 0} w_{q p d 0}}\end{array}$ & Actual Reduction \\
no lens & 1.35 & 1.46 & 1.43 & 1.58 & $1.3-1.5$ & $0.76-1.1$ & $1 \mathrm{X}$ & $1 \mathrm{X}$ \\
$1 \mathrm{~m}$ lens in & 0.324 & 0.341 & 0.886 & 0.891 & $2.2-3.1$ & $2.5-4.3$ & $\mathrm{~N} / \mathrm{A}$ & Not Realigned \\
$1 \mathrm{~m}$ lens in & 0.324 & 0.341 & 0.886 & 0.891 & $0.29-0.27$ & $0.24-0.09$ & $7 \mathrm{X}$ & $5.2 \mathrm{X}$ \\
$-75 \mathrm{~cm}$ lens in & 0.324 & 0.341 & 2.06 & 1.907 & $0.47-0.54$ & $0.33-0.36$ & $3 \mathrm{X}$ & $2.7 \mathrm{X}$ \\
\hline
\end{tabular}
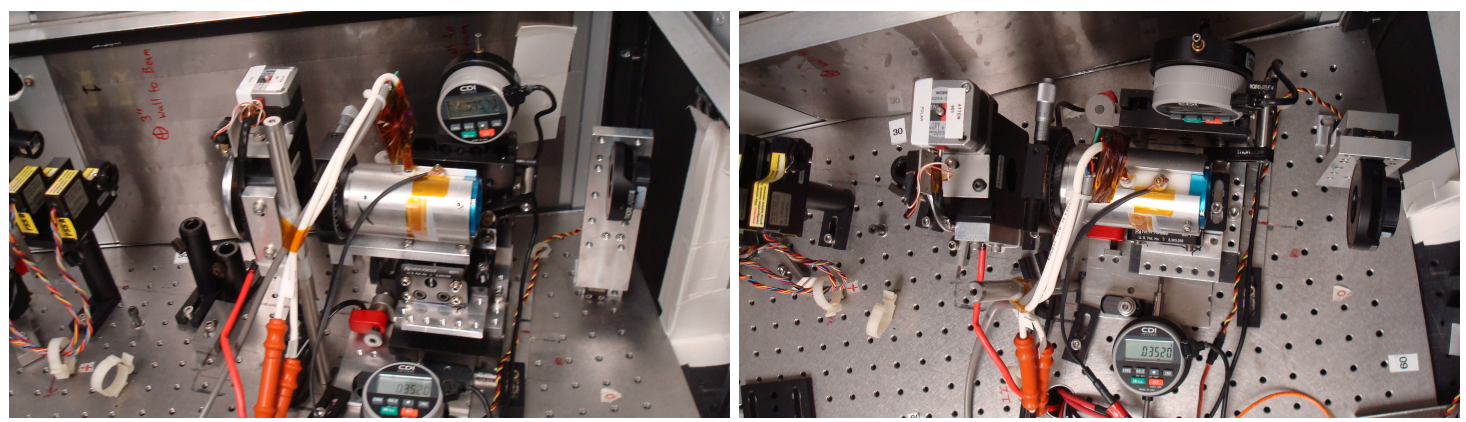

Figure D-24: JLab Pockels Cell. 
Spot-size studies were also performed at JLab by analogous insertion of a $1 \mathrm{~m}$ lens upstream and changing the downstream steering lens from $2 \mathrm{~m}$ focal length to $0.75 \mathrm{~m}$ focal length. It was demonstrated that reducing the spot-size on the cathode by a factor of $2 \mathrm{X}$, by changing the steering lens, reduced the position differences in the injector beamline by $\sim 2 \mathrm{X}$.

\section{Layout (Conceptual)}

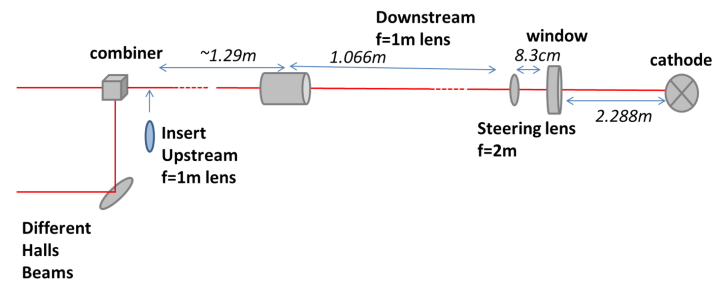

Figure D-25: JLab Spot Size Study Table Layout.

The results of the JLab spot-size studies are summarized in the following table:

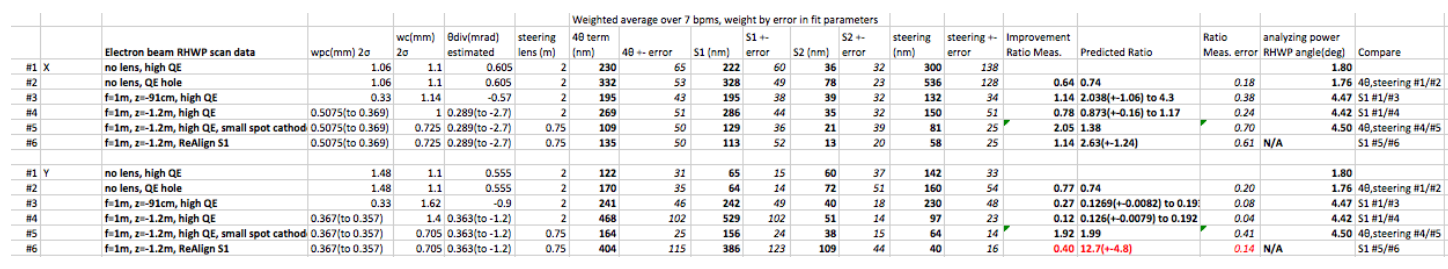

Figure D-26: JLab Spot Size Study Results.

There is a life-time cost to cathode spot-size reduction (Polog 3473865) as the smaller spot results in faster QE degradation. If one wishes to improve the cathode lifetime by expanding the beam at the cathode, the spot-size at the Pockels cell must be reduced to make up for it and keep the position differences small. Thus it was also important to explore spot-size reduction at the Pockels cell position, instead of at the cathode. While UVa studies definitively proved spot-size reduction at the Pockels cell reduces position differences, the JLab studies did not show such reduction (see table above). This was due to the divergence of the laser.

The JLab Hall A laser differed from the UVa laser in an important way: it was more divergent and it was astigmatic. In addition to being very large (2mm waist 
in $\mathrm{x}, 1.7 \mathrm{~mm}$ waist in $\mathrm{y}$ at the $\mathrm{PC}$ ), the Hall $\mathrm{A}$ laser divergence was measured to be $0.58 \mathrm{mrad}$ in $\mathrm{x}$ and $0.44 \mathrm{mrad}$ in $\mathrm{y}$ initially, which was $2-5 \mathrm{X}$ larger than the UVa laser (Polog 3473285). The $\mathrm{x} / \mathrm{y}$ divergences of the HallA laser differed and the laser was heavily astigmatic so that the divergence, especially along the y axis, was large at the crystal position when the lens was inserted. The laser was picked off and profiled, M-squared was and the astigmatism was measured with a $50 \mathrm{~cm}$ lens. The astigmatism was measured to be $42 \mathrm{~cm}$ coming out the the telescope by the PPLN crystal (Polog 3473180)

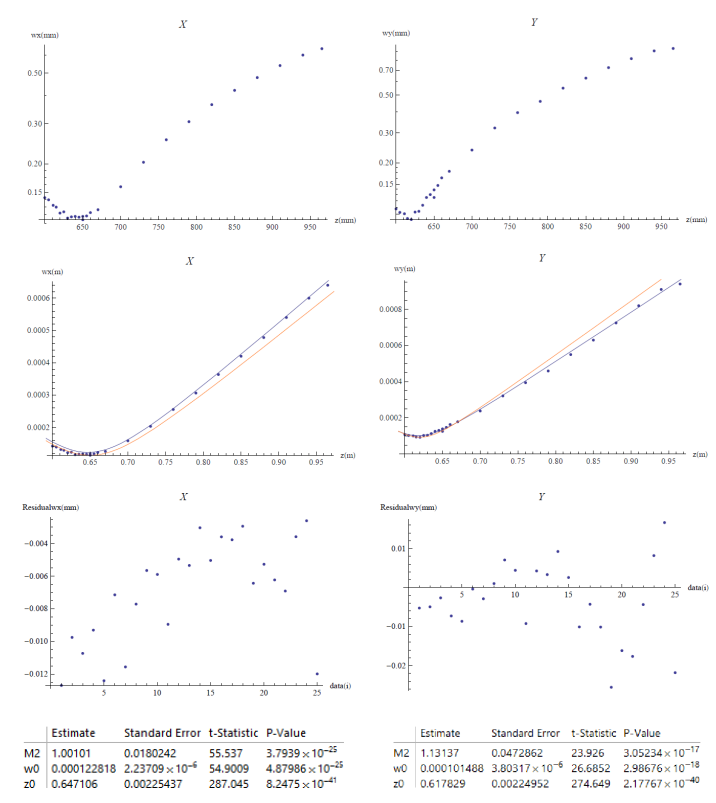

Figure D-27: M2 measurement of Hall A laser. Polog 3473100.

This JLab study demonstrates the very great need to have both small laser spot-size and small divergence at the Pockels in order to achieve small position differences and Parity Quality Beam. 


\section{Bibliography}

[1] The MOLLER Collaboration, "The MOLLER Experiment Measurement Of a Lepton Lepton Electroweak Reaction: An Ultra-precise Measurement of the Weak Mixing Angle using Moller Scattering - Pre-Conceptual Design Report", December 1, 2016.

[2] The MOLLER Collaboration, "The MOLLER Experiment Measurement Of a Lepton Lepton Electroweak Reaction: An Ultra-precise Measurement of the Weak Mixing Angle using Moller Scattering - An MIE proposal toThe United States Department of Energy", September 12, 2011.

[3] K. S. Kumar et al., "A precision measurement of the weak mixing angle in Møller scattering", SLAC-Proposal-E158 (1997)

[4] http://cnu-jefferson-lab.wikia.com/wiki/CEBAF

[5] A. Czarnecki and W. J. Marciano, Phys. Rev. D 53, 1066 (1996) [arXiv:hep$\mathrm{ph} / 9507420]$.

[6] A. Czarnecki and W. J. Marciano, Int. J. Mod. Phys. A 15, 2365 (2000) [arXiv:hep-ph/0003049].

[7] J. Erler and M. J. Ramsey-Musolf, Phys. Rev. D 72, 073003 (2005) [arXiv:hep-ph/0409169].

[8] A. Aleksejevs, S. Barkanova, Y. Kolomensky, E. Kuraev and V. Zykunov, Phys. Rev. D 85, 013007 (2012) [arXiv:1110.1750 [hep-ph]]. 
[9] A. G. Aleksejevs, S. G. Barkanova, Y. M. Bystritskiy, A. N. Ilyichev, E. A. Kuraev and V. A. Zykunov, Eur. Phys. J. C 72, 2249 (2012).

[10] A. G. Aleksejevs, S. G. Barkanova, V. A. Zykunov and E. A. Kuraev, Phys. Atom. Nucl. 76, 888 (2013) [Yad. Fiz. 76, 942 (2013)].

[11] The MOLLER Collaboration, "Uncertainties in the Theoretical Calculation of the Parity-Violating Asymmetry in Møller Scattering", MOLLER Collaboration report submitted to DOE, September 15, 2016:

[12] K. Kumar, S. Mantry. , W. Marciano, P. Sounder, Ann. Rev. Nucl. Part. Sci. 63(2013), 237-267.

[13] P.C. Rowson, Dong Su, and Stephane Willocq, "Highlights of the SLD physics program at the SLAC Linear Collider", Ann. Rev. Nucl. Part. Sci. 51: 345-412, 2001. (see references contained therein).

[14] The LEP Collaborations et al.,"A Combination of Preliminary Electroweak Measurements and Constraints on the Standard Model", Technical Report CERN-EP/2001-98, 2001 (hep-ex/0112021)

[15] The Jefferson Lab Qweak Collaboration, "Precision measurement of the weak charge of the proton", Nature volume 557, pages 207-211 (2018)

[16] SLAC E158 Collaboration: P. L. Anthony et al., "Precision Measurement of the Weak Mixing Angle in Møller Scattering", Phys. Rev. Lett. 95, 081601 $(2005)$

[17] S. C. Bennett and C. E. Wieman, "Measurement of the 6S-7S transition polarizablility in atomic cesium and an improved test of the standard model", Phys. Rev. Lett. 82, 2484 (1999) [Erratum-ibid. 83, 889 (1999)] [hep-ex/9903022]. 
[18] V. A. Dzuba, J. C. Berengut, V. V. Flambaum and B. Roberts, "Revisiting parity non-conservation in cesium", Phys. Rev. Lett. 109, 203003 (2012) [arXiv:1207.5864 [hep-ph]].

[19] Qweak Collaboration: D. Androic et al. , "First Determination of the Weak Charge of the Proton", Phys. Rev. Lett. 111, 141803 (2013) [arXiv:1307.5275 [nuclex]].

[20] H. Davoudiasl, H. -S. Lee and W. J. Marciano, " "Dark" Z implications for Parity Violation, Rare Meson Decays, and Higgs Physics", Phys. Rev. D 85, 115019 (2012) [arXiv:1203.2947 [hep-ph]].

[21] H. Davoudiasl, H. -S. Lee and W. J. Marciano, "Muon Anomaly and Dark Parity Violation", Phys. Rev. Lett. 109, 031802 (2012) [arXiv:1205.2709[hep$\mathrm{ph}] \mid$.

[22] A. Bodek, "Standard Model Precision Electroweak Measurements at HLLHC and Future Hadron Colliders", CIPANP2015-EW-Bodek, CMS CR2015/201 [arXiv:1510.02006v2 [hep-ex]]

[23] W. Chao, H. Li, M. J. Ramsey-Musolf, and S. Su, in preparation

[24] V. Cirigliano, A. Kurylov, M. J. Ramsey-Musolf and P. Vogel, "Lepton Flavor Violation without Supersymmetry", Phys. Rev. D 70, 075007 (2004) [arXiv:hep-ph/0404233].

[25] A. Kurylov, M. J. Ramsey-Musolf and S. Su, Phys. Rev. D 68, 035008 (2003) [arXiv:hep-ph/0303026].

[26] M. J. Ramsey-Musolf and S. Su, "Probing Supersymmetry with ParityViolating Electron Scattering", Phys. Rept. 456, 1 (2008) [arXiv:hep$\mathrm{ph} / 0612057]$. 
[27] J. Erler, P. Langacker, S. Munir and E. Rojas, "Z' Bosons from E6:Collider and Electroweak Constraints", 2011 [arXiv:1108.0685v1 [hep-ph]].

[28] ATLAS Collaboration, "New ATLAS measurement of the Higgs Boson mass", Physics Briefing, 6th July 2017

[29] C.Y. Prescott et. al., "Parity Non-Conservation in Inelastic Electron Scattering", Phys.Lett. B77 (1978) 347-352

[30] C.Y. Prescott et al., "Further Measurements of Parity Nonconservation in Inelastic Electron Scattering", Phys. Lett. B, 84:524-528, 1979.

[31] F.J. Hasert et al., "Observation of neutrino-like interactions without muon or electron in the Gargamelle neutrino experiment", Phys. Lett. 46B (1973) 138

[32] Griffiths, David J., Introduction to elementary particles; 2nd rev. version, New York, NY, Wiley, 2008

[33] J. Benesch et al., "An Ultra-precise Measurement of the Weak Mixing Angle using Møller Scattering", Proposal, 2008

[34] William R. Leo, "Techniques for Nuclear and Particle Physics Experiments: A How-to Approach", Berlin ; New York : Springer-Verlag, c1987.

[35] Erler et al., "Weak Polarized Electron Scattering", Ann. Rev. Nucl. Part. Sci., Vol. 64:269-298 (2014)

[36] E. Eichten, K. D. Lane and M. E. Peskin, "New Tests for Quark and Lepton Substructure", Phys. Rev. Lett. 50, 811 (1983).

[37] The ALEPH Collaboration et al., "Precision Electroweak Measurements on the Z Resonance", Phys. Rept. 427, 257 (2006) [arXiv:hep-ex/0509008]. 
[38] W. J. Marciano, "The weak mixing angle and new physics (a tale of two numbers)", AIP Conf. Proc. 870, 236 (2006).

[39] Y. Li, F. Petriello and S. Quackenbush, "Reconstructing a Z' Lagrangian using the LHC and low-energy data", Phys. Rev. D 80, 055018 (2009) [arXiv:0906.4132 [hep-ph]].

[40] F. Petriello, S. Quackenbush, Y. Li, private communication.

[41] H.Davoudiasl, H. Lee, and W. J. Marciano," "Dark" Z implications for parity violation, rare meson decays, and Higgs physics", Phys. Rev. D85 (2012) 115019

[42] H.Davoudiasl, H. Lee, and W. J. Marciano, "Muon Anomaly and Dark Parity Violation", Phys.Rev.Lett. 109 (2012) 031802

[43] H.Davoudiasl, H. Lee, and W. J. Marciano, "Muon g-2, rare kaon decays, and parity violation from dark bosons", Phys.Rev. D89 (2014) 9, 095006

[44] H.Davoudiasl, H. Lee, and W. J. Marciano, "Low $Q^{2}$ weak mixing angle measurements and rare Higgs decays",Phys.Rev. D92 (2015) 5, 055005

[45] M. Pitt, Overview of MOLLER Subsystems, DOE Science Review, Sep. 2014

[46] T. B. Humensky, "Probing the Standard Model and Nucleon Structure via Parity-Violating Electron Scattering", Ph. D. Thesis (2003)

[47] F. Halzen and A. Martin, Quarks and Leptons, John Wiley and Sons, New York, New York, 1984. See pp. 105-106, 126.

[48] E. Derman and W. J. Marciano, "Parity Violating Asymmetries in Polarized Electron Scattering", ANNALS OF PHYSICS 121, 147-180 (1979)

[49] A. Bodek et al., "Using Drell-Yan forward-backward asymmetry to reduce PDF uncertainties in the measurement of electroweak parameters", Eur.Phys.J. C76 (2016) no.3, 115 (2016-03-02) 
[50] BR Martin, Nuclear and Particle Physics: An Introduction, 2nd edition, Wiley, Mar 2009

[51] E. Noether, "Invariante Variationsprobleme", Nachr. v. d. Ges. d. Wiss. zu Gottingen, Mathematisch-Physikalische Klasse, pages S. 235-257, 1918.

[52] T.D. Lee and C.N. Yang, "Question of Parity Conservation in Weak Interactions", Phys. Rev., 1-4:254-258, 1956.

[53] C.S. Wu, E. Ambler, R.W. Hayward, D.D. Hoppes, and R.P. Hudson, "Experimental Test of Parity Conservation in Beta Decay", Phys. Rev., 105:1413-1415, 1957.

[54] Carlo Rubbia, Seminar announcing six candidate W events for UA1, January 20, 1983.

[55] M.Hauger, "A high-precision polarimeter", Nuclear Instruments and Methods in Physics Research Section A: Accelerators, Spectrometers, Detectors and Associated Equipment, Volume 462, Issue 3, 21 April 2001, Pages 382392

[56] E. Kargiantoulakis, "A Precision Test of the Standard Model via ParityViolating Electron Scattering in the Qweak Experiment", Ph.D. Thesis, University of Virginia (2015).

[57] Kent Paschke, "Looking for New Physics with the Weak Interaction: Recent Results from Qweak, and Future Perspectives", UVa Physics Colloquium, October 6, 2017.

[58] M. Cadeddu and F. Dordei, "Reinterpreting the weak mixing angle from atomic parity violation in view of the Cs neutron rms radius measurement from COHERENT", 2018. [arXiv:1808.10202 [hep-ph]] 
[59] J. Mammei, "MOLLER Spectrometer Update", Møller Collaboration Meeting, Oct. 8, 2018.

[60] D. Jones, "Moller Polarimetry Update", Moller Collaboration Meeting, Oct. 7, 2018 .

[61] Kent Paschke, "Compton Polarimetry Update", Moller Collaboration Meeting, Oct. 8, 2018.

[62] Kent Paschke, Status Update on "Parity Quality" Beam for MOLLER, Moller Collaboration Meeting, Oct. 8, 2018.

[63] O. O. Versolato, "Atomic Parity Violation in a single trapped radium ion", TCP 2010, Saariselka, April 12th, 2010.

[64] D. M. Meekhof, P. A. Vetter, P. K. Majumder, S. K. Lamoreaux, and E. N. Fortson, Phys. Rev. Lett. 71, 3442 (1993).

[65] S. J. Phipp, N. H. Edwards, P. E. G. Baird, and S. Nakayama, J. Phys. B 29, 1861 (1996).

[66] M. J. Ramsey-Musolf, "Low-energy parity-violation and new physics", Phys. Rev. C, 60(1):015501, 1999.

[67] S.G. Porsev, K. Beloy, and A. Derevianko, "Precision determination of electroweak coupling from atomic parity violation and implications for particle physics", Physical Review Letters, 102(18):181601, 2009.

[68] NuTeV Collaboration: G. P. Zeller et al., "A Precise Determination of Electroweak Parameters in Neutrino-Nucleon Scattering", Phys. Rev. Lett. 88, 091802 (2002) [Erratum-ibid. 90, 239902 (2003)] [arXiv:hep-ex/0110059].

[69] www.alternativephysics.org 
[70] Kent Paschke, "Measurements of Parity Violation in Electron Scattering", DNP 2016 Fall Meeting Vancouver, Bri.sh Columbia October 13, 2016.

[71] M. Pitt, "Overview of MOLLER Subsystems", DOE Nuclear Physics MOLLER Science Review, U. Mass. Amherst September 10, 2014

[72] Stanford Linear Accelerator Center, hrefhttps://phys.org/news/2005-07scientists-landmark-weak.htmlphys.org/news, "Scientists make landmark observations about weak force", July 13, 2005.

[73] Kent Paschke, "Recent Results in Parity-Violating Electron Scattering at Jefferson Lab: PREX and HAPPEX-III", APS Spring Meeting, Anaheim, CA, May 1(2011).

[74] T.Allison et al, "The Qweak experimental apparatus", Nuclear Instruments and Methods in Physics Research Section A: Accelerators, Spectrometers, Detectors and Associated Equipment Volume 781, 1 May 2015, Pages 105133.

[75] www.jlab.org/Hall-C/Qweak

[76] B. Frois et al and I. Sick, "High-Momentum-Transfer Electron Scattering from 208Pb", PRL Vol. 38 Num. 4, 24 Jan. 1977.

[77] C. J. Horowitz, S. J. Pollock, P. A. Souder and R. Michaels, "Parity-Violating Measurements of Neutron Densities", Phys. Rev. C 63, 025501 (2001). 5, 28

[78] T.W. Donnelly, J. Dubach, I. Sick, "Isospin Dependences in Parity-Violating Electron Scattering",Nuclear Physics A503 (1989) 589-631

[79] R. Silwal, "Probing the Strangeness Content of the Proton and the Neutron Radius of 208Pb using Parity-Violating Electron Scattering", Ph. D. Thesis, University of Virginia (2012). pg. 27-28. 
[80] G. A. Lalazissis, J. Konig, P. Ring, "A New Parameterization for the Lagrangian Density of Relativistic Mean Field Theory", Phys. Rev. C 55, 540 (1997) [arXiv:9607039 [nucl-th]].

[81] B. G. Todd-Rutel and J. Piekarewicz, "Neutron-Rich Nuclei and Neutron Stars: A New Accurately Calibrated Interaction for the Study of NeutronRich Matter", Phys. Rev. Lett. 95, 122501 (2005).

[82] M. Beiner, H. Flocard, N. van Giai, P. Quentin, "Nuclear ground-state properties and self-consistent calculations with the skyrme interaction: (I). Spherical description", Nucl. Phys. A 238, 29 (1975).

[83] E. Chabanat, P. Bonche, P. Haensel, J. Meyer, R. Schaefer, "A Skyrme parametrization from subnuclear to neutron star densities Part II. Nuclei far from stabilities", Nucl. Phys. A 635, 231 (1998).

[84] D. Vautherin, D. M. Brink, "Hartree-Fock Calculations with Skyrme's Interaction. I. Spherical Nuclei", Phys. Rev. C 5, 626 (1972).

[85] R. Silwal, Probing the Strangeness Content of the Proton and the Neutron Radius of 208Pb using Parity-Violating Electron Scattering, Ph. D. Thesis, University of Virginia (2012), pg. 203.

[86] R. Silwal, Probing the Strangeness Content of the Proton and the Neutron Radius of 208Pb using Parity-Violating Electron Scattering, Ph. D. Thesis, University of Virginia (2012), Section 3.14.3.

[87] Juan Carlos Cornejo, Compton Photon Detector for PREX2/CREX, PREX Collaboration Meeting, Summer 2018.

[88] Juan Carlos Cornejo, "Compton Scattering Polarimetry For The Determination Of The Proton'S Weak Charge Through Measurements Of The ParityViolating Asymmetry Of 1H(E,e')P", Ph.D. Thesis, College of William and Mary (2016). 
[89] R. Silwal, Probing the Strangeness Content of the Proton and the Neutron Radius of 208Pb using Parity-Violating Electron Scattering, Ph. D. Thesis, University of Virginia (2012), Section 4.9.

[90] R. Silwal, Probing the Strangeness Content of the Proton and the Neutron Radius of 208Pb using Parity-Violating Electron Scattering, Ph. D. Thesis, University of Virginia (2012), pg. 85.

[91] O. Glamazdin, Hall A Møller Polarimeter after Upgrade, June 10 (2010).

[92] R. Silwal, Probing the Strangeness Content of the Proton and the Neutron Radius of 208Pb using Parity-Violating Electron Scattering, Ph. D. Thesis, University of Virginia (2012), pg. 63-65.

[93] J. Napolitano, "Moller Polarimetry Status and Plans", PREX/CREX Collaboration Meeting Sunday 25 February 2018.

[94] R. Silwal, Probing the Strangeness Content of the Proton and the Neutron Radius of 208Pb using Parity-Violating Electron Scattering, Ph. D. Thesis, University of Virginia (2012), pg. 38.

[95] C. Palatchi, "Measurements of the Neutron Skin of 208Pb and 48Ca", JLab Hall A Collaboration Meeting, January 18, 2017. (reproduced from Don Jones' slide)

[96] D.W. Higinbotham, T. Keppel, 2017 Version: Jefferson Lab Hall A Standard Equipment Manual, November 2017

[97] P. Gueye, Status of the actual Beam Position Monitors in the Hall C Beamline, December, 1995.

[98] P. Zhu et al, Beam Position Reconstruction for the g2p Experiment in Hall A at Jefferson Lab Nuclear Instruments and Methods in Physics Research Sec- 
tion A: Accelerators, Spectrometers, Detectors and Associated Equipment, Volume 808, 1 February 2016, Pages 1-10.

[99] P. Forck, P. Kowina, and D. Liakin, Beam Position Monitor, CERN-2009005, p 187, 2009.

[100] C. Hyde-Wright, L. Todor, G. Laveissiere, Beam Position Studies for E93050, 1999.

[101] M.Spata, T. Allison, K. Cole, J. Musson, J. Yan, Evaluation and Correction of the Non-linear Distortion of Cebaf Beam Position Monitors, Proceedings of IPAC2011, San Sebastian, Spain, TUPC172, pg.1440-1442.

[102] A. Freyberger, M. Keesee, D. Napier, BPM Systems Training (Ops) Hardware and Software, 2005.

[103] W. Barry, A general analysis of thin wire pickups for high frequency beam position monitors, Nuclear Instruments and Methods in Physics Research Section A: Accelerators, Spectrometers, Detectors and Associated Equipment, Volume 301, Issue 3, 15 March 1991, Pages 407-416.

[104] M. Spata, "Application of Chebyshev Formalism to Identify Nonlinear Magnetic Field Components in Beam Transport Systems", Ph. D. Thesis, Old Dominion University (2012).

[105] John Musson, Private Communication, Feb. 1, 2018.

[106] T. Allison, B. Bevins, K. Cole, R. Flood, O.r Garza, J. Musson, D. Williams, S. Windham, Overview and Expected Performance of RF Beam Diagnostics, August 6, 2015.

[107] Kent Paschke, Private Communication, 2017

[108] E. Forman, Hall A song sheet, 2014. 
[109] Bob Michaels, Private Communication, Feb 2, 2018

[110] R. Michaels, "Beamline Instrumentation for Future Parity-Violation Experiments", Oct 16, 2014. [arXiv:1410.4514v1 [nucl-ex]]

[111] J. Benesch et al., "The MOLLER Experiment: Pre-Conceptual Design Report", December 1, 2016.

[112] S. J. Russell, J. D. Gilpatrick, J. E Power and R. B Shurter, Characterization of Beam Position Monitors for Measurement of Second Moment, In Proceedings of the 16th Particle Accelerator Conference Dallas, TX, 1995, pages $2580-2582$.

[113] J. Musson, J. Koros, Modified M20 Beam Position Monitor Testing, 2017 Fall Meeting of the APS Division of Nuclear Physics Poster, Oct. 2017.

[114] John Musson, Private Communication, Feb 21, 2018

[115] https://ace.phys.virginia.edu:80/HAPPEX/3525

[116] John Musson, Private Communication, July 25, 2018

[117] X. Roca-Maza, M. Centelles, X. Vinas, and M. Warda, "Neutron skin of $208 \mathrm{~Pb}$, nuclear symmetry energy, and the parity radius experiment", Phys. Rev. Lett. 106252501 (2011)

[118] B.A. Brown , "Neutron Radii in Nuclei and the Neutron Equation of State", PRL 85, 5296 (2000)

[119] S. Abrahamyan et al. (PREX Collaboration), "Measurement of the Neutron Radius of 208Pb through Parity Violation in Electron Scattering", Phys Rev Let. 108, 112502 (2012)

[120] C. J. Horowitz et al., "Weak charge form factor and radius of 208Pb through parity violation in electron scattering", Phys. Rev. C 85, 032501(R) (2012) 
[121] K. Kumar, P. A. Souder, R. Michaels, and G. M. Urciuoli, http://hallaweb.jlab.org/parity/prex (see section 'Status and Plans' for latest updates).

[122] C. J. Horowitz and J. Piekarewicz, "Neutron Star Structure and the Neutron Radius of 208Pb", PRL Vol. 86, Num. 25, 18 June (2001).

[123] F. J. Fattoyev, J. Piekarewicz, and C. J. Horowitz "Neutron skins and neutron stars in the multi-messenger era", December 18, (2017). [arXiv:1711.06615v2 [nucl-th]]

[124] F. J. Fattoyev and J. Piekarewicz, "Has a thick neutron skin in 208Pb been ruled out?", August 28, 2018. [arXiv:1306.6034v1 [nucl-th]]

[125] F. J. Fattoyev, C. J. Horowitz, J. Piekarewicz, and G. Shen, "Relativistic effective interaction for nuclei, giant resonances, and neutron stars", May 30 (2018). [arXiv:1008.3030v1 [nucl-th]]

[126] P. Ring et al., Nucl. Phys. A624, 349 (1997).

[127] C. Olmer et al., "Elastic And Inelastic Scattering Of 162-mev Pions By Si-28, Ni-58, And Pb-208", Phys. Rev. C 21, 254 (1980).

[128] S.J. Pollock, E.N. Fortson, and L. Wilets, "Atomic parity nonconservation: Electroweak parameters and nuclear structure", Phys. Rev. C 46, 2587(1992).

[129] S.J. Pollock and M.C. Welliver, "Effects of Neutron Spatial Distributions on Atomic Parity Nonconservation in Cesium", Phys. Lett. B 464, 177 (1999).

[130] J.A. Nolen, Jr. and J.P. Schiffer, Phys. Lett. 29B, 396 (1969); Annu. Rev. Nucl. Sci. 19, 471 (1969)

[131] L. Ray and G.W. Hoffmann, "Relativistic and nonrelativistic impulse approximation descriptions of 300-1000 MeV proton + nucleus elastic scattering", Phys. Rev. C 31, 538 (1985) 
[132] C.J. Horowitz, "Parity violating elastic electron scattering and Coulomb distortions", Phys. Rev. C 57, 3430 (1998)

[133] R. Silwal, Probing the Strangeness Content of the Proton and the Neutron Radius of 208Pb using Parity-Violating Electron Scattering, Ph. D. Thesis, University of Virginia (2012), pg. 249

[134] S. Riordan, "PREX-II/CREX Welcome and Overview", PREX/CREX Collaboration Meeting, July 25, 2018.

[135] B. Alex Brown, "New Skyrme interaction for normal and exotic nuclei", Phys. Rev. C 58, 220 (1998)

[136] C.J. Horowitz and B.D. Serot, Nucl. Phys. A368, 503 (1981)

[137] D. Vretenar, P. Finelli, A. Ventura, G.A. Lalazissis, and P. Ring, "Parity violating elastic electron scattering and neutron density distributions in the Relativistic Hartree-Bogoliubov model", Phys. Rev. C 61, 064307 (2000). [nucl-th/9911024]

[138] Relativistic optical code RUNT; E. D. Cooper (private communication)

[139] B.C. Clark (private communication)

[140] B. Mueller et al., "Measurement of the Proton's Neutral Weak Magnetic Form Factor", Phys. Rev. Lett. 78, 3824 (1997).

[141] K.A. Aniol et al., "Measurement of the neutral weak form factors of the proton", Phys. Rev. Lett. 82, 1096 (1999).

[142] B. Povh, "Hadronic radii", Nucl. Phys. A532, 133 (1991).

[143] S. Riordan, "208Pb and 48Ca Targets", ERR, May 2017.

[144] PREX/CREX Collaboration, PREX/CREX Design Document Version 3.0, May, 2017. 
[145] S. C. Dusa, "PREX2/CREX Target Chamber", ERR, May 2017.

[146] R. Silwal, Probing the Strangeness Content of the Proton and the Neutron Radius of 208Pb using Parity-Violating Electron Scattering, Ph. D. Thesis, University of Virginia (2012), pg. 87.

[147] C. Palatchi, "Measurements of the Neutron Skin of 208Pb and 48Ca", JLab Hall A Collaboration Meeting, January 18, 2017.

[148] James M. Lattimer and Andrew W. Steiner, "Constraints on the symmetry energy using the mass-radius relation of neutron stars", Eur. Phys. J. A (2014).

[149] J.M. Lattimer, Y. Lim, "Constraining the Symmetry Parameters of the Nuclear Interaction", Astrophys. J. 771, 51 (2013).

[150] S. Gandolfi, J. Carlson, S. Reddy, Phys. Rev. C 85, 032801(2012).

[151] K. Hebeler, J.M. Lattimer, C.J. Pethick, A. Schwenk, Phys. Rev. Lett. 105, $161102(2010)$.

[152] R. Silwal, Probing the Strangeness Content of the Proton and the Neutron Radius of 208Pb using Parity-Violating Electron Scattering, Ph. D. Thesis, University of Virginia (2012), pg. 69

[153] T. Kutz,"Collimator design and error estimate", PREX/CREX collaboration meeting July 25, 2018.

[154] J. Mammei, "Septum Magnet", PREX/CREX Experimental Readiness Review, May 17, 2017.

[155] A. Gavalya, Septum Beam Pipe Drawing, 2018.

[156] R. Silwal, Probing the Strangeness Content of the Proton and the Neutron Radius of 208Pb using Parity-Violating Electron Scattering, Ph. D. Thesis, University of Virginia (2012), pg. 73 
[157] https://ace.phys.virginia.edu:80/HAPPEX/3444

[158] https://ace.phys.virginia.edu:80/HAPPEX/3532

[159] K. Paschke, "Overview of PREX/CREX Experiments", PREX/CREX Collaboration Meeting, February 25, 2018.

[160] R. Silwal, Probing the Strangeness Content of the Proton and the Neutron Radius of 208Pb using Parity-Violating Electron Scattering, Ph. D. Thesis, University of Virginia (2012), pg. 74

[161] R. Silwal, Probing the Strangeness Content of the Proton and the Neutron Radius of 208Pb using Parity-Violating Electron Scattering, Ph. D. Thesis, University of Virginia (2012), pg. 246

[162] R. Silwal, Probing the Strangeness Content of the Proton and the Neutron Radius of 208Pb using Parity-Violating Electron Scattering, Ph. D. Thesis, University of Virginia (2012), Fig. 6.7.5

[163] R. Silwal, Probing the Strangeness Content of the Proton and the Neutron Radius of 208Pb using Parity-Violating Electron Scattering, Ph. D. Thesis, University of Virginia (2012).

[164] K. Paschke, K. Kumar, et al, "Proposal to Jefferson Lab PAC 38 PREXII: PRECISION PARITY-VIOLATING MEASUREMENT OF THE NEUTRON SKIN OF LEAD".

[165] C. Garcia-Recio, J. Nieves and E. Oset, Nucl. Phys. A 547, 473 (1992).

[166] L. Ray, W. R. Coker, G. W. Hoffmann, Phys. Rev. C 18, 2641 (1978).

[167] V. E. Starodubsky, N. M. Hintz, Phys. Rev. C 49, 2118 (1994).

[168] B. C. Clark, L. J. Kerr, S. Hama, Phys. Rev. C 67, 054605 (2003).

[169] A. Trzcinska et al., Phys. Rev. Lett. 87 , 082501 (2001). 
[170] H. Lenske, Hyperfine Interact (2009) 194: 277. [https://doi.org/10.1007/s10751-009-0079-5]

[171] R. Silwal, Probing the Strangeness Content of the Proton and the Neutron Radius of 208Pb using Parity-Violating Electron Scattering, Ph. D. Thesis, University of Virginia (2012), pg. 280

[172] X. Roca-Maza, M. Brenna, G. Colo, M. Centelles, X. Vinas et al., Phys. Rev. C 88, 024316 (2013).

[173] A. Tamii, I. Poltoratska, P. von Neumann-Cosel, Y. Fujita, T. Adachi et al., Phys. Rev. Lett. 107, 062502 (2011).

[174] P. Danielewicz, J. Lee, Nucl. Phys. A 922, 1 (2014).

[175] L. Trippa, G. Colo, E. Vigezzi, Phys. Rev. C 77, 061304 (2008).

[176] M.B. Tsang, Y. Zhang, P. Danielewicz, M. Famiano, Z. Li,W.G. Lynch, A.W. Steiner, Phys. Rev. Lett. 102, 122701 (2009).

[177] L.-W. Chen, C.M. Ko, B.-A. Li, J. Xu, Phys. Rev. C 82, 024321 (2010).

[178] C. Gal, "ERR variance", PREX/CREX Collaboration Meeting, July 2018.

[179] K. Paschke, "PREX/CREX Radiation Shielding", PREX/CREX Experimental Readiness Review, May 17, 2017.

[180] https://ace.phys.virginia.edu:80/HAPPEX/3487

[181] R. Silwal, Probing the Strangeness Content of the Proton and the Neutron Radius of 208Pb using Parity-Violating Electron Scattering, Ph. D. Thesis, University of Virginia (2012), pg. 283

[182] https://ace.phys.virginia.edu:80/HAPPEX/3259

[183] C. Palatchi "Parity Beam Studies", Hall A weekly meeting, June 28, 2016. 
[184] J. Musson, "A Functional Description of Algorithms Used in Digital Receivers", Old Dominion University, ECE 652.

[185] John Musson, Functional Description of Algorithms Used in Digital Receivers, private communication

[186] A. Steiner, "The Equation of State of Dense Matter and Neutron Star Obervations", NNPSS Lectures, 2016.

[187] Korobkin O., Rosswog S., Arcones A., Winteler C., "On the astrophysical robustness of the neutron star merger r-process", Monthly Notices of the Royal Astronomical Society, Volume 426, Issue 3, 1 November 2012, Pages 1940-1949 (2012)

[188] Jonas Lippuner, http://jonaslippuner.com/research/skynet/

[189] F. Foucart, "From Chirps to Jets: The extreme world of Black Holes and Neutron Stars", University of Virginia, March 1st 2017.

[190] Del'Pozzo et al., "Demonstrating the Feasibility of Probing the Neutron-Star Equation of State with Second-Generation Gravitational-Wave Detectors", Phys. Rev. Lett. 111, 071101 (2013)

[191] B. Lackey, L. Wade. PRD 91, 043002 (2015)

[192] Lackey et al., PRD 89, 043009 (2014)

[193] T. Hinderer, B. D. Lackey, R. N. Lang, and J. S. Read, Phys. Rev. D81, $123016(2010)$.

[194] R. Fernandez et al., "Dynamics, nucleosynthesis, and kilonova signature of black hole neutron star merger ejecta", Classical and Quantum Gravity, Volume 34, Number 15 (2017).

[195] J. Lippuner and L. Roberts, Abundances from SkyNet 
[196] John Musson, Functional Description of Algorithms Used in Digital Receivers

[197] Oechslin et al.,"Relativistic neutron star merger simulations with non-zero temperature equations of state I. Variation of binary parameters and equation of state", A\&A Volume 467, Number 2, May IV (2007).

[198] Roberts et al., "Electromagnetic transients powered by nuclear decay in the tidal tails of coalescing compact binaries", The Astrophysical Journal Letters, Volume 736, Number 1 (2011).

[199] Michael Tiefenback, JLab CLAS Collaboration Meeting, Feb 23-26 2016.

[200] R. Silwal, Probing the Strangeness Content of the Proton and the Neutron Radius of 208Pb using Parity-Violating Electron Scattering, Ph. D. Thesis, University of Virginia (2012), pg. 110

[201] D. Price and S. Rosswog, "Producing ultra-strong magnetic fields in neutron star mergers", Science 2006.

[202] A.L. Bowley, The Standard Deviation of the Correlation Coefficient, Journal of the American Statistical Association Vol. 23, No. 161 (Mar., 1928), pp. $31-34$

[203] A. Esser and colleagues, "Beam Normal Single Spin Asymmetry Measurement", September 21, 2017 (private communication).

[204] A. Kabir, "Determination of the Charge Radii of Several Light Nuclei From Precision, High-Energy Electron Elastic Scattering", Ph. D. Thesis, Kent State University (2015)

[205] E. A. J. M. Offermann et al., "Energy dependence of the form factor for elastic electron scattering from 12C", Phys. Rev. C 44, 1096 (1991) 
[206] Mikhail Gorchtein and Charles J. Horowitz, "Analyzing power in elastic scattering of the electrons off a spin-0 target", Phys. Rev. C 77, 044606 (2008)

[207] D. B. Rios, "Measurement of the two photon exchange asymmetry in the quasielastic scattering of transversely polarized electrons off unpolarized deuterium", Ph.D. Thesis, Institut fur Kernphysik Johannes GutenbergUniversitat Mainz (2008)

[208] A. Esser et al., "First Measurement of the Q2 Dependence of the BeamNormal Single Spin Asymmetry for Elastic Scattering off Carbon", Phys. Rev. Lett. 121, 022503 (2018).

[209] S. Abrahamyan et al. (HAPPEX, PREX), Phys. Rev. 340 Lett. 109, 192501 (2012) [arXiv:1208.6164 [nucl-ex]].

[210] M. Gorchtein, C. J. Horowitz, Phys. Rev. C77, 044606 (2008).

[211] A. V. Afanasev, S. J. Brodsky, C. E. Carlson, Y. -C. Chen, M. Vanderhaeghen, Phys. Rev. D72, 013008 (2005).

[212] E. D. Cooper, C. J. Horowitz, Phys. Rev. C72, 034602 (2005).

[213] http://wwwa1.kph.uni-mainz.de/A1/

[214] K. I. Blomqvist et al., Nucl. Instrum. Meth. A403, 263 (1998)

[215] B.S. Schlimme et al, "Vertical Beam Polarization at MAMI", Nuclear Instruments and Methods in Physics Research A 850 54-60 (2017)

[216] K. Aulenbacher et al., "The MAMI source of polarized electrons" ,Nucl. Instrum. Meth. A391, 498 (1997).

[217] K. Aulenbacher, Eur. Phys. J. ST 198, 361 (2011). 
[218] F. E. Maas et al. (A4), Phys. Rev. Lett. 93, 022002 (2004) [arXiv:nuclex/0401019 [nucl-ex]]

[219] http://www.netzfrequenzmessung.de/

[220] A. Esser, private communication (9/2016)

[221] M. Dehn, H. Euteneuer, A. Jankowiak, Longitudinal Beam Dynamics In The HDSM at MAMI, Proceedings of PAC09, Vancouver, BC, Canada, TH5RFP063

[222] K.Blomqvist et al, "The three-spectrometer facility at the Mainz microtron MAMI", Nuclear Instruments and Methods in Physics Research Section A: Accelerators, Spectrometers, Detectors and Associated Equipment Volume 403, Issues 2-3, Pages 263-30, 1 February 1998.

[223] M. Seidl, "Untersuchungen zur Strahldynamik am Minzer Mikrotron", Ph.D. Thesis, Johannes Gutenberg-Universitat in Mainz (2003).

[224] https://ace.phys.virginia.edu:80/A1parity/50

[225] https://ace.phys.virginia.edu:80/A1parity/78

[226] https://ace.phys.virginia.edu:80/A1parity/114

[227] A. Esser, Recalibration procedure, Internal Document, (March 2018)

[228] http://www.cristal-laser.com/en/rtp-q-switch.html

[229] R. Silwal, Probing the Strangeness Content of the Proton and the Neutron Radius of 208Pb using Parity-Violating Electron Scattering, Ph. D. Thesis, University of Virginia (2012), pg. 113

[230] A. Brachmann, "SLAC's polarized electron source laser system for the E-158 parity violation experiment", SLAC PUB 9145, March 2002. 
[231] https://ace.phys.virginia.edu:80/Lab26/841

[232] https://ace.phys.virginia.edu:80/Lab26/798

[233] https://ace.phys.virginia.edu:80/Lab26/804

[234] https://ace.phys.virginia.edu:80/Lab26/493

[235] https://ace.phys.virginia.edu:80/Lab26/508

[236] hhttp://www.redoptronics.com/RTP-crystal.html (March 2010).

[237] H. Albrecht, C. Bonnin, Y. Gromfeld, M. A. Herrmann, "Characterization of RbTiOPO4 crystal for non-linear and electro-optic applications", Proc. SPIE 6100, Solid State Lasers XV: Technology and Devices, 61001F (28 February 2006)

[238] M. Roth, "Ferroelectric phase transition temperatures of self-flux-grown RbTiOPO4 crystals", Optical Materials 26(4):465-470 (September 2004).

[239] M Roth, E Samoka, E Mojaev, M Tseitlin, "RTP Crystals For Electro-Optic Q-Switching"

[240] C. Palatchi, Lab book, July 2017-2018, pg. 46

[241] https://ace.phys.virginia.edu:80/Lab26/861

[242] https://logbooks.jlab.org/entry/3581096 Run3934

[243] https://ace.phys.virginia.edu:80/Lab26/860

[244] https://ace.phys.virginia.edu:80/Lab26/858

[245] https://ace.phys.virginia.edu:80/Lab26/851

[246] Raicol Catalog

[247] https://ace.phys.virginia.edu:80/Lab26/514 
[248] https://ace.phys.virginia.edu:80/Lab26/860

[249] Chris Mueller, The Electro-Optic Effect: Reading Notes, Dept. of Physics, University of Florida, 27 March, 2010

[250] Olivier Gobert, Linear Electro Optic Effect for High Repetition Rate Carrier Envelope Phase Control of Ultra Short Laser Pulses, Appl. Sci. 2013, 3, 168188; doi:10.3390/app3010168

[251] K.D. Paschke, "Controlling helicity-correlated beam asymmetries in a polarized electron source", Eur. Phys. J. A 32, 549-553 (2007).

[252] https://en.wikipedia.org/wiki/Anti-reflective-coating

[253] http://raicol.com/rtp/rtp-for-eo

[254] https://ace.phys.virginia.edu:80/Lab26/855

[255] https://logbooks.jlab.org/entry/3641908

[256] https://logbooks.jlab.org/entry/3482486

[257] https://logbooks.jlab.org/entry/3584281

[258] https://logbooks.jlab.org/entry/3646394

[259] R. Silwal, Probing the Strangeness Content of the Proton and the Neutron Radius of 208Pb using Parity-Violating Electron Scattering, Ph. D. Thesis, University of Virginia (2012), pg. 304-305.

[260] R. Silwal, Probing the Strangeness Content of the Proton and the Neutron Radius of 208Pb using Parity-Violating Electron Scattering, Ph. D. Thesis, University of Virginia (2012), pg. 308-310.

[261] https://logbooks.jlab.org/entry/3641295

[262] https://logbooks.jlab.org/entry/3642279 
[263] Roger Flood, Scott Higgins, and Riad Suleiman, HelicityControlBoard User's Guide, February 4, 2010.

[264] https://ace.phys.virginia.edu:80/Lab26/448 https://ace.phys.virginia.edu:80/Lab26/459

[265] https://ace.phys.virginia.edu:80/Lab26/508 785WOPK04.C05

[266] https://ace.phys.virginia.edu:80/Lab26/846

[267] https://ace.phys.virginia.edu:80/Lab26/847

[268] Yutsis, "Temperature dependent dispersion relations for RTP and RTA", App Phys B (2004).

[269] https://logbooks.jlab.org/entry/3581049

[270] R. Silwal, Probing the Strangeness Content of the Proton and the Neutron Radius of 208Pb using Parity-Violating Electron Scattering, Ph. D. Thesis, University of Virginia (2012), pg. 110.

[271] Matsui et al., "Parallel and antiparallel angular momentum transfer of circularly polarized light to photoelectrons and Auger electrons at the NiL3 absorption threshold", Phys. Rev. B 97, 035424, January 18, 2018

[272] https://logbooks.jlab.org/entry/3645813

[273] https://ace.phys.virginia.edu:80/Lab26/782

[274] https://ace.phys.virginia.edu:80/MOLLER/3

[275] https://ace.phys.virginia.edu:80/Lab26/423

[276] C. Palatchi and K. D. Paschke, to be submitted (2019)

[277] Beth Schaefer, Edward Collett, Robert Smyth, Daniel Barrett, and Beth Fraher, "Measuring the Stokes polarization parameters", American Journal of Physics 75, 163 (2007) 
[278] https://ace.phys.virginia.edu:80/Lab26/575

[279] Thorlabs Beam Displacers

[280] polog3482499 Run3248, Run3250 (08/26/2017)

[281] polog3482831

[282] https://ace.phys.virginia.edu:80/Lab26/474

[283] https://ace.phys.virginia.edu:80/Lab26/843

[284] https://ace.phys.virginia.edu:80/Lab26/626

[285] https://ace.phys.virginia.edu:80/Lab26/871

[286] https://ace.phys.virginia.edu:80/Lab26/870

[287] https://ace.phys.virginia.edu:80/Lab26/869

[288] M. Abarkan et al., "Frequency and wavelength dependences of electro-optic coefficients in inorganic crystals", Appl. Phys. B 76, 765-769 (2003)

[289] G. E. Peterson and P. M. Bridenbaugh, "Time Resolution of Acoustic Mode Patterns in KDP Crystals", Applied Optics Vol. 4, Issue 12, pp. 1655-1659 (1965)

[290] https://ace.phys.virginia.edu:80/Lab26/866

[291] https://ace.phys.virginia.edu:80/Lab26/868

[292] http://www.edn.com/electronics-blogs/bogatin-s-rules-ofthumb/4424573/Rule-of-Thumb-1-The-bandwidth-of-a-signal-from-itsrise-time

[293] https://ace.phys.virginia.edu:80/Lab26/793 
[294] Simeon J. Krumbein, "Metallic Electromigration phenomena", Technical paper Presented at the 33rd Meeting of the IEEE Holm Conference on Electrical Contacts, Chicago, Illinois September 21-23, 1987.

[295] David J. Griffiths, Introduction to Electrodynamics. Upper Saddle River, N.J.: Prentice Hall, 1999.

[296] https://ace.phys.virginia.edu:80/Lab26/734

[297] https://ace.phys.virginia.edu:80/Lab26/779

[298] L. J. Kaufman, Precision Measurement of the Proton Neutral Weak Form Factors at $Q^{2} \sim 0.1 \mathrm{GeV}^{2}$, Ph.D. Thesis, University of Massachusetts Amherst, (2007).

[299] https://ace.phys.virginia.edu:80/Lab26/740https://ace.phys.virginia.edu:80/Lab26/740ht

[300] https://ace.phys.virginia.edu:80/Lab26/762

[301] https://ace.phys.virginia.edu:80/Lab26/838

[302] https://ace.phys.virginia.edu:80/MOLLER/2

[303] E. Kargiantoulakis, "A Precision Test of the Standard Model via ParityViolating Electron Scattering in the Qweak Experiment", Ph.D. Thesis, University of Virginia (2015), pg. 92.

[304] C. Palatchi, Lab book, 09/2017, pg. 65

[305] J. Benesch, verbal communication (10/12017)

[306] - Paul A. Souder and Kent Paschke, Beam Spot Size Sensitivity, August 27, 2008 .

[307] https://ace.phys.virginia.edu:80/Lab26/304

[308] http://www.unitedcrystals.com/KDPProp.html 
[309] http://www.cristal-laser.com/UserFiles/File/brochures-techniques/rtp.pdf

[310] https://en.wikipedia.org/wiki/Birefringence

[311] D.W. Higinbotham, T. Keppel, 2017 Version: Jefferson Lab Hall A Standard Equipment Manual, November 2017.

[312] Chris Mueller, "The Electro-Optic Effect Reading Notes", Internal document, University of Florida, 27 March, 2010.

[313] http://www.fastpulse.com/pdf/1040.pdf

[314] https://ace.phys.virginia.edu:80/Lab26/414

[315] https://ace.phys.virginia.edu:80/Lab26/339

[316] R. Silwal, Probing the Strangeness Content of the Proton and the Neutron Radius of 208Pb using Parity-Violating Electron Scattering, Ph. D. Thesis, University of Virginia (2012), pg. 124.

[317] Moshe Shapira, Raicol, Private Communication, July 17, 2015.

[318] POLOG 3482381

[319] POLOG 3482511

[320] Lab26 ELOG 749

[321] Lab26 ELOG 828

[322] Lab26 ELOG 849

[323] Lab26 ELOG 826

[324] Lab26 ELOG 474, Run 2344

[325] Lab26 ELOG 499 
[326] N. Mavalvala et al, Lasers and optics: looking towards third generation gravitational wave detectors, Gen. Relativ. Gravit., Volume 43, 2011, Pages 569-592.

[327] http://www.redoptronics.com/RTP-crystal.html 2/20/2018

[328] Cristal Laser, Private Communication, Feb 20, 2018

[329] POLOG 3482236

[330] POLOG 3470277

[331] G. Mueller et al, "Method for compensation of thermally induced modal distortions in the input optical components of gravitational wave interferometers", Class. Quantum Grav., Volume 19, 2002, 1793.

[332] T. Toyoda, M. Yabe, The temperature dependence of the refractive indices of fused silica and crystal quartz, J. Phys. D: Appl. Phys., Volume 16, 1983, L97-L100.

[333] R. Silwal, Probing the Strangeness Content of the Proton and the Neutron Radius of 208Pb using Parity-Violating Electron Scattering, Ph. D. Thesis, University of Virginia (2012), pg. 61.

[334] R. Silwal, Probing the Strangeness Content of the Proton and the Neutron Radius of 208Pb using Parity-Violating Electron Scattering, Ph. D. Thesis, University of Virginia (2012). pg. 119.

[335] R. Silwal, Probing the Strangeness Content of the Proton and the Neutron Radius of 208Pb using Parity-Violating Electron Scattering, Ph. D. Thesis, University of Virginia (2012). pg. 314.

[336] R. Silwal, Probing the Strangeness Content of the Proton and the Neutron Radius of 208Pb using Parity-Violating Electron Scattering, Ph. D. Thesis, University of Virginia (2012). pg.160. 
[337] R. Beminiwattha, "A Measurement of the Weak Charge of the Proton through Parity Violating Electron Scattering using the Qweak Apparatus", Ph. D. Thesis, Ohio University (2013).

[338] E. Kargiantoulakis, A Precision Test of the Standard Model via ParityViolating Electron Scattering in the Qweak Experiment, Ph. D. Thesis, University of Virginia (2015), pg. 94.

[339] E. Kargiantoulakis, A Precision Test of the Standard Model via ParityViolating Electron Scattering in the Qweak Experiment, Ph. D. Thesis, University of Virginia (2015), pg. 77. 